\title{
The Crosswell Electromagnetic Response of Layered Media
}

\author{
Maria Deszcz-Pan \\ Ph.D. Thesis \\ Department of Materials Science and Mineral Engineering \\ University of California \\ and \\ Earth Sciences Division \\ Lawrence Berkeley Laboratory \\ University of California \\ Berkeley, CA 94720
}

April 1994

This work was supported, in part, by the Director, Office of Energy Research, Office of Basic Energy Sciences, Geosciences Program, of the U.S. Department of Energy under Contract No. DE-AC03-76SF00098.

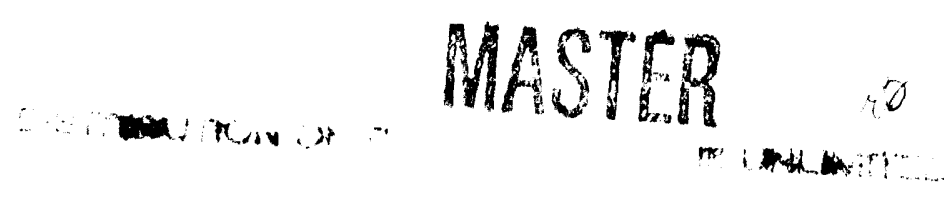




\section{DISCLAIMER}

This report was prepared as an account of work sponsored by an agency of the United States Government. Neither the United States Government nor any agency Thereof, nor any of their employees, makes any warranty, express or implied, or assumes any legal liability or responsibility for the accuracy, completeness, or usefulness of any information, apparatus, product, or process disclosed, or represents that its use would not infringe privately owned rights. Reference herein to any specific commercial product, process, or service by trade name, trademark, manufacturer, or otherwise does not necessarily constitute or imply its endorsement, recommendation, or favoring by the United States Government or any agency thereof. The views and opinions of authors expressed herein do not necessarily state or reflect those of the United States Government or any agency thereof. 


\section{DISCLAIMER}

Portions of this document may be illegible in electronic image products. Images are produced from the best available original document. 


\title{
THE CROSSWELL ELECTROMAGNETIC RESPONSE OF LAYERED MEDIA
}

\author{
Maria Deszcz-Pan
}

\begin{abstract}
Crosswell electromagnetic measurements are a promising new geophysical technique for mapping subsurface electrical conductivity. Because the conductivity of sedimentary rocks depends on the conductivity of the fluid that fills the rock pores, the variations in conductivity can provide information about the subsurface distribution of water, oil or steam.

In this work the fields from a low frequency vertical magnetic dipole have been examined from the specific point of view of their application to the determination of the conductivity of a layered medium. Since it was established that the sensitivity of the fields to the conductivity distribution is higher when the source and the receiver are located inside the medium they were placed inside two separate boreholes.

The range of penetration of such a crosswell system for typical earth resistivities and for currently available transmitter and receiver technologies was found to be up to 1000 meters so problems in ground water and petroleum reservoir characteristics can be practically examined. The parameters that affect the conductivity estimates have been assessed using a whole-space approximation to simulate the conditions around the boreholes. It was established that the sensitivity of the fields to the variations in conductivity depends on the transmitter-receiver geometry and that the regions where fields change sign or vary rapidly with distance are subject to large errors caused by the possible misplacement of the transmitter with respect to the receiver.

An analysis of the behavior of the magnetic fields at the boundary between two half-spaces showed that the horizontal magnetic field component, $\mathrm{H}_{\mathrm{p}}$, and the vertical derivative of a vertical component, $\delta \mathrm{H}_{\mathrm{z}} / \delta \mathrm{z}$, are more sensitive to conductivity variations than $\mathrm{H}_{\mathrm{z}}$. The analysis of derivatives led to the concept of measuring the conductivity directly using a second vertical derivative of $\mathrm{H}_{\mathrm{z}}$. Conductivity profiles interpreted from field data using this technique reproduced accurately the electrical logs for a test site near Devine, Texas.
\end{abstract}


Inversion of crosswell data using least squares techniques for multilayered models showed a high sensitivity of crosswell measurements to the layer parameters. An analysis of error patterns between the field and numerical data revealed the possibility of using the patterns as an aid in detecting problems with the measuring system such as calibration errors or position errors between transmitter and receiver. Inversion results also underscored the fact that the repeatability of the measurements within a given error margin does not necessarily guarantee that the accuracy of the whole experiment satisfies the same limits.

It was found in this study that the inversion techniques are more stable when the first vertical derivative of $\mathrm{H}_{\mathrm{z}}$ is used rather than $\mathrm{H}_{\mathrm{z}}$ itself. Using data from a salt water injection experiment at the Richmond Field test site in Berkeley it was also found that these robust layer inversions were successful in identifying the preferential flow direction of the injected brine to four boreholes surrounding the injection well.

$$
\text { NJM/oman } 11 / 21 / 93
$$


This thesis is dedicated to parents:

Hwei-Chen and In-Chang Pan

and

Jozefa and Jan Deszcz 


\section{Table of contents}

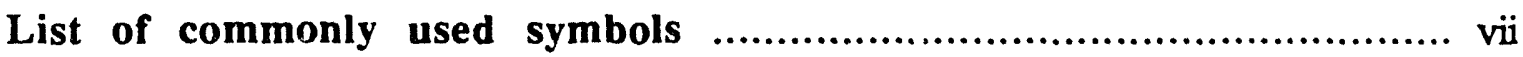

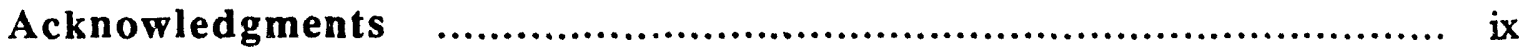

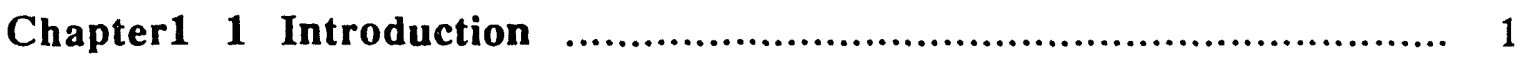

Introduction $\quad$................................................................. 1

1.1 Range of conductivity variations ................................... 1

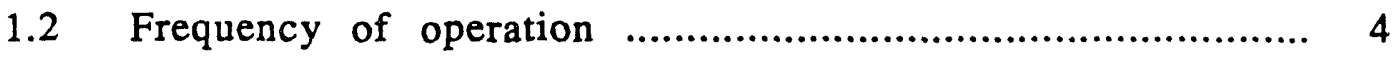

1.3 Sources of electromagnetic noise inside boreholes...................... 5

1.4 Borehole systems ................................................. 5

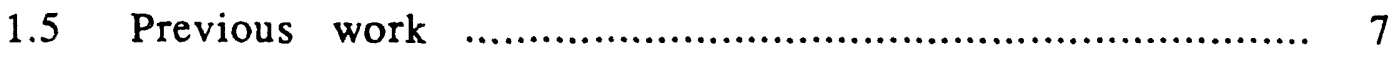

1.6 Objective of this work............................................ 11

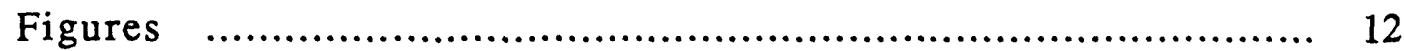

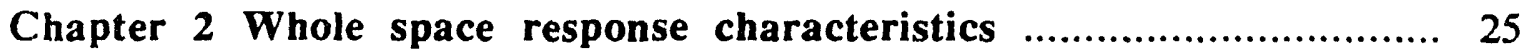

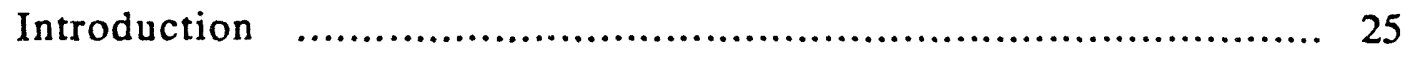

2.1 Vertical magnetic dipole fields ........................................ 25

2.2 Sensitivity of $\mathrm{H}_{2}$ and $\mathrm{H}_{\mathrm{p}}$ to the conductivity variations in a whole

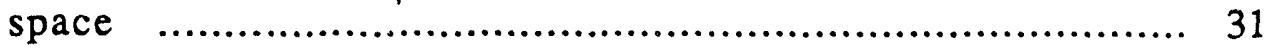

2.3 Sensitivity of $\mathrm{H}_{2}$ and $\mathrm{H}_{\rho}$ measurements to position errors ............... 32

2.4 Conditions for simplified relationships between magnetic field

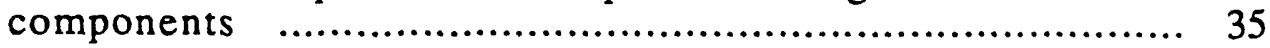

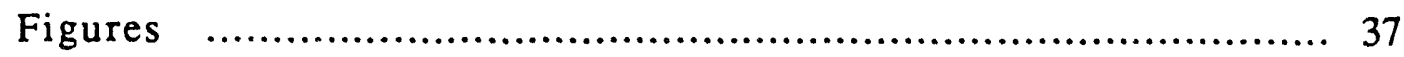

Chapter 3 Characteristics of the response near the boundary .............. 62

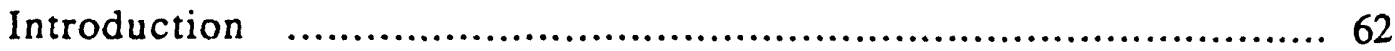

3.1 Transmitter and receiver on the interface ............................. 62

3.1.1 $\mathrm{H}_{\mathrm{z}}$ component .............................................. 64

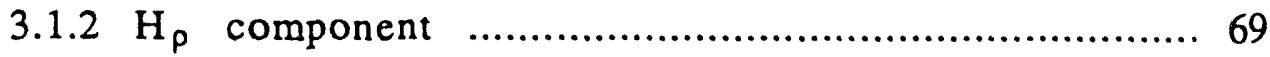

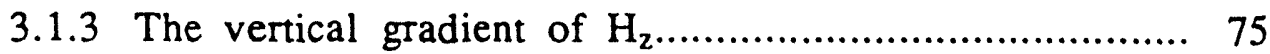

3.1.4 Evaluation of $\delta^{2} \mathrm{H}_{2} / \delta z^{2}$ on the interface ....................... 77

3.2 Properties of the solution when transmitter and receiver are both above the interface............................................................. 79

3.2.1 Asymptotic expressions when transmitter and receiver are both above the interface valid for $k \rho \gg 1$.......................... 81

3.2.2 Asymptotic expressions when transmitter and receiver approach boundary at the same height ........................... 82

3.3 Expressions when transmitter is above and receiver is below the interface 
3.3.1 Asymptotic expressions when transmitter is above and receiver is below the interface ........................................... 87

3.4 Characteristics of the response near a layer ............................. 88

3.4.1 Transmitter located outside layer ................................ 88

3.4.2 Transmitter located inside layer .................................. 90

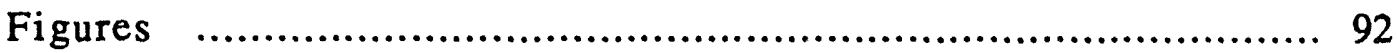

Chapter 4 Direct methods to obtain apparent conductivity profiles in a one-dimensional medium ................................. 97

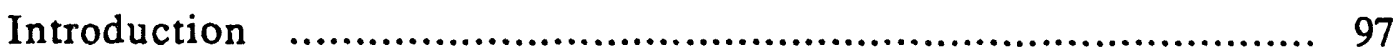

4.1 Surface to borehole conductivity profiles ................................ 97

4.2 Crosshole conductivity profiles ........................................ 102

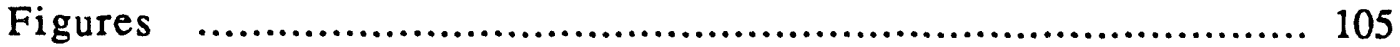

Chapter 5 One-dimensional least squares inversion of field data .......... 119

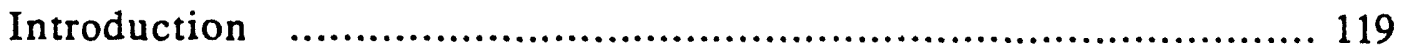

5.1 NLSEM1D inversion program.................................... 119

5.2 Least-squares inversion of model tank data ............................ 120

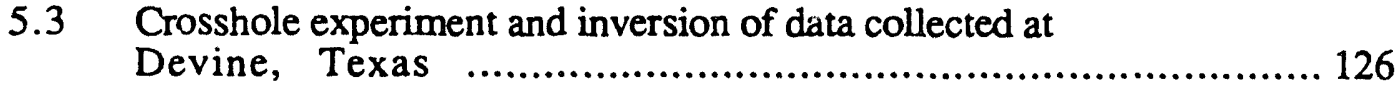

5.3.1 Inversion of data from upper level survey between 213.66 and $335.28 \mathrm{~m}$ (700 and $1100 \mathrm{ft}$ ) ...................... 128

5.3.1 Inversion of data from lower level survey between

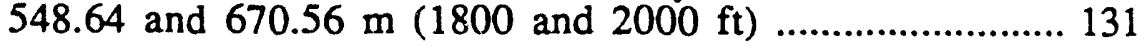

5.3.3 One-dimensional inversion using the first vertical derivative

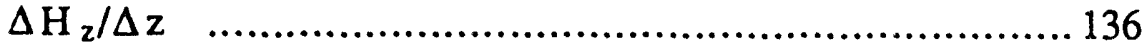

5.4 The Richmond Field Station experiment .................................. 138

5.4.1 One-dimensional inversion of data collected in Richmond,

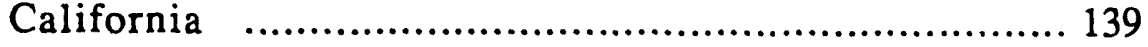

$5.5 \quad$ Summary.................................................... 142

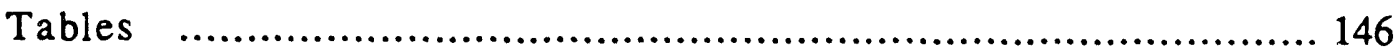

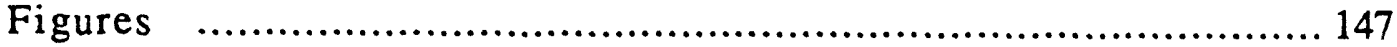

Chapter 6 Conclusions and recomendations for future research ............ 200

6.1 Conclusions ..................................................... 200

6.2 Recomendations for future research ................................. 202

References $\quad$................................................................ 203

Appendix A .Mathematical derivations.......................................... 210

A.1 General solution ................................................ 210

A.2 Whole space ................................................... 213

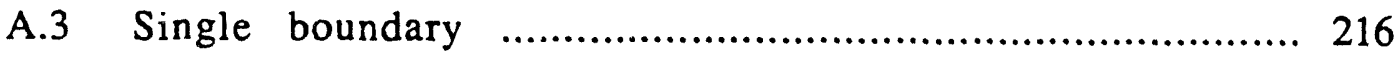


A.3-1 Exact expressions when transmitter and receiver are both above the interface

A.3-2 Exact integral expressions when transmitter is above and receiver isbelow the interface

A.4 Exact expressions when transmitter is below the layer .................. 220

A.5 Exact expressions when transmitter is inside the layer ................... 223

Appendix $B$

B.1 Evaluation of the integrals by the method of steepest descent

Appendix C

C. 1 Summary of the relationships used in the text

230

C. 2 Integrals that can be reduced to whole space fields 231

C.3a Explicit expressions for whole space field $\mathrm{H}_{\mathrm{z}}$

C.3b Explicit expressions for whole space field $\mathrm{H}_{\rho}$ 233

C.4 Explicit expressions for higher order vertical derivatives of $e^{-i k s R} / R \quad$... 234 


\section{List of commonly used symbols}

$\Delta \mathrm{H}_{2} / \Delta \mathrm{z}, \Delta \mathrm{H}_{\mathrm{z}} / \Delta \rho \quad$ finite difference first vertical and horizontal derivatives

$\delta \mathrm{H}_{\mathcal{z}} / \delta \mathrm{z}, \delta \mathrm{H}_{\mathcal{z}} / \delta \rho \quad$ partial vertical and horizontal derivatives

f

frequency

$\mathrm{H}_{\mathrm{z}}(\mathrm{k}, \mathrm{R}), \mathrm{H}_{\rho}(\mathrm{k}, \mathrm{R}) \quad$ whole space $\mathrm{H}_{\mathrm{z}}$ and $\mathrm{H}_{\mathrm{\rho}}$ components in the medium

with propagation constant $\mathrm{k}$ and $\mathrm{R}=\left(\rho^{2}+z^{2}\right)^{1 / 2}$

$\mathrm{H}_{\mathrm{z}}, \mathrm{H}_{\mathrm{\rho}}$

$\mathrm{H}_{\mathrm{z}}$ and $\mathrm{H}_{\rho}$ componets in a medium (not restricted to the whole space)

$\mathrm{H}_{0}^{(2)}(\lambda \rho), \mathrm{H}_{0}^{(1)}(\lambda \rho)$

Hankel functions of zero order

$I_{v}(z), K_{v}(z)$

modified Bessel functions of the first and second kind of complex

$\mathrm{J}_{0}(\lambda \rho), \mathrm{J}_{1}(\lambda \rho)$ arguments

$\mathrm{k}=\sqrt{\omega^{2} \mu \varepsilon-\mathrm{i} \omega \mu \sigma}$

Bessel functions of zero and first order

$k_{s}=\sqrt{\omega^{2} \mu \varepsilon-i \omega \mu \sigma_{s}}$

propagation constant of the general medium

$k_{s-1}=\sqrt{\omega^{2} \mu \varepsilon-i \omega \mu \sigma_{s-1}}$

propagation constant of the medium with source

$k_{s+1}=\sqrt{\omega^{2} \mu \varepsilon-i \omega \mu \sigma_{s+1}}$

propagation constant of the layer above the source

$\mathrm{M}$

propagation constant of the layer below the source

magnetic dipole moment

$R=\left(\rho^{2}+z^{2}\right)^{1 / 2}$

total distance between the transmitter and the reciever

$R_{1}=\left(\rho^{2}+z_{1}^{2}\right)^{1 / 2}$

total distance between the receiver and the "image" source

$\mathbf{z}$

$z_{s}$

$\mathbf{z}_{\mathrm{s}+1}$

$z_{s-1}$

$z_{1}$

$\Delta$

$\varepsilon$

$\gamma_{\mathrm{s}}=\sqrt{\mathrm{k}_{\mathrm{s}}^{2}-\lambda^{2}}$

$\gamma_{s+1}=\sqrt{k_{s+1}^{2}-\lambda^{2}}$

$\gamma_{s-1}=\sqrt{\mathrm{k}_{s-1}^{2}-\lambda^{2}}$

$\Pi_{z}^{*}$

$\lambda$

$\mu$

$\pi$

$\rho$ - receiver depth

source depth

depth of the first boundary below the source

depth of the first boundary above the source

vertical distance between the receiver and the "image" source

finite difference

dielectric constant

propagation constant in the medium with the source

propagation constant in the first layer below the source

propagation constant in the first layer above the source

z-component of the magnetic Hertz vector, Hertz potential

Hankel transform wavenumber

magnetic permeability

$\mathrm{Pi}=3.14$

horizontal transmitter-receiver separation 
$\sigma_{\mathrm{s}}$

$\sigma_{\mathrm{s}+1}$

$\sigma_{\mathrm{s}-1}$

$\left(\sigma_{s} f\right)^{1 / 2} \rho$

$\left(\sigma_{s+1} f\right)^{1 / 2} \rho$

$\left(\omega \mu \sigma_{s+1}\right)^{1 / 2} \rho$

$\left(\sigma_{\mathrm{s}} \mathrm{f}\right)^{1 / 2} \mathrm{z}$

$\omega$ electrical conductivity of the medium with a source

electrical conductivity of the first layer below the source

electrical conductivity of the first layer above the source

horizontal induction parameter in the medium with the source

horizontal induction parameter in the medium below the source

horizontal induction number in the medium below the source

vertical induction parameter in the medium with the source

radial frequency equal to $2 \pi f$ 


\section{Acknowledgments}

This certainly was a long stay in Berkeley. It would not be possible without the infinite patience of professor H. Frank Morrison - only he knows how much of his enthusiasm and ideas were poured on me during this time, only to be trickled to the drizzle and eventually evaporated without a trace. I will never forget his support that lasted for so many years. Professor Alex Becker was invaluable in keeping me on the ground with his cold but necessary showers. Over the years I have enjoyed his sense of humor, and respected his judgment.

My warmest thanks to my friends and mentors: Ki Ha Lee and Mike Wilt, for their support that went beyond all limits. For their wisdom and scientific ideas that they shared with me, and the gentle (too soft) pushes that made it possible to finish this work.

During my stay in Berkeley I enjoyed the friendship of many people that made life easier and a place where you found support and understanding. My friends from Engineering Geoscience: I owe you !!! Thank you Dave Alumbaugh, Ted Asch, Guan Cheng, Ricardo Fernandez, Mike Hoversten, Dave Kennedy, Vic Labson, Nancy Nadel, Ed Nichols, Mark Meadows, Ed Moseley, Francesca Quercia, Cliff Schenkel, Carlos Torres-Verdin, Hung Wen (Ocean) Tseng, Qiang Zhou.

I am also grateful to professor Shimon Coen who was a great inspiration at the beginning of my Berkeley experience, Jim Doheny for his help during the field experiments, and Rich Hardman for checking the equations.

Outside Engineering Geoscience I was blessed by friends like Renee Romero, Kinga Potocki and Grazyna Odyniec; thank you for patting, praying and poking (respectively!).

And at the end, but not the least: my Family. This work would have never been finished if not for the unyielding support of my parents in-law who spend many months taking care of every aspect of our daily life so I could complete this work. My parents and my sisters have been with me all these years and their love and support gave me strength and courage.

These years were the hardest for my husband - Chun Pan, who had to spend countless lonely nights and weekends, this work is his as much as mine. I am very lucky to have somebody like him. For all his support, understanding, and love I can only say thank you.

I am grateful for the financial support I received during the years to the department of MSME, LBL and LLNL. The work was also supported by an industrial 
research consortium that includes MOBI, SCHLUMBERGER, SHELL, NORANDA, EXXON, NITETSU MINING CORPORATION, BRITISH PETROLEUM, TEXACO and AMOCO, and by the Director, Office of Energy Research, Office of Basic Energy Sciences, Geosciences Program, under contract no. DE-AC03-76SF00098. 


\section{Chapter 1}

\section{Introduction}

The use of low frequency electromagnetic (EM) fields to determine electrical conductivity between boreholes is an emerging new technique in geophysics. Its development has been stimulated by recent interest in the monitoring of hazardous waste plumes and remobilized petroleum. These applications demand an accurate estimate of conductivity over distances of several hundred meters. The use of high resolution induction logs is not suitable for this purpose because of the associated short range of penetration which extends at most few meters into the medium around the borehole. While low frequency electromagnetic measurements on the surface of the earth have been used in geophysics for many decades to locate conductive targets, they lack the resolution required for monitoring of small conductivity changes in the medium. By lowering the transmitter or receiver, or both, down the borehole, the sensors come closer to the target and at the same time further away from distorting surface effects and noise, so a greater sensitivity to the conductivity and structure of the medium is obtained.

The objective of this work is to understand the behavior and characteristics of EM fields in the ground, and how measurements in boreholes differ from surface measurements in providing information about the conductivity of a one-dimensional, lav'red medium. Here, the layered medium response is important, because it determines the strength of the signal that should be used in a particular experiment. Estimates of the layer response that has to be removed from data used in EM tomography to image inhomogeneities are equally important. Layer conductivities derived from cross-hole measurements correspond to the bulk conductivity of the medium that is not affected by the presence of the drill-fluid and the nonuniform walls of the borehole.

\subsection{Range of conductivity variations}

Electromagnetic methods in geophysics have been used for many decades to determine the electrical conductivity distribution in the earth. The conductivity can be related to physical parameters of the medium and thus provide information about the processes that occur below the surface of the earth. For example by monitoring the changes in conductivity with time it is possible to trace the movement of conductive fluids (Asch and Morrison, 1989; Bevc and Morrison, 1991, Wilt et al., 1992), steam fronts in the earth (Ranganayaki et al., 1992; Wilt and Schenkel, 1992), or combustion zones 
(Bartel and Ranganayaki, 1989), because these processes are known to locally alter the ambient conductivity values.

The relationship between the physical properties of rocks and the conductivity has been a subject of studies for many years. It was shown that although the materials that build rocks can vary by several orders of magnitude in conductivity (Parkhomenko, 1967), the rock matrix does not play a significant role in conductivity if the rock is porous and filled with fluid. In general, in sedimentary, porous rocks, where the current conduction is mostly electrolytic, the conductivity depends on pore fluid conductivity, porosity, saturation, temperature, pressure, pore geometry, wettability, and clay content (Ward and Fraser, 1967). Inside clear, fully saturated sands widely used Archie's Law (Archie, 1942) relates the overall conductivity of the medium, $\sigma_{\mathrm{f}}$, to the conductivity of the pore fluid, $\sigma_{\mathrm{p}}$, and porosity, $\phi$, via:

$$
\sigma_{\mathrm{f}}=\sigma_{\mathrm{p}} \phi^{\mathrm{n}}
$$

The exponent $\mathrm{n}$ and can take values between 1.8 and 2.2 depending on the rock matrix.

If the clay particles are present inside the rock matrix then the process of conductivity complicates significantly because of the presence of exchange cations on the boundary between clay and water (Ward and Fraser, 1967). The conductive properties of clay-contaminated shaly sands were described by Waxman-Smits model (1968) which assumed that the conductance of the shaly sand formations can by represented by two resistors in parallel: one resistor representing the matrix, the other the fluid filling the pores. Bussian (1983) proposed a model that relates the formation conductivity to the matrix and fluid conductivities at any frequency. Sen and Goode (1988) developed the mathematical model of conductivity in shaly sands for low and high water salinity.

Recently temperature dependence of rock conductivity has been extensively studied in connection with the monitoring of the enhanced oil recovery processes and with the application to geothermal exploration. It has been established that at low temperatures up to about 200 degrees the resistivities of rocks and saturating fluid have similar temperature dependence: the increase in conductivity with increasing temperature up to about $200^{\circ} \mathrm{C}$ (Llera et al., 1990). This is caused by an increased mobility of ions with the increase of temperature (Sen and Goode, 1992). Above $200^{\circ} \mathrm{C}$ the chemical reactions and development of microcracks become more important (Llera et al. 1990). 
The significance of pore fluid conductivity in overall conductivity is an important factor in determining the success of electromagnetic methods in monitoring movements of fluid. Because the conductivity of a pore fluid can vary over several orders of magnitude the conductivity of a formation will also vary significantly. For example, Hearst and Nelson (1985) show that the conductivity of water alone can vary by seven orders of magnitude (Figure 1.1-1) between resistive pure water and a conductive brine. Therefore, according to Archie's Law the overall conductivity of high porosity $(\phi=.3)$ sandstone can also vary by seven orders of magnitude depending on the salinity of pore water. By monitoring the variation in conductivity with time it may be possible to map the movement of the boundary between a fresh water and brine.

Similarly, it may be possible to monitor the movement of oil-water boundary in the water-flooding process. In this case the contrast between water and oil saturated rock is expected to reach four orders of magnitude as suggested by laboratory measurements of the conductivity of oil-shale rocks by Duba (1982). His results present the variation of conductivity with temperature and oil-water saturation (Figure 1.1.2). At room temperature the three samples had widely varying conductivities that depended on their oil-water saturation: from $10^{-2} \mathrm{~S} / \mathrm{m}$ for sample with oil saturation of $3 \mathrm{gal} / \mathrm{ton}$ to $10^{-6} \mathrm{~S} / \mathrm{m}$ for sample with $56 \mathrm{gal} / \mathrm{ton}$ oil saturation.

In an enhanced oil recovery process the monitoring of the steam front is of crucial importance. The pumping of steam into an oil bearing formation is a complicated, not well understood process. But a recent studies (Mansure, 1990; Spies and Greaves, 1991), indicate that the conductivity of the steaming zone can increase by a factor between 5 to 8 from its presteaming values. This increase is caused by the fact that, although the steam itself is resistive, only between of $60 \%$ to $80 \%$ of the steam actually enters the medium. The rest is distributed as a hot water. The high temperature significantly increases the solubility of minerals and as a result increases the conductivity of the water as it passes through rocks. Mansure and Meldau (1990) estimated this change in conductivity using the Archie's law. They considered the magnitude of the change in the resistivity of a formation caused by the change in salinity (Figure 1.1-3a), temperature (Figure 1.1-3b) and saturation (Figure 1.1-3c). As can be observed salinity variations have the largest effect on the variations in resistivity, followed by the effect of change in saturation. The change in temperature had the smallest effect, but nevertheless is very significant since it can cause a factor of five increase of resistivity during the steam flooding process. 
The conductivity variations caused by the change in the porosity, saturation and temperature are significantly larger than the similar variations in velocity. This can be observed in Figure 1.1-4 that compares the two quantities for the case of an unconsolidated sandstone. Figure is based on the Schlumberger logging charts and shows that the use of electrical methods in the monitoring of the processes that involve the changes in the pore fluid properties might be better than the use of the seismic methods.

\subsection{Frequency of operation}

Traditional electromagnetic measurements inside boreholes are performed with an induction logging tool that employs high frequencies $(10-100 \mathrm{~Hz})$ and short transmitterreceiver separations (1-10 m) which limits the depth of investigation to several meters around the borehole. If the region of interest extends from several meters to several hundred meters as in monitoring of contaminants, or enhanced oil recovery processes, then an increase in transmitter-receiver separation and use of lower frequencies is necessary. The frequency of operation is determined by the desired depth of penetration, source strength and the sensitivity of the receiver. Figure 1.2-1 shows how the conductivity and frequency are related to the depth of penetration that is given in terms of skin depth, $\delta$ :

$$
\delta=\sqrt{\frac{2}{\sigma \omega \mu}} \approx \frac{500}{\sqrt{\sigma f}}
$$

The dashed line shows the boundary between regions where displacement or conductive currents dominate. In regions where conductive currents dominate, i.e. where,

$$
\omega \epsilon \ll \sigma
$$

the diffusion process is more important than wave propagation. Because the most typical conductivities of the sedimentary rocks vary between $1.0 \mathrm{~S} / \mathrm{m}$ and $0.001 \mathrm{~S} / \mathrm{m}$, and the distance of penetration we are interested in is between $5.0 \mathrm{~m}$ and $1000.0 \mathrm{~m}$, most of our work will deal with relatively low frequencies between $1 \mathrm{~Hz}$ and $100 \mathrm{~Hz}$. In this region the conduction currents dominate the displacement currents. 


\subsection{Sources of electromagnetic noise inside boreholes}

The electromagnetic fields that exist inside boreholes, not created by a given controlled source, are considered to be electromagnetic noise. There are numerous manmade sources of electromagnetic noise, but the sources of natural EM fields in the $1.0 \mathrm{~Hz}$ to $100.0 \mathrm{~Hz}$ band are lightning discharges in the earth ionosphere cavity.

An example of the spectrum of natural horizontal, $\mathrm{H}_{\mathrm{x}}$ component field (on the surface of the earth), measured by Labson (1985) is given in Figure 1.3-1. Figure 1.3-2 shows how this noise in the $\mathrm{H}_{\mathrm{x}}$ field will diminish inside a conductive half-space. This figure is based on the assumption that the fields attenuate exponentially as they propagate through a conductive medium according to the relation:

$$
S_{z}(\omega)=e^{-z / \delta} S_{\text {surfacd }}(\omega)
$$

where $S_{z}(\omega)$ is a spectral density of magnetic field measured at depth $z, S_{\text {surface }}(\omega)$ is a spectral density of magnetic field measured at a surface and $\delta$ is the skin depth defined above. The curve parameter is $\sqrt{\sigma} \mathrm{z}$. Inside boreholes the vertical component of magnetic field is usually measured, and since the vertical component $\mathrm{H}_{z}$ of natural electromagnetic fields is zero in a one-dimensional medium (Vozoff, 1972), then there is no natural noise in borehole measurements of $\mathrm{H}_{\mathbf{z}}$.

The man-made noise depends strongly on the location and has to be estimated individually for each particular site. This noise is centered around $60 \mathrm{~Hz}$ and its harmonics. Seunghee Lee (personal communication) measured this noise in one of the worst possible cases inside an active oil producing field, with a high concentration of conductive pipes, oil pumps and during the steam flooding operation. His measurements of $\mathrm{H}_{z}$ component at $60 \mathrm{~Hz}$ were at a level of $0.02 \mathrm{nT} /(\mathrm{Hz})^{1 / 2}$ at depth of $90.0 \mathrm{~m}$. Our own measurements in Richmond Field Station in May of 1992, indicate that the noise level at a depth of $5.0 \mathrm{~m}$ and at frequency $18500 \mathrm{~Hz}$ is around $0.001 \mathrm{nT} /(\mathrm{Hz})^{1 / 2}$.

\subsection{Borehole systems}

In traditional surface systems the transmitter and receiver are located at the same level and the frequency of operation or the transmitter-receiver separation is varied. With the availability of the boreholes, one more dimension is open for exploration and this creates new options for different transmitter-receiver combinations.

Different combinations of transmitter and receiver positions are sensitive to different features of the medium. To illustrate some typical responses and different 
configurations we considered how different systems respond to the same model. The examples show typical curves obtained by the traditional, well proven techniques: measurements on the surface of the earth and induction logs in boreholes. For simplicity we presented only the $\mathrm{H}_{\mathrm{z}}$ quadrature response of each system. The model chosen is a 30 $\mathrm{m}$ thick conductive layer located at depth of $300 \mathrm{~m}$ inside a more resistive half space. The conductivity of the layer is $0.1 \mathrm{~S} / \mathrm{m}$, and the conductivity of half space is $0.001 \mathrm{~S} / \mathrm{m}$. The frequency used is $1000 \mathrm{~Hz}$.

Figure 1.4-1 presents the simulation of the surface EM techniques: the geometric sounding where the separation between the transmitter and receiver increases and the frequency is kept constant. The transmitter and receiver are both located on the surface. The two curves represent the quadrature $\mathrm{H}_{\mathrm{z}}$ response with and without a layer for comparison. The field strength is given on vertical axis in nT. The solid black line shows the half-space response without layer, the gray line shows the response with a layer. To make comparison easier, all values of $\mathrm{H}_{\mathrm{z}}$ quadrature are presented on a logarithmic scale (except in Figure 1.4-6) that spans seven orders of magnitude.

Figure 1.4-2 shows a typical induction logging response where the transmitter and receiver are moving together vertically along the borehole. The spacing between the transmitter and receiver is $2 \mathrm{~m}$ and is much shorter than the layer thickness. In this case it is possible to recover the conductivity of the layer directly from the measurements, and the thickness of the layer can be correctly estimated.

If the transmitter is kept at the surface and coaxial with the borehole and the receiver is allowed to move inside the borehole then one simulates surface-to-borehole electromagnetic logging, and as the transmitter-receiver separation is increased, a larger area around the borehole is examined. The typical response in this case is shown in Figure 1.4-3.

Another version of surface to borehole EM is obtained if transmitter is located on the surface and the receiver moves down the hole off the axis of the transmitter (Fig. 1.44). This system can be useful if only one borehole is available or when a strong magnetic source is needed. Such a strong source can only be provided with a large loop of current carrying wire laying on the surface of the earth.

By separating the transmitter and receiver horizontally and putting them in two different boreholes a new version of the technique is created: crosshole EM. An example of the response when the transmitter and receiver are located at the sarne depth and move simultaneously down two boreholes is given in Figure 1.4-5. 
Finally the most complete se of data in the crosshole measurements is the socalled tomographic survey. In this method the transmitter is placed in one borehole and the receiver moves along a profile in the second borehole. Then the transmitter is moved to another position in the first borehole and the receiver travels along the same profile. The process is repeated until all required combinations of transmitter-receiver positions are covered. Since tomographic surveys involve a large number of data sets, a new form of data presentation is given in Figure 1.4.6. In this form the position of the transmitter coil is on the horizontal axis and the vertical axis represents the receiver location.

As can be observed from the above figures, there is a significant change in response for all the systems considered, for models with and without a layer in. This is also true for the measurements on the surface and that may suggest that there is no significant advantage for measurements in boreholes. Measurements on the surface however can only resolve the conductivity-thickness product of the layer, while measurements in boreholes can provide an exact location of boundaries. Measurements of the second vertical derivative (Chapter 3 ) can be used for this purpose.

\subsection{Previous work}

There are few reports of surface-to-borehole or crosshole electromagnetic measurements at low frequencies and large transmitter-receiver separations. Since 1989 measurements have been made in boreholes at several sites by a National Laboratories and Engineering Geoscience team. The measurements were performed with different field configurations and in various geologic settings. The interpretation of some of these measurements is included in Chapters 5 and 6. Prior to these experiments the only large scale borehole measurements were conducted in mineral exploration primarily to detect conductors that were missed by the borehole. An early review of these methods was given by Dyck (1975). Model studies in the frequency domain measurements in boreholes with a surface transmitter (Hohmann et al., 1978) confirmed, that downhole receivers are very sensitive to the deep conductors that could not be detected from the surface. A variation of the method with a downhole electrode as the source and a surface magnetic field receiver also showed good diagnostic possibilities.

Since then the use of downhole receivers and surface transmitters has become a standard procedure in mining geophysics to detect highly conductive sulphides in a relatively resistive medium that were missed by a drillhole. Work has been conducted especially by Australian and Canadian researchers in time-domain with a large surface loop as a source. The results of the research in Australia were summarized in a 
published special issue of Exploration Geophysics, (1987) dedicated to downhole methods. This review shows that the work emphasizes on the search for sulphides in a typical Australian geological conditions: highly resistive medium with a conductive overburden. The field experiments proved that downhole receivers help to detect and localize conductive zones otherwise undetectable from the surface. This conclusion was reached by Raiche and Bennett, (1987) who considered the response of the layered earth model. By comparing the responses of two models with and without the conductive layer, they showed that the downhole receiver significantly enhanced the detection of the conductive layer in comparison with the surface receiver. However the same calculations for the resistive layer showed that resistive layer can be a difficult target even with a downhole receiver.

A variation of the downhole measurements in which the receiver was fixed and transmitter was moved along the surface of the earth was considered by Coggon and Clarke (1987). The method was used to detect a finite conductor which had a dipolar type magnetic field anomaly which could be interpreted with simple loop model.

Dyck and West (1984) describe several field measurements with the wide-band time domain systems. Their results show that it is possible to detect large bodies that are off the axis of the borehole and interpret them using simple models based on current filaments whose location provides informatior on the shape of the conductor. They also suggest introducing asymmetry to the problem (for example by moving the transmitter loop off the axis of the borehole) to resolve the ambiguity in the location of the conductor and to use the multicomponent receiver.

The problems encountered in the interpretation of downhole time domain measurements were examined by Macnae and Staltari, (1987). They describe different causes of the sign reversal of the recorded signal along the downhole profile. Eaton and Hohmann (1984) worked in the time domain and showed that the conductive overburden delays and attenuates the amplitudes of the anomalies measured inside boreholes, but does not change the shape of the anomaly at late times. The time constant obtained from decay curves in conductive terrains can be grossly overestimated giving conductivitythickness products that are too high. They also showed that there exists optimum time window for target detection.

Several authors examined other options in performing measurements inside boreholes. For example the use of a multicomponent receiver was reported by Cull and Cobcroft, (1986), who also suggest the use of ellipticity which is invariant to the prot orientation. Because ellipticity is based on a three component sensor, at least one of the 
components will be well coupled with the secondary fields from conductor. The authors also suggested that the ellipticity is more sensitive to conductors off the axis of the borehole then the vertical component.

Measurements of the horizontal component, $\mathrm{H}_{\rho}$, were performed by Worthington et al., (1981). They point out that this component of the magnetic field, when measured on the axis of the vertical magnetic dipole transmitter, should be sensitive to the lateral inhomogeneities because in a one-dimensional medium $\mathrm{H}_{\rho}$ vanishes.

The possibility of measuring the magnetic field inside a cased borehole was examined by Augustine et al., (1989) who showed that the use of very low frequencies (below $10 \mathrm{~Hz}$ ) allows the measurement of fields inside cased boreholes. This is very important, because once the borehole is cased, the higher frequency electromagnetic fields can not be used to monitor the medium outside the casing, due to the strong attenuation from iron casing.

Because borehole electromagnetic measurements are performed inside a conductive medium, many results for borehole measurements can be obtained from a vast body of research in the area of electromagnetic wave propagation in dissipative media. At lower frequencies which are needed for propagation through the earth the pioneering derivations by J. Wait $(1970,1972,1981)$ form the basis on which many later developments were founded.

Some theoretical results from other applications can also be adopted to borehole conditions, in particular let us cite th development of methods to measure ocean floor condincrivity. The propagation of electromagnetic waves near the ocean bottom-earth irterface can approximate the conditions that exist in cross-hole measurements when the transruitter and receiver are crossing the boundary. Mahmound et al. (1979), Bubenik and Fraser-Smith (1978), King et al. (1979), Kauffman and Keller (1983) examined EM modes of propagation inside such medium. Coggon and Morrison (1970) considered the possibilities of measuring the conductivity of the sea floor by using the EM fields around the vertical magnetic dipole located on the sea floor. Recently this subject was examined by Edwards and Chave (1986), Cheesman at al. (1987), Edwards and Cheesman (1987) and Chave et al. (1990) who consider different transmitter-receiver configurations and give a set of analytic solutions for frequency and time domain responses on the boundary between the two conductive media. Their research points to several important aspects of measurements inside conductive media in contrast to measurements on the surface. For example, the conductive environment permits a vertical current flow through the horizontal boundary for all the electromagnetic dipole sources except the vertical magnetic 
dipole source (VMD). Because of this, fields created by a horizontal magnetic dipole (HMD) and horizontal electric dipole (HED) are sensitive even to resistive regions (Cheesman, 1987). The same author concludes, that with increased separation and frequencies the possibility of detecting resistive layers also increases and that the best configurations for this purpose are coaxial magnetic dipole (HRHR) and coaxial electric dipole-dipole (ERER) sources. In time domain, the results show that early times are indicative of resistive zones, late times of conductive. Furthermore Cheesman at al. (1987) showed that if a conductive outcropping dyke is located between the transmitter and receiver at the ocean-subflor interface, then the response is insensitive to the particular location of the dyke, but the delay time is linearly proportional to the conductance (conductivity-thickness product) of the dike.

Many of the previously mentioned papers point out the difficulties encountered during the interpretation of the field results. Interpretation based on simple models represented by current filaments was frequently sufficient in search for the sulphides in a resistive medium. However in monitoring the movements of fluids this is not the case. Here the conductivity contrasts are small, the anomalous zone shape is frequently highly irregular and the only option that yields reliable conductivity distributions is a tomographic picture of the medium. Every possibility of obtaining additional information around the boreholes should be utilized to create that tomographic picture. Crosshole measurements offer this possibility wherever there is more than one drillhole.

One of the first experiments in which electromagnetic methods were used to monitor movements of fluids was conducted by Lytle et al. (1981) of the Lawrence Livermore Laboratory, as part of a coal gasification experiment. The experiment was conducted at high frequency ( $10 \mathrm{MHz}$ ) which limited the separation between boreholes to just $4.6 \mathrm{~m}$ but allowed to use the optic ray approximation to construct the tomographic image of conductivity.

The possibilities of using the measurements inside boreholes for reservoir monitoring were explored by Spies and Greaves (1991), Spies (1992), and Greaves at et al. (1991) who argued that borehole measurements are capable to detect the changes in the reservoir. Asch and Morrison (1989) and Bevc and Morrison (1991) presented the results of field experiments using DC resistivity methods. The use of EM induction for steam-flooding monitoring in boreholes was reported by Wilt and Schenkel (1992) and the use of the EM induction for tracing the salt-water plume was described by Wilt et al. (1991). 
The first low frequency diffusion tomography algorithm to create a conductivity image with a specific purpose of detecting small conductivity variations in the medium was developed by Zhou (1989). The first interpretation of actual field data using an extension of this work for axisymmetric geometry was performed by Alumbaugh (1993).

\subsection{The objective of this work.}

This work has two objectives: 1) to examine some of the characteristics of the electromagnetic fields in a borehole environment that contains a vertical magnetic dipole in a one-dimensional medium, and 2) to interpret crosshole EM measurements in a onedimensional layered medium.

In Chapter 2 we examine the electromagnetic fields in a conductive unbounded whole space. This chapter explains certain relations between the EM field components, examines the sensitivity of the response to the conductivity of the medium, and evaluates the accuracy in position needed for particular measurements.

Chapter 3 deals with the boundary between two conductive half-spaces. It shows that the approximate relations obtained from asymptotic expansions for large induction numbers are consistent with numerical results. Then the vertical magnetic field is examined as a function of transmitter-receiver separation and distances to the boundary. The results are then compared to the conclusions reached by King et al. (1979). Next the fields near a layer that separates two half-spaces are considered to find out how the layer thickness and transmitter-receiver separation affect the detectability of the layer.

Chapter 4 opens the second part of the work that deals mostly with the interpretation of electromagnetic measurements in a borehole. It examines the possibilities of utilizing the second vertical derivatives of $\mathrm{H}_{\mathrm{z}}$ to determine the conductivity profile directly from the measurements.

Chapter 5 describes the results of least squares inversion of field data in layered medium. It also shows the inversion results in which the first vertical derivative of $\mathrm{H}_{\mathrm{z}}$ was used as an input to the inversion routine. 
Pure water
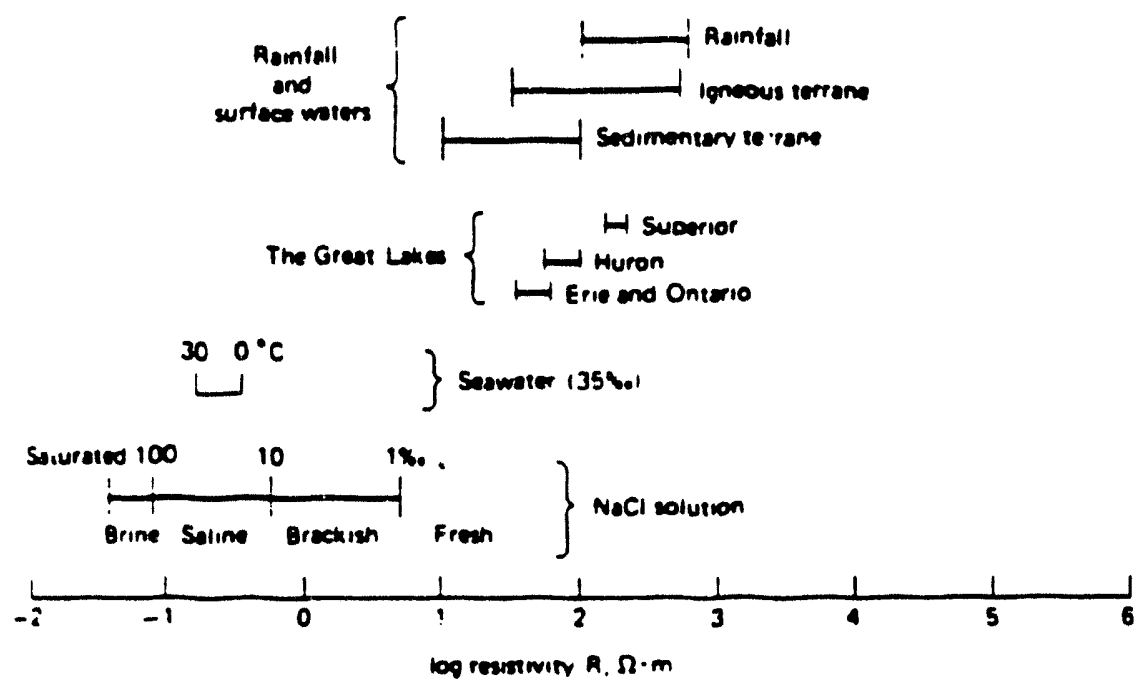

Fig. 1.1-1 The electrical resistivity of natural water (Hearst and Nelson, 1985). 


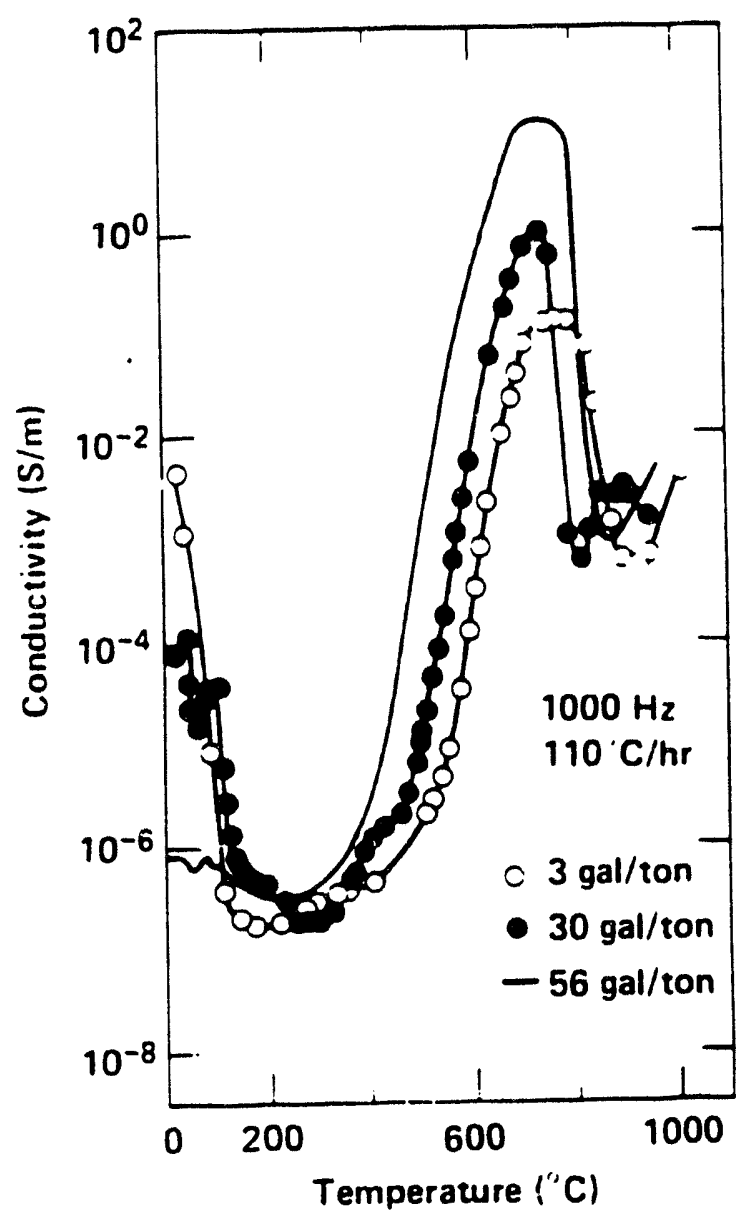

Fig. 1.1-2 Electrical conductivity of oil-shale as a function of temperature and content of kerogen measured at $1000 \mathrm{~Hz}$. The samples are water saturated at room temperature (Duba, 1982). 


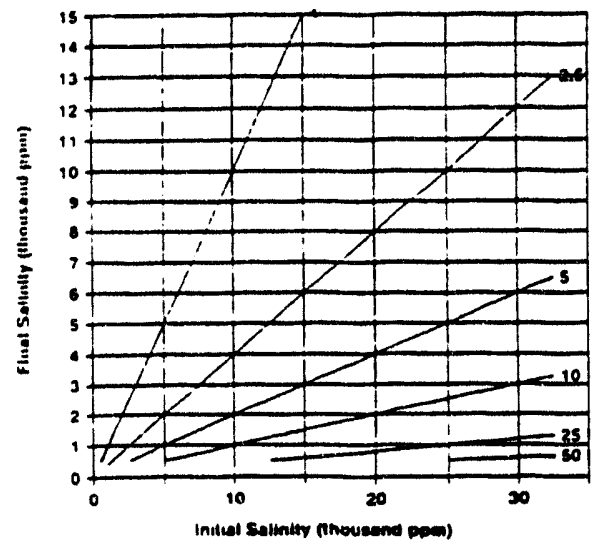

a )

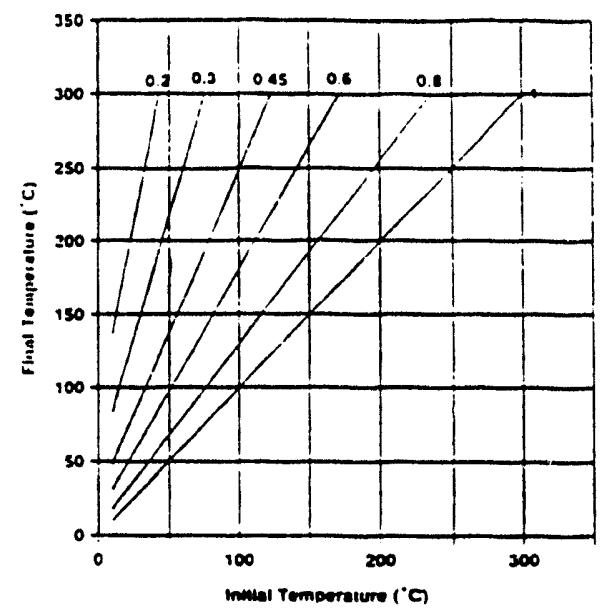

b )

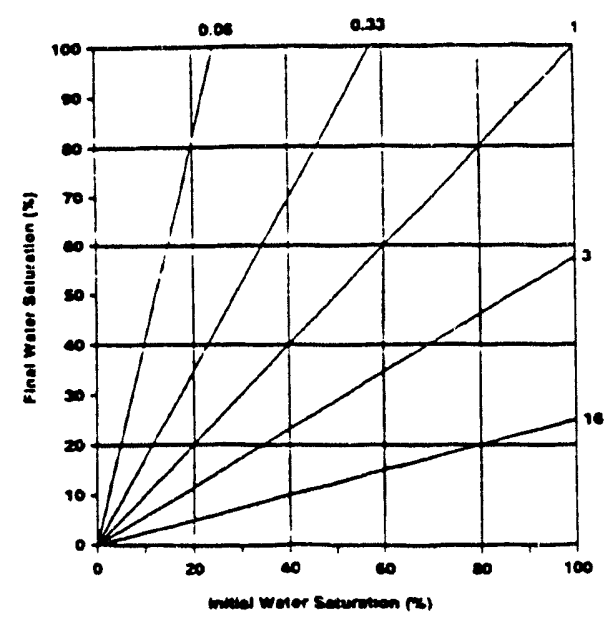

c)

Fig. 1.1-3 Ratio of formation resistivity after a salinity change at constant temperature and saturation to initial resistivity (a), ratio of formation resistivity after a temperature change at constant salinity and saturation to initial resistivity (b), ratio of formation resistivity after a saturation change at constant salinity and temperature to initial resistivity (c) (Mansure and Meldau, 1990). 


\section{Resistivity and velocity as a function} of porosity, $\Phi$, water saturation, $S_{w}$, and temperature, $\circ \mathrm{C}$.
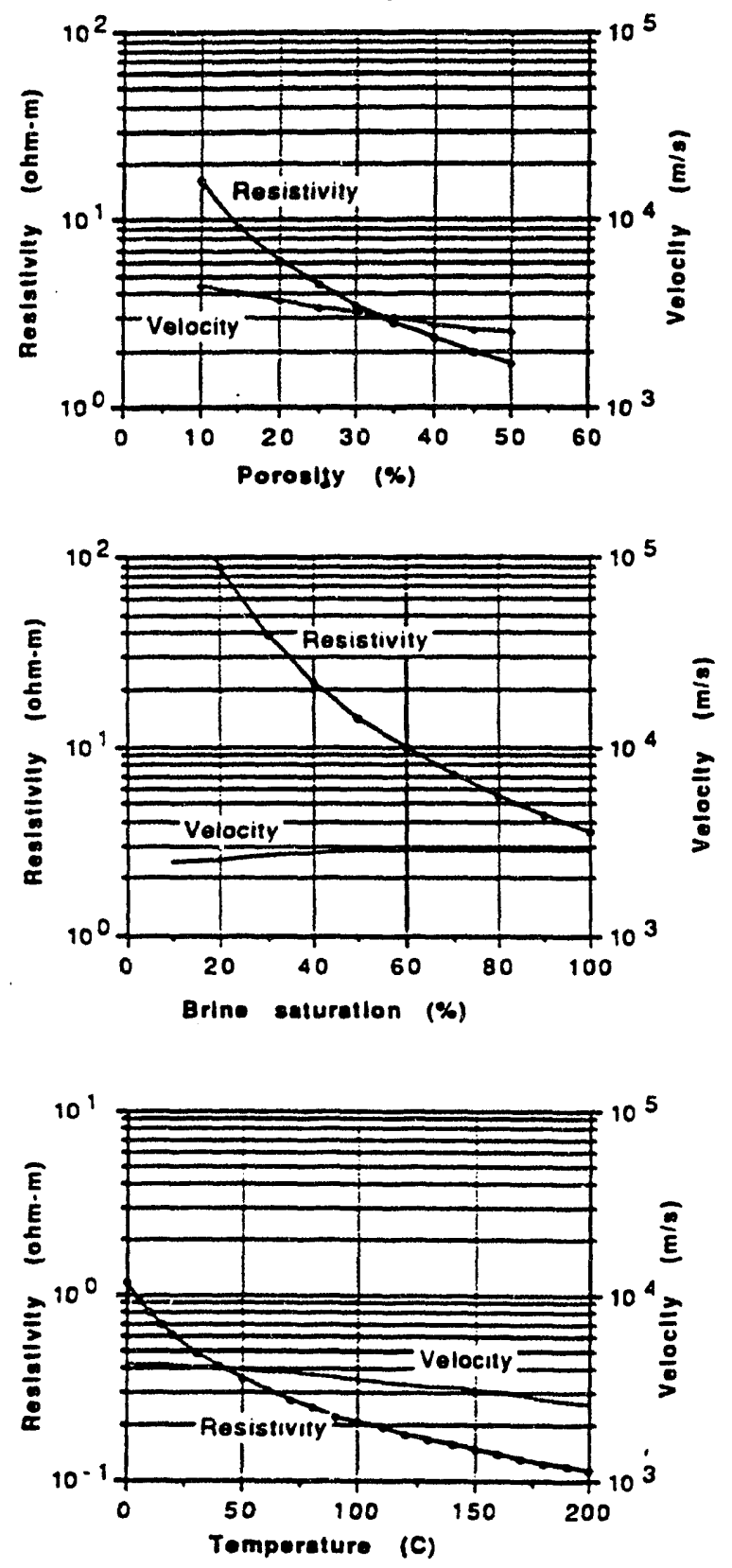

Unconsolidated sandstone $p_{w}=0.65$ onm-m at $20^{\circ} \mathrm{C}$ $\Phi=30 \%$

Fig. 1.1-4 The comparison of resistivity and velocity variations due to the changes in porosity, sanuration and temperature for the unconsolidated sandstone. 
Depth of propagation as a function of trequency and conductivity

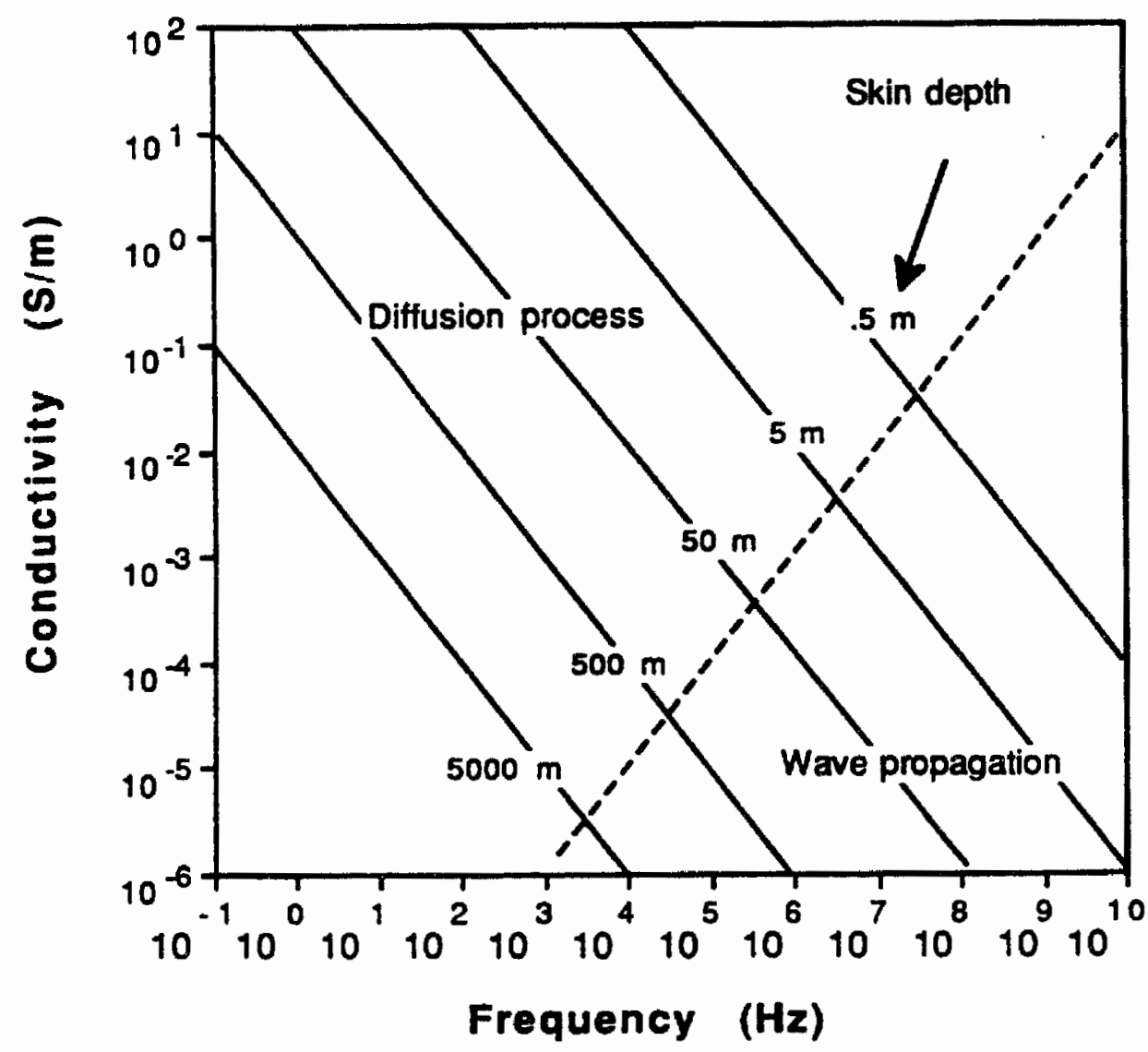

Fig. 1.2-1 Depth of penetration as a function of conductivity and frequency. 


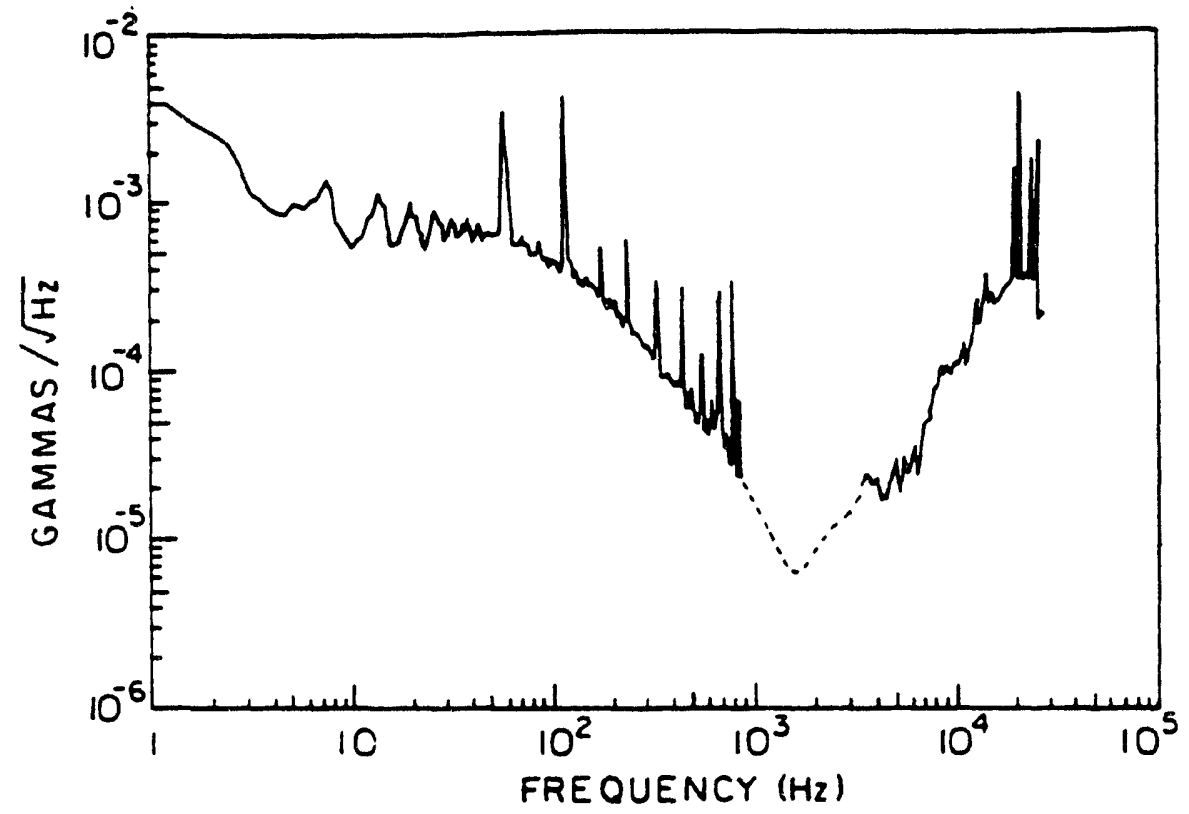

Fig. 1.3-1 A typical spectrum of natural $\mathrm{H}_{\mathrm{x}}$ field on the surface of the earth at San Antonio Valley, California (Labson, 1985). 
ATTENUATION OF NATURAL NOISE WITH DEPTH AND CONDUCTIVITY

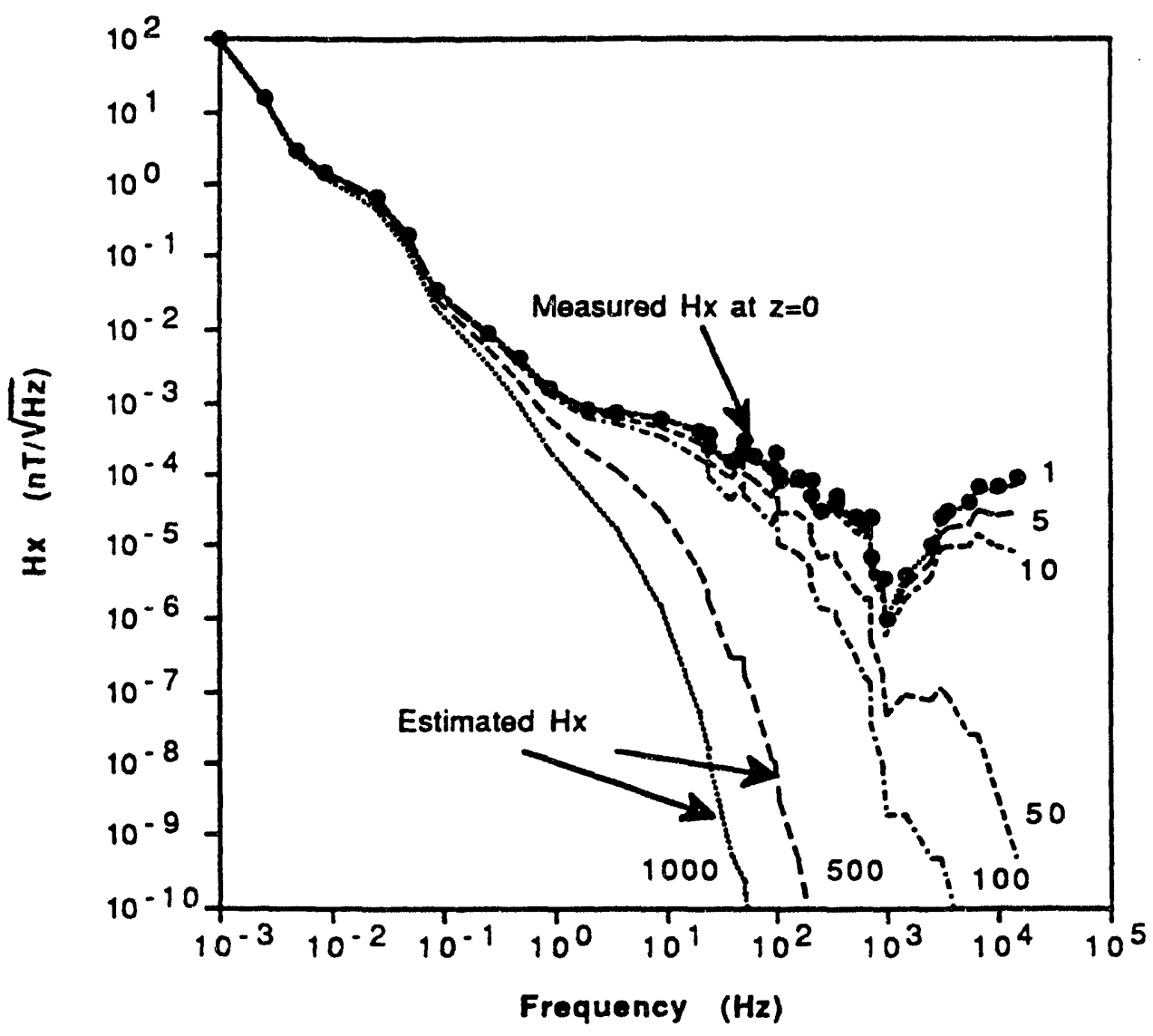

Fig. 1.3-2 Attenuation of natural electromagnetic noise in the $\mathrm{H}_{\mathrm{x}}$ field with conductivity-depth product. The curve parameter is $\sigma^{1 / 2} \mathrm{z}$ product. 


\section{GEOMETRIC SOUNDING ON THE SURFACE}
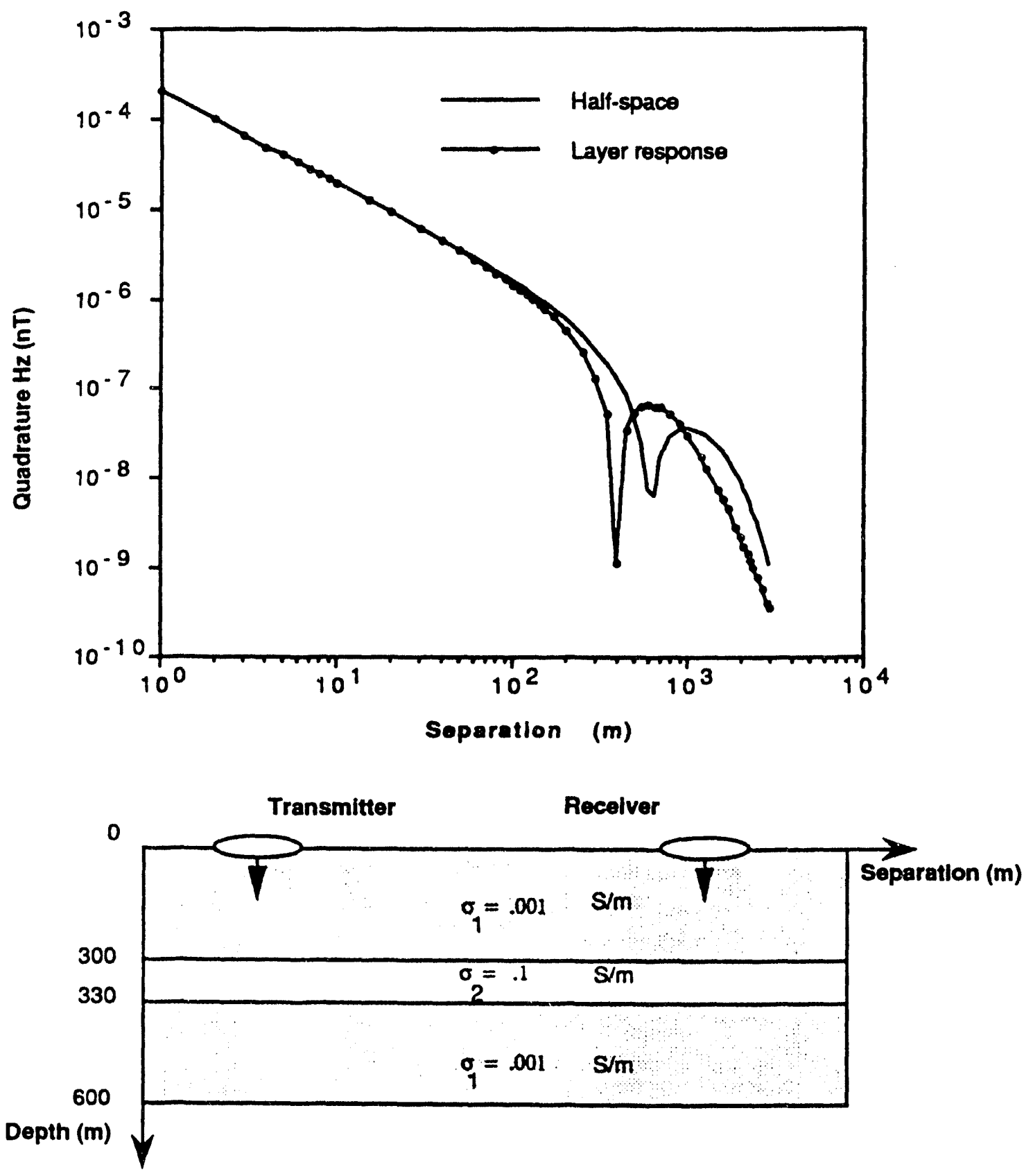

Fig. 1.4-1 Simulation of the geometric sounding for transmitter and receiver on the surface. Source frequency is $1000 \mathrm{~Hz}$. The model consists of the $30.0 \mathrm{~m}$ thick layer that has a conductivity of $0.1 \mathrm{~S} / \mathrm{m}$. The layer is inside a half-space of conductivity 0.001 $\mathrm{S} / \mathrm{m}$. 


\section{LOGGING GEOMETRY}

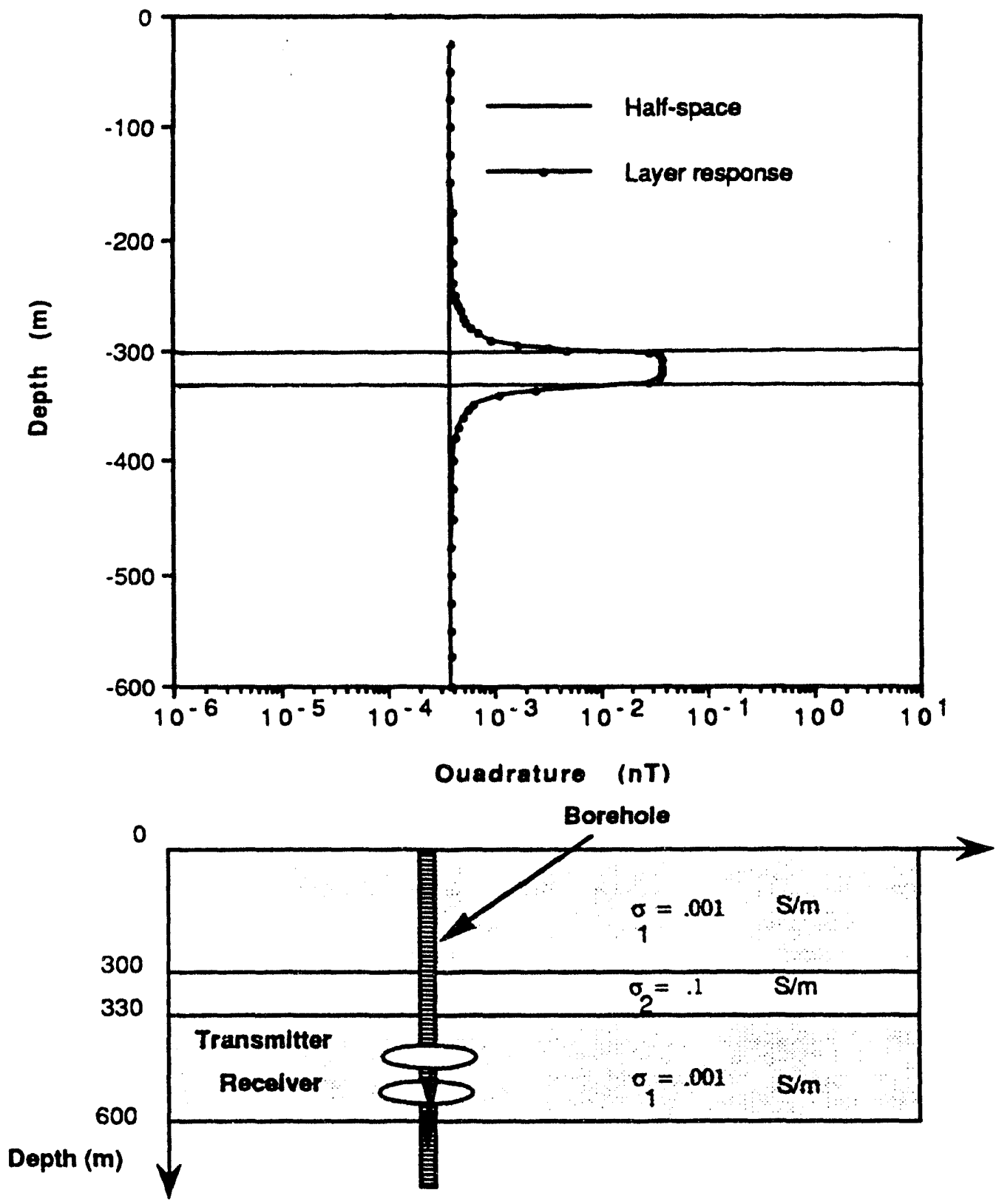

Fig. 1.4-2 Simulation of the induction logging: the transmitter and the receiver move simultaneously in one borehole and the transmitter is placed $2.0 \mathrm{~m}$ above the receiver. Source frequency is $1000 \mathrm{~Hz}$. The model consists of the $30.0 \mathrm{~m}$ thick layer that has a conductivity of $0.1 \mathrm{~S} / \mathrm{m}$. The layer is inside a half-space of conductivity $0.001 \mathrm{~S} / \mathrm{m}$. 
SURFACE-TO-BOREHOLE RESPONSE
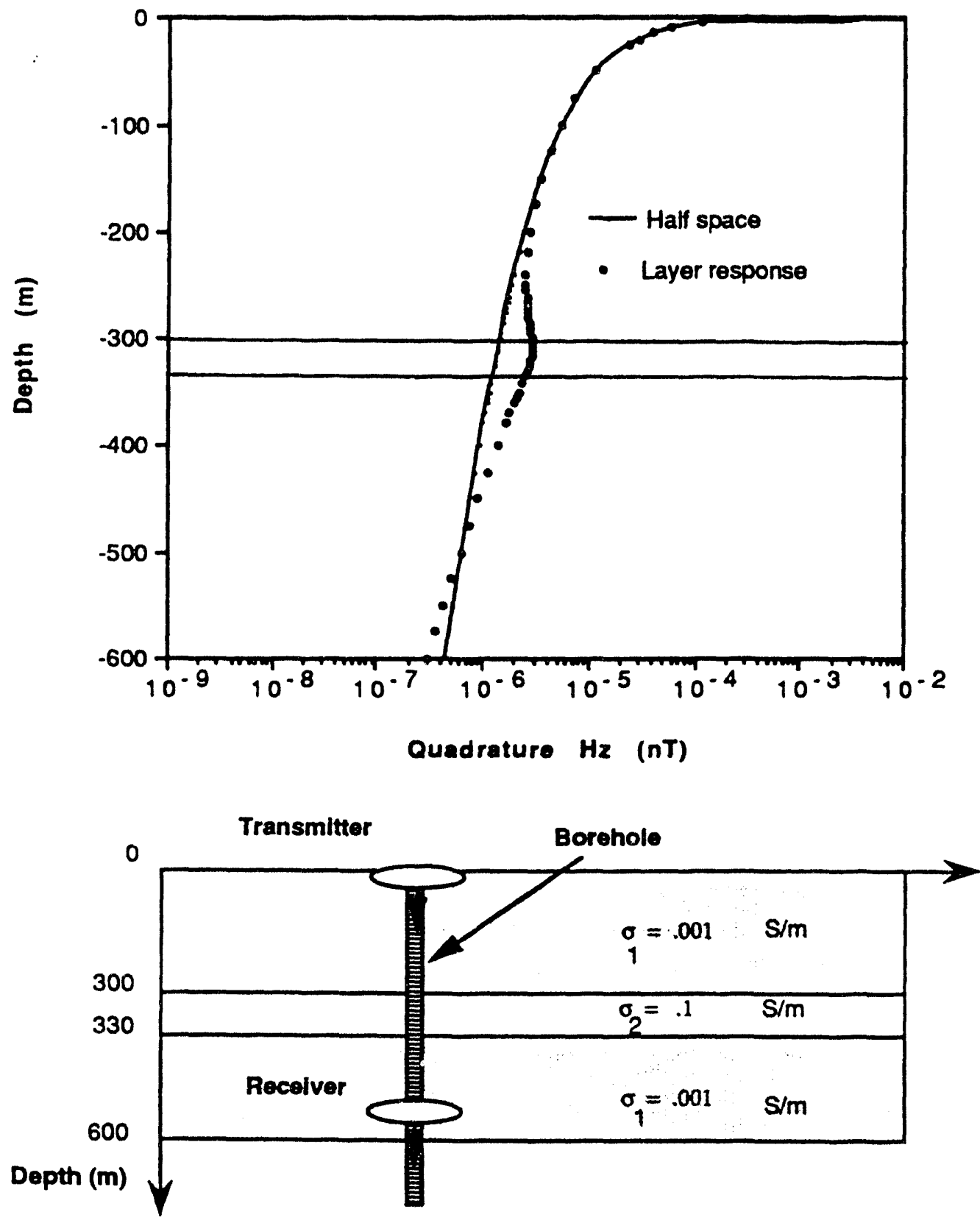

Fig. 1.4-3 Simulation of the surface-to-borehole electromagnetic response for transmitter on the surface and receiver moving down the borehole below the transmitter. Source frequency is $1000 \mathrm{~Hz}$. The model consists of the $30.0 \mathrm{~m}$ thick layer that has a conductivity of $0.1 \mathrm{~S} / \mathrm{m}$. The layer is inside a half-space of conductivity $0.001 \mathrm{~S} / \mathrm{m}$. 


\section{SURFACE-TO-BOREHOLE RESPONSE}
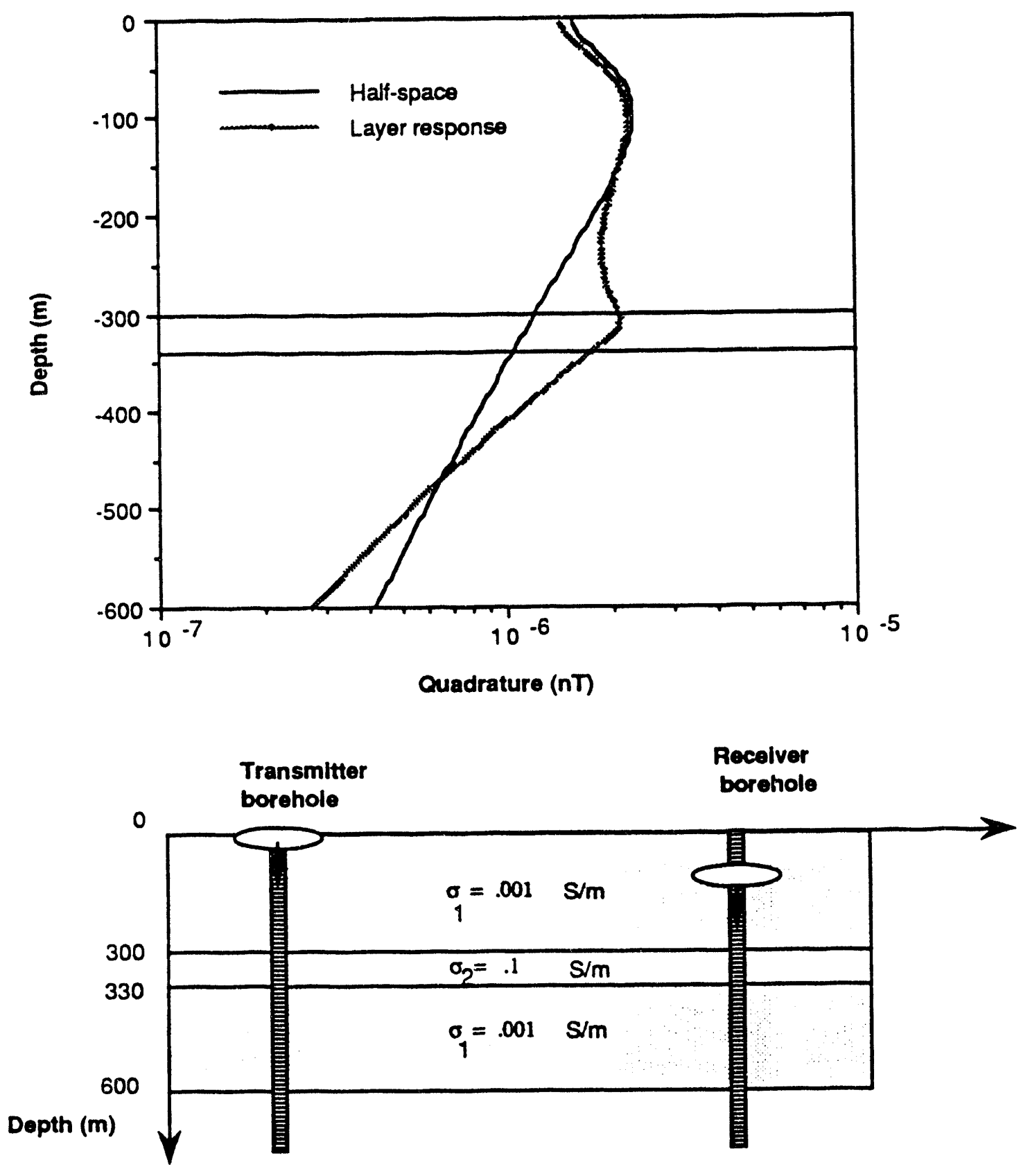

Fig. 1.4-4 Simulation of the surface-to-borehole electromagnetic response where transmitter is on the surface and receiver is moving down a borehole. The horizontal transmitter-receiver separation is $100 \mathrm{~m}$. The model consists of the $30.0 \mathrm{~m}$ thick layer that has a conductivity of $0.1 \mathrm{~S} / \mathrm{m}$. The layer is inside a half-space of conductivity $0.001 \mathrm{~S} / \mathrm{m}$. 
CROSSHOLE RESPONSE
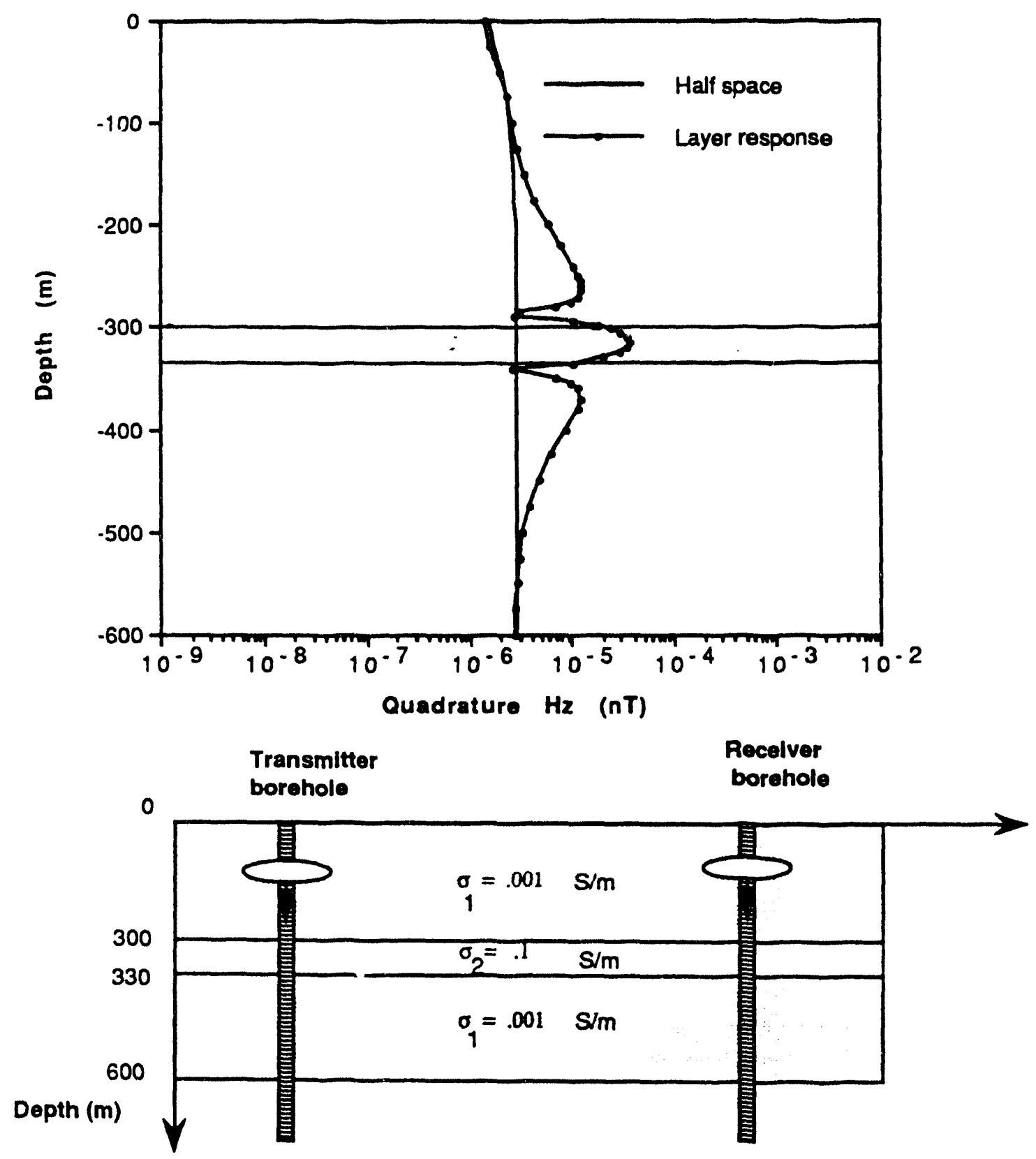

Fig. 1.4-5 Simulation of the crosshole electromagnetic response for transmitter and receiver moving down two boreholes at the same depth. The transmitter-receiver separation is $100 \mathrm{~m}$. The model consists of the $30.0 \mathrm{~m}$ thick layer that has a conductivity of $0.1 \mathrm{~S} / \mathrm{m}$. The layer is inside a half-space of conductivity $0.001 \mathrm{~S} / \mathrm{m}$. 


\section{CROSSHOLE TOMOGRAPHY}
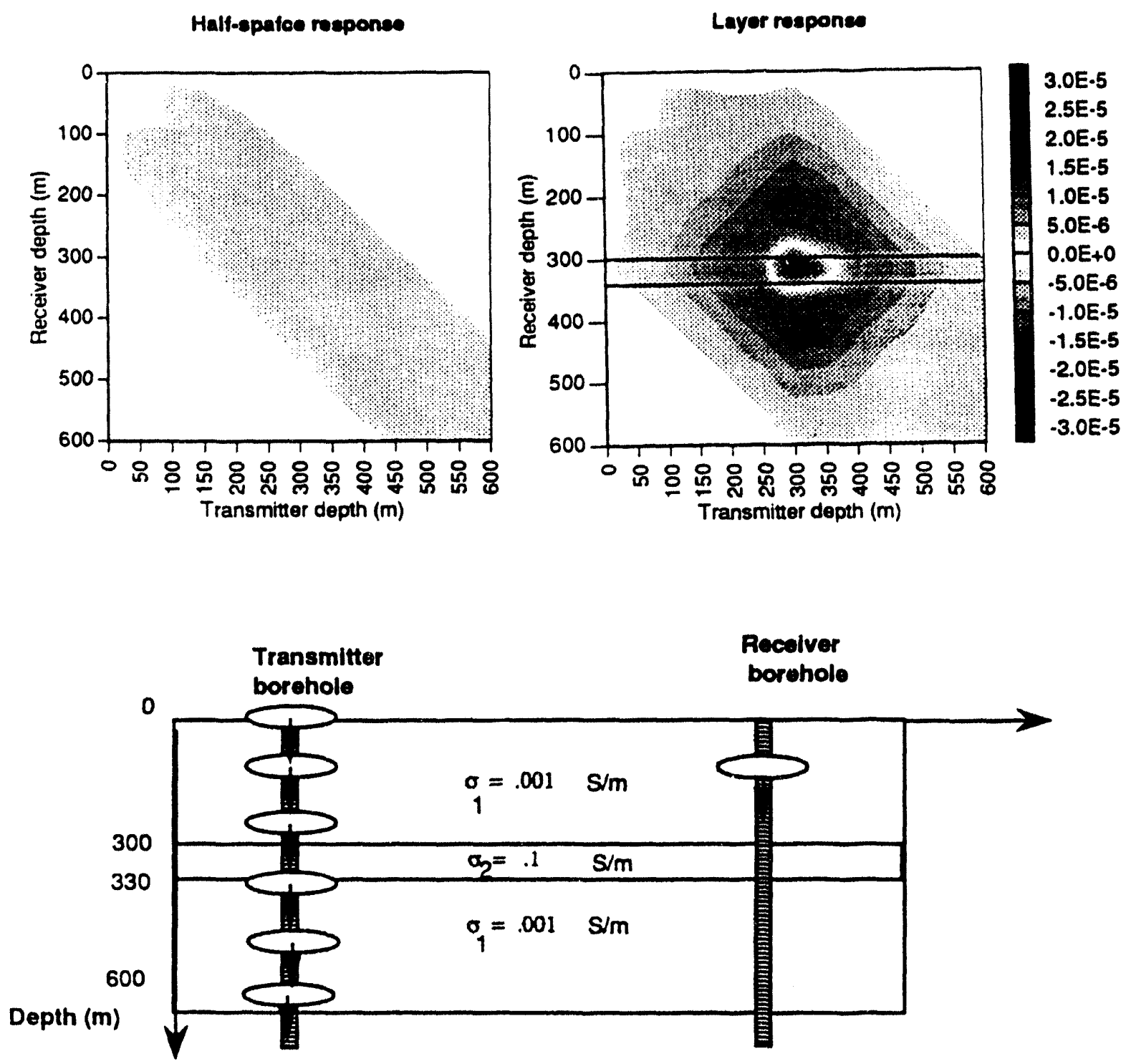

Fig. 1.4-6 Simulation of crosshole electromagnetic tomography where transmitter is in one borehole and a receiver is moving down another borehole. The horizontal transmitter-receiver separation is $100 \mathrm{~m}$. The model consists of the $30.0 \mathrm{~m}$ thick layer that has a conductivity of $0.1 \mathrm{~S} / \mathrm{m}$. The layer is inside a half-space of conductivity $0.001 \mathrm{~S} / \mathrm{m}$. The horizontal axes describes the locations of the transmitter inside one borehole, the vertical axes describes the location of a receiver inside the second borehole. 


\section{Chapter 2 \\ Whole space response characteristics.}

The uniform, homogeneous, whole space plays the same role in downhole electromagnetic measurements as the half-space model in the surface geophysics. This whole space model is the crudest approximation of the medium that exists around the borehole, but it can be used to estimate the effects of conductivity, frequency and geometry on the observed magnetic field values and to establish the basic required parameters of a given experiment. In this chapter we will consider the radial $\left(\mathrm{H}_{\mathrm{p}}\right)$ and vertical $\left(\mathrm{H}_{2}\right)$ components of magnetic field produced by a vertical magnetic dipole source. In particular we will examine the range of penetration, the sensitivity to whole space conductivity changes and the errors in measurement caused by misplacement of the receiver with respect to the transmitter. We will illustrate our analysis with field data collected in Richmond, California.

\subsection{Vertical magnetic dipole fields}

The magnetic fields around the vertical magnetic dipole in a uniform homogeneous whole space are given by (Kauffman and Keller, 1983):

$$
\begin{aligned}
& H_{z}\left(k_{s}, R\right)=\frac{M}{4 \pi} \frac{e^{-i k_{s} R}}{R^{5}}\left\{\left(1+i k_{s} R\right)\left[-\rho^{2}+2\left(z-z_{s}\right)^{2}\right]+\left(i k_{s} R\right)^{2}\left(-\rho^{2}\right)\right\}, \\
& H_{\rho}\left(k_{s} R\right)=\frac{M}{4 \pi} \frac{e^{-k_{s} R}}{R^{5}} \rho\left(z-z_{s}\right)\left[3\left(1+i k_{s} R\right)+\left(i k_{s} R\right)^{2}\right],
\end{aligned}
$$

where $M$ is the magnetic dipole moment, $z_{s}$ is source depth, $z$ is the depth of the observation, $\rho$ is a horizontal separation, $R=\left[\rho^{2}+\left(z-z_{s}\right)^{2}\right]^{1 / 2}$ is the total separation between the transmitter and the receiver, $k_{s}$ is the fropagation constant. The subscript "s" refers to the medium in which the magnetic dipole source is placed. Assuming an $e^{+i \omega l}$ time dependence, $k_{s}$ is given as:

$$
k_{s}=\sqrt{\omega^{2} \mu \varepsilon-i \omega \mu \sigma_{s}}
$$

where $\sigma_{s}$ describes the conductivity of the medium, $\omega$ is angular frequency of the source, $\varepsilon$ dielectric constant and $\mu$ magnetic permeability. 
To visualize these fields we plotted the magnetic fields inside a conductive whole space in several different ways as presented in Figures (2.1-1) and (2.1-2) for the $\mathrm{H}_{2}$ and the $\mathrm{H}_{\rho}$ components respectively. We started with a case of a specific whole space conductivity and source frequency, then considered more general case for varying of products and finally we examined the general case of normalized fields.

First we considered the fields in a whole space of conductivity $0.043 \mathrm{~S} / \mathrm{m}$ excited by a magnetic dipole source oscillating at $18.5 \mathrm{kHz}$. These parameters are representative of an actual experiment conducted in Richmond, CA, in the spring of 1992 . In this experiment cross hole measurements were performed before and after injection of a salt water plume. The central injection borehole and four other boreholes (NW, NE, SE and $\mathrm{SW}$ ) were separated by 20.0 or $25.0 \mathrm{~m}$. The transmitter frequency was $18,500 \mathrm{~Hz}$. The initial estimate of the background conductivity in Richmond was $0.043 \mathrm{~S} / \mathrm{m}$ (Alumbaugh, 1993). We used this source frequency and medium conductivity to plot the magnetic fields around the magnetic dipole inside a conductive whole space. The in-phase, quadrature, amplitude and phase of $\mathrm{H}_{z}$ and $\mathrm{H}_{\mathrm{p}}$ components are presented in Figures (2.11a) and (2.1-2a) respectively. These figures are constructed for a specific conductivity and frequency, but equations $(2.1-1)$ and (2.1-2) suggest that when the displacement currents can be neglected (i.e. $\omega \varepsilon \ll \sigma_{s}$ ) one would obtain an identical set of plots for different conductivity and frequency as long as the same $\sigma_{8} f(=795.5)$ product is retained. The two vertical lines on the Figure 2.1-1a show the location of the profiles where the field data for the $\mathrm{H}_{2}$ component were collected in Richmond.

Lets consider the $\mathrm{H}_{2}$ component first. As can be observed the character of the curves is different in each region of space. Close to the transmitter fields vary rapidly with distance because when $R$ is small, even a small variation in $R$ causes a large change in fields. For example the $\mathrm{H}_{2}$ component diminishes from $100 \mathrm{nT}$ to $0.46 \mathrm{nT}$ when the horizontal separation $\rho$ increases from 1 to $6 \mathrm{~m}$ (and $\left|z-z_{s}\right|=0$ ), i.e. the field decays more than two orders of magnitude. But the fields drop from $1.25^{*} 10^{-2} \mathrm{nT}$ to $6.4^{*} 10^{-3} \mathrm{nT}$ over the same $5.0 \mathrm{~m}$, distance, i.e. less than $50 \%$ when measured 20 and $25 \mathrm{~m}$ away from the transmitter. Further away from the transmitter the fields vary more slowly with distance except in regions where the in-phase and quadrature change sign.

Notice, that both in-phase and quadrature components of $\mathrm{H}_{2}$ field change sign, but this sign change occurs at different locations for each component. As a result the $\mathrm{H}_{\mathbf{z}}$ field does not vanish at any point inside a whole space as long as the propagation constant in this medium is finite (and the quadrature component does not vanish). Only in the DC 
limit, where the propagation constant is zero, does the $\mathrm{H}_{\mathbf{z}}$ component vanish along lines where $\rho=\sqrt{2}\left(z-z_{s}\right)$.

To confirm this behavior we present two of the more than 50 profiles collected in Richmond in June of 1993 superimposed on the numerical whole-space profiles in Figure 2.1-1b. The transmitter was moved inside a vertical borehole from 4.0 to $60.0 \mathrm{~m}$ below the surface. The receiver was stationary and placed at a depth of $30.0 \mathrm{~m}$ (marked by a thick horizontal line on the plots). The profiles below the thick line are further away from the surface and are less affected by the earth-air interface, therefore numerical and field profiles agree better. In a uniform whole space the profiles would be symmetric around this line. As can be observed the maximum $\mathrm{H}_{2}$ field is measured at a closest transmitterreceiver separation i.e. when both tools are at a depth of $30.0 \mathrm{~m}$. From this point the field diminishes but the variation with distance is greater for the closer $(20 \mathrm{~m})$ profile. Notice also that the quadrature component changes sign. The sign change occurs deeper for larger transmitter-receiver horizontal separations. This behavior closely corresponds to the quadrature field in Figure 2.1-1a.

Next we considered similar characteristics of the $\mathrm{H}_{\rho}$ component but without the examples of field data since in the Richmond experiment only the $\mathrm{H}_{\mathrm{z}}$ component was measured. As can be observed from these figures the $\mathrm{H}_{2}$ and $\mathrm{H}_{\rho}$ components behave quite differently in the region considered. The $\mathrm{H}_{\rho}$ component inside the whole space presented in Figure 2.1-2a is different because the in-phase and quadrature components of this field vanish where either $\rho$ or $\left(z-z_{s}\right)$ is zero. As a result the $\mathrm{H}_{\mathrm{p}}$ field in a whole space also vanishes at these points independently of the value of the propagation constant.

In surface geophysics the fields are plotted on the surface of a half-space as a function of separation or, more generally, of the half-space induction number $(\omega \mu \sigma)^{1 / 2} \rho$. By analogy we plotted the $\mathrm{H}_{z}$ and $\mathrm{H}_{\rho}$ components inside a conductive whole space as a function of separation in the following cases: for the $\mathrm{H}_{2}$ component when the transmitter is at the same depth as receiver and when it is $100.0 \mathrm{~m}$ above the receiver in Figures 2.1$1 \mathrm{c}$ and 2.1-1d respectively; for the $\mathrm{H}_{\mathrm{p}}$ component we considered the transmitter $1.0 \mathrm{~m}$ above the receiver and $100.0 \mathrm{~m}$ above the receiver in Figures $2.1-2 \mathrm{~b}$ and $2.1-2 \mathrm{c}$ respectively. In all the figures the curve parameter is the $\sigma_{\mathrm{s}} \mathrm{f}$ product where $\mathrm{f}$ is the frequency of the current in a transmitter and $\sigma_{s}$ is the conductivity of a whole space. The vertical axis presents the field values in $\mathrm{nT}$ (or degrees for phase) for a dipole moment of $1.0 \mathrm{Am}^{2}$. Notice that the minimum value on the vertical axis is $10-12 \mathrm{nT}$. This is an extreme limit of measurable fields assuming the maximum sensitivity of the receiver coil to be $10^{-8} \mathrm{nT}$ and the maximum source strength of $104 \mathrm{Am}^{2}$. For borehole sources the 
current maximum moment is less than $10^{3} \mathrm{Am}^{2}$ and the best sensors have sensitivity of 10-7 nT. This figure proves, that it is possible to obtain a measurable signal inside boreholes separated by more than $1000 \mathrm{~m}$ if the $\sigma_{\mathrm{s}} \mathrm{f}$ product is sufficiently low (below 10).

Figures 2.1-1c and 2.1-2b also illustrate different zones that are characterized by varying decay rates of the fields which are determined by the terms that dominate the response in equations (2.1-1) and (2.1-2) (Kaufman and Keller,1983). In the near field zone the induction number $\mathrm{ik}_{\mathbf{g}} \mathrm{R} \ll 1$ and the fields are proportional to $1 / \mathrm{R}^{\mathrm{n}}$ (because the $\left.e^{-i k_{S} R} \sim 1\right)$. In particular the in phase and amplitude components of $\mathrm{H}_{\mathrm{z}}$ are proportional to $1 / R^{3}$, and quadrature is proportional to $1 / R$, the in phase and amplitude components of $H_{\rho}$ are proportional to $1 / R^{4}$, and quadrature is proportional to $1 / R^{2}$ as can be seen from the figures. These observations can be verified by comparing the in-phase and quadrature of the fields at small induction numbers (Kaufman and Keller, 1983). At small induction numbers we assume that $i k_{s} R \ll 1.0$ which corresponds to $\left(\sigma_{s} f\right)^{1 / 2} R \ll 503$. If we neglect the displacement currents, then at small induction number the real and imaginary parts are given by:

$$
\begin{gathered}
H_{z}\left(k_{s}, R\right) \approx \\
\frac{M}{4 \pi} \frac{1}{R^{5}}\left\{\left[\left(-R^{2}+3\left(z-z_{s}\right)^{2}\right)-\frac{\sqrt{2}}{3}\left(\omega \mu \sigma_{s}\right)^{3 / 2} R^{5}+\frac{1}{8}\left(\omega \mu \sigma_{s}\right)^{2} R^{4}\left(3 R^{2}-\left(z-z_{s}\right)^{2}\right)\right]+\right. \\
\left.i\left[\frac{1}{2} \omega \mu \sigma_{s} R^{2}\left(R^{2}+\left(z-z_{s}\right)^{2}\right)-\frac{\sqrt{2}}{3}\left(\omega \mu \sigma_{s}\right)^{3 / 2} R^{5}\right]\right\} \\
H_{p}\left(k_{s s} R\right) \approx \frac{M}{4 \pi} \frac{p\left(z-z_{s}\right)}{R^{5}}\left\{3-\frac{1}{8}\left(\omega \mu \sigma_{s}\right)^{2} R^{4}+i \frac{1}{2} \omega \mu \sigma_{s} R^{2}\right\}
\end{gathered}
$$

The extent of the near field zone from the transmitter depends on the induction number $i \mathrm{k}_{\mathrm{s}} \mathrm{R}$ and corresponds to the straight part of the decay curves. In the near field only the quadrature component is proportional to the $\sigma_{\mathrm{s}} \mathrm{f}$ product, the in-phase component consists mainly of the free-space field. The Richmond experiment was conducted almost in the near field since the $\sigma_{\mathrm{s}} \mathrm{f}$ product was 795.5 and the maximum separation was $25.0 \mathrm{~m}$ which resulted in $\mathrm{ik}_{\mathrm{s}} \mathrm{R}=1.4$. A true near field condition would exist for separation $\mathrm{R} \ll$ $17.8 \mathrm{~m}$.

In the far field zone $i k_{s} R \gg 1$ and the $e^{-i k_{s} R}$ term dominates the decay; the fields decay exponentially with increasing induction number.

The plots in Figures $(2.1-1 \mathrm{a}, \mathrm{c}, \mathrm{d})$ and $(2.1-2 \mathrm{a}, \mathrm{b}, \mathrm{c})$ describe the fields for a specific $\sigma_{s} f$ product. For the general case consider fields normalized by $R^{3}$ (where $R$ is a 
total transmitter-receiver distance). If we neglect the displacement currents, then the normalized fields for all transmitter-receiver combinations and $\sigma_{\mathrm{s}} f$ products can be presented on a single plot. To see this multiply $\mathrm{H}_{2}$ and $\mathrm{H}_{\mathrm{p}}$ by $\mathrm{R}^{3}$, and rearrange the variables in equations (2.1-1) and (2.1-2) by multiplying and dividing $\mathrm{H}_{2}$ and $\mathrm{H}_{p}$ by $\mathrm{k}_{s}{ }^{2}$. The resulting expressions are (Kauffman and Keller, 1983):

$$
\begin{aligned}
& H_{z}\left(k_{s}, R\right) R^{3}=\frac{M}{4 \pi} \frac{e^{-i k_{s} R}}{k_{s}^{2} R^{2}}\left[\left(1+i k_{s} R\right)\left(-k_{s}^{2} \rho^{2}+2 k_{s}^{2}\left(z-z_{s}\right)^{2}\right)+\left(i k_{s} R\right)^{2}\left(k_{s}^{2} \rho^{2}\right)\right], \\
& H_{\rho}\left(k_{s}, R\right) R^{3}=\frac{M}{4 \pi} \frac{e^{-i k_{s} R}}{k_{s}^{2} R^{2}} k_{s}^{2} \rho\left(z-z_{s}\right)\left[3\left(1+i k_{s} R\right)+\left(i k_{s} R\right)^{2}\right],
\end{aligned}
$$

The right hand side of the above equations depends only on the $k_{s} \rho$ and $k_{s}\left(z-z_{s}\right)$. If the displacement currents are neglected at low frequencies where $\omega^{2} \mu \varepsilon<<\omega \mu \sigma_{s}$, then $k_{s}=\left(-i \omega \mu \sigma_{s}\right)^{1 / 2}$ and each component can be presented on the single plot with the $z$-axis scaled by $\left(\sigma_{s} f\right)^{1 / 2}\left(z-z_{s}\right)$ and $x$-axis scaled by $\left(\sigma_{s} f\right)^{1 / 2} \rho$. The examples of such plots are given in Figures (2.1-1e) and (2.1-2d) for $\mathrm{H}_{2}$ and $\mathrm{H}_{\rho}$ respectively. A detailed contour section for the last two plots is given in Figures (2.1-1f) and (2.1-2e) These plots of normalized values can be used to estimate field strengths anywhere in the whole space medium. However we can use these figures to delineate the regions that are the most sensitive to the variations in induction parameters and to estimate the location of sign reversal inside a whole space.

We observe that the most rapid variations in fields occur at induction parameters, $\left(\sigma_{s} f\right)^{1 / 2}\left(z-z_{s}\right)$ or $\left(\sigma_{s} f\right)^{1 / 2} \rho$, above 1000 (i.e. induction numbers $\left(\omega \mu \sigma_{s}\right)^{1 / 2} \rho$ or $\left(\omega \mu \sigma_{s}\right)^{1 / 2}\left(z-z_{s}\right)$ above 1) and these are the regions that are the most sensitive to conductivity variations. In the Richmond experiment we measured the $\mathrm{H}_{\mathrm{z}}$ component at the horizontal separation $\rho=25.0 \mathrm{~m}$ which gives the $\left(\sigma_{\mathrm{s}} f\right)^{1 / 2} \rho=705$ (i.e. induction number $\left.\left(\omega \mu \sigma_{\mathrm{s}}\right)^{1 / 2} \rho=0.8\right)$. The measurements extended to $50.0 \mathrm{~m}$ below the transmitter, which gives $\left(\sigma_{s} f\right)^{1 / 2} z=1410$ (i.e. induction number $\left.\left(\omega \mu \sigma_{s}\right)^{1 / 2} \rho=1.6\right)$. So these measurements barely reached the regions where the fields were the most sensitive to conductivity.

From Figures 2.1-1a,c,d,e,f to 2.1-2a,b,c,d,e it can be observed that the regions where DC fields dominate are not sensitive to the variation of conductivity-frequency product. As a result the regions of high sensitivity to the conductivity for in-phase, amplitude and phase components occur where the curves depart from the lines that 
describe the primary DC fields. Only the quadrature component is strongly dependent on of variations but at low values of $\left(\sigma_{g}\right)^{1 / 2} R$ it is very much smaller from the in-phase component.

The phases and amplitudes of the $\mathrm{H}_{\mathrm{z}}$ and $\mathrm{H}_{\mathrm{p}}$ components are different at each point of space. This means that the components do not achieve the maximum or minimum values at the same time resulting in a total vector that does not have a fixed direction in space but rotates with the frequency of the current in the transmitter (Harrington, 1961). The tip of this total vector describes an ellipse and thus in a conductive medium we have an elliptically polarized wave. The ellipse is characterized by two parameters: ellipticity and tilt angle that are related to the field parameters by the following relations:

$$
\begin{aligned}
& \varepsilon=\frac{2 R_{H} \sin \delta}{1+R_{H}^{2}+\sqrt{4 R_{H}^{2} \cos \delta+\left(1-R_{H}\right)}} . \\
& \tan 2 \theta=\frac{2 R_{H} \cos \delta}{1-R_{H}^{2}} .
\end{aligned}
$$

where $R_{H}=\left|H_{z}\right| /\left|H_{\rho}\right|, \delta$ is a phase shift between $H_{z}$ and $H_{\rho}$ components. The plots of the ellipricity and tilt angle are given in Figures (2.1-3abc).

In particular Figure 2.1-3a shows the ellipticity and tilt angle inside a conductive whole space where $\sigma_{\mathrm{s}} \mathrm{f}=795.5$. As can be observed the fields in a whole space are elliptically polarized, except in the regions in where $\mathrm{H}_{\rho}$ component vanishes. The ellipticity is small where $H_{\rho}$ component is also small. For a large depth and a big conductivity-frequency product the $\mathrm{H}_{\mathrm{p}}$ component is large and ellipticity approaches 0.5 . Furthermore close to the transmitter axis the $\mathrm{H}_{\mathrm{z}}$ component dominates, resulting in a polarization ellipse with an almost vertical principal axis. The $\mathrm{H}_{\mathbf{z}}$ field also dominates in regions coplanar with transmitter which results in small ellipticity and tilt angle close to 90.0 degrees. As the induction number increases the ellipticity goes back toward zero. The fields at this point are becoming planar with no component in the direction of the advancing "wave front". However because of the very great amplitude attenuation that has taken place it is unlikely that fields in this asymptotic regime could be measured with practical equipment. 


\subsection{Sensitivity of $H_{z}$ and $H_{\rho}$ to the conductivity variations in a whole space.}

As we observed before in Figures (2.1-1) and (2.1-2), the sensitivity to conductivity of the medium depends on the transmitter-receiver geometry and on the induction parameter. To quantify this observation we considered the change in the fields inside a whole space medium caused by the change in conductivity. We considered the percent change caused by a $10 \%$ increase in the conductivity. We used the same parameters as in the Richmond experiment and compared how an increase in conductivity from $0.043 \mathrm{~S} / \mathrm{m}$ to $0.0473 \mathrm{~S} / \mathrm{m}$ changed the response. The percent difference in measurements are given in Figures (2.2-1) and (2.2-2) for $\mathrm{H}_{z}$ and $\mathrm{H}_{\mathrm{p}}$ fields respectively. The above plots represent the change caused by $10 \%$ change in conductivity, but the same set of curves would be obtained for the same percent increase in frequency as long as displacement currents can be neglected. As can be observed it is not easy to determine which component is the most sensitive to conductivity changes in a whole space. The sensitivity of the $\mathrm{H}_{\mathrm{z}}$ component at low induction numbers is very strongly influenced by the location of transmitter with respect to receiver. In contrast the sensitivity of $\mathrm{H}_{\rho}$ component depends on the total transmitter-receiver separation as can be seen in a circular pattern in Figure (2.2-2). These observations can be verified by comparing the in-phase and quadrature of the fields at small induction numbers (Kaufman et al., 1983) given in equations (2.1-4) and (2.1-5).

We immediately see that for the $\mathrm{H}_{\rho}$ component at low induction number the conductivity enters only as a factor in a product with $R$ i.e. with total transmitter-receiver separation which is consistent with Figure (2.2-2). The $\mathrm{H}_{2}$ component however shows that the sensitivity to conductivity is strongly dependent on the geometrical configuration between transmitter and receiver. As can be observed the leading terms of the real components for $\mathrm{H}_{z}$, and $\mathrm{H}_{\mathrm{p}}$ are the free-space fields that do not provide any information about the medium conductivity. In general both components exhibit larger percent change at larger separations which is intuitively obvious since larger volumes of the medium are affected.

At large induction numbers where $i k_{s} R \gg 1$, or $\left(\sigma_{s} f\right)^{1 / 2} R \gg 503$ all components decrease exponentially with induction numbers and show a strong dependence on conductivity. 


\subsection{Sensitivity of $H_{z}$ and $H_{p}$ measurements to position errors.}

The whole space fields can also provide information about the sensitivity of the measurements to the misplacement of the receiver with respect to the transmitter position. To estimate of the absolute differences in field quantities we calculated the vertical and horizontal derivatives for $\mathrm{H}_{2}$ and $\mathrm{H}_{p}$ components and plotted them in Figures (2.3-1a) to (2.3-4a). And thus Figure (2.3-1a) represents the in-phase and quadrature of $\delta \mathrm{H}_{z} / \delta z$, as well as $\delta\left|\mathrm{H}_{z}\right| / \delta \mathrm{z}$ and $\delta$ phase $\left(\mathrm{H}_{\mathrm{z}}\right) / \delta \mathrm{z}$. Figure (2.3-2a) represents the in-phase and quadrature of $\delta \mathrm{H}_{2} / \delta \rho$, as well as $\delta \mid \mathrm{H}_{z} V / \delta \rho$ and $\delta$ phase $\left(\mathrm{H}_{z}\right) / \delta \rho$. Figures (2.3-3a) and (2.3-4a) give similar quantities for $\mathrm{H}_{\rho}$ component. To obtain an actual value of the diffe ance in fields, the required value must be multiplied by the appropriate shift in horizontal or vertical direction.

Since in Richmond experiment we measured the $\mathrm{H}_{2}$ fields along the vertical profile in $0.5 \mathrm{~m}$ intervals we calculated the finite differences: $\Delta\left(\operatorname{ReH}_{\mathrm{z}}\right)(/ \Delta \mathrm{z}), \Delta\left(\mathrm{ImH}_{\mathrm{z}}\right) / \Delta \mathrm{z}$, $\Delta \mid \mathrm{H}_{\mathrm{z}} \mathrm{l} / \Delta \mathrm{z}$ and $\Delta\left(\right.$ phase $\left.\mathrm{H}_{\mathrm{z}}\right) / \Delta \mathrm{z}$ and plotted them in Figure 2.3-1b superimposed on the corresponding whole space derivatives. We used the same data set as before with the receiver placed $30 \mathrm{~m}$ below surface. As can be seen from the theoretical whole space figures and the field examples for boreholes separated by $25 \mathrm{~m}$ around $10 \mathrm{~m}$ below and above the receiver the fields vary rapidly with vertical distance and for this reason can be susceptible to errors caused by misplacement of receiver with respect to the transmitter. However the plots of percent difference in Figures 2.3-1c, 2.3-2b, 2.3-3b and 2.3-4b show that the most important factor that determines the size of any positioning error for the in-phase and the quadrature is caused by the sign change of the fields. The phase is not sensitive to position errors. This can be seen in the field example presented in Figure 2.3-5 that shows the percent error in measurements along the same profiles but taken over a period of several days. As can be seen the accuracy in measuring the quadrature fields is very bad in areas where the quadrature changes sign. The in phase component worsens significantly below $40 \mathrm{~m}$ ( $10 \mathrm{~m}$ below the receiver) possibly because this is the region where in-phase component approaches the sign-change region as can be seen in Figure 2.1-1a.

In Figures (2.3-1c), (2.3-2b), (2.3-3b) and (2.3-4b) we plotted the percent differences in the $\mathrm{H}_{z}$ and the $\mathrm{H}_{p}$ components caused by $0.1 \mathrm{~m}$ change in the receiver positions. Figure (2.3-1c) presents the percent change in the $\mathrm{H}_{\mathrm{z}}$ component where the receiver is misplaced in the vertical direction, Figure (2.3-2b) presents the situation where the receiver is misplaced in the horizontal direction also by $0.1 \mathrm{~m}$. Figures (2.3-3b) and (2.3-4b) show similar errors for the $\mathrm{H}_{p}$ component. These figures show that in the 
Richmond experiment the shift of the transmitter by $0.1 \mathrm{~m}$ with respect to the receiver in either vertical or horizontal direction (for example by moving the transmitter from one side to the another side of the 8 " well) could cause up to $2 \%$ errors in amplitude measurements and significantly larger errors for the in-phase and quadrature measurements. The phase however was not sensitive to the position errors.

Since in later chapters we will use the vertical derivatives to calculate the conductivity of the medium we also considered how the error in the $\mathrm{H}_{2}$ measurements propagates into the measurements of the first vertical derivative. We used the first vertical derivative that was shown in Figure 2.3-1b and compared it with the one measured several days later. We calculated the percent error in $\left(\operatorname{ReH}_{z}\right)(\mid \Delta z), \Delta\left(\operatorname{lmH}_{z}\right) / \Delta z, \Delta\left|H_{z}\right| / \Delta z$ and $\Delta\left(\right.$ phase $\left._{z}\right) / \Delta z$ and plotted the differences in Figure 2.3-6. As can be seen the error that was in the range of $2 \%$ for in-phase and amplitude (see Figure 2.3-5) translates to the $+-10 \%$ error in vertical derivatives (except just below and above the receiver) i.e. 5 10 fold increase in the error.

We will estimate these errors quantitatively at low induction numbers at first for the in-hole geometry and then in the crosshole geometry.

For in-hole measurements, where $\rho=.0$, and $R=z-z_{s}$ and where $i k_{s} R \ll 1$, we have:

$$
\frac{\partial}{\partial z} H_{z}\left(k_{s}, R\right)=\frac{M}{4 \pi} \frac{1}{\left(z-z_{s}\right)^{4}}\left\{-6+\frac{2}{8}\left(\omega \mu \sigma_{s}\right)^{2}\left(z-z_{s}\right)^{4}-i \frac{1}{2} \omega \mu \sigma_{s}\left(z-z_{s}\right)^{2}\right\}
$$

So the absolute error in measurements in the in-phase component caused by the misplacement of receiver by $\Delta z$ is as follows:

$$
\text { In phase } \Delta H_{z} \approx \frac{M}{4 \pi} \frac{\Delta z}{\left(z-z_{s}\right)^{4}}\left[-6+\frac{1}{4}\left(\omega \mu \sigma_{s}\right)^{2}\left(z-z_{s}\right)^{4}\right]
$$

The corresponding error in the quadrature component is given by:

$$
\text { Quadrature } \Delta \mathrm{H}_{\mathrm{z}}=\frac{\mathrm{M}}{4 \pi} \frac{\Delta \mathrm{z}}{\left(\mathrm{z}-\mathrm{z}_{\mathrm{s}}\right)^{2}}\left(-\omega \mu \sigma_{\mathrm{s}}\right)
$$

It can be observed that both errors decrease rapidly with increasing transmitterreceiver separation, but the quadrature component is smaller since $i_{s} R \ll 1$. The quadrature component depends also on the of product and is less susceptible to errors in a less conductive medium. To obtain the relative error, divide the above expressions by the 
in-phase and quadrature components $o_{s} f$ whole space fields at low induction numbers. We obtain:

$$
\begin{aligned}
& \frac{\text { In phase } \Delta \mathrm{H}_{\mathrm{z}}}{\text { In phase } \mathrm{H}_{\mathrm{z}}} \approx \frac{-3 \Delta \mathrm{z}}{\left(\mathrm{z}-\mathrm{z}_{\mathrm{s}}\right)} \\
& \frac{\text { Quadrature } \Delta \mathrm{H}_{\mathrm{z}}}{\text { Quadrature } \mathrm{H}_{\mathrm{z}}} \approx \frac{\Delta \mathrm{z}}{\left(\mathrm{z}-\mathrm{z}_{\mathrm{s}}\right)}
\end{aligned}
$$

From these expressions we see that the relative error in measuring the in-phase component is three times bigger then in quadrature component.

Similar analysis can be performed for crosshole measurements where $\mathrm{z}-\mathrm{z}_{\mathrm{s}}=0.0$ and $R=\rho$ and where $i k_{s} R \ll 1$. We have:

$$
\frac{\partial}{\partial z} H_{z} \approx \frac{M}{4 \pi} \frac{\left(z-z_{s}\right)}{\rho^{5}}\left\{6+\frac{1}{8}\left(\omega \mu \sigma_{s}\right)^{2} \rho^{4}-i \frac{1}{2} \omega \mu \sigma_{s} \rho^{2}\right\}
$$

So the absolute error in measurements of the in-phase component when the receiver is misplaced by $\Delta z$ from the horizontal position is given by:

$$
\text { In phase } \Delta H_{2} \approx \frac{M}{4 \pi} \frac{\left(z-z_{s}\right)}{\rho^{5}} 6 \Delta z
$$

and similarly:

$$
\text { Quadrature } \Delta \mathrm{H}_{\mathrm{z}} \approx \frac{\mathrm{M}}{4 \pi} \frac{\left(\mathrm{z}-\mathrm{z}_{\mathrm{s}}\right)}{\rho^{3}}\left(-\frac{1}{2} \omega \mu \sigma_{\mathrm{s}}\right) \Delta \mathrm{z}
$$

In this case when the transmitter and the receiver are moved down two boreholes simultaneously, the ideal situation occurs when $z-z_{s}=0$; any other values of $z-z_{s}$ represent the error in vertical position, i.e. $z-z_{s}=\Delta z$

The relative errors for in-phase and quadrature components when $z-z_{s} \rightarrow 0$ are given by:

$$
\frac{\text { In phase } \Delta H_{z}}{\text { In phase } H_{z}} \approx \frac{6 \Delta z^{2}}{\rho^{2}}
$$




$$
\frac{\text { Quadrature } \Delta \mathrm{H}_{\mathrm{z}}}{\text { Quadrature } \mathrm{H}_{\mathrm{z}}} \approx \frac{\Delta \mathrm{z}^{2}}{\rho^{2}}
$$

So the in-phase component is more susceptible to errors in position than the quadrature component but in the geometry where the transmitter and receiver are located at the same depth the error is much smaller than in a coaxial geometry.

\subsection{Conditions for simplified relationships between magnetic field components}

Maxwell's equations describe the relationships between components that involve various derivative combinations. By measuring all of the derivatives prescribed by Maxwell's equations we could determine the conductivity of the medium. However usually only the vertical component is measured inside boreholes. So we posed a question: under what conditions it is possible to approximate $\mathrm{H}_{\rho}$ by $\delta \mathrm{H}_{2} / \delta \mathrm{z}$ derivative?

To obtain some understanding, let's consider the ratio of whole space fields around a magnetic dipole source:

$$
\frac{\frac{\partial}{\partial z} H_{z}}{H_{p}}=\frac{1}{\rho}\left[3-5 \frac{z^{2}}{R^{2}}+\frac{\left(i k_{s} R\right)^{2} \frac{\rho^{2}}{R^{2}}}{3+\frac{\left(i_{s} R\right)^{2}}{1+\left(i_{s} R\right)}}\right]
$$

where $R=\left[\rho^{2}+\left(z-z_{s}\right)^{2}\right]^{1 / 2}$. This ratio can be examined for several cases: if $\rho^{2} \ll z^{2}$, then $R \approx z$ and

$$
\frac{\frac{\partial}{\partial z} H_{z}}{H_{\rho}} \approx \frac{1}{\rho}\left[-2+\frac{\left(i k_{s} R\right)^{2} \frac{\rho^{2}}{z^{2}}}{3+\frac{\left(i k_{s} R\right)^{2}}{1+\left(i k_{s} R\right)}}\right] \approx(-) \frac{2}{\rho},
$$

if $\rho^{2} \gg z^{2}$, then $R \approx \rho$ and

$$
\frac{\frac{\partial}{\partial z} H_{z}}{H_{\rho}}=\frac{1}{\rho}\left[3+\frac{\left(\mathrm{ik}_{s} R\right)^{2}}{3+\frac{\left(\mathrm{ik}_{s} R\right)^{2}}{1+\left(\mathrm{ik}_{s} R\right)}}\right]
$$

which leads to 


$$
\frac{\frac{\partial}{\partial z} H_{2}}{H_{p}}=\frac{3}{\rho} \quad \text { if } \quad\left(i_{s} R\right)^{2} \ll 1
$$

if $\rho \approx z$, then

$$
\frac{\frac{\partial}{\partial z} H_{z}}{H_{\rho}} \approx \frac{1}{\rho}\left[-2+\frac{\left(i_{s} R\right)^{2}}{3+\frac{\left(\text { ikg }_{s} R\right)^{2}}{1+\left(\text { ikg }_{s} R\right)}}\right] \text {. }
$$

This simple analysis show that $\delta \mathrm{H}_{z} / \delta \mathrm{z}$ is indeed proportional to $-2 / \rho \mathrm{H}_{\rho}$ as long as $\rho^{2} \ll z^{2}$ i.e. the receiver remains close to the axis of the dipole. This relation is independent of induction number of the medium and because of this the presence of the layered medium does not disturb the proportionality. When $\rho^{2} \gg z^{2}, \delta \mathrm{H}_{z} / \delta \mathrm{z}$ is proportional to $3 / \rho \mathrm{H}_{\rho}$ if $\left|\mathrm{ik}_{\mathrm{s}} \mathrm{R}\right|^{2} \ll 1$ is satisfied. 

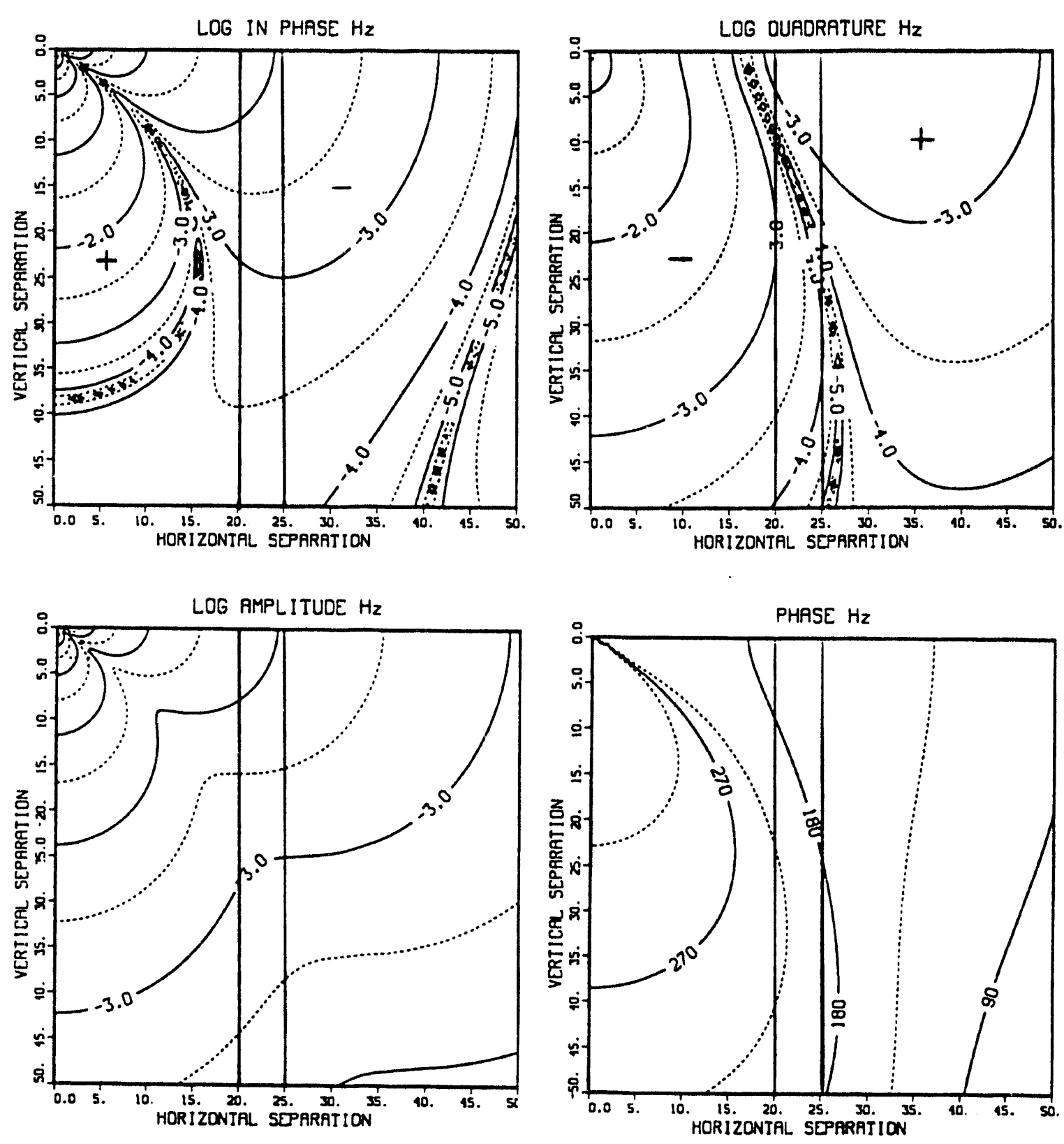

Fig. 2.1-1a The in-phase, quadrature, amplitude and phase of $\mathrm{H}_{\mathrm{z}}$ in a whole space. The axes are horizontal separation, $\rho$, and vertical separation, $\left|z-z_{s}\right|$, between transmitter and receiver. The numbers on the curves are $\log _{10} \mathrm{H}_{z}$ in $n T$ for in-phase, quadrature and amplitude data and degrees for phase data. The $\sigma_{s} f$ product is 795.5. The vertical lines locate the crosshole profiles collected in Richmond. 

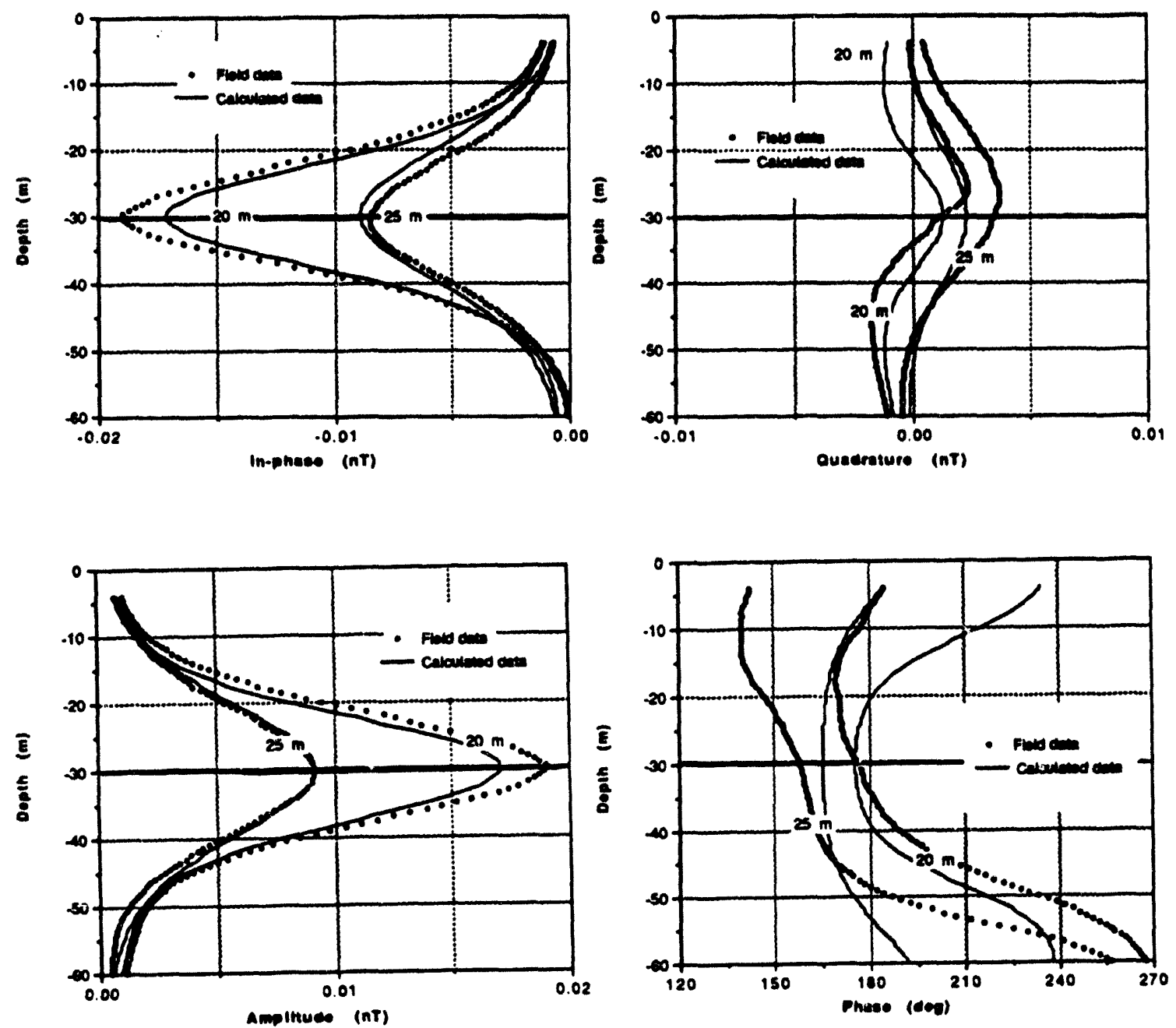

Fig. 2.1-1b Samples oi trosshole measurements of $\mathrm{H}_{\mathrm{z}}$ component from Richmond, California superimposed on the numerical profiles for a whole space. The measurements were taken along the vertical lines marked in Figure 2.1-1a. The receiver is placed 30.0 $m$ below the surface, and is marked by a thick line on the figures. The number on the curves is horizontal separation between wells. The source frequency is $18,500 \mathrm{~Hz}$. The conductivity of the whole-space is $0.043 \mathrm{~S} / \mathrm{m}$. 

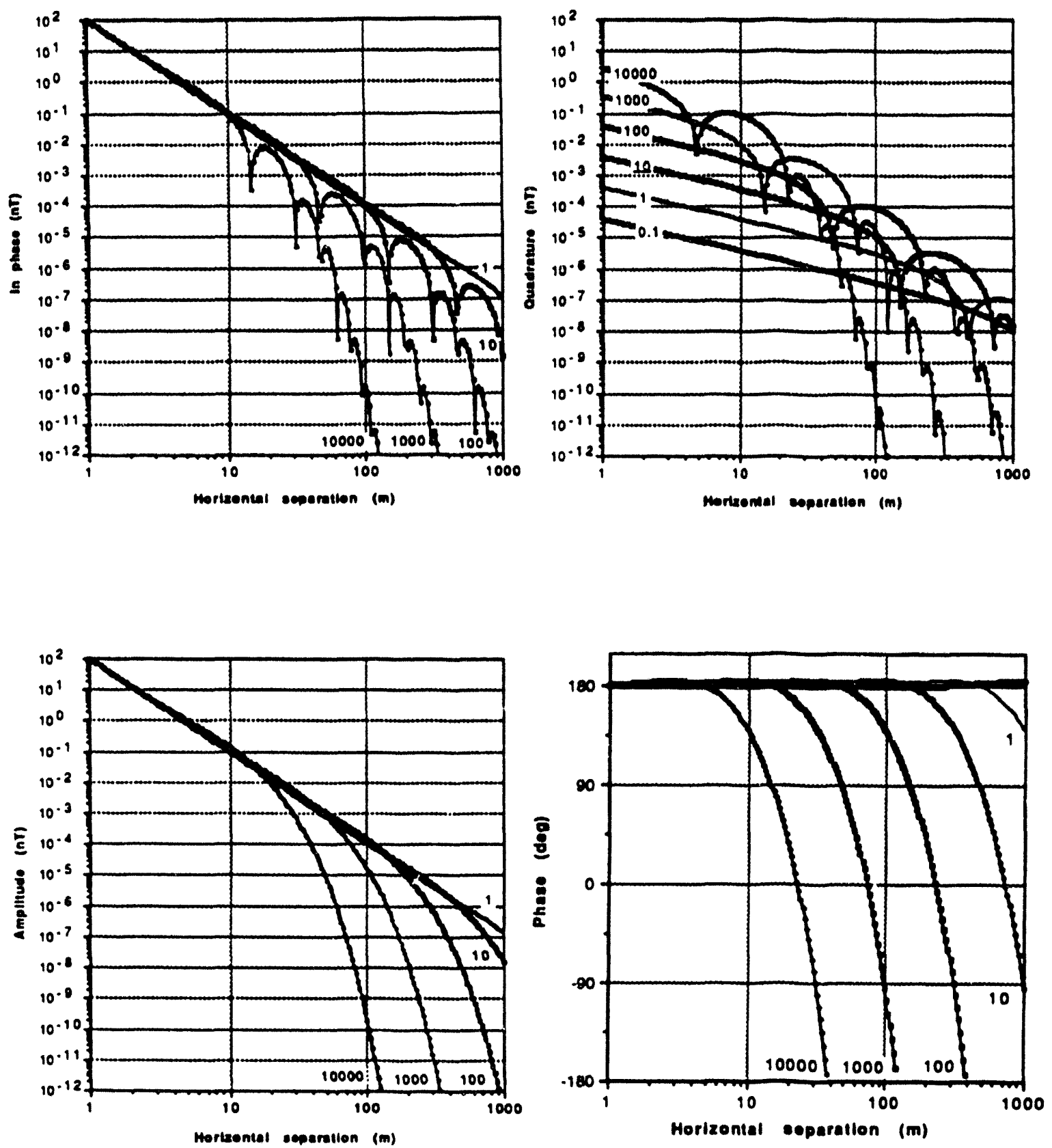

Fig. 2.1-1c The in-phase, quadrature, amplitude and phase of $\mathrm{H}_{\mathrm{z}}$ in a whole space as a function of horizontal separation for transmitter and receiver at the same depth. Curve parameter is the $\sigma_{s} f$ product. 

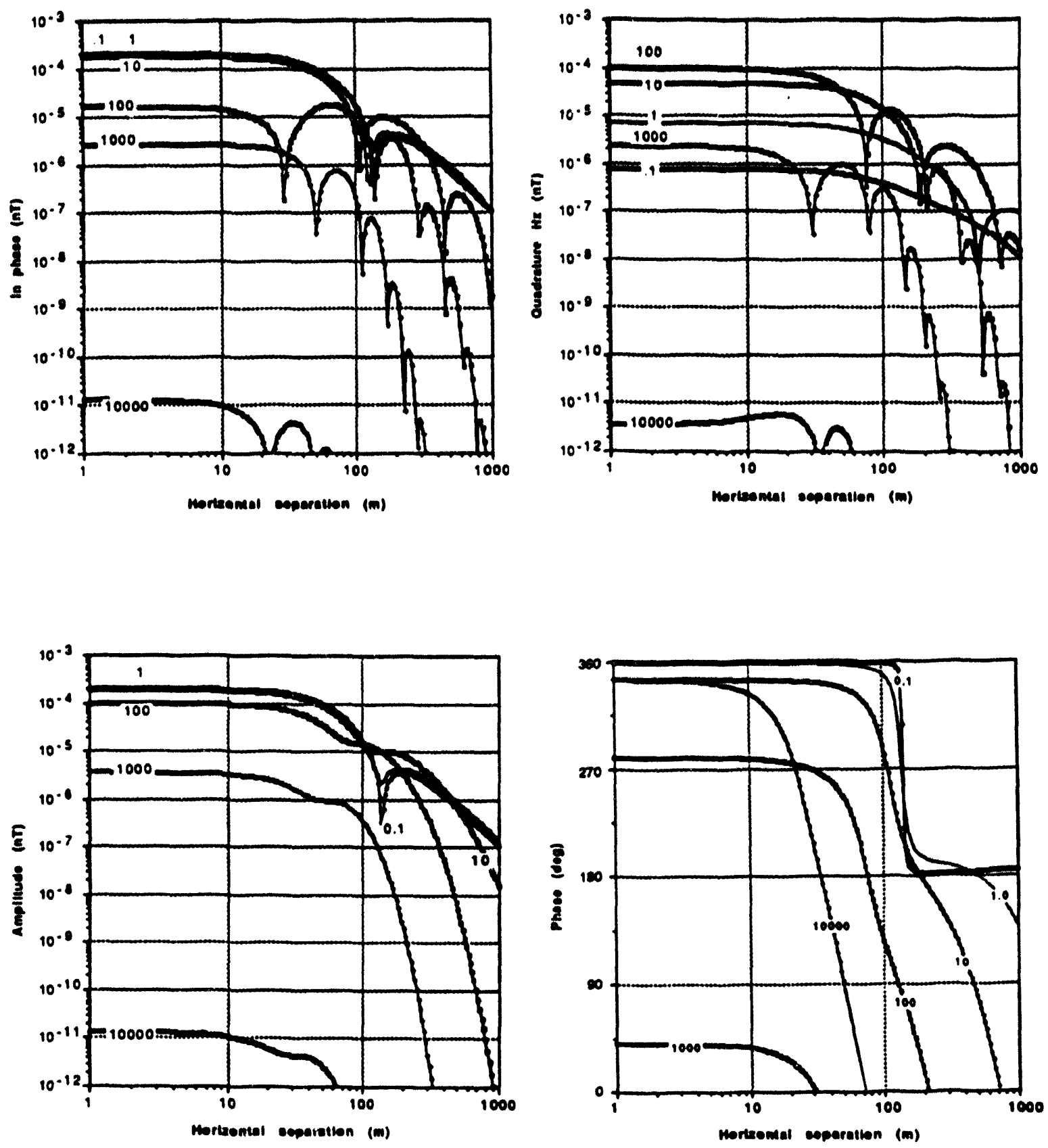

Fig. 2.1-1d The in-phase, quadrature, amplitude and phase of $\mathrm{H}_{2}$ in a whole space as a function of horizontal separation for transmitter placed $100 \mathrm{~m}$ above the receiver. Curve parameter is the $\sigma_{s} f$ product. 

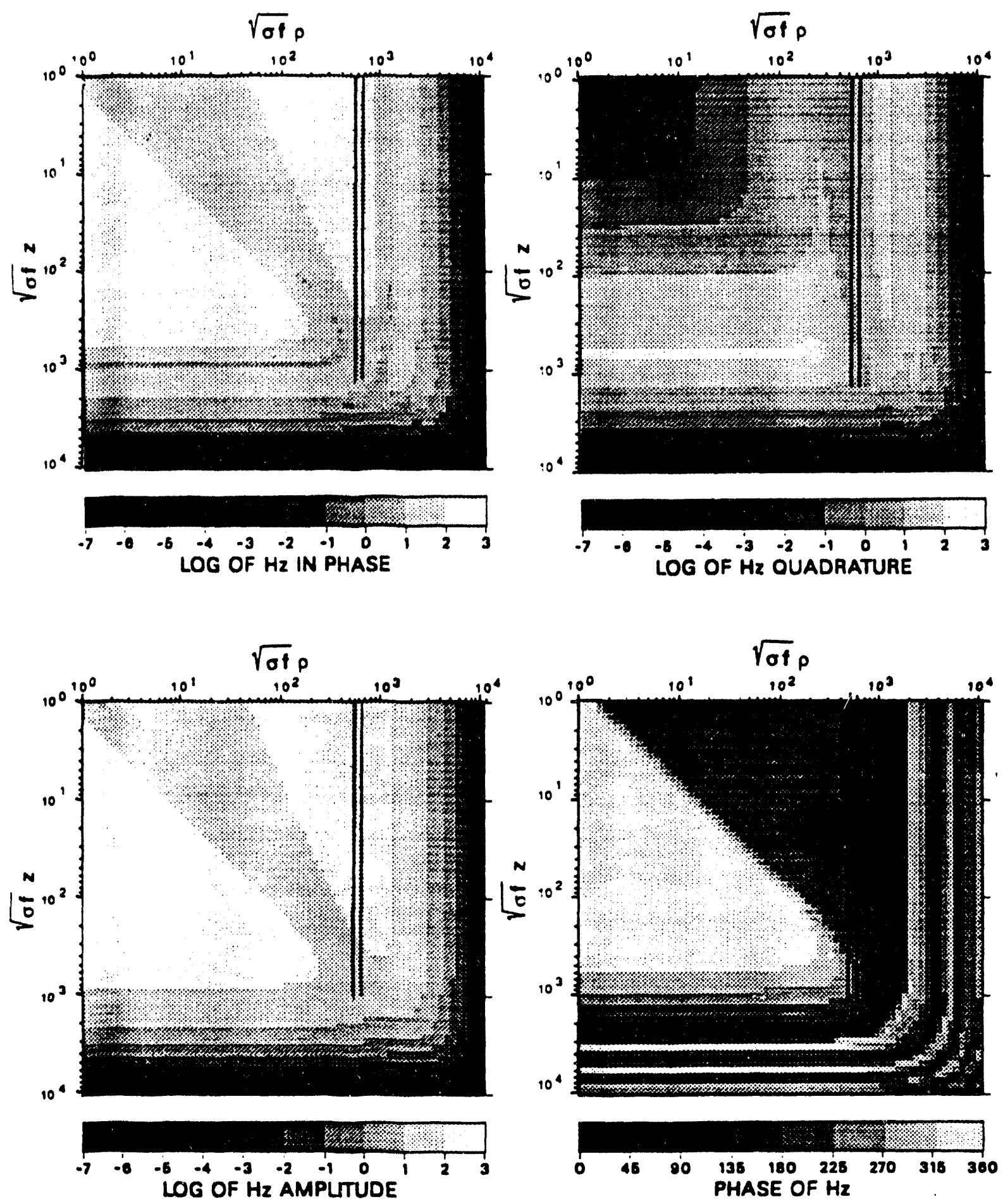

Fig. 2.1-1e The normalized in-phase, quadrature, amplitude and phase of $\mathrm{H}_{2}$ in a whole space. The axes are $\left(\sigma_{s} f\right)^{1 / 2} \rho$ and $\left(\sigma_{s} f\right)^{1 / 2}\left|z-z_{s}\right|$. The numbers on the curves are $\log _{10}\left(\mathrm{H}_{2} \mathrm{R}^{3}\right)$ in $\mathrm{nTm}^{3}$ and degrees for phase data. The vertical lines locate the crosshole profiles collected in Richmond. 

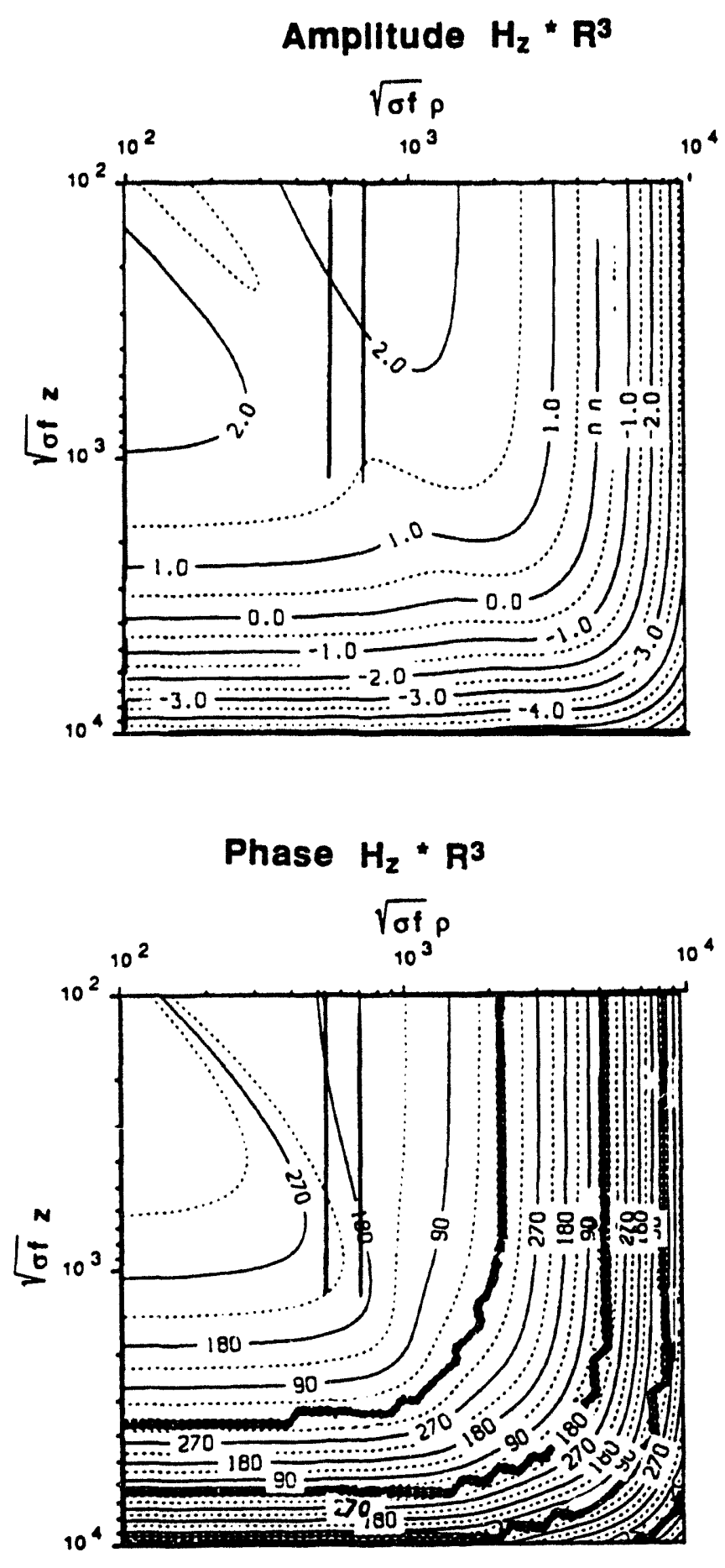

Fig. 2.1-1f A detail from Figure 2.1-1d: the normalized amplitude and phase of $\mathrm{H}_{z}$ in a whole space. The axes are $\left(\sigma_{s} f\right)^{1 / 2} \rho$ and $\left(\sigma_{s} f\right)^{1 / 2}\left|z-z_{s}\right|$. The numbers on the curves are $\log _{10}\left(\mathrm{H}_{2} \mathrm{R}^{3}\right)$ in $n \mathrm{Tm}^{3}$ and degrees for phase data. The vertical lines locate the crosshole profiles collected in Richmond. 

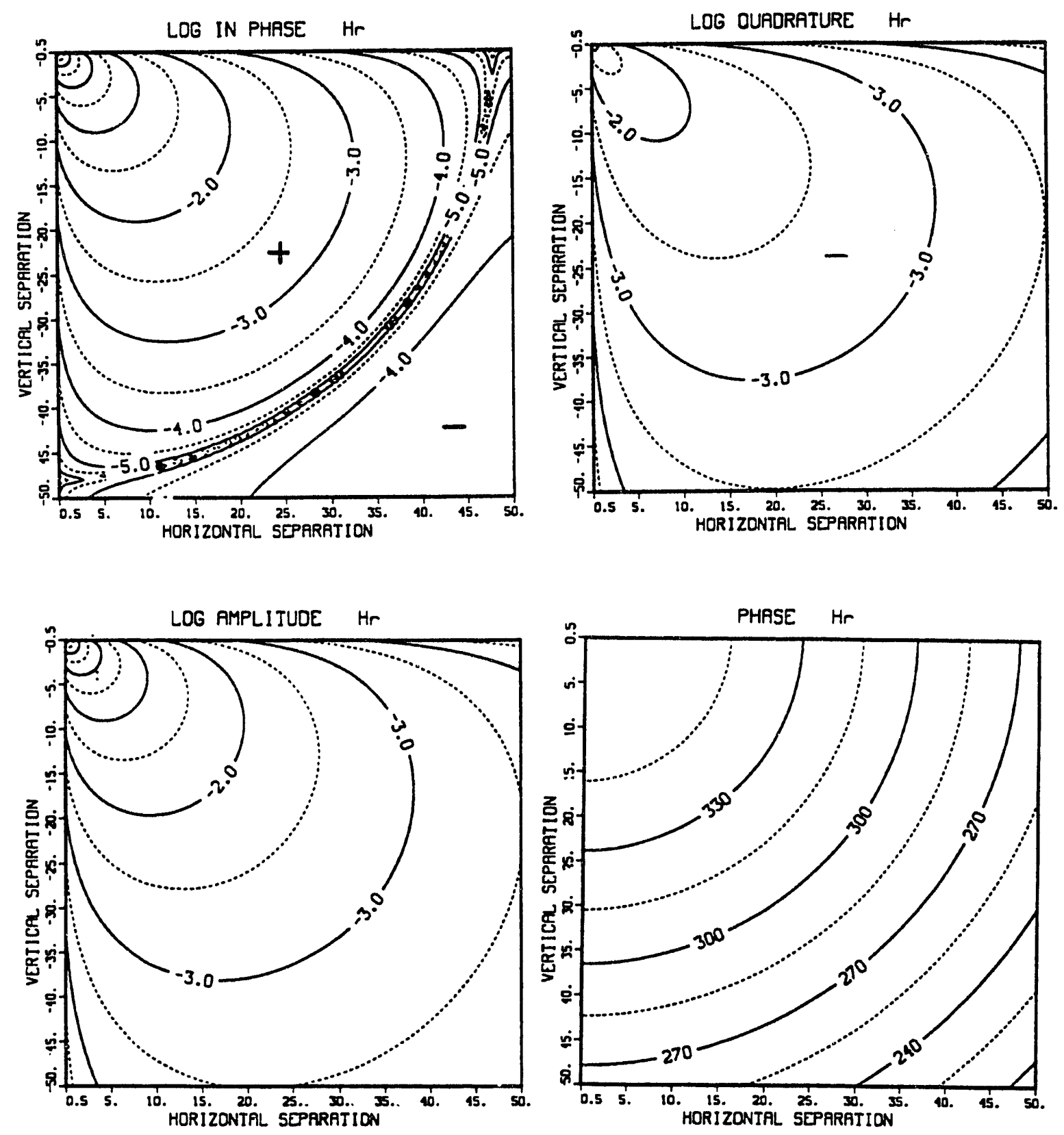

Fig. 2.1-2a The in-phase, quadrature, amplitude and phase of $\mathrm{H}_{\mathrm{p}}$ in a whole space. The axes are horizontal separation, $\rho$, and vertical separation, $\left|z-z_{s}\right|$, between transmitter and receiver. The numbers on the curves are $\log _{10} \mathrm{H}_{\rho}$ in $\mathrm{nT}$ for in-phase, quadrature and amplitude data and degrees for phase data. The $\sigma_{\mathrm{s}} \mathrm{f}$ product is 795.5. 

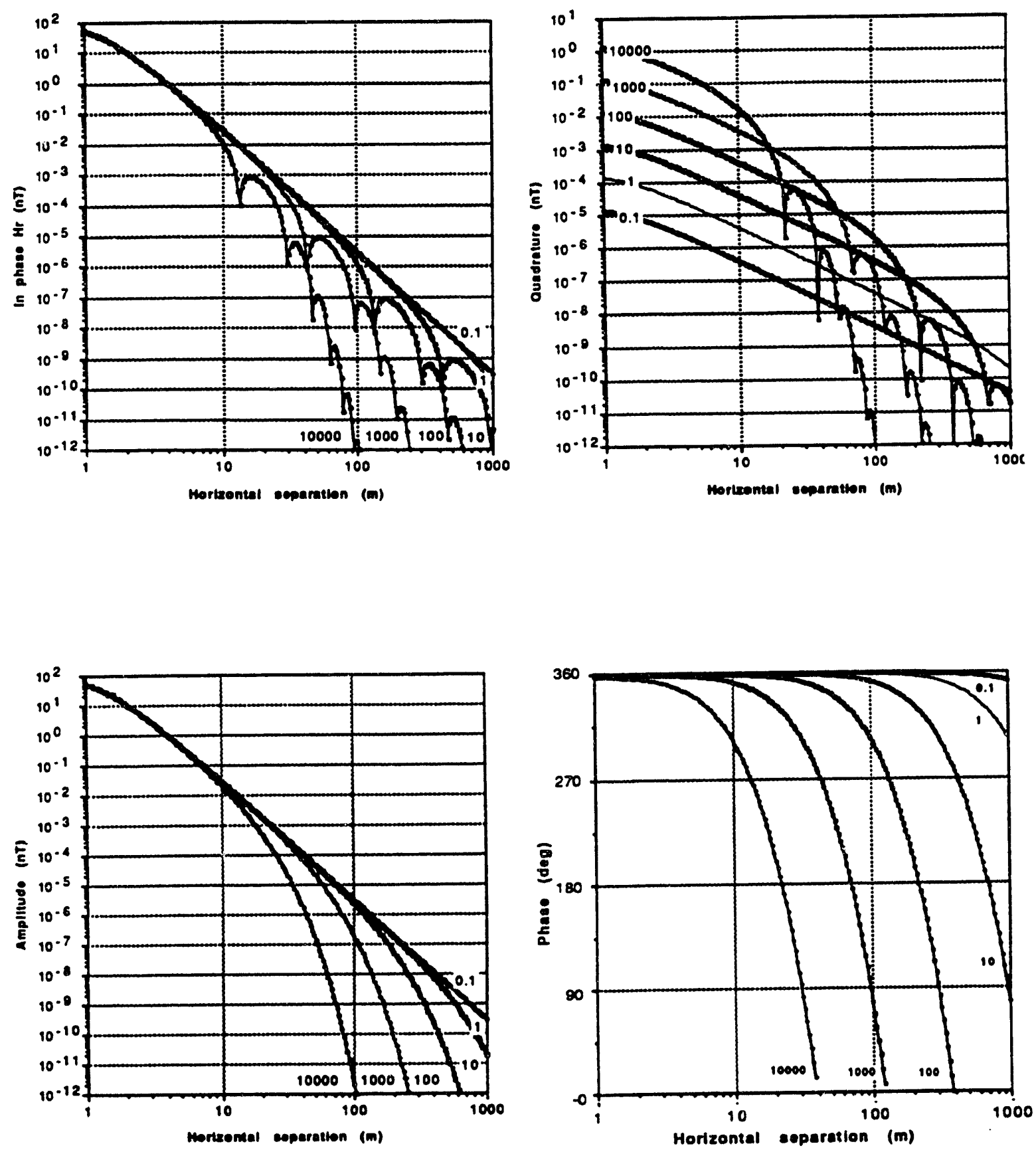

Fig. 2.1-2b The in-phase, quadrature, amplitude and phase of $\mathrm{H}_{\mathrm{p}}$ in a whole space as a function of horizontal separation for transmitter placed $1.0 \mathrm{~m}$ above receiver. Curve parameter is the $\sigma_{\mathrm{s}} \mathrm{f}$ product. 

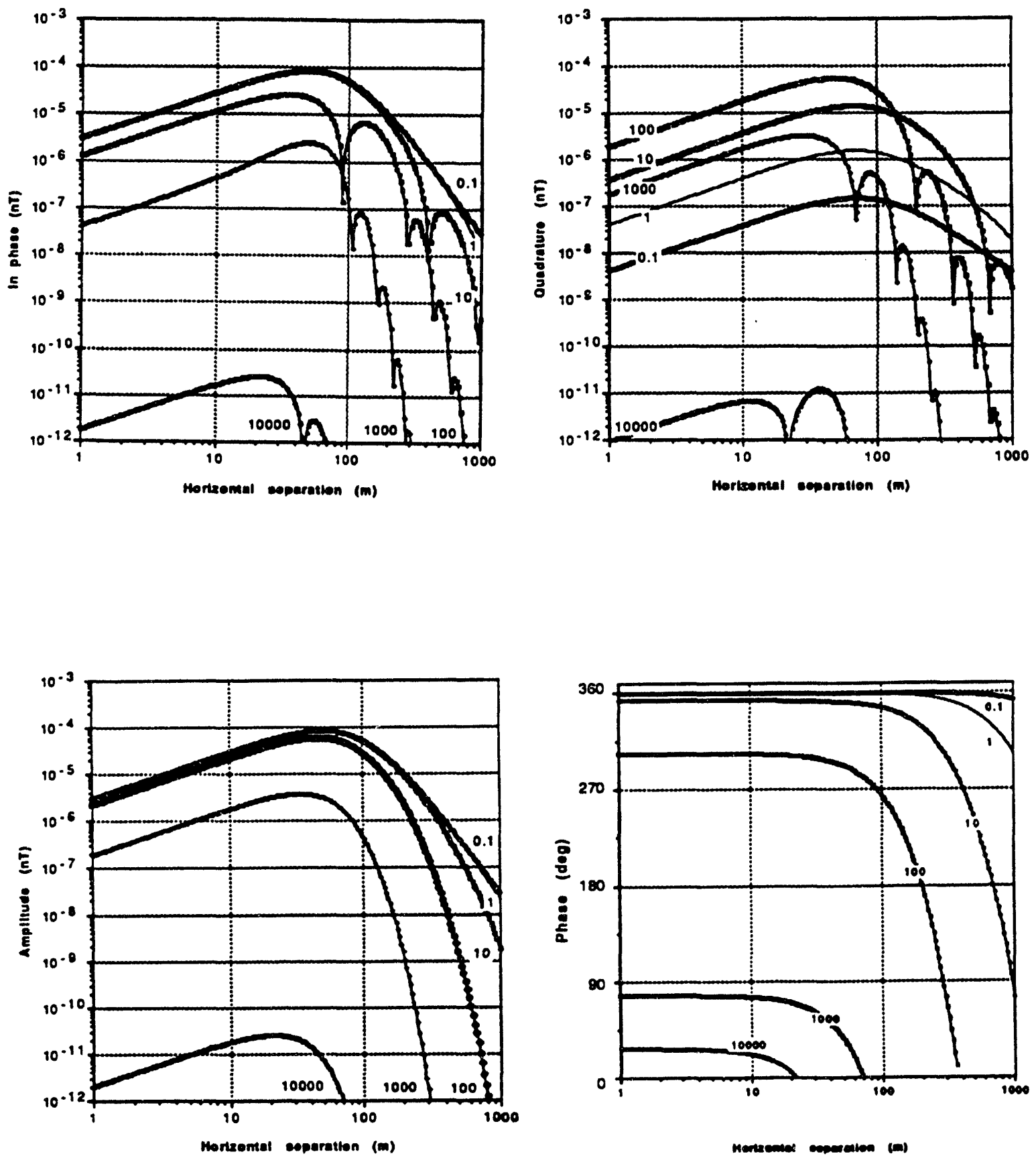

Fig. 2.1-2c The in-phase, quadrature, amplitude and phase of $\mathrm{H}_{\mathrm{p}}$ in a whole space as a function of horizontal separation for transmitter placed $100.0 \mathrm{~m}$ above receiver. Curve parameter is the $\sigma_{\mathrm{s}} \mathrm{f}$ product. 

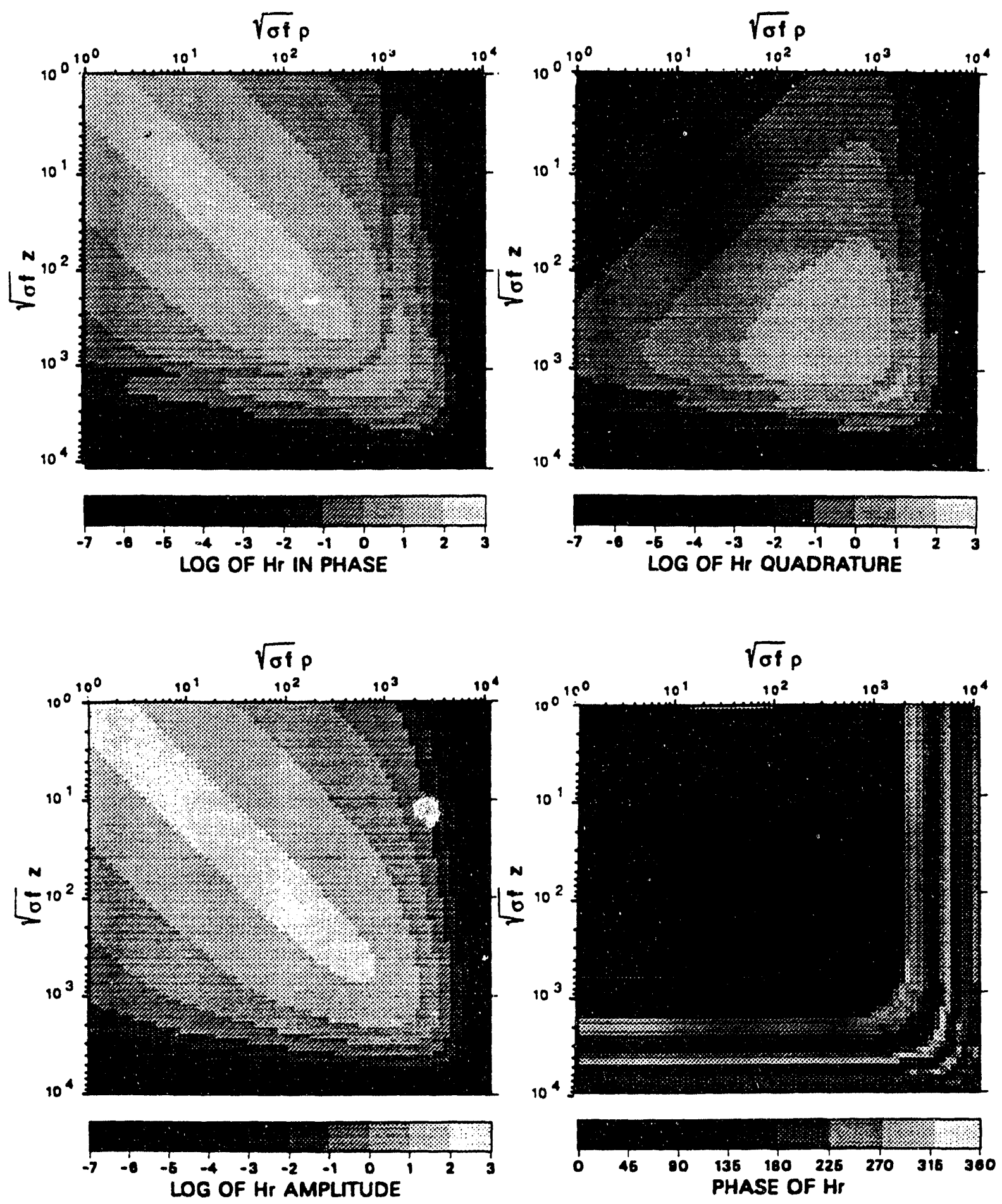

Fig. 2.1-2d The normalized in-phase, quadrature, amplitude and phase of $\mathrm{H}_{\mathrm{p}}$ in a whole space. The axes are $\left(\sigma_{s} f\right)^{1 / 2} \rho$ and $\left(\sigma_{s} f\right)^{1 / 2}\left|z-z_{s}\right|$. The numbers on the curves are $\log _{10}\left(\mathrm{H}_{\rho} \mathrm{R}^{3}\right)$ in $n \mathrm{Tm}^{3}$ and degrees for phase data. 

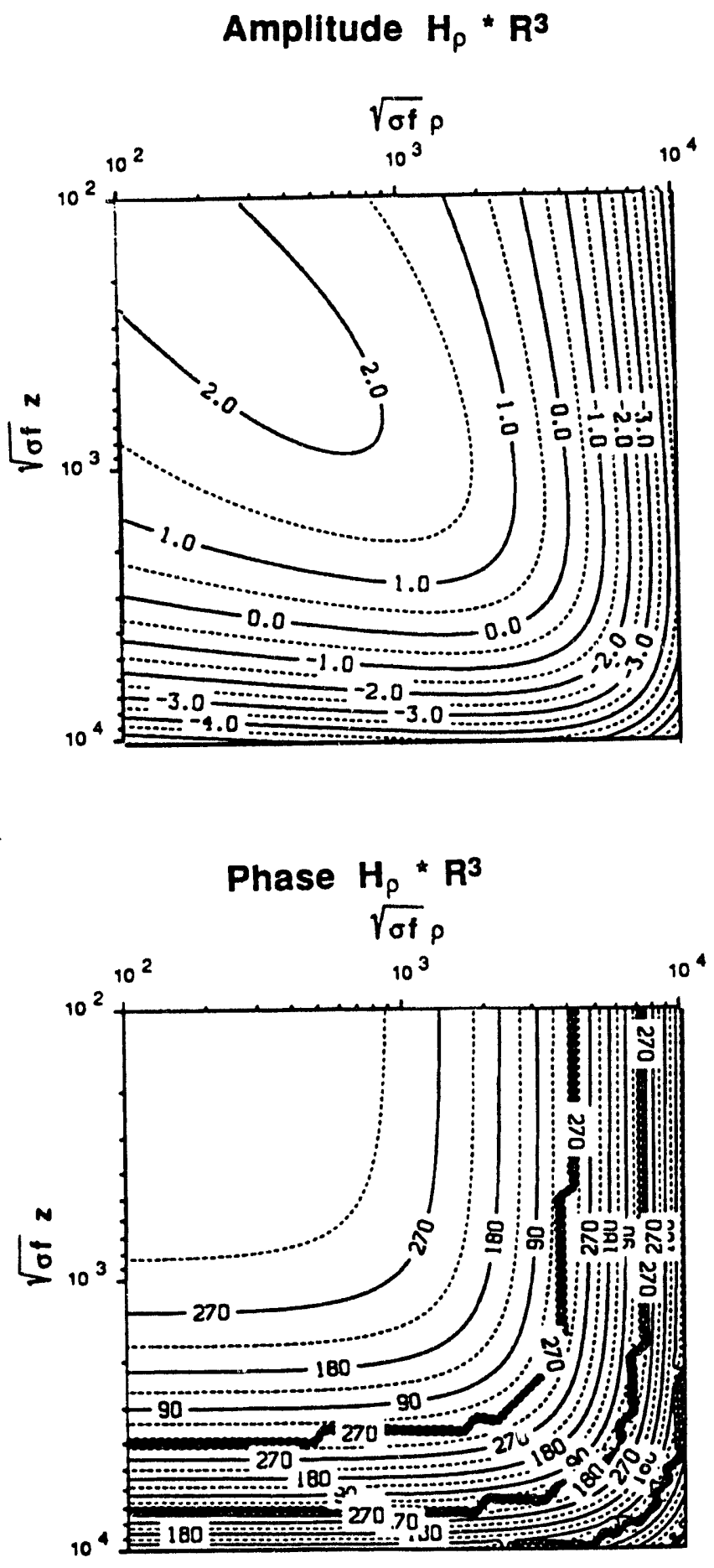

Fig. 2.1-2e A detail from Figure 2.1-2d: the normalized amplitude and phase of $\mathrm{H}_{\mathrm{p}}$ in a whole space. The axes are $\left(\sigma_{s} f\right)^{1 / 2} \rho$ and $\left(\sigma_{s} f\right)^{1 / 2}\left|z-z_{s}\right|$. The numbers on the curves are $\log _{10}\left(\mathrm{H}_{\mathrm{p}} \mathrm{R}^{3}\right)$ in $n \mathrm{Tm}^{3}$ and degrees for phase data. 

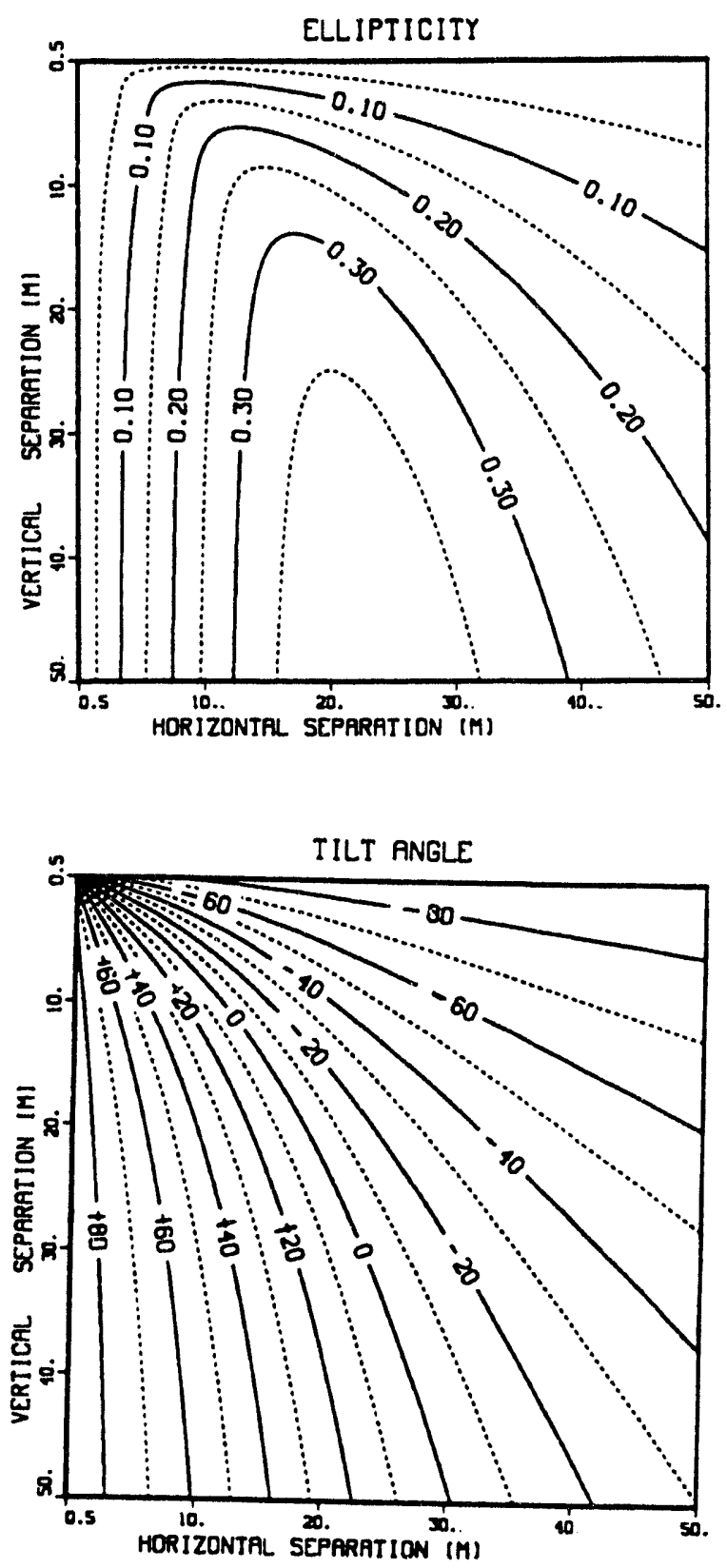

Fig. 2.1-3a The ellipticity and tilt angle in a whole space. The axes are horizontal separation, $\rho$ and vertical separation, $\left|z-z_{s}\right|$, between transmitter and receiver. The $\sigma_{s} f$ product is 795.5 . 

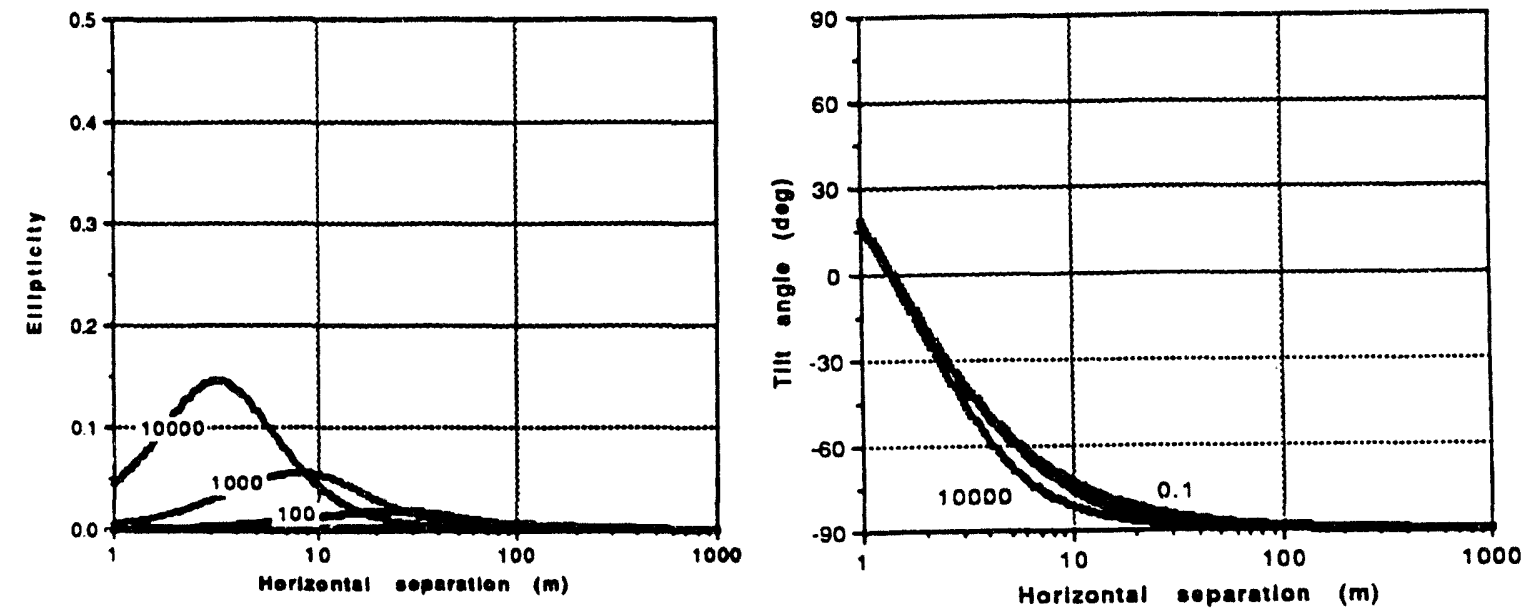

Fig. 2.1-3b The ellipticity and tilt angle in a whole space as a function of horizontal separation for transmitter placed $1.0 \mathrm{~m}$ above receiver. Curve parameter is the $\sigma_{\mathrm{s}} \mathrm{f}$ product.
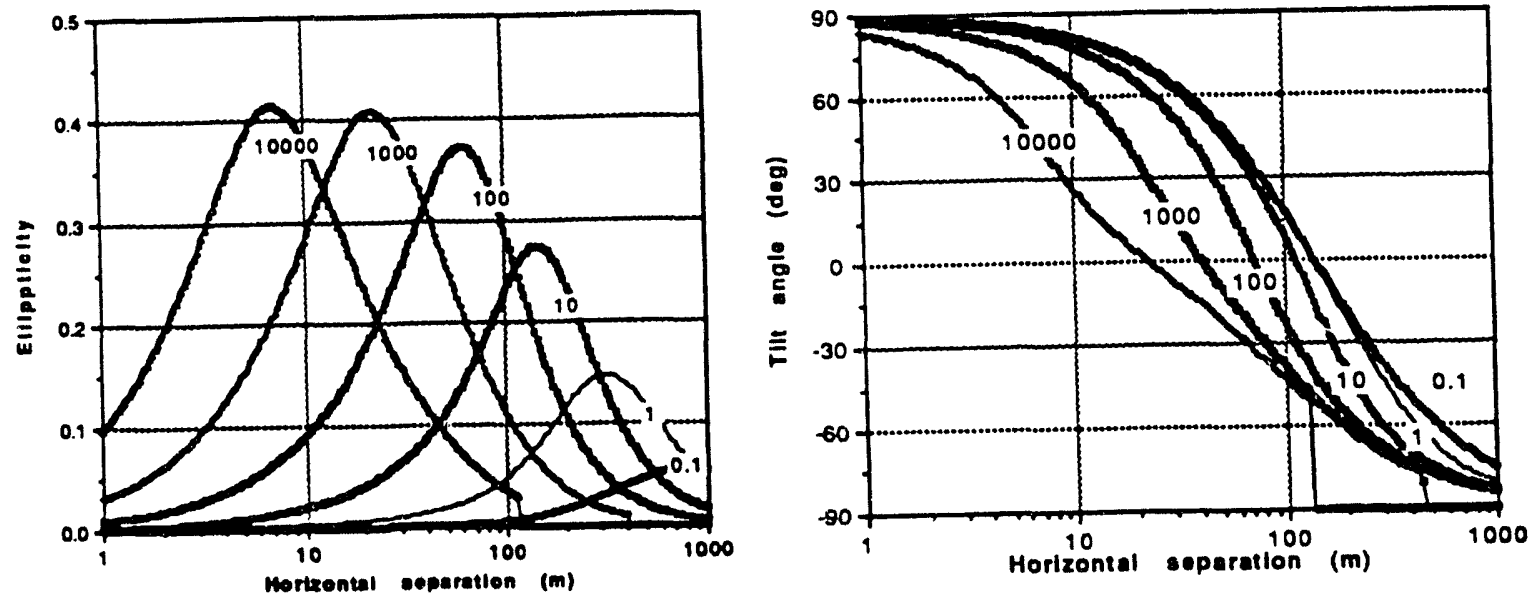

Fig. 2.1-3c The ellipticity and tilt angle in a whole space as a function of horizontal separation for transmitter placed $100.0 \mathrm{~m}$ above receiver. Curve parameter is the $\sigma_{\mathrm{s}} \mathrm{f}$ product. 

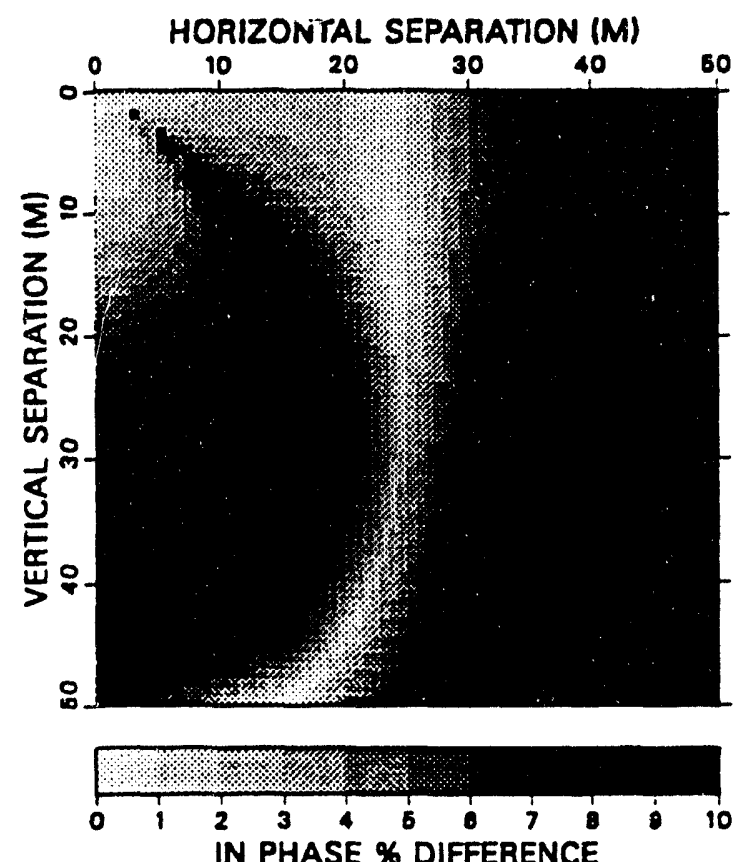

IN PHASE \% DIFFERENCE
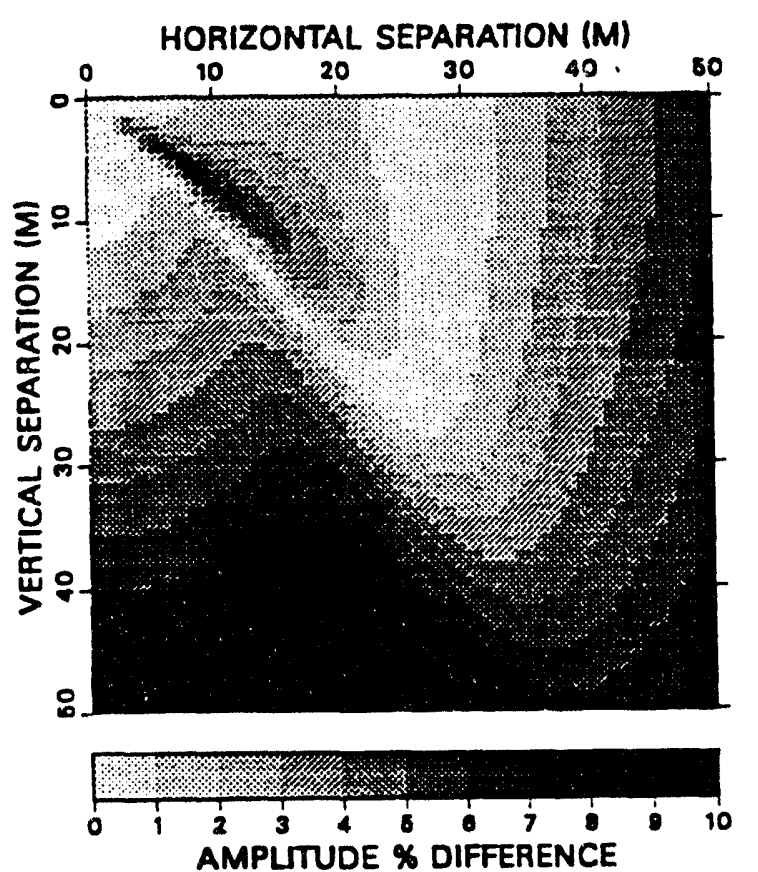
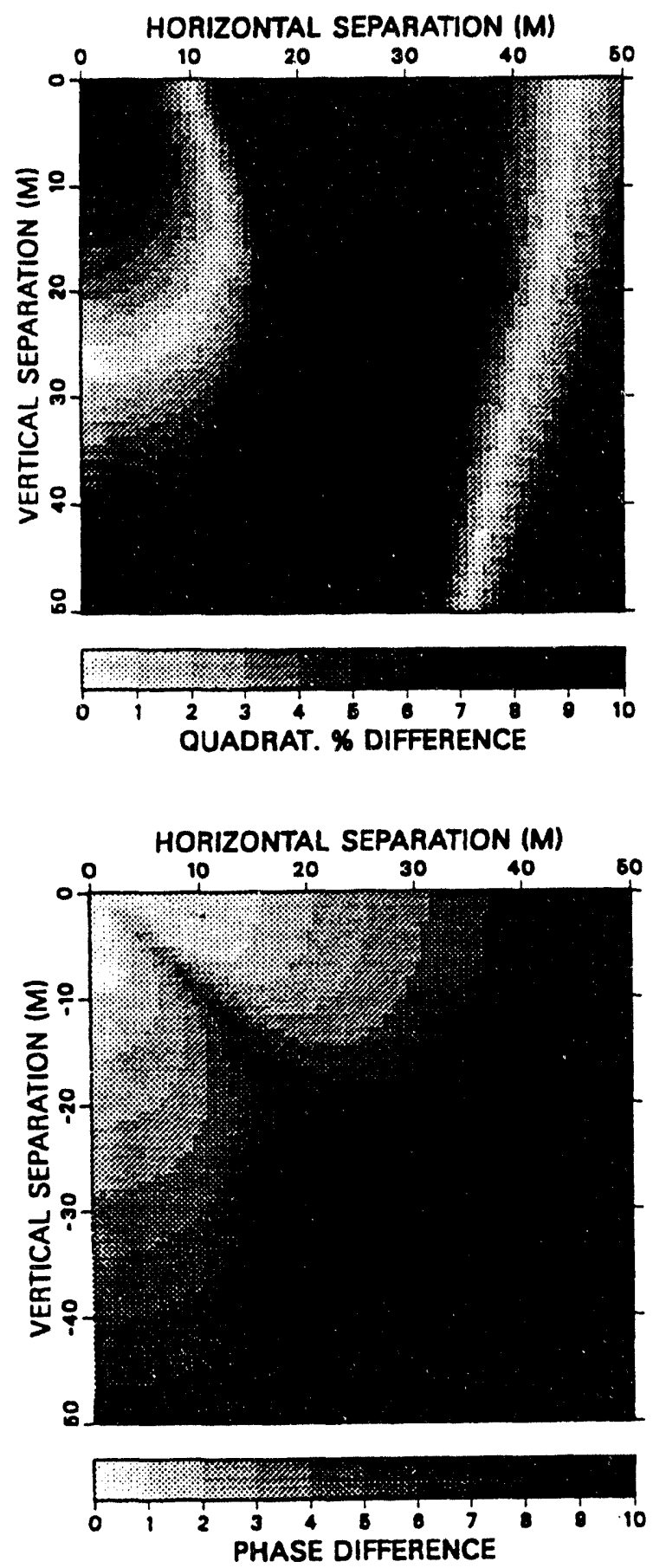

Fig. 2.2-1 In-phase, quadrature and amplitude percent change and phase shift of $\mathrm{H}_{\mathrm{z}}$ component due to conductivity change from $0.043 \mathrm{~S} / \mathrm{m}$ to $0.047 \mathrm{~S} / \mathrm{m}$ in a whole space where $\sigma_{\mathrm{s}} \mathrm{f}=795.5$. The vertical lines locate the crosshole profiles collected in Richmond. 


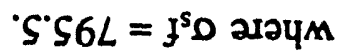

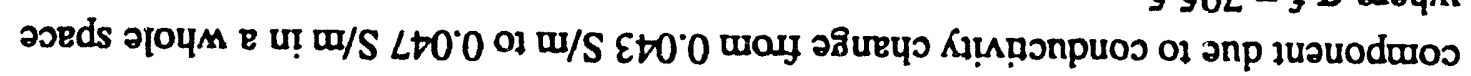

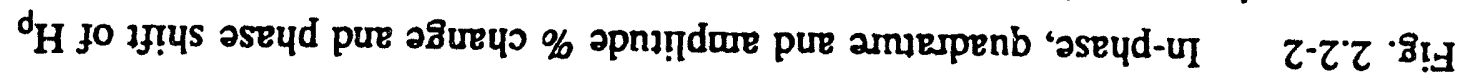
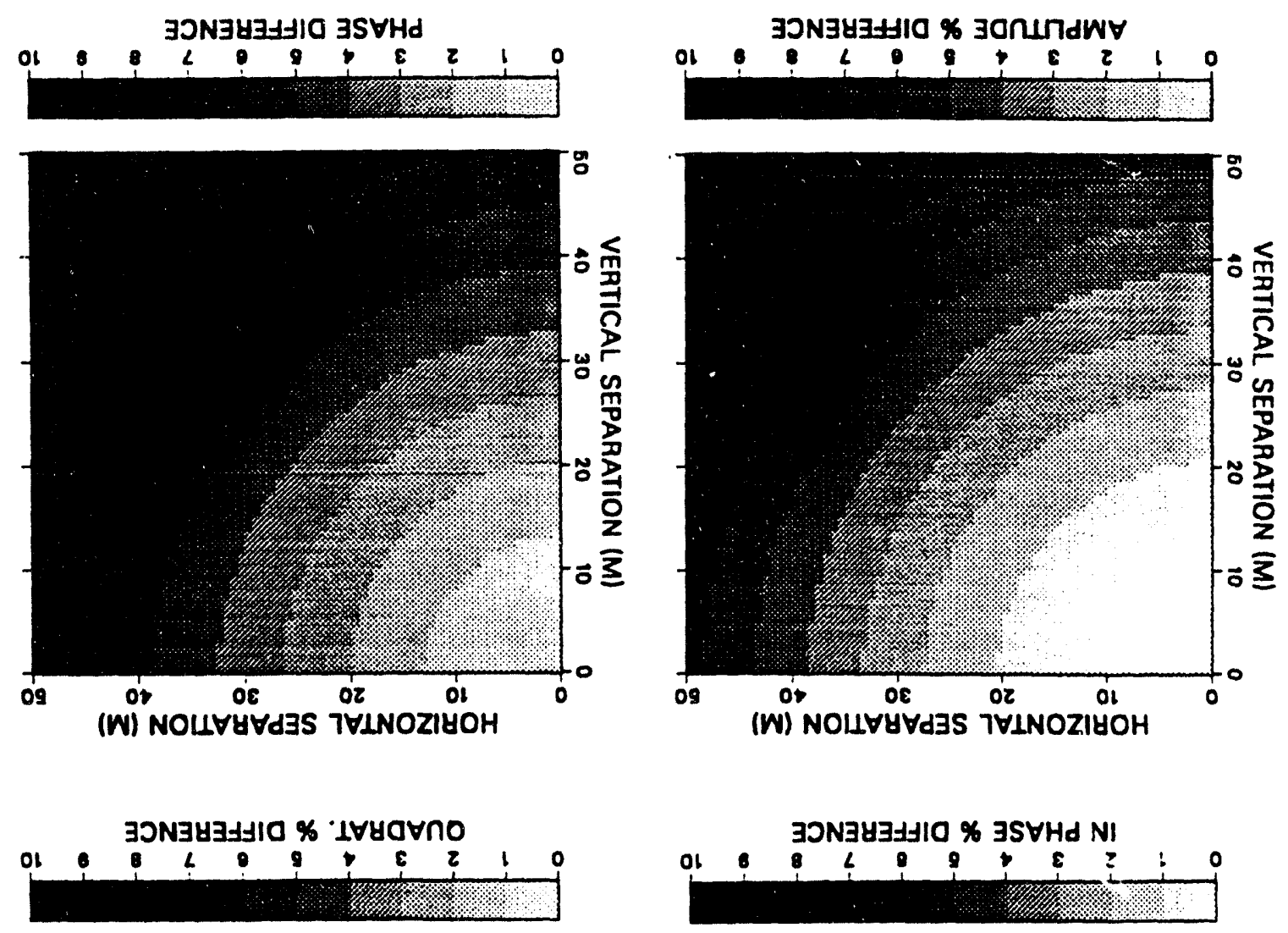

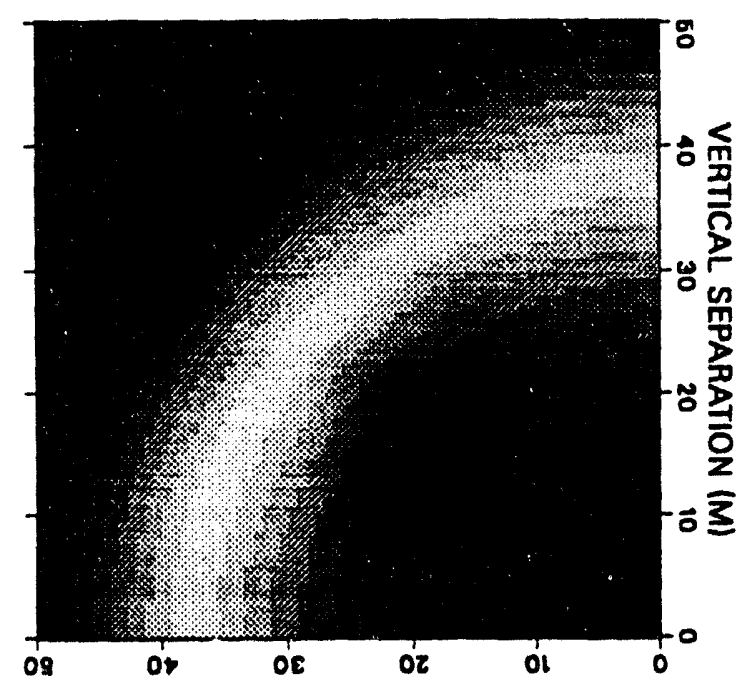

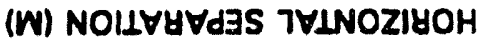

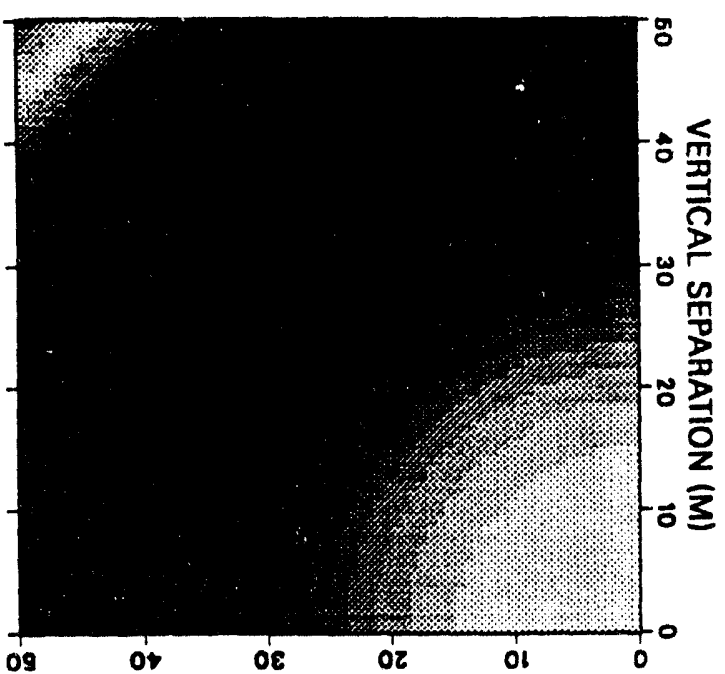

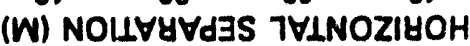


Log in minst da/da

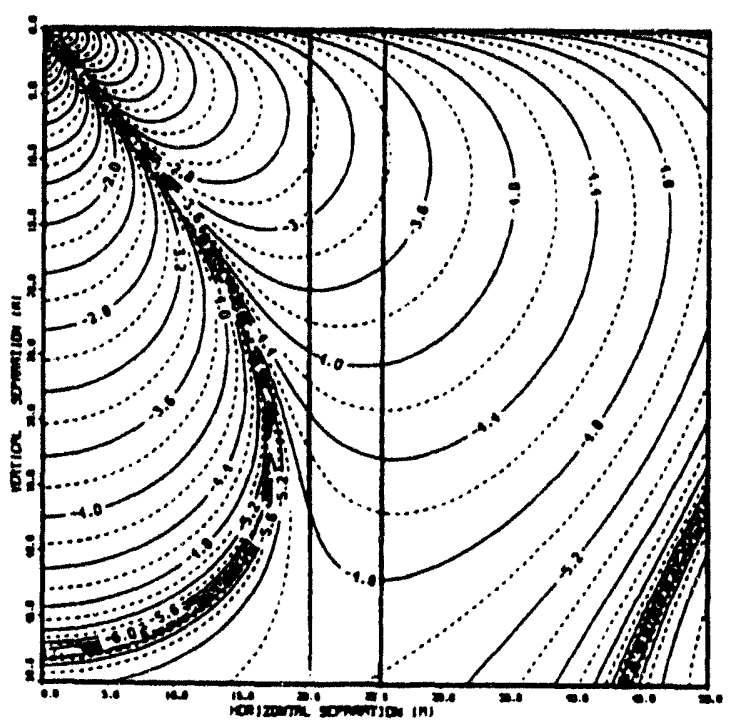

Los mitroe etroz

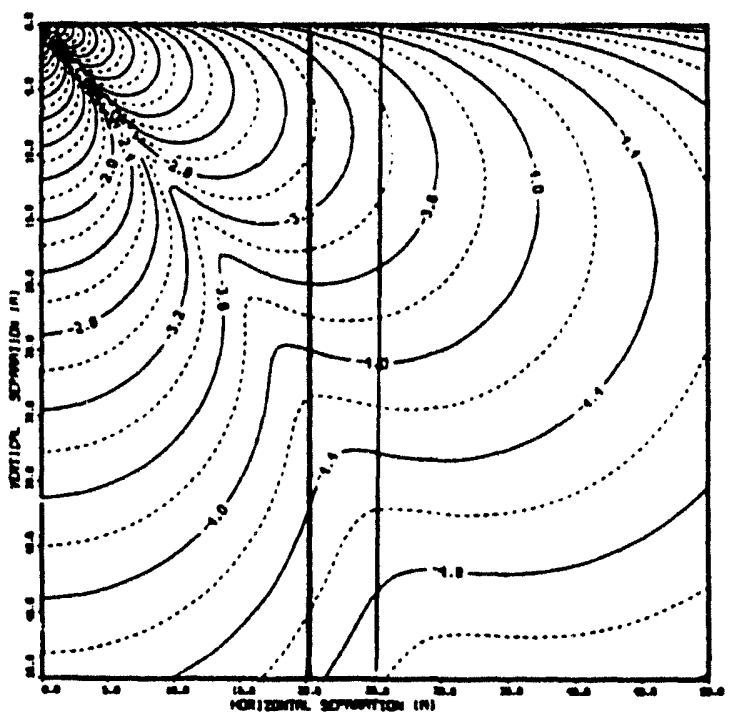

Los andownune arads

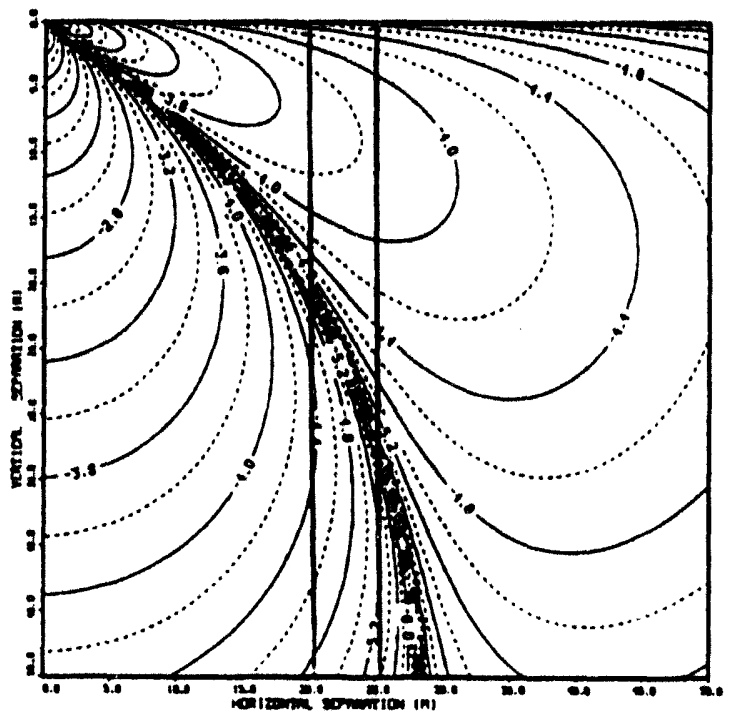

20

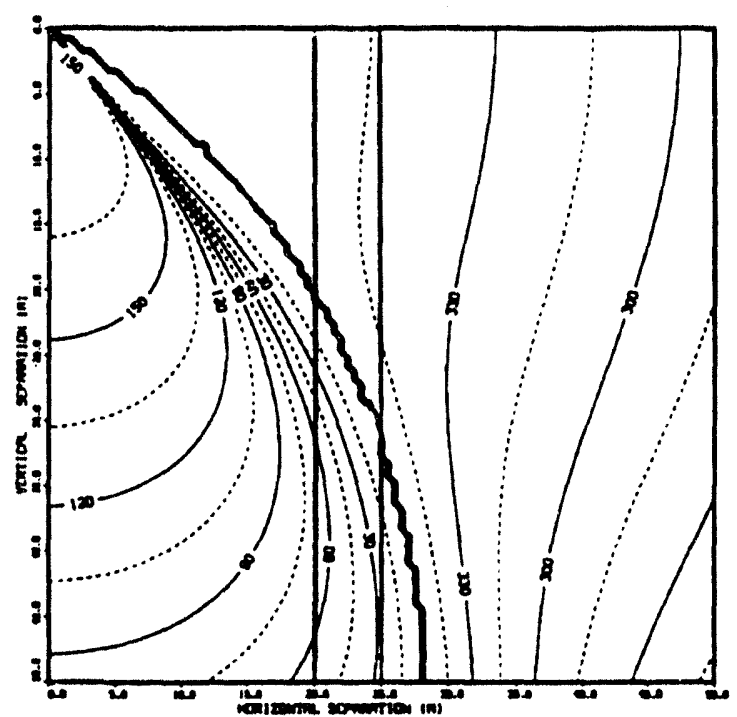

Fig. 2.3-1a The in-phase, quadrature, amplitude and phase of $\delta \mathrm{H}_{2} / \delta \mathrm{z}$ in a whole space. The axes are horizontal separation, $\rho$, and vertical separation, $\left|z-z_{s}\right|$, between transmitter and receiver. The numbers on the curves are $\log _{10}$ for in-phase, quadrature and amplitude data in $\mathrm{nT} / \mathrm{m}$ and degrees for phase data. The $\sigma_{8} f$ product is 795.5. The vertical lines locate the crosshole profiles collected in Richmond. 

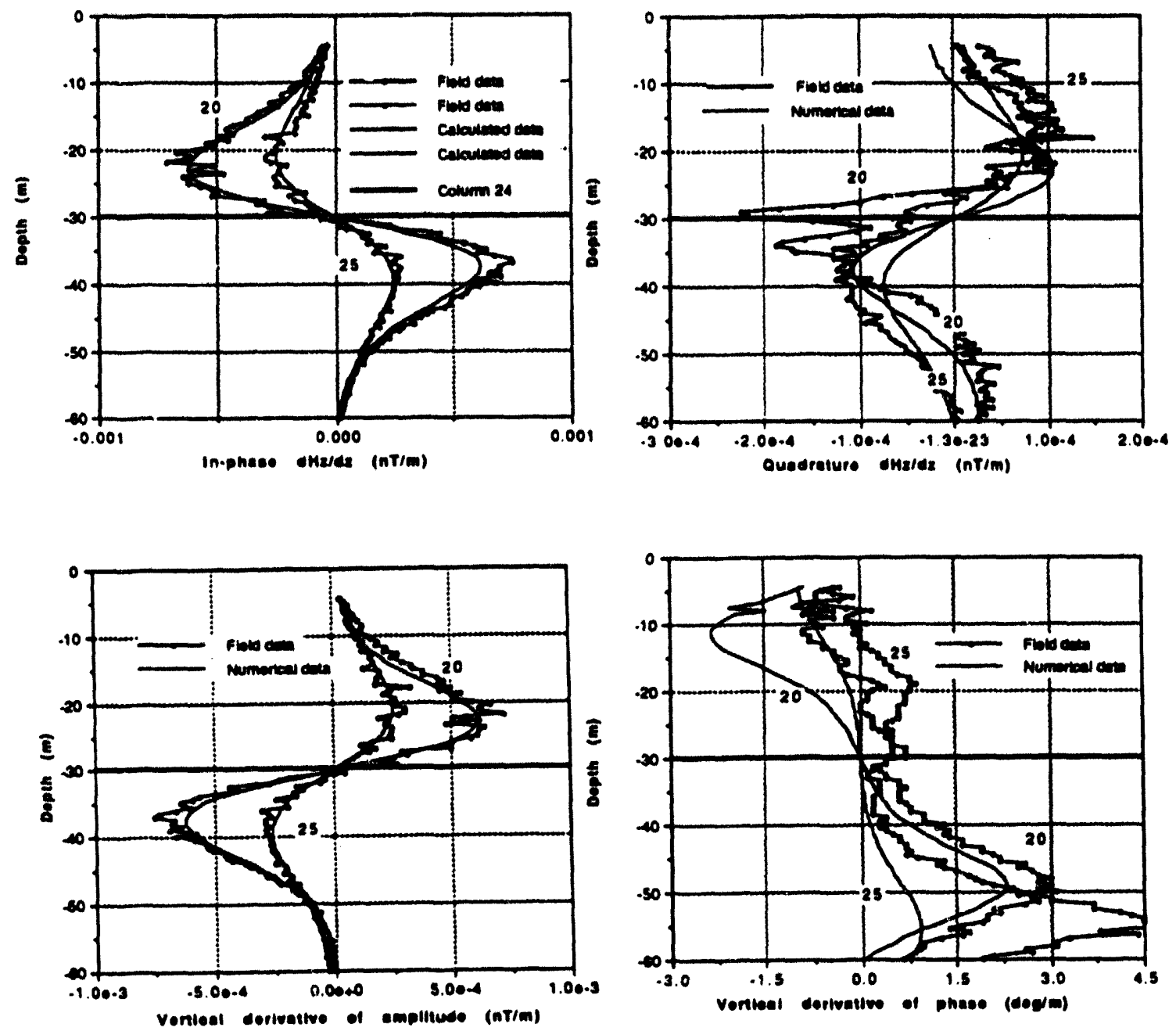

Fig. 2.3-1b Samples of the vertical derivatives from crosshole measurements from Richmond, California superimposed on the numerical profiles for a whole-space. The measurements were taken along the vertical lines marked in Figure 2.3-1a. The curves are $\Delta\left(\operatorname{ReH}_{z}\right) / \Delta z, \Delta\left(\operatorname{ImH}_{z}\right) / \Delta z, \Delta\left(\left(\mathrm{H}_{z}\right)\right) / \Delta z, \Delta\left(\right.$ phaseH $\left._{z}\right) / \Delta z$. The receiver is placed 30.0 $\mathrm{m}$ below the surface, and is marked by a thick line on the figures. The number on the curves is horizontal separation between wells. The source frequency is $18,500 \mathrm{~Hz}$. 
puomyग!y

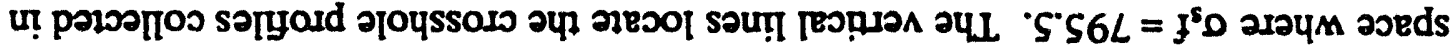

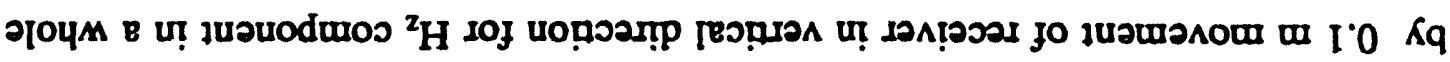

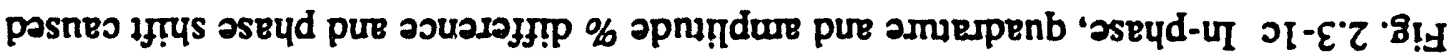
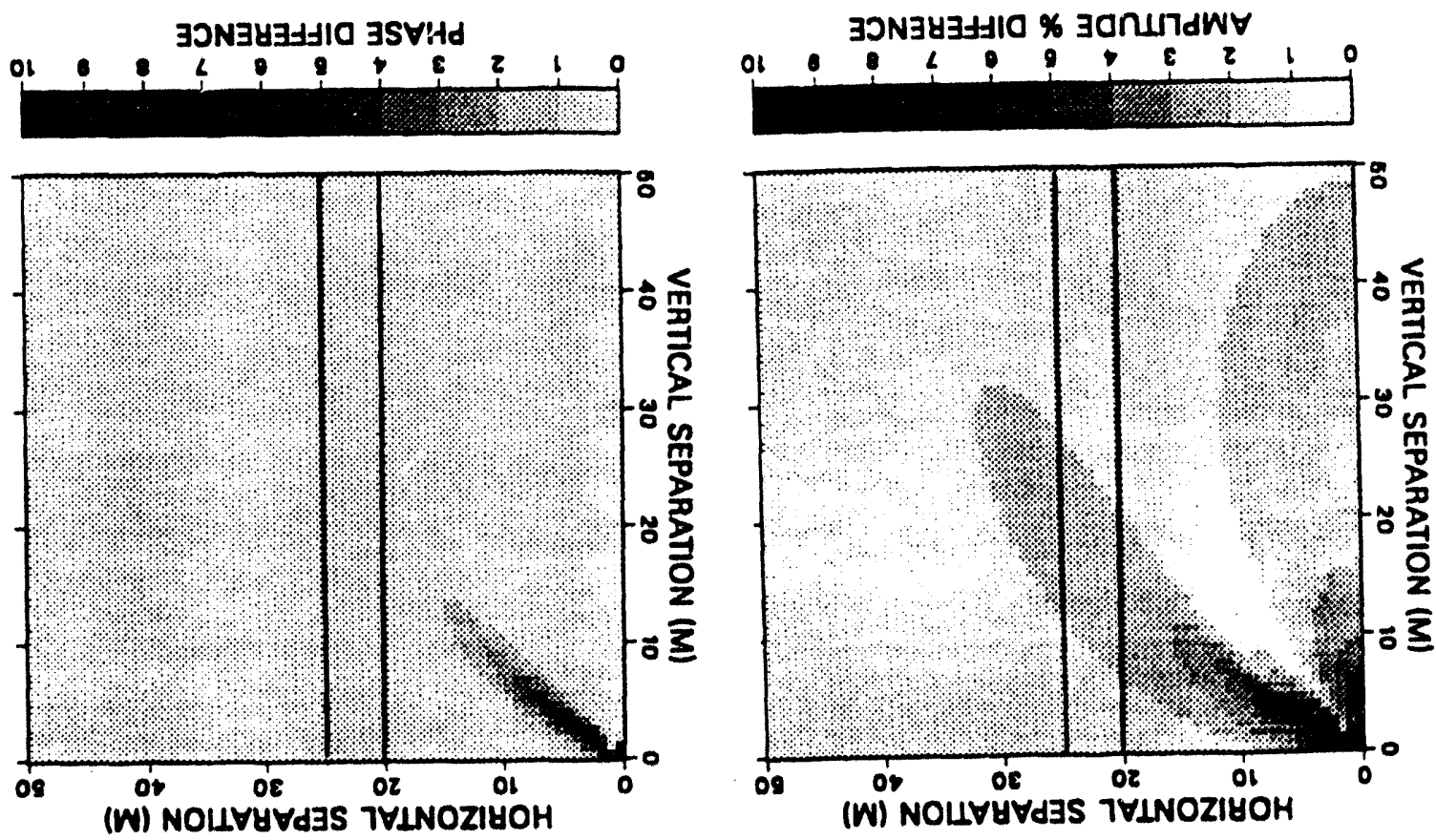

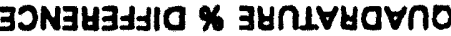
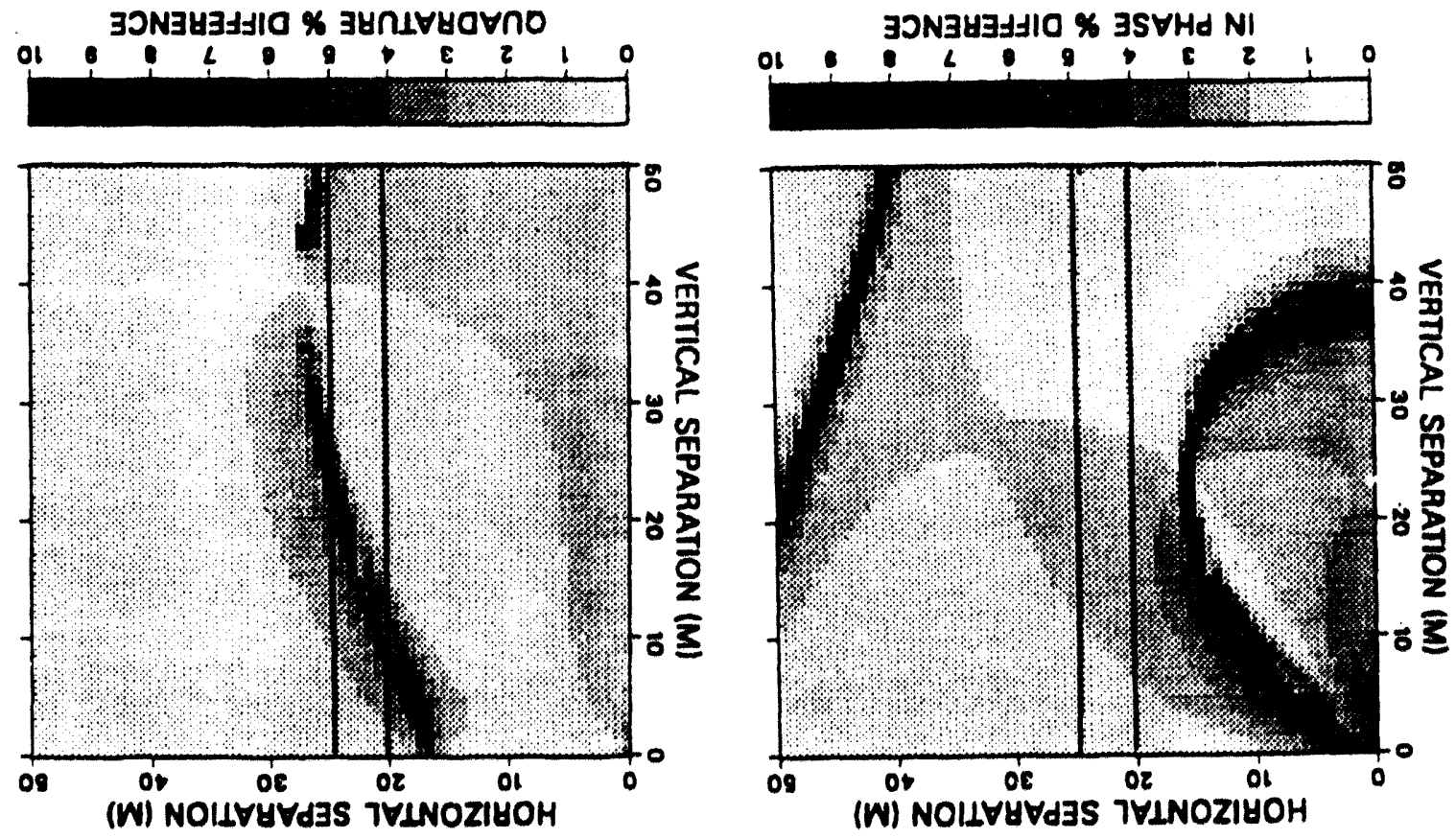

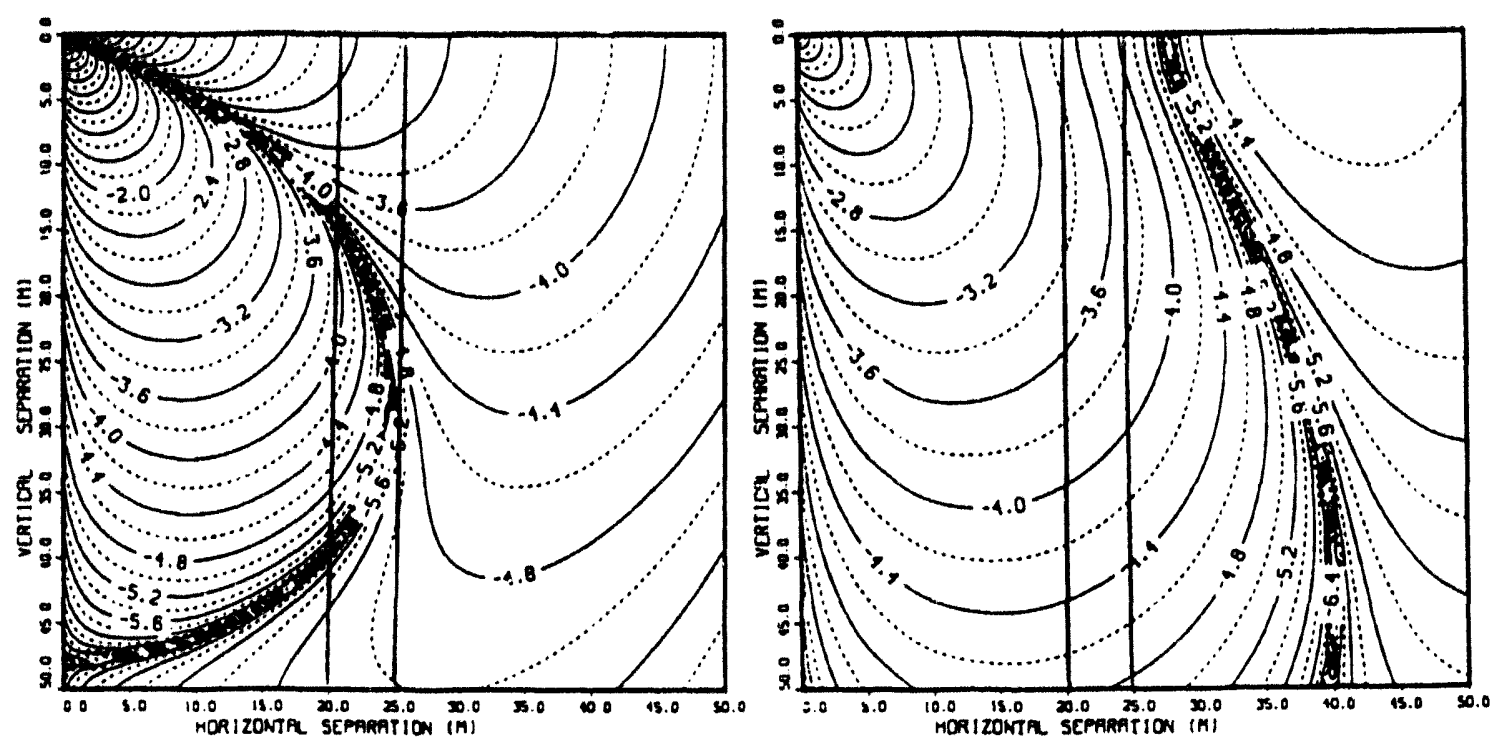

LOC MPLITUOC anzor

PMasconz/de
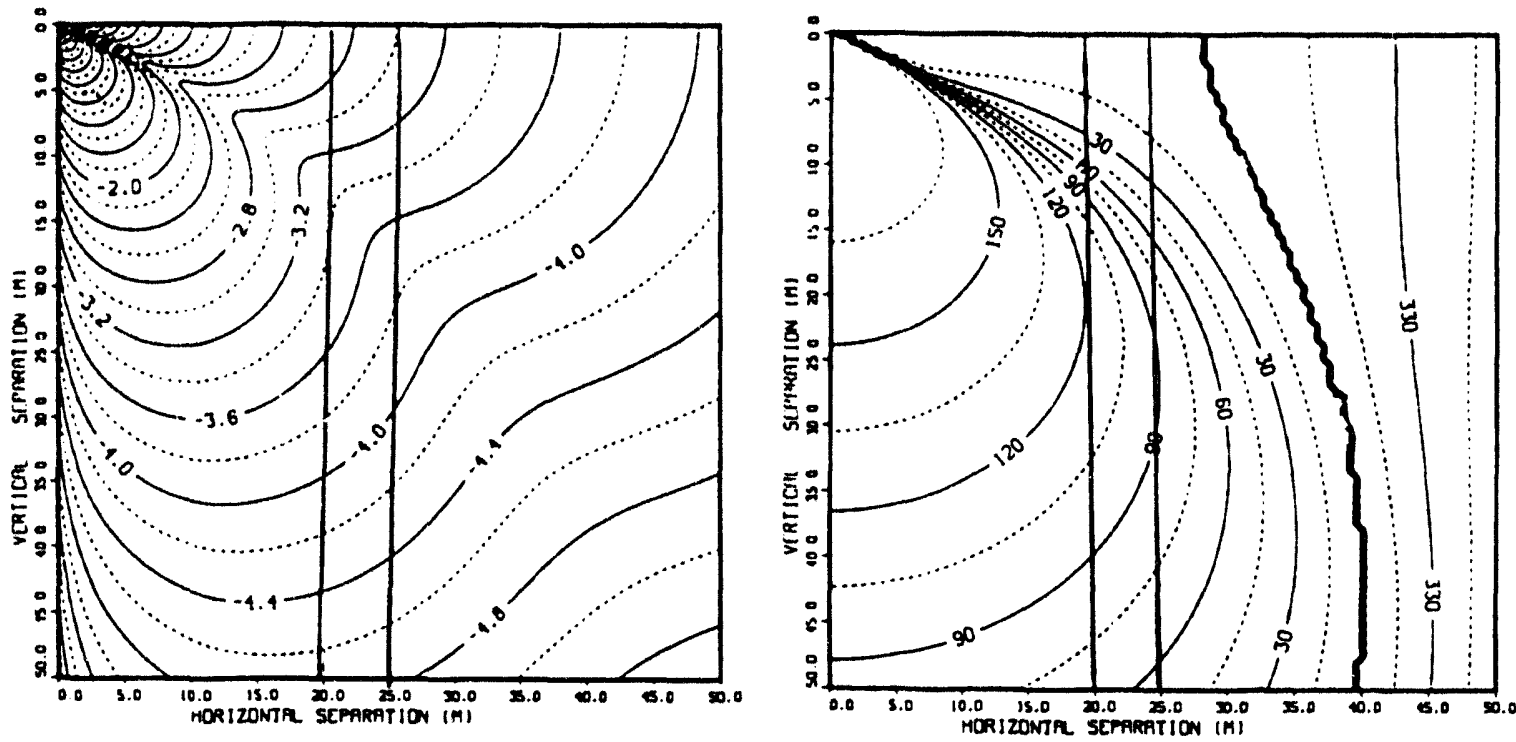

Fig. 2.3-2a The in-phase, quadrature, amplitude and phase of $\delta \mathrm{H}_{2} / \delta \rho$ in a whole space. The axes are horizontal separation, $\rho$, and vertical separation, $\left|z-z_{s}\right|$, between transmitter and receiver. The numbers on the curves are $\log _{10}$ for in-phase, quadrature and amplitude data in $\mathrm{nT} / \mathrm{m}$ and degrees for phase data. The $\sigma_{\mathrm{s}} \mathrm{f}$ product is 795.5. The vertical lines locate the crosshole profiles collected in Richmond. 

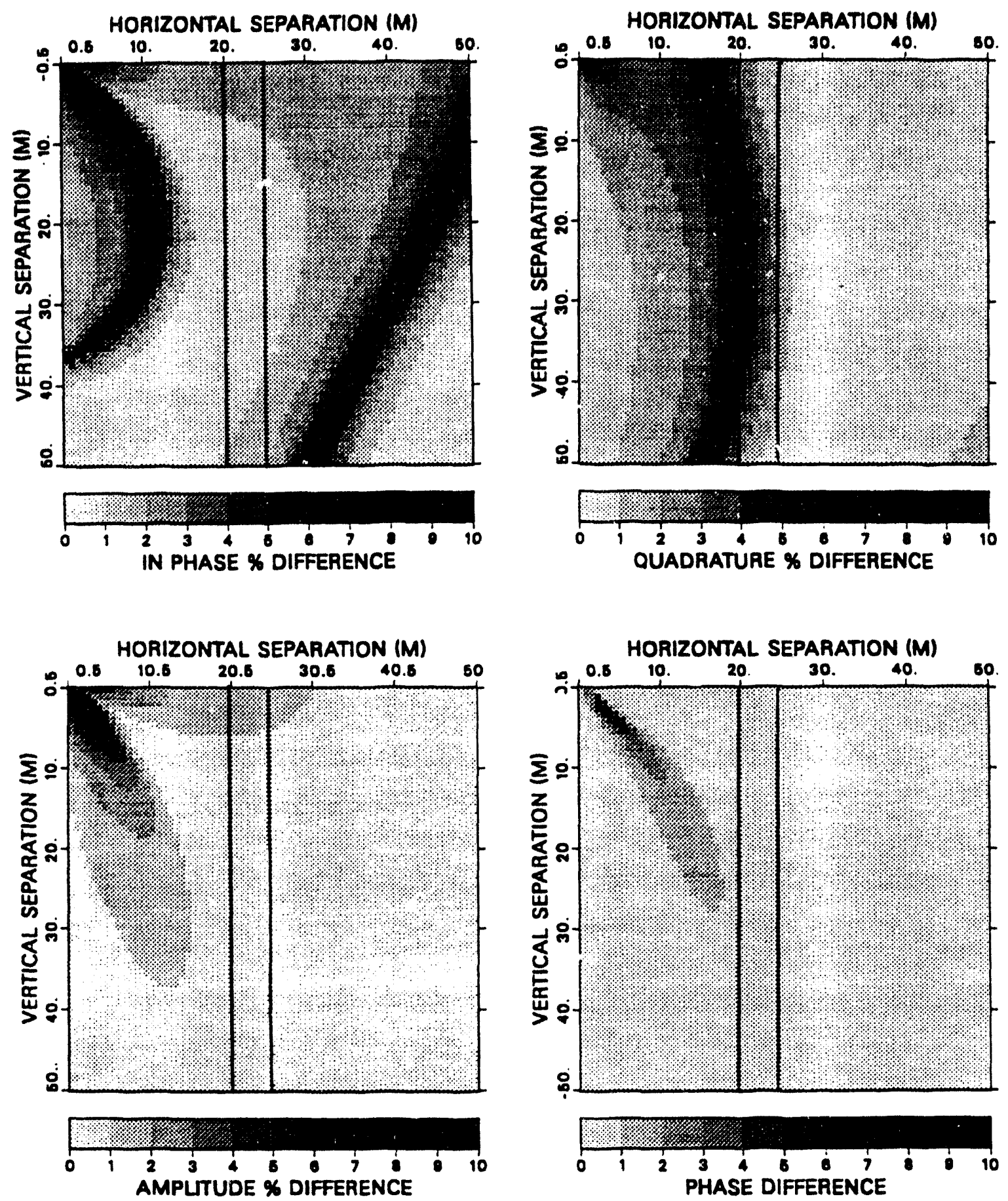

Fig. 2.3-2b In-phase, quadrature and amplitude \% difference and phase shift caused by $0.1 \mathrm{~m}$ movement of the receiver in the horizontal direction for the $\mathrm{H}_{2}$ component in a whole space where $\sigma_{s} f=795.5$. The vertical lines locate the crosshole profiles collected in Richmond. 
LOG IN PMASE ort 12

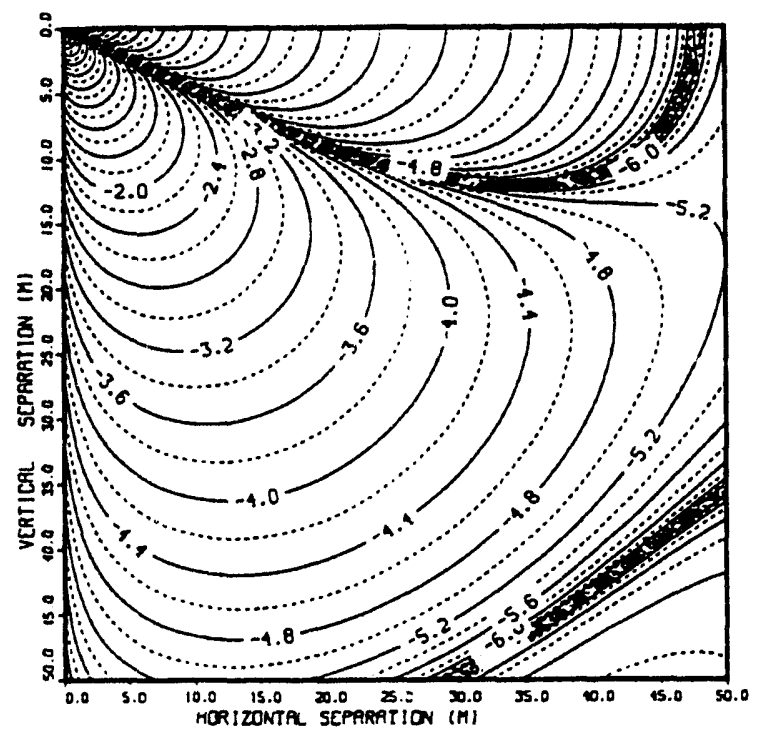

LD AMPLITIES otro/dz

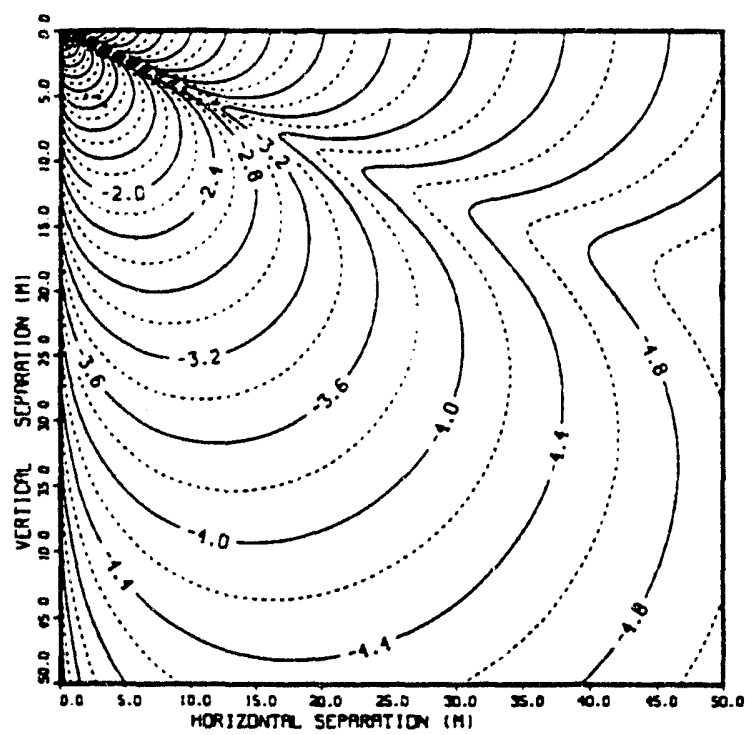

LOG OUFORATURE AHRO/dZ

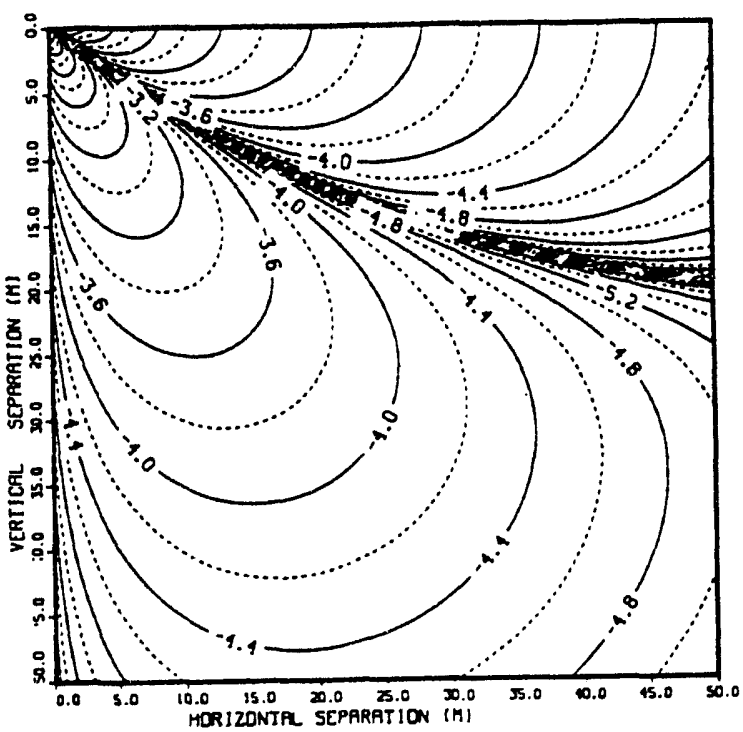

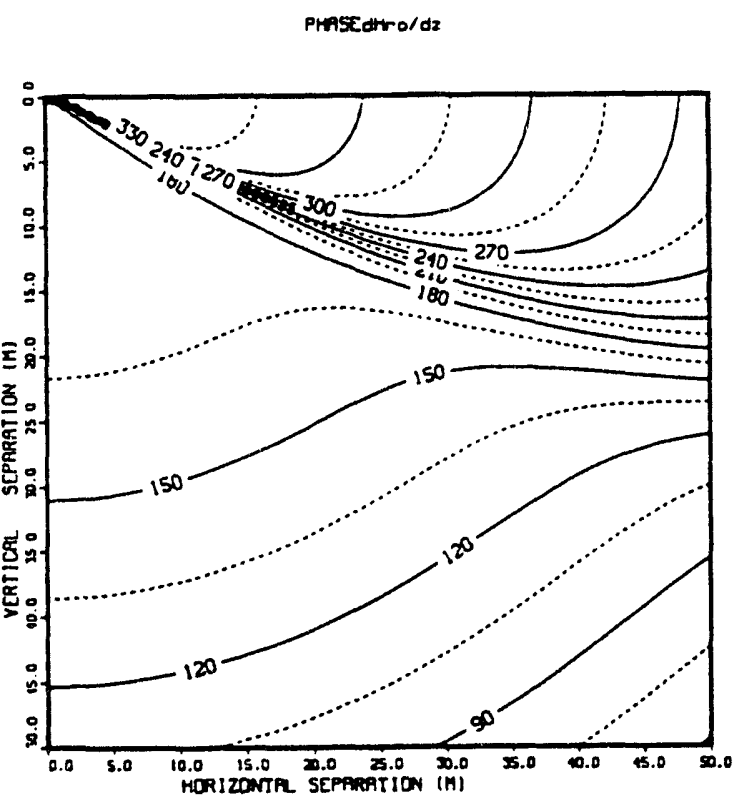

Fig. 2.3-3a The in-phase, quadrature, amplitude and phase of $\delta \mathrm{H}_{\rho} / \delta \mathrm{z}$ in a whole space. The axes are horizontal separation, $\rho$, and vertical separation, $\left|z-z_{s}\right|$, between transmitter and receiver. The numbers on the curves are $\log _{10}$ for in-phase, quadrature and amplitude data in $\mathrm{nT} / \mathrm{m}$ and degrees for phase data. The $\sigma_{\mathrm{s}} \mathrm{f}$ product is 795.5 . 

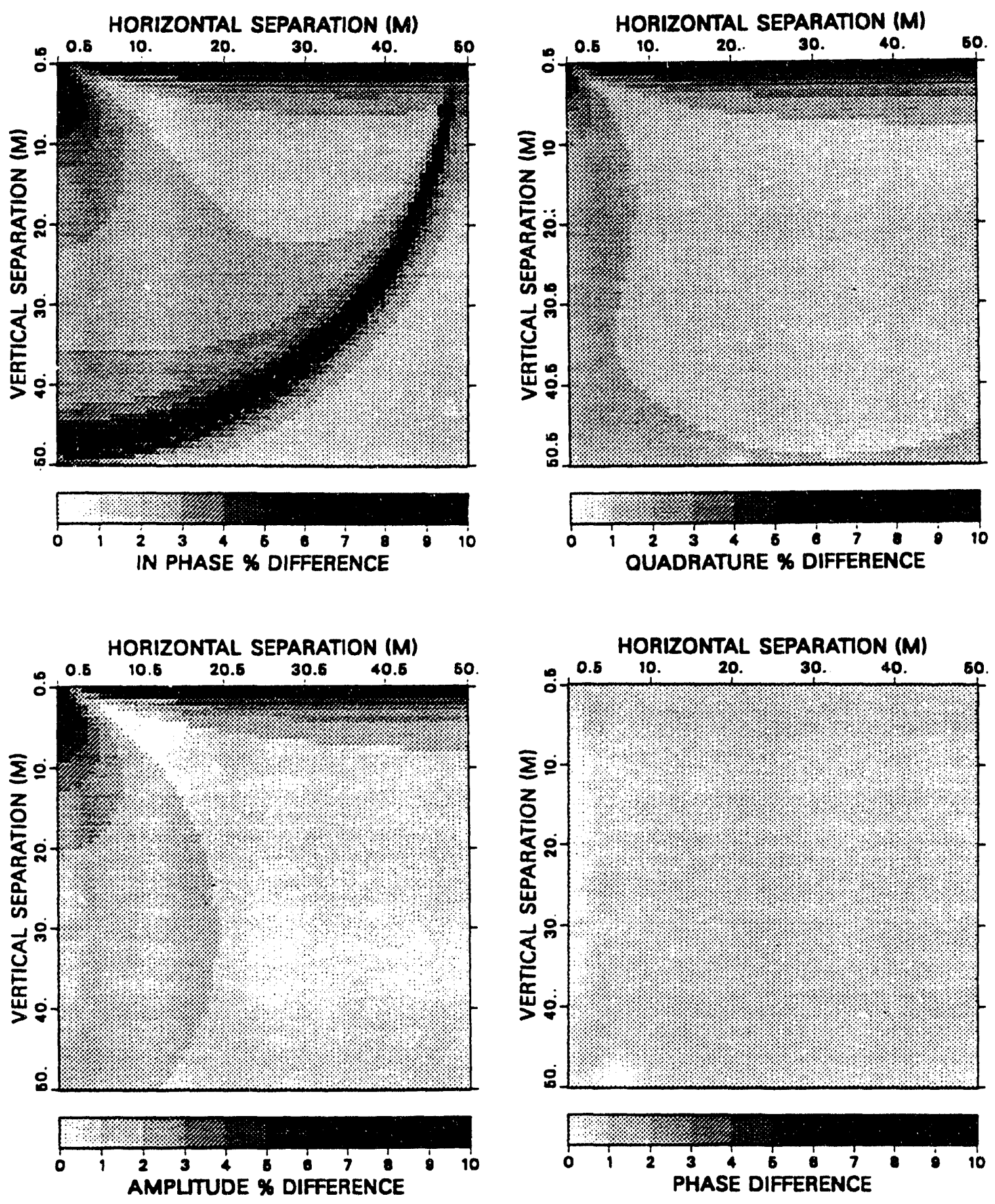

Fig. 2.3-3b In-phase, quadrature and amplitude percent difference and phase shift caused by $0.1 \mathrm{~m}$ movement of the receiver in the vertical direction for the $\mathrm{H}_{\mathrm{p}}$ component in a whole space where $\sigma_{\mathrm{s}} \mathrm{f}=\mathbf{7 9 5 . 5}$. 

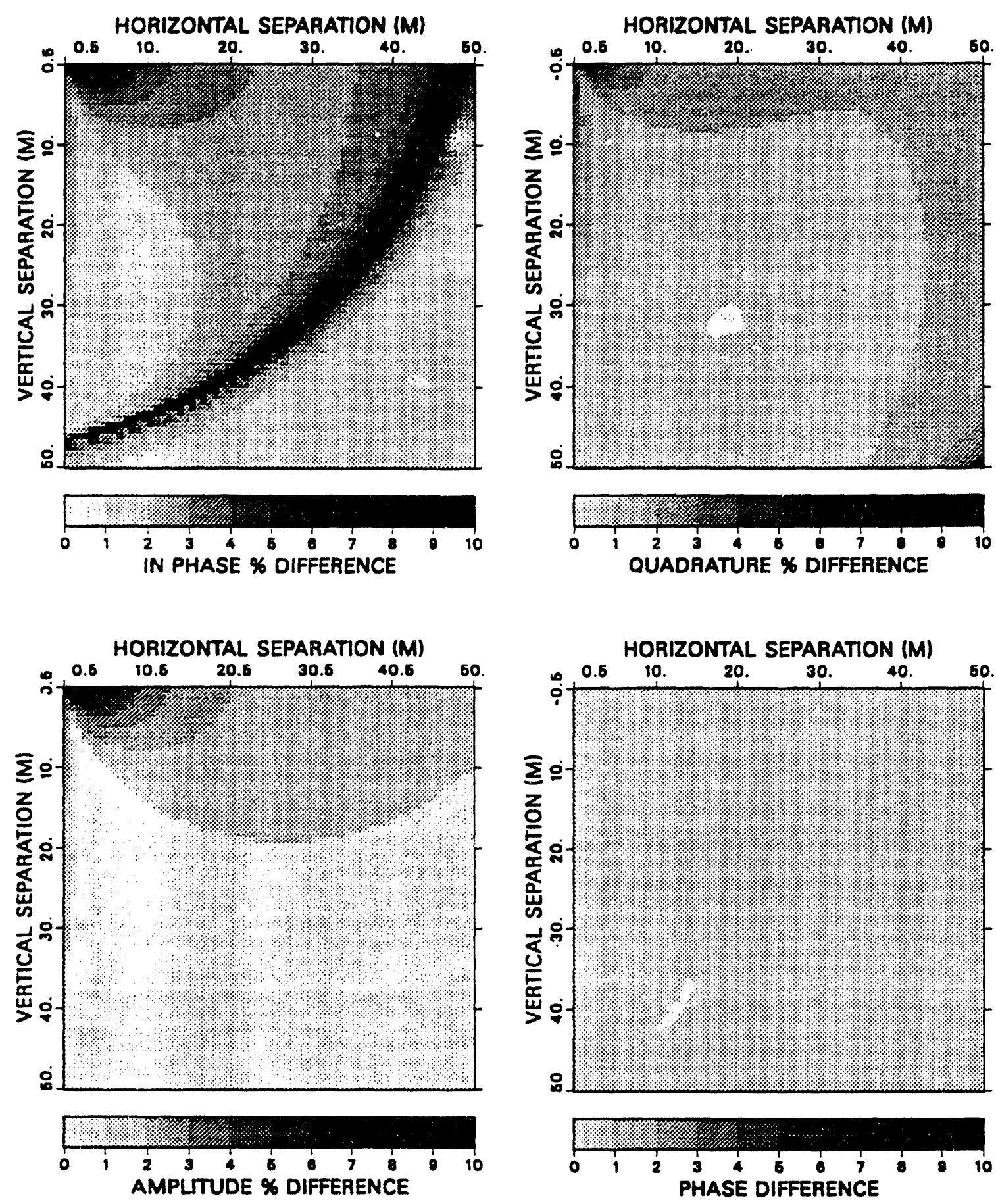

Fig. 2.3-4b In-phase, quadrature and amplitude percent difference and phase shift caused by $0.1 \mathrm{~m}$ movement of the receiver in the horizontal direction for the $\mathrm{H}_{\rho}$ component in a whole space where $\sigma_{s} f=795.5$. 


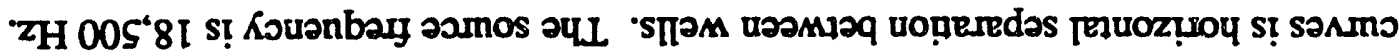

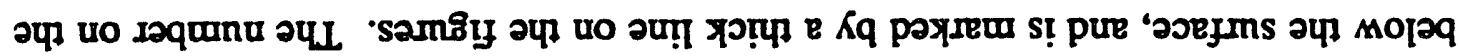

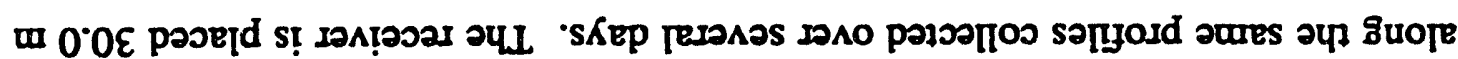

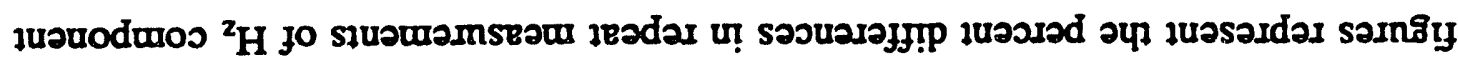

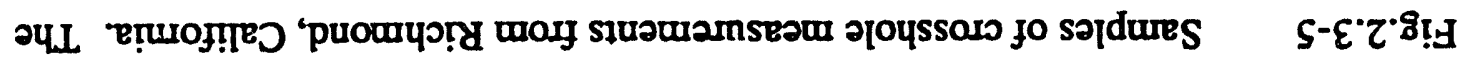
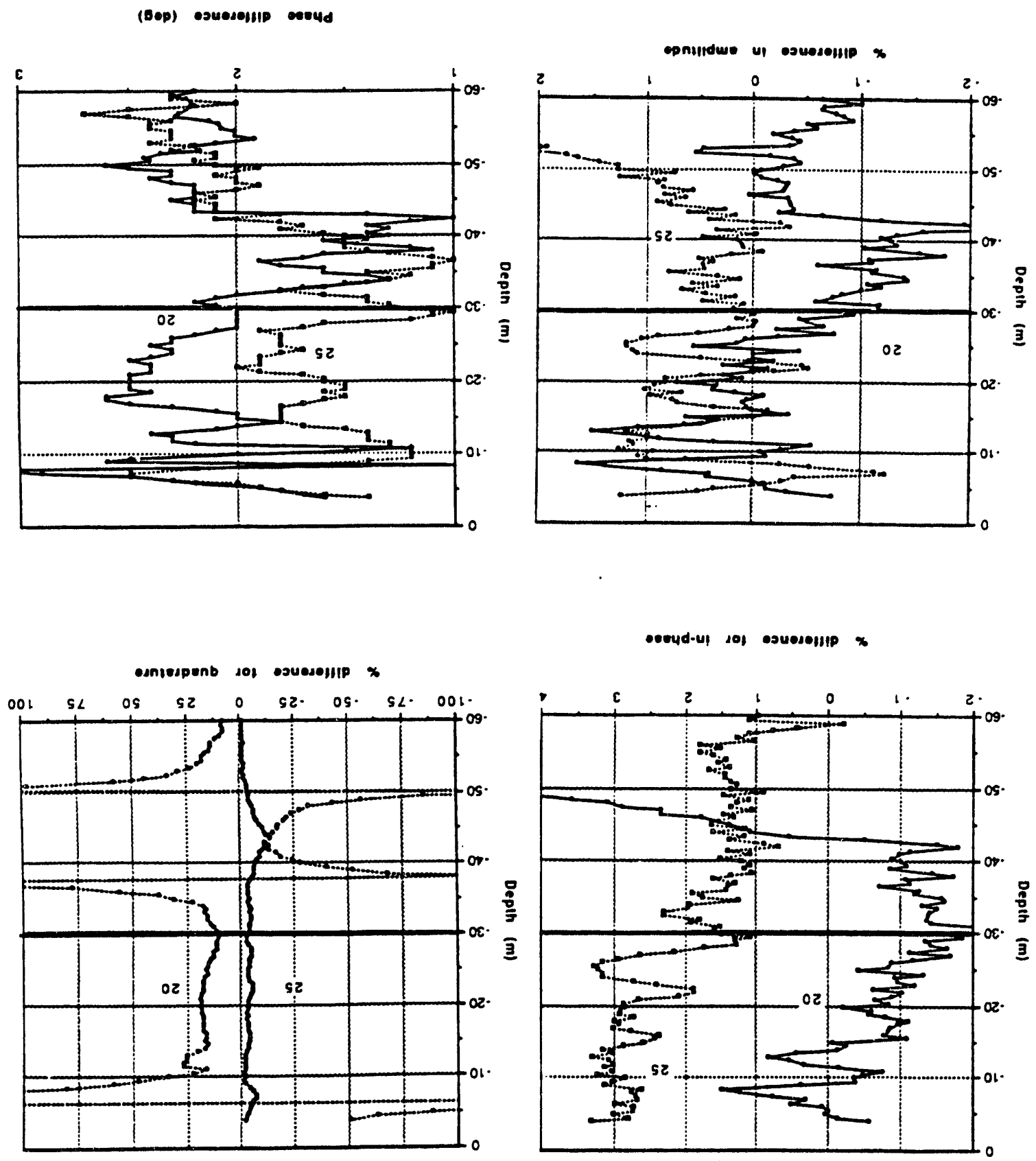

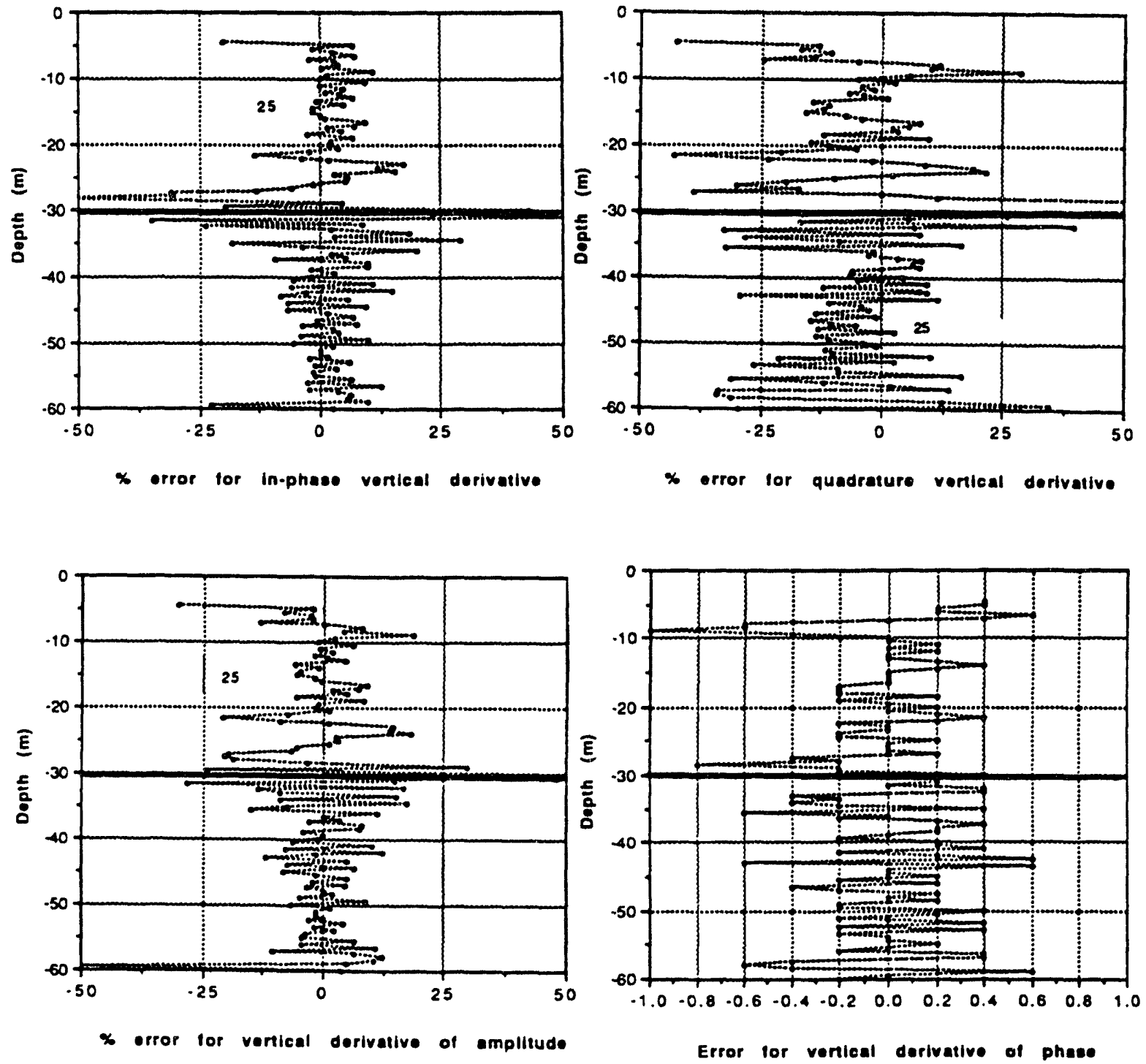

Fig.2.3-6 Samples of crosshole measurements from Richmond, California. The figures represent the error in finite difference derivatives of $\mathrm{Hz}$ component: $\mathrm{D}\left(\mathrm{ReH}_{\mathrm{z}}\right) / \Delta \mathrm{z}, \Delta\left(\mathrm{ImH}_{\mathrm{z}}\right) / \Delta \mathrm{z}, \Delta\left(\mathrm{IH}_{\mathrm{z}} \mathrm{l}\right) / \Delta \mathrm{z}, \Delta\left(\mathrm{phaseH}_{\mathrm{z}}\right) / \Delta \mathrm{z}$ measured on different days. The receiver is placed $30.0 \mathrm{~m}$ below the surface, and is marked by a thick line on the figures. The number on the curves is horizontal separation between wells. The source frequency is $18,500 \mathrm{~Hz}$. 


\section{Chapter 3 \\ Characteristics of the response near the boundary.}

The flat infinite boundary is the simplest case of inhomogeneity within a dissipative medium, but even such simple case does not have a general analytic solution and integrals must be calculated numerically or various approximations implemented to obtain the analytic solution.

In general the pertainent expressions reveal a complicated pattern of interdependence between conductivities, frequencies separations and distances to the boundary. Only in few special cases can one obtain exact, analytic expressions.

In this chapter I will consider the special case when both the transmitter and the receiver are located on the boundary. I will show that the $\mathrm{H}_{\mathrm{z}}$ component depends on the average conductivity of the two half-spaces, whereas $\mathrm{H}_{\rho}$ and $\delta \mathrm{H}_{2} / \delta \mathrm{z}$ depend on the difference in conductivities. This is important, since it suggests that $\mathrm{H}_{\rho}$ or $\delta \mathrm{H}_{2} / \delta \mathrm{z}$ are more sensitive to the conductivity variations inside a one-dimensional medium. Then I will examine the exact expressions involving the infinite integrals and their asymptotic approximations. These approximate solutions will be further simplified by applying restrictions on conductivity or geometry. From these expressions I will extract some response characteristics that can be used in interpretation and experiment design.

Finally I will consider the layer between two conductive half-spaces. I will develop the expressions for the transmitter located inside the layer and outside of the layer to gain the insight about the parameters that dictate the response from the layer.

This chapter follows the works of Kauffman and Keller (1983), Bannister (1968), Kraichman (1970) and King et al. (1979) to mention just a few authors that worked with the dipoles submerged inside the conductive medium.

\subsection{Transmitter and receiver on the interface.}

This is an important case in the analysis of the responses near the boundary. First of all, in this geometry, exact analytic solutions exist. Secondly, this is a limiting case for sensing the medium without actually entering it, and provides an estimate for the limits of resolution in other cases, where transmitter and receiver are off the boundary.

Some fundamental properties of fields and their derivatives on the interface, outside of sources, can be obtained from boundary conditions and Maxwell's equations. The boundary conditions in non magnetic media assure the continuity of $\mathrm{E}_{\phi}, \mathrm{H}_{\rho}$ and $\mathrm{H}_{\mathrm{z}}$ on the interface. Because fields are continuous, then all $\rho$-derivatives: $\delta \mathrm{H}_{z} / \delta \rho, \delta \mathrm{H}_{\rho} / \delta \rho$ and $\delta \mathrm{E}_{\phi} / \delta \rho$ are also continuous across interface if there is no variation in conductivity in 
the horizontal direction For the same reason the higher order $\rho$ derivatives are also continuous.

If there is no discontinuity in magnetic permeability, then because $\mathrm{H}_{\rho}$ is continuous, $\delta \mathrm{E}_{\phi} / \delta \mathrm{z}$ must also be continuous as deducted from Maxwell's equation (see equation (A.1-7) in Appendix A):

$$
\frac{\partial}{\partial z} E_{\phi}=i \omega \mu H_{\rho}
$$

$\delta \mathrm{H}_{\rho} / \delta \mathrm{z}$ is discontinuous across the boundary by the amount $\Delta(\sigma+i \omega \varepsilon) \mathrm{E}_{\phi}$ because in another Maxwell's equation (see equation (A.1-9) in Appendix A):

$$
\frac{\partial}{\partial z} H_{\rho}-\frac{\partial}{\partial \rho} H_{z}=(\sigma+i \omega \varepsilon) E_{\phi} .
$$

$\mathrm{E}_{\phi}$ and $\delta \mathrm{H}_{2} / \delta \rho$ are continuous and $\sigma$ is discontinuous.

The continuity of $\delta \mathrm{H}_{z} / \delta \mathrm{z}$ on a boundary is assured from:

$$
\nabla \cdot H=0=\frac{\partial}{\partial \rho} H_{\rho}+\frac{1}{\rho} H_{\rho}+\frac{\partial}{\partial z} H_{z}
$$

and the fact, that $\mathrm{H}_{\rho}$ and $\delta \mathrm{H}_{\rho} / \delta \rho$ are continuous on the boundary if $\mu=\mu_{0}$.

The second z-derivative of $\mathrm{H}_{\mathrm{z}}$ is discontinuous by $\Delta \mathrm{k}^{2} \mathrm{H}_{\mathrm{z}}$ because in the diffusion equation:

$$
\frac{\partial^{2}}{\partial \rho^{2}} H_{z}+\frac{1}{\rho} \frac{\partial}{\partial \rho} H_{z}+\frac{\partial^{2}}{\partial z^{2}} H_{z}+k^{2} H_{z}=0 .
$$

$\mathrm{H}_{\mathrm{z}}$ and its radial derivatives are continuous across the boundary. The derivative $\delta^{2} E_{\phi} \delta \rho \delta z$ is continuous on the boundary because taking the $z$ - derivative of Maxwell's equation (equation (A.1-8), Appendix A) results in :

$$
\frac{\partial 2}{\partial z \partial \rho} E_{\phi}=-i \omega \mu \frac{\partial}{\partial z} H_{z}-\frac{1}{\rho} \frac{\partial}{\partial z} E_{\phi}
$$

and as we showed before $\delta \mathrm{H}_{z} / \delta \mathrm{z}$ and $\delta \mathrm{E}_{\phi} / \delta \mathrm{z}$ are continuous. 
$\delta^{2} E_{\phi} / \delta z^{2}$ is discontinuous by $\Delta k^{2} E_{\phi}$ because after taking the $z$-derivative of equation (3.1-1) and using equation (3.1-2) to substitute for $\delta \mathrm{H}_{\rho} / \delta \mathrm{z}$ we have:

$$
\frac{\partial^{2}}{\partial z^{2}} E_{\phi}=+i \omega \mu \frac{\partial}{\partial \rho} H_{z}-\left(\omega^{2} \mu \varepsilon-i \omega \mu \sigma\right) E_{\phi}
$$

$\delta^{2} \mathrm{H}_{2} / \delta \rho^{2}$ is continuous because $\delta \mathrm{H}_{2} / \delta \rho$ is continuous and conductivity is constant in horizontal direction.

\subsection{1 $\mathrm{H}_{2}$ component}

The magnetic field near the interface when both transmitter and receiver are above the interface is given by the following equation derived in Appendix A:

$$
\begin{aligned}
& H_{z_{0+0}}=H_{z}\left(k_{s}, R\right)-H_{z}\left(k_{s}, R_{1}\right) \\
& -\frac{2}{k_{t}^{2}-k_{s+1}^{2}}\left[\frac{\partial^{2}}{\partial z_{1}^{2}} H_{z}\left(k_{s}, R_{1}\right)-\frac{M}{4 \pi} \int_{0}^{-} \lambda^{3}\left(i \gamma_{s+1}\right) e^{-i \gamma_{s} z_{1}} J_{0}\left(\lambda \rho_{j}\right) d \lambda\right]
\end{aligned}
$$

where, $z$ is the receiver depth, $z_{s}$ is the transmitter depth, $R$ is the total transmitter-receiver separation given by $R=\left(\rho^{2}+\left(z-z_{s}\right)^{2}\right)^{1 / 2}, R_{1}=\left(\rho^{2}+z_{1}^{2}\right)^{1 / 2}$ is the separation between the receiver and the "image" source (see Figure A.3-1 in Appendix A), $M$ is the transmitter moment and $\mathrm{H}_{z}\left(k_{s}, R\right)$ and $H_{z}\left(k_{s}, R_{1}\right)$ are whole space fields as given by appendix C.3-a. Furthemore:

$$
\begin{aligned}
& \gamma_{s}=\sqrt{k_{s}^{2}-\lambda^{2}} \\
& \gamma_{s+1}=\sqrt{k_{s+1}^{2}-\lambda^{2}} .
\end{aligned}
$$

When the transmitter and the receiver are both located on the boundary, than the first two terms in equation (3.1.1-1) for $H_{2}$ cancel out because then $R=R_{1}$. The last integral can be written as (equation (C.2-4) in Appendix C):

$$
I=\left[\frac{M}{4 \pi} \frac{\partial}{\partial z} \int_{0}^{-} \lambda^{3} e^{-i y_{+1} z} J_{d}(\lambda \rho) d \lambda\right] I_{z=0}=\frac{\partial^{2}}{\partial z^{2}} H_{d}\left(k_{s+1}, R\right) I_{z=0},
$$


where $\mathrm{H}_{\mathbf{z}}\left(\mathrm{k}_{\mathrm{s}+1}, \mathrm{R}\right)$ is a whole space field in a medium with a propagation constant $\mathbf{k}_{\mathrm{s}+1}$. This term at $\mathrm{z}=0$ has a form (equation (C.3-3) in Appendix C):

$$
\begin{gathered}
\frac{\partial^{2}}{\partial z^{2}} H_{z}\left(k_{s+1}, R\right) l_{z=0}= \\
\frac{M}{4 \pi} \frac{1}{\rho^{5}} e^{-i k_{s+1} \rho}\left[9+9 i_{s+1} \rho+4\left(i_{s+1} \rho\right)^{2}+\left(i k_{s+1} \rho\right)^{3}\right]
\end{gathered}
$$

Similarly the $\delta^{2} \mathrm{H}_{\mathrm{z}}\left(\mathrm{k}_{\mathrm{s}}, \mathrm{R}\right) / \delta \mathrm{z}^{2}$ derivative is given by:

$$
\begin{gathered}
\frac{\partial^{2}}{\partial z^{2}} H_{z}\left(k_{s}, R\right) l_{z=0}= \\
\frac{M}{4 \pi} \frac{1}{\rho^{5}} e^{-i k_{s} \rho}\left[9+9 i_{s} \rho+4\left(i_{s} \rho\right)^{2}+\left(i k_{s} \rho\right)^{3}\right]
\end{gathered}
$$

The exact, analytic equation for $\mathrm{H}_{\mathrm{z}}$ field on the interface between two dissipative half-spaces is given by (Kaufman and Keller, 1983):

$$
\begin{aligned}
H_{z_{+\infty}+0}= & -\frac{M}{4 \pi} \frac{2}{k_{c}^{2}-k_{s+1}^{2}} \frac{1}{\rho^{5}}\left\{e^{-i k_{s} \rho}\left[9+9 i k_{s} \rho+4\left(i k_{s} \rho\right)^{2}+\left(i k_{s} \rho\right)^{3}\right]\right. \\
& \left.-e^{-i k_{s+1} \rho}\left[9+9 i k_{s+1} \rho+4\left(i k_{s+1} \rho\right)^{2}+\left(i k_{s+1} \rho\right)^{3}\right]\right\}
\end{aligned}
$$

After multiplying the above equation by $\rho^{3}$ we have:

$$
\begin{aligned}
\mathrm{H}_{z_{s+0}} \rho^{3}= & -\frac{M}{4 \pi} \frac{2}{k_{s}^{2} \rho^{2}-k_{s+1}^{2} \rho^{2}}\left\{e^{-i k_{s} \rho}\left[9+9 i k_{s} \rho+4\left(i k_{s} \rho\right)^{2}+\left(i k_{s} \rho\right)^{3}\right]\right. \\
& \left.-e^{-i k_{s+1} \rho}\left[9+9 i_{s+1} \rho+4\left(i k_{s+1} \rho\right)^{2}+\left(i k_{s+1} \rho\right)^{3}\right]\right\},
\end{aligned}
$$

It can be seen, that the right hand side depends only on $k_{s} \rho$ and $k_{s+1} \rho$. Thus, $\left|k_{s} \rho\right|$ and $\left|k_{s+1} \rho\right|$ are the two induction numbers for this particular geometry and the $\mathrm{H}_{2}$ field on the boundary, normalized by $\rho^{3}$, depends only on the products of conductivity, frequency and distance $\rho$ (under the assumption that the displacement currents are neglected).

It is then possible to present all cases of this specific geometry on a single plot which is realized in Figure 3.1.1-1. The plot shows the normalized in phase, quadrature, 
amplitude and phase of $\mathrm{H}_{2}$ component. The horizontal axis describes the lower medium, the vertical axis describes the upper medium. The numbers on the axis are the induction parameters: $\left(\sigma_{s} f\right)^{1 / 2} p$ of the upper medium and $\left(\sigma_{s+1} f\right)^{1 / 2} p$ of the lower medium. The axis on the top and on the right are in terms of the skin depth in the upper and lower medium: $\rho / \delta_{s+1}$ and $\rho / \delta_{s}$. The skin depth in the upper medium is $\delta_{s} \approx 500 /\left(\sigma_{s} f\right)^{1 / 2}$. Similarly the skin depth in the lower medium is $\delta_{s+1} \approx 500 /\left(\sigma_{s+1} f\right)^{1 / 2}$.

The plots can be interpreted in two ways. If the conductivities of both media are known, than by following the lines parallel to the diagonal the normalized $\mathrm{H}_{2}$ response can be observed with the changing frequency or horizontal distance. The diagonal line represents the zero conductivity contrast between the two media, and the numbers read from it give the normalized fields inside a whole space for the transmitter located at the same height as the receiver. Lines above or below the diagonal on Figure (3.1.1-1) represent media with different conductivities, where the difference in conductivities increases with increasing distance from the diagonal. Each line parallel to diagonal represents different conductivity contrast between the two media.

This can be seen by comparing the values on the diagonal of Figure (3.1.1.1-1) with values in Figure 2.1-9. For example from Figure 3.1.1-1c we see that the normalized amplitude $\mathrm{H}_{2}$ does not change significatly unless the induction parameter reaches $(\sigma f)^{1 / 2} \rho=10^{3}$ (i.e. induction number: $\left.(\omega \mu \sigma)^{1 / 2} \rho \sim 1\right)$. So in the whole space medium of conductivity $0.1 \mathrm{~S} / \mathrm{m}$, examining boreholes separated by $100 \mathrm{~m}$, we have to use frequencies above $1000 \mathrm{~Hz}$ to reach this induction number which marks the region of higher sensitivity to conductivity. At this point our normalized amplitude would be $\mathrm{H}_{2} \rho^{3}$ $=10^{2}$ which translates to the $10^{-4} \mathrm{nT}$ for the true value of the $\mathrm{H}_{2}$ component. The same result can be obtained from Figure 2.1-9 using $\rho=100 \mathrm{~m}$ and a curve with of product $=$ $10^{2}$.Another way to use the plots is as a tool in sensitivity analysis. The plots show what range of induction parameters (frequencies or separations) must be used to detect changes in conductivity of the upper or lower half space. Straight parts of the curves on the plot correspond to the regions insensitive to the conductivity, because along this lines the fields remain the same independently of the conductivities of the other medium.

Notice two most striking features of these plots. First, that with exception of quadrature component all other components are insensitive to conductivity change in the medium unless the induction parameter in this medium reaches $10^{3}$. Secondly, all components, including the quadrature, are not sensitive to conductivity of the more resistive half-space unless the induction number in this resistive half-space is comparable to the induction number in a conductive half -space. 
It can be observed that the response is dominated by the more conductive medium and it is impossible to detect even the large changes in conductivity of the more resistive medium if this medium has a conductivity that fells on the straight part of the curve.

The plots also show why this problem does not exist in surface electromagnetics: in this case the lower medium $\left(\sigma_{8+1} f\right)^{1 / 2} \rho$ is always more conductive than the upper one: $\left(\sigma_{s} f\right)^{1 / 2} \rho=0$ and the changes in this conductive half-space are easily detected at all induction numbers.

To quantify the properties of the $\mathrm{H}_{\mathrm{z}}$ field on the boundary between two halfspaces we will consider some special cases following the analysis from Kauffman and Keller (1983).

If $\left|k_{s} \rho\right|$ and $\left|k_{s+1} \rho\right| \ll 1$, which occur at very low frequencies or small separation (and are below $(\sigma f)^{1 / 2} \rho=5 \times 10^{2}$ on the plots), then the expansion of $\theta^{-i k p}$ and $e^{-i k_{*}+p}$ into a power series leads to:

$$
e^{-i k_{s+1} \rho}\left[9+9 i k_{s+1} \rho+4\left(i_{s+1} \rho\right)^{2}+\left(i k_{s+1} \rho\right)^{3}\right]=9-\frac{1}{2}\left(i k_{s+1} \rho\right)^{2}-\frac{1}{8}\left(i k_{s+1} \rho\right)^{4}
$$

and

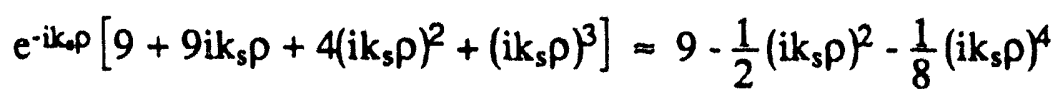

As a result we have:

$$
H_{z_{0+0}}=-\frac{M}{4 \pi} \frac{1}{\rho^{3}}\left[1+\frac{i \omega \mu \rho^{2}}{4}\left(\sigma_{s}+\sigma_{s+1}\right)\right] .
$$

In this equation the real part is independent of the conductivity of the medium to the first order of approximation, whereas the imaginary part depends only on the average of both conductivities. If one of the conductivities, say $\sigma_{s+1}$ for example, is much higher than the other, then this conductivity dominates the imaginary response.

In case where $\left|k_{s} \rho\right|>1$ and $\sigma_{s} \gg \sigma_{s+1}$, the term in equation (3.1.1-7) with a smaller exponential attenuation dominates: $e^{-i k_{n+1} p} \gg e^{-i k p}$ and the expression takes the form:

$$
H_{z_{s+0}} \approx \frac{M}{4 \pi} \frac{2}{k_{t}^{2}} \frac{1}{\rho^{5}} e^{-i k_{s+1} \rho}\left[9+9 \mathrm{ik}_{s+1} \rho+4\left(\mathrm{ik}_{s+1} \rho\right)^{2}+\left(\mathrm{ik}_{s+1} \rho\right)^{3}\right]
$$


This equation shows, that most of the received signal propagates through the resistive medium, although the exponential attenuation with distance in this resistive medium also takes place.

Notice, that by taking the ratio at two frequencies the conductivity of the more resistive medium can be obtained by solving the following equation for $\sigma_{s+1}$ :

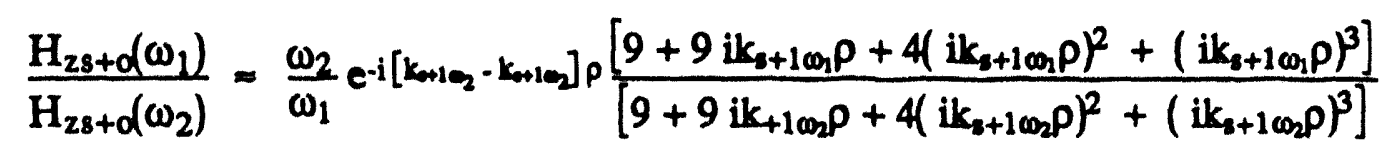

where $k_{s+1 \omega 1}$ and $k_{s+1002}$ denote the induction numbers of the more resistive medium at frequency $\omega_{1}$ and $\omega_{2}$ respectively.

A slightly different pattern emerges if the upper medium is non conductive (air). Then $k_{8} \rho=0$, and from equation (3.1.1-8) we have:

$$
H_{z+0}=\frac{M}{4 \pi} \frac{2}{k_{m+1}^{2}} \frac{1}{\rho^{5}}\left\{9-e^{-i k_{s+1} \rho}\left[9+9 i k_{s+1} \rho+4\left(i k_{s+1} \rho\right)^{2}+\left(i k_{s+1} \rho\right)^{3}\right]\right\} \text {. }
$$

The last equation is a well known solution for surface electromagnetic techniques (Sinha and Bhattaharya, 1966).

At high frequencies, where $k_{8+1} \rho \gg 1$, the second term in the above expression decays exponentially and:

$$
H_{z+0}=\frac{M}{4 \pi} \frac{18}{k_{s+1}^{2}} \frac{1}{\rho^{5}}=\frac{M}{4 \pi} \frac{i}{\rho^{5}} \frac{18}{\omega \mu \sigma_{s+1}} .
$$

At these high frequencies $\mathrm{H}_{\mathrm{z}}$ is strongly dependent upon conductivity of the earth.

From the plots and from the analysis above, the following conclusions emerge: 1 ) at low induction numbers where $\mid \mathrm{kpl}<1$ (which translates to $\left(\sigma_{\mathrm{s}} \mathrm{f}\right)^{1 / 2} \rho<5 \times 10^{2}$ in our figures), it is possible to detect changes of conductivity in the more resistive medium but only if the induction numbers in this resistive medium are not much smaller than in conductive medium, and 2) changes in the more resistive medium are more easily detected if the induction number in this medium: $|\mathrm{kp}|>1$. 


\subsection{2 $\mathrm{H}_{\mathrm{p}}$ component}

To calculate $\mathrm{H}_{p}$ on the boundary let's take the $\mathrm{z}$-derivative of the Hertz potential above the interface as derived in Appendix A (equation A.3-5):

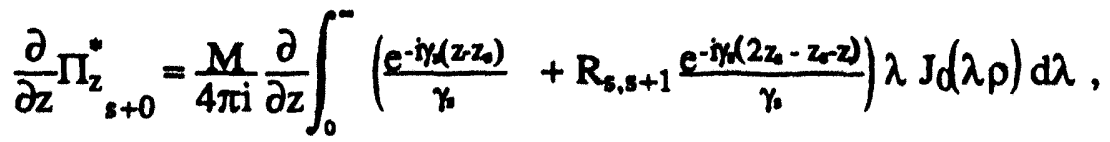

and evaluate it at $z=z_{s}=z_{s+1}$. The $z$-derivative of the first term is zero at $z=z_{s}$ and we are left with:

$$
\left.\frac{\partial}{\partial z} \Pi_{s+0}^{*} l_{z=2=2+2+1}=\frac{M}{4 \pi} \int_{0}^{\infty} R_{s, s+1} \lambda J d \lambda \rho\right) d \lambda .
$$

The reflection coefficient can be written (Kauffman and Keller, 1983, p. 415) as:

$$
R_{s, s+1}=\frac{\gamma_{s}-\gamma_{s+1}}{\gamma_{s}+\gamma_{s+1}}=\frac{1}{\gamma_{s} \gamma_{s+1}} \frac{k_{s}^{2}-k_{s+1}^{2}}{4}\left[1-\left(\frac{\gamma_{s}-\gamma_{s+1}}{\gamma_{s}+\gamma_{s+1}}\right)^{2}\right]
$$

which gives:

$$
\frac{\partial}{\partial z} \Pi_{s+0}^{*} l_{z=z_{s}=z_{n+1}}=\frac{M}{4 \pi} \int_{0}^{-} \frac{1}{\gamma_{s} \gamma_{s+1}} \frac{k_{s}^{2}-k_{s+1}^{2}}{4}\left[1-\left(\frac{\gamma_{s}-\gamma_{s+1}}{\gamma_{s}+\gamma_{s+1}}\right)^{2}\right] \lambda J_{0}(\lambda \rho) d \lambda
$$

and because (Kauffman and Keller, 1983, p.415):

$$
\left.\int_{0}^{\infty} \frac{\lambda}{\gamma_{s} \gamma_{s+1}}\left(\frac{\gamma_{s}-\gamma_{s+1}}{\gamma_{s}+\gamma_{s+1}}\right)^{\nu} J d \lambda \rho\right) d \lambda=I V\left(\rho \frac{i k_{s}-i k_{s+1}}{2}\right) K_{V}\left(\rho \frac{\mathrm{ik}_{s}+i k_{s+1}}{2}\right)
$$

under the condition that $\operatorname{Re}\left(\mathrm{ik}_{\mathrm{s}}\right)>\operatorname{Re}\left(\mathrm{k}_{\mathrm{s}+1}\right) \geq 1$, we have:

$$
\begin{aligned}
\frac{\partial}{\partial z} \Pi_{s+0}^{*} l_{z=z_{s}=z_{s+1}} & \left.=\frac{M}{4 \pi} \frac{k_{s}^{2}-k_{s+1}^{2}}{4}\left[I d \rho \frac{i k_{s}-i k_{s+1}}{2}\right) K d \rho \frac{i k_{s}+i k_{s+1}}{2}\right) \\
& \left.\left.-I \frac{i k_{s}-i k_{s+1}}{2}\right) K_{2}\left(\rho \frac{i k_{s}+i k_{s+1}}{2}\right)\right]
\end{aligned}
$$


To obtain $\mathrm{H}_{p}$ we need the $p$-derivatives of the products of modified Bessel functions of different arguments:

$$
\frac{\partial}{\partial \rho} I_{d}\left(\rho \text { b) } K_{d}(\rho a)=I_{1}(\rho b) b K_{d}(\rho a)-I_{d}\left(\rho \text { b) a } K_{1}(\rho a)\right.\right.
$$

$\frac{\partial}{\partial \rho} I_{2}(\rho b) K_{2}(\rho a)=\left[I_{1}(\rho b)-\frac{2}{\rho b} I_{2}(\rho b)\right] b K_{2}(\rho a)-I_{2}(\rho b) a\left[K_{1}(\rho a)+\frac{2}{\rho a} K_{2}(\rho a)\right]$

where:

$$
a=\frac{i k_{s}+i k_{s+1}}{2}
$$

and

$$
\mathrm{b}=\frac{\mathrm{ik_{s }}-\mathbf{i k _ { s + 1 }}}{2} \text {. }
$$

Using the recurrence relation (9.6.26) from Abramovitz and Stegun (1970) we finally obtain the exact, analytic expression for $\mathrm{H}_{\rho}$ when the transmitter and receiver are located on the boundary between two dissipative half-spaces (Kauffman and Keller, 1983):

$$
\begin{aligned}
H_{\rho} & =-\frac{M}{4 \pi} \frac{k_{s}^{2}-k_{s+1}^{2}}{\rho}\left[I / \rho \frac{i k_{s}-i k_{s+1}}{2}\right) K_{2}\left(\rho \frac{i k_{s}+i k_{s+1}}{2}\right) \\
& \left.-\frac{k_{s}^{2}+k_{s+1}^{2}}{k_{s}^{2}-k_{s+1}^{2}} I_{1}\left(\rho \frac{i k_{s}-i k_{s+1}}{2}\right) K_{1}\left(\rho \frac{i k_{s}+i k_{s+1}}{2}\right)\right]
\end{aligned}
$$

If we multiply both sides of equation $(3.1 .2-11)$ by $r 3$, then we have:

$$
\begin{aligned}
H_{\rho} \rho^{3}= & -\frac{M}{4 \pi}\left(k_{s}^{2} \rho^{2}-k_{s+1}^{2} \rho^{2}\right)\left[I_{2}\left(\rho \frac{i k_{s}-i k_{s+1}}{2}\right) K_{2}\left(\rho \frac{i k_{s}+i k_{s+1}}{2}\right)\right. \\
& \left.-\frac{k_{s}^{2}+k_{s+1}^{2}}{k_{s}^{2}-k_{s+1}^{2}} I_{1}\left(\rho \frac{i k_{s}-i k_{s+1}}{2}\right) K_{1}\left(\rho \frac{i k_{s}+i k_{s+1}}{2}\right)\right]
\end{aligned}
$$

Notice that the normalized $\mathrm{H}_{\rho} \rho^{3}$ on a boundary is a function of $k_{s} \rho$ and $k_{s+1} \rho$ only, as we already observed for a normalized $\mathrm{H}_{2} \rho^{3}$ component. It is then possible to 
plot these normalized values on the boundary for all combinations of induction numbers on the single plot, which is given in Figure 3.1.2-1.

As can be observed by comparing the Figure 3.1.2-1 with 3.1.1-1, the $\mathrm{H}_{\rho}$ field is very different from the $\mathrm{H}_{2}$ field. First of all notice, that the $\mathrm{H}_{\mathrm{p}}$ component vanishes on the diagonals of the plots where the conductivities of the upper and lower half-spaces are identical, i.e. the two conductive half-spaces become a uniform whole space. We already observed this before in Figures 2.1-16. Secondly, all $\mathrm{H}_{\rho}$ components (except the phase) increase rapidly with increasing induction numbers, reaching the maximum around $(\sigma f)^{1 / 2} \rho \sim 10^{3}$, proving that the $\mathrm{H}_{\rho}$ field is sensitive to the conductive medium at all induction numbers. Finally, the $\mathrm{H}_{p}$ can detect conductivity changes in the less conductive medium, but only if the contrast in conductivity between the two half-spaces is not large.

To quantify our observations we need to evaluate the $\mathrm{H}_{\mathrm{p}}$ field on the boundary.

In general, the evaluation of the values for $\mathrm{H}_{p}$ component (equation 3.1.2-11) has to be performed numerically even in this case, where the analytic solution exists. The approximate values can be obtained by expanding the modified Bessel functions in series for large and small arguments.

At small induction numbers where the argument $\mathrm{z} \sim 0$, the modified Bessel functions are given by (Abramovitz and Stegun, 1970, relations (9.6.7) and (9.6.9)):

$$
\begin{aligned}
& I_{1}(z) \approx \frac{1}{2} z \\
& K_{1}(z) \approx \frac{1}{z} \\
& I_{2}(z) \approx \frac{z^{2}}{8} \\
& K_{2}(z) \approx \frac{2}{z^{2}}
\end{aligned}
$$

Substituting these values into equation (3.1.2-11) we get:

$$
H_{p} \approx-\frac{M}{4 \pi} \frac{a b}{\rho}=-\frac{M}{4 \pi} \frac{1}{\rho} \frac{i \omega \mu\left(\sigma_{s}-\sigma_{s+1}\right)}{4}
$$

So at low induction numbers the quadrature $\mathrm{H}_{\mathrm{p}}$ component is proportional to the difference in conductivities between the two media. This is in contrast to the quadrature $\mathrm{H}_{\mathrm{z}}$ component that was proportional to the average conductivity between the two media. This is an important observation, that explains why the profiles of $\mathrm{H}_{\mathrm{z}}$ field are smooth and without much of a character when observed on both sides of the boundary. 
The above approximation involves only terms that are of the second order. The in phase $\mathrm{H}_{\rho}$ component is smaller, and thus does not enter the asymptotic expression. However the comparison of figures for real $\mathrm{H}_{p}$ and imaginary $\mathrm{H}_{p}$ components on the boundary shows that the real $\mathrm{H}_{\rho}$ is indeed smaller, but more sensitive to the conductivity than imaginary $\mathrm{H}_{p}$, and is proportional to:

$$
H_{p} \approx\left[\cos \left(\sigma_{s}-\sigma_{s+1}\right)\right]^{2}
$$

To evaluate $\mathrm{H}_{\rho}$ on the boundary for large values of the argument, at least a threeterm expansions are needed, otherwise the contradictory results occur. Furthermore, all of the expansions of modified Bessel functions have to be performed with the assumption that $k_{s}>k_{s+1}$, because this condition was imposed on the integral leading to the analytic solution on the boundary (equation (3.1.2-5)).

Expanding the modified Bessel functions in series for large arguments (Abramovitz and Stegun, 1970) leads to:

$$
\begin{aligned}
& I_{1}(\rho b) \approx \frac{e-p b}{\sqrt{2 \pi \rho b}}\left\{1-\frac{3}{8 \rho b}-\frac{5 \times 3}{2 \times 8^{2} \rho^{2} b^{2}}-\frac{15 \times 7}{2 \times 8^{3} \rho^{3} b^{3}}+\ldots\right\} \\
& K_{1}(\rho a) \approx e^{-\rho a} \sqrt{\frac{\pi}{2 \rho a}}\left\{1+\frac{3}{8 \rho a}-\frac{5 \times 3}{2 \times 8^{2} \rho^{2} a^{2}}+\frac{15 \times 7}{2 \times 8^{3} p^{3} a^{3}}+\ldots\right\} \\
& I_{2}(\rho b):=\frac{e^{-\rho b}}{\sqrt{2 \pi \rho b}}\left\{1-\frac{15}{8 \rho b}+\frac{15 \times 7}{2 \times 8^{2} p^{2} b^{2}}+\frac{5 \times 7 \times 9}{2 \times 8^{3} p^{3} b^{3}}+\ldots\right\} \\
& K_{2}(\rho a) \approx e^{-\rho a} \sqrt{\frac{\pi}{2 \rho a}}\left\{1+\frac{15}{8 \rho a}+\frac{15 \times 7}{2 \times 8^{2} \rho^{2} a^{2}}-\frac{5 \times 7 \times 9}{2 \times 8^{3} \rho^{3} a^{3}}+\ldots\right\}
\end{aligned}
$$

The products of modified Bessel functions are given by:

$$
\begin{aligned}
I_{1}(\rho b) K_{1}(\rho a) & =\frac{e-p(a-b)}{\rho \sqrt{4 a b}}\left\{1-\frac{3}{8 \rho} \frac{(a-b)}{a b}-\frac{15}{2 \times 8^{2} p^{2}} \frac{\left(a^{2}+b^{2}\right)}{a^{2} b^{2}}-\frac{9}{8^{2} \rho^{2}} \frac{1}{a b}\right. \\
& \left.-\frac{5 \times 7 \times 3}{2 \times 8^{3} \rho^{3}} \frac{\left(a^{3}-b^{3}\right)}{a^{3} b^{3}}-\frac{3 \times 15}{2 \times 8^{3} \rho^{3}} \frac{(a-b)}{a^{2} b^{2}}+\ldots\right\}
\end{aligned}
$$




$$
\begin{aligned}
I_{2}(p b) K_{2}(\rho a) & =\frac{e-p(a-b)}{\rho \sqrt{4 a b}}\left\{1-\frac{15}{8 \rho} \frac{(a-b)}{a b}+\frac{15 \times 7}{2 \times 8^{2} p^{2}} \frac{\left(a^{2}+b^{2}\right)}{a^{2} b^{2}}-\frac{15 \times 15}{8^{2} \rho^{2}} \frac{1}{a b}\right. \\
& \left.+\frac{15 \times 7 \times 3}{2 \times 8^{3} \rho^{3}} \frac{\left(a^{3}-b^{3}\right)}{a^{3} b^{3}}+\frac{15 \times 15 \times 7}{2 \times 8^{3} \rho^{3}} \frac{(a-b)}{a^{2} b^{2}}+\ldots\right\}
\end{aligned}
$$

Finally, the estimate for $\mathrm{H}_{\mathrm{p}}$ on the boundary for the case where $k_{s}>k_{s+1}$, and both:

$$
|\rho|=\left|\rho \frac{i k_{s}+i k_{s+1}}{2}\right| \text { and }|\rho b|=\left|\rho \frac{i k_{s}-i k_{s+1}}{2}\right|
$$

are large is given by:

$$
\begin{gathered}
H_{\rho} \approx \frac{M}{4 \pi} \frac{i}{\sqrt{k_{s}^{2}-k_{s+1}^{2}}} \frac{e^{-k_{s+1} \rho}}{\rho^{4}}\left[2\left(i_{s+1} \rho\right)^{2}-\frac{3}{2} i_{s+1} \rho\left(-5+r_{s, s+1}\right)-\frac{15}{16} r_{s, s+1}\left(7+r_{s, s+1}\right)\right. \\
-\frac{9}{16}\left(-25+r_{s, s+1}\right)-\frac{5 \times 21}{16 \times 4} \frac{\left(i k_{s+1} \rho\right)}{\rho^{2}\left(k_{s}^{2}-k_{s+1}^{2}\right)^{2}}\left(-7 k_{s+1}^{2}+3 k_{s}^{2}\right)\left(3+r_{s, s+1}\right) \\
\left.+\frac{3 \times 15}{16 \times 4} \frac{\left(i k_{s+1} \rho\right)}{\rho^{2}\left(k_{s}^{2}-k_{s+1}^{2}\right)}\left(35+r_{s, s+1}\right)\right]
\end{gathered}
$$

where

$$
\mathbf{r}_{\mathrm{s}, \mathrm{s}+1}=\frac{\mathbf{k}_{\mathrm{s}}^{2}+\mathbf{k}_{\mathrm{s}+1}^{2}}{\mathbf{k}_{\mathrm{s}}^{2}-\mathbf{k}_{\mathrm{s}+1}^{2}}
$$

The conditions from equation (3.1.2-18) are quite restrictive, because they imply the large contrast between $k_{s}$ and $k_{s+1}$ in order to satisfy the requirements for large argument of $I_{2}(\rho b)$ and $I_{1}(\rho b)$.

An example of the medium that satisfies the above conditions is an air - earth interface where $k_{s+1}=0$. In this case equation (3.1.2-19) takes a form:

$$
H_{\rho}=\frac{M}{4 \pi} \frac{i}{k_{s}} \frac{6}{\rho^{4}} \approx \frac{M}{4 \pi} \frac{i-1}{\sqrt{2 \omega \mu \sigma_{s}}} \frac{6}{\rho^{4}}
$$

which agrees with the analogous expression in Kauffman and Keller (1983).

For $k_{s} \gg k_{s+1}$, but with $k_{s+1}$ finite the coefficient $r_{s, s+1} \sim 1, k_{s}^{2}-k_{s+1}{ }^{2} \approx k_{s}^{2}$ and after neglecting the term with $k_{s}{ }^{2}$ in denominator of equation (3.1.2-19) we obtain: 


$$
H_{\rho}=\frac{M}{4 \pi} \underset{k_{s}}{2 i} \frac{e^{-i k_{s+1} \rho}}{\rho^{4}}\left[3+3 i k_{s+1} \rho+\left(i k_{s+1} \rho\right)^{2}\right]
$$

As can be observed this expression has a similar appearance as $\mathbf{H}_{\mathbf{z}}$. Similar results, easier to analyze, can be obtained by using an asymptotic evaluation of the integrals. In order to do so, lets take the z-derivative of the Hertz potential (equation (A.3-12) in Appendix A):

$$
\begin{gathered}
\frac{\partial}{\partial z} \Pi_{s+0}^{*}=\frac{M}{4 \pi} \frac{\partial}{\partial z} \frac{e^{-i k_{s} R}}{R}-\frac{M}{4 \pi} \frac{\partial}{\partial z} \frac{e^{-i k_{0} R_{1}}}{R_{1}} \\
-\frac{2}{k_{s}^{2}-k_{s+1}^{2}} \frac{M}{4 \pi}\left[\frac{\partial}{\partial z} \frac{\partial^{2}}{\partial z_{1}^{2}} \frac{e^{-i k_{s} R_{1}}}{R_{1}}-\frac{\partial}{\partial z} \int_{0}^{-} \lambda\left(i \gamma_{s+1}\right) e^{-i \gamma_{s} z_{1}} J_{0}(\lambda \rho) d \lambda\right]
\end{gathered}
$$

and evaluate it at the interface where $R=R_{1}$ and $z_{1}=0$. In this case the first three terms in above equation are zero and we are left with:

$$
\left.\frac{\partial}{\partial z} \Pi_{s+0}^{*}=\frac{2}{k^{2}-k_{s+1}^{2}} \frac{M}{4 \pi} \int_{0}^{-} \lambda\left(i \gamma_{s+1}\right)\left(i \gamma_{s}\right) J d \lambda \rho\right) d \lambda
$$

Using integral $\mathrm{I}_{5}$ and relations $(\mathrm{B}-4)$ and $(\mathrm{B}-7)$ from the Appendix $\mathrm{B}$ we have:

$$
\frac{\partial}{\partial z} \Pi_{s+0}^{*}=\frac{2}{k_{s}^{2}-k_{s+1}^{2}}\left[\left.i \sqrt{k_{s+1}^{2}-k_{s}^{2}} \frac{\partial^{2}}{\partial z_{1}^{2}} \frac{e^{-k+R_{1}}}{R_{1}}\right|_{z=0}+\left.i \sqrt{k_{s}^{2}-k_{s+1}^{2}} \frac{\partial^{2}}{\partial z^{2}} \frac{e^{-k_{s+1} R}}{R}\right|_{z=0}\right]
$$

Taking the derivative with respect to $\rho$ and using:

$$
\frac{\partial^{2} e^{-k_{c} R_{1}}}{\partial z_{1} \partial \rho R_{1}}=H_{p}\left(k_{8}, R_{1}\right)
$$

and

$$
\frac{\partial^{2} e^{-k_{+1} R} R}{\partial z \partial \rho R}=H_{p}\left(k_{s+1}, R\right)
$$


where $H\left(k_{s}, R_{1}\right)$ and $H\left(k_{s+1}, R\right)$ are whole space fields (Appendix C.3.b), we obtain:

$$
H_{p}=\frac{2}{k_{s}^{2}-k_{s+1}^{2}}\left[i \sqrt{k_{s+1}^{2}-k_{s}^{2}} \frac{\partial}{\partial z_{1}} H_{p}\left(k_{s}, R_{1}\right) I_{z_{1}=0}+i \sqrt{k_{s}^{2}-k_{s+1}^{2}} \frac{\partial}{\partial z} H_{p}\left(k_{s+1}, R\right) I_{z=0}\right] .
$$

The final, expression for the $\mathrm{H}_{\mathrm{p}}$ on the boundary between two half-spaces under conditions that the horizontal separation is large is given by:

$$
\begin{aligned}
H_{\rho}= & \frac{M}{4 \pi} \frac{2}{\sqrt{k_{s}^{2}-k_{s+1}^{2}}} \frac{1}{\rho^{4}}\left\{e^{-i k_{s} \rho}\left[3+3 i k_{s} \rho+\left(i k_{s} \rho\right)^{2}\right]\right. \\
& +i e^{\left.-i k_{s+1} \rho\left[3+3 i k_{s+1} \rho+\left(i k_{s+1} \rho\right)^{2}\right]\right\}}
\end{aligned}
$$

This expression is somewhat similar to the $\mathrm{H}_{\mathrm{z}}$ component on the boundary as given by equation (3.1.1-7). Once more we see that the term describing propagation in the more resistive medium will dominate; if $\left|k_{s} \rho\right|>1$, and if $k_{s} \gg k_{s+1}$, then the first term in brackets is negligible in comparison with the second term and gives:

$$
H_{\rho} \approx \frac{M}{4 \pi} \frac{2 i}{k_{s}} \frac{1}{\rho^{4}} e^{-i k_{s+1} \rho\left[3+3 i k_{s+1} \rho+\left(i k_{s+1} \rho\right)^{2}\right]}
$$

that has the same form as the equation (3.1.2-22) obtained from analytic solution.

In the limiting case where one of the media is free space, say $k_{s+1}$ for example, then:

$$
H_{\rho}=\frac{M}{4 \pi} \frac{1}{k_{4}} \frac{6 i}{\rho^{4}}=\frac{M}{4 \pi} \frac{(i-1)}{\sqrt{2 \omega \mu \sigma_{s}}} \frac{6}{\rho^{4}}
$$

and we obtain the same expression as equation (3.1.2-21) that was developed from the analytic solution. So at this high induction numbers the $\mathrm{H}_{p}$ component is less sensitive to the conductivity of the medium that the $\mathrm{H}_{2}$ component.

\subsubsection{The vertical gradient of $\mathbf{H}_{2}$.}

$\delta \mathrm{H}_{2} / \delta \mathrm{z}$ can be calculated easily from the $\mathrm{H}_{\mathrm{p}}$ using equation (3.1-3): 


$$
\frac{\partial}{\partial z} \mathrm{H}_{\mathrm{z}}=-\left(\frac{1}{\rho} \mathrm{H}_{\rho}+\frac{\partial}{\partial \rho} \mathrm{H}_{\rho}\right)
$$

The computations can be simplified if we notice that $\mathrm{H}_{\mathrm{p}}$ field on a boundary can be written in a following form:

$$
H_{\rho}=\frac{1}{\rho} F(\rho)
$$

in which the $F(\rho)$ function is given by equation (3.1.2-11) with the $1 / \rho$ term factored out. Then we can rewrite equation (3.1.3-1) as:

$$
\frac{\partial}{\partial z} H_{z}=-\frac{1}{\rho} \frac{\partial}{\partial \rho} F(\rho)
$$

As a result of taking the derivative of equation (3.1.2-11) we get:

$$
\begin{gathered}
\frac{\partial}{\partial z} H_{z}=\frac{M}{4 \pi} \frac{k_{s}^{2}-k_{s+1}^{2}}{\rho^{2}}\left\{4 I_{2}(\rho b) K_{2}(\rho a)-I_{1}(\rho b) K_{2}(\rho a)(\rho b)+I_{2}(\rho b) K_{1}(\rho a)(\rho a)\right. \\
\left.+\frac{k_{s}^{2}+k_{s+1}^{2}}{k_{s}^{2}-k_{s+1}^{2}}\left[I_{d}(\rho b) K_{1}(\rho a)(\rho b)-I_{1}(\rho b) K_{d}(\rho a)(\rho a)-2 I_{1}(\rho b) K_{1}(\rho a)\right]\right\}
\end{gathered}
$$

The evaluation of this expression with all the functions involved can be accomplished only if the arguments of modified Bessel functions are large or small, as was the case with $\mathrm{H}_{\mathrm{p}}$ component.

At small induction numbers the evaluation of the $\delta \mathrm{H}_{2} / \delta \mathrm{z}$ component follows the procedure described for $\mathrm{H}_{\mathrm{p}}$, with a help of two additional expressions:

$$
\begin{aligned}
& I_{d}(z) \approx 1 \\
& K_{d}(z) \approx-\ln \left(\frac{z}{2}\right)
\end{aligned}
$$

In this case

$$
\begin{gathered}
\frac{\partial}{\partial z} H_{z} \approx \frac{M}{4 \pi} 4 a b\left[\frac{b^{2}}{8}+\frac{a^{2}+b^{2}}{4} \ln (\rho a)\right] \\
\approx-\frac{M}{4 \pi} \frac{(\omega \mu)^{2}\left(\sigma_{s}^{2}-\sigma_{s+1}^{2}\right)}{8}\left[1-\frac{2 \sqrt{\sigma_{s} \sigma_{s+1}}}{\left(\sigma_{s}+\sigma_{s+1}\right)} \ln \left(\frac{\rho}{2} \sqrt{\frac{\omega \mu}{2}}\left(\sqrt{\sigma_{s}}+\sqrt{\sigma_{s+1}}\right)\right]+i \frac{\pi}{4}\right]
\end{gathered}
$$


with $a$ and $b$ given by equations (3.1.2-9) and (3.1.2-10), respectively.

So both real and imaginary $\delta \mathrm{H}_{z} / \delta \mathrm{z}$ components are very sensitive to conductivity contrast. Comparing the real $\mathrm{H}_{\rho}$ component with both real and imaginary $\delta \mathrm{H}_{z} / \delta \mathrm{z}$ component (Figure 3.1.3-1) we can see that real $\mathrm{H}_{\mathrm{p}}$ is as sensitive to conductivity as both $\delta \mathrm{H}_{2} / \delta \mathrm{z}$ derivative components.

The computations for large induction numbers are very involved and face the same limitations as encountered in $\mathrm{H}_{\rho}$ component.

Simpler and more meaningful results are obtained by differentiating the expression for asymptotic $\mathrm{H}_{\rho}$ on the boundary, equation (3.1.2-29), with the help of equation (3.1.3-3). As a result we obtain:

$$
\begin{aligned}
\frac{\partial}{\partial z} H_{z}= & \frac{M}{4 \pi} \frac{2}{\sqrt{k_{s}^{2}-k_{s+1}^{2}}} \frac{1}{\rho^{5}}\left\{\mathrm{e}^{-i k_{s} \rho\left[9+9 i k_{s} \rho+4\left(i k_{s} \rho\right)^{2}+\left(i k_{s} \rho\right)^{3}\right]}\right. \\
& \left.\left.+i e^{-i k_{s+1} \rho[9+9} i_{s+1} \rho+4\left(i k_{s+1} \rho\right)^{2}+\left(i k_{s+1} \rho\right)^{3}\right]\right\}
\end{aligned}
$$

\subsubsection{Evaluation of $\delta^{2} \mathrm{H}_{z} / \delta z^{2}$ on the interface.}

The evaluation of $\delta^{2} \mathrm{H}_{z} / \delta z^{2}$ on a boundary can be made easy if we use the wave equation in cylindrical coordinates:

$$
\frac{1}{\rho} \frac{\partial}{\partial \rho} \rho \frac{\partial}{\partial \rho} \mathrm{H}_{\mathbf{z}}+\frac{\partial^{2}}{\partial \mathrm{z}^{2}} \mathrm{H}_{\mathrm{z}}+\mathrm{k}^{2} \mathrm{H}_{\mathrm{z}}=0
$$

to express of $\delta^{2} \mathrm{H}_{2} / \delta z^{2}$ in terms of of $\delta \mathrm{H}_{2} / \delta \rho$ :

$$
\frac{\partial^{2}}{\partial z^{2}} H_{z}=-k^{2} H_{z}-\frac{1}{\rho} \frac{\partial}{\partial \rho} \rho \frac{\partial}{\partial \rho} H_{z}
$$

The value of the $\mathrm{H}_{\mathrm{z}}$ on the boundary is given by equation (3.1.1-7). After taking the prescribed derivatives we have: 


$$
\begin{gathered}
\frac{\partial 2}{\partial z^{2}} H_{z}=-k_{s, s+1}^{2} H_{z}+\frac{M}{4 \pi} \frac{2}{k_{s}^{2}-k_{s+1}^{2}} \frac{1}{\rho^{7}} \\
\left\{e ^ { - i k _ { s } \rho } \left[225+225 i k_{s} \rho+108\left(i k_{s} \rho\right)^{2}\right.\right. \\
\left.+33\left(i_{s} \rho\right)^{3}+7\left(i k_{s} \rho\right)^{4}+\left(i k_{s} \rho\right)^{5}\right] \\
-e^{-i k_{s+1} \rho\left[225+225 i k_{s+1} \rho+108\left(i k_{s+1} \rho\right)^{2}\right.} \\
\left.\left.+33\left(i k_{s+1} \rho\right)^{3}+7\left(i k_{s+1} \rho\right)^{4}+\left(i k_{s+1} \rho\right)^{5}\right]\right\}
\end{gathered}
$$

In the above equation the symbol $k_{s, s+1}$ represents the propagation constant in the medium below or above boundary depending on the direction from which the boundary is approached. As can be observed, this is the only term in equation (3.1.4-3) that is discontinuous on a boundary. It shows, that while crossing the boundary the discontinuity in second vertical derivative is proportional to the difference in of product multiplied by $\mathrm{H}_{2}$.

The above analysis and plots show that $\mathrm{H}_{\rho}$ and $\delta \mathrm{H}_{2} / \delta \mathrm{z}$ show similar pattern of behavior near the boundary. Since it was frequently suggested to measure $\mathrm{H}_{p}$ component as more sensitive to conductivity variations, we propose to use $\delta H_{2} / \delta \mathrm{z}$ in cases where $\mathrm{H}_{\mathrm{p}}$ is not available. 


\subsection{Properties of the solution when transmitter and receiver are both above the interface.}

When transmitter and receiver are located away from the boundary, then its effect diminishes with increasing distance from the boundary. We will now estimate how far the influence of the boundary extends into a conductive medium as a function of the of product, and the transmitter-receiver separation, $p$. We will prove that this effect extends roughly to the depth of $\rho / 2$ from the boundary if transmitter and receiver are located at the same depth.

If source and receiver are both located above the interface, the exact expressions for $\mathrm{H}_{\mathrm{z}}, \mathrm{H}_{\mathrm{p}}$ and $\delta \mathrm{H}_{2} / \delta \mathrm{z}$ (derived in Appendix A.3) have the form:

$$
\begin{aligned}
H_{z_{0+0}}= & H_{z}\left(k_{s}, R\right)-H_{z}\left(k_{s}, R_{1}\right) \\
& -\frac{2}{k_{0}^{2}-k_{s+1}^{2}}\left[\frac{\partial^{2}}{\partial z_{1}^{2}} H_{z}\left(k_{s}, R_{1}\right)-\frac{M}{4 \pi} \int_{0}^{-} \lambda^{3}\left(i \gamma_{s+1}\right) e^{-i \gamma_{s} z_{1}} J_{d}(\lambda \rho) d \lambda\right] \\
H_{\rho_{s+0}}= & H_{\rho}\left(k_{s}, R\right)+H_{\rho}\left(k_{s}, R_{1}\right) \\
& +\frac{2}{k_{s}^{2}-k_{s+1}^{2}}\left[\frac{\partial^{2}}{\partial z_{1}^{2}} H_{\rho}\left(k_{s}, R_{1}\right)+\frac{M}{4 \pi} \frac{\partial}{\partial \rho} \int_{0}^{\infty} \lambda\left(i \gamma_{s+1}\right)\left(i \gamma_{s}\right) e^{-i \gamma_{s} z_{1}} J_{d}(\lambda \rho) d \lambda\right] \\
\frac{\partial}{\partial z} H_{z_{0+0}} & =\frac{\partial}{\partial z} H_{z}\left(k_{s}, R\right)-\frac{\partial}{\partial z} H_{z}\left(k_{s}, R_{1}\right) \\
& +\frac{2}{k_{0}^{2}-k_{o+1}^{2}}\left[\frac{\partial^{3}}{\partial z_{1}^{3}} H_{z}\left(k_{s}, R_{1}\right)+\frac{M}{4 \pi} \int_{0}^{-} \lambda^{3}\left(i \gamma_{s+1}\right)\left(i \gamma_{s}\right) e^{-i \gamma_{s} z_{1}} J_{0}(\lambda \rho) d \lambda\right] .
\end{aligned}
$$

In the above equations the $\mathrm{H}_{z}\left(\mathrm{k}_{\mathrm{s}}, \mathrm{R}\right), \mathrm{H}_{\mathrm{z}}\left(\mathrm{k}_{\mathrm{s}}, \mathrm{R}\right), \mathrm{H}_{\rho}\left(\mathrm{k}_{s}, \mathrm{R}_{1}\right), \mathrm{H}_{\rho}\left(\mathrm{k}_{s}, \mathrm{R}_{1}\right)$ are whole space fields, $z$ is receiver depth, $z_{s}$ is the source (transmitter) depth, and $R=\left(p^{2}+(z-\right.$ $\left.\left.z_{s}\right)^{2}\right)^{1 / 2}, R_{1}=\left(p^{2}+z_{1}^{2}\right)^{1 / 2}$. Here $z_{1}$ represents the sum of separations between the transmitter and the boundary and between the receiver and the boundary: $z_{1}=\left(z_{s+1}-z_{s}\right)+$ $\left(z_{s+1}-z\right)$, (Figure A.3-1a in Appendix A).

Observe that the first term in the equations for fields above the interface represents the direct wave between transmitter and receiver. The second term can be interpreted as the contribution from the source placed at the distance $\left|z_{s+1}-z_{s}\right|$ below the boundary. This kind of contribution is often referred to as an image source. This "image" term and the terms in square brackets constitute the reflected wave. Notice, that in the reflected wave the distance to the boundary is included in the $z_{1}$ parameter only. Thus the 
exponential vertical attenuation of reflected wave does not depend on how close transmitter (or receiver) is to boundary, but on the sum of their distances to the boundary. It is possible then, to write (Brekhowskikh, 1960):

$$
\Psi\left(k_{s}, k_{s+1}, \rho, R, R_{1}\right)=\Psi^{H}\left(k_{s}, \rho, R\right)+\Psi^{\text {refl }}\left(k_{s}, k_{s+1}, \rho, R_{1}\right)
$$

where $\Psi$ can represent $\Pi^{*}, \mathrm{H}_{2}, \mathrm{H}_{\rho}, \delta \mathrm{H}_{z} / \delta \mathrm{z}$, or $\delta^{2} \mathrm{H}_{z} / \delta \mathrm{z}^{2}$, with $\Psi^{\mathrm{H}}$ being a whole space field and $\Psi$ refl representing the 'reflected wave'.

This property has important implications. It states, that if the transmitter is moved away from the boundary and the receiver is moved towards the boundary by the same distance, $\Delta z$, then the total distance of transmitter and receiver to the boundary remains the same and the reflected field does not change (Figure 3.2-1). The total field changes however, since it includes the primary whole space field that varies with the transmitter receiver separation.
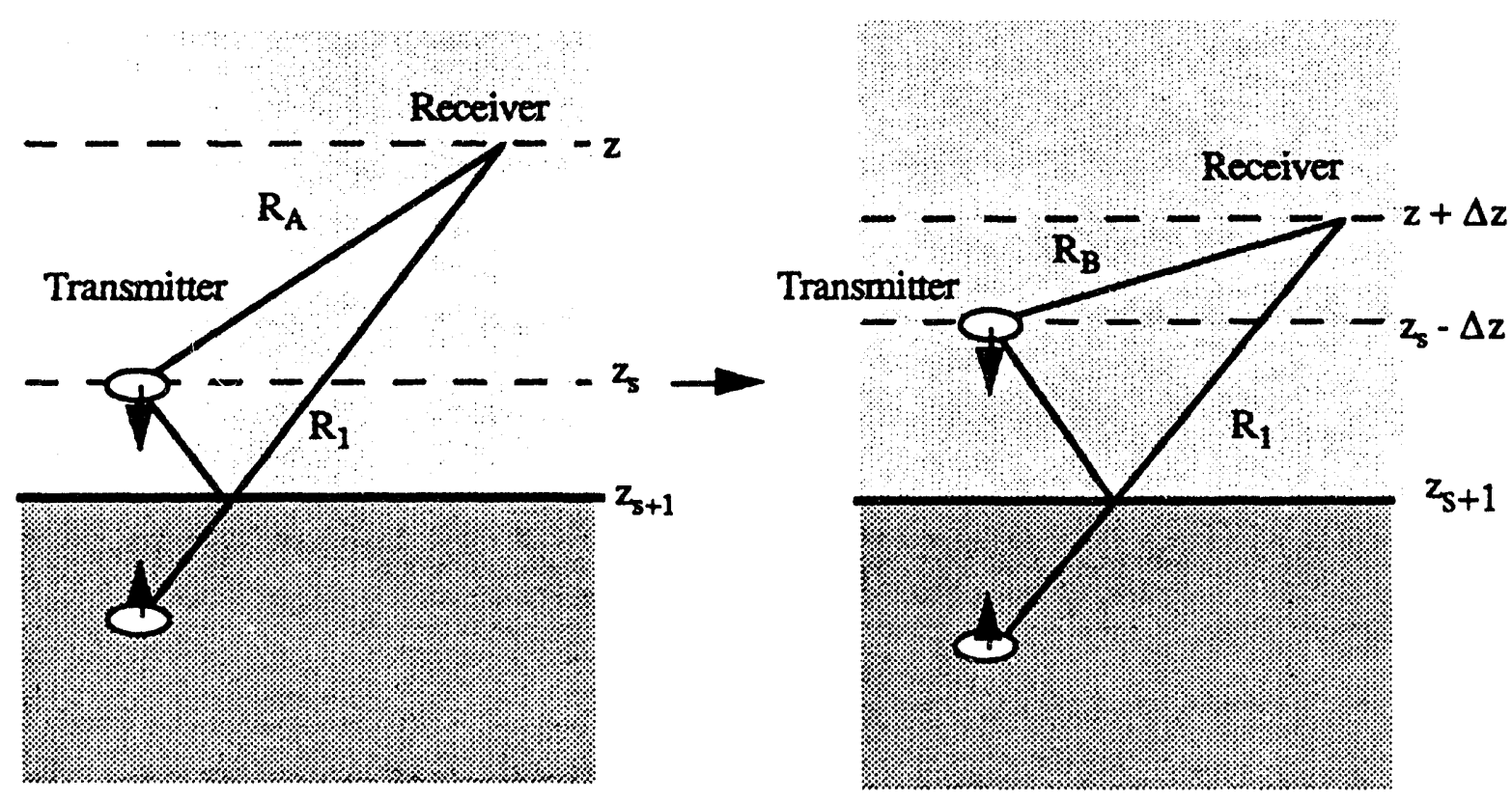

Fig. 3.2-1 Reflected field in this two configurations is the same. The primary filed changes because the transmitter - receiver separations are different in both cases. 
This property can be useful in obtaining the conductivity of the medium in which the source and receiver are located. The conductivity of the medium can be obtained by subtracting the measurements taken at two positions: $A$ and $B$ under the condition that the $z_{1}$ parameter is constant (Figure 3.2-1). Then:

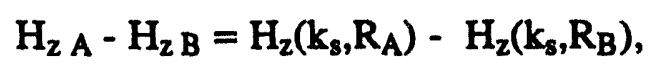

where: $\mathrm{H}_{2 A}$ and $\mathrm{H}_{\mathbf{2 B}}$ are fields measured at positions $A$ and $B, \mathrm{H}_{\mathbf{z}}\left(\mathrm{k}_{\mathbf{S}}, \mathrm{R}_{\mathbf{A}}\right)$ and $\mathrm{H}_{\mathbf{Z}}\left(\mathrm{k}_{\mathbf{5}}, \mathrm{R}_{\mathbf{B}}\right)$ are whole space primary fields, $R_{A}$ and $R_{B}$ are total transmitter-receiver separations at positions $A$ and $B$ respectively and $k_{s}$ is the induction parameter: $\omega^{2} \mu \varepsilon-i \omega \mu \sigma_{s}$. The only unknown in the above equation is $\sigma_{s}$ that can obtained by solving the above equation numerically.

Furthermore, by writing the whole space fields in an explicit form (see Appendix C, equations (C.3-1), (C.3-2), (C.3-3), (C.3-6) and (C.3-8) with $k_{s}$ or $k_{s+1}$ in place of $k$ and $z_{1}$ in place of $z$ ) it can be observed that the distance to the boundary, $z_{1}$, is included within a product $k_{s} z_{1}$. So the influence of the boundary depends on the product of $k_{s}$ and the sum of distances: transmitter to the boundary and receiver to the boundary.

Let's consider some properties of these solutions. The first important observation is that all these expressions depend on the of product, but not separately on conductivity and frequency. This property is true as long as the displacement currents can be neglected, i.e. as long as $\omega \varepsilon \ll \sigma$.

\subsubsection{Asymptotic analytic expressions when transmitter and receiver are both above the interface valid for $k \rho \gg 1$.}

The integrals in the exact expressions must be calculated numericallv or approximated by analytical methods. The approximations depend on the prubiem. In most surface geophysical methods it is frequently assumed that the propagation parameter in the air is zero. With this approximation the exponent in the integrand becomes a real number: $\gamma_{s}=\sqrt{k_{s}^{2}-\lambda^{2}} \approx \pm i \lambda$ and many analytic solutions exist. This quasi static condition was used by Sinha and Bhattaharya (1966) Wait, Kraichman (1970), Bannister (1977) and Banos (1966) to mention just the few authors that worked with dipoles embeded in conductive medium. In crosshole electromagnetic measurements we can not apply this condition because both media are conductive. 
In this case some useful results can be obtained in the far field region where $k \rho \gg$ 1 (Kaufman et al., 1983). The derivation of asymptotic forms for this condition is accomplished with the help of integrals given in Appendix $B: I_{1}$ for integral from equation (3.2-1), I5 for equations (3.2-2) and (3.2-3). These asymptotic solutions for transmitter and receiver off the boundary converge to the analytic ones on the boundary and are as follows:

$$
\begin{aligned}
& \mathrm{H}_{\mathrm{z}_{\mathrm{p}+0}} \approx \mathrm{H}_{\mathrm{z}}\left(\mathrm{k}_{\mathrm{s}}, \mathrm{R}\right)-\mathrm{H}_{\mathrm{z}}\left(\mathrm{k}_{\mathrm{s}}, \mathrm{R}_{1}\right)-\frac{2}{\mathrm{k}_{\mathrm{s}}^{2}-\mathrm{k}_{\mathrm{s}+1}^{2}}\left\{\frac{\partial^{2}}{\partial \mathrm{z}_{1}^{2}} \mathrm{H}_{\mathrm{z}}\left(\mathrm{k}_{\mathrm{s}}, \mathrm{R}_{1}\right)\right. \\
& \left.-\left[e^{-i \sqrt{k_{t}^{2}-k_{s+1}^{2}} z_{1}} \frac{\partial^{2}}{\partial z^{2}} H_{z}\left(k_{s+1}, R\right) I_{z=0}-i \sqrt{k_{s+1}^{2}-k_{t}^{2}} \frac{\partial}{\partial z_{1}} H_{z}\left(k_{s}, R_{1}\right)\right]\right\} \\
& H_{p_{s+0}} \approx H_{\rho}\left(k_{s}, R\right)+H_{p}\left(k_{s}, R_{1}\right)+\frac{2}{k_{s}^{2}-k_{s+1}^{2}}\left\{\frac{\partial^{2}}{\partial z_{1}^{2}} H_{p}\left(k_{s}, R_{1}\right)\right. \\
& \left.+\left.i \sqrt{k_{s}^{2}-k_{s+1}^{2}} e^{-i \sqrt{k_{s}^{2}-k_{s+1}^{2} z_{1}}} \frac{\partial}{\partial z} H_{p}\left(k_{s+1}, R\right)\right|_{z=0}+i \sqrt{k_{s+1}^{2}-k_{t}^{2}} \frac{\partial}{\partial z_{1}} H_{p}\left(k_{s}, R_{1}\right)\right\} \\
& \frac{\partial}{\partial z} H_{z_{0+0}}=\frac{\partial}{\partial z} H_{z}\left(k_{s}, R\right)-\frac{\partial}{\partial z} H_{z}\left(k_{s}, R_{1}\right)+\frac{2}{k_{s}^{2}-k_{s+1}^{2}}\left\{\frac{\partial^{3}}{\partial z_{1}^{3}} H_{z}\left(k_{s}, R_{1}\right)\right. \\
& \left.+\left[\left.i \sqrt{k_{s}^{2}-k_{s+1}^{2}} e^{-i \sqrt{k_{s}^{2}-k_{s+1}^{2}} z_{1}} \frac{\partial^{2}}{\partial z^{2}} H_{z}\left(k_{s+1}, R\right)\right|_{z=0}+i \sqrt{k_{s+1}^{2}-k_{s}^{2}} \frac{\partial^{2}}{\partial z_{1}^{2}} H_{z}\left(k_{s}, R_{1}\right)\right]\right\}
\end{aligned}
$$

where $H_{z}\left(k_{s}, R\right), H_{z}\left(k_{s+1}, R_{1}\right), H_{\rho}\left(k_{s}, R\right), H_{p}\left(k_{s+1}, R_{1}\right)$ are whole space fields.

\subsubsection{Transmitter and receiver approach boundary at the same height.}

In this case the depth variables take the values: $z=z_{s}, z_{1}=2\left(z_{s+1}-z_{s}\right), R=\rho$, and when implemented in equations from Section 3.2.1 give: 


$$
\begin{aligned}
& H_{z_{*+0}} \approx H_{2}\left(k_{s}, R\right)-H_{z}\left(k_{s}, R_{1}\right)-\frac{2}{k_{s}^{2}-k_{p+1}^{2}}\left\{\frac{\partial^{2}}{\partial z_{1}^{2}} H_{z}\left(k_{s}, R_{1}\right)\right. \\
& \left.-\left[e^{-i \sqrt{k_{s}^{2}-k_{s+1}^{2}} z_{1}} \frac{\partial^{2}}{\partial z^{2}} H_{z}\left(k_{s+1}, R\right) I_{z=0}-i \sqrt{k_{s+1}^{2}-k_{s}^{2}} \frac{\partial}{\partial z_{1}} H_{z}\left(k_{s}, R_{1}\right)\right]\right\}
\end{aligned}
$$

Writing the above expression in the explicit form we have:

$$
\begin{gathered}
H_{z s+0} \approx \\
\frac{M e^{-i k_{s} \rho}}{4 \pi} \rho^{3}(-)\left[1+i k_{s} \rho+\left(i k_{s} \rho\right)^{2}\right] \\
-\frac{M e^{-i k_{s}} R_{1}}{4 \pi} R_{1}^{5}\left[\left(1+i k_{s} R_{1}\right)\left(-\rho^{2}+2 z_{1}^{2}\right)+\left(i k_{s} R_{1}\right)^{2}(-) p^{2}\right] \\
-\frac{2}{k_{s}^{2}-k_{s+1}^{2}} \frac{M}{4 \pi}\left\{\frac { e ^ { - i k _ { s } } R _ { 1 } } { R _ { 1 } ^ { 9 } } \left[\left(1+i k_{s} R_{1}\right) 3\left(3 \rho^{4}-24 \rho^{2} z_{1}^{2}+8 z_{1}^{4}\right)\right.\right. \\
\left.+\left(i k_{s} R_{1}\right)^{2}\left(4 \rho^{4}-31 \rho^{2} z_{1}^{2}+10 z_{1}^{4}\right)+\left(i k_{s} R_{1}\right)^{3}\left(\rho^{4}-7 \rho^{2} z_{1}^{2}+28 z_{1}^{4}\right)+\left(i k_{s} R_{1}\right)^{4}\left(-\rho^{2} z_{1}^{2}\right)\right] \\
-i \sqrt{k_{s}^{2}-k_{s+1}^{2}} z_{1} \frac{1}{\rho^{5}} e^{-i k_{s+1} \rho}\left[9+9 i k_{s+1} \rho+4\left(i k_{s i-1} \rho\right)^{2}+\left(i k_{s+1} \rho\right)^{3}\right] \\
+i \sqrt{k_{s+1}^{2}-k_{s}^{2}} z_{1} \frac{e^{-i k_{s}} R_{1}}{R_{1}^{7}} \\
\left.\left[\left(1+i k_{s} R_{1}\right) 3\left(3 \rho^{2}-2 z_{1}^{2}\right)+\left(i k_{s} R_{1}\right)^{2} 2\left(2 \rho^{2}-z_{1}^{2}\right)+\left(i k_{s} R_{1}\right)^{3} \rho^{2}\right]\right\}
\end{gathered}
$$

This expression is still complicated, although it can provide some insight into the behavior of fields when transmitter and receiver move simultaneously towards the boundary. It can be observed that since the $R_{1}$ variable is a function of distance to the boundary, than and as long as $z_{1} \ll p$, the $R_{1}$ variable is dominated by $p$. In this case exponents $e^{-i k_{s} R_{1}} \approx e^{-i k_{s} \rho}$ do not change with $z_{1}$ for the fixed transmitter-receiver separation as long as $z_{1} \ll \rho$. When $z_{1} \gg \rho$ (and $\rho \gg>1$ ), the $e^{-i k_{s} R_{1}} \approx e^{-i k_{s} z_{1}}$ all terms with large $z_{1}$ in exponent can be neglected leaving only primary field that is not affected by the distance to the boundary. So we can assume that at high induction numbers, the boundary affects the response roughly up to the depth $p / 2$ because when $\rho \approx z_{1}=2\left(z_{s+1}\right.$ $z_{s}$ ), $R_{1}$ starts to be dominated by $z_{1}$ which introduces an exponential decay into the reflected wave with increasing separation to the boundary. 
To prove that indeed the effect of the boundary extends roughly to the depth of $h$ $=p / 2$ we constructed Figure 3.2-2a which shows the \% differences in amplitude $\mathrm{H}_{2}$ measured inside a conductive earth with and without the air-earth interface. The horizontal axis is an induction parameter for the conductive earth, $(\sigma f)^{1 / 2} \rho$. The vertical axis is the $z / p$ ratio where $h$ is the depth of transmitter (receiver) to the boundary. The transmitter and receiver are located at the same depth i.e. $z=z_{\mathbf{z}}$. The curve parameter is the percent difference between half-space and whole space response: $\left(\mathrm{H}_{2}\right.$ half-space $-\mathrm{H}_{2}$ whole space)/ $\mathrm{H}_{2}$ whole space ${ }^{*} 100 \%$. As can be observed the difference between whole space amplitudes and half-space amplitudes increases for induction parameter value above 1000. However this increase occurs only for the $z / \rho<0.5$, beyond which it rapidly decreases to zero. At this point the amplitude measured inside conductive earth (halfspace) can not be distinguished from the whole space field.

When transmitter (or receiver) is located on the surface of the earth (as in the surface to borehole geometry) then $z_{s+1}=z_{s}$ and $z_{1}=\left(z_{s+1}-z\right)$ and the effect of the boundary extends deeper into the earth (see Figure 3.2-2b). In this case difference between whole space amplitudes and half-space amplitudes also increases for induction parameter value above 1000 . However in this case the increase occurs to the depth $z / p=$ 1 , beyond which the effect of the boundary diminishes but is not smaller that $1 \%$.

To obtain an more quantitative descriptions of fields near the boundary consider some even more restrictive cases.

A significant simplification occurs if the horizontal separation is much greater than the distance from the boundary:

if $\rho \gg z_{1}$, then:

$$
\begin{gathered}
H_{z_{s+0}} \approx \frac{2}{k_{s}^{2}-k_{s+1}^{2}} \frac{M}{4 \pi} \frac{1}{\rho^{5}} \\
\left\{e^{-i \sqrt{k_{s}^{2}-k_{s+1}^{2}} 2\left(z_{s+1}-z_{s}\right)} e^{-i k_{s+1} \rho\left[9+9 i k_{s+1} \rho+4\left(i k_{s+1} \rho\right)^{2}+\left(i k_{s+1} \rho\right)^{3}\right]}\right. \\
\left.-\left(1+i \sqrt{k_{s+1}^{2}-k_{s}^{2}} 2\left(z_{s+1}-z_{s}\right)\right) e^{-i k_{s} \rho}\left[9+9 i k_{s} \rho+4\left(i k_{s} \rho\right)^{2}+\left(i k_{s} \rho\right)^{3}\right]\right\} .
\end{gathered}
$$

and we have an expression that resembles equation (3.1.1-7) for the $H_{z}$ field on a boundary between two dissipative half-spaces, with the exception of the terms are influenced by the distance to the boundary. 
If in addition to $\rho \gg z_{1}$, the conductivity of the source layer: $k_{s} \gg k_{s+1}$ then $e$ $-i k_{s} \rho \ll e^{-i k_{s}+1 \rho}$ and:

$$
\begin{gathered}
\mathrm{H}_{z_{s+0}} \approx \frac{2}{k_{s}^{2}} \frac{M}{4 \pi} \frac{1}{\rho^{5}} \\
e^{-i k_{s} 2\left(z_{s+1}-z_{s}\right)} \mathrm{e}^{-i k_{s+1} \rho\left[9+9 i k_{s+1} \rho+4\left(i k_{s+1} \rho\right)^{2}+\left(i k_{s+1} \rho\right)^{3}\right]}
\end{gathered}
$$

and the $\mathrm{H}_{\mathrm{z}}$ increases exponentially (for a fixed $\mathrm{k}_{s+1} \rho$ ) with the transmitter and receiver approaching the boundary, i.e. when $z_{1}=\left(z_{s+1}-z_{s}\right)$ decreases.

On the other hand if the conductivity of the source layer: $k_{s}<<k_{s+1}$ then $e^{-i k_{s p}} \gg>$ $e^{-i k s+1 \rho}$ and:

$$
\begin{gathered}
\mathrm{H}_{\mathrm{z}_{\mathrm{o}+0}} \approx \frac{2}{\mathrm{k}_{\mathrm{s}+1}^{2}} \frac{\mathrm{M}}{4 \pi} \frac{1}{\rho^{5}} \\
\left(1+\mathrm{ik}_{\mathrm{s}+1} 2\left(\mathrm{z}_{\mathrm{s}+1}-\mathrm{z}_{\mathrm{s}}\right)\right) \mathrm{e}^{-\mathrm{i}_{\mathrm{s}} \rho}\left[9+9 \mathrm{ik_{s } \rho}+4\left(\mathrm{ik}_{\mathrm{s}} \rho\right)^{2}+\left(\mathrm{ik}_{\mathrm{s}} \rho\right)^{3}\right] .
\end{gathered}
$$

and the $\mathrm{H}_{\mathrm{z}}$ decreases linearly as $\mathrm{k}_{s+1} 2\left(\mathrm{z}_{\mathrm{s}+1}-\mathrm{z}_{\mathrm{s}}\right)$ when the system approaches the boundary.

Let's summarize the above observations. If the medium in which the source is located is much more conductive then the other half-space, then the response increases exponentially as $\mathrm{e}^{- \text {-ikmore conductive } 2(2 s+1-2 s)}$ with diminishing distance to the boundary, i.e. when $\left(z_{s+1}-z_{s}\right)$ becomes smaller. In the lateral direction, the more resistive medium dominates with an exponential attenuation as $e^{-j e m o r e ~ r e s i s t i v e ~} p$. If the source medium is less conductive than the other half-space, the fields decrease while approaching the boundary, proportional to $\left(1+i k_{s+1} 2\left(z_{s+1}-z_{s}\right)\right)$ and the lateral attenuation is still governed by the $e^{-i k m o r e ~ r e s i s t i v e ~} P$. So the lateral attenuation when transmitter and receiver are near the boundary is governed by $e^{-i k m o r e ~ r e s i s t i v e ~} \rho$ factor independently whether the transmitter is in the resistive medium or not. Another way to look at this problem is to observe that lateral propagation is through the resistive layer. The same conclusions were reached in the paper by King et al. (1979) for a horizontal electric dipole. For the magnetic dipole the conclusions are the same because the magnetic dipole at a distance " is equivalent to four electric dipoles arranged in square ... and although the superimposed fields suffer great reduction, their modal structure is not changed".

Thus we observe the same pattern that took place for the transmitter and receiver located at the interface: the field is dominated by the propagation in the more resistive medium. For transmitter and receiver located off the boundary the exponential attenuation 
with distance to the boundary depends on the total transmitter-boundary and receiverboundary distance.

Examples of how the $\mathrm{H}_{2}$ field changes when the transmitter and receiver approach the air-earth boundary at the same height are given in Figure 3.2-3 for in-phase, quadrature, amplitudse and phase of $\mathrm{H}_{2}$ component. The transmitter-receiver horizontal separation is $20 \mathrm{~m}$. The conductivity of the lower half-space is $0.043 \mathrm{~S} / \mathrm{m}$, transmitterreceiver horizontal separation is $20.0 \mathrm{~m}$. The vertical axis gives the distance from the boundary. The three curves show the effect of frequency: curve $A$ is for frequancy $18,5000 \mathrm{~Hz}$ (to simulate the Richmond experiment), curve $B$ is for a lower frequency $1,850 \mathrm{~Hz}$, curve $\mathrm{C}$ for a higher frequency $180,500 \mathrm{~Hz}$. As can be seen from these figures the distance at which the boundary starts to be noticeable is of the order of $\rho / 2$ at large induction numbers. It is not so for small induction parameters, where the effect of the boundary can be observed over depths that are larger then the horizontal transmitterreceiver separation.

\subsection{Expressions when transmitter is above and receiver is below the interface.}

For sources and receivers on opposite side of the boundary, the exact, analytical expression for $\mathrm{H}_{\mathrm{z}}, \mathrm{H}_{\mathrm{p}}$ and $\mathrm{dH}_{\mathrm{z}} / \mathrm{dz}$ are given by the following:

$$
\begin{aligned}
& H_{z_{s+1}}=-\frac{2}{k_{0}^{2}-k_{s+1}^{2}} \frac{M}{4 \pi} \int_{0}^{-} \lambda^{3}\left(i \gamma_{s}-i \gamma_{s+1}\right) e^{-i \gamma_{s+1}\left(z-z_{s+1}\right)} e^{-i \gamma_{s}\left(z_{s+1}-z_{s}\right)} J_{0}(\lambda \rho) d \lambda \\
& H_{\rho s+1}=\frac{2}{k_{s}^{2}-k_{s+1}^{2}} \frac{M}{4 \pi} \frac{\partial}{\partial \rho} \int_{0}^{-} \lambda\left[\left(i \gamma_{s}\right)\left(i \gamma_{s+1}\right)-\left(i \gamma_{s+1}\right)^{2}\right] e^{-i \gamma_{s+1}\left(z-z_{o+1}\right)} e^{-i \gamma_{s}\left(z_{s+1}-z_{s}\right)} J_{0}(\lambda \rho) d \lambda
\end{aligned}
$$

$$
\frac{\partial}{\partial z} H_{z_{s+1}}=\frac{2}{k_{s}^{2}-k_{s+1}^{2}} \frac{M}{4 \pi} \int_{0}^{-} \lambda^{3}\left[\left(i \gamma_{s}\right)\left(i \gamma_{s+1}\right)-\left(i \gamma_{s+1}\right)^{2}\right] e^{-i \gamma_{s+1}\left(z-z_{o+1}\right)} e^{-i \gamma_{t}\left(z_{s+1}-z_{s}\right)} J_{0}(\lambda \rho) d \lambda
$$

In this case the response is influenced separately by the distance of transmitter to the boundary and by the distance of the receiver to the boundary on the opposite side. Thus in this case the effect of the boundary depends on the products: $k_{s}\left(z_{s+1}-z_{s}\right)$ and $k_{s+1}\left(z-z_{s+1}\right)$. The symbols used in the above equations are given in Figure a.3-1b. 


\subsubsection{Asymptotic expressions when transmitter is above and receiver is} below the interface.

The asymptotic evaluation of integrals in this case is performed with the help of integral $\mathrm{I}_{2}$ and $\mathrm{I}_{3}$ from the Appendix $\mathrm{B}$ (equation (b-12). Using different combinations of values for $z_{2}$ and $z_{3}$, all combinations of radicals and exponents encountered in the equations (3.3-1) to (3.3-3) that describe fields for this situation are covered. The $z_{2}=$ $z_{s+1}-z_{s}$ variable describes the vertical distance between boundary and the source above, and $z_{3}=z_{s}-z_{s+1}$ describes the vertical distance between the receiver and the boundary. We then have:

$$
\begin{aligned}
& H_{z_{s+1}}=\frac{2}{k_{s}^{2}-k_{s+1}^{2}}\left\{e^{-i \sqrt{k_{s}^{2}-k_{s+1}^{2}} z_{2}}\left[i \sqrt{k_{s}^{2}-k_{s+1}^{2}} \frac{\partial}{\partial z_{3}} H_{z}\left(k_{s+1}, R_{3}\right)+\frac{\partial^{2}}{\partial z_{3}^{2}} H_{z}\left(k_{s+1}, R_{3}\right)\right]\right. \\
& \left.-e^{-i \sqrt{k_{s+1}^{2}-k_{s}^{2}} z_{3}}\left[i \sqrt{k_{p+1}^{2}-k_{i}^{2}} \frac{\partial}{\partial z_{2}} H_{z}\left(k_{s}, R_{2}\right)+\frac{\partial^{2}}{\partial z_{2}^{2}} H_{z}\left(k_{s}, R_{2}\right)\right]\right\} \\
& H_{\rho_{s+1}} \approx \frac{2}{k_{*}^{2}-k_{s+1}^{2}}\left\{\mathrm { e } ^ { - i \sqrt { k _ { s } ^ { 2 } - k _ { o + 1 } ^ { 2 } } z _ { 2 } } \left[i \sqrt{k_{t}^{2}-k_{o+1}^{2}} \frac{\partial}{\partial z_{3}} H_{p}\left(k_{s+1}, R_{3}\right)\right.\right. \\
& \left.+\left(-k_{s+1}^{2}+\frac{1}{\rho^{2}}\right) H_{\rho}\left(k_{s+1}, R_{3}\right)-\frac{\partial^{2}}{\partial \rho^{2}} H_{\rho}\left(k_{s+1}, R_{3}\right)\right] \\
& \left.-e^{-i} \sqrt{k_{o+1}^{2}-k_{s}^{2}} z_{3}\left[i \sqrt{k_{s+1}^{2}-k_{s}^{2}} \frac{\partial}{\partial z_{2}} H_{\rho}\left(k_{s}, R_{2}\right)+\left(-k_{\delta+1}^{2}+\frac{1}{\rho^{2}}\right) H_{\rho}\left(k_{s}, R_{2}\right)-\frac{\partial^{2}}{\partial \rho^{2}} H_{\rho}\left(k_{s}, R_{2}\right)\right]\right\} \\
& \frac{\partial}{\partial z} H_{z_{s+1}}=\frac{2}{k_{s}^{2}-k_{s+1}^{2}}\left\{e^{-i \sqrt{k_{s}^{2}-k_{s+1}^{2}} z_{2}}\left[i \sqrt{k_{s}^{2}-k_{s+1}^{2}} \frac{\partial^{2}}{\partial z_{3}^{2}} H_{z}\left(k_{s+1}, R_{3}\right)+\frac{\partial^{3}}{\partial z_{3}^{3}} H_{z}\left(k_{s+1}, R_{3}\right)\right]\right. \\
& \left.+e^{-i} \sqrt{k_{t+1}^{2}-k_{i}^{2}} z_{3}\left[i \sqrt{k_{s+1}^{2}-k_{2}^{2}} \frac{\partial^{2}}{\partial z_{2}^{2}} H_{z}\left(k_{s}, R_{2}\right)+\frac{\partial^{3}}{\partial z_{2}^{3}} H_{z}\left(k_{s}, R_{2}\right)+\left(k_{s}^{2}-k_{s+1}^{2}\right) \frac{\partial}{\partial z_{2}} H_{z}\left(k_{s}, R_{2}\right)\right]\right\}
\end{aligned}
$$

where $z_{2}$ and $z_{3}$ variables were described before. 


\subsection{Characteristics of the response near a layer.}

In this part we will write explicit equations for the layer sandwiched between two half-spaces to examine which parameters determine the layer response inside the conductive medium.

\subsubsection{Transmitter located outside layer}

When the transmitter and receiver are below the layer then the $\mathrm{H}_{\mathrm{z}}$ component is given by equation (A.4-13), Appendix A. By rewriting this equation in a slightly different form we obtain:

$$
\begin{aligned}
& \mathrm{H}_{\mathrm{zs}-0}=\mathrm{H}_{\mathrm{d}}\left(\mathbf{k}_{\mathrm{s}}, \mathrm{z}_{\mathrm{s}}-\mathbf{z}\right)
\end{aligned}
$$

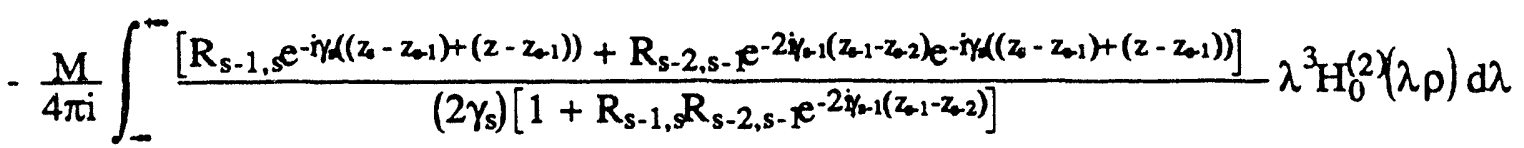

where: $\gamma_{s}=\sqrt{k_{s}^{2}-\lambda^{2}}, \gamma_{s-1}=\sqrt{k_{s-1}^{2}-\lambda^{2}}, \gamma_{s-2}=\sqrt{k_{s-2}^{2}-\lambda^{2}}$, and $R_{s-1,2}$ and $R_{s-2, s-1}$ are reflection coefficients from lower and upper boundary given in Appendix A. Figure 3.41 shows the geometry of the problem and symbols used.

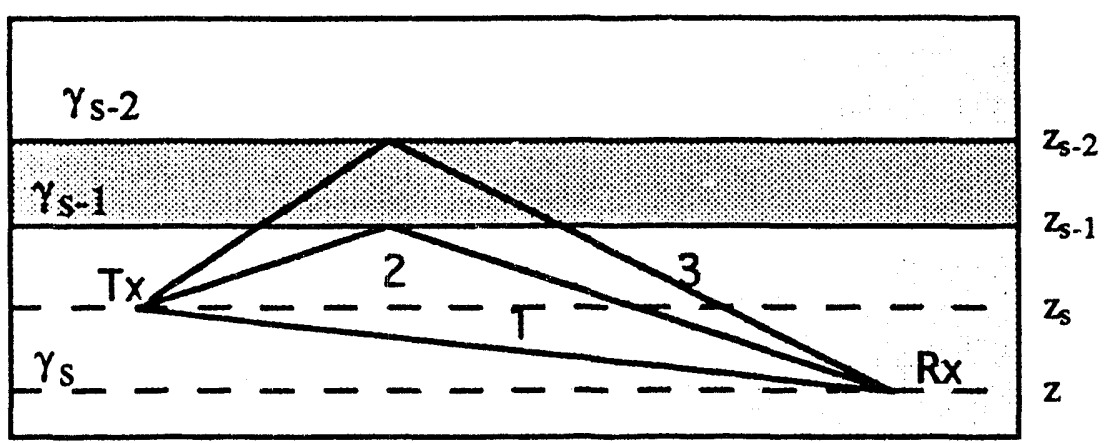

Fig. 3.4-1 Contributions from different boundaries when transmitter and receiver are below the interface: 1 - direct, whole space field, 2 - reflection from lower boundary, 3reflection from upper boundary and transmission through the layer

It can be observed that below the layer the received signal consists of two parts: primary whole-space field and secondary signal (expressed by the integral term), that is caused by the presence of the layer. 
The secondary response consists of two terms: the first term under the integral represents the reflection from the lower boundary, the second term describes the reflection from the upper boundary.

In particular the $\mathrm{H}_{z}\left(\mathrm{k}_{\mathrm{s}}, \mathrm{R}\right)$ term is a whole space primary field that depends on $\mathrm{k}_{\mathrm{s}}$ and $R$. The second contribution is characterized by the exponential attenuation e-is $\left(z_{s}-z_{s-1}\right)$ of signal in vertical direction and depends on the sum of distances: transmitter to the lower boundary and receiver to the lower boundary. The amplitude of this term is modified by the reflection coefficient from the lower boundary. The contribution from third term is characterized by the two-way attenuation in the layer (between $\mathrm{z}_{\mathrm{s}-1}$ and $\mathrm{z}_{\mathrm{s}-2}$ ) combined with the attenuation in the medium below the layer. The amplitude of this term depends on the reflection coefficient from the upper boundary. The denominator of all terms is modified by the signal "trapped" in the layer. The figure below shows how each term contributes to the total signal at receiver.

When the transmitter is below the layer, but the receiver moves inside the layer, then the response consists of the following contributions (see equation A.4-2 in Appendix A):

$$
\begin{aligned}
& H_{z s-1}=\frac{M}{2 \pi i} \int_{0}^{-}\left[-\frac{R_{s-2, s-1} e^{-i \gamma_{s}\left(z_{s}-z_{+-1}\right)} e^{-i \gamma_{s-1}\left(\left(z_{s-1}-z_{s-2}\right)+\left(z-z_{-2}\right)\right)}}{\left(\gamma_{s-0}+\gamma_{s-1}\right)\left[1+R_{s-1, s} R_{s-2, s-1} e^{-2 i \gamma_{s-1}\left(z_{-1-1}-z_{-2}\right)}\right]}\right. \\
& \left.+\frac{e^{-i \gamma_{\alpha}\left(z_{-}-z_{s-1}\right)} e^{-i \gamma_{-1}\left(z_{s-1}-z\right)}}{\left(\gamma_{s-0}+\gamma_{s-1}\right)\left[1+R_{s-1, s} R_{s-2, s 1} e^{\left.-2 i \gamma_{-1}\left(z_{s-1}-z_{-2}\right)\right]}\right.}\right] \lambda^{3} J_{0}(\lambda \rho) d \lambda
\end{aligned}
$$

The Figure 3.4-1 below suggest how each term can be visualized.

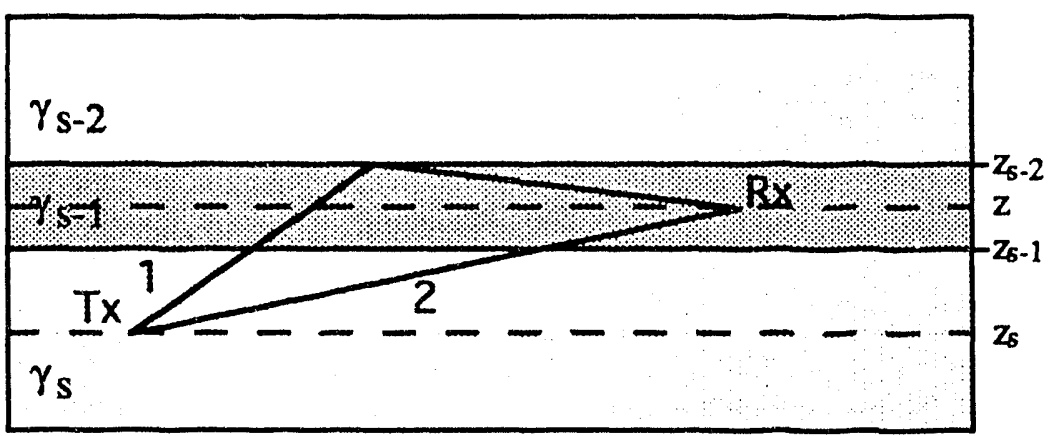

Fig. 3.4-2 Contributions from different boundaries when transmitter is below and receiver is inside a layer. 
The first term describes the signal at receiver that is reflected from the upper boundary. The second term is attenuated in lower medium over the distance $\left(z_{s}-z_{s-1}\right)$, then passes through the layer over the distance $\left(\mathrm{z}_{\mathrm{s}-1}-\mathrm{z}\right)$ to reach the receiver.

When transmitter and receiver are on the opposite sides of the layer than the oneway vertical attenuation depends on the sum of the distances over each portion of the path between transmitter and receiver. This can be seen from (see equation (A.4-1) and A.4-5 in appendix A):

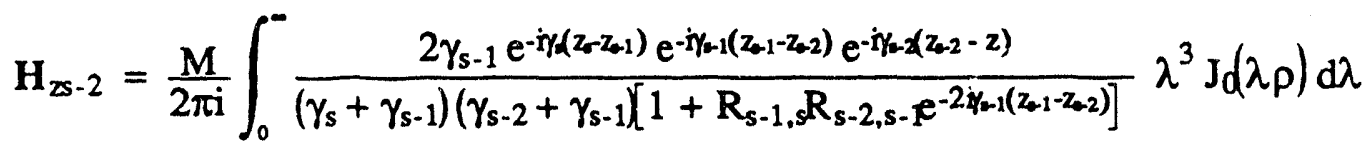

The intuitive interpretation of this expression is given in the Figure 3.4-3.

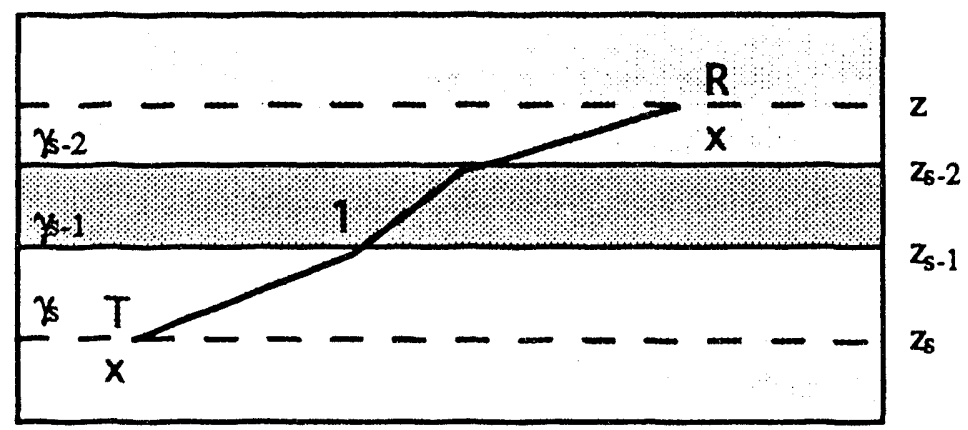

Fig. 3.4-3 Contributions from different boundaries when transmitter is below the layer

\subsubsection{Transmitter located inside the layer}

When the transmitter and receiver are inside the layer then the response consists of the following contributions (see equations (A.5-2), (A.5-6) and (A.5-7) from Appendix A): 


$$
\begin{aligned}
& H_{z s-0}=\frac{M}{4 \pi i} \int_{0}^{\infty}\left[\frac{R_{s-1, s} e^{-i \gamma_{s}\left(\left(z_{s}-z_{s+1}\right)+\left(z_{s+1}-z\right)\right)}}{\gamma_{s}\left[1+R_{s, s+1} R_{s-1, s} e^{\left.-2 i \gamma_{s}\left(z_{s+1}-z_{s-1}\right)\right]}\right.}\right. \\
& +\frac{R_{s, s+1} R_{s-1, s} e^{-\dot{\gamma} \gamma_{s}\left(2\left(z_{s+1}-z_{s}\right)+\left(z-z_{s}\right)\right)}}{\gamma_{s}\left[1+R_{s, s+1} R_{s-1, s} e^{\left.-2 i \gamma_{s}\left(z_{o+1}-z_{+1}\right)\right]}\right.} \\
& +\frac{e^{-\dot{\gamma_{p}}\left(z_{s}-z\right)}}{\gamma_{s}\left[1+R_{s, s+1} R_{s-1, s} e^{\left.-2 i \gamma_{s}\left(z_{s+1}-z_{s-1}\right)\right]}\right.} \\
& \left.\left.+\frac{R_{s, s+1} e^{-\dot{\gamma}_{s}\left(\left(z_{s+1}-z_{s}\right)+\left(z_{s+1}-z\right)\right)}}{\gamma_{s}\left[1+R_{s, s+1} R_{s-1, s} e^{\left.-2 i \gamma_{s}\left(z_{o+1}-z_{s-1}\right)\right]}\right.}\right] \lambda^{3} J d \lambda \rho\right) d \lambda \text {, }
\end{aligned}
$$

and we assumed that the receiver is above the transmitter just for illustrative purposes. The geometry of the problem is shown below.

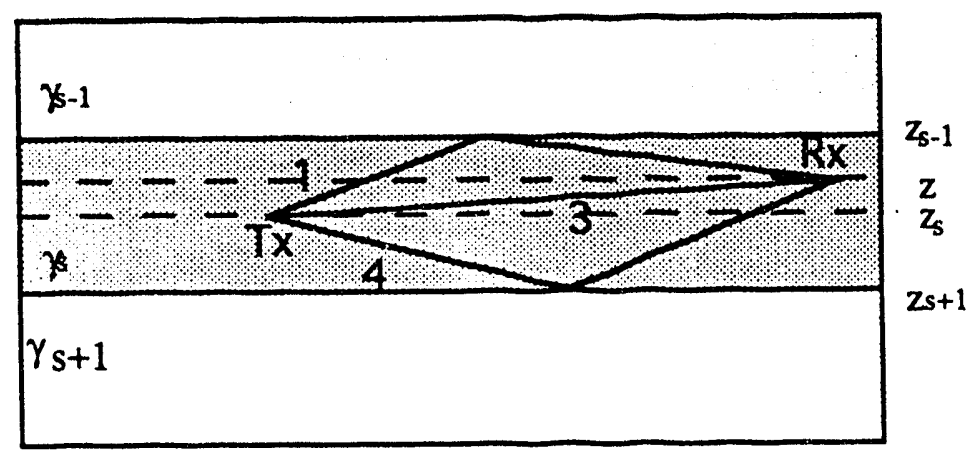

Fig. 3.4-4 Three main contributions to the signal when transmitter and receiver are inside the layer. The term that represents the interaction between layers is not shown.

Each term in the above equation is modified by the same expression in the denominator as in the case when transmitter was located outside layer. If the reflection coefficients are large, then a wave guide mode can be created. However in the case when the reflection coefficients are small, then their products are also small and we can visualize the signal contribution at the receiver as shown in Figure (3.4.2-1).

The first term in the equation (3.4.2-1) can be interpreted as a reflection from upper boundary, the last term as a reflection from lower boundary, the third term is a direct primary field between transmitter and receiver. The second term represents the interaction between the boundaries. 

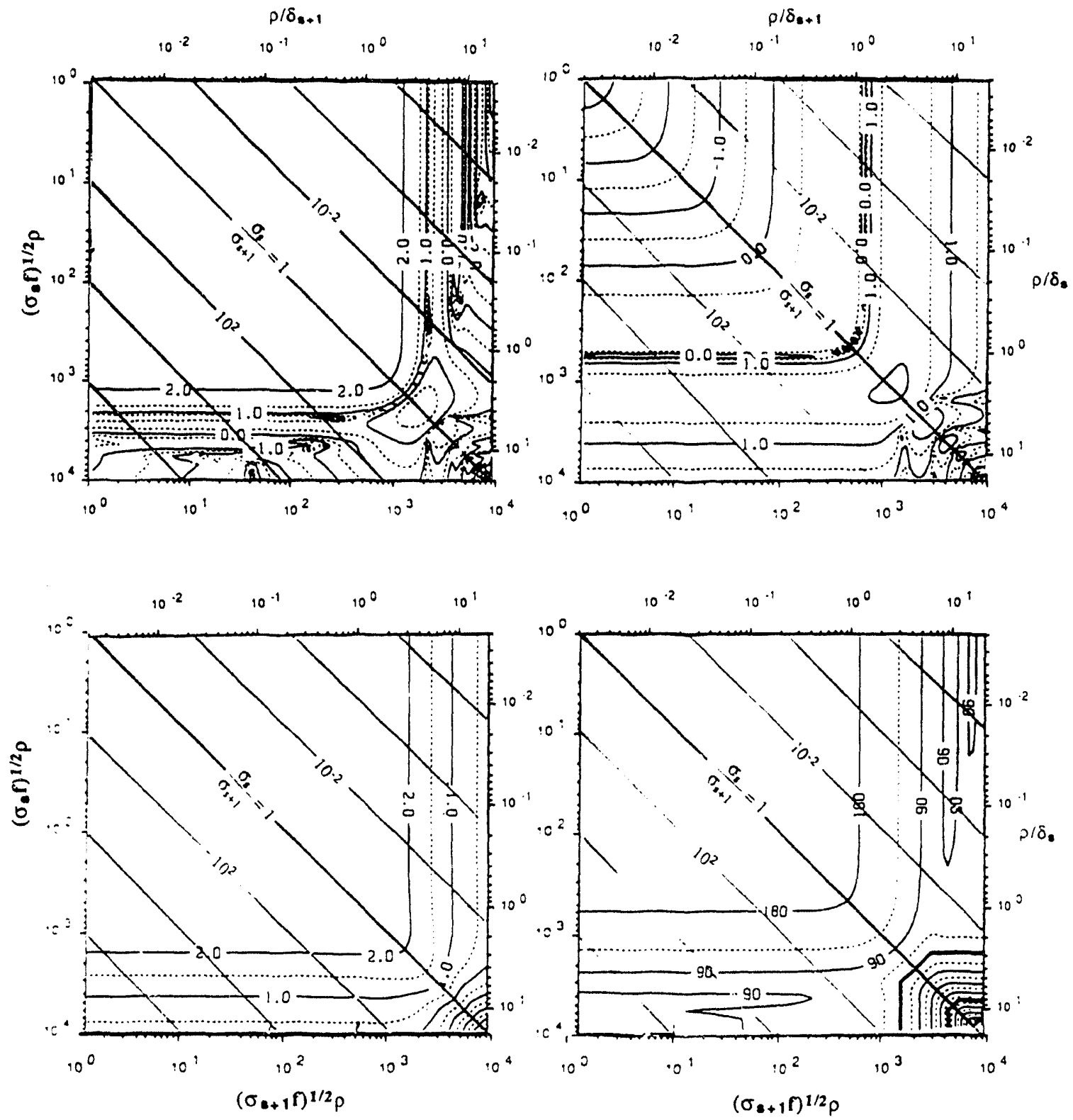

Fig. 3.1.1-1 Normalized response for $\mathrm{H}_{z} \rho^{3}$ : a) in-phase, b) quadrature, c) amplitude and d) phase for transmitter and receiver on the boundary. The curve parameters are the logarithms of normalized field strength in $\left(\mathrm{nTm}^{3}\right)$. The axes on the left and bottom of each figure are upper and lower half space induction parameters: $\left(\sigma_{s} f\right)^{1 / 2} \rho$ and $\left(\sigma_{s+1} f\right)^{1 / 2} \rho$, respectively. The axes on top and right are in terms of skin depth: $\rho / \delta_{s+1}$ and $\rho / \delta_{s}$ respectively. The parallel lines represent constant conductivity contrasts between the two half-spaces, with no contrast on the diagonal. 

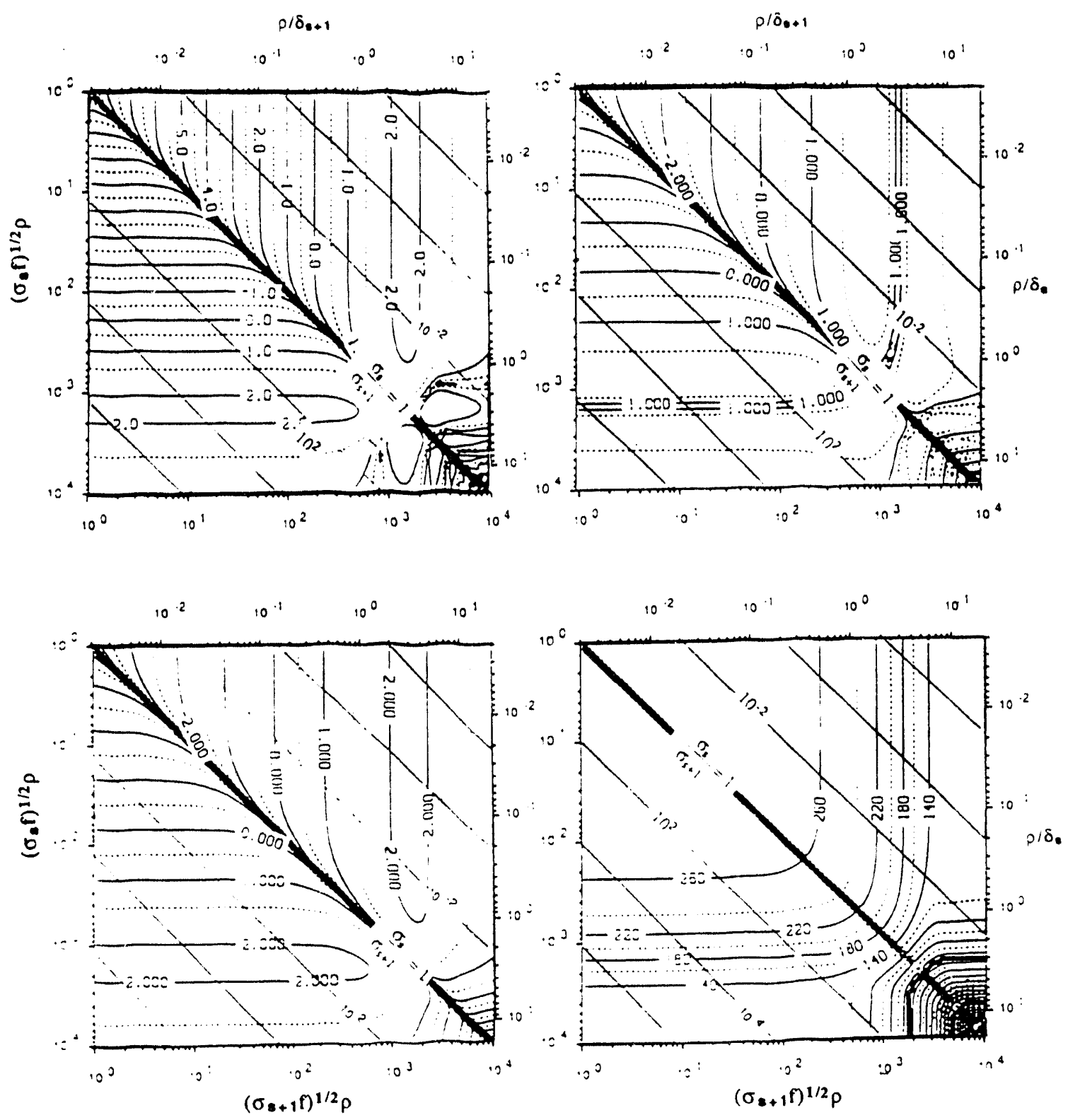

Fig. 3.1.2-1 Normalized response for $\mathrm{H}_{\rho} \rho^{3}$ : a) in-phase, b) quadrature, c) amplitude and $d$ ) phase $\mathrm{H}_{\rho} \rho^{3}$ for transmitter and receiver on the boundary. The curve parameters are the logarithms of normalized field strength in $\left(\mathrm{nTm}^{3}\right)$. The axes on the left and bottom of each figure are upper and lower half space induction parameters: $\left(\sigma_{s} f\right)^{1 / 2} \rho$ and $\left(\sigma_{s+1} f\right)^{1 / 2} \rho$, respectively. The axes on top and right are in terms of skin depth: $\rho / \delta_{s+1}$ and $\rho / \delta_{s}$ respectively. The parallel lines represent constant conductivity contrasts between the two half-spaces, with no contrast on the diagonai. 

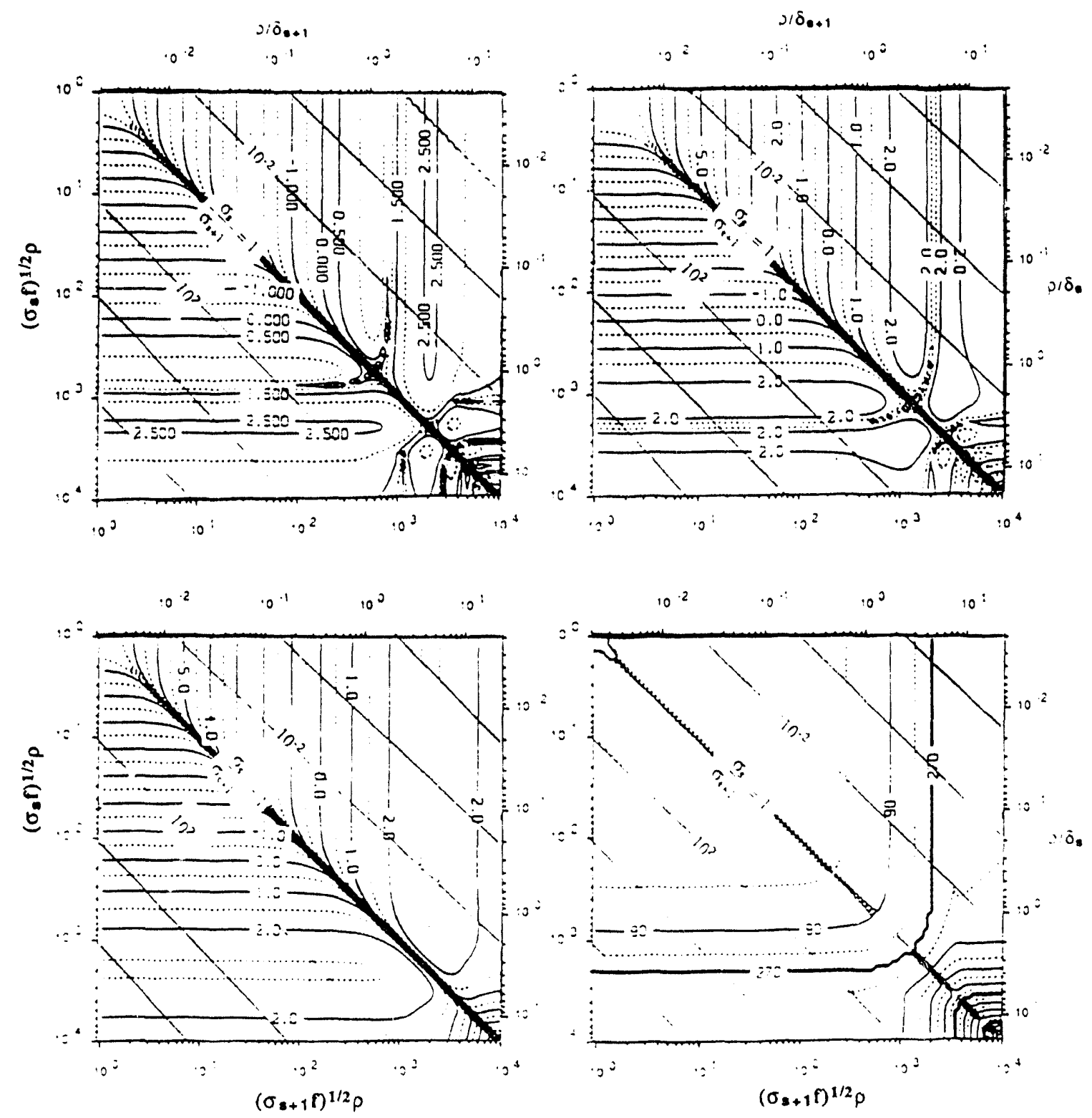

Fig. 3.1.3-1 Normalized response for $\left(\delta \mathrm{H}_{z} / \delta z\right) p^{4}$ : a) in-phase, b) quadrature, c) amplitude and d) phase for transmitter and receiver on the boundary. The curve parameters are the logarithms of normalized field strength in $\left(n \mathrm{Tm}^{3}\right)$. The axes on the left and bottom of each figure are upper and lower half space induction parameters: $\left(\sigma_{s} f\right)^{1 / 2} \rho$ and $\left(\sigma_{s+1} f\right)^{1 / 2} \rho$, respectively. The axes on top and right are in terms of skin depth: $\rho / \delta_{s+1}$ and $\rho / \delta_{s}$ respectively. The parallel lines represent constant conductivity contrasts between the two half-spaces, with no contrast on the diagonal. 

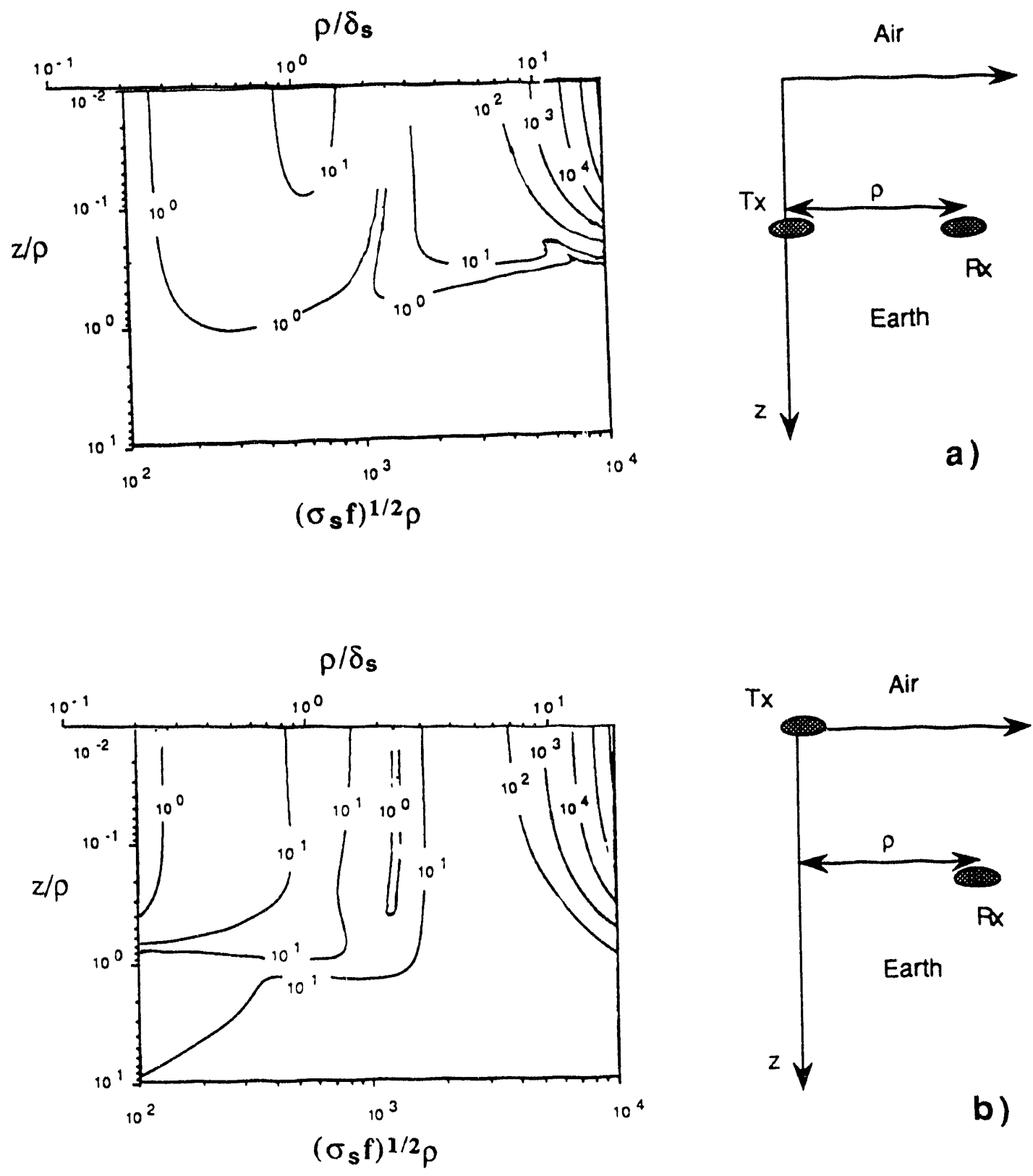

Fig. 3.2-2 The depth extent of the air-earth interface in crosshole geometry when the transmitter is at the same depth as the receiver (a), and in surface to borehole geometry,(b). The curve parameter is the percent difference in $\mathrm{H}_{\mathrm{z}}$ amplitude between the whole space and a half-space. The vertical axis is $z / \rho$ i.e. distance from the boundary over separation. The horizontal axis on a bottom in an induction parameter: $\left(\sigma_{s} f\right)^{1 / 2} \rho$, the horizontal axis on the top is given in terms of skin depth: $\rho / \delta$. 

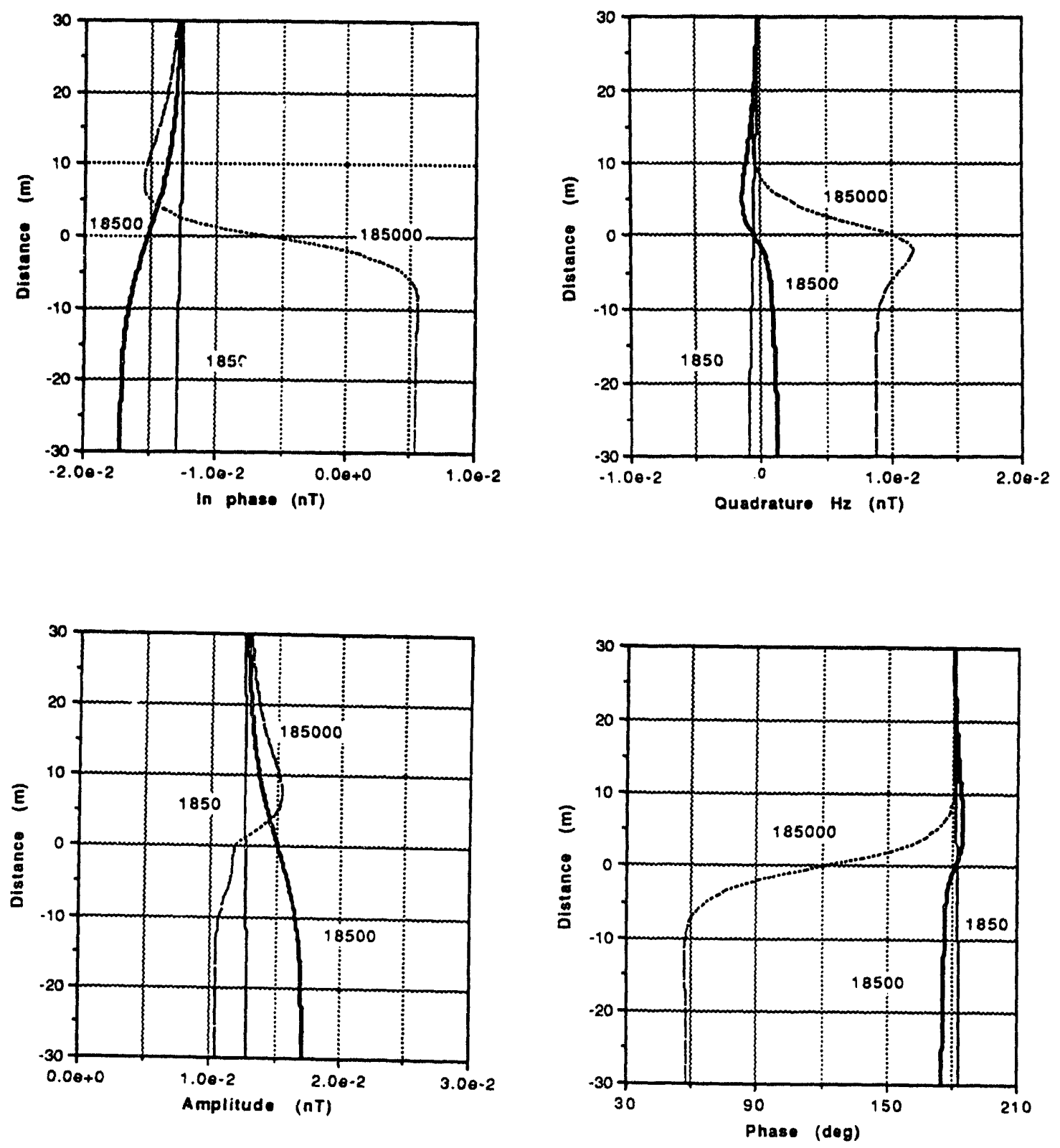

Fig. 3.2-3 The in-phase, quadrature, amplitude and phase $\mathrm{H}_{z}$ field near the interface between air and conductive half-space with conductivity $0.043 \mathrm{~S} / \mathrm{m}$. The transmitter and receiver move simultaneously at the same depth separated by $20 \mathrm{~m}$. Curve $A$ is for frequency $18,500 \mathrm{~Hz}$, curve $B$ for frequency $1,850 \mathrm{~Hz}$, curve $\mathrm{C}$ for frequency $185,000 \mathrm{~Hz}$. 


\section{Chapter 4}

\section{Direct methods to obtain apparent conductivity profiles in a one-} dimensional medium.

In this chapter we analyze simple techniques that give estimates of the conductivity directly, without the need for inversion. We will show that by using the vertical derivatives of the vertical fields we can recover the apparent conductivity of a onedimensional medium directly from the borehole measurements of electromagnetic fields. We will start with the method suggested by $\mathrm{Ki} \mathrm{Ha}$ Lee (Lee et al., 1992) that utilizes the second vertical derivative of the vertical magnetic field component measured on the axis of the transmitter. Then we will develop a method to estimate the conductivity in the cross-hole environment. Finally, using the field data we will show how the conductivity obtained from the cross-hole measurements corresponds to the conductivity obtained with conventional induction logs.

\subsection{Surface to borehole conductivity profiles}

Inside a one-dimensional medium the diffusion equation can be easily converted to provide the conductivity of the medium:

$$
\sigma=\frac{1}{i \omega \mu} \frac{\frac{\partial^{2} \mathrm{H}_{z}}{\partial x^{2}}+\frac{\partial^{2} \mathrm{H}_{z}}{\partial \mathrm{y}^{2}}+\frac{\partial^{2} \mathrm{H}_{z}}{\partial \mathrm{z}^{2}}}{\mathrm{H}_{\mathrm{z}}}
$$

It is evident that if all vertical and horizontal derivatives of the field components were known, then the conductivity of the medium at the measurement point could be calculated from equation (4.1-1). However in the narrow dimensions of the boreholes the horizontal derivatives cannot be measured, and $\mathrm{Ki} \mathrm{Ha}$ Lee (1992) suggested approximating the horizontal derivatives in the following way.

In general, we can always write:

$$
\frac{\partial H_{z}}{\partial x}=\frac{\partial H_{z}}{\partial R} \frac{\partial R}{\partial x}
$$

where $H_{z}=H_{z}(x, y, z)=H_{z}(R, z)$ and $R=x^{2}+y^{2}+z^{2}=\rho^{2}+z^{2}$ is the total transmitterreceiver separation (shown in the sketch on the next page). Taking the second horizontal derivative we have: 


$$
\frac{\partial^{2} \mathrm{H}_{\mathrm{z}}}{\partial \mathrm{x}^{2}}=\frac{\partial}{\partial \mathrm{x}}\left(\frac{\partial \mathrm{H}_{\mathrm{z}}}{\partial \mathrm{R}} \frac{\partial \mathrm{R}}{\partial \mathrm{x}}\right)=\left(\frac{1}{\mathrm{R}}-\frac{\mathrm{x}}{\mathrm{R}^{2}}\right) \frac{\partial \mathrm{H}_{\mathrm{z}}}{\partial \mathrm{R}}+\frac{\partial}{\partial \mathrm{R}} \frac{\partial \mathrm{H}_{\mathrm{z}}}{\partial \mathrm{R}}\left(\frac{\partial \mathrm{R}}{\partial \mathrm{x}}\right)^{2}
$$

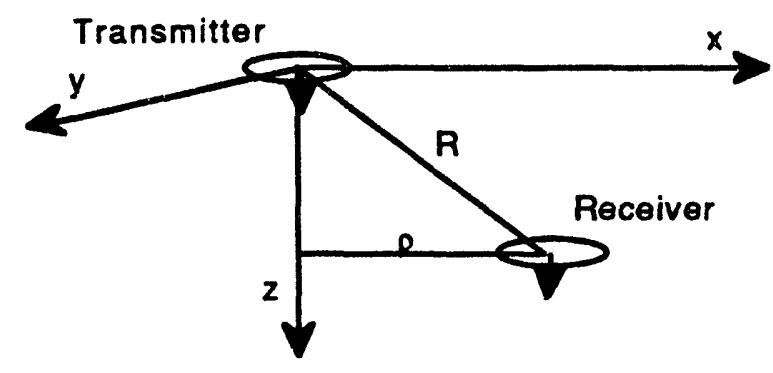

On the axis of the transmitter where $x=0$ and $\delta R / \delta x=0$ the second horizontal derivative reduces to:

$$
\frac{\partial^{2} H_{z}}{\partial x^{2}}=\frac{1}{R} \frac{\partial H_{z}}{\partial R}
$$

Similarly the derivative in the $y$ direction has the form:

$$
\frac{\partial^{2} \mathrm{H}_{\mathrm{z}}}{\partial \mathrm{y}^{2}}=\frac{1}{\mathrm{R}} \frac{\partial \mathrm{H}_{\mathrm{z}}}{\partial \mathrm{R}}
$$

As a result, equation (4.1-1) can be written as:

$$
\sigma=\frac{1}{i \omega \mu} \frac{\frac{2}{R} \frac{\partial H_{z}}{\partial R}+\frac{\partial^{2} H_{z}}{\partial z^{2}}}{H_{z}}
$$

This expression indicates that if we could measure both derivatives of the vertical magnetic field, then the conductivity would be determined. However, inside boreholes it is not possible to measure the $\delta \mathrm{H}_{2} / \delta \mathrm{R}$ derivative, and for this reason we express this derivative in terms of the $\delta \mathrm{H}_{2} / \delta \mathrm{z}$ derivative by applying the chain rule.

We have: 


$$
\frac{\partial \mathrm{H}_{z}(\mathrm{R}, \mathrm{z})}{\partial \mathrm{z}}=\frac{\partial \mathrm{H}_{\mathrm{z}}(\mathrm{R}, \mathrm{z})}{\partial \mathrm{z}} \mathrm{I}_{\mathrm{R}}+\frac{\partial \mathrm{H}_{\mathrm{z}}(\mathrm{R}, \mathrm{z})}{\partial \mathrm{R}} \frac{\partial \mathrm{R}}{\partial \mathrm{z}}
$$

where $I_{R}$ denotes the partial derivative at a constant $R$. Rearranging the terms in the above equation leads to:

$$
\frac{\partial H_{z}(R, z)}{\partial R}=\left[\frac{\partial H_{z}(R, z)}{\partial z}-\left.\frac{\partial H_{z}(R, z)}{\partial z}\right|_{R}\right] \frac{\partial z}{\partial R}
$$

which provides the $\delta \mathrm{H}_{\mathbf{Z}} / \delta \mathrm{R}$ derivative in terms of $\delta \mathrm{H}_{\mathbf{Z}} / \delta \mathrm{z}$ derivative.

Up to this moment we were dealing with a general one-dimensional medium. But in order to use equation (4.1-9) we need a specific expression for the $\mathrm{H}_{\mathrm{z}}$ field. Since inside a uniform whole space the $\mathrm{H}_{2}$ component has a simple analytic form we will use it to express the $\delta \mathrm{H}_{z} / \delta \mathrm{R}$ derivative in terms of $\delta \mathrm{H}_{z} / \delta \mathrm{z}$. The whole space fields are given in equations (C.3-1) and (C.3-2) in Appendix C. After substituting $\rho^{2}=R^{2}-z^{2}$ they assume the following form:

$$
\begin{gathered}
H_{z}(R, z)=\frac{M}{4 \pi} \frac{e^{-i k R}}{R^{5}}\left[(1+i k R)\left(-R^{2}+3 z^{2}\right)+(i k R)^{2}\left(-R^{2}+z^{2}\right)\right] \\
\frac{\partial H_{z}(R, z)}{\partial z}=\frac{M}{4 \pi} \frac{e^{-i k R}}{R^{7}} z\left[(1+i k R) 3\left(3 R^{2}-5 z^{2}\right)\right. \\
+(i k R)^{2} 2\left(2 R^{2}-3 z^{2}\right) \\
\left.+(i k R)^{3}\left(R^{2}-z^{2}\right)\right]
\end{gathered}
$$

Calculating $\delta \mathrm{H}_{2} / \delta \mathrm{z}_{\mathrm{R}}$ from equation (4.1-9) we obtain:

$$
\left.\frac{\partial H_{2}(R, z)}{\partial z}\right|_{R}=\frac{M}{4 \pi} \frac{e^{-i k R}}{R^{5}} 2 z\left[3(1+i k R)+(i k R)^{2}\right]
$$

After substituting equations (4.1-10) and (4.1-11) into equation (4.1-8), on the axis of the transmitter where $\rho=0$ and $R=z$, we obtain:

$$
\frac{\partial \mathrm{H}_{\mathrm{z}}(\mathrm{R}, \mathrm{z})}{\partial \mathrm{R}}=2 \frac{\partial \mathrm{H}_{\mathrm{z}}(\mathrm{R}, \mathrm{z})}{\partial \mathrm{z}}
$$

The final expression for the conductivity on the axis of the borehole is given by: 


$$
\sigma=\frac{1}{i \cos \mu} \frac{\frac{4}{\mathrm{z}} \frac{\partial \mathrm{H}_{\mathrm{z}}}{\partial \mathrm{z}}+\frac{\partial^{2} \mathrm{H}_{\mathrm{z}}}{\partial \mathrm{z}^{2}}}{\mathrm{H}_{\mathrm{z}}}
$$

The above expression gives the whole - space conductivity from the measurement of the vertical derivative of the vertical field on the axis of a borehole. For more complex conductivity distributions it provides an apparent conductivity.

To test the method we used a numerical code to calculate the conductivity for three models: half-space, layered half-space and a layered half-space. The conductivity of the whole space and half-space was $0.043 \mathrm{~S} / \mathrm{m}$ (the same as in Chapter 2), the conductivities of the layered half space were obtained from a one-dimensional least - squares inversion of the field data collected in Richmond and described in Chapter 5. The vertical profiles of the $\mathrm{H}_{2}$ component were collected at depths of 4.0 to $60.0 \mathrm{~m}$. The transmitter was placed on the surface, except in one case when it was traveling with receiver as in the logging geometry (described later).

Our first model was a half-space to test how the calculated conductivity is affected by the presence of a free space - earth boundary. Figure 4.1-1 shows the resulting conductivity at two frequencies: $100 \mathrm{~Hz}$ and $18500 \mathrm{~Hz}$ respectively. As can be observed the recovered conductivity is very strongly affected by a presence of a boundary especially at low frequencies, where the effect of the free space extends deep into the medium and causes over $25 \%$ errors in the estimate of conductivity. The skin depth at the lower frequency was 242. m and was three times larger than our depth of observation. The skin depth for the high frequency was $18 . \mathrm{m}$ and was smaller than the depth of observation. We thus prove, that using the whole space model to approximate the effect of horizontal derivatives provides only a rough estimate of the conductivity for a half-space.

Next we applied the method to the layered half-space. As can be observed in Figure 4.1-2 the method provided a very accurate location of the boundaries between layers, however the layer conductivities were only approximate, although the ratio of successive layer conductivities is correct. Figure 4.1-2 also shows that the higher frequency recovered the conductivity better than the lower frequency.

Finally we examined how the method would work deep inside boreholes where the effect of the air - earth interface would be sunaller and the conditions closer to the whole space model. Our model was the same as before with an exception of the upper half-space that assumed the conductivity of $0.1 \mathrm{~S} / \mathrm{m}$. The results for frequency $100 \mathrm{~Hz}$ 
and $18500 \mathrm{~Hz}$ are given in Figure 4.1-3. As can be observed deep inside a conductive earth the conductivity of a layered medium is also biased especially at larger vertical transmitter-receiver separations and lower frequency, but close to the transmitter the results improved significantly, and at low frequency are also satisfactory. The fact that at low frequency the conductivity of the layered medium can be recovered in a limited region close to the transmitter has an important practical application, since it promises a correct recovery of conductivity inside cased boreholes. Measurements inside cased boreholes are possible, as shown in Augustine et. al (1987) but only at low frequencies.

To test these possibilities we used the same layered earth model and applied our method to a situation that simulated the induction log geometry: transmitter was placed $2.6 \mathrm{~m}$ above the three receiver coils separated by $0.1 \mathrm{~m}$. The measurements were taken every $0.5 \mathrm{~m}$ with transmitter and receiver traveling simultaneously down the borehole. The results for two frequencies are given in Figure 4.1-4. As can be observed the apparent conductivity although not exact, follows the true conductivity quite accurately.

This layered half-space model showed that it is possible to use the derivatives to obtain an approximat conductivity distribution of a one dimensional medium. This estimate however deteriorates in the surface to borehole situation when transmitter is fixed on the surface. To improve the agreement between true and calculated conductivities in this situation we calculated the conductivity in two steps. First we obtained a rough estimate of conductivity by matching the in-phase and quadrature of $\mathrm{H}_{2}$ profiles with the whole-space conductivity, then we averaged the results and used the averaged value to calculate numerically the contributions from the horizontal derivatives using a half-space model of averaged conductivity. As a result we obtained an apparent conductivity of a half-space that closely resembles a true one as can be seen in Figure 4.1-5 for frequencies 100 and $18500 \mathrm{~Hz}$ respectively.

For the layered model the apparent conductivity is presented in Figure 4.1-6. The conductivity was also calculated in two steps: first we obtained a rough estimate of conductivity (the same as in Figure 4.1-2), then we averaged the results and used the averaged value to calculate numericaly the contributions from the horizontal derivatives. As can be observed the results are better at high frequencies where the recovered conductivity closely follows a true model. At low frequency the difference between calculated and true model increases with increasing transmitter-receiver sefaration.

In conclusion we observe that the second derivative is very accurate in locating the layer boundary. It also predicts the correct trend in conductivity, i.e. whether the 
conductivity increases or decreases with distance. However the estimate of the layer conductivities itself is not always accurate. In general the conductivities are recovered better at close transmitter-receiver vertical separation. At larger transmitter-receiver separations the recovered conductivity is biased, especially at low frequencies. The error is especially large in surface to borehole geometry (where the transmitter is located on the surface of the earth) and when the operating frequencies are low.

\subsection{Crosshole conductivity profiles}

When the measurements are performed in a crosshole environment, not on the axis of the transmitter, equation (4.1-6) is no longer valid. In this case equation (4.1-1) can still provide the conductivity estimate, but one must find another method to evaluate the contribution from the second horizontal derivatives. We propose to approximate these contributions numerically using the conductivity of a half-space that produces the smallest least-squares error in amplitude fit between numerical half-space data and measured data.

We start by examining several parameters that are crucial for the success of the method, such as the choice of background conductivity, effect of frequency, and the use of multiple transmitters. To reach the conclusions we use numerical data obtained with the half-space model of conductivity $0.043 \mathrm{~S} / \mathrm{m}$. Horizontal spacing between transmitter and receiver is $20.0 \mathrm{~m}$, the depth of measurements is between 4.0 and $60.0 \mathrm{~m}$. The frequency was 100 and $18500 \mathrm{~Hz}$.

We found that to recover the conductivity profile that corresponds to the induction logs, several factors had to be considered.

At first we considered the effect of the error in estimating the background conductivity of a half-space at two frequencies: $100 \mathrm{~Hz}$ and $18500 \mathrm{~Hz}$. Using numerical data obtained with a half-space model of conductivity $0.043 \mathrm{~S} / \mathrm{m}$ we changed the reference half space conductivity to $0.040 \mathrm{~S} / \mathrm{m}$. As can be observed in Figure 4.2-1a,b for frequencies 100 and $18500 \mathrm{~Hz}$, respectively, the conductivity of the half-space was recovered best at $18500 \mathrm{~Hz}$ and when the vertical distance between the transmitter and the receiver was small. With increasing separation, the difference between the true and calculated conductivity also increased. This observation proved that using only one transmitter we could recover the conductivity but only for the limited vertical transmitterreceiver separation. At low frequency the conductivity was recovered correctly only in close proximity to the transmitter.

To solve this problem we considered several (eleven) transmitters and averaged the results from different transmitters but only if the vertical separation between particular 
transmitter and receiver was not greater than $5.0 \mathrm{~m}$ i.e. not greater than the vertical spacing of transmitter locations. Using this technique we recovered the conductivity of the half-space perfectly and the results for the layered half-space seemed satisfactory. The results for the layered half-space can be observed in Figure 4.2-2 which shows the tue model and the recovered one at frequency $100 \mathrm{~Hz}$. In particular Figure $4.2-2 \mathrm{~b}$ shows how the reconstructed conductivity of the layered half-space behaves when transmitters located at different depth from the source were used. Figure 4.2-2a presents the final results, in which the conductivities obtained from different transmitters were averaged, but only for points which had vertical transmitter-receiver separations less than $5.0 \mathrm{~m}$. Figure 4.3-3 shows the same results bout for the frequency of $18500 \mathrm{~Hz}$.

In both cases the layer boundaries are found correctly, but conductivities were biased especially at low frequencies.

So in principle we proved that it is possible to obtain the layer boundaries and estimate the conductivity of the one-dimensional model in cross-hole environment without the need of an inversion.

The final test was to apply our method to the field data. We used field data from Devine and from Richmond. The Devine data were collected in a strictly one-dimensional environment at two frequencies: 512 and $2048 \mathrm{~Hz}$, the Richmond data were collected in a complicated, three-dimensional medium at frequency $18500 \mathrm{~Hz}$. Figure $4.2-4 \mathrm{a}, \mathrm{b}$ presents the induction $\log$ and the calculated conductivity for Devine data at 100 and 2048 $\mathrm{Hz}$, respectively. Figures $4.2-5$ to $4.2-8$ present the results for the Richmond data.

The conductivities were calculated in the same manner as described above. Each figure presents the recovered conductivity (curve B) and the induction log (curve A) collected at the same well in which the cross-hole measurements were taken. Curve $\mathrm{C}$ represents the background conductivity of a half-space that was obtained by matching the measured in-phase and quadrature with numerical values from a half-space over a certain depth interval below and above the fixed receiver. That interval was $20.0 \mathrm{~m}$ for Richmond data and $50.0 \mathrm{~m}$ for Devine data. The choice of the depth inteval was dictated by frequency and separation. We wanted the conductivity of a half-space that represents the averaged conductivities the best in the region of receiver location. We did not use a whole length of a profile to fit conductivity because the data at larger vertical-transmitter separations have more error. We considered using just one point at maximum amplitude, but this method would be very sensitive to errors in location and measurements errors. As a compromise we decided to fit values over a distance which 
was larger at lower frequencies such as in Devine and smaller at higher frequencies as in Richmond.

In addition for Richmond $\mathrm{da}_{\mathrm{m}}$ we plotted the difference in conductivities measured before and after the injection of salt water plume (Figures 4.2-5b to 4.2-8b). In particular curve A shows the conductivity difference as measured by induction logs before and after the injection, curve B shows the difference calculated from the crosshole data.

As can be observed there exist a strong correlation between the induction logs and the conductivity calculated from the derivatives. The best results we obtained were for the Devine data collected at $2048 \mathrm{~Hz}$, despite the fact that we considered this data more noisy than at $512 \mathrm{~Hz}$. This confirms our observation that high frequencies produce fields that vary faster with distance making the derivative calculations more accurate. We also observe that although the results in Richmond produce the conductivity variations that correlate with the induction logs, the recovered conductivity shows an offset in the lower part of the profile that is not present in Devine data. We assumed that this offset is caused by the calibration of the logging tool - it was not calibrated properly when the conductivities were very low - and also by the errors in the estimate of the background conductivity of the half-space. This background error is especially evident in the data collected in wells SW and SE where we observe a sudden jump in conductivity that can not be justified by induction logs. Furthermore, by comparing the differences in conductiviri- alculated using second vertical derivatives (curve B in figures 4.2-5b to 4.2-8u), tefore and after injection of salt water we notice that wells NW and NE show a strong positive anomaly at a depth of $30.0 \mathrm{~m}$ that is not present in data from SE and SW wells. This agrees with the conclusions reached by D. Alumbaugh et al. (1993) that places the salt water plume in the NW direction from the injection well.

We proved, that by taking data along vertical profiles at closely space intervals we can recover the layer boundaries very accurately. We also showed that the conductivities correlate with induction logs providing another method of conductivity estimate. Furthermore by comparing the difference in conductivities measured before and after injection from different observation wells, one can get a preliminary estimate of the direction of plume propagation. The process gives better results at higher frequencies because fast variations in field strength with distance allow to obtain more accurate derivative calculations. 


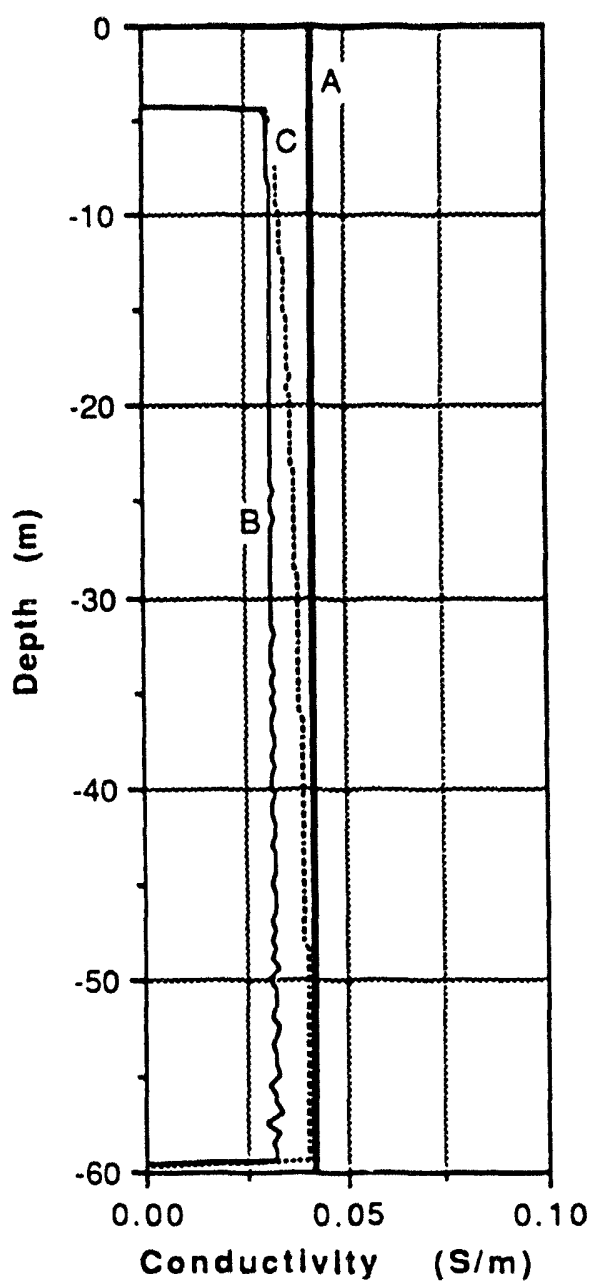

Fig. 4.1-1 The true and recovered conductivity of a half-space at $100 \mathrm{~Hz}$ (curve B) and $18500 \mathrm{~Hz}$ (curve C). The thick line (curve A) is the true half-space conductivity equal to $0.043 \mathrm{~S} / \mathrm{m}$. 


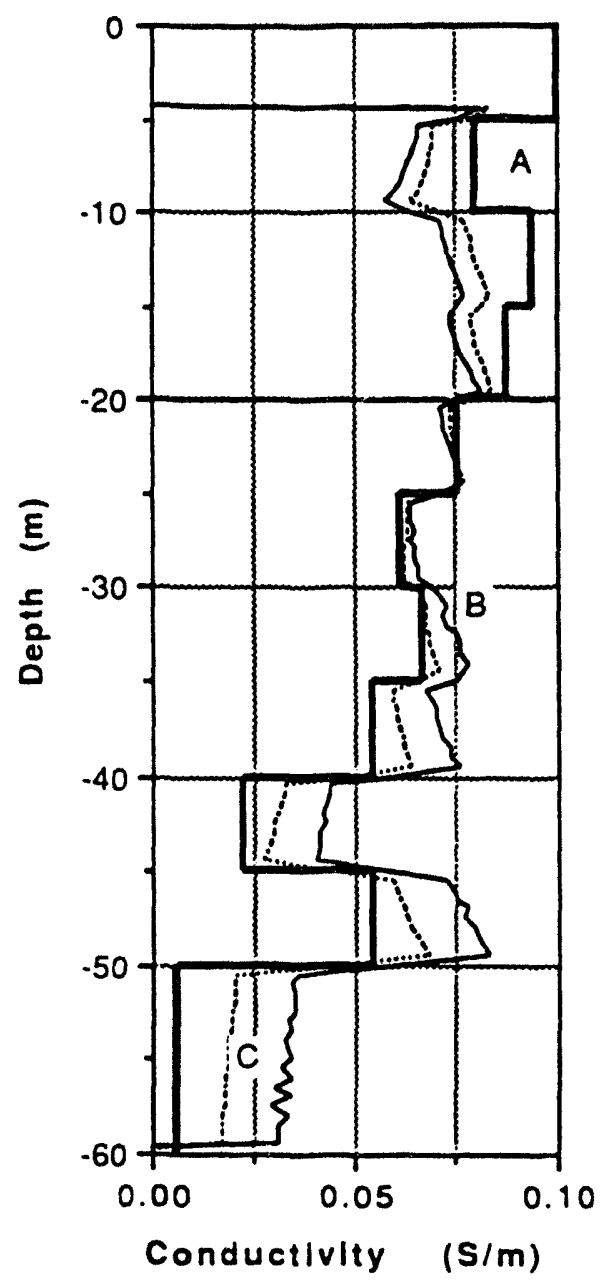

Fig. 4.1-2 The true and recovered conductivity of a layered half-space at $100 \mathrm{~Hz}$ (curve B), and $18500 \mathrm{~Hz}$ (curve $\mathrm{C}$ ). The thick line is the true conductivity (curve A). 


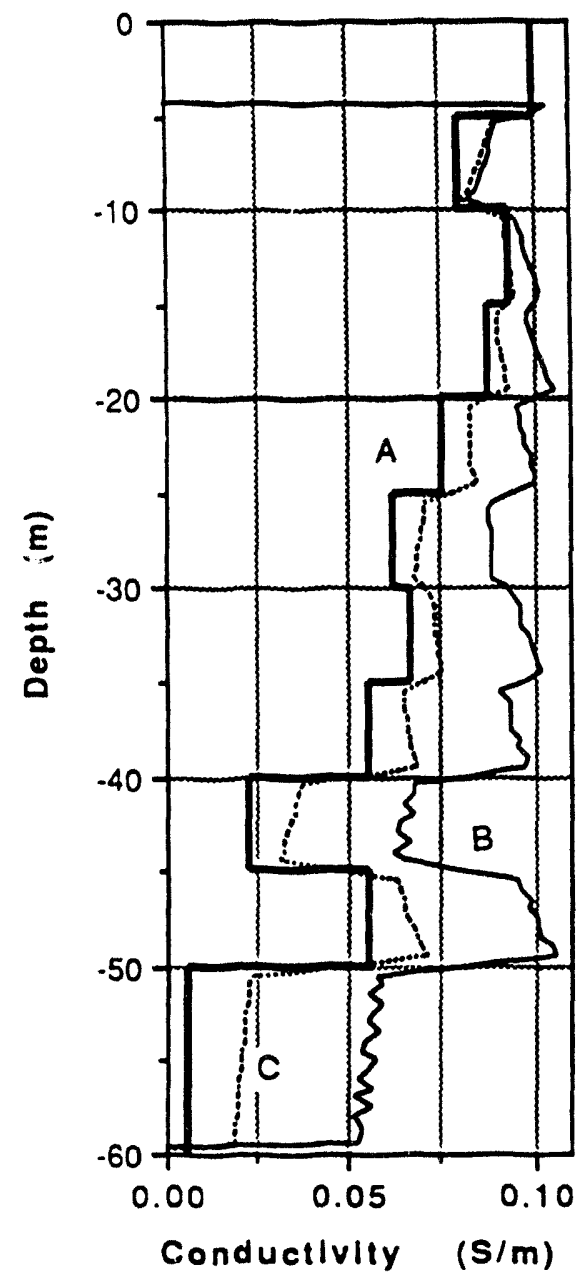

Fig. 4.1-3 The true and recovered conductivity of a layered half-space under a conductive overburden at $100 \mathrm{~Hz}$ (curve B), and $18500 \mathrm{~Hz}$ (curve C). The thick line is the true model conductivity (curve A). 


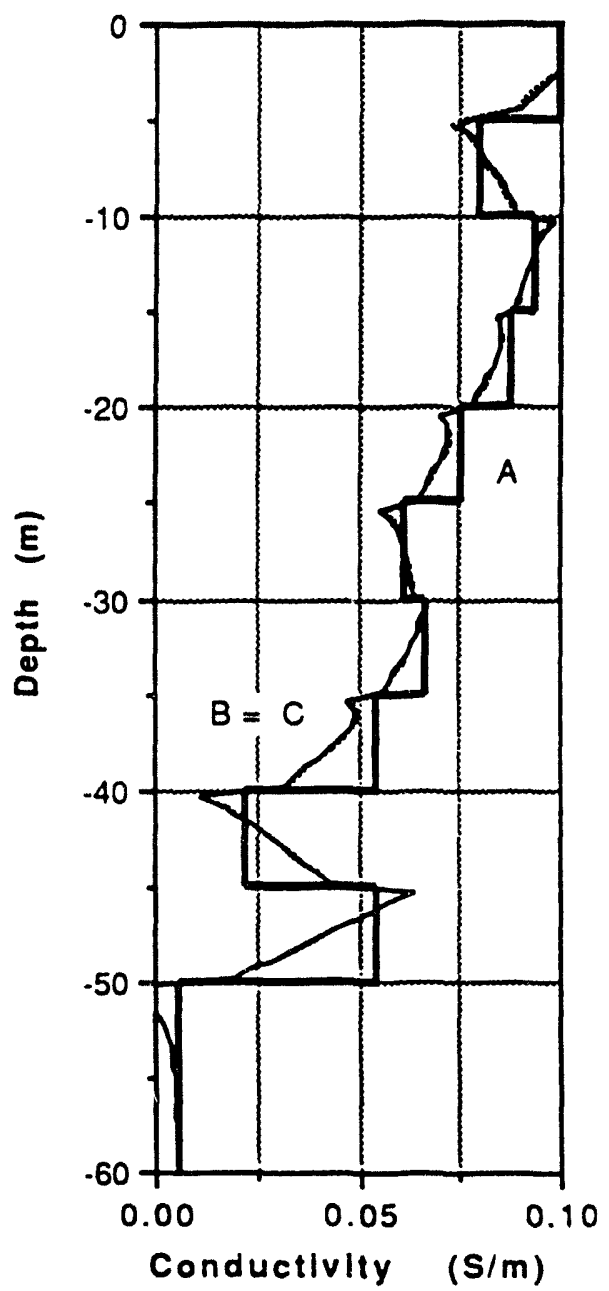

Fig. 4.1-4 The true and recovered conductivity of a layered half-space at $100 \mathrm{~Hz}$ (curve B), and $18500 \mathrm{~Hz}$ (curve $\mathrm{C}$ ) in a simulated induction log geometry. The thick line is the true conductivity (curve A). The transmitter is located $2.6 \mathrm{~m}$ above the three receivers separated by $0.1 \mathrm{~m}$. The measurements are taken every $0.5 \mathrm{~m}$ along the vertical profile. 


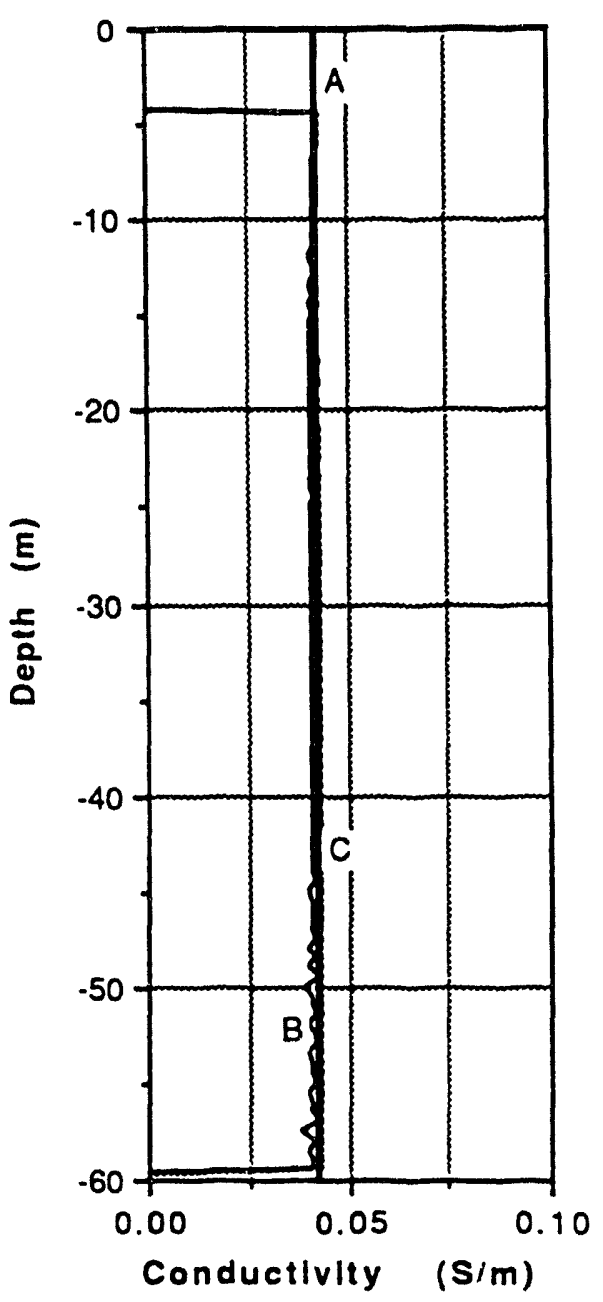

Fig. 4.1-5 The true and recovered conductivity of a half-space at $100 \mathrm{~Hz}$ (curve B) and $18500 \mathrm{~Hz}$ (curve C), using numerical estimate of horizontal derivative contributions. The thick line (curve $C$ ) is the true conductivity of a half-space equal to $0.43 \mathrm{~S} / \mathrm{m}$. 


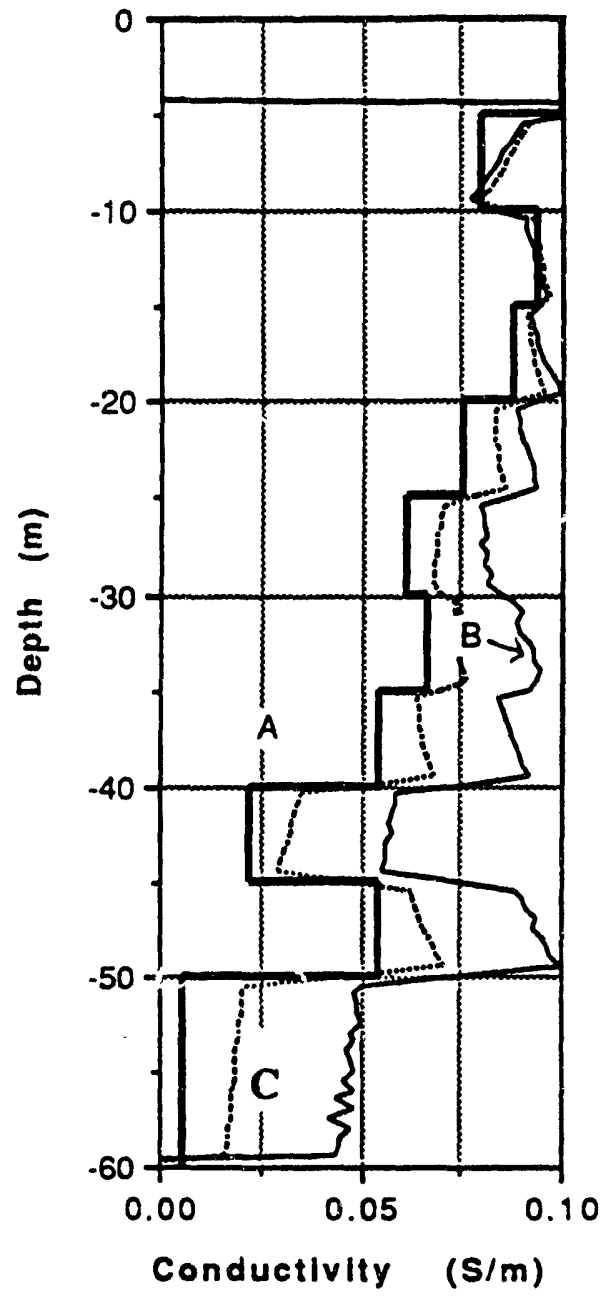

Fig. 4.1-6 The true and recovered conductivity of a layered half-space at $100 \mathrm{~Hz}$ (curve B) and $18500 \mathrm{~Hz}$ (curve $\mathrm{C}$ ), using numerical estimate of horizontal derivative contributions. The thick line (curve A) i; the true conductivity of a layered half-space 

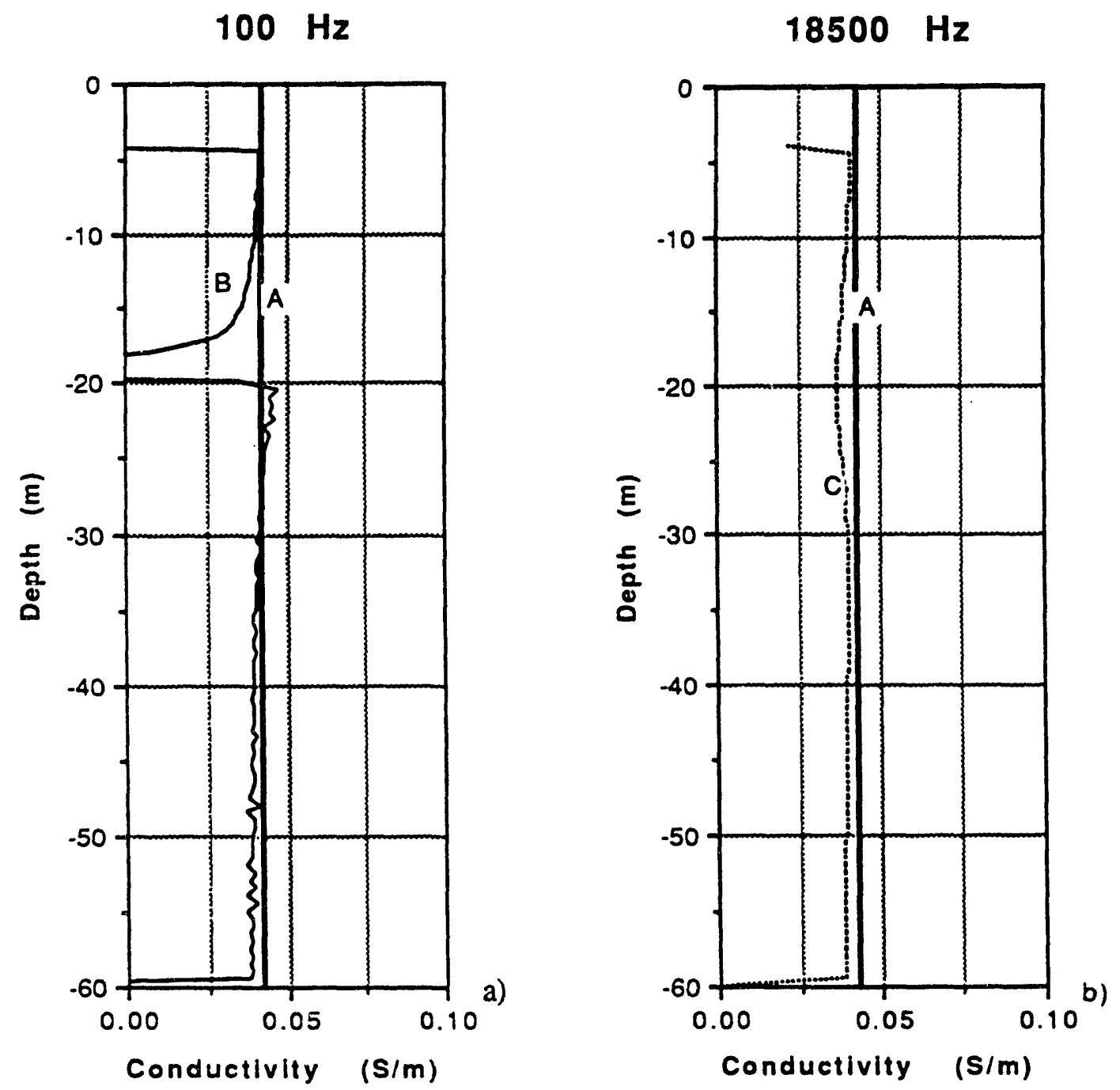

Fig. 4.2-1 The effect of error in the background conductivity estimate on the recovered conductivity of the half-space using the background conductivity of $0.040 \mathrm{~S} / \mathrm{m}$ at frequencies $100 \mathrm{~Hz}$ (curve B) and $18500 \mathrm{~Hz}$ (curve C). The thick line (curve A) is the true conductivity of a half-space equal to $0.043 \mathrm{~S} / \mathrm{m}$. 

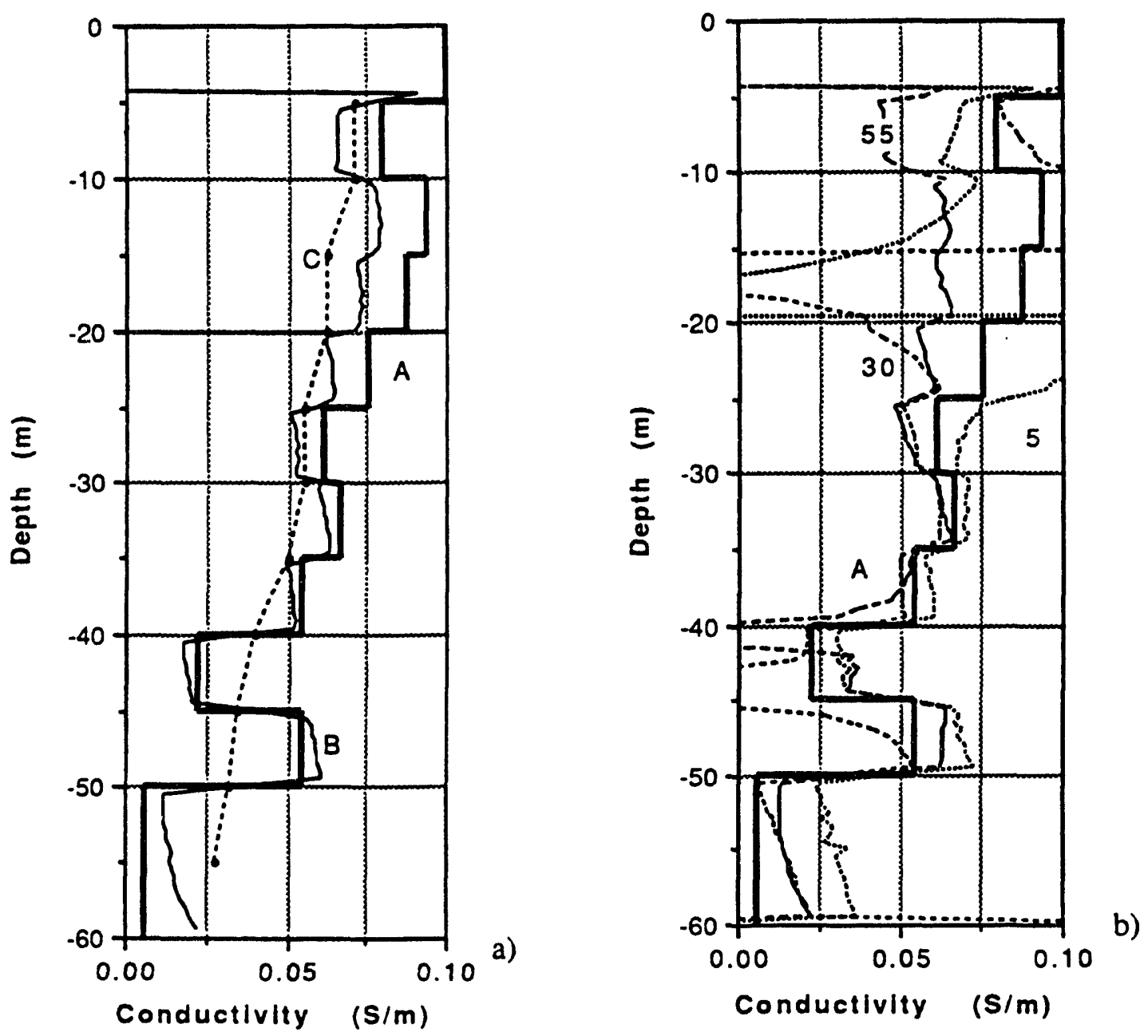

Fig. 4.2-2 The true and recovered conductivity of the layered half-space at $100 \mathrm{~Hz}$. In particular panel a) shows the conductivity profile obtained by averaging the conductivities from two adjoining transmitters over $5.0 \mathrm{~m}$ interval; panel b) shows a sample of conductivities recovered using only single transmitter located at $5.0,30.0$ and $55.0 \mathrm{~m}$. Curve A represents true model, curve B shows calculated conductivity, curve $C$ represents the estimated background conductivity of a half-space. 

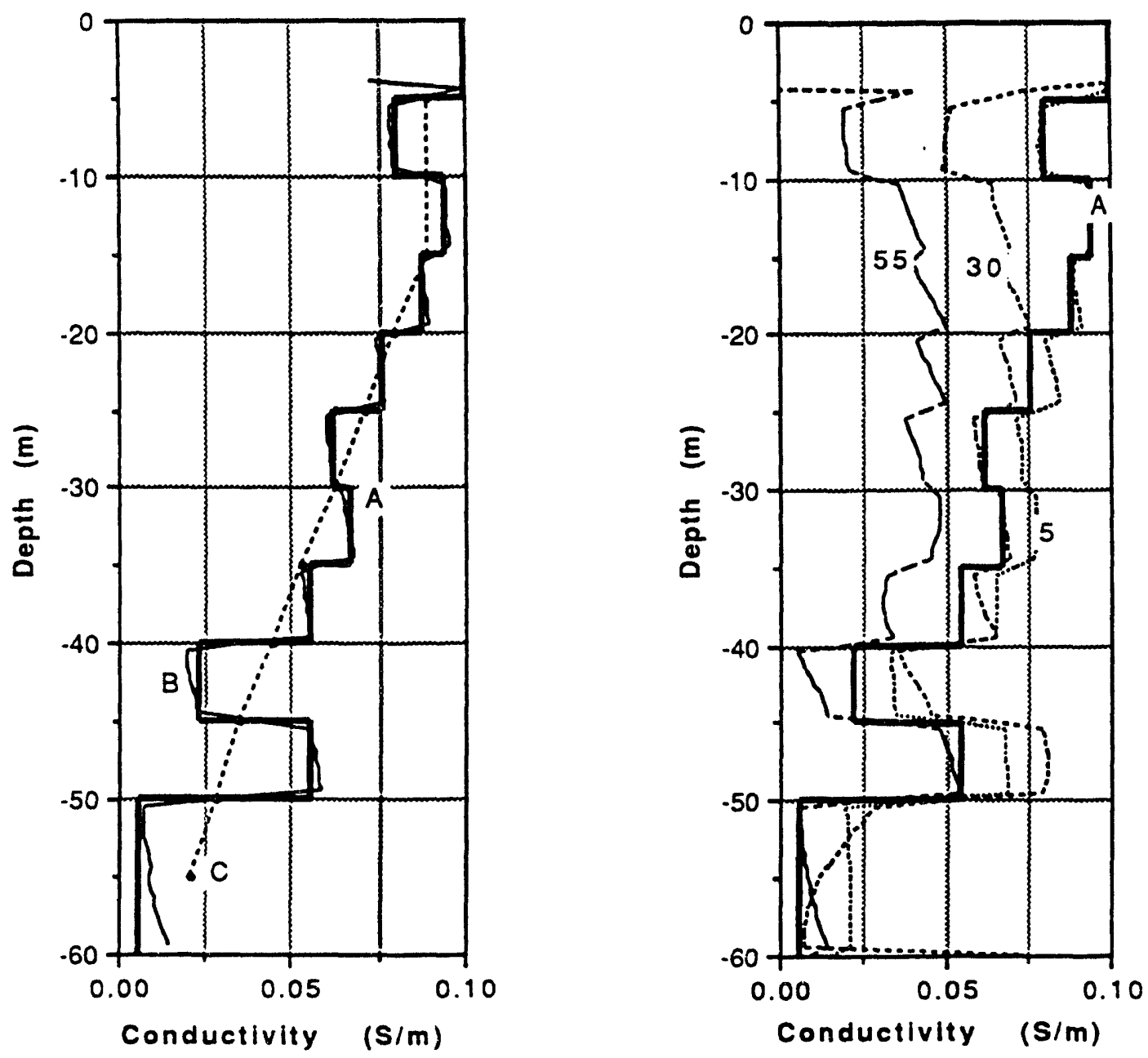

Fig. 4.2-3 The true and recovered conductivity of the layered half-space at $18500 \mathrm{~Hz}$. In particular panel a) shows the conductivity profile obtained by averaging the conductivities from two adjoining transmitters over $5.0 \mathrm{~m}$ interval; panel b) shows a sample of conductivities recovered using only single transmitter located at $5.0,30.0$ and $55.0 \mathrm{~m}$. Curve A represents true model, curve B shows calculated conductivity, curve $C$ represents the estimated background conductivity of a half-space. 

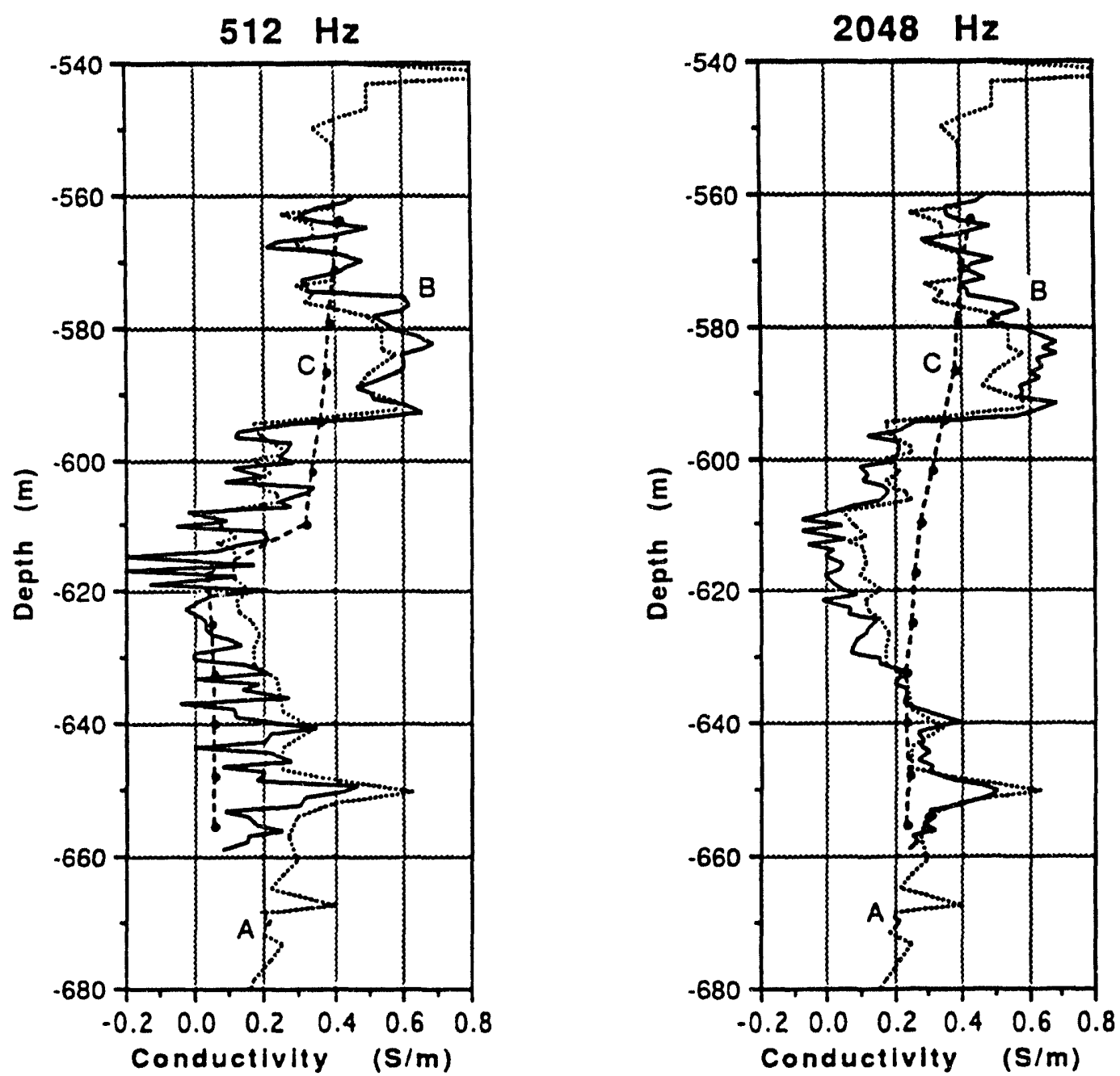

Fig. 4.2-4 The induction log (curve A), recovered conductivity curve (B) and background conductivity of a half-space (curve C) for the dala from Devine, TX: a) at frequency $512 \mathrm{~Hz}$ and: b) at frequency $2048 \mathrm{~Hz}$. 

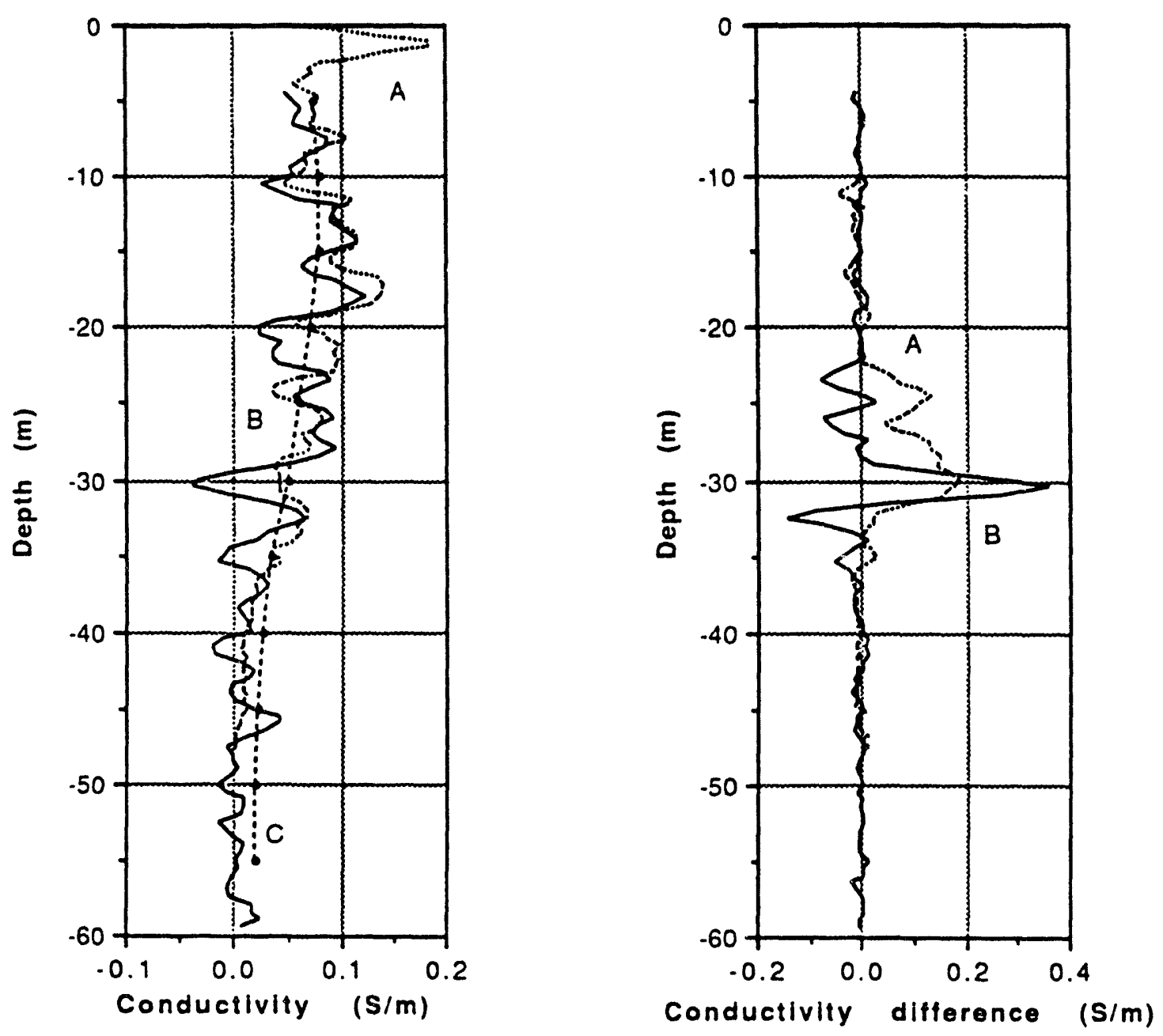

Fig. 4.2-5 The induction log (curve A), recovered conductivity curve (B) and background conductivity of a half-space (curve $\mathrm{C}$ ) for the data from Richmond, CA, using crosshole data between INJ1 and NW wells. The frequency is $18500 \mathrm{~Hz}$. 

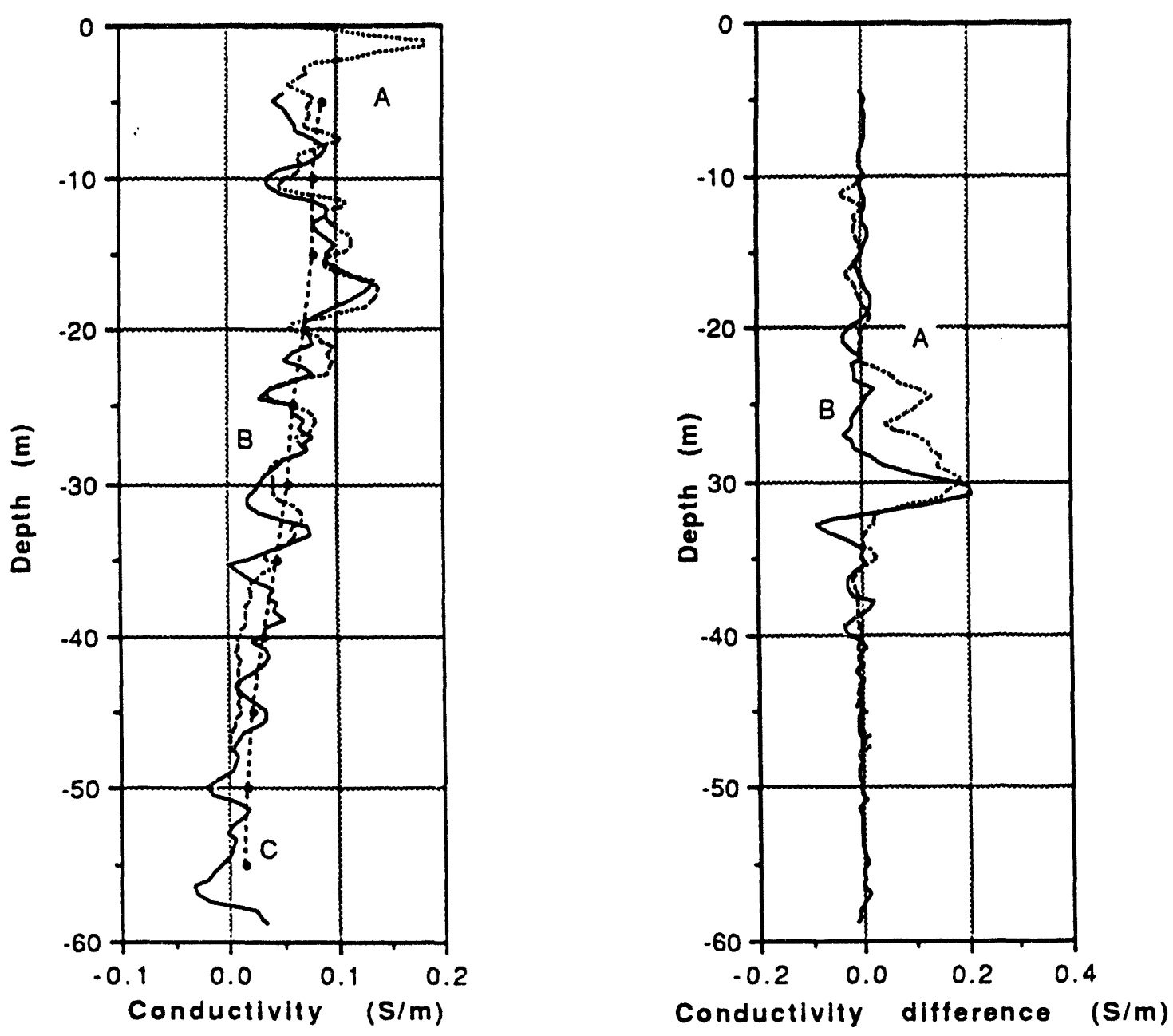

Fig. 4.2-6 The induction log (curve A), recovered conductivity curve (B) and background conductivity of a half-space (curve $C$ ) for the data from Richmond, CA, using crosshole data between INJI and NE wells. The frequency is $18500 \mathrm{~Hz}$. 

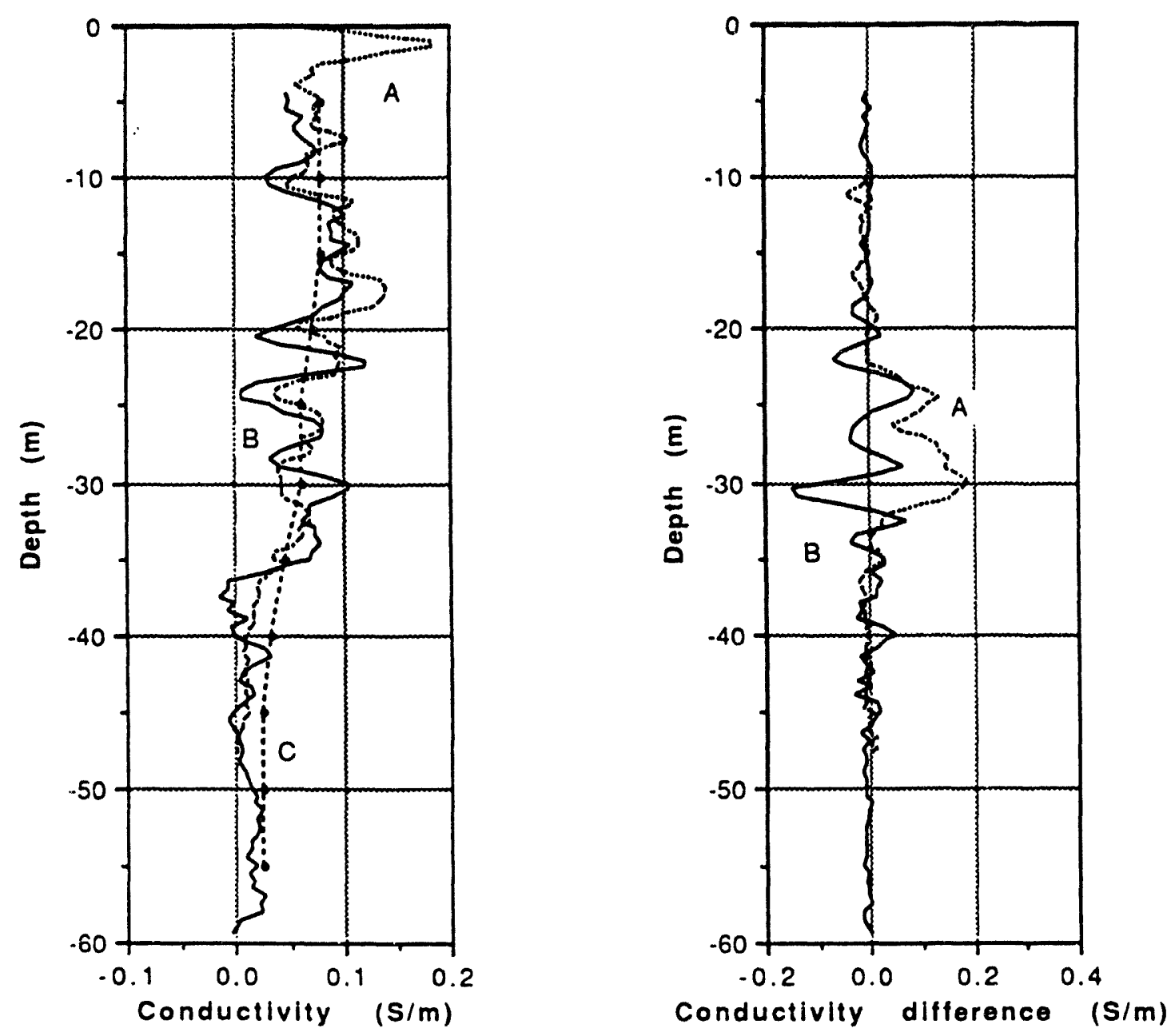

Fig. 4.2-7 The induction log (curve A), recovered conductivity curve (B) and background conductivity of a half-space (curve $\mathrm{C}$ ) for the data from Richmond, $\mathrm{CA}$, using crosshole data between INJ1 and SE wells. The frequency is $18500 \mathrm{~Hz}$. 

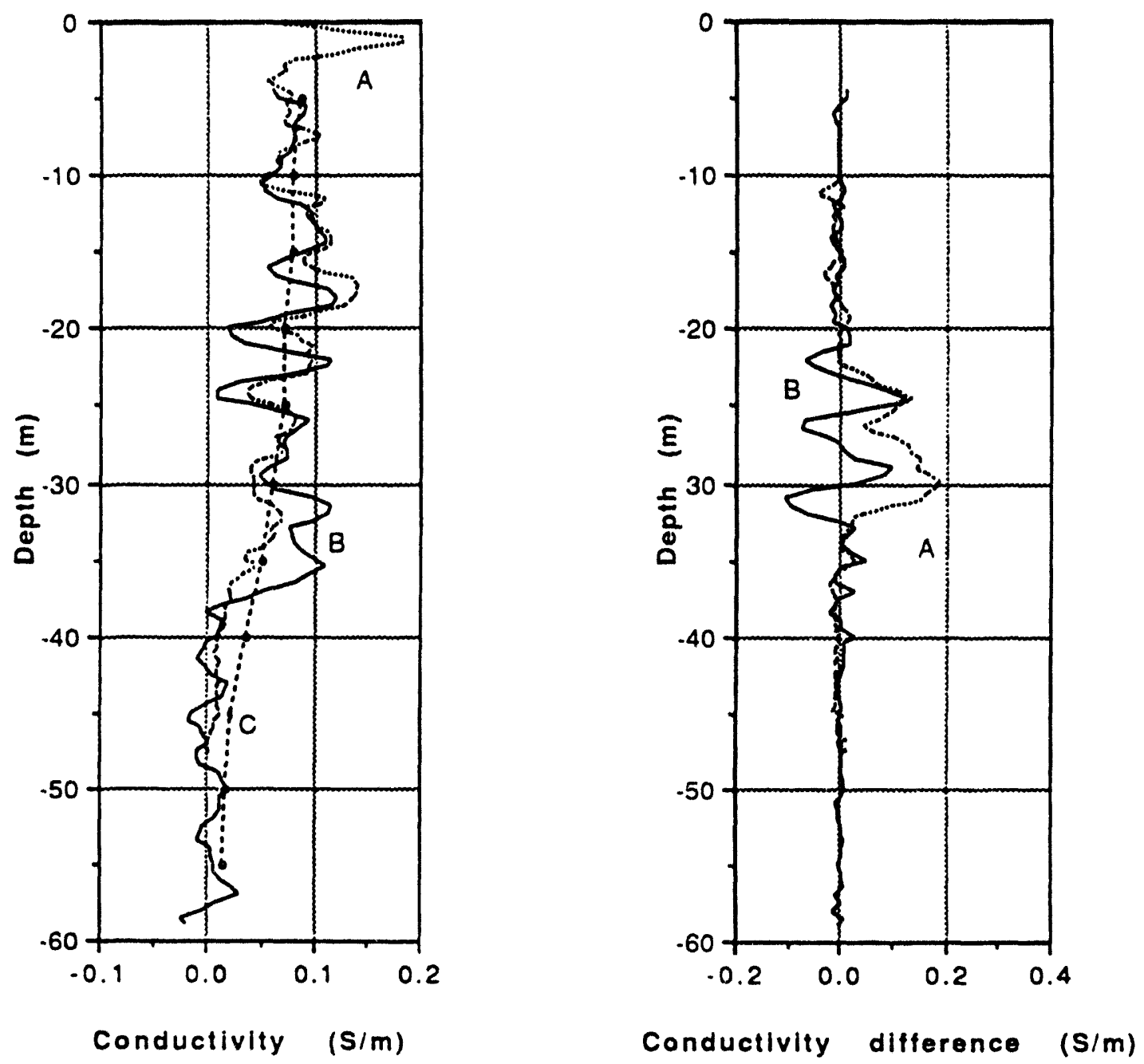

Fig. 4.2-8 The induction log (curve A), recovered conductivity curve (B) and background conductivity of a half-space (curve $\mathrm{C}$ ) for the data from Richmond, $\mathrm{CA}$, using crosshole data between INJ1 and SW wells. The frequency is $18500 \mathrm{~Hz}$. 


\section{Chapter 5 \\ One-dimensional least-squares inversion of field data.}

The objective of least-squares inversion of crosshole data is to provide an estimate of the overall, background, layered conductivity of the medium. It does not attempt to substitute for induction logging which provides a detailed conductivity structure close to the borehole or for crosshole tomography that resolves structures between boreholes. However the results from crosshole inversion can help in tomographic inversion which needs an accurate estimate of background conductivities. This in turn can indicate inhomogeneities between boreholes that are not visible on induction logs.

In this chapter I outline the basic principles behind the NLSEM1D inversion program and discuss its practical application in three experiments. The simplest case involved the conductivity estimate of the water in a model tank experiment from the measurements of the vertical magnetic field component. In the second case inversion was used to obtain the background conductivity from cross-hole measurements in a site that had a simple, one-dimensional conductivity distribution. Finally the inversion was applied to crosshole measurements for determining the direction of the propagation of a conductive salt water plume in a complicated conductivity structure.

We will also show that using the first vertical derivative, $\Delta \mathrm{H}_{\mathrm{z}} / \Delta \mathrm{z}$, instead of the measured $\mathrm{H}_{2}$ field improves inversion process because it then converges faster and is less dependent on the initial model.

\subsection{NLSEM1D inversion program.}

To obtain an estimate of the conductivity of a one-dimensional medium from measurements performed inside boreholes a nonlinear least-squares inversion program called NLSEM1D was developed. It is based on double precision versions of three algorithms called NL2SNO, NLSOL and EM1D.

The subroutine NL2SNO solves the adaptive, unconstrained, nonlinear inverse problem using a finite difference Jacobian. The routine provides a flexible, unconstrained least squares algorithm that "is more reliable than the Gauss-Newton or the Levenberg-Marquardt method and is more efficient than the secant or variable metric algorithms" (Dennis et. al, 1981).

A subroutine, NLSOL, written by W. Anderson (1982) extends this original unconstrained version to include constrained and fixed parameters as well as different options for data normalization. 
The forward problem is calculated with the program EMID written by Ki Ha Lee. Because of the flexibility of the forward routine many different combinations of frequency and geometrical soundings are available. The program can take any component of an EM field radiated by either electric or magnetic dipoles and invert on real and imaginary components together, or separately. The same can be done with amplitude and phase. The dipole sources can be oriented horizontally or vertically and can be placed anywhere inside or above the conductive medium. The receivers can also be placed in arbitrary locations.

Although the program is flexible and can take any number of model parameters and data points, practical considerations limited the number of data points to about 700 and number of parameters to about 15 . If the number of parameters is much higher, the program still converges but frequently not to a realistic model.

Since in practice the calibration constants of the field equipment may be unknown or uncertain, we added the option to invert for the amplitude and phase calibration constants. We found this feature to be useful in testing the calibration of the field systems (Wilt et al., 1992; Alumbaugh, 1993).

\subsection{Least-squares inversion of model tank data}

The first practical application of least-squares inversion was related to a the model tank experiment conducted by David Alumbaugh in Richmond (Alumbaugh and Becker, 1990). The objective of the experiment was to simulate the crosshole EM response of a conductive body in a uniform background to provide a set of reliable data that could be used in a new scheme of tomographic inversion described by Zhou (1989).

The crucial part of the experiment was to obtain accurate measurements of the primary fields, i.e. the fields measured in the conductive medium without the conductive body. These primary fields were then subtracted from total fields (i.e. fields measured with the conductive body) to obtain the secondary fields (i.e. fields due only to the conductive body). The secondary fields were used in the tomographic inversion. We used the primary fields to obtain an independent estimate of the water conductivity in the tank. This conductivity was needed as a background conductivity for the tomography and also to check the accuracy of measurements with existing three-dimensional numerical codes.

The measurements were performed with the experimental set-up shown in Figure 5.2-1. The medium was energized by a vertical magnetic dipole source moving along a vertical line simulating one borehole and the vertical magnetic field was measured by a 
stationary receiver on another vertical line simulating the other borehole. Then the receiver was moved to the next position and the whole process was repeated to obtain a complete coverage for all transmitter and roceiver positions along the two boreholes. The operating frequency was $98.6 \mathrm{kHz}$. The repeatability of the measurements was within $1 \%$.

All the measured values of the $\mathrm{H}_{2}$ component along the downhole profiles were normalized by the response on the surface, so the absolute values of the amplitude and phase of the magnetic field were not known. On the surface the quadrature component was zeroed, and in-phase component provided the normalization.

Laboratory measurements gave $14.45 \mathrm{~S} / \mathrm{m}$ for the conductivity of the water in a tank. We wanted to compare the laboratory measurements of conductivity with the conductivity obtained from the measurements of the $\mathrm{H}_{2}$ component.

The first trial and error fit of experimental data to the EM1D half-space results showed a reasonable agreement for a half-space of conductivity $12.0 \mathrm{~S} / \mathrm{m}$ for the first data set collected. We then used the inversion to help in a more accurate determination of the conductivity of the water. Our objective was to find the conductivity of a half-space that would fit the data within the repeatability error, i.e. below the $1 \%$ error. Since the absolute value of fields and the phase shift were not known, the inversion had to be performed not only for the conductivity, but also for the calibration constants in amplitude and phase.

The inversion was performed on two data sets collected within a period of two months, during summer of 1990 . Both data sets had repeatability below $1.0 \%$. However the first set of measurements was performed with a receiver that fenched to stick on the measuring rod at the same location. We will show how a small mechanical malfunction of equipment affects the estimated conductivity of the water in the tank and how in this case the repeatability of measurements did not guarantee that the absolute accuracy of the experiment was indeed within $1 \%$.

The inversion on first data set was performed using amplitude and phase components for three downhole profiles recorded with the receiver on the surface, and at $0.1 \mathrm{~m}$ and $0.5 \mathrm{~m}$ below the surface of the water. The examples of measured amplitude and phase at each of these locations are given in Figure 5.2-2. Data were inverted for conductivity (assuming a half-space model) and for the amplitude and phase calibration i.e. amplitude and phase of the magnetic field on the surface which were unknown.

At these three receiver positions the inversions of the amplitude and phase of $\mathrm{Hz}_{\mathrm{Z}}$ gave the conductivity of the half-spare to be $12.35+0.005 \mathrm{~S} / \mathrm{m}, 12.30+0.003 \mathrm{~S} / \mathrm{m}$ and 
$12.90+0.06 \mathrm{~S} / \mathrm{m}$ respectively. The results, although close to each other did not produce a least-squares fit to the measured data below $1 \%$ for the first two receiver positions (Figure 5.2-3). The attempts to account for possible differences in the conductivity of the water at different depths by using a three layer model gave much worse results. D. Alumbaugh (personal communication) suggested the use of two layer model to fit the data: the top layer representing the water in the tank and the bottom layer representing the air below the tank (see sketch below).

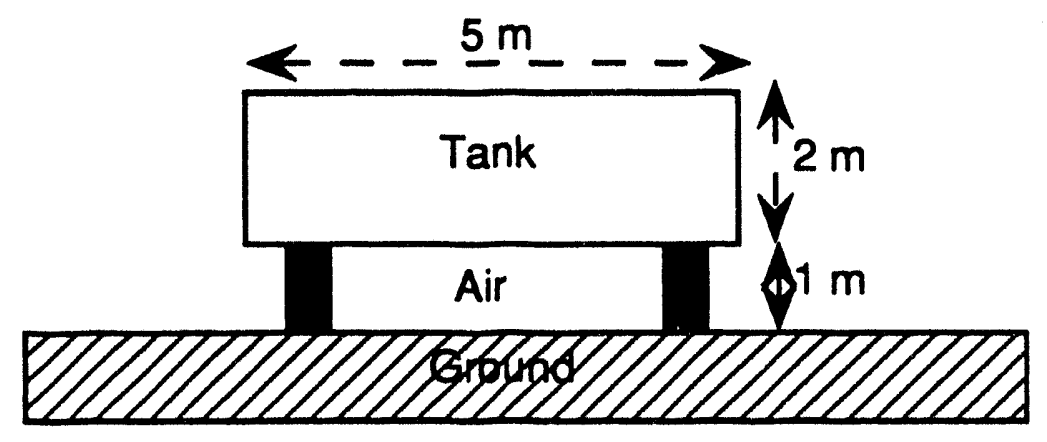

With this model, the inversion gave conductivities of: $12.43,12.40,12.90 \mathrm{~S} / \mathrm{m}$ for three receiver positions with a least-squares fit of experimental and numerical data within $1 \%$ (with the exception of three points for the surface receiver). The fit between measured and calculated values is given in Figure 5.2-4 and shows a significant improvement compared to the half-space result. This improvement is especially pronounced in the upper profiles. But the error in the least-squares fit for two receiver positions: at 0.1 and $0.5 \mathrm{~m}$ displays a sudden jump around a depth of $0.5 \mathrm{~m}$. This kind of misfit can not be caused by a sudden change in model parameters because the $\mathrm{H}_{\mathbf{Z}}$ response is continuous across a boundary. Since the fit to the data for the receiver on the surface does not show this abrupt change in least-squares error, we suspect that between the surface measurements and the two later ones something changed in the measurement conditions when the transmitter reached the depth of $0.2 \mathrm{~m}$.. D. Alumbaugh (personal communication) explained this pattern of error by a problem with the transmitter sticking on the guiding rod at this depth. This example shows that sometimes it is possible to detect problems in the experiment (that otherwise might not be detected) by analyzing the error pattern. A small linear trend in the error pattern that remains after applying the corrections can be caused by several reasons: a slightly different distances between transmitter and receiver than assumed in the experiment (discussed later) or possible 
response from the conductive ground over which a whole tank model is placed (see sketch above).

The inversion for the second data set was performed not only on amplitude and phase but on in-phase and quadrature components as well. In this case the receiver was located at four positions: at the surface, and at $0.2,0.4$ and $0.575 \mathrm{~m}$ below the surface. The inversion was performed on three parameters: the conductivity of the water and the quantities used to normalize the data i.e. the amplitude and phase of the $\mathrm{H}_{\mathrm{Z}}$ component at the surface. The initial guess for the conductivity of the water in a tank was $12.8 \mathrm{~S} / \mathrm{m}$ (i.e. the conductivity of the layer that gave the best trial and error fit to in phase and quadrature measured components obtained by D. Alumbaugh, 1990). The initial guess for the amplitude was usually set at 0.1 . This value is close to the amplitude of $\mathrm{H}_{\mathrm{z}}$ field in $\mathrm{A} / \mathrm{m}$ measured on the surface of a half-space of conductivity $12.8 \mathrm{~S} / \mathrm{m}$ at $1.0 \mathrm{~m}$ separation assuming a unit dipole moment for the transmitter. The initial guess for phase shift varied, but usually was set to $160^{\circ}$, i.e. the expected phase on the half-space.

The results of the inversion are given in Tables 1 and 2. In Table 1 we present the results of the inversion for the half-space model, i.e. without taking into consideration the finite depth to the tank bottom. In Table 2 the inversion results are given for the model in which the water layer is sandwiched between two nonconductive half-spaces. In the tables the lower number represents the variance in parameter estimates.

\begin{tabular}{|c|c|c|c|c|c|c|c|c|}
\hline \multirow[b]{2}{*}{$\begin{array}{l}\text { Depth } \\
\text { (m) } \\
\end{array}$} & \multicolumn{4}{|c|}{ In-phase and quadrature inversion } & \multicolumn{4}{|c|}{ Amplitude and phase inversion } \\
\hline & $\begin{array}{l}\text { Cond. } \\
(\mathrm{S} / \mathrm{m})\end{array}$ & $\begin{array}{l}\text { Amp. } \\
\text { (A/m) }\end{array}$ & $\begin{array}{r}\text { Phase } \\
\text { (deg) }\end{array}$ & $\begin{array}{l}\text { Ave } \\
\% \text { \%err }\end{array}$ & $\begin{array}{l}\text { Cond. } \\
(\mathrm{S} / \mathrm{m})\end{array}$ & $\begin{array}{l}\text { Amp. } \\
(\mathrm{A} / \mathrm{m})\end{array}$ & $\begin{array}{r}\text { Phase } \\
\text { (deg) }\end{array}$ & $\begin{array}{l}\text { Ave } \\
\% \text { ent }\end{array}$ \\
\hline 0.0 & $\begin{array}{l}12.78 \\
0.0088\end{array}$ & $\begin{array}{l}0.102 \\
0.049\end{array}$ & $\begin{array}{l}157.43 \\
0.008\end{array}$ & 1.41 & $\begin{array}{l}12.51 \\
0.0013\end{array}$ & $\begin{array}{l}0.1024 \\
0.0003\end{array}$ & $\begin{array}{l}158.58 \\
0.0004\end{array}$ & 0.83 \\
\hline 20.0 & $\begin{array}{l}12.55 \\
0.0098\end{array}$ & $\begin{array}{l}0.1042 \\
0.0026\end{array}$ & $\begin{array}{l}158.6 \\
0.0015\end{array}$ & 0.57 & $\begin{array}{l}12.47 \\
0.0012\end{array}$ & $\begin{array}{l}0.1043 \\
0.0001\end{array}$ & $\begin{array}{l}158.98 \\
0.002\end{array}$ & 0.31 \\
\hline 40.0 & $\begin{array}{l}11.68 \\
0.021\end{array}$ & $\begin{array}{l}0.1062 \\
0.0035\end{array}$ & $\begin{array}{l}162.34 \\
0.0034\end{array}$ & 0.42 & $\begin{array}{l}11.85 \\
0.0081\end{array}$ & $\begin{array}{l}0.106 \\
0.0036\end{array}$ & $\begin{array}{l}161.60 \\
0.0014\end{array}$ & 0.25 \\
\hline 60.0 & $\begin{array}{l}13.08 \\
0.29\end{array}$ & $\begin{array}{l}0.1045 \\
0.0184\end{array}$ & $\begin{array}{l}156.60 \\
0.0088\end{array}$ & 0.78 & $\begin{array}{l}13.52 \\
0.04\end{array}$ & $\begin{array}{l}0.1037 \\
0.0018\end{array}$ & $\begin{array}{l}154.50 \\
0.0096\end{array}$ & 0.35 \\
\hline Avrg. & 12.59 & 0.1042 & 158.80 & & 12.52 & 0.1041 & 158.48 & \\
\hline
\end{tabular}


Table 1 Results for least-squares inversion for half-space model.

\begin{tabular}{|c|c|c|c|c|c|c|c|c|}
\hline \multirow[b]{2}{*}{$\begin{array}{l}\text { Depth } \\
\text { (m) }\end{array}$} & \multicolumn{4}{|c|}{ In-phase and quadrature inversion } & \multicolumn{4}{|c|}{ Amplitude and phase inversion } \\
\hline & $\begin{array}{l}\text { Cond. } \\
(\mathrm{S} / \mathrm{m})\end{array}$ & $\begin{array}{l}\text { Amp. } \\
(\mathrm{A} / \mathrm{m})\end{array}$ & $\begin{array}{l}\text { Phase } \\
\text { (deg) }\end{array}$ & $\begin{array}{l}\text { Ave } \\
\text { \%err }\end{array}$ & $\begin{array}{l}\text { Cond. } \\
(\mathrm{S} / \mathrm{m})\end{array}$ & $\begin{array}{l}\text { Amp. } \\
(\mathrm{A} / \mathrm{m})\end{array}$ & $\begin{array}{r}\text { Phase } \\
\text { (deg) }\end{array}$ & $\begin{array}{l}\text { Ave } \\
\text { \%err }\end{array}$ \\
\hline 0.0 & $\begin{array}{l}12.77 \\
0.006\end{array}$ & $\begin{array}{l}0.1022 \\
0.0034\end{array}$ & $\begin{array}{l}157.43 \\
0.0005\end{array}$ & 0.93 & $\begin{array}{l}12.59 \\
0.01\end{array}$ & $\begin{array}{l}0.1025 \\
0.002\end{array}$ & $\begin{array}{l}158.19 \\
0.0002\end{array}$ & 0.49 \\
\hline 20.0 & $\begin{array}{l}12.64 \\
0.0048\end{array}$ & $\begin{array}{l}0.1044 \\
0.0013\end{array}$ & $\begin{array}{l}158.2 \\
0.0007\end{array}$ & 0.21 & $\begin{array}{l}12.65 \\
0.0014\end{array}$ & $\begin{array}{l}0.1044 \\
0.0016\end{array}$ & $\begin{array}{l}158.14 \\
0.0003\end{array}$ & 0.14 \\
\hline 40.0 & $\begin{array}{l}12.24 \\
0.015\end{array}$ & $\begin{array}{l}0.1058 \\
0.0031\end{array}$ & $\begin{array}{l}160.06 \\
0.0025\end{array}$ & 0.32 & $\begin{array}{l}12.38 \\
0.0089\end{array}$ & $\begin{array}{l}0.1055 \\
0.0045\end{array}$ & $\begin{array}{l}159.42 \\
0.0015\end{array}$ & 0.21 \\
\hline 60.0 & $\begin{array}{l}13.24 \\
0.029\end{array}$ & $\begin{array}{l}0.1047 \\
0.0068\end{array}$ & $\begin{array}{l}155.88 \\
0.0045\end{array}$ & 0.39 & $\begin{array}{l}13.38 \\
0.031\end{array}$ & $\begin{array}{l}0.1044 \\
0.0099\end{array}$ & $\begin{array}{l}155.22 \\
0.0048\end{array}$ & 0.17 \\
\hline Avrg. & 12.72 & 0.1042 & 157.90 & & 12.75 & 0.1042 & 157.75 & \\
\hline
\end{tabular}

Table 2 Results for the least squares inversion for layer sandwiched between two nonconductive half-spaces.

As can be observed the averaged cumulative least-squares error given by:

$$
\text { Ave \%ert }=\frac{1}{n} \sum_{i=1}^{n} \frac{\left(\mathrm{H}_{\text {obs }}-\mathrm{H}_{\mathrm{calc}}\right)}{\mathrm{H}_{\text {calc }}} 100 \%
$$

is always smaller for the layer than for the corresponding half-space model. Thus the uncertainty in the parameter estimate is lower for a layer than a half-space. Furthermore the estimated conductivity of the half-space is always (with one exception) lower than the estimated conductivity of the layer.

The above analysis shows that the measured data in the tank are affected by the finite dimensions of the tank. From Chapter 3, Figure 3.2-2 we estimate that the amplitude $\mathrm{H}_{2}$ with transmitter and receiver at the same depth would be affected by less then $1 \%$ by the air-water interface if the system is more than $0.7 \mathrm{~m}$ away from the interface ( $1 \%$ difference in amplitude for induction parameter of the scale model: $(\sigma)^{1 / 2} \rho$ $=1120$ is at $\mathrm{h} / \mathrm{\rho} \sim 0.7$ ). The smallest distance between the tank bottom and the lowest 
point on the profile is $0.5 \mathrm{~m}$. At this depth, by the same reasoning, the amplitude can be affected by the interface up to few percent. The effects of the side walls should be much smaller than the effect of a bottom, because the tank is $3.0 \mathrm{~m}$ wide and $5.0 \mathrm{~m} \mathrm{long}$, and the measuring set-up is in the middle: the closest side wall is about $1.5 \mathrm{~m}$ from the transmitter or receiver.

Taking the averaged conductivity estimated from the four receiver positions we obtained $12.52 \mathrm{~S} / \mathrm{m}$ for the conductivity of a half-space and $12.74 \mathrm{~S} / \mathrm{m}$ for the conductivity of the layer, which is very close to $12.8 \mathrm{~S} / \mathrm{m}$, the trial and error fit obtained by $D$. Alumbaugh. To see how this layer of conductivity $12.74 \mathrm{~S} / \mathrm{m}$ fits the measured profiles, we kept the conductivity of the layer constant and inverted for amplitude and phase using amplitude and phase as well as in-phase and quadrature components.

The inversion resulted in an amplitude of between 0.1022 and $0.1057 \mathrm{~A} / \mathrm{m}$ depending on the receiver location, and phase between 158.24 and 157.46 degrees. The average amplitude for all measurements is $0.1043 \mathrm{~A} / \mathrm{m}$ and the averaged phase is 157.79 degrees. Figure 5.2-5 gives the \% enror in amplitude and phase shift in least-squares fit between the measured and calculated data obtained using a layer of constant conductivity fixed at $12.74 \mathrm{~S} / \mathrm{m}$. Figure 5.2.-6 gives the \% error for in-phase and quadrature. The amplitude percent error in least squares fit is less than $1 \%$ for all receiver positions except when the receiver is located on the surface. Comparing the error plots for all receiver positions we observe that with the exception of the receiver on the surface, all curves show patterns of errors as the transmitter moves down a profile. We tried to find out what (besides an inadequate model) could cause this kind of error. One possibility was that the experimental set-up was not perfectly vertical or horizontal, but slightly twisted, resulting in the data that repeated to within $1 \%$, but which involved measurement points which were not exactly at the assumed locations. The sketch below explains the problem:

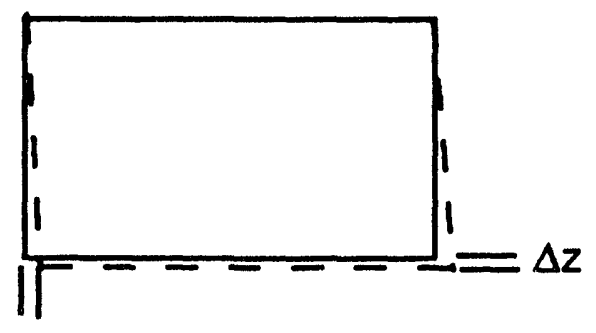

$\Delta x$

To estimate the errors we calculated the differences between the "perfect" alignment and profiles shifted horizontally by $\Delta x$ or vertically by $\Delta z$. Figure 5.2-7 
shows the results for amplitude and phase when $\Delta$ equals $0.002 \mathrm{~m}, 0.005 \mathrm{~m}$ and $0.01 \mathrm{~m}$, Figure 5.2-8 shows the $\%$ differences for in-phase and quadrature components. The upper panels are for the transmitter on the surface, the lower panels are for the receiver located $0.6 \mathrm{~m}$ below the surface. The numbers on the curves describe the horizontal or vertical shift of an entire profile in meters. As can be observed even a $2 \mathrm{~mm}$ difference between the true and assumed separation can cause errors close to $1 \%$.

To check whether the error observed in Figures 5.2-5 and 5.2-6 could be caused by slightly different geometry of the experimental set-up than that assumed, we calculated the error in least-squares fit when the receiver was moved from the surface to $0.007 \mathrm{~m}$ below the surface, from 0.2 to $0.202 \mathrm{~m}$ and from 0.4 to 0.402 below the surface. Figures 5.2-9 presents a new misfit for amplitude and phase data, Figure 5.2-10 shows the misfit for in-phase and quadrature data. The fit improved significantly for all components, except quadrature which was worse. It thus seems possible that at least part of the systematic error can be caused by slight differences in geometry between transmitter and receiver than these that were assumed.

This inversion study provided some additional information besides the conductivity of the water in a tank and helped to assess the problems that might be encountered in other experiments. In passing we notice that the higher conductivity of the water in a tank obtained from laboratory measurements may have been caused by increased temperature of the water samples when they reached the laboratory several hours later on a very hot day (D. Alumbaugh, personal communication).

\subsection{The crosshole experiment and inversion of data collected at Devine, Texas.}

The first successful crosshole low frequency electromagnetic measurements were performed in Devine, Texas in September, 1990. The project was conducted by LLNL and Engineering Geoscience. The objective of the experiment was to obtain highly accurate measurements of the vertical magnetic field component between boreholes that were repeatable to within $1 \%$ for amplitude and 1 degree for phase. This accuracy was required for crosshole low frequency electromagnetic tomography as described by Zhou (1989). The experimental site is shown in Figure 5.3.1. The two wells that were chosen for the crosshole experiment are marked \#2 and \#4 on the plan. They have fiberglass casing and are separated by $100.6 \mathrm{~m}$. The receiver was placed in well \#2, the transmitter moved along well \#4. Transmitter frequencies of $128 \mathrm{~Hz}, 512 \mathrm{~Hz}$ and 2048 $\mathrm{Hz}$ were used. The measurements were taken every $0.95 \mathrm{~m}(3 \mathrm{ft})$. 
The Devine site was chosen for several reasons, but one of the most important was that the geology in Devine is strictly one-dimensional and thus provides a perfect environment for testing the field equipment and theoretical calculations. The onedimensionality of the region was evident from a comparison of three induction logs measured in three different experimental wells. These logs matched perfectly over the whole depth. Figure 5.3.2 shows an example of an induction log together with a geologic cross-section.

The detailed crosshole measurements were performed at two different sections of the well. These sections were chosen because of their induction log characteristics (Figure 5.3.2). The upper section extending from $213.36 \mathrm{~m}$ to $335.28 \mathrm{~m}$ (700.0-1100.0 $\mathrm{ft}$ ) was chosen because it was assumed that the conductivity of this region could be approximated by a uniform $0.3 \mathrm{~S} / \mathrm{m}$ layer. The lower section between $548.64 \mathrm{~m}$ and $670.56 \mathrm{~m}(1800.0-2200.0 \mathrm{ft})$, was chosen to examine how well the contrasts in conductivity around $600 \mathrm{~m}$ could be resolved with crosshole measurements.

Figure 5.3.2a shows the simplified, initial model of conductivity based on the induction logs. This model was used for preliminary studies to estimate the required transmitter moment and operating frequency. However when we applied this model to calculate the forward solution and compared the calculated and measured data they differed significantly and the need for inversion became obvious.

An ideal one-dimensional inversion in a one-dimensional medium should produce a model that fits all frequencies and transmitter-receiver combinations within the measurement error. If our data were exact, then by assuming a model consisting of the same number of layers as the number of data points we should be able to obtain a conductivity distribution that fits all data combinations. However, in reality this is never the case. We were faced with the practical limitations that our data were not perfect and furthermore putting over 100 layers into the inversion program would produce a highly unstable solution and would tie-up the computer until the next millennium. For this reason we had to limit the number of layers and data points and we had to try many different combinations of models and data arrangements.

The inversion proceeded in two stages. At the beginning we performed a few initial inversions on raw data collected over the upper section of the profile - these inversions helped to calibrate the system and proved that the data gathering system was working correctly. Later on we concentrated on the lower section and tried to obtain the conductivity model for this section using different combinations of data and model parameters. 
During all inversions we were constantly faced with the old dilemma of uncertainty vs. resolution. At first our objective was to fit the data within $1 \%$ in amplitude and 1 degree in phase between the observed and calculated values, since these were the estimates of the measurement accuracy. But we were faced with a predictable set of problems: different data sets (amplitude, phase, in-phase and quadrature) at different frequencies and receiver locations produced different models that looked reasonable when compared with the induction conductivity log, but did not produce a close fit to the data obtained at other frequencies or receiver location. We were able to fit individual profiles collected with a single receiver to within $1.0 \%$ for amplitude and 1.0 degrees for phase data. However since the solution is not unique the inversions of the data for different receiver positions were different from each other even in this simple, strictly one-dimensional medium. Similarly the inversions of the amplitude data did not produce the same model as the phase data inversion. For these reasons we tried to invert jointly all profiles, for practical reasons using only every fifth point along each vertical profile ( 52 points for each of the 13 receiver locations). We started with an inversion of data collected at lower frequencies ( 128 and $512 \mathrm{~Hz}$ ). The resulting model was then used as an initial guess at higher frequencies. Using this procedure we were not able to fit the $2048 \mathrm{~Hz}$ data within $1 \%$ in amplitude and 1 degrees in phase, but within $15 \%$ for the amplitude and +-5 degrees for the phase data. The $512 \mathrm{~Hz}$ fit was within $2 \%$ for the amplitude and within 1 degrees for the phase data. We tested how this model changes if data collected at $512 \mathrm{~Hz}$ and at 2048 were inverted jointly for a particular receiver location and using every amplitude and phase data point. Then we tested how this model changes if we simultaneously invert profiles collected at $2048 \mathrm{~Hz}$ at two receiver locations: 566.88 $\mathrm{m}(1850.0 \mathrm{ft})$ and $655.32 \mathrm{~m}(2150.0 \mathrm{ft})$. And finally we used the first vertical derivatives, $\Delta \mathrm{H}_{\mathrm{z}} / \Delta \mathrm{z}$ as an input to the inversion instead of our usual $\mathrm{H}_{\mathrm{z}}$ fields and we discovered that this is the easiest method to obtain a one-dimensional conductivity profiles from the least-squares inversion because it is not sensitive to the conductivities of the initial model. We also showed that assigning slightly different amplitude and phase corrections to each profile improves the fit between observed and calculated data. Below we will detail some of the inversion results.

\subsubsection{Inversion of data from upper level survey between 213.36 and $335.28 \mathrm{~m}$ (700 and $1100 \mathrm{ft})$.}

$128 \mathrm{~Hz}$. The first data set collected at Devine was obtained at $128 \mathrm{~Hz}$ for the upper section with a receiver located at a depth of $243.84 \mathrm{~m}(800 \mathrm{ft})$. At this frequency 
the system was not calibrated and the true amplitude and phase of the data were unknown. For this reason the data had to be inverted not only for parameters of the medium, but also for the phase shift calibration and amplitude calibration.

At first we approximated the conductivity of this section by a uniform half-space because the induction logs of this section did not show significant contrasts in conductivity. The least-squares inversion of phase data resulted in a half-space of conductivity $0.28 \mathrm{~S} / \mathrm{m}$. The phase shift was calculated to be $-15.0^{\circ}$. Since phase is more sensitive to the conductivity of the medium than the amplitude (because at low induction numbers it strongly depends on quadrature, see Chapter 2) we fixed the conductivity of the half-space to $0.28 \mathrm{~S} / \mathrm{m}$ and inverted the field data for the amplitude calibration. The amplitude calibration was 0.00254 (to convert measured values in $\mathrm{mV}$ to $\mathrm{nT}$ ). The results of this preliminary inversion are shown in Figure 5.3.1-1 which presents the comparison between the amplitude and phase of field data with numerical data obtained using inversion model. Figure also presents the percent error in amplitude and phase difference between observed and calculated values. The percent error in amplitude between the calculated and observed data is given by:

$$
\text { \% Ërror }=\frac{\text { observed data }- \text { analytic data }}{\text { analytic data }} \times 100.0
$$

The random errors in the amplitude and phase fit are superimposed on a general trend that in the upper portion of the profile reaches close to $4.0 \%$ for amplitude and 2 degrees for phase. These inversion results prove that a simple half-space model is not adequate to produce the response that matches the values obtained from crosshole measurements even in this simple one-dimensional geology and using low frequency data.

Since the higher frequencies are more sensitive to the conductivity variations in the model, the inversion of data collected at higher frequencies should produce models that would fit lower frequencies as well. With this in mind we concentrated on the inversion of data collected at $512 \mathrm{~Hz}$ (described later in this section) that resulted in a five layer model. To check whether this five layer model is consistent with the data obtained at $128 \mathrm{~Hz}$ we constructed Figure 5.3.1-2 which gives the amplitude and phase fit between measured and calculated data at $128 \mathrm{~Hz}$. As can be observed this new, five layer, model lowers significantly the systematic error in amplitude and phase roughly by a factor of two with phase fit being well below the 1 degree limit. With the new model the amplitude calibration at $128 \mathrm{~Hz}$ was $2.48 * 10^{-3}$ and phase shift was $-11.96^{\circ}$. 
$512 \mathrm{~Hz}$. The inversion of data collected over the same section of the profile but at $512 \mathrm{~Hz}$ showed much more sensitivity to conductivity and needed a five layer model to fit the data within the measurement error. We reached this conclusion after trying to fit the data with simpler models but we failed. We started with the inversion of the phase data assuming a half-space model. The inversion produced a half-space of conductivity $0.26 \mathrm{~S} / \mathrm{m}$ but the misfit between the observed and calculated phase data reached 7.00. This error was significantly larger that the expected error in measurements. We added a second layer and performed the inversion on two data sets with the receiver located at $240.79 \mathrm{~m}(790.0 \mathrm{ft})$ and $289.56(950.0 \mathrm{ft})$. The fit to the data improved significantly and was close to $1 \%$ for amplitude and 1 degrees for phase. Both data sets produced similar models when phase data were inverted. Similarly, both data sets produced almost the same model when amplitude data were inverted. However the two models, one obtained from phase inversions, another obtained from amplitude inversions, were not quite the same: phase inversions produced a model of conductivities $0.24 / 0.31 \mathrm{~S} / \mathrm{m}$, whereas amplitude inversions produced model close to $0.18 / 0.41 \mathrm{~S} / \mathrm{m}$. A possible reason for this inconsistency could be a difference in sensitivity of amplitude and phase to the conductivity of the medium and to the misplacement of the transmitter and receiver: amplitude is more sensitive to misplacement errors and less sensitive to the conductivity (see Figures 2.3-1c and 2.3-2b). The inversion for phase shift calibration was negligible which confirmed the calibration results for the system at $512 \mathrm{~Hz}$ (M. Wilt et al., 1991).

To reconcile the differences we jointly inverted amplitude and phase for the profile recorded with the receiver placed at depth $289.5 \mathrm{~m}(950.0 \mathrm{ft})$. The inversion resulted in the conductivity of the upper layer of $0.23 \mathrm{~S} / \mathrm{m}$ and gave $0.31 \mathrm{~S} / \mathrm{m}$ for the lower layer. The boundary between layers was located at 289.5. As can be observed in Figure 5.3.1-3 the errors in amplitude and phase show a systematic trend (straight line for amplitude and sinusoidal pattern for phase) that exceed the largest experimental errors especially for amplitude inversion. Hoping that these systematic errors are caused by a model that is not sufficiently detailed we added additional layers to the profile.

Since the calibration of the system showed a negligible phase shift for $512 \mathrm{~Hz}$, the initial guess at this frequency for the phase shift was always set to zero. The initial guess for amplitude calibration was usually set at 0.002 to obtain the $\mathrm{H}_{\mathrm{Z}}$ field in $\mathrm{nT}$ for unit source dipole moment.

After several attempts by trial and error with different numbers of layers we chose a five layer model. The inversion of data for this five layer model fitted the phase of field data within 1 degree. The phase shift was negligible for both data sets which confirmed 
the results of system calibration. To check the model for consistency with other data sets we performed an inversion of phase data assuming a five layer model but using data from a receiver placed at $240.79 \mathrm{~m}(790.0 \mathrm{ft})$. The inversion produced an almost identical model. An inversion of amplitude data for the same model produced a fit between the field data and analytic data that exceeded $1 \%$. This error was reduced significantly to below $1 \%$ when we assumed that the location of transmitter with respect to receiver is not exactly correct and inverted amplitude data for the correction in vertical distance between transmitter with respect to receiver. The two inversions on data sets where receiver was placed at $240.8 \mathrm{~m}(790.0 \mathrm{ft})$ and $289.6 \mathrm{~m}$ (950.0 ft) produced the following amplitude calibrations: $2.165^{*} 10^{-3}$ from the first data set and $2.201 * 10^{-3}$ from the second set. Similarly the correction for transmitter position produced $-1.52 \mathrm{~m}$ for the first, and -1.84 $\mathrm{m}$ for the second data set. The final inversion results for the upper data set are plotted in Figures 5.3.1-4. The five layer model for the upper section of the Devine experiment is plotted in Figure 5.3.1-5.

As can be observed this model is different from our initial assumption of a uniform layer of a fixed conductivity and prove that the crosshole measurements are more sensitive to conductivity variations than was originally assumed. With this in mind we turn our attention to the data collected over the region with high conductivity contrasts.

\subsubsection{Inversion of data from lower section between 548.64 and $670.56 \mathrm{~m}$ (1800 and $2200 \mathrm{ft}$ ).}

$512 \mathrm{~Hz}$. The detailed inversion for the lower part of the profile was performed at two frequencies: $512 \mathrm{~Hz}$ and $2048 \mathrm{~Hz}$. We started with a data set at $512 \mathrm{~Hz}$ in which the receiver was located at a depth of $594.5 \mathrm{~m}$ (1950 ft). As before we started an inversion by assuming a simple half-space model. The first least-squares inversion of phase data produced the half-space model of conductivity $0.28 \mathrm{~S} / \mathrm{m}$ with a large RMS error, and proved that a more detailed model was needed. A six layer model based on the resistivity logs was then used as an initial guess for inversions. Figure 5.3.2-1 shows the results of the inversion of phase data. This result was obtained in several steps in which some of the parameters, such as thickness or conductivities were kept fixed and the results from the best fits were used as initial guess for subsequent trials. The final six layer model was obtained by letting all depths and conductivities (except the top layer below the surface) vary.

As can be observed in Figure 5.3.2-1 the fit to the data is almost within $1^{\circ}$ and the model could be accepted if not for two observations: first from the calibration of the 
system at $512 \mathrm{~Hz}$ and from the results of the upper level inversion, we know that the instrumental phase shift should be close to zero at this frequency. This is not a case here - the results presented in Figure 5.3.2-1 are obtained with -6.30 phase shift. Furthermore, in the upper part of the section we observe a sinusoidal pattern suggesting that the model is more complicated than just six layers. Inserting one more layer into the upper part into a region with the largest sinusoidal variations in misfit error, dramatically reduces the RMS error and decreases the phase shift to zero. Figure 5.3.2-2 presents the results of amplitude and phase inversion for an eight layer model The amplitude data were obtained with all model parameters fixed to the ones obtained from phase inversion. The misfit error in phase does not show the sinusoidal pattern and confirms that the phase shift is equal to zero. The amplitude calibration for this case is $2.23 * 10^{-3}$. However the error in amplitude exceeds the measurement error and as before, we assumed it is caused by the misplacement of receiver with respect to transmitter location. Moving the receiver $0.47 \mathrm{~m}$ decreased the amplitude error to within $1.0 \%$. The new amplitude fit with percentage error is given in Figure 5.3.2-3. Figure 5.3.2-4 presents the resulting 8 layer conductivity model. The model is based on a phase inversion with all conductivities and depths (except the last one) varying. This model was used to calibrate the data collected at Devine.

To check how different data sets obtained with receivers placed at different depths confirm the 8 layer section we inverted the phase data for each individual profile separately and we allowed all parameters i.e. conductivities and layer depths to vary. The results were plotted by M. Wilt et al. (1991) in Figure 5.3.2-5. As can be observed the separate inversions produced models that were clearly one dimensional, and fitted the phase within 1 degree.

In conclusion: the inversions of data at $512 \mathrm{~Hz}$ produced eight layer models that fitted individual profiles within 1 degree. The models for different receiver locations did not vary significantly, however the same models needed corrections for absolute transmitter-receiver separations to fit the amplitude data within $1 \%$.

$2048 \mathrm{~Hz}$. Fitting the data at higher frequencies was much more difficult. Our inversions proved once more that higher frequencies need a model that is more detailed than the model that fits the data at lower frequencies.

This can be observed in Figure 5.3.2-6. The figure represents the fit between field and analytic data at $2048 \mathrm{~Hz}$ for the model interpreted using data at $512 \mathrm{~Hz}$ (see Figure 5.3.2-4). As can be observed the error in amplitude fit exceeds $3 \%$, the phase 
difference reaches 2.5 degrees. The amplitude calibration was 1.653 and phase calibration was $\mathbf{- 2 2 . 8 8}$.

The misfit error in Figure 5.3.2-6 shows a certain pattern: a large general trend in both amplitude and phase over which are superimposed smaller errors that show a mixture of random and sinusiodal errors. To examine the possible causes of these types of errors we constructed a set of numerical data at frequency 512 and $2048 \mathrm{~Hz}$ and considered three cases: 1) when inversion model is less detailed than the true one, 2) when the boundary of one layer is moved from the true position and 3) when the vertical location of the receiver with respect to the transmitter profile is in error.

In the first case we checked how frequency affects the misfit error and we examined two numerically generated data sets in which the inversion model did not correspond to the model for which the data were calculated. For this purpose we constructed phase data at 512 and $2048 \mathrm{~Hz}$ using a ten layer model. The ten layer model was based on the 8 layer inversion model (Figure 5.3.2-4) with an extra layer inserted at a depth of $650 \mathrm{~m}$. We inverted this data set assuming an eight layer model. The results are given in Figure 5.3.2-7 and show that the phase difference exhibits the sinusoidal pattern of misfit error at both frequencies but at the higher frequency the error was close to 1 degree whereas at lower frequency the phase difference was less than 0.25 degrees. In conclusion: by using the smaller number of layers in inversion one observes the sinusoidal misfit error around the faulty model and also the averaging of the layer conductivities in the region where the true model is more detailed than the inversion model. The errors are larger at higher frequencies.

To examine how sensitive the measurements are to changes in the medium parameters we varied the thicknesses of one layer in our previously described eight-layer model (shown in Figure 5.3.2-4). The variation in sensitivity can be seen in Figure 5.3.2-8 which presents the percent change in amplitude and phase difference at 512 and $2048 \mathrm{~Hz}$. The model boundaries and conductivities are superimposed on the lower left panel describing the percent error in amplitude. A variation in layer thickness as small as $1.0 \mathrm{~m}$ can cause a $2 \%$ change in amplitude and up to $1.0{ }^{\circ}$ shift in phase at $2048 \mathrm{~Hz}$.

Finally we examined the size of the error expected when the assumed location of the receiver with respect to the transmitter profile is not correct. Figure 5.3.2-8b shows that even a two foot error in the mutual distance between transmitter and receiver can cause up to $5 \%$ error in amplitude.

This short study shows that the misfit observed in Figure 5.3.2-6 can be caused by a combination of the above reasons. 
To check our model and procedure we then examined how it fits the data collected at other receiver locations and were quite disappointed: even in this one-dimensional medium the model interpreted from a single receiver location did not fit the data collected at other locations within measurement accuracy.

After many attempts to reconcile the differences we decided to jointly invert the amplitude and phase data for all receiver positions, but for practical reasons (computer time) using only every fifth point along the transmitter profile. This gave us a total of 676 data points: 52 data points of amplitude and phase data for each of the 13 receiver profiles. In the figures that follow each of the thirteen segments has 52 points and represents a profile recorded with receiver placed at different depth inside borehole. The receiver depth for which the profile was recorded is given on the right hand side of each panel.

The subsequent inversions were performed on calibrated data and for this reason we will talk about eventual corrections to the provided amplitudes and phase values whenever they were calculated.

We tried several options for weighting data however the best convergence was obtained when amplitude and phase data were not weighted even if their values differed by several orders of magnitude. Not weighting the amplitude and phase data put more significance on phase data that were more sensitive to conductivity and less sensitive to position errors (especially at higher frequencies). Furthermore, amplitude decreases with separation, providing an automatic weighting factor for amplitude data because it gives less significance to noisier data collected at larger separations. When the convergence failed we weighted the amplitude and phase data by the square root of their values. This operation put more weight on the smaller numbers (amplitude) and decreased the influence of the larger values (phase) and frequently resulted in a better convergence. Assigning equal importance to amplitude and phase data by normalizing each data point by its value frequently produced models that were not physically feasible. Figure 5.3.2$8 \mathrm{c}$ shows the pattern of misfit errors obtained from numerical data using different weighting options. The true model that provided the numerical data had 14 layers, the inversion was performed assuming 8 layers. As can be observed from the error pattern of misfit between observed and numerical data only the first two weighting options resulted in the same model (shown in Figure 5.3.2-12). The model obtained in the third case was not realistic and produced a different misfit error pattern. 
We also tried many models with different numbers of layers of varying conductivity and thicknesses, but using the layers of fixed thicknesses and varying only the conductivities provided the fastest convergence and more consistent models.

Our final model for Devine consisted of 14 layers, each $10.0 \mathrm{~m}$ thick. The joint inversion of amplitude and phase at $512.0 \mathrm{~Hz}$ for all receiver positions provided a good fit to the data: amplitude was fit within $2.0 \%$, phase was fit within $1.0 \mathrm{deg}$ as can be observed in Figure 5.3.2-9. At $2048.0 \mathrm{~Hz}$ (Figure 5.3.2-10) the same model did not fit the data as well: phase fit was within 6.0 degrees and amplitude within 15.0\%. Figure 5.3.2-11 shows the model.

A glance at the error pattern in amplitude and phase reveals a systematic trend at both frequencies. To test whether this error pattern can be caused by a model that is not sufficiently detailed we constructed two analytic data sets at both frequencies for a fourteen layer model obtained from the joint inversion. These numerical data were then inverted assuming an eight layer model. The fit to the data and the misfit errors for $\mathbf{5 1 2}$ and $2048 \mathrm{~Hz}$ are given in Figures 5.3.2-12 and 5.3.2-13 respectively. As can be observed by comparing Figures 5.3.2-9 and 5.3.2-10, the error pattern, although not the same, looks similar and shows a systematic trend proving that a more detailed, made with more than fourteen layers model could fit the data better. However, a model with many layers takes a lot of computational time and poses a danger of fitting the noise. This experiment also proved (see Figure 5.3.2-14) that inversion gives an averaged conductivity of the adjacent layers that closely approximate the true conductivity distribution.

At this point we could to continue to increase the number of layers to fit both frequencies within the measurement errors, however we decided against it for two reasons: first of all we did not know how accurate were the measurements of the absolute distances between the transmitter and receiver positions - we only knew (from the $1 \%$ repeatability of the measurements) that the distance between the successive measurements did not change by more than few centimeters. But the absolute measurements of distances between transmitter and receivers were not independently checked, so we did not know whether stretching a cable or possible tilt of the borehole from the vertical affected our distances. If this was the case, then our model obtained at $512 \mathrm{~Hz}$ assuming a perfect knowledge of the geometry would be biased. Our suspicion about the absolute geometry between transmitter and receiver increased when (as described in Chapter 4) we had to shift the position of our data by $5 \mathrm{~m}$ in order to correlate the induction logs with the second vertical derivative results. To check how the 
change in the geometry affects the fit of the data we fixed the model obtained from the $512 \mathrm{~Hz}$ inversion and inverted the data at $2048 \mathrm{~Hz}$ for the errors in distance between the receiver and the location of the all transmitter profiles. The fit improved and gave us an error of $-0.74 \mathrm{~m}$ for horizontal transmitter receiver separation and $0.34 \mathrm{~m}$ for vertical transmitter-receiver separation, but increased the error when we applied this position correction to the $512 \mathrm{~Hz}$ data. This is understandable since the model was obtained at $512 \mathrm{~Hz}$ assuming a perfect measurements of transmitter location with respect to the receiver. This location need not to be the same for both frequencies, furthermore the sensitivity of the system to errors in location and conductivity variations in the medium varies with frequency (Figure 5.3.2-8).

Secondly a model consisting of 14 layers takes a lot of computer time to invert for all transmitter - receiver combinations.

From the above analysis it is evident that we were not able to obtain a model that would fit all data within $1 \%$ and 1 degree however we were able to fit individual profiles within these limits relatively easily and the models did not differ significantly.

\subsubsection{One-dimensional inversion using the first vertical derivative $\Delta \mathbf{H}_{\mathbf{z}} / \Delta \mathbf{z}$}

In Chapter 3 we observed that the first vertical derivative of the vertical magnetic field is as sensitive to the location of the boundary as the horizontal component. Since our measurements were taken along vertical profiles, and the spacing between the measurements was small $(0.95 \mathrm{~m})$ it was easy to approximate the vertical derivative by a finite central difference and use this data as an input in the least-squares inversion. Because by taking the derivatives we increase the noise roughly by a factor of ten (see Figures 2.3-5 and 2.3-6) we accepted a $20 \%$ error in amplitude and 10 degrees error in phase as a good fit to the derivative data.

Figures 5.3.3-1 to 5.3.3-4 present the results of the least-squares inversions using first vertical derivative as an input to the inversion routine at two frequencies (512 and $2048 \mathrm{~Hz}$ ). In all figures the model had fourteen layers. The receiver was placed at $609.6 \mathrm{~m}(2000 \mathrm{ft})$ in the middle of the section for all profiles. The inversions were performed jointly on amplitude and phase of the vertical derivative (not on the derivative of the amplitude and phase) or on the in-phase and quadrature derivative. All inversions produced similar models (shown in Figure 5.3.3-5) but we found that joint inversion of in-phase and quadrature components was the most stable and the least-sensitive to the choice of the initial model. Although the initial model for in-phase and quadrature 
inversions was totally arbitrary (intermediate layers of 0.1 and $0.2 \mathrm{~S} / \mathrm{m}$ ) it produced similar model at both frequencies as can be seen in Figure 5.3.3-5. The initial model using amplitude and phase of the vertical derivative had to be closer to reality to produce the required convergence.

To check how the model obtained from the single profile but using the vertical derivatives fits the data collected at other receiver locations we constructed Figures 5.3.36 and 5.3.3-7 for frequencies 512 and $2048 \mathrm{~Hz}$ respectively. These figures represent the misfit error between observed and numerical data for a fixed fourteen layer model obtained at $512 \mathrm{~Hz}$ from the in-phase and quadrature inversion of $\Delta \mathrm{H}_{\mathrm{Z}} / \Delta \mathrm{z}$ data. The error pattern shows a systematic bias at both frequencies. The most probable cause of the error is that our model is not the best, because it is based on the single profile. If the data were perfect, then in a one-dimensional medium the data should be consistent with the model. Since we can not be sure that there is no systematic error in the data we considered what happens if we fix that model and invert the data for each receiver location for amplitude and phase correction. The results are given in Figure 5.3.3-8 for data at $512 \mathrm{~Hz}$. Table 5.3-1 shows the values of the amplitude and phase corrections and compares them with the corrections obtained by D. Alumbaugh (1993). As can be observed assigning slightly different corrections to the data lowers the systematic error in both amplitude and phase and produces the fit that is almost within the prescribed measurement accuracy.

\begin{tabular}{|c|c|c|c|c|}
\hline & \multicolumn{2}{|c|}{ Least-squares corrections } & \multicolumn{2}{c|}{ D. Alumbaugh's corrections } \\
\hline $\begin{array}{c}\text { Receiver } \\
\text { depth (m) }\end{array}$ & $\begin{array}{c}\text { Implitude } \\
\text { correction }\end{array}$ & $\begin{array}{c}\text { Phase } \\
\text { correction }\end{array}$ & $\begin{array}{c}\text { Amplitude } \\
\text { correction }\end{array}$ & $\begin{array}{c}\text { Phase } \\
\text { correction }\end{array}$ \\
\hline 564 & 1.03 & 3.44 & 1.06 & 5.7 \\
571 & 1.01 & 2.72 & 1.05 & 5.2 \\
579 & 1.01 & 2.28 & 1.04 & 4.3 \\
586 & 1.01 & 1.60 & 1.04 & 3.2 \\
594 & 1.01 & 1.12 & 1.05 & 3.6 \\
602 & 1.01 & 1.02 & 1.04 & 1.6 \\
609 & 1.01 & 1.41 & 1.03 & 1.5 \\
617 & 1.00 & 1.59 & 1.02 & 1.6 \\
625 & 1.00 & 1.65 & 1.01 & 1.3 \\
632 & 0.99 & 1.50 & 1.00 & 0.8 \\
640 & 0.99 & 1.43 & 1.00 & 0.3 \\
647 & 1.00 & 1.61 & 1.00 & 0.4 \\
655 & 1.00 & 1.04 & 1.00 & -0.6 \\
\hline
\end{tabular}

Table 5.3-1 The amplitude and phase corrections obtained by least-squares for a fixed model that was obtained from the inversion of the first vertical derivatives at $512 \mathrm{~Hz}$ and 
compared to the corrections obtained by D. Alumbaugh (1993) using an eight layer model.

A summary of our inversion efforts is given in Figure 5.3.3-9 for the lower section of the crosshole measurements in Devine. We found, that different inversions produced similar models (except joint inversions at 512 and $2048 \mathrm{~Hz}$ using single profile) that fit the data within reasonable limits especially at lower frequency of $512 \mathrm{~Hz}$. However the data were not fit within $1 \%$ and degree for phase because this would require a larger number of layers and also the introduction of additional corrections for amplitude and phase data different for each of the separate profiles.

\subsection{The Richmond Field Station Experiment}

In this section we present the one-dimensional interpretation of data from the Richmond Field Station (RFS), California. The experiment in RFS was conducted as a joint project between LLNL, LBL and Engineering Geoscience in the spring of 1992. The objective of the experiment was to prove that the movement of fluids between boreholes can be monitored using electromagnetic measurements between them. To accomplish this, we collected two sets of measurements: one set of measurements was performed in May of 1992 to establish the baseline values. The second set of measurements was conducted in June 1992 after the 250,000 liters of conductive salt water were injected into the ground. The water was pumped into the central injection well (Figure 5.4.1). Measurements were made between the injection well and four observation wells around it: NW, NE, SE, and SW. Wells NW and SE were placed 20 $\mathrm{m}$ away from the injection well, wells $\mathrm{NE}$ and $\mathrm{SW}$ were $25 \mathrm{~m}$ away. The stationary receivers were placed in observation wells and were moved in $5.0 \mathrm{~m}$ intervals starting form $5.0 \mathrm{~m}$ below the surface to $55 \mathrm{~m}$ below the surface. The transmitter traversed the injection well taking measurements every $0.5 \mathrm{~m}$ from $4.0 \mathrm{~m}$ to $60.0 \mathrm{~m}$ below the surface. The operating frequency was $18.5 \mathrm{kHz}$.

The geology of the Richmond Field Station is presented in Figure 5.4.2. The upper section is 35 to $40 \mathrm{~m}$ uhick and is a mixture of unconsolidated sands, gravel and sills. The basement in the lower section consists of sandstones or shales. The upper section is more conductive, about $0.075 \mathrm{~S} / \mathrm{m}$, the basement conductivity is around 0.02 $\mathrm{S} / \mathrm{m}$. The injected salt had conductivity $1 \mathrm{~S} / \mathrm{m}$.

Our goal in this interpretation was not to recover the conductivity distribution in Richmond (which is quite complicated and definitely not one-dimensional), but to find an 
equivalent conductivity model which would show the conductivity variations before and after salt water injection. We also hoped that the conductivity variations between boreholes would indicate the possible direction of the subsurface water propagation. We accomplished this task, but only after many trials using different number of layers, weighting options and combinations of data used for inversion.

\subsubsection{One-dimensional inversion of data collected in Richmond, California.}

The geology of RFS is not one dimensional as can be seen by comparing the induction logs from different boreholes presented in Figure 5.4-2.

The inversion was further complicated by the fact that at the initial stage of interpretation the inversion was performed on raw, field data for which the true amplitude and phase were not known, introducing two additional unknown parameters. The inversion in this case produced an independent estimate of the calibration parameters and was used to check the system calibration.

Based on our previous experience we abandoned the separate inversions of ampiitude and phase data and concentrated instead on joint amplitude and phase inversions.

The first attempt involved the joint inversion of amplitude and phase data but for each individual receiver position. After many different attempts we settled for a five layer model where each layer had a constant $10.0 \mathrm{~m}$ thickness. We hoped that the $10.0 \mathrm{~m}$ interval would produce sufficiently detailed conductivity distribution to fit the data within reasonable accuracy and at the same time correspond to the conductivities obtained from the induction logs. The inversion results for all four wells before and after injection of salt water are summarized in Tables 5.4-1 to 5.4-4. In these tables the first column describes the name of the inverted profile where the last digits describe the receiver depth. The inversions of the data collected in May from the NW borehole produced a good fit between observed and calculated data for receiver positions of 10.0, 15.0, 20.0, 25.0 and $30.0 \mathrm{~m}$, however the fit at other receiver positions was significantly worse. By the 'good' fit in this case we understand the fit within $10 \%$ in amplitude and within $10 \mathrm{deg}$ in phase. This very poor fit between measured and calculated data in comparison with Devine data can be caused not only by a complicated geometry, but also by the frequency of transmitter current: the frequency in Richmond was $18.5 \mathrm{kHz}$ whereas the frequencies in Devine were 512 and $2048 \mathrm{~Hz}$ making the Richmond system far more sensitive to conductivity but at the same time much more difficult to match to a layered model. Even 
this "good" fits produced models that roughly fit within the conductivities obtained from induction logs, but that were not the same for each inversion. The inversion of data from the other wells produced similar pattern where the best fits were obtained for the same depths $(10.0,15.0,20.0,25.030 .0 \mathrm{~m})$ of the receiver. This result may be understandable if we realize that the conductivities in the upper part of the profile (up to the depth of roughly $40 \mathrm{~m}$ ) are much higher than in the deeper regions and therefore are better resolved that in the lower part. Nevertheless this form of inversion did not produce a consistent estimate of conductivity. Furthermore, the inversion of June data was also inconsistent with the inversion of May data. In Figure 5.4-3 we showed the variations in conductivity profiles obtained for individual receiver locations.

To obtain a more stable conductivity estimate and better calibrations for amplitude and phase we took the averages of the best fitting results (that had the misfit error below $2.5 \%)$ and models that looked reasonable when compared with induction logs and summarized the results in Table 5.4-5. As can be observed the amplitude and phase calibrations in May are less stable than the same calibrations obtained for the June data.

To eliminate the confusion resulting from different results obtained for different receiver positions we decided to jointly invert amplitude and phase data for all receiver positions. To shorten the time needed for inversion we picked up every fifth point along profiles for each receiver position. So instead of inverting on all 113 data points measured every $0.5 \mathrm{~m}$ for each receiver position, we inverted 23 data points spaced 2.5 $\mathrm{m}$ apart. This gave us total of $\mathbf{5 0 6}$ data points of amplitude and phase data. Our initial model consisted of the five layers of equal $10.0 \mathrm{~m}$ thicknesses but the misfit error was large and we had to increase the number of layers to 11 and decrease the thickness of each layer to $5.0 \mathrm{~m}$.

We inverted for the layer conductivities and for amplitude and phase calibrations.

In Table 5.4-6 we compared the calibration values obtained from inversions with calibrations based on the system analysis described by $D$. Alumbaugh (1993). As can be observed the biggest difference between calibration values occurs for the SE well where it reaches $8.3 \%$ for amplitude and 8.6 degrees for phase.

Our final fit to the data was worse then that for individual profiles: the misfit error reached $40.0-50.0 \%$ in amplitude for some of the receiver locations (usually at 5.0, $45.0,50.0$ and $55.0 \mathrm{~m}$ depth), but usually was within $10 \%$ for the remaining receiver locations. We used the model obtained with data collected in May as an initial guess for the June data inversions. To illustrate the fit between measured and numerical data we plotted the observed and calculated phase in Figure 5.4-4 for each of the observation 
wells obtained from the pre injection data. As can be observed the best phase fit was obtained in the middle of the section for receivers located at depth 15,20,25 and $30 \mathrm{~m}$.

The conductivity models for each observation were based on the joint inversion of amplitude and phase data for all receiver locations are given in Figure 5.4-5. The curves on the plot represent the induction logs before injection and the model obtained from data before injection and model obtained from data after injection. It can be seen that the numerical model traces the conductivities obtained from the induction logs reasonably well except for the 5-10 m section for the NW well where the model fails. The differences in conductivity obtained by subtracting the inversion results from before and after the injection of salt water are given in Figure 5.4-6. It can be seen that the largest variations in conductivity between May and June occurred around the area where the salt water was injected in the NW and NE wells.

Next based on our experience with the Devine data we decided to perform inversions not on the $\mathrm{H}_{\mathrm{Z}}$ field itself, but on the vertical space derivative of $\mathrm{Hz}$ component. The data points were collected every $0.5 \mathrm{~m}$ and this was the spacing of our data points in taking the derivatives. At first we started by inverting the amplitude and phase data of the $\Delta \mathrm{H}_{\mathbf{Z}} / \Delta \mathrm{z}$ (using as an initial guess the best model obtained from $\mathrm{H}_{\mathbf{Z}}$ inversion), but after few trials we realized that inversion of in-phase and quadrature of $\Delta \mathrm{H}_{\mathrm{z}} / \Delta \mathrm{z}$ was much faster (the convergence usually occurred after 4-10 iterations) that we abandoned the first amplitude and phase attempts and settled for the in-phase and quadrature inversion.

At first we inverted just a single profile from the receiver placed $30.0 \mathrm{~m}$ below the surface for all four wells surveyed. This produced model that agreed with induction logs in three cases, but was not realistic for one well. When we added the second data set collected with receiver located $15 \mathrm{~m}$ below the surface, and used as an initial guess an uniform half-space of conductivity $0.043 \mathrm{~S} / \mathrm{m}$, we obtained results presented in Figures 5.4- 7, 5.4-8, 5.4-9 and 5.4-10 for NW, NE, SE and SW wells respectively. These figures show: a) the models obtained using the data collected before and after the salt water injection superimposed on the induction $\log$ from the injection well, b) conductivity difference in models before and after the injection, c) and d) in-phase and quadrature of observed and calculated derivatives together with the misfit error for data collected before and after injection, respectively. The top 224 points on panels $c$ and $d$ represent profiles collected with the receiver placed at $15 \mathrm{~m}$ from the surface, points below were collected using the receiver placed at $30 \mathrm{~m}$ below the surface. These results were obtained without changing the initial guess, or putting any restrictions on 
conductivities (except our usual constraints that conductivity had to be between 0 and 1 $\mathrm{S} / \mathrm{m}$ ). The convergence was usually accomplished in less than 5 iterations. As can be observed the largest conductivity contrast occurs in NW and NE wells suggesting that this is a direction of fluid propagation. The variations in southern wells are much smaller. These results support the conclusions reached by D. Alumbaugh (1993) about the direction of fluid propagation. However, the conductivity contrast obtained by D. Alumbaugh was much larger: $0.15 \mathrm{~S} / \mathrm{m}$. This is understandable, since his model is twodimensional and therefore more closely resembles the actual conductivity distribution. In a one-dimensional model the conductivity variations had to be distributed over the whole layer (not just inside a smaller disk) resulting in a lower overall conductivity. In passing we notice that the second vertical derivative method (Chapter 4 ) produced conductivity variations of the order of $0.2 \mathrm{~S} / \mathrm{m}$, i.e. even higher than D. Alumbaugh (1993) estimate but closer to the conductivity measurements of borehole fluid obtained by $\mathrm{O}$. Tseng (personal communication) which was $1.0 \mathrm{~S} / \mathrm{m}$. This result can be explained by the fact that the second derivative method is most sensitive to the medium in the vicinity of measurement point.

\subsection{Summary}

The inversion results from the three very different experiments revealed several important aspects of electromagnetic measurements in conductive media. The inversion of the data showed that the sensitivity of the measurements to the conductivity of the medium is higher that we anticipated and that derived calibration factors for the system are strongly dependent on the model and on the geometry of the transmitter with respect to the receiver.

The inversion results also indicated that the repeatability measurements do not necessarily guarantee the accuracy of the measurements. If the measurements are repeatable, but other parameters (like frequency or separations) are measured incorrectly, then even in the simple one dimensional geology it is impossible to obtain a model that fits all data within a repeatability error and is consistent with other data sets. However in the monitoring of movements of fluids we are mostly interested in detection of the fluid propagation and the absolute values of conductivity are of a lesser importance. Our inversion shows, that even a very rough model that fits the data only in the least-squares sense and is not even close to the true conductivity distribution is capable of detecting the direction of fluid propagation. The inversions proved that even in a complicated geology it is possible to detect changes in the apparent conductivity that are indicative of the 
direction of the propagation of fluids. We found, that at low frequencies it is much easier to obtain a good fit to the data, but that the resolution of the model is worse.

The separate inversions of amplitude and phase data produced different models for in phase and quadrature as well as amplitude and phase data. Furthermore the in phase and quadrature data are more susceptible to position errors and therefore an accurate fit of a one-dimensional model and calculated data (if possible at all) would pose a danger of fitting the model to the noise. For this reason we looked at the joint inversion of amplitude and phase data. We found out that amplitude is sensitive to position errors, but not very sensitive to the model parameters. Phase data shows opposite characteristics: it is more sensitive to layer conductivities, but not as sensitive to position errors. We tried to use the sensitivity of the amplitude data to position errors to find out the corrections in distances of transmitter with respect to receiver, but this approach only proved the necessity of having a very accurate locations of transmitter and receiver. If we tried to invert for position errors the results proved to be confusing and the models were not consistent. The joint inversion of amplitude and phase data for all receiver locations is important in providing the consistent model of conductivity even if there is larger misfit between calculated and field observations. Furthermore the accurate knowledge of transmitter location with respect to receiver is crucial especially at higher frequencies and smaller transmitter-receiver separations.

Positioning is very important. During the inversion procedure we observed that different results were obtained with depth and separation corrections fixed or when treated as an unknown. Frequently even $0.3 \mathrm{~m}$ shift in vertical or horizontal position meant the difference between good convergence and failure of inversion. It is also very important to keep a record of changing transmitter-receiver geometry i.e. whether the vertical and horizontal separations are indeed what we are assuming. For example a cable that lengthens with depth or a tilted well can produce high quality data with very good repeatability because the conditions of the experiment are kept constant, but can introduce a lot of uncertainty and frustration during the inversion since the final model depends on the assumed geometry of transmitter with respect to the receiver.

It is very important to weight the data properly. In our 1-D least squares inversion we tried different ways to weight the data. The data have to be weighted with respect to their measurement accuracy and sensitivity to conductivity. Since the amplitude is not very sensitive to conductivity and has a larger error associated with the transmitter - receiver distance, amplitude must have less importance than phase in determining the inversion model. Since our final results were based on joint amplitude 
and phase inversions that varied by several orders of magnitude we tried three weighting procedures: not weighting the data, weighting them by the square root of their value and finally assigning an equal importance to all data points. The first approach left the amplitude and phase of the field data unchanged and since the phase is two to four orders of magnitude greater then amplitude this method resulted in placing a large emphasis on phase, and much less importance on the amplitude. Phase data are more sensitive to conductivity and at the same time less sensitive to position errors than the amplitude. Furthermore not weighting the amplitude data puts more importance on data measured at the closest transmitter-receiver separation where amplitude is the largest and the least sensitive to the position errors. In the second approach weighting the data by the square root of the data value decreased the ratio between the small and large values giving more importance to amplitude and less importance to phase data, but still kept the phase data larger. We used this second weighting procedure when the inversion without weighting the data failed. The third weighting option considered was to divide amplitude and phase data by their absolute values. This approach assigned the same significance to amplitude and phase data and also to the data collecred at large transmitter receiver separations. We found out, that the fastest convergence in most cases was when the amplitude and phase data were not weighted at all, despite the large difference in their absolute values. We assumed that this fast convergence was caused by the properties of the data themselves: the decrease in amplitude with distance and at the same time increase of noise put less emphasis on amplitude data collected far away from the transmitter. The phase data had the same "importance" far away and close to the transmitter as long as the phase did not go through zero. In situations where we had problems with convergence we weighted the amplitude and phase data by the square root of their value. This approach increases the importance of amplitude with respect to the phase data and in several cases allowed the inversion to converge.

The most important conclusion of our inversion work is that the inversion based on the first vertical derivative is a valuable tool in recovering the conductivity even in a complicated conductivity structure. It also appears that the type of inversion works better on in-phase and quadrature data because it is less dependent on the initial guess model. Furthermore using the first vertical derivative produced consistent models at higher and lower frequencies which was not always the case when we used the $\mathrm{H}_{z}$ field alone.

Below we will highlight some other conclusions that were the result of our inversion work:

1. Repeatability is not a replacement for the accuracy of the measurements 
2. In-phase and quadrature $\mathrm{H}_{\mathbf{z}}$ field components are noisy in regions where they change sigit and for this reason were more difficult to invert on.

3. Amplitude and phase data should be inverted simultaneously to get consistent inversion results because the sensitivity to model and noise is different in these two quantities.

4. Weighting of the data should correspond to the noise in the data, i.e. do not weight amplitude and phase data used in joint inversion.

5. The layers that are not detailed enough show a sinusoidal error pattern around the :missing layer"

6. The conductivity provided by the least-squares inversion gives the averaged conductivity distribution over the neighboring layers.

7. The error in position shows-up as a general trend in the error pattern

8. A sudden jump in the measurement conditions (such as a change in geometry or range switch on the lock-in amplifier) shows as a sudden jump that "levels off" after a certain distance.

9. Derivative inversion of in-phase and quadrature is not sensitive to the initial guess and converges very fast to the reasonable models that are consistent with other data sets and with other frequencies.

10. Use of in-phase and quadrature of $\Delta \mathrm{Hz} / \Delta \mathrm{z}$ works better possibly because they are the sarne order of magnitude and converge better than $|\Delta \mathrm{Hz} / \Delta \mathrm{z}|$ and phase $|\Delta \mathrm{Hz} / \Delta \mathrm{z}|$.

11. The error in derivatives is ten times larger than in the $\mathrm{H}_{\mathrm{z}}$ component. 


\begin{tabular}{|l|lllll|l|l|l|l|}
\hline Well & $\sigma_{1}$ & $\sigma_{1}$ & $\sigma_{1}$ & $\sigma_{1}$ & $\sigma_{1}$ & $\begin{array}{l}\text { Ampl. } \\
* 10-04\end{array}$ & $\begin{array}{l}\text { Phase } \\
(\mathrm{deg})\end{array}$ & $\%$ & RMS \\
\hline 5SC1NW5 & .13 & .095 & .065 & .039 & .013 & 1.24 & 41.4 & 3.6 & 4.7 \\
55C1NW10 & .083 & .11 & .059 & .067 & .007 & 1.22 & 53.2 & 1.6 & 1.1 \\
5SC1NW15 & .068 & .1 & .048 & .073 & .015 & 1.18 & 56.9 & 1.6 & .94 \\
5SC1NW20 & .085 & .095 & .059 & .056 & .022 & 1.19 & 56.7 & 1.3 & .9 \\
55C1NW25 & .096 & .091 & .072 & .031 & .03 & 1.16 & 56.5 & 1.1 & .1 \\
5SC1NW30 & .09 & .11 & .08 & .016 & .031 & 1.10 & 56.4 & 1.9 & .86 \\
56C1NW35 & .073 & .113 & .085 & .041 & .016 & 1.05 & 55.1 & 5.2 & 3.9 \\
56C1NW40 & .075 & .095 & .096 & .055 & .0092 & 1.04 & 53.75 & 7.3 & 4.3 \\
56C1NW45 & .1 & .06 & .09 & .07 & .056 & 1.05 & 53.9 & 8.3 & 5.12 \\
56C1NW50 & .12 & .068 & .061 & .090 & .009 & 1.08 & 58.0 & 8.2 & 5.5 \\
56C2NW55 & .1 & .1 & .03 & .08 & .017 & 1.10 & 59.4 & 5.2 & 4.4 \\
\hline AVERAGE & .084 & .1 & .064 & .056 & .0212 & 1.13 & 54.66 & & \\
\hline
\end{tabular}

\begin{tabular}{|l|lllll|l|l|l|l|}
\hline Well & $\sigma_{1}$ & $\sigma_{1}$ & $\sigma_{1}$ & $\sigma_{1}$ & $\sigma_{1}$ & $\begin{array}{l}\text { Ampl. } \\
* 104\end{array}$ & $\begin{array}{l}\text { Phase } \\
\text { (deg) }\end{array}$ & $\%$ & RMS \\
\hline 65 C1NW5 & .12 & .11 & .06 & .04 & .005 & 1.34 & 49.2 & 3.2 & 2.5 \\
65C1NW10 & .085 & .087 & .089 & .043 & .009 & 1.34 & 54.8 & 1.4 & .91 \\
65C1NW15 & .1 & .068 & .084 & .046 & .018 & 1.31 & 58.9 & 1.5 & 1.0 \\
68C1NW20 & .1 & .07 & .091 & .027 & .024 & 1.29 & 55.4 & 1.5 & 1.2 \\
68 C1NW25 & .1 & .073 & .086 & .011 & .031 & 1.25 & 57.5 & 1.6 & 1.6 \\
68C1NW30 & .088 & .094 & .082 & .016 & .027 & 1.19 & 58.8 & 2.7 & .19 \\
68C1NW35 & .072 & .11 & .085 & .039 & .017 & 1.14 & 54.7 & 4.9 & 3.8 \\
68C1NW40 & .071 & .092 & .098 & .056 & .01 & 1.15 & 51.2 & 5.6 & 3.7 \\
68C1NW45 & .096 & .067 & .093 & .08 & .006 & 1.15 & 50.8 & 6.0 & 4.3 \\
68C1NW50 & .11 & .07 & .063 & .099 & .008 & 1.17 & 52.7 & 5.6 & 4.4 \\
68C1NW55 & .097 & .087 & .042 & .082 & .019 & 1.22 & 52.6 & 4.3 & 3.6 \\
\hline AVERAGE & .095 & .078 & .086 & .029 & .022 & 1.29 & 56.6 & & \\
\hline
\end{tabular}

Table 5.4-1 The results of the joint amplitude and phase least-squares inversion of individual profiles from EMNW well for data before injection (top) and after injection (bottom). The model consists of five $10 \mathrm{~m}$ thick layers. Amplitude calibration unit is $(\mathrm{A} / \mathrm{m}) / \mathrm{V}$ per unit moment. 


\begin{tabular}{|l|lllll|l|l|l|l|}
\hline & $\sigma_{1}$ & $\sigma_{2}$ & $\sigma 3$ & $\sigma 4$ & $\sigma 5$ & $\begin{array}{l}\text { Ampl. } \\
* 104\end{array}$ & $\begin{array}{l}\text { Phase } \\
(\mathrm{deg})\end{array}$ & $\%$ & RMS \\
\hline 54C1NE5 & .041 & .077 & .066 & .076 & .015 & 1.16 & 77.3 & 2.6 & 1.2 \\
54C1NE10 & .087 & .11 & .061 & .057 & .016 & 1.17 & 53.7 & 2.2 & .69 \\
54C1NE15 & .098 & .14 & .074 & .056 & .01 & 1.15 & 41.7 & 2.0 & .74 \\
54C1NE20 & .08 & .13 & .078 & .060 & .003 & 1.18 & 45.1 & 2.1 & 1.0 \\
54C1NE25 & .02 & .16 & .055 & .071 & .003 & 1.21 & 51.4 & 2.6 & 1.9 \\
54C1NE30 & .021 & .16 & .044 & .067 & .0 & 1.27 & 57.2 & 3.0 & 2.2 \\
54C1NE35 & .027 & .18 & .021 & .06 & .0 & 1.26 & 61.5 & 3.6 & 2.8 \\
54C1NE40 & .156 & .04 & .124 & .0 & .008 & 1.16 & 64.7 & 3.2 & 3.2 \\
54C1NE45 & .083 & .14 & .026 & .0083 & .0 & 1.26 & 55.7 & 3.2 & 2.0 \\
54C1NE50 & & & & & & & & & \\
54C2NE55 & .11 & .08 & .059 & .007 & .018 & 1.15 & 48.9 & 5 & 2.8 \\
\hline AVERAGE & .103 & .121 & .061 & .046 & .008 & 1.19 & 55.7 & & \\
\hline
\end{tabular}

\begin{tabular}{|l|lllll|l|l|l|l|}
\hline & $\sigma_{1}$ & $\sigma_{2}$ & $\sigma_{3}$ & $\sigma_{4}$ & $\sigma_{5}$ & $\begin{array}{l}\text { Ampl. } \\
* 10-4\end{array}$ & $\begin{array}{l}\text { Phase } \\
\text { (deg) }\end{array}$ & $\%$ & RMS \\
\hline 63 CINE5 & .02 & .068 & .086 & .078 & .016 & 1.23 & 83.0 & 2.5 & 1.5 \\
63C1NE15 & .084 & .089 & .083 & .051 & .013 & 1.28 & 57.6 & 1.7 & 1.1 \\
63C1NE15 & .108 & .117 & .103 & .046 & .005 & 1.25 & 43.6 & 1.8 & 1.4 \\
63C1NE20 & .094 & .099 & .094 & .051 & 0 & 1.31 & 50.3 & 2.2 & 1.5 \\
63C1NE25 & .065 & .11 & .063 & .061 & .0001 & 1.34 & 57.7 & 2.6 & 1.5 \\
63C1NE30 & .015 & .15 & .048 & .054 & 0 & 1.37 & 63.3 & 3.7 & 2.6 \\
63C1NE35 & .0076 & .17 & .041 & .045 & .005 & 1.34 & 68.3 & 3.4 & 3.3 \\
64CINE40 & .16 & .0 & .140 & .020 & .006 & 1.28 & 60.8 & 3.7 & 3.9 \\
64CINE45 & .14 & .005 & .150 & .046 & 0 & 1.31 & 56.2 & 3.7 & 2.5 \\
648CINE50 & .11 & .07 & .080 & .096 & .002 & 1.26 & 52.1 & 2.7 & 1.6 \\
64CINES5 & .098 & .071 & .062 & .080 & .017 & 1.23 & 49.9 & 3.8 & 2.7 \\
\hline AVERAGE & .093 & .086 & .086 & .057 & .006 & 1.29 & 58.5 & & \\
\hline
\end{tabular}

Table 5.4-2 The results of the joint amplitude and phase least-squares inversion of individual profiles from EMNE well for data before injection (top) and after injection (bottom). The model consists of five $10 \mathrm{~m}$ thick layers. Amplitude calibration unit is $(\mathrm{A} / \mathrm{m}) / \mathrm{V}$ per unit moment. 


\begin{tabular}{|l|lllll|l|l|l|l|}
\hline & $\sigma_{1}$ & $\sigma 2$ & $\sigma 3$ & $\sigma_{4}$ & $\sigma 5$ & $\begin{array}{l}\text { Ampl. } \\
+10-4\end{array}$ & $\begin{array}{l}\text { Phase } \\
\text { (deg) }\end{array}$ & $\%$ & RMS \\
\hline 58C1SES & & & & & & & & & \\
58C1SE10 & .07 & .12 & .02 & .081 & .018 & 1.25 & 58.1 & 2.4 & 2 \\
58C1SE15 & .089 & .085 & .09 & .009 & .027 & 1.30 & 59.7 & 1.9 & 1.9 \\
58C1SE20 & .077 & .086 & .071 & .026 & .026 & 1.26 & 60.8 & 1.9 & 1.9 \\
58C1SE25 & .089 & .085 & .065 & .038 & .024 & 1.21 & 61.2 & 1.8 & 2.0 \\
58C1SE30 & .088 & .088 & .09 & .029 & .018 & 1.17 & 59.3 & 2.1 & 1.7 \\
58C1SE35 & .091 & .1 & .09 & .035 & .011 & 1.13 & 57.65 & 2 & 2.5 \\
58C1SE40 & .102 & .1 & .097 & .029 & .007 & 1.10 & 57.06 & 3.6 & 3.0 \\
58C1SE45 & .098 & .083 & .097 & .058 & .001 & 1.12 & 54.4 & 3.8 & 5.0 \\
58C1SE50 & .095 & .095 & .053 & .095 & .003 & 1.10 & 54.5 & 3.9 & 2.9 \\
511C1SES5 & .095 & .089 & .094 & .012 & .02 & 1.11 & 55.2 & 1.8 & 1.2 \\
\hline AVERAGE & .089 & .093 & .077 & .041 & .016 & 1.17 & 57.8 & & \\
\hline
\end{tabular}

\begin{tabular}{|l|lllll|l|l|l|l|}
\hline & $\sigma_{1}$ & $\sigma_{2}$ & $\sigma_{3}$ & $\sigma_{4}$ & $\sigma_{5}$ & $\begin{array}{l}\text { Ampl. } \\
+10-4\end{array}$ & $\begin{array}{l}\text { Phase } \\
\text { (deg) }\end{array}$ & $\%$ & RMS \\
\hline 64 C1SES & .028 & .085 & .1 & .085 & .016 & 1.15 & 69.2 & 2.6 & 2.3 \\
64 C1SE15 & .081 & .094 & .079 & .047 & .024 & 1.26 & 55.5 & 1 & .5 \\
$645 C 1 S E 15$ & .075 & .11 & .079 & .032 & .023 & 1.26 & 55.5 & 1.4 & 1.3 \\
64 C1SE20 & .059 & .1 & .074 & .031 & .023 & 1.24 & 55.3 & 1.5 & 1.6 \\
64C1SE25 & & & & & & & & & \\
65C1SE30 & & & & & & & & & \\
65C1SE35 & .093 & .092 & .1 & .037 & .009 & 1.15 & 52.8 & 2.6 & 2.3 \\
65C1SE40 & .1 & .1 & .12 & .021 & .006 & 1.11 & 53.4 & 3.4 & 3.4 \\
65C1SE45 & .11 & .06 & .1 & .07 & .004 & 1.13 & 48.8 & 5.3 & 5.8 \\
658C1SE50 & .23 & .083 & .052 & .094 & .026 & 1.11 & 50.5 & 3.2 & 5.1 \\
65C1SES5 & .098 & .13 & .051 & .072 & .078 & 1.07 & 51.6 & 3.2 & 3 \\
\hline AVERAGE & .097 & .095 & .084 & .054 & .023 & 1.12 & 54.7 & & \\
\hline
\end{tabular}

Table 5.4-3 The results of the joint amplitude and phase least-squares inversion of individual profiles from EMSE well for data before injection (top) and after injection (bottom). The model consists of five $10 \mathrm{~m}$ thick layers. Amplitude calibration unit is ( $/ / \mathrm{m}) / \mathrm{V}$ per unit moment. 


\begin{tabular}{|l|lllll|l|l|l|l|}
\hline & $\sigma_{1}$ & $\sigma 2$ & $\sigma 3$ & $\sigma 4$ & $\sigma 5$ & $\begin{array}{l}\text { Ampl. } \\
* 10-4\end{array}$ & $\begin{array}{l}\text { Phase } \\
(\mathrm{deg})\end{array}$ & $\%$ & RMS \\
\hline s11C1SW5 & .1 & .09 & .086 & .00 & .0038 & 1.32 & 55.9 & 3.7 & 2.4 \\
s11C1SW10 & .1 & .078 & .11 & .007 & .011 & 1.25 & 56.2 & 3.0 & 2.0 \\
s11C1SW15 & .11 & .075 & .073 & .056 & .012 & 1.28 & 61.4 & 1.9 & 1.1 \\
511C1SW20 & .003 & .075 & .073 & .056 & .012 & 1.28 & 61.4 & 1.9 & 1.1 \\
s11C1SW25 & .085 & .079 & .073 & .067 & .012 & 1.27 & 61 & 1.7 & 1.2 \\
511C1SW30 & .86 & .078 & .071 & .057 & .015 & 1.26 & 66.4 & 1.1 & 1.5 \\
512C1SW35 & .087 & .081 & .088 & .067 & .017 & 1.26 & 57.5 & 1.2 & 1.2 \\
512C1SW40 & .082 & .084 & .083 & .078 & .017 & 1.26 & 54 & 1.8 & .97 \\
512C1SW45 & .074 & .09 & .078 & .068 & .017 & 1.25 & 56.5 & 2.6 & 1.7 \\
512C1SW50 & .086 & .09 & .11 & .041 & .008 & 1.16 & 61.9 & 3.2 & 2.5 \\
512C2SW55 & .1 & .08 & .14 & .052 & .000 & 1.08 & 61.9 & 2.9 & 2.5 \\
\hline AVERAGE & .1 & .08 & .09 & .057 & .013 & 1.25 & 59.9 & & \\
\hline
\end{tabular}

\begin{tabular}{|l|lllll|l|l|l|l|}
\hline & $\sigma_{1}$ & $\sigma_{2}$ & $\sigma_{3}$ & $\sigma_{4}$ & $\sigma_{5}$ & $\begin{array}{l}\text { Ampl. } \\
* 10-4\end{array}$ & $\begin{array}{l}\text { Phase } \\
\text { (deg) }\end{array}$ & $\%$ & RMS \\
\hline 65C1NW5 & .124 & .09 & .116 & .00 & .0037 & 1.26 & 42.7 & 1.5 & 1.2 \\
65C1NW10 & .119 & .102 & .11 & .024 & .0081 & 1.17 & 41.6 & 1.9 & .89 \\
65C1NW15 & .127 & .102 & .133 & .022 & .006 & 1.22 & 40.2 & 2.3 & 1.9 \\
68C1NW20 & .098 & .087 & .098 & .059 & .007 & 1.21 & 47.4 & 2.1 & 1.5 \\
68C1NW25 & .085 & .084 & .086 & .076 & .0076 & 1.32 & 51.4 & 1.7 & 1.5 \\
68C1NW30 & .087 & .08 & .084 & .07 & .012 & 1.32 & 56.1 & 1.5 & 1.2 \\
68C1NW35 & .088 & .082 & .087 & .061 & .013 & 1.29 & 58.0 & 1.8 & 1.1 \\
68C1NW40 & .079 & .083 & .088 & .074 & .015 & 1.29 & 52.3 & 1.3 & .78 \\
68C1NW45 & .069 & .091 & .083 & .069 & .017 & 1.29 & 52.1 & 1.9 & 1.2 \\
68C1NW50 & & & & & & & & & \\
68C1NW55 & .095 & .085 & .1 & .070 & .003 & 1.23 & 56.4 & 1.1 & 1.9 \\
\hline AVERAGE & .088 & .09 & .084 & .056 & .01 & 1.26 & 49.7 & & \\
\hline
\end{tabular}

Table 5.4-4 The results of the joint amplitude and phase least-squares inversion of individual profiles from EMSW well for data before injection (top) and after injection (bottom). The model consists of five $10 \mathrm{~m}$ thick layers. Amplitude calibration unit is $(\mathrm{A} / \mathrm{m}) / \mathrm{V}$ per unit moment. 


\begin{tabular}{|c|c|c|c|c|}
\hline WELL & DATE & $\begin{array}{l}\text { Receiver depths } \\
\text { for the best } \\
\text { fiting profiles }\end{array}$ & $\begin{array}{l}\text { Amplitude } \\
\text { calibration: } \\
\text { V }>\mathrm{Am}\end{array}$ & $\begin{array}{l}\text { Phase } \\
\text { calibration } \\
\text { (deg) }\end{array}$ \\
\hline \multirow[t]{4}{*}{$\overline{N W}$} & \multirow[t]{2}{*}{ MAY } & $\begin{array}{l}30 \\
25 \\
20 \\
15 \\
10\end{array}$ & $\begin{array}{l}1.10^{*} 10^{-4} \\
1.16^{*} 10^{-4} \\
1.19 * 10^{-4} \\
1.18 * 10^{-4} \\
1.22 * 10^{-4}\end{array}$ & $\begin{array}{l}56.4 \\
56.5 \\
56.7 \\
56.9 \\
53.2\end{array}$ \\
\hline & & AVERAGE & $1.17 * 10^{4}$ & 55.94 \\
\hline & \multirow[t]{2}{*}{ JUNE } & $\begin{array}{l}25 \\
20 \\
15 \\
10\end{array}$ & $\begin{array}{l}1.25 * 10^{4} \\
1.29 * 10^{-4} \\
1.31 * 10^{-4} \\
1.27 * 10^{-4}\end{array}$ & $\begin{array}{l}57.5 \\
55.4 \\
58.9 \\
54.8\end{array}$ \\
\hline & & AVERAGE & $1.29 * 10^{4}$ & 56.6 \\
\hline \multirow[t]{4}{*}{ NE } & \multirow{4}{*}{$\begin{array}{l}\text { MAY } \\
\text { JUNE }\end{array}$} & 10 & 1.18 & 53.7 \\
\hline & & AVERAGE & $1.17 * 10^{-4}$ & 53.7 \\
\hline & & $\begin{array}{l}25 \\
20 \\
15 \\
10\end{array}$ & $\begin{array}{l}1.33^{* 1} 10^{-4} \\
1.30^{*} 10^{-4} \\
1.25 * 10^{-4} \\
1.28 * 10^{-4}\end{array}$ & $\begin{array}{l}57.7 \\
50.3 \\
43.6 \\
57.6\end{array}$ \\
\hline & & AVERAGE & $1.31 * 10^{4}$ & 52.3 \\
\hline \multirow[t]{4}{*}{ SE } & \multirow[t]{2}{*}{ MAY } & $\begin{array}{l}35 \\
30 \\
25 \\
20 \\
15\end{array}$ & $\begin{array}{l}1.14^{*} 10^{-4} \\
1.17^{*} 10^{-4} \\
1.21^{*} 10^{-4} \\
1.26^{*} 10^{-4} \\
1.30^{*} 10^{-4}\end{array}$ & $\begin{array}{l}57.7 \\
59.3 \\
61.2 \\
60.8 \\
59.7\end{array}$ \\
\hline & & AVERAGE & $1.22 * 10^{-4}$ & 59.7 \\
\hline & \multirow[t]{2}{*}{ JUNE } & $\begin{array}{l}20 \\
15 \\
10\end{array}$ & $\begin{array}{l}1.24^{*} 10^{-4} \\
1.26^{*} 10^{-4} \\
1.26^{*} 10^{-4}\end{array}$ & $\begin{array}{l}55.3 \\
51.2 \\
55.5\end{array}$ \\
\hline & & AVERAGE & $1.27 * 10^{-4}$ & 54.0 \\
\hline \multirow[t]{4}{*}{ SW } & \multirow[t]{2}{*}{ MAY } & $\begin{array}{l}40 \\
35 \\
30 \\
25 \\
20\end{array}$ & $\begin{array}{l}1.26^{*} 10^{-4} \\
1.26^{*} 10^{-4} \\
1.26^{*} 10^{-4} \\
1.27^{*} 10^{-4} \\
1.29 * 10^{-4}\end{array}$ & $\begin{array}{l}54.0 \\
57.5 \\
66.4 \\
61.0 \\
61.4\end{array}$ \\
\hline & & AVERAGE & $1.27 * 10^{4}$ & 60.6 \\
\hline & \multirow[t]{2}{*}{ JUNE } & $\begin{array}{l}45 \\
40 \\
35 \\
30 \\
25 \\
20\end{array}$ & $\begin{array}{l}1.29 * 10^{-4} \\
1.29 * 10^{-4} \\
1.30^{*} 10^{-4} \\
1.35^{*} 10^{-4} \\
1.34^{*} 10^{-4} \\
1.23^{*} 10^{-4}\end{array}$ & $\begin{array}{l}52.3 \\
52.4 \\
58.0 \\
56.1 \\
51.4 \\
47.4\end{array}$ \\
\hline & & AVERAGE & $1.31 * 10^{-4}$ & 53.98 \\
\hline
\end{tabular}

Table 5.4-5 The amplitude and phase calibrations obtained by averaging the calibrations from the best fitting least-squares inversions of individual profiles and assuming a unit dipole moment. 


\begin{tabular}{|c|c|l|l|l|l|l|l|}
\hline Well & Date & $\begin{array}{l}\text { Amplitude } \\
\text { calibration } \\
\text { for joint } \\
\text { inversion } \\
\text { of all } \\
\text { profiles }\end{array}$ & $\begin{array}{l}\text { Phase } \\
\text { calibrationf } \\
\text { for joint } \\
\text { inversion } \\
\text { (deg) }\end{array}$ & $\begin{array}{l}\text { Amplitude } \\
\text { calibration } \\
\text { V->A/m } \\
\text { from } \\
\text { individual } \\
\text { inversions }\end{array}$ & $\begin{array}{l}\text { Phase } \\
\text { calibration } \\
\text { (deg) }\end{array}$ & $\begin{array}{l}\text { Amplitude } \\
\text { calibration } \\
\text { from D. } \\
\text { Alumbaug } \\
\text { h }\end{array}$ & $\begin{array}{l}\text { Phase } \\
\text { calibration } \\
\text { (deg) }\end{array}$ \\
\hline NW & May & $1.19 * 10-4$ & 51.4 & $1.17 * 10-4$ & 55.9 & $1.23 * 10-4$ & 60 \\
\hline & June & $1.27 * 10-4$ & 50.0 & $1.29 * 10-4$ & 56.6 & $1.30 * 10-4$ & 58 \\
\hline NE & May & $1.23 * 10-4$ & 54.4 & $1.17 * 10-4$ & 53.7 & $1.23 * 10-4$ & 59 \\
\hline & June & $1.31 * 10-4$ & 54.7 & $1.31 * 10-4$ & 55.2 & $1.30 * 10-4$ & 58 \\
\hline SE & May & $1.18 * 10-4$ & 53.9 & $1.22 * 10-4$ & 59.7 & $1.25 * 10-4$ & 60 \\
\hline & June & $1.20 * 10-4$ & 49.4 & $1.25 * 10-4$ & 54.0 & $1.30 * 10-4$ & 58 \\
\hline SW & May & $1.24 * 10-4$ & 61.9 & $1.27 * 10-4$ & 60.6 & $1.27 * 10-4$ & 63 \\
\hline & June & $1.31 * 10-4$ & 58.6 & $1.31 * 10-4$ & 54.0 & $1.30 * 10-4$ & 58 \\
\hline
\end{tabular}

Table 5.4-6 The comparison of amplitude and phase calibrations obtained by inversions with the results obtained by D. Alumbaugh (1993). Amplitude calibration unit is $(\mathrm{A} / \mathrm{m}) / \mathrm{V}$ per unit moment. 


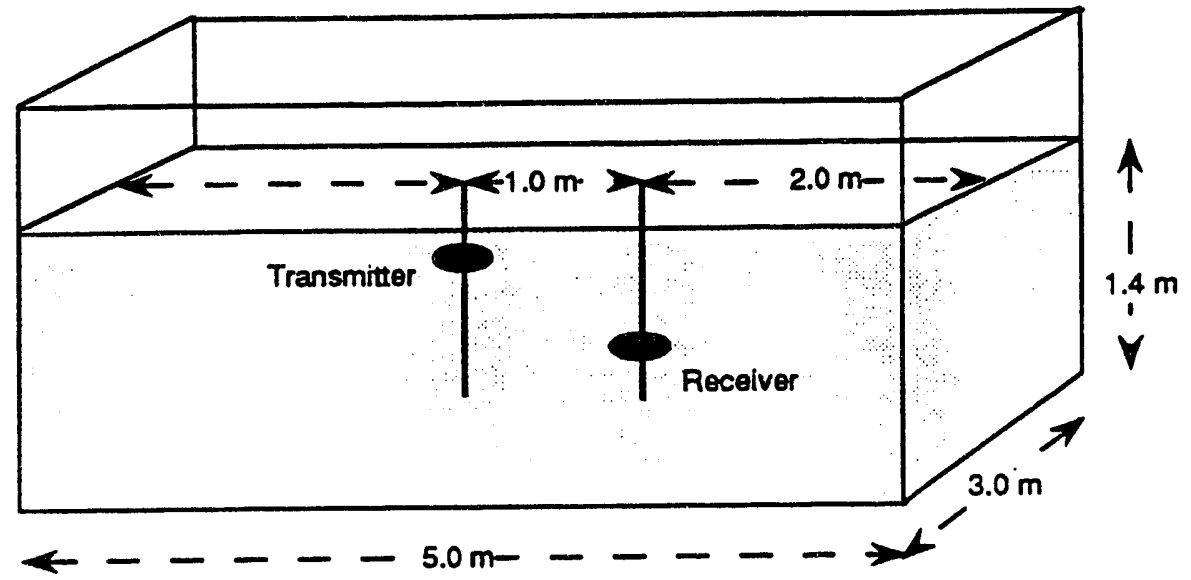

Operating frequency:98.6 kHz

Fig. 5.2-1 The geometry of the model tank experiment (after D. Alumbaugh, 1990). 

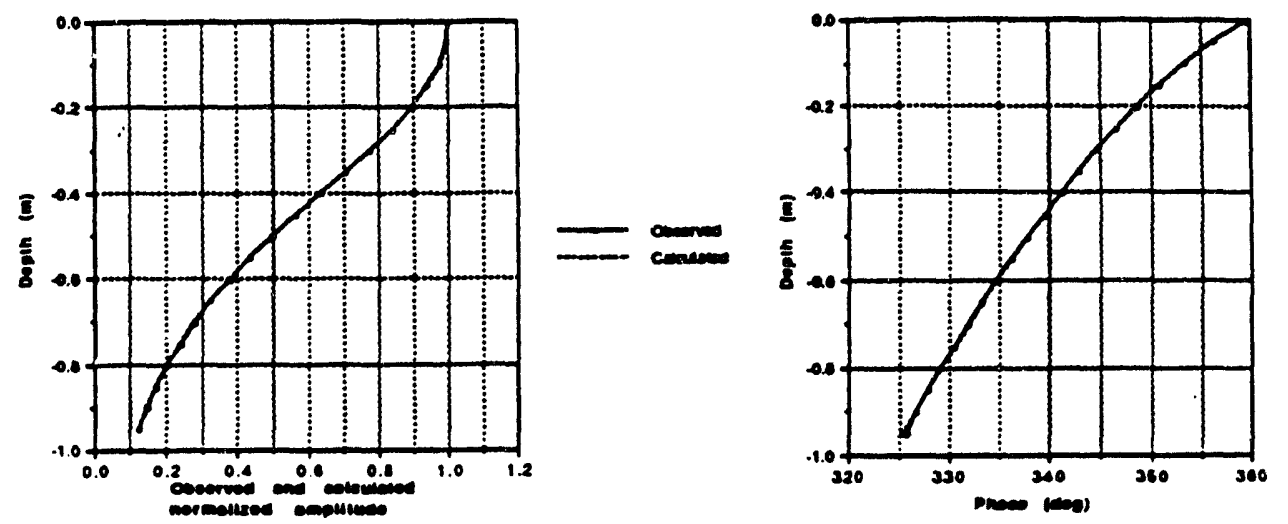

a)
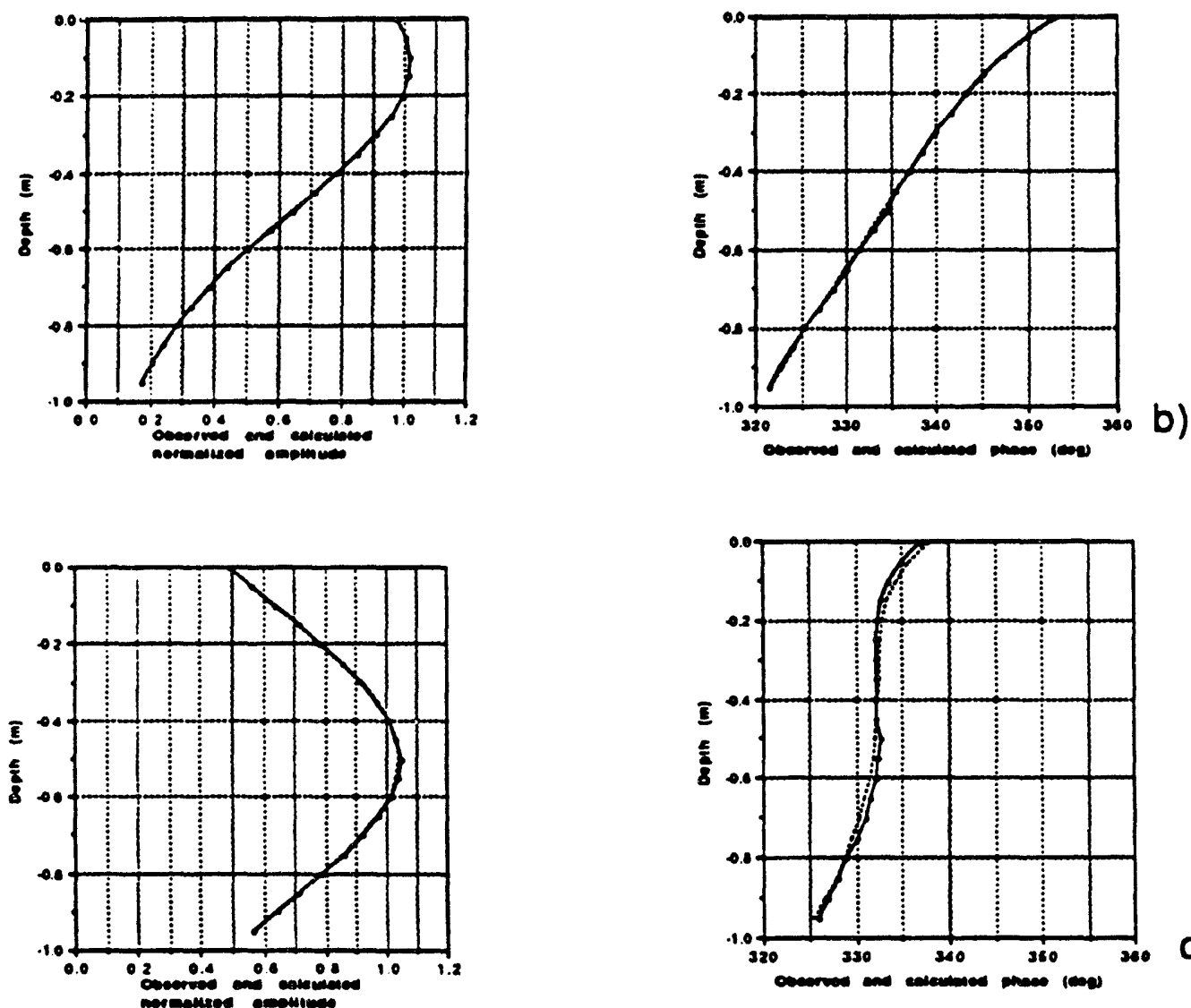

c)

Fig. 5.2-2 The examples of the measured normalized amplitude and phase profiles for the model tank experiment. The stationary receiver located: (a) on a surface; (b) at a depth of $0.1 \mathrm{~m}$, and (c) at a depth of $0.5 \mathrm{~m}$. The calculated profiles for a half-space model overlap the experimental profiles with the exception of phase in (c). 

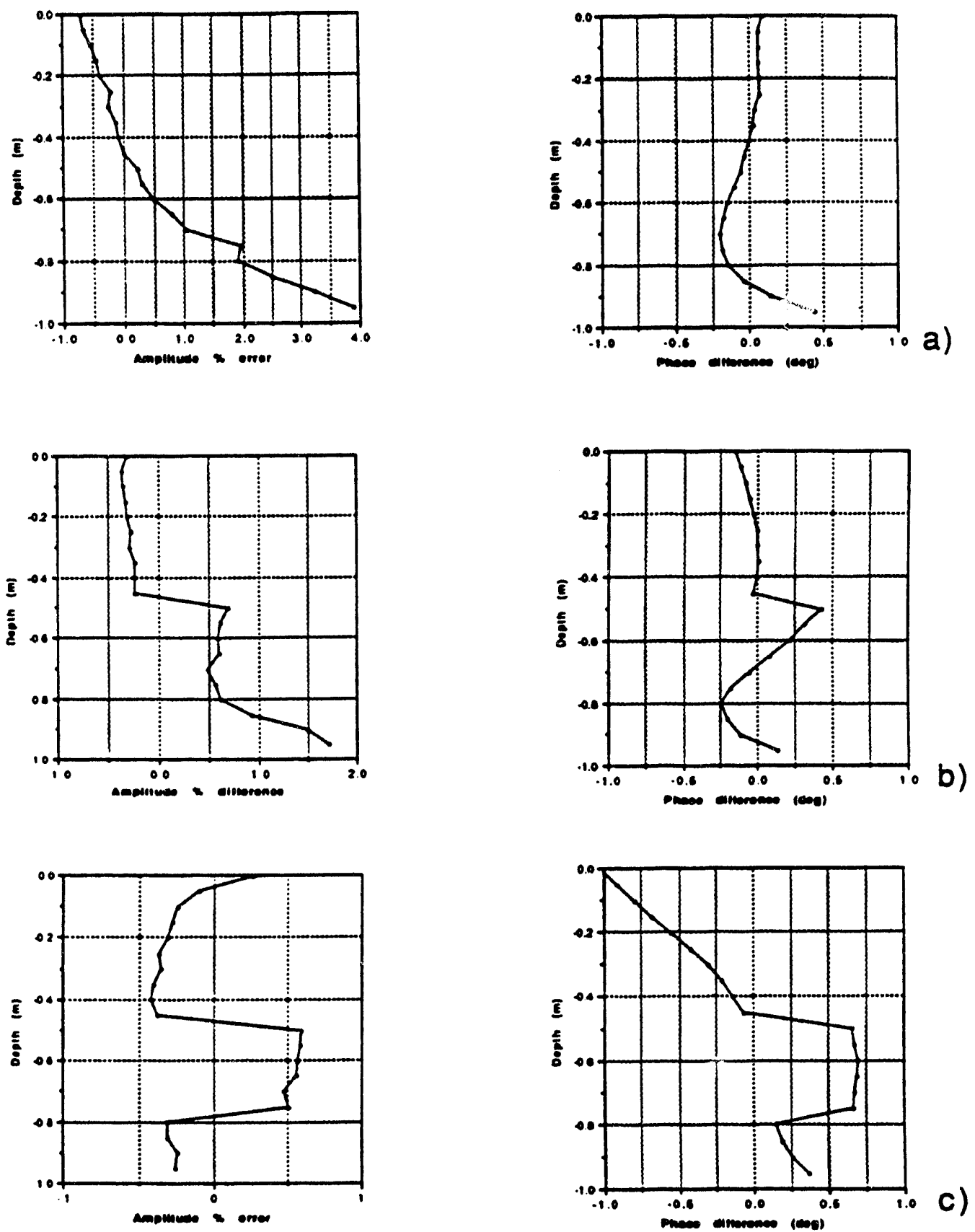

Fig. 5.2-3 The percent difference in amplitude and phase shift between observed and calculated data for a half-space model. The receiver is located: (a) on a surface; (b) at depth of $0.1 \mathrm{~m}$, and (c) at depth $0.5 \mathrm{~m}$ below the surface. The plots illustrate different pattern of errors: one associated with a sudden jump in transmitter location that is superimposed on the general trend of unknown origin. 

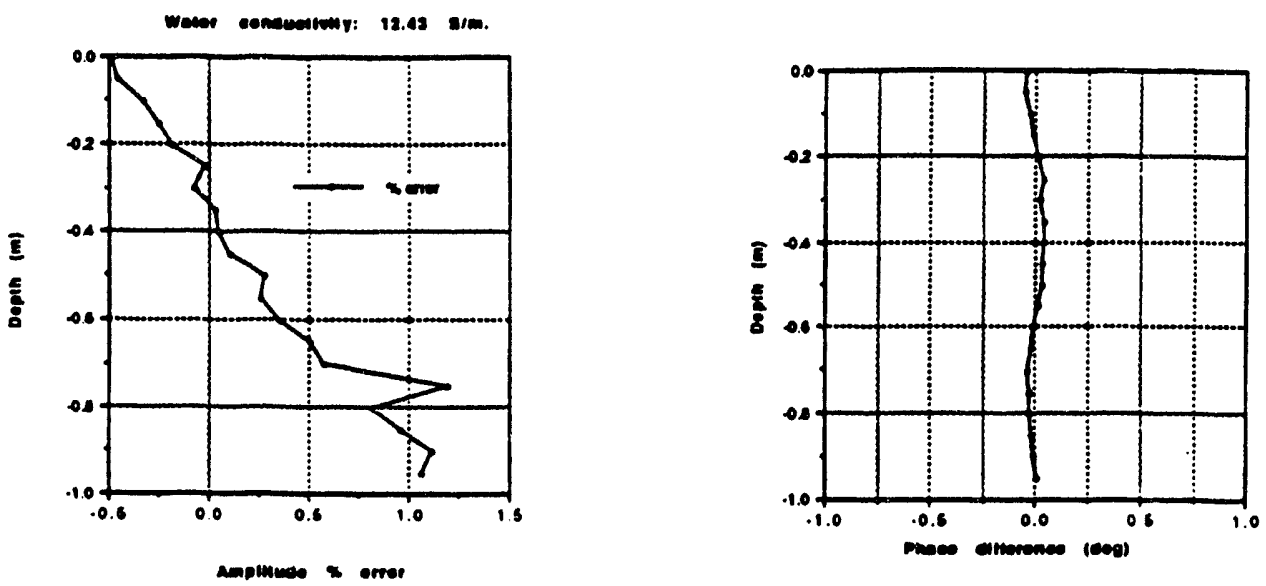

a)
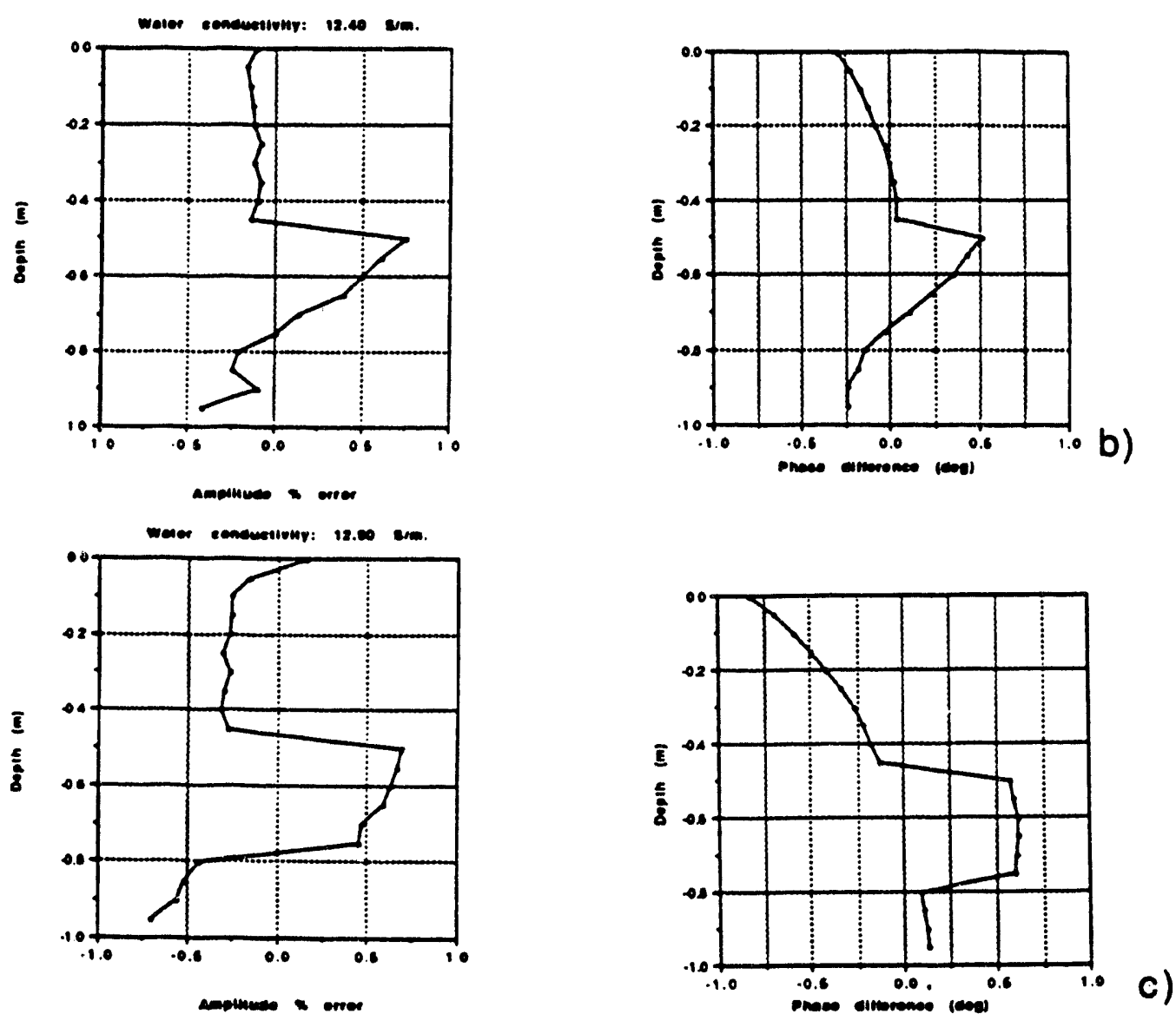

Fig. 5.2-4 The percent difference in amplitude and phase shift between observed and calculated data for a two-layer model The receiver is located: (a) on a surface; (b) at depth $0.1 \mathrm{~m}$, and (c) at depth $0.5 \mathrm{~m}$ below the surface. The plots illustrate how the general trend in error decreased [in comparison with Figure (5.2.-3)] when the model changed from a half-space to the finite conductive layer. 

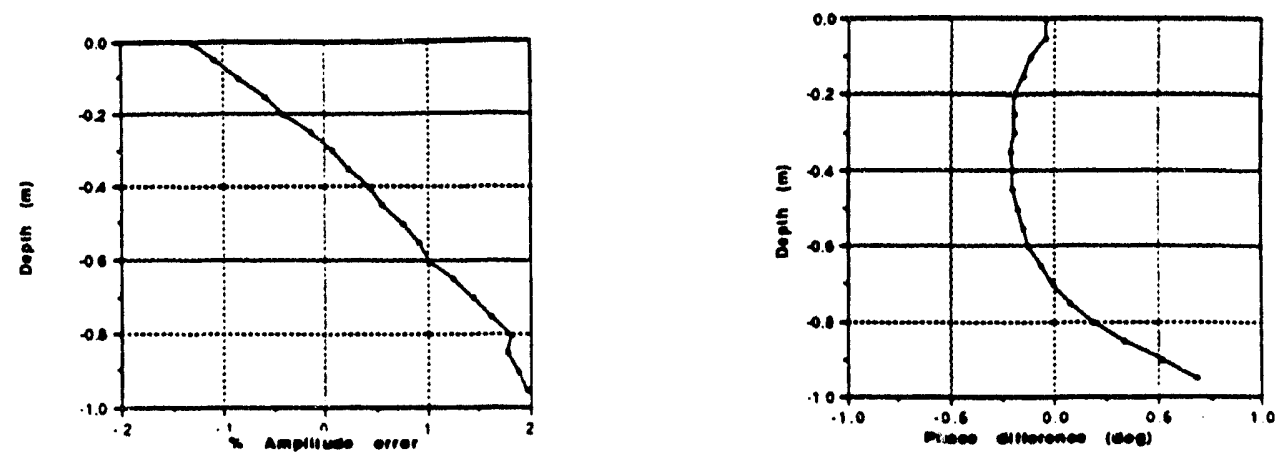

a)
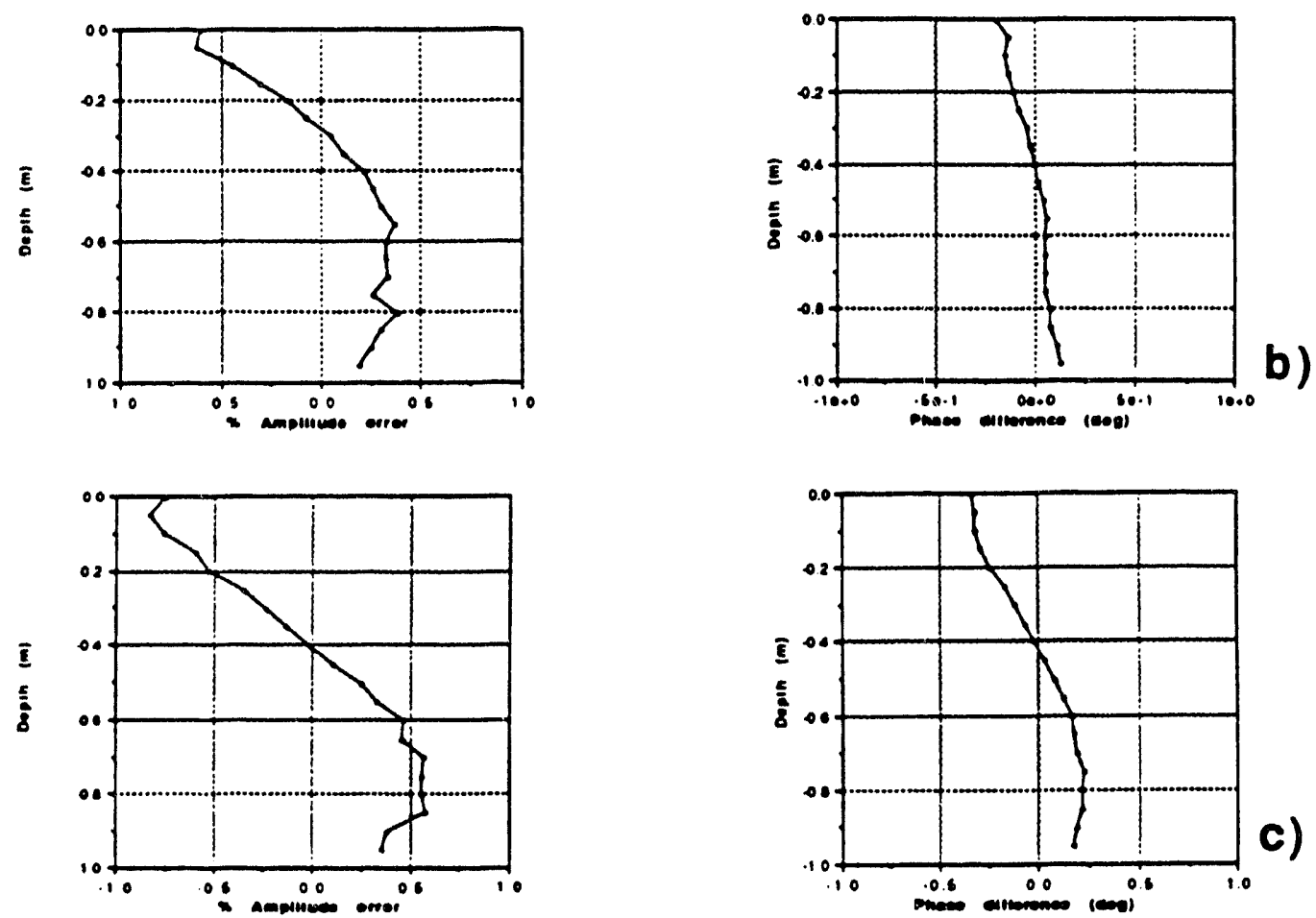

C)
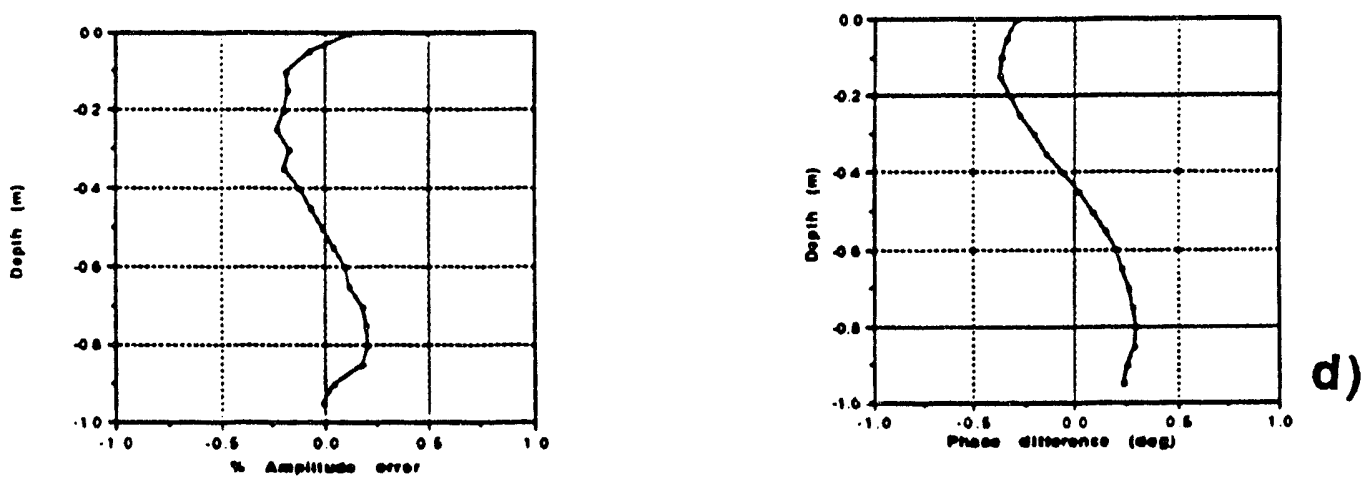

Fig. 5.2-5 The percent difference in amplitude and phase shift between observed and calculated data. The model is a layer of conductivity $12.74 \mathrm{~S} / \mathrm{m}$ embedded in a nonconductive whole-space. The receiver is located: (a) on a surface; (b) at depth $0.2 \mathrm{~m}$; (c) at depth $0.4 \mathrm{~m}$, and (d) $0.6 \mathrm{~m}$ below the surface. 

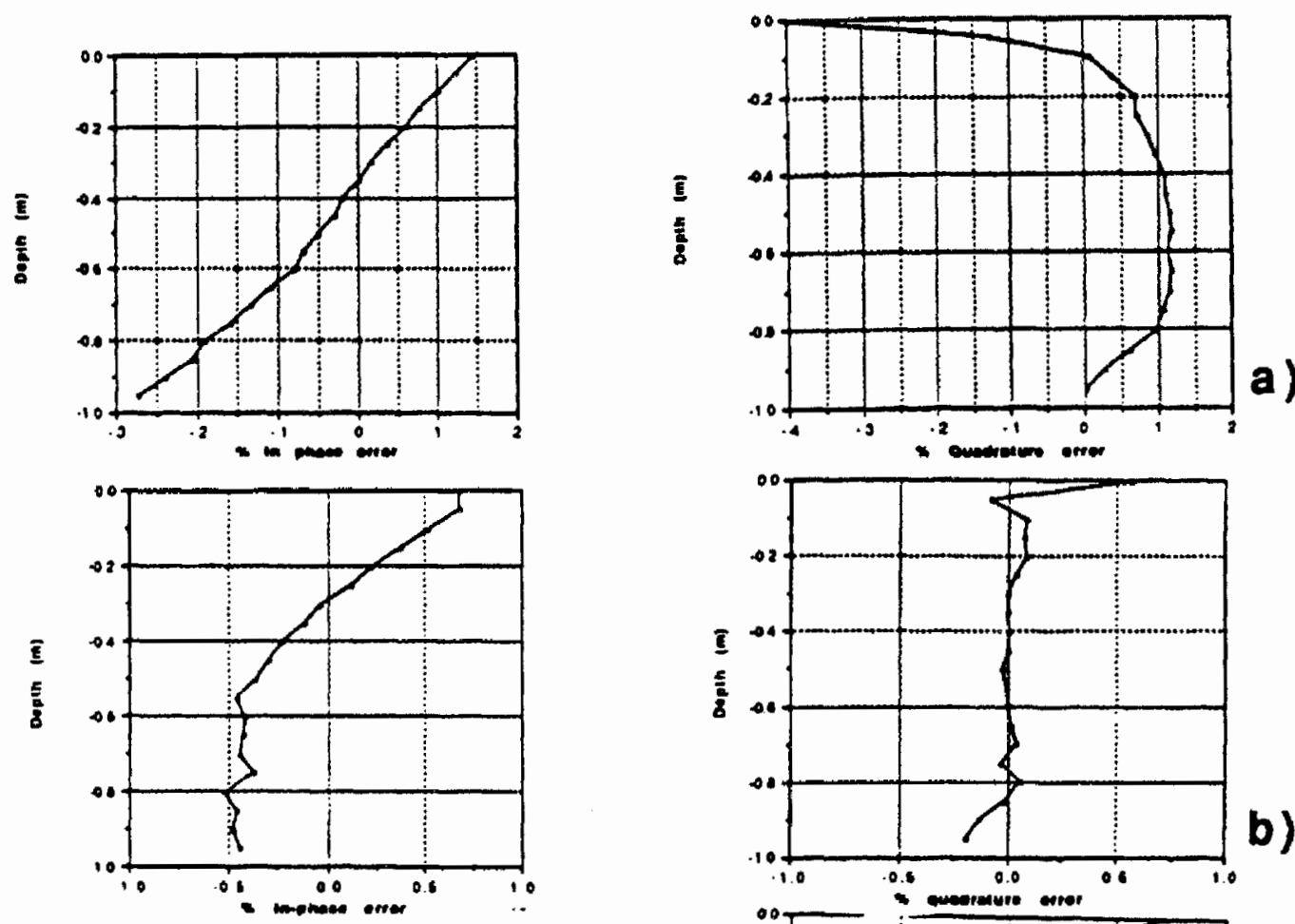

b)
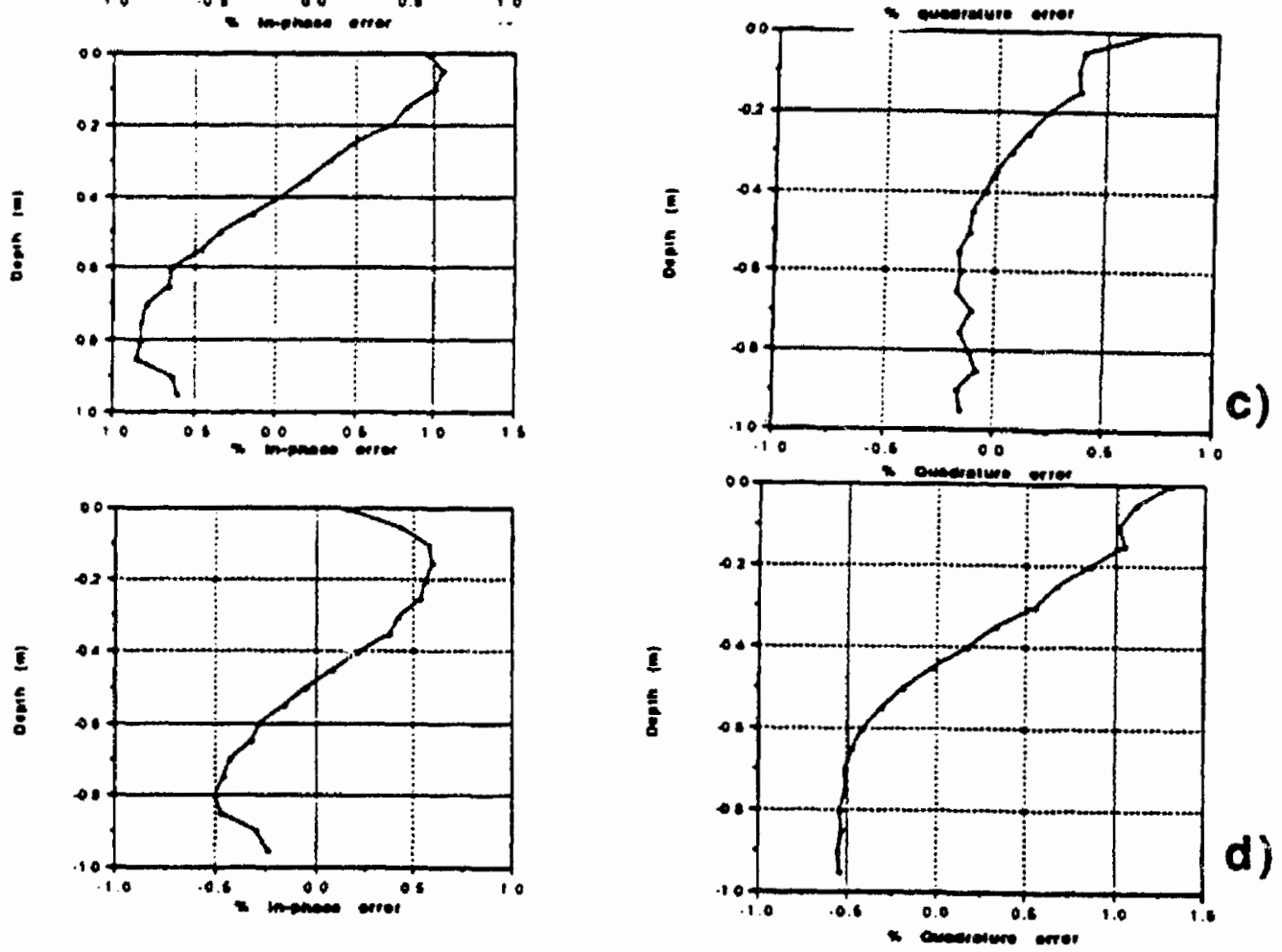

Fig. 5.2-6 The percent difference for in-phase and quadrature between observed and calculated data. The model is a layer of conductivity $12.74 \mathrm{~S} / \mathrm{m}$ embedded in a nonconductive whole-space. The receiver is located: (a) on a surface; (b) at depth $0.2 \mathrm{~m}$; (c) at depth $0.4 \mathrm{~m}$, and (d) $0.6 \mathrm{~m}$ below the surface. 

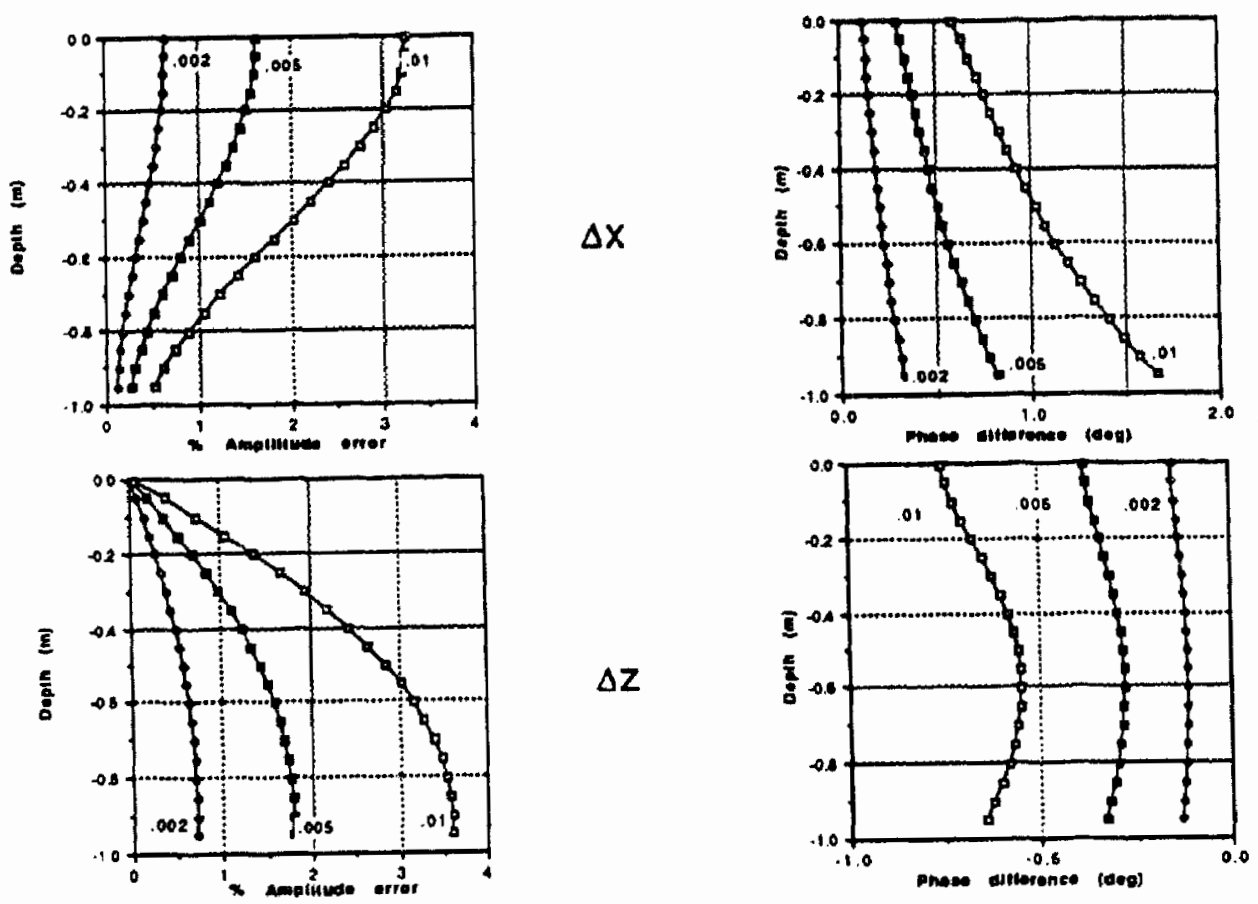

a)

$\Delta \mathbf{Z}$
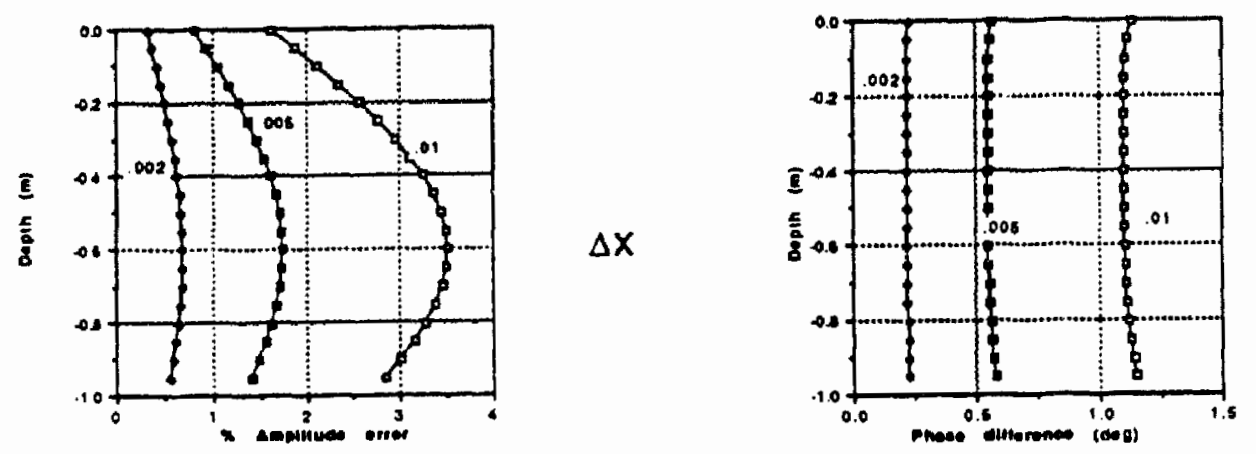

d)
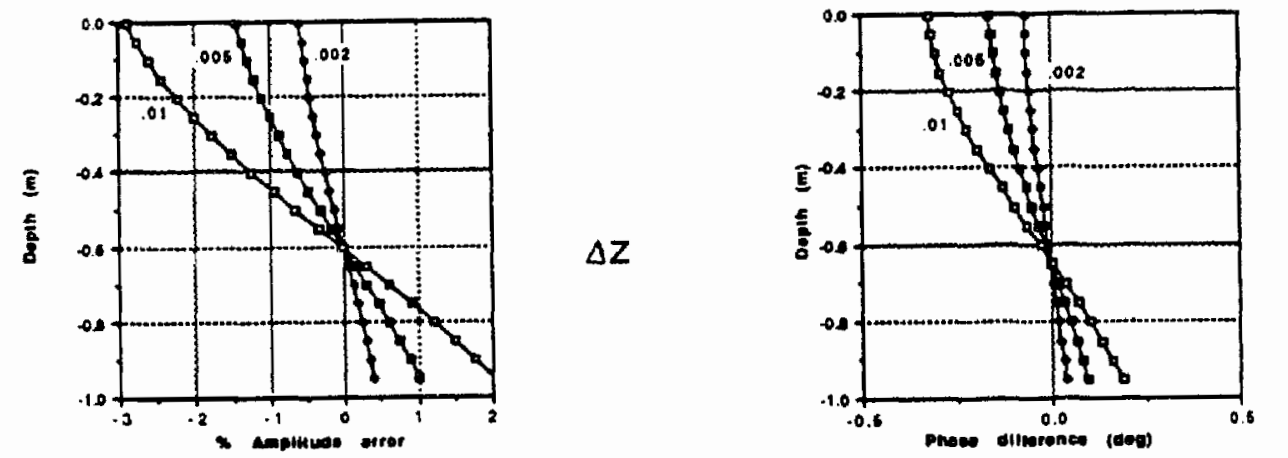

Fig. 5.2-7 The errors in amplitude and phase data when the estimated vertical: $\Delta z$ or horizontal: $\Delta x$ distances are not exact for receiver located at a surface (a); and at depth $0.6 \mathrm{~m}$ (d); below the surface. The model is a layer of conductivity $12.74 \mathrm{~S} / \mathrm{m}$ embedded in a nonconductive whole-space. 


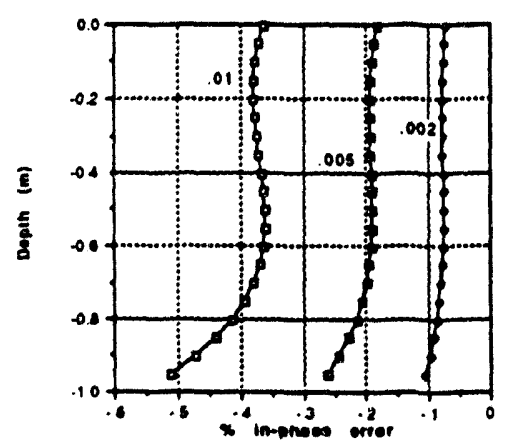

$\Delta x$
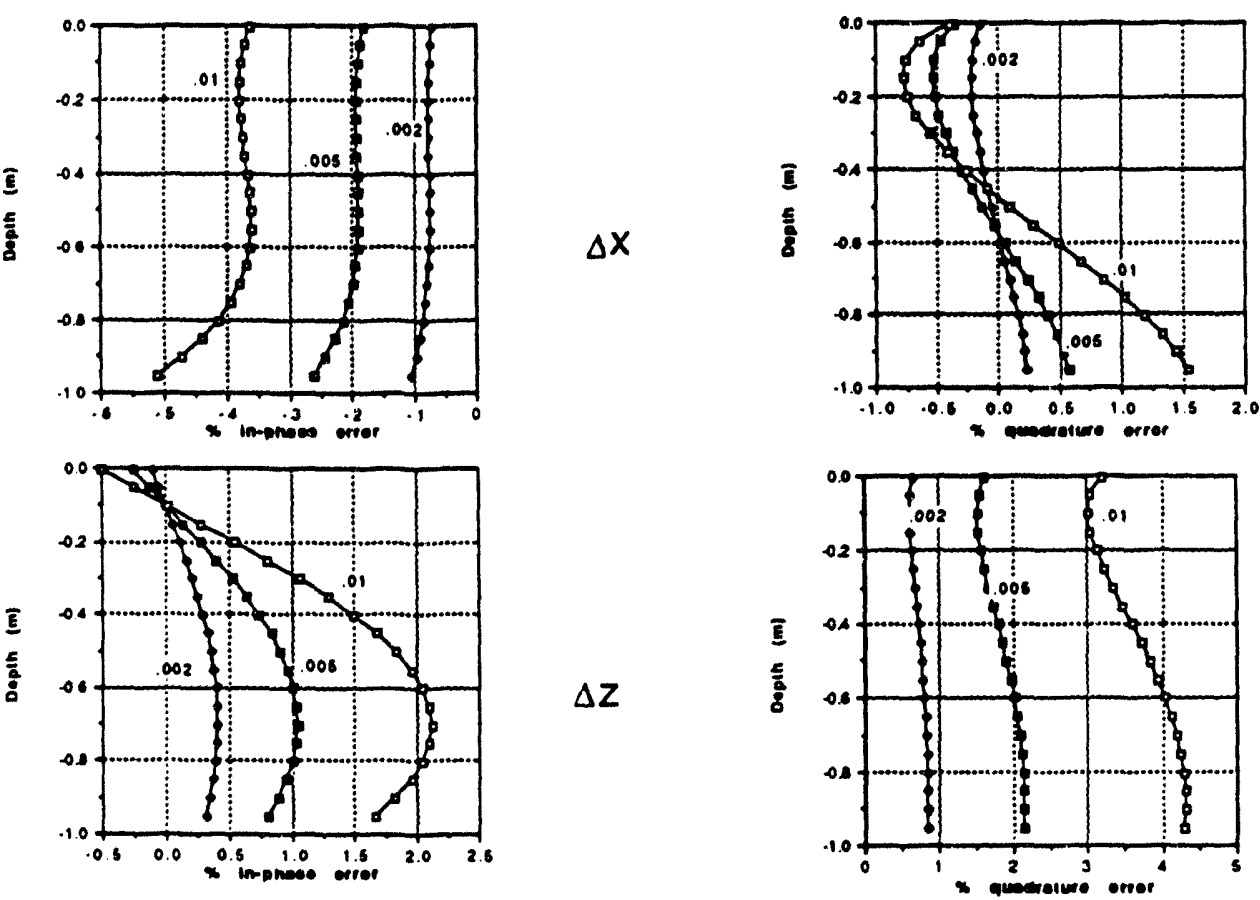

$\Delta \mathbf{Z}$

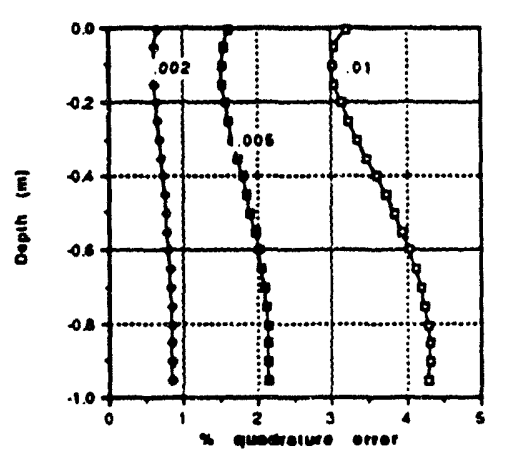

a)
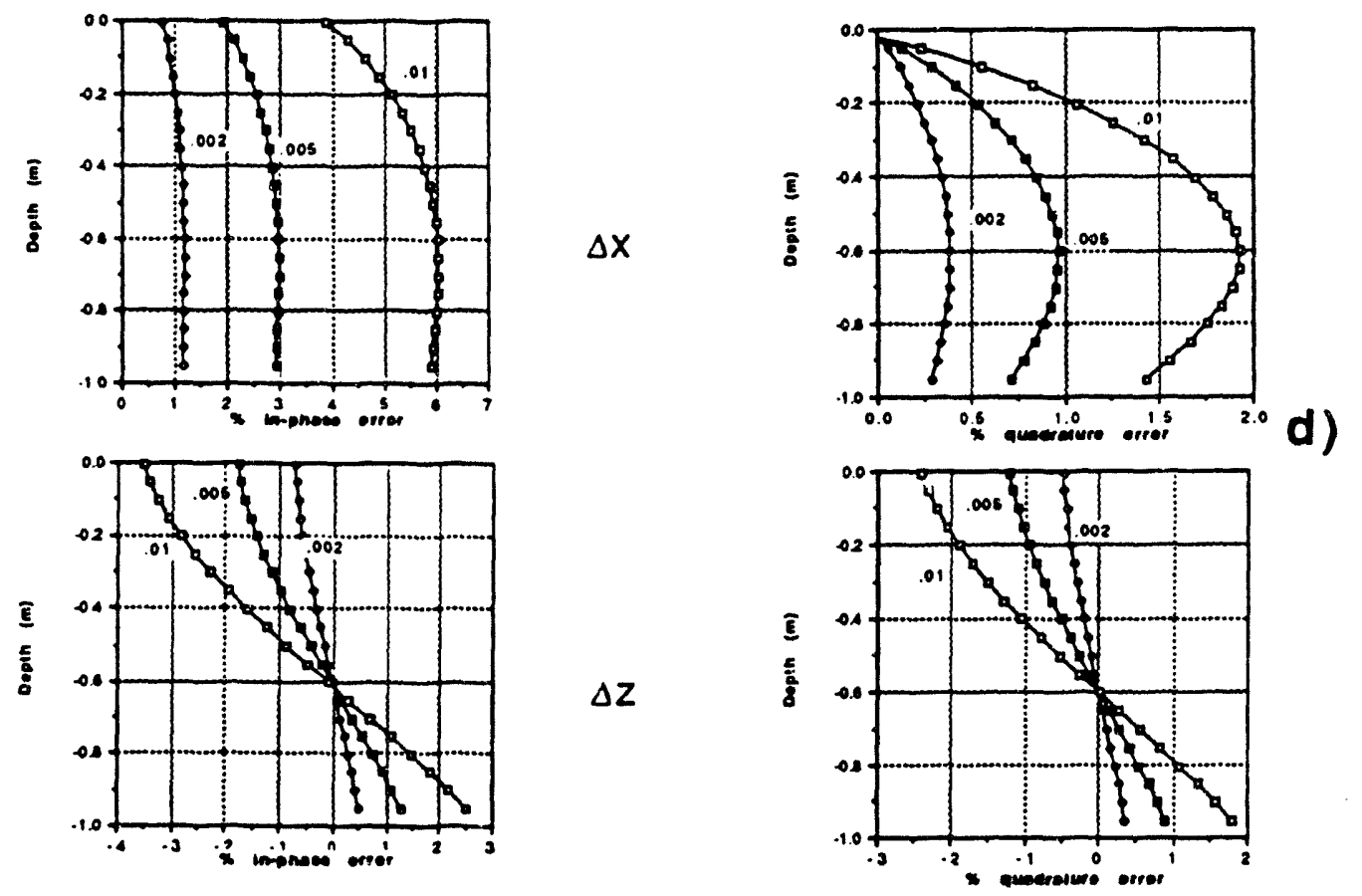

Fig. 5.2-8 The errors for in-phase and quadrature data when the estimated vertical: $\Delta z$ or horizontal: $\Delta x$ distances are not exact for receiver located at a surface (a); and at depth $0.6 \mathrm{~m}$ (d); below the surface. The model is a layer of conductivity $12.74 \mathrm{~S} / \mathrm{m}$ embedded in a nonconductive whole-space. 

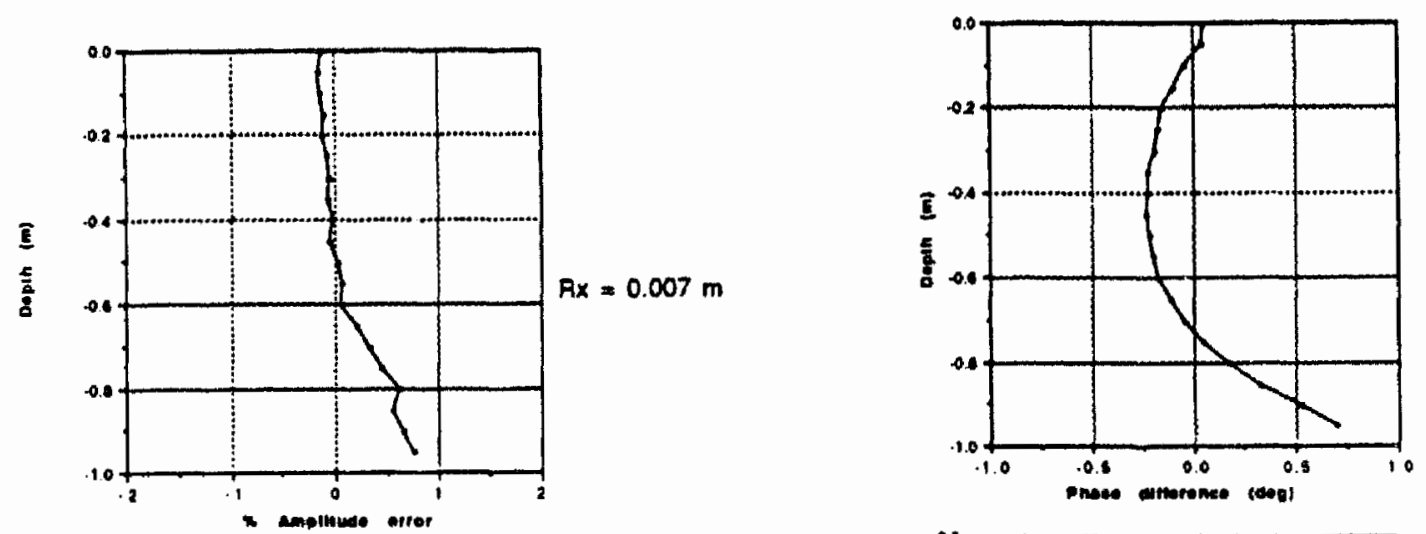

a)
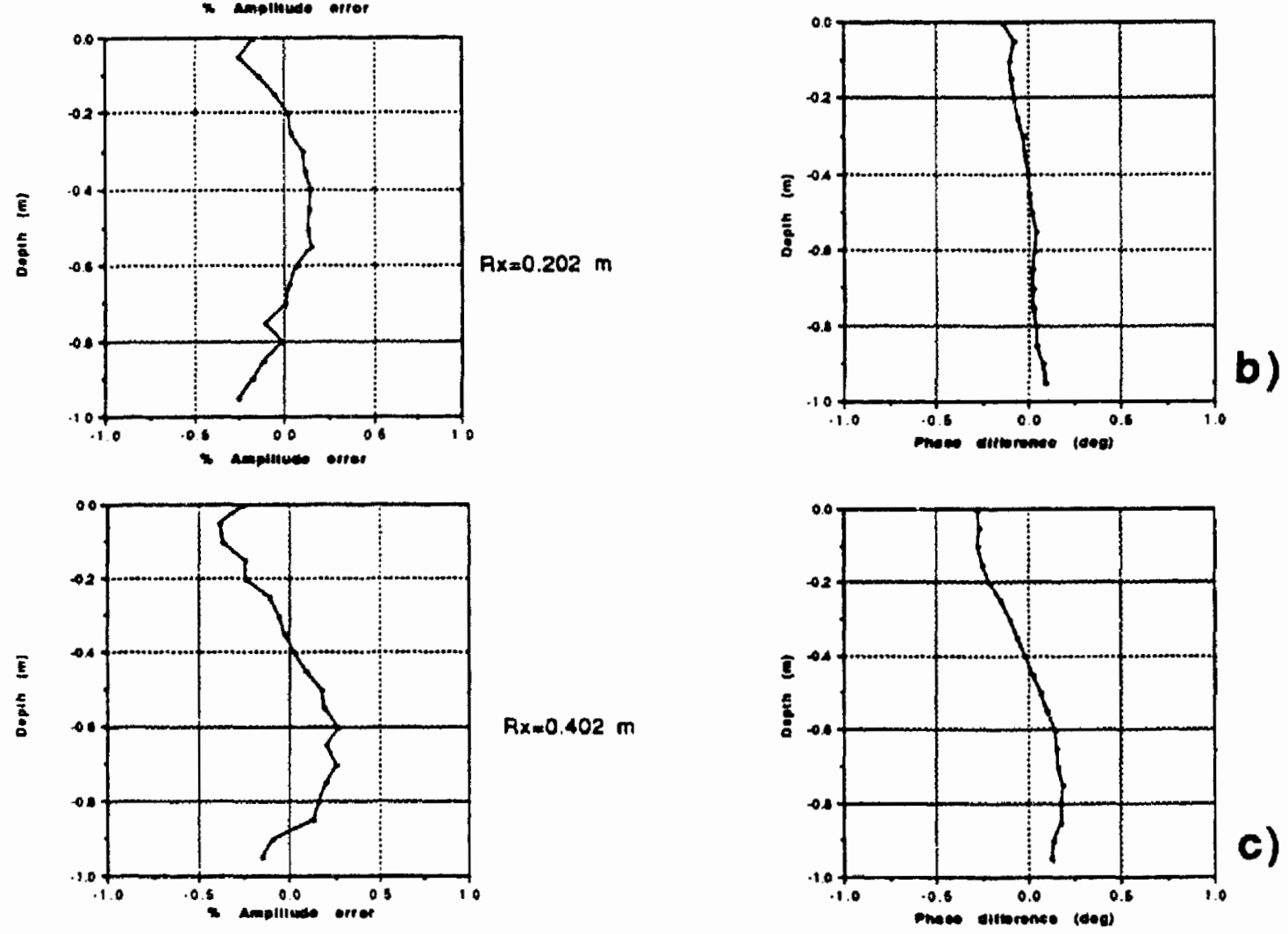

Fig. 5.2-9 The change in error pattems between observed and calculated amplitude and phase data when the estimated vertical distances are changed: (a) for receiver located at a surface by $0.007 \mathrm{~m}$; (b) for receiver located at depth of 0.2 by $0.002 \mathrm{~m}$; and (c) for receiver located at depth $0.4 \mathrm{~m}$ also by $0.002 \mathrm{~m}$. The model is a layer of conductivity 12.74 S/m embedded in a nonconductive whole-space. 

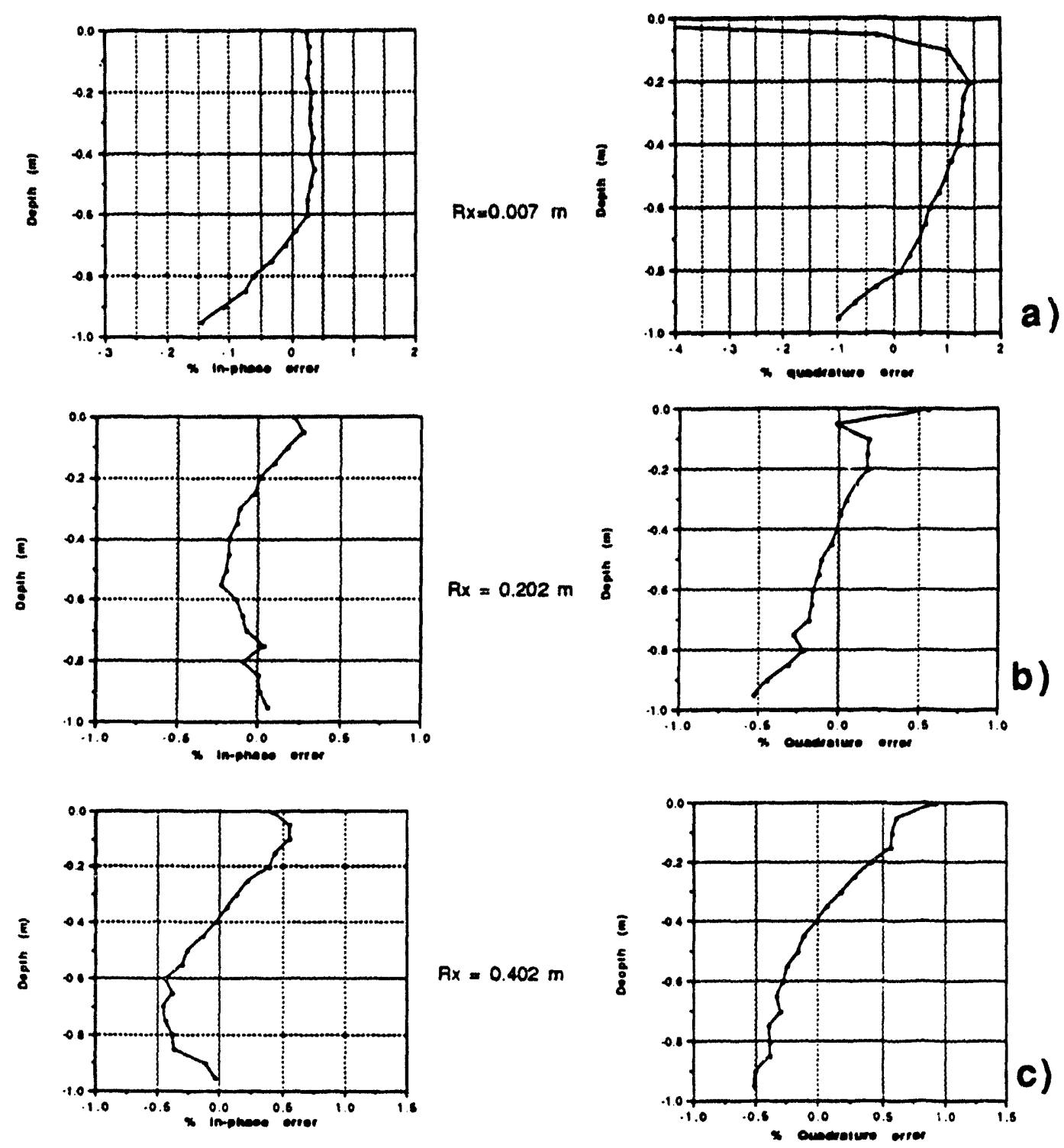

Fig. 5.2-10 The change in error patterns between observed and calculated in-phase and quadrature data when the estimated vertical distances are changed: (a) for receiver located at a surface by $0.007 \mathrm{~m}$; (b) for receiver located at depth of 0.2 by $0.002 \mathrm{~m}$; and (c) for receiver located at depth $0.4 \mathrm{~m}$ also by $0.002 \mathrm{~m}$. The model is a layer of conductivity $12.74 \mathrm{~S} / \mathrm{m}$ embedded in a nonconductive whole-space. 


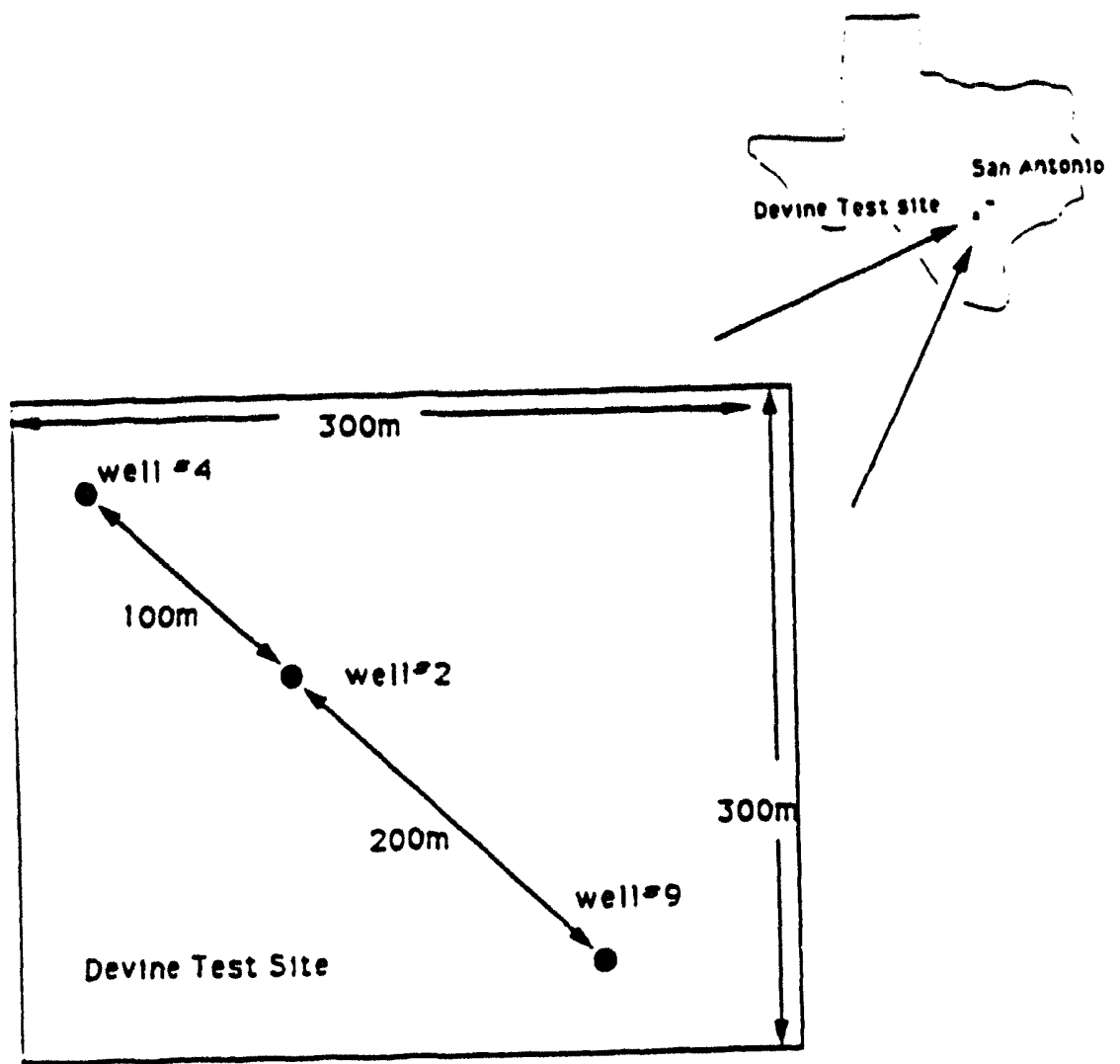

Fig. 5.3-1 The location of the Devine Test Site in Texas. 

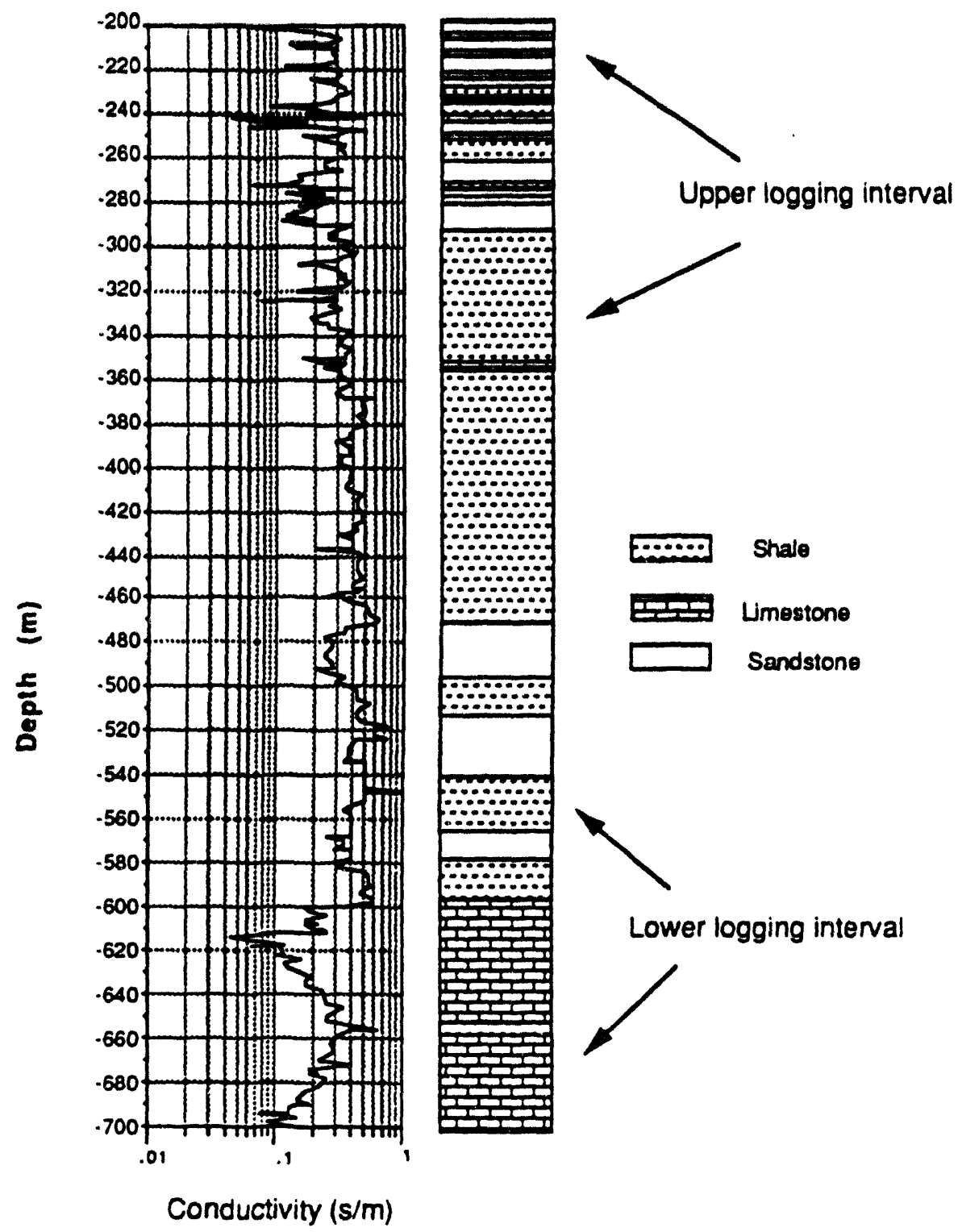

Lower logging interval

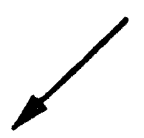

Fig. 5.3-2 The borehole induction logs and the geologic sections of the Devine Test Site. 


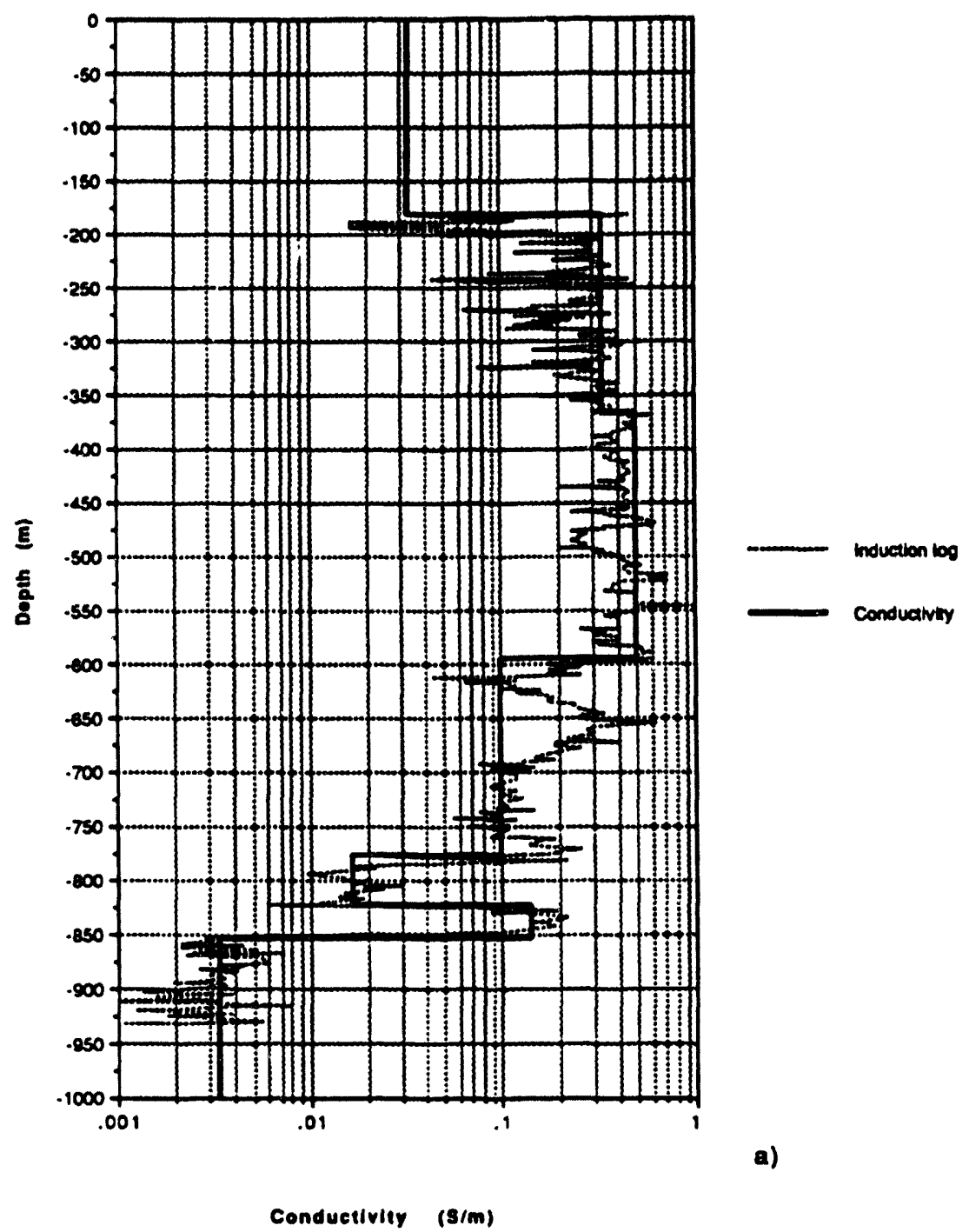

Fig. 5.3.-2a A simplified conductivity profile based on induction logs. This model was used for preliminary studies. 

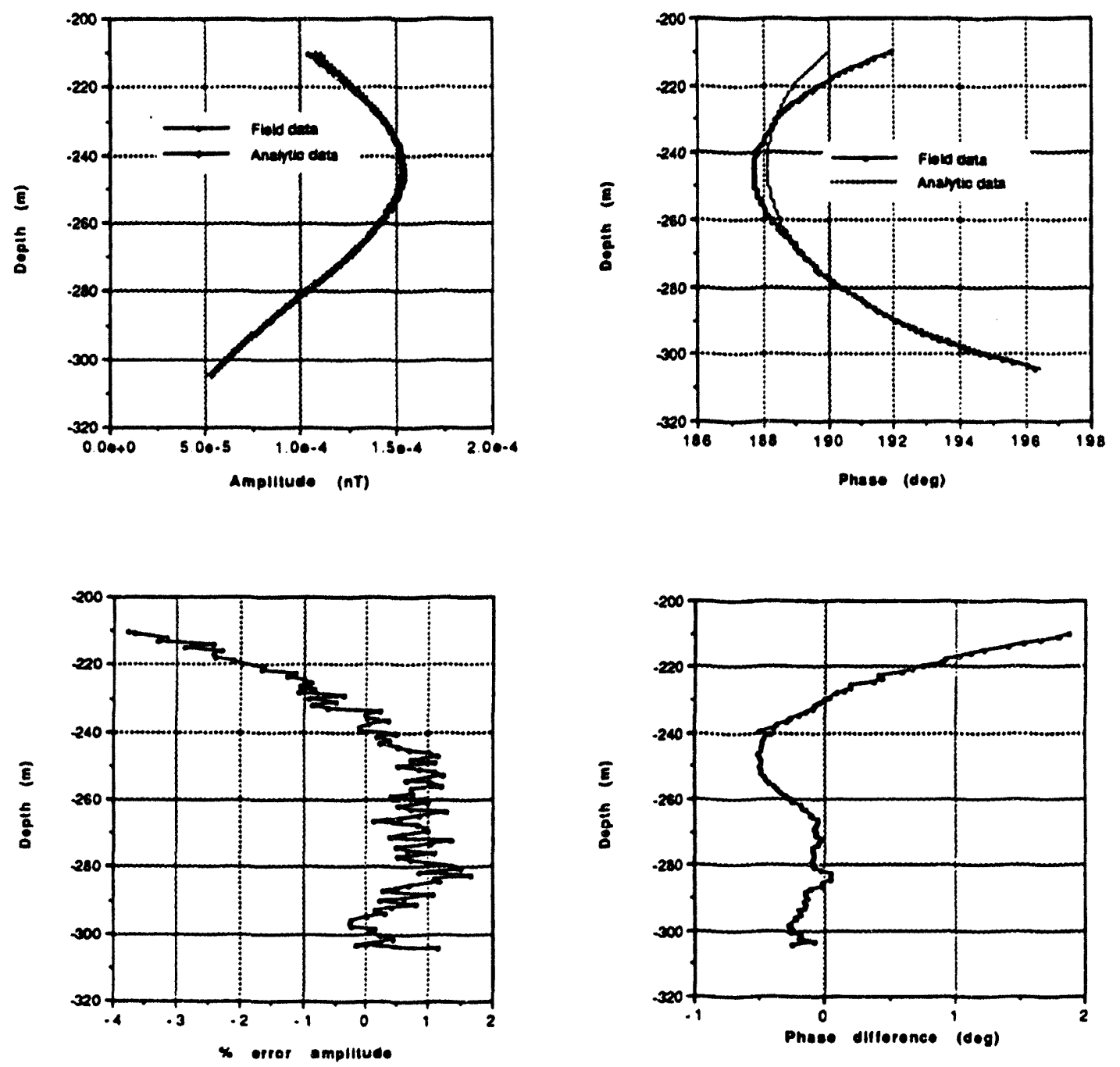

Fig. 5.3.1-1 The least squares inversion of amplitude and phase data for a half-space model. The frequency is $128 \mathrm{~Hz}$. Receiver is at $243.84 \mathrm{~m}$ (800 ft). Amplitude calibration was $2.54^{*} 10^{-3}$, phase shift calibration was $-15^{\circ}$. 

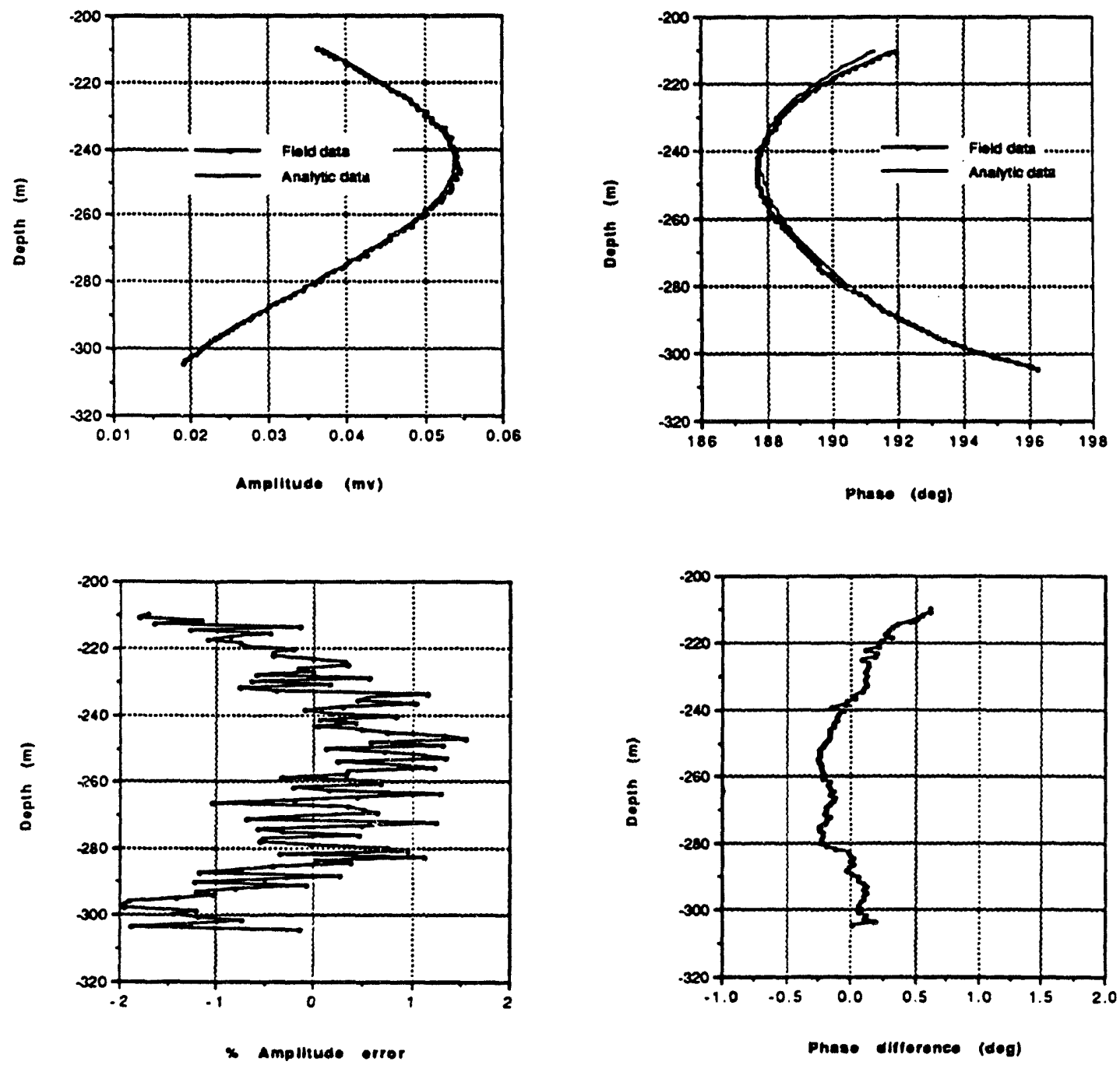

Fig. 5.3.1-2 The least squares fit of amplitude and phase data at frequency $128 \mathrm{~Hz}$ for a five layer model based on data inversion at $512 \mathrm{~Hz}$. Receiver is at $243.84 \mathrm{~m}(800 \mathrm{ft})$. Amplitude calibration was $2.48^{*} 10^{-3}$, phase shift calibration was $-11.96^{\circ}$. 

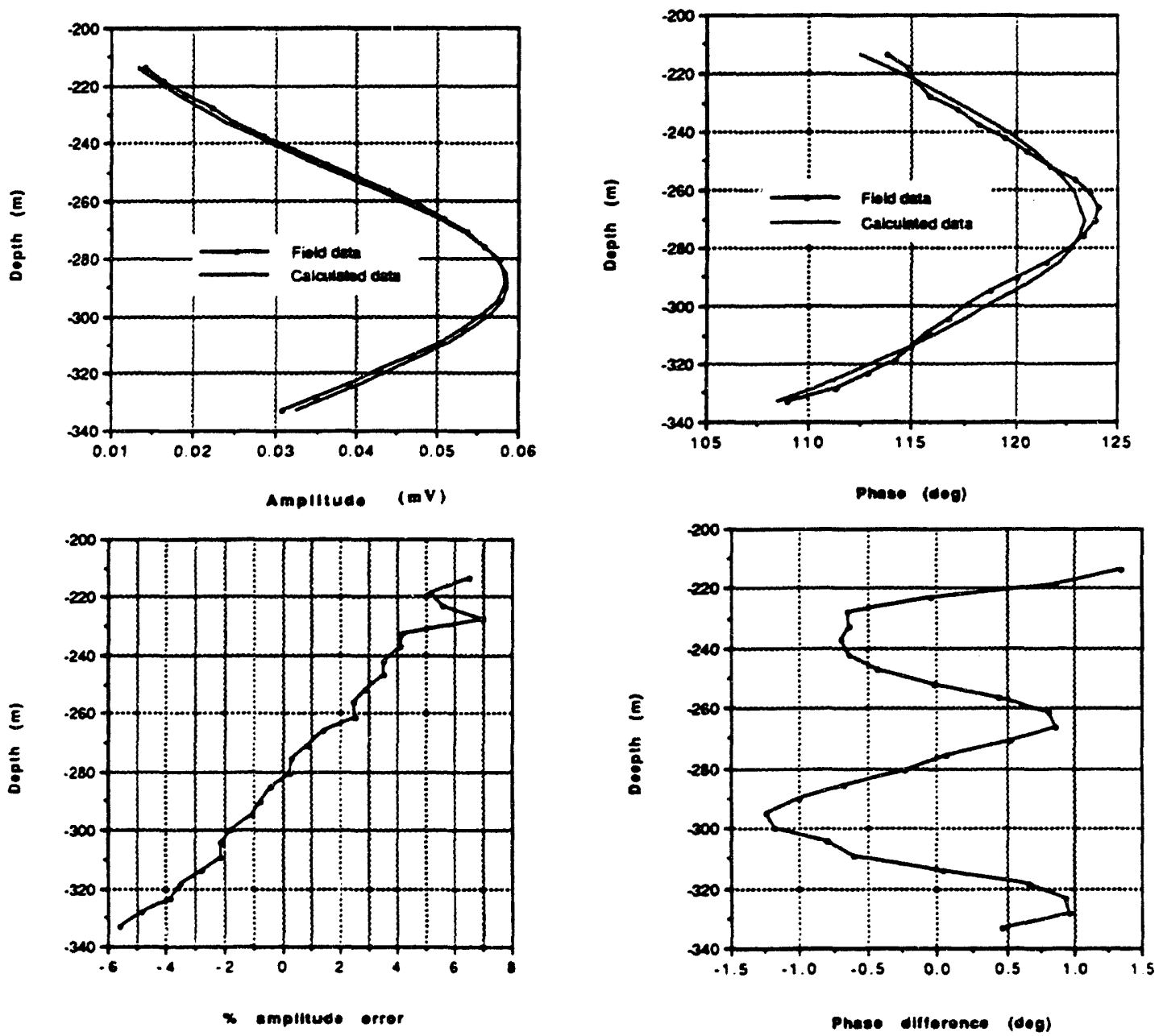

Fig. 5.3.1-3 The least squares fit for joint inversion of amplitude and phase data for a two layer model at frequency $512 \mathrm{~Hz}$. Receiver is at $289.56 \mathrm{~m}$ (950 ft). Amplitude calibration was $2.19^{*} 10^{-3}$, phase shift calibration was $0.0^{\circ}$. 

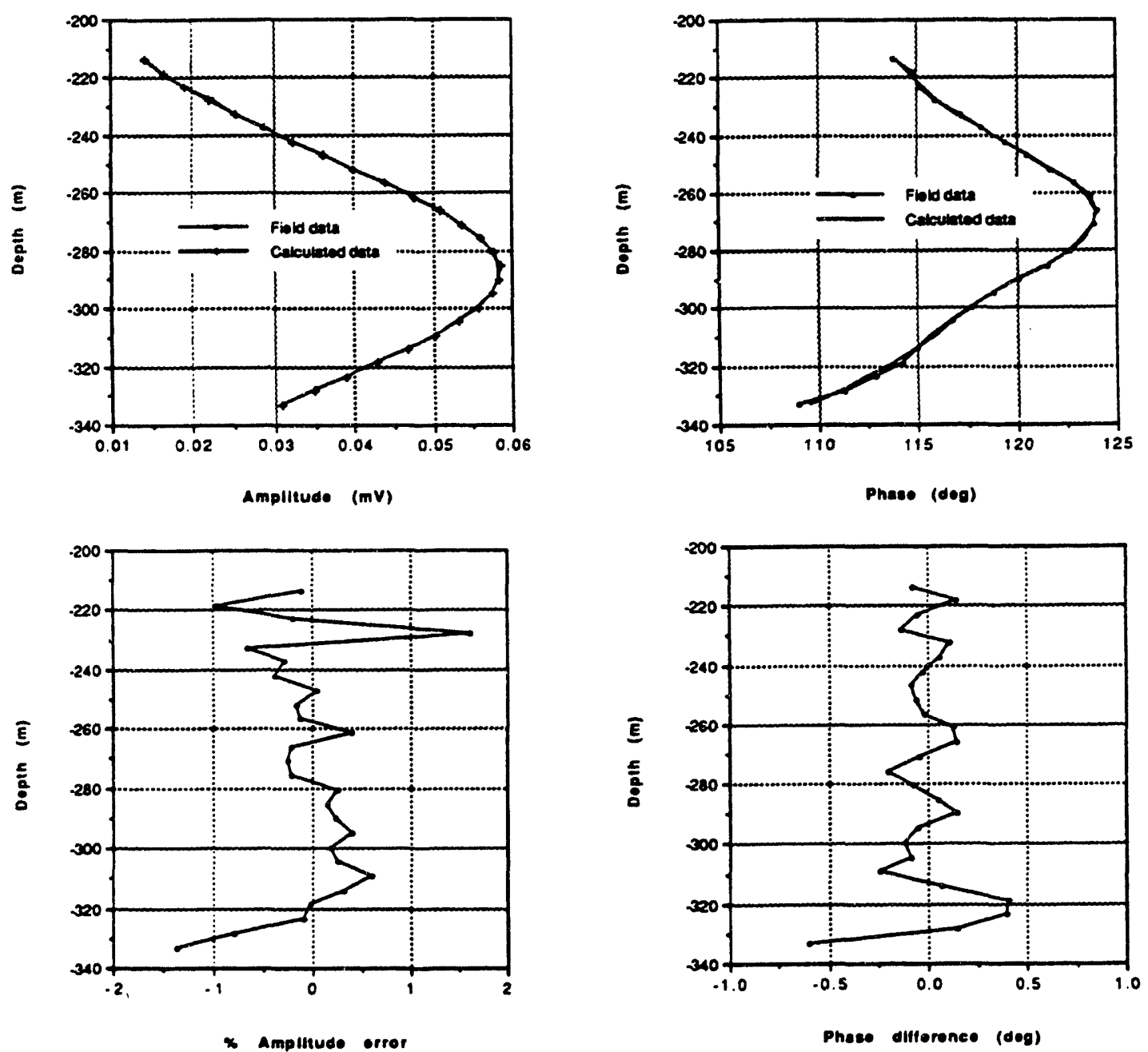

Fig. 5.3.1-4 The least squares fit of amplitude and phase data for a five layer model at frequency $512 \mathrm{~Hz}$. Receiver is at $289.56 \mathrm{~m}$ (950 ft). Amplitude calibration was $2.19^{*} 10^{-3}$, phase shift calibration was $0.0^{\circ}$. 


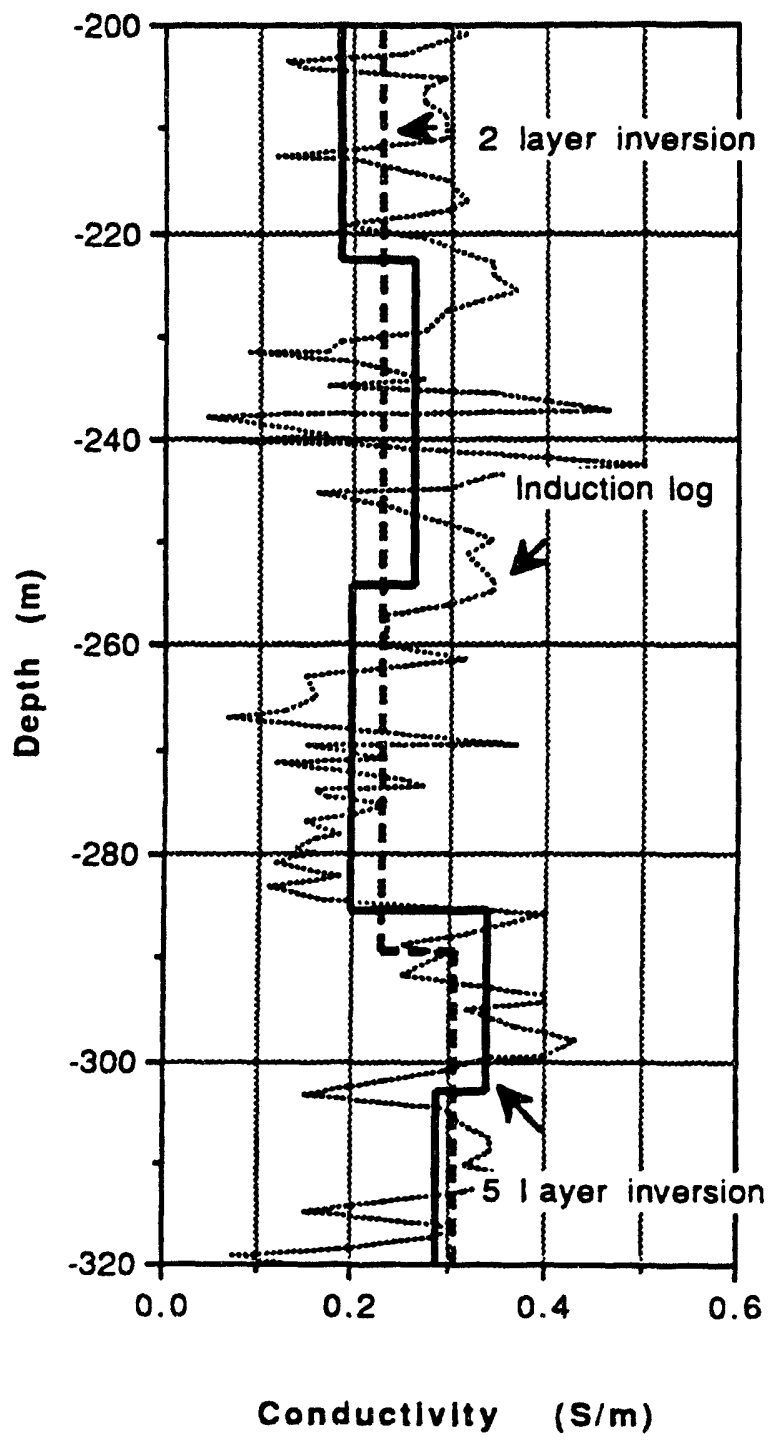

Fig. 5.3.1-5 The conductivity model based on least squares inversion for the upper section in Devine, Texas. 

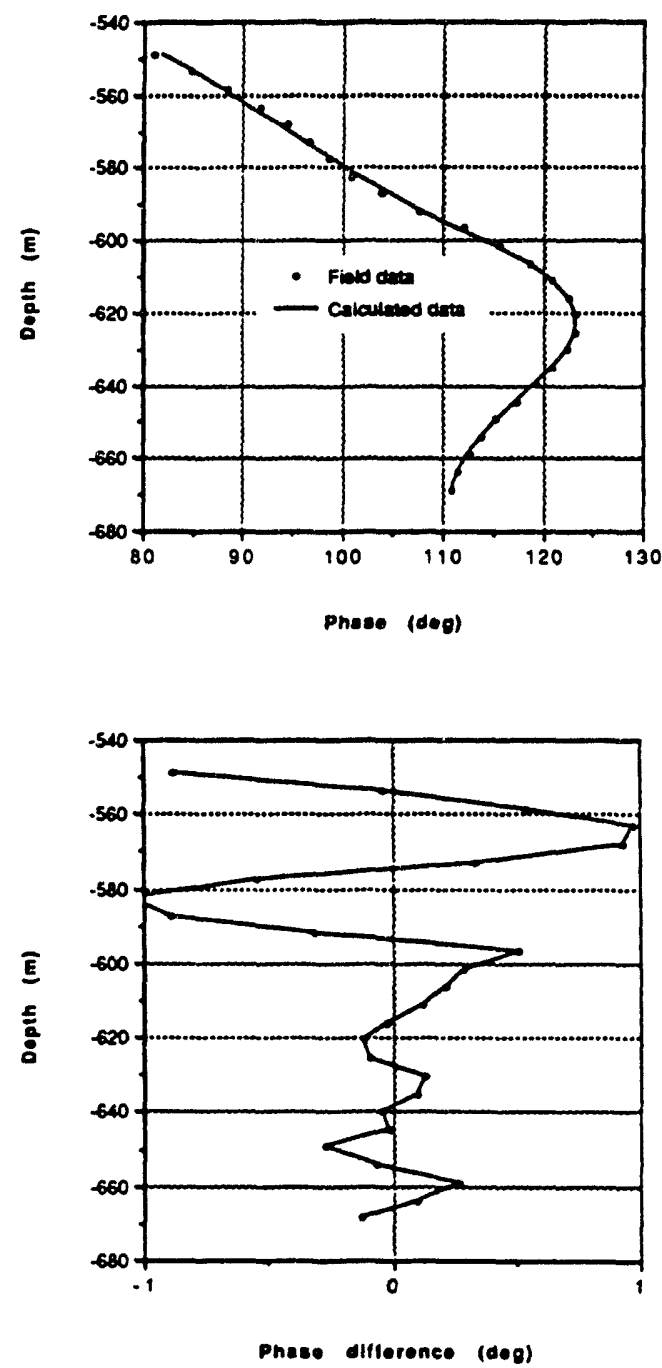

Fig. 5.3.2-1 The least squares fit of phase data for a six layer model at frequency 512 $\mathrm{Hz}$. Receiver is at $594.5 \mathrm{~m}(1950 \mathrm{ft})$. Phase shift calibration: $-6.3^{\circ}$. 

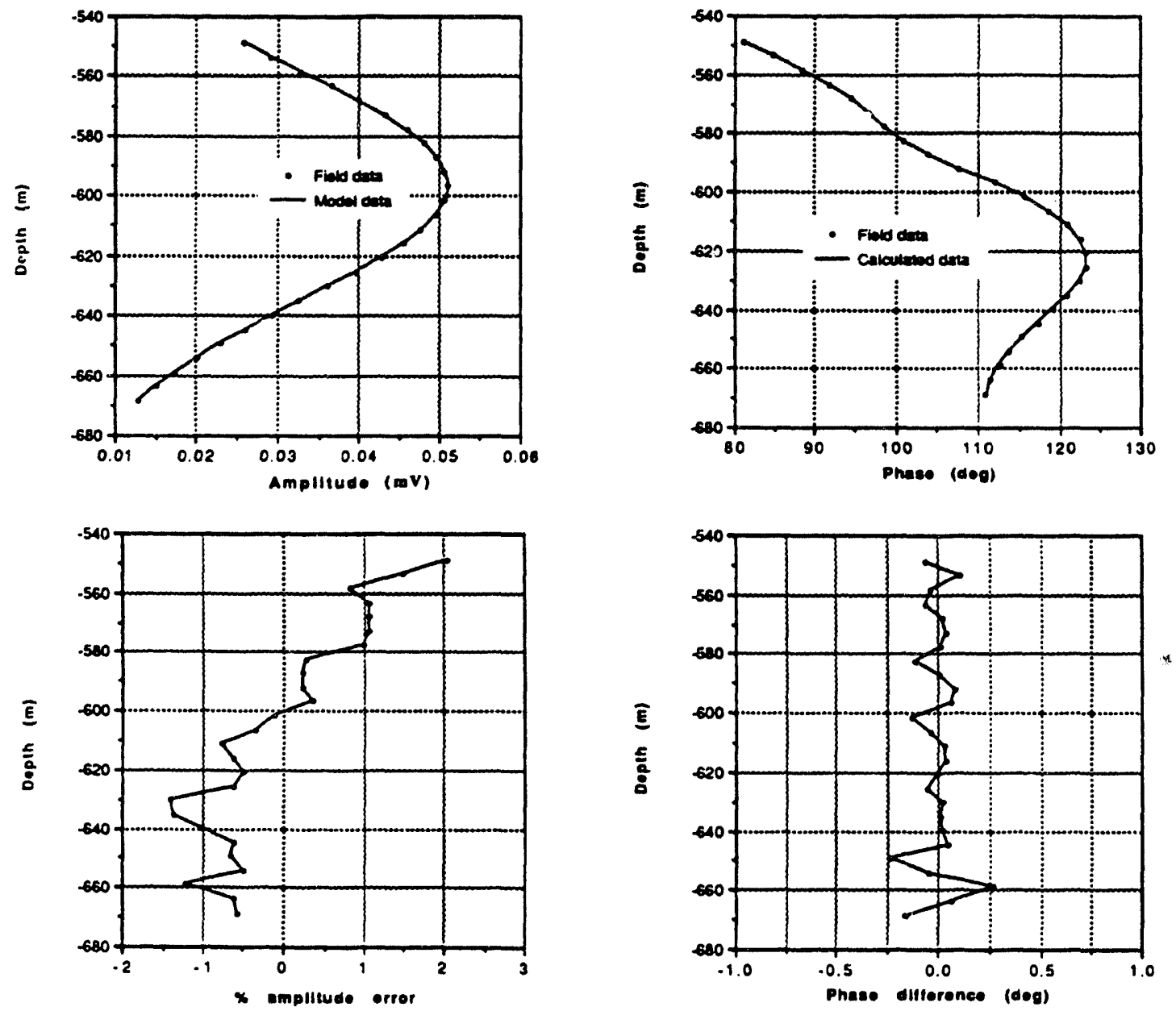

Fig. 5.3.2-2 The least squares fit of amplitude and phase data for an eight layer model at frequency $512 \mathrm{~Hz}$. Receiver is at $594.5 \mathrm{~m}$ (1950 ft). Amplitude calibration: $2.23^{*} 10^{-3}$, phase shift calibration: $0.0^{\circ}$. 

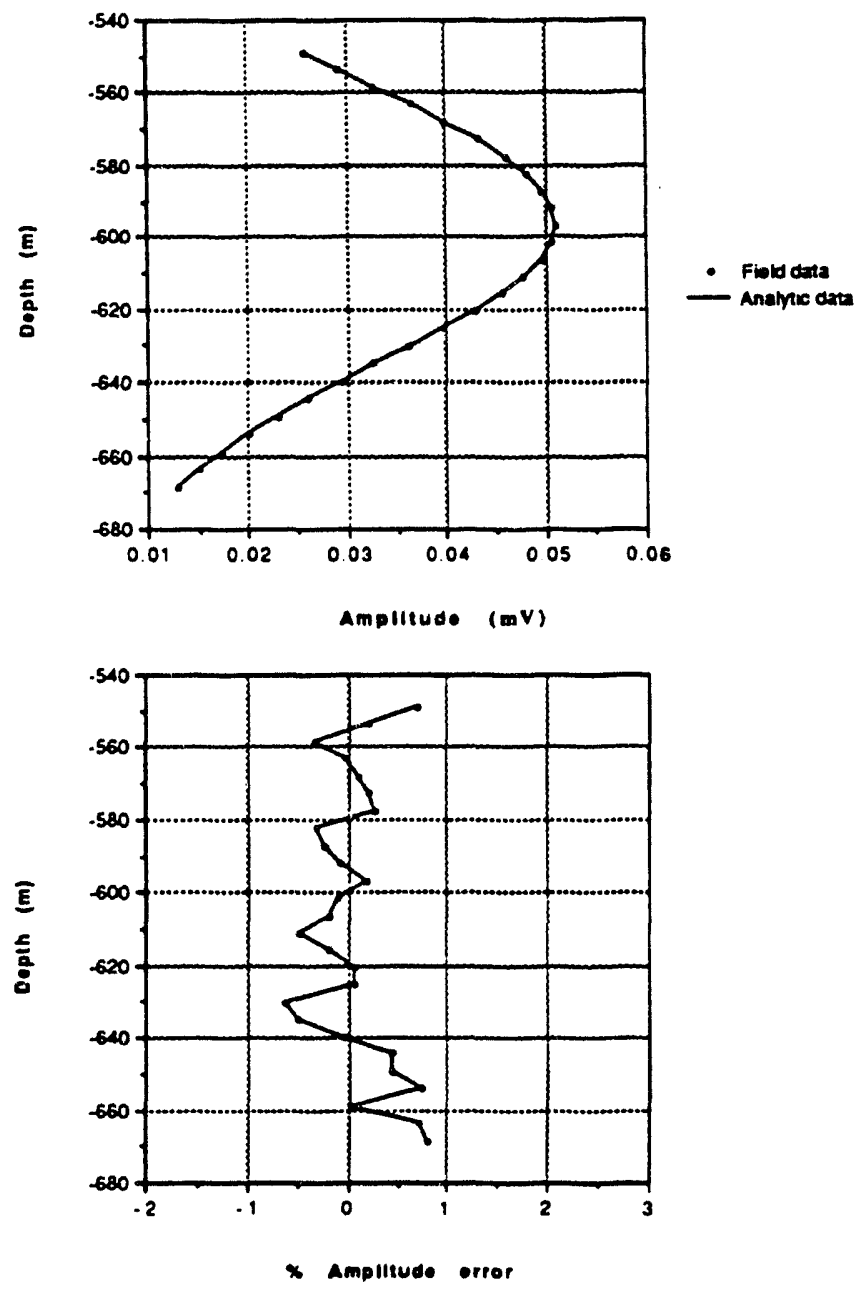

Fig. 5.3.2-3 The least squares inversion of amplitude data at $512 \mathrm{~Hz}$ for the same eight layer model as in Figure 5.3.2-2 assuming the error in the receiver location with respect to the depth of a whole profile. The inversion produced the correction in receiver location equal to $-0.47 \mathrm{~m}$. Receiver was at $594.5 \mathrm{~m}$ (1950 ft). 


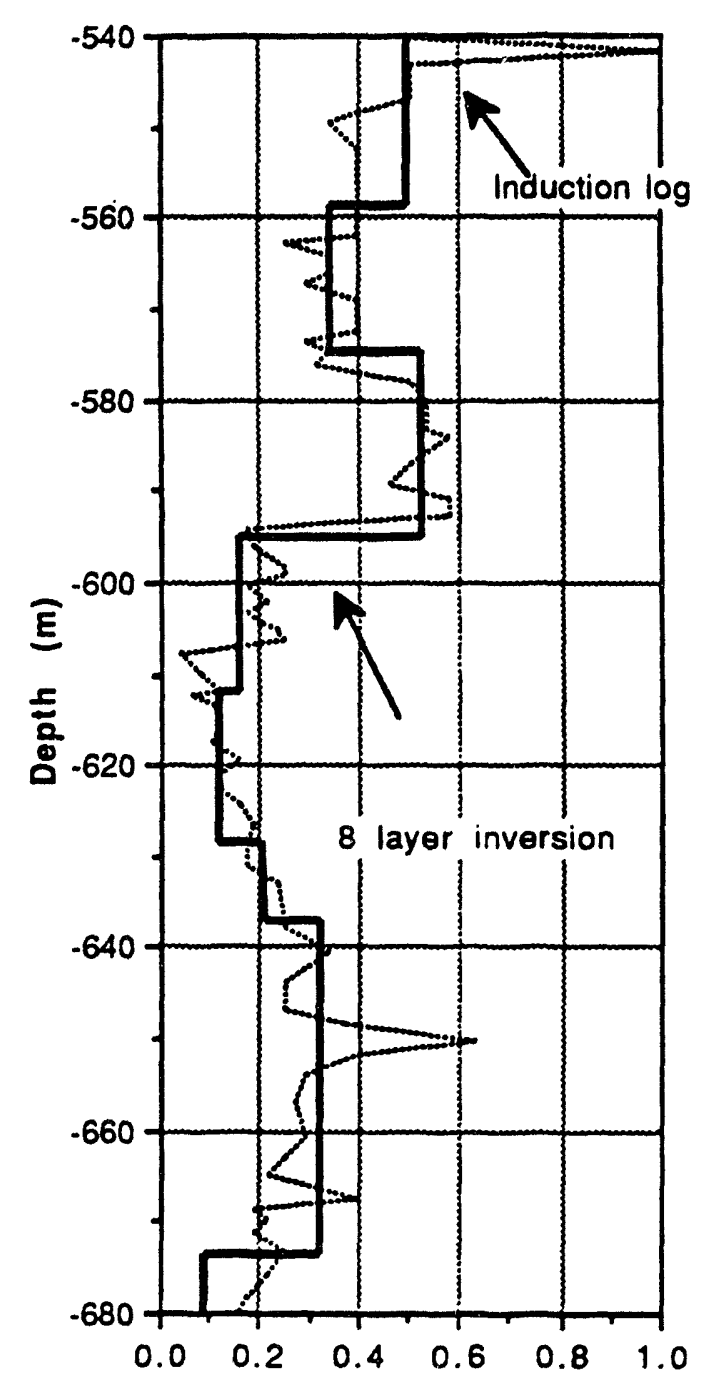

Conductivity $(5 / m)$

Fig. 5.3.2-4 The eight layer model based the inversion of phase data at $512 \mathrm{~Hz}$ from receiver located at $594.5 \mathrm{~m}$. 


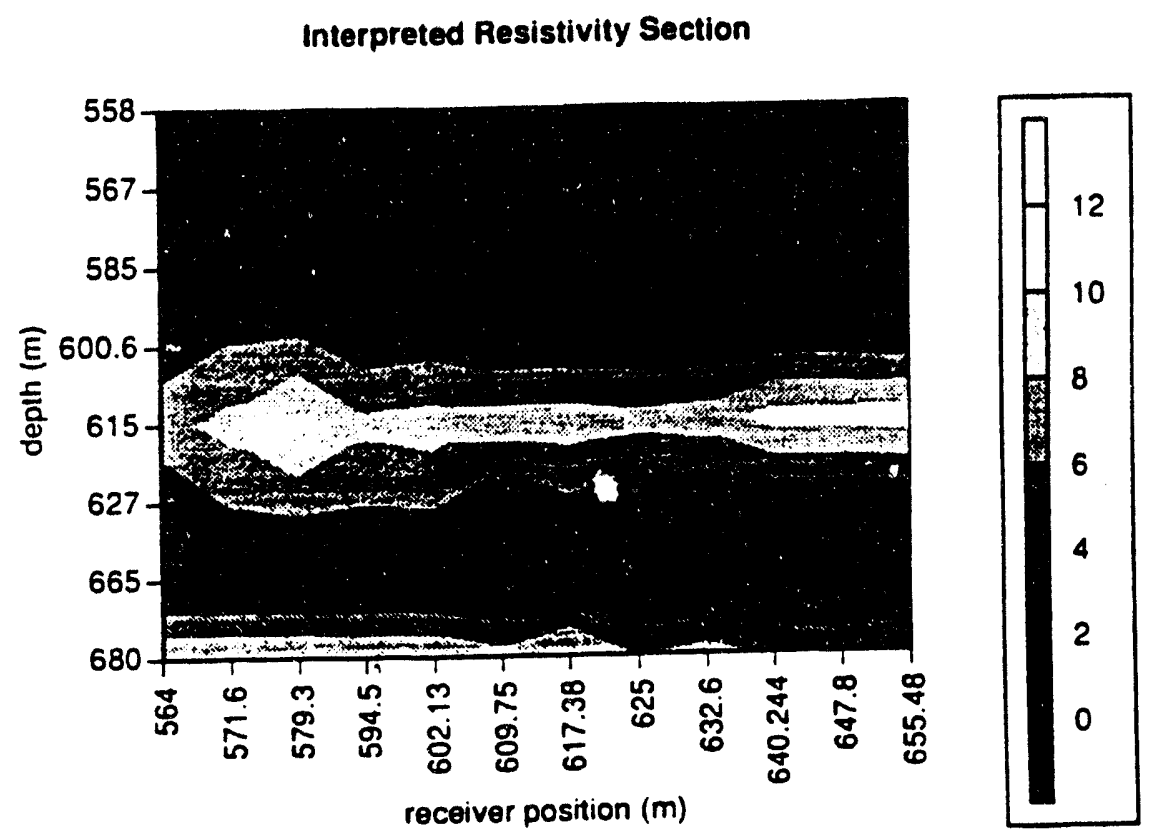

Phase difference (degrees)

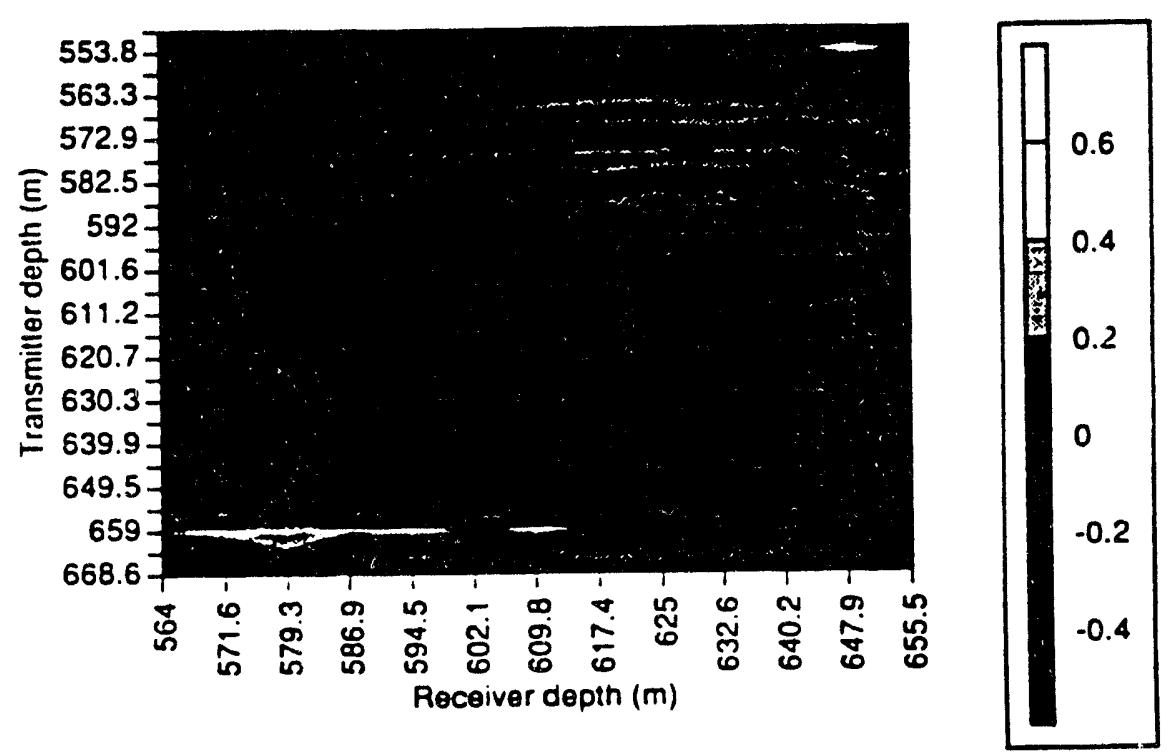

Fig. 5.3.2-5 The separate inversions at $512 \mathrm{~Hz}$ for each receiver location assuming the eight layer model. The figure was constructed by M. Wilt (1991). 

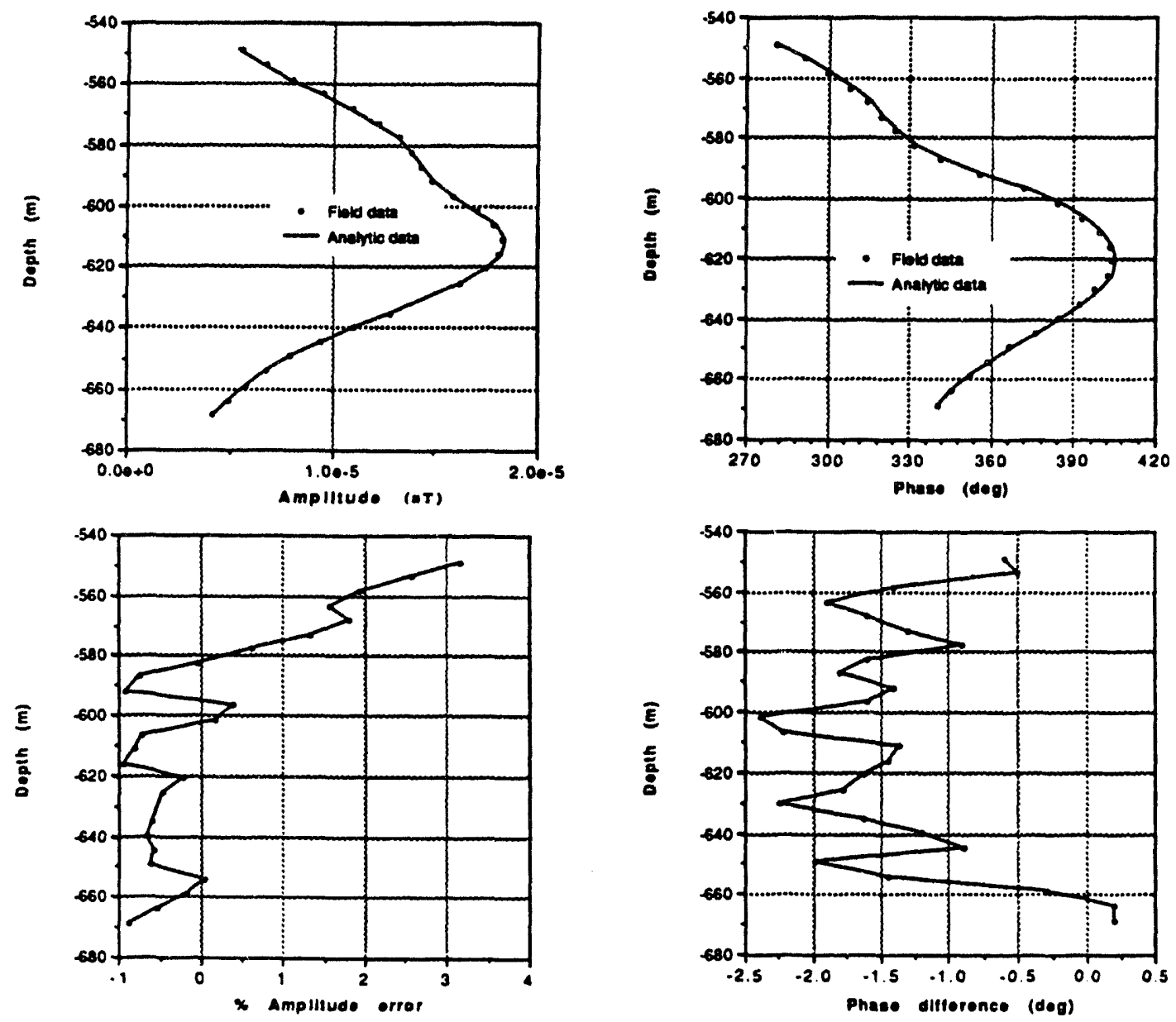

Fig. 5.3.2-6 The inversion of amplitude and phase data at $2048 \mathrm{~Hz}$ using 8 layer model obtained from the inversion of data collected at $512 \mathrm{~Hz}$ and shown in Figure 5.3.2-4. Amplitude calibration was 1.653 , phase shift calibration was $\mathbf{- 2 2 . 8 8}$ o. 
True model: 10 layers Inversion model: 8 layers

Frequencies: 512 and $2048 \mathrm{~Hz}$
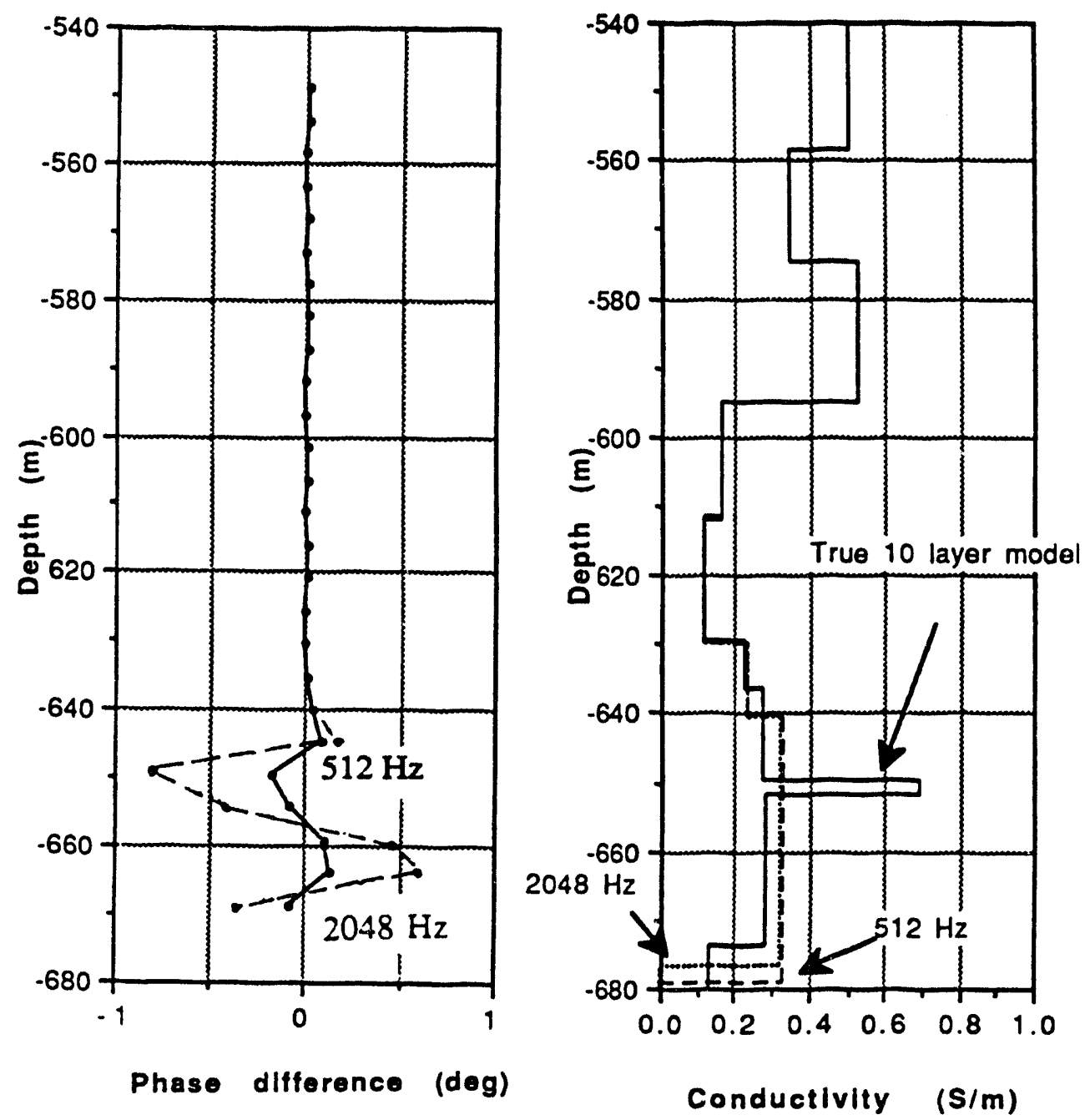

Fig. 5.3.2-7 The inversion of analytic data calculated for a 10 layer model but assuming an 8 layer model for inversion. The phase difference at $512 \mathrm{~Hz}$ shows the sirusoidal pattern around the depth where the layer was inserted. The phase difference at $2048 \mathrm{~Hz}$ had the same pattern but was larger and reached 1 degree. 

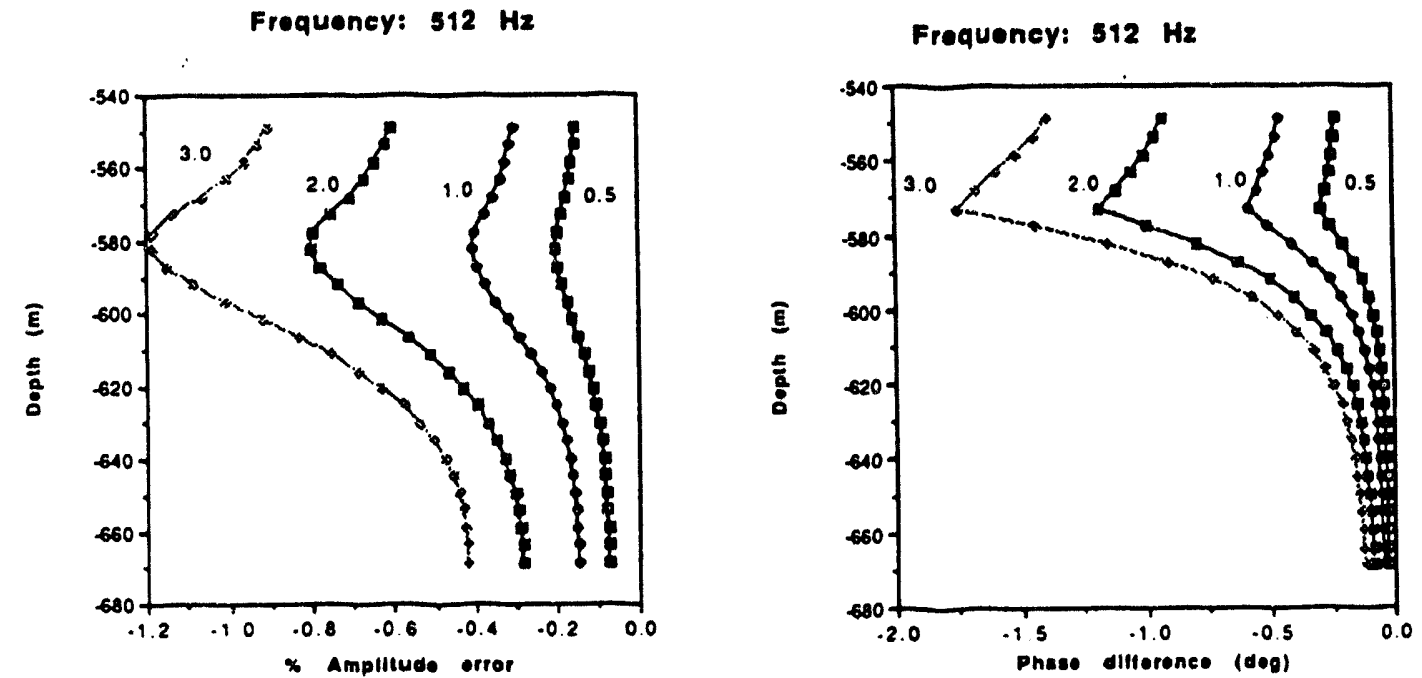

Frequency: $2048 \mathrm{~Hz}$

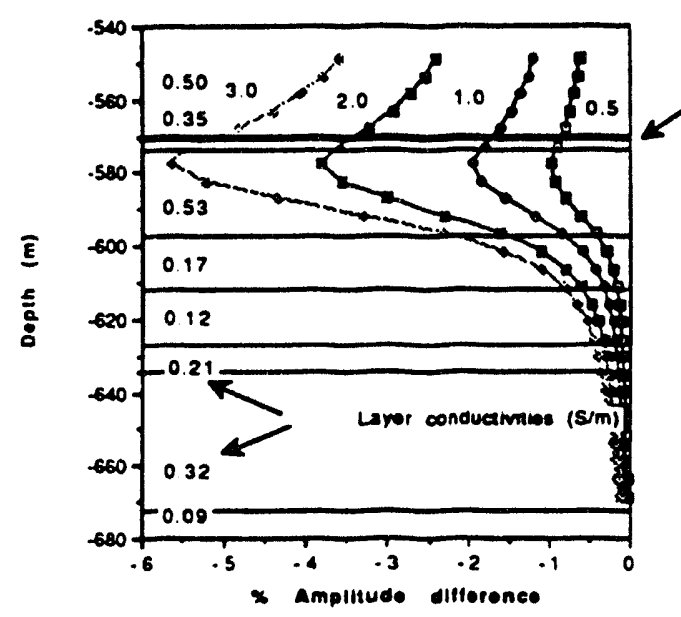

Frequency: $2048 \mathrm{~Hz}$.

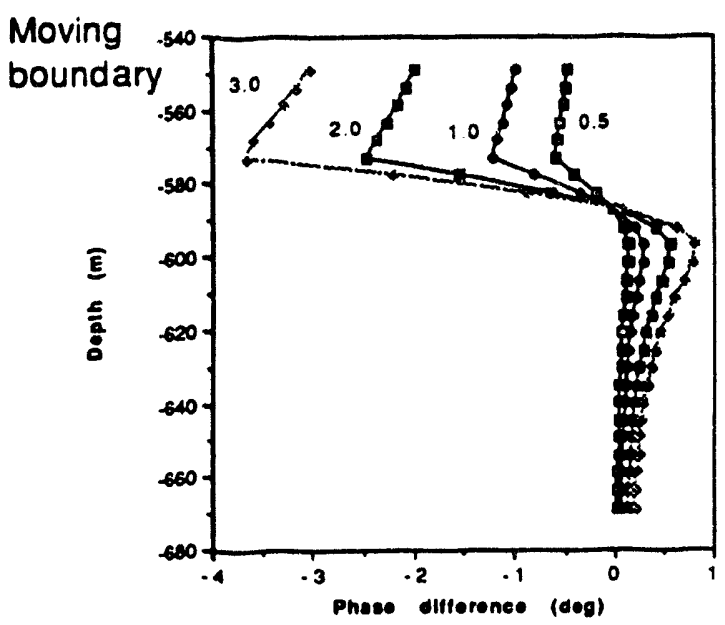

Fig. 5.3.2-8 The sensitivity analysis of amplitude and phase to the variations in layer thickness at $512 \mathrm{~Hz}$ and $2048 \mathrm{~Hz}$. The lower left panel at $2048 \mathrm{~Hz}$ shows the location of the layer whose upper boundary moves up by an amount $(m)$ indicated on the curves. 
Freq.: $4096 \mathrm{~Hz}$.

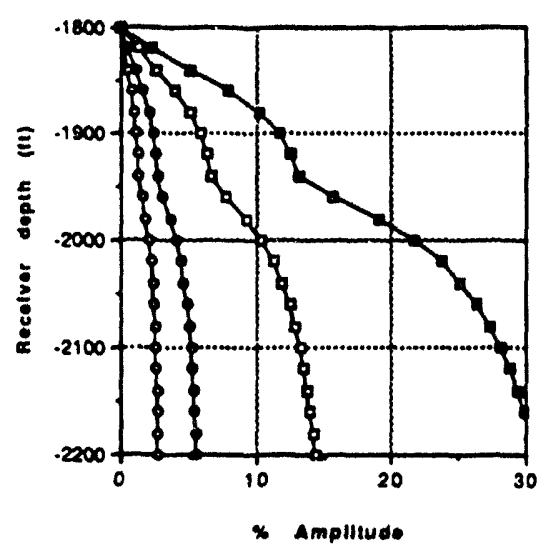

* Ampiliude

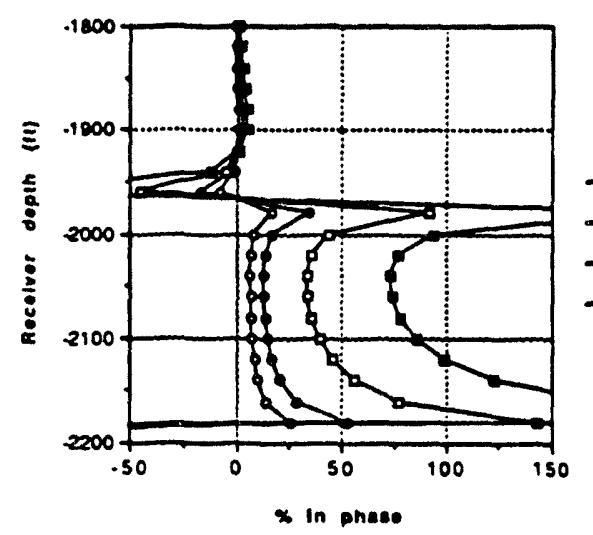

Freq.: $4096 \mathrm{~Hz}$.

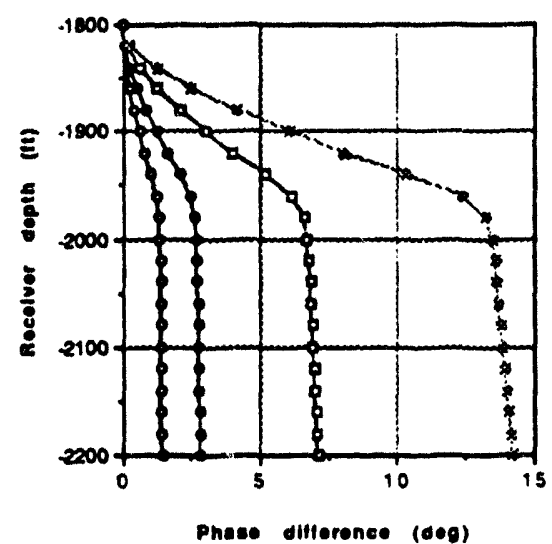

Phase difterence (deg)

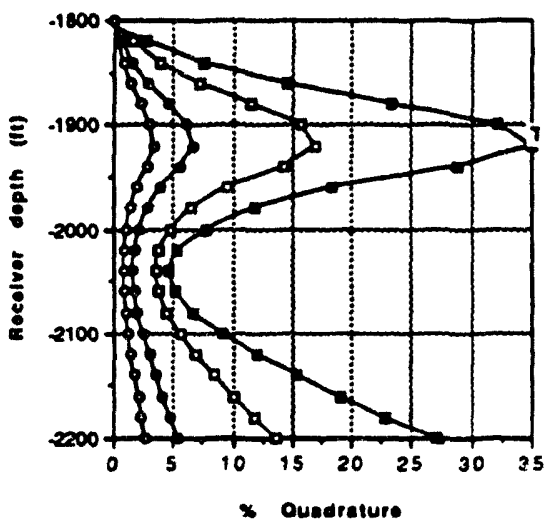

Fig. 5.3.2-8b The sensitivity analysis of the amplitude, phase, in-phase and quadrature components to the error in the estimate of the vertical separation between transmitter and receiver when the assumed separation is different from the true one by $1,2,5$ and 10 feet. The error was computed by taking the differences in response for the receiver located at $1800 \mathrm{ft}$ and between $1801,1802,1805$ and $1810 \mathrm{ft}$ at $4096 \mathrm{~Hz}$. 

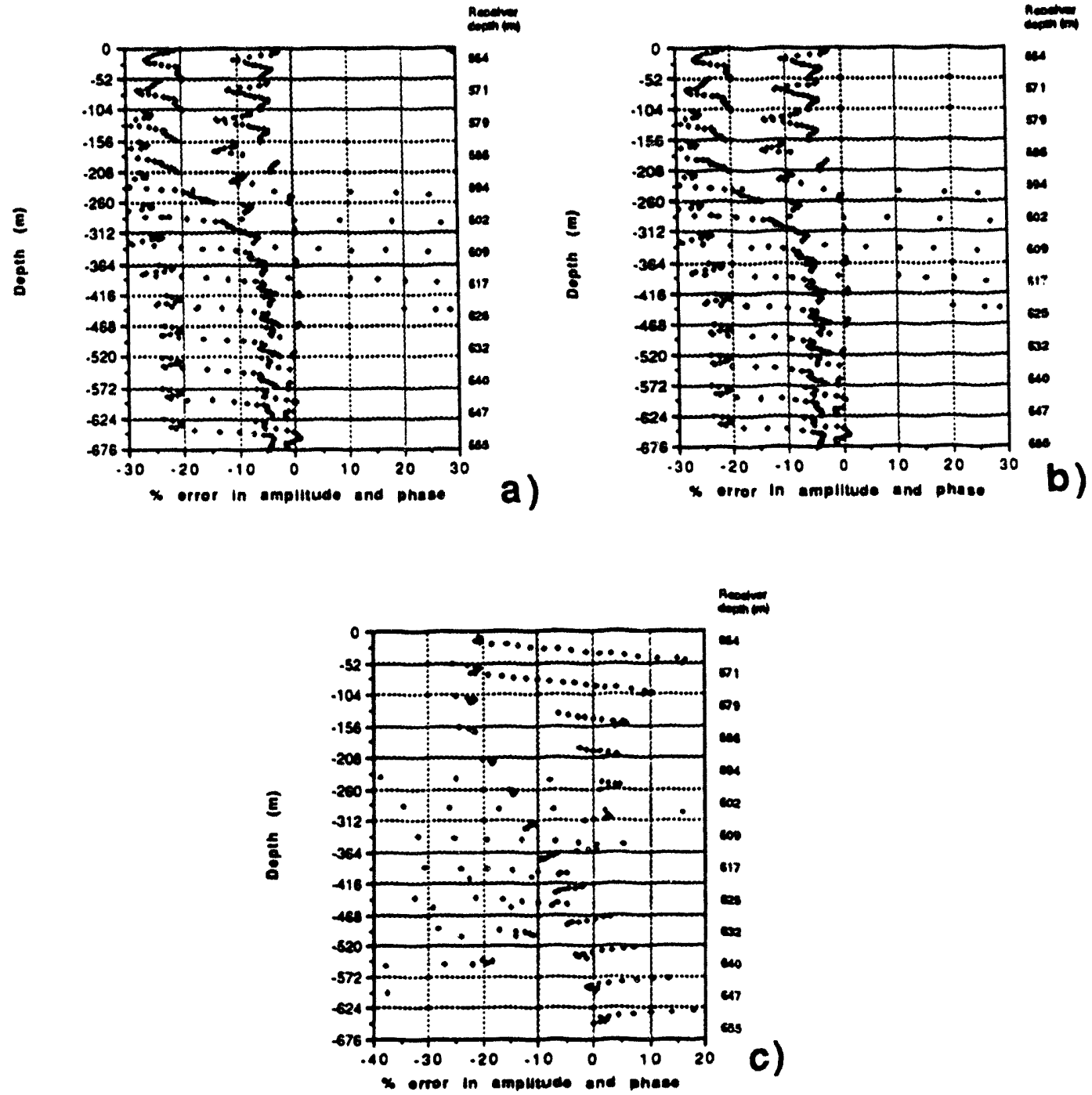

Fig. 5.3.2-8c The sensitivity of the least squares fit of joint inversions of amplitude and phase data to the choice of weighting options: a) amplitude and phase are not weighted, b) amplitude and phase are weighted by their square root value, and c) amplitude and phase data are weighted by their value. The data for inversions are calculated using analytic response from a 14 layer model and assuming 8 layer model in inversion. 

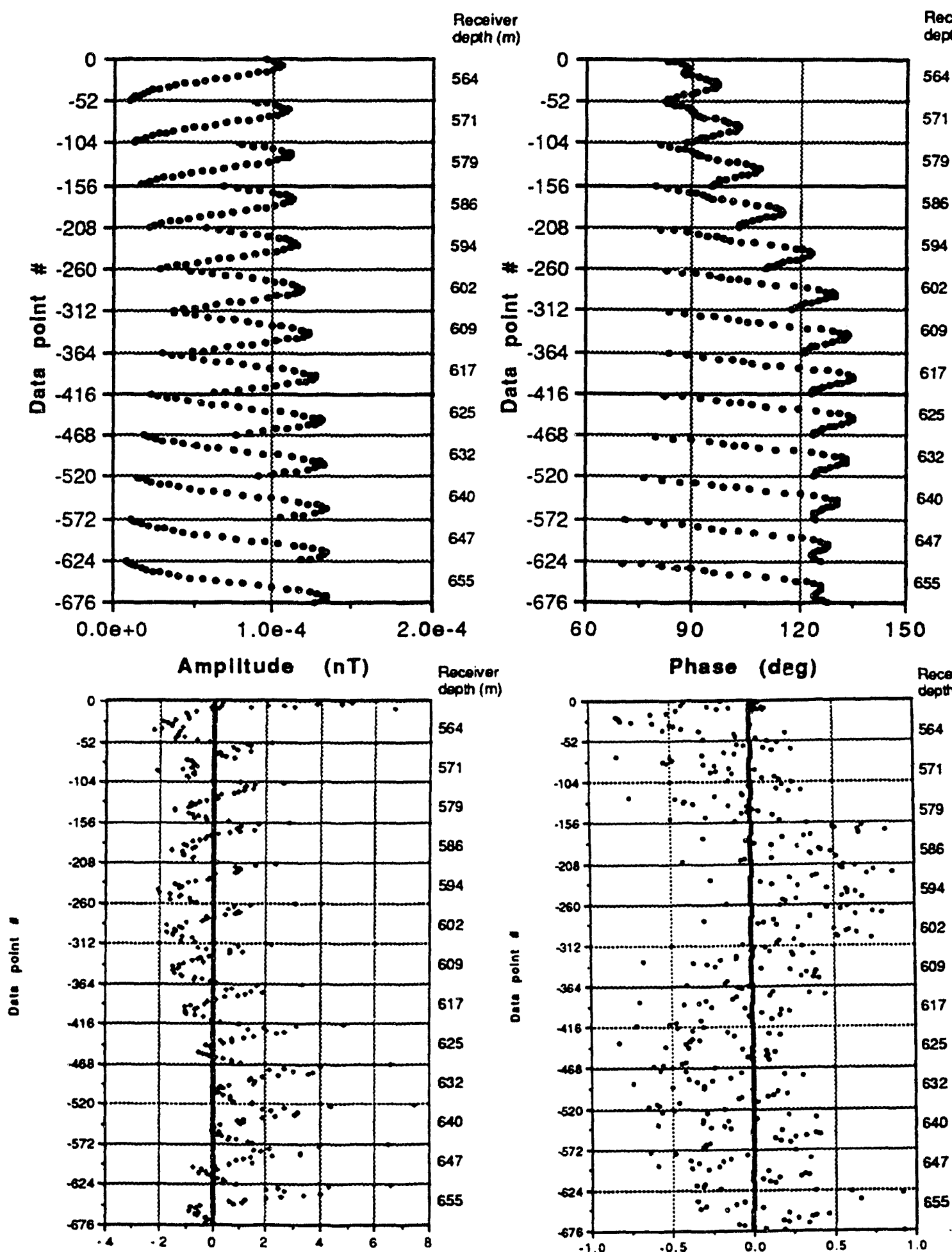

* error in amplleude

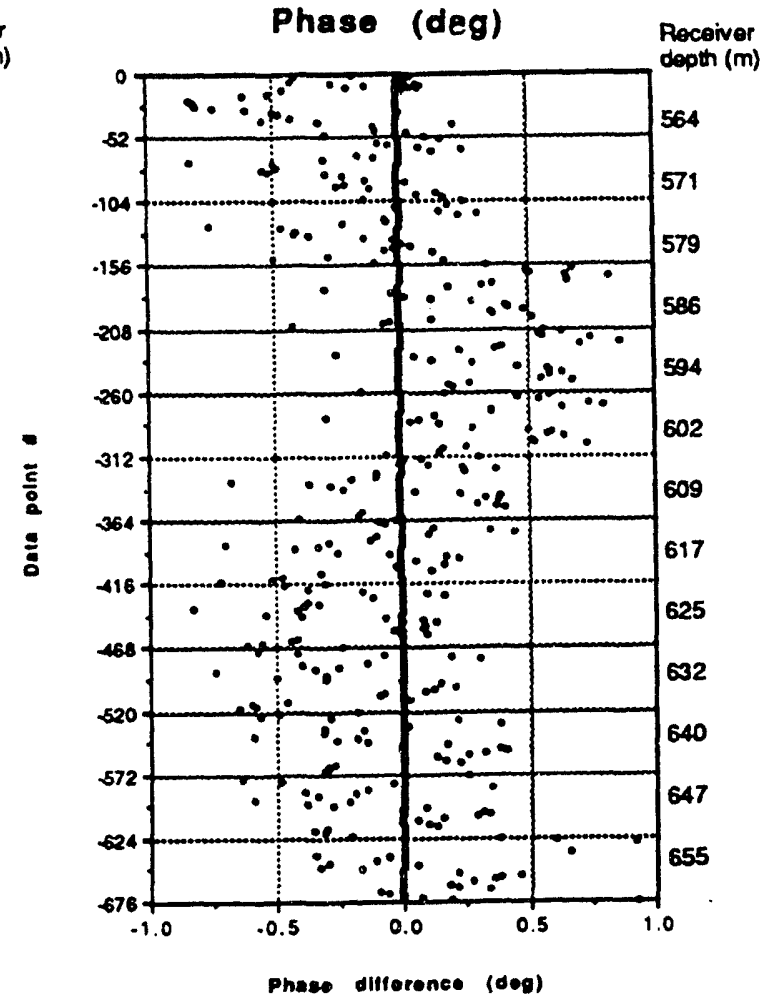

Fig. 5.3.2-9 The joint inversion of amplitude and phase data at $512 \mathrm{~Hz}$ for all receiver locations using every fifth point and assuming 14 layer model. 

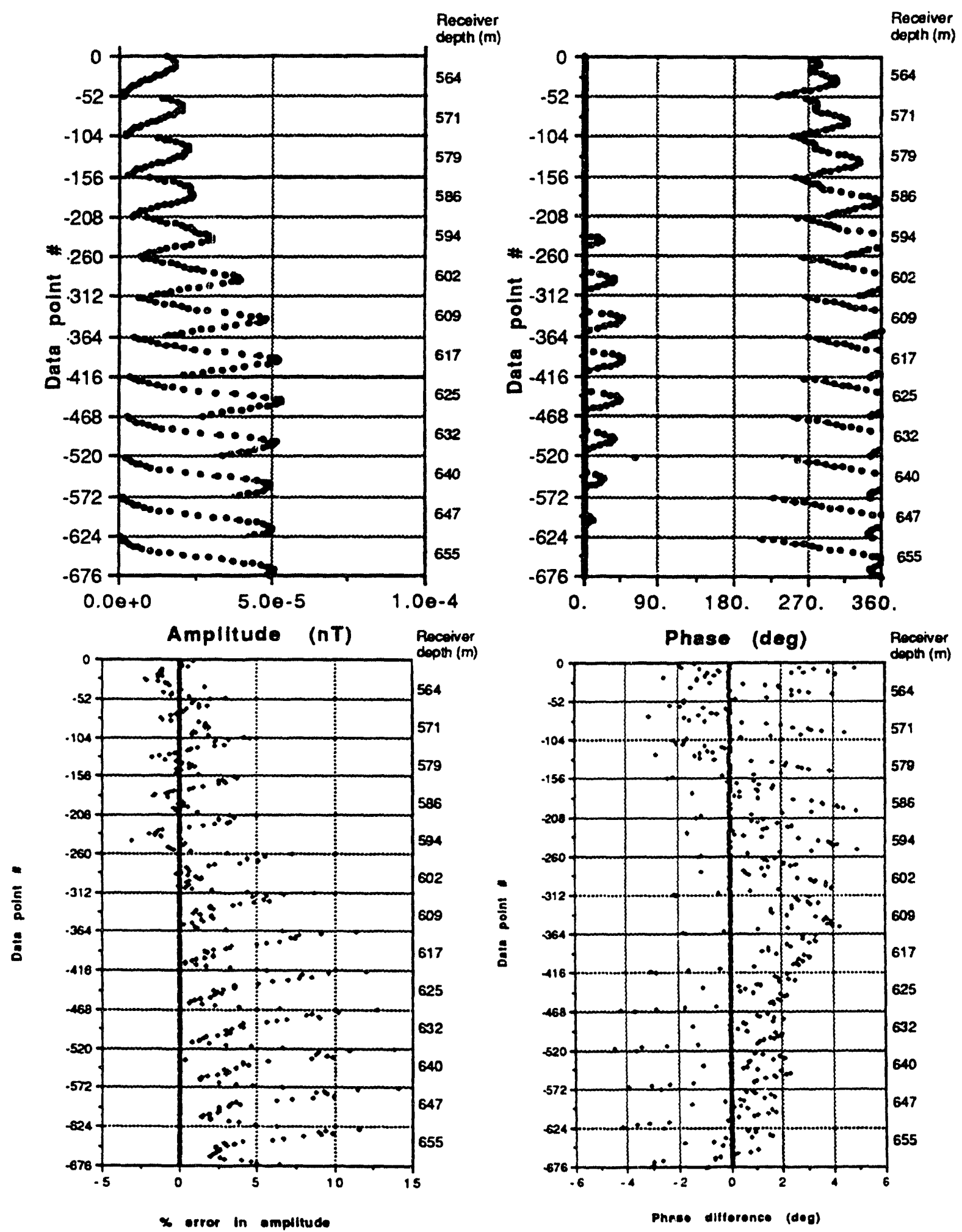

Fig. 5.3.2-10 The joint inversion of amplitude and phase data at $2048 \mathrm{~Hz}$ for all receiver locations using every fifth point and assuming 14 layer model. 


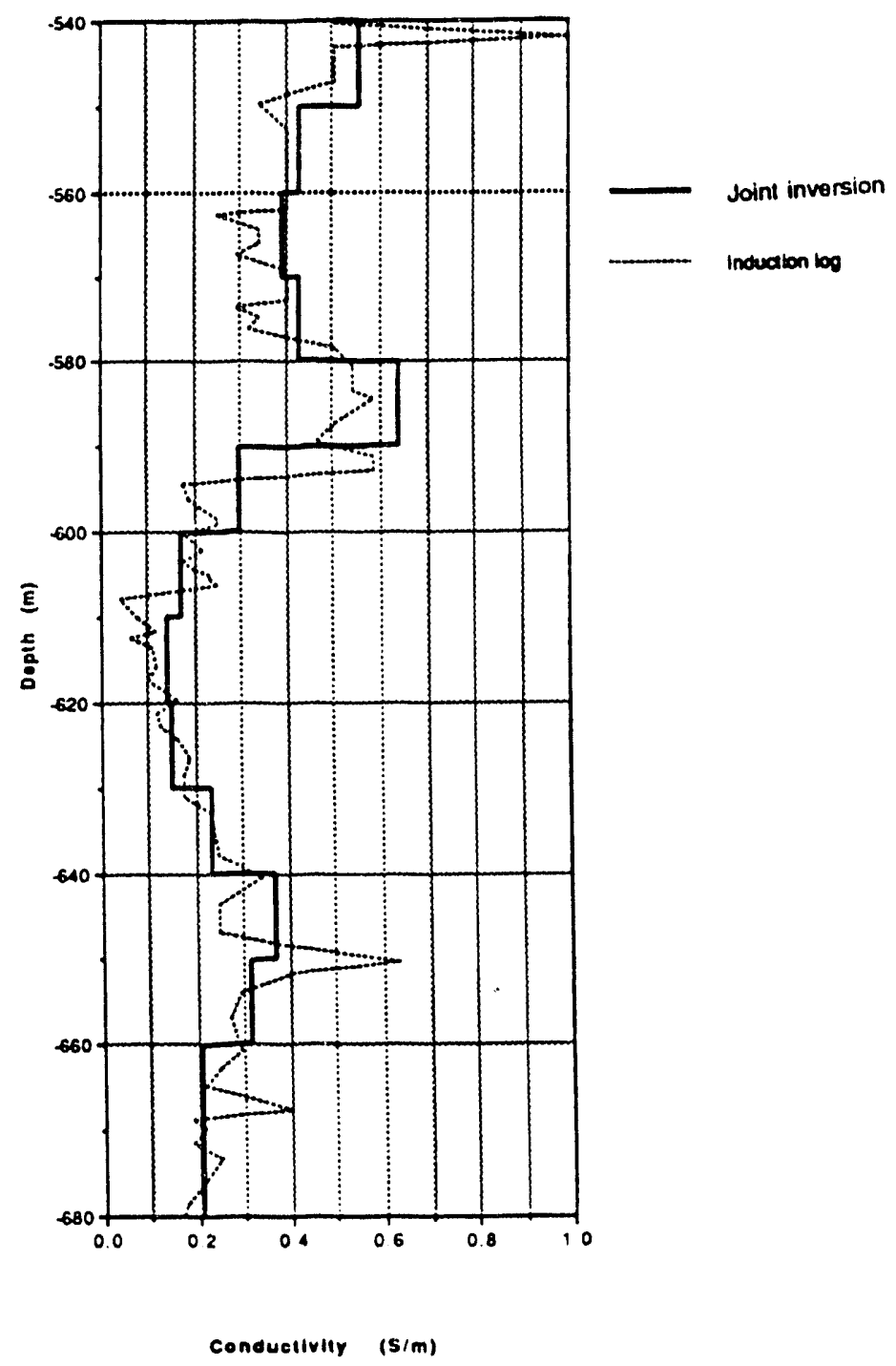

Fig. 5.3.2-11 The fourteen layer model of conductivity distribution in Devine obtained using joint inversion of amplitude and phase data at $512 \mathrm{~Hz}$ 

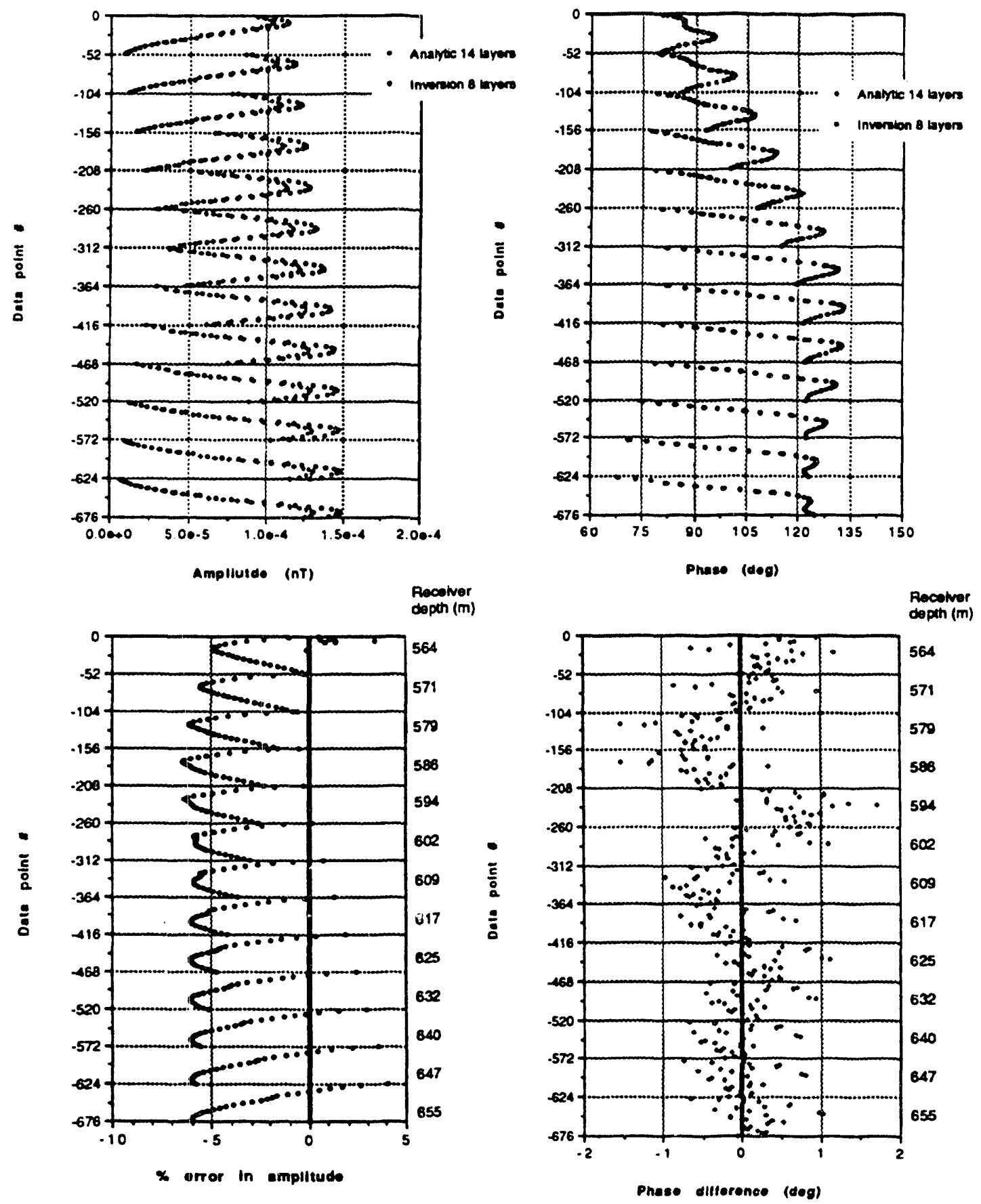

Fig. 5.3.2-12 The inversion of analytic data calculated using a 14 layer model and assuming 8 layer model in inversion at $512 \mathrm{~Hz}$. The inversion was performed jointly on amplitude and phase data using ali 13 receiver location and points spaced $4.5 \mathrm{~m}$ apart. 

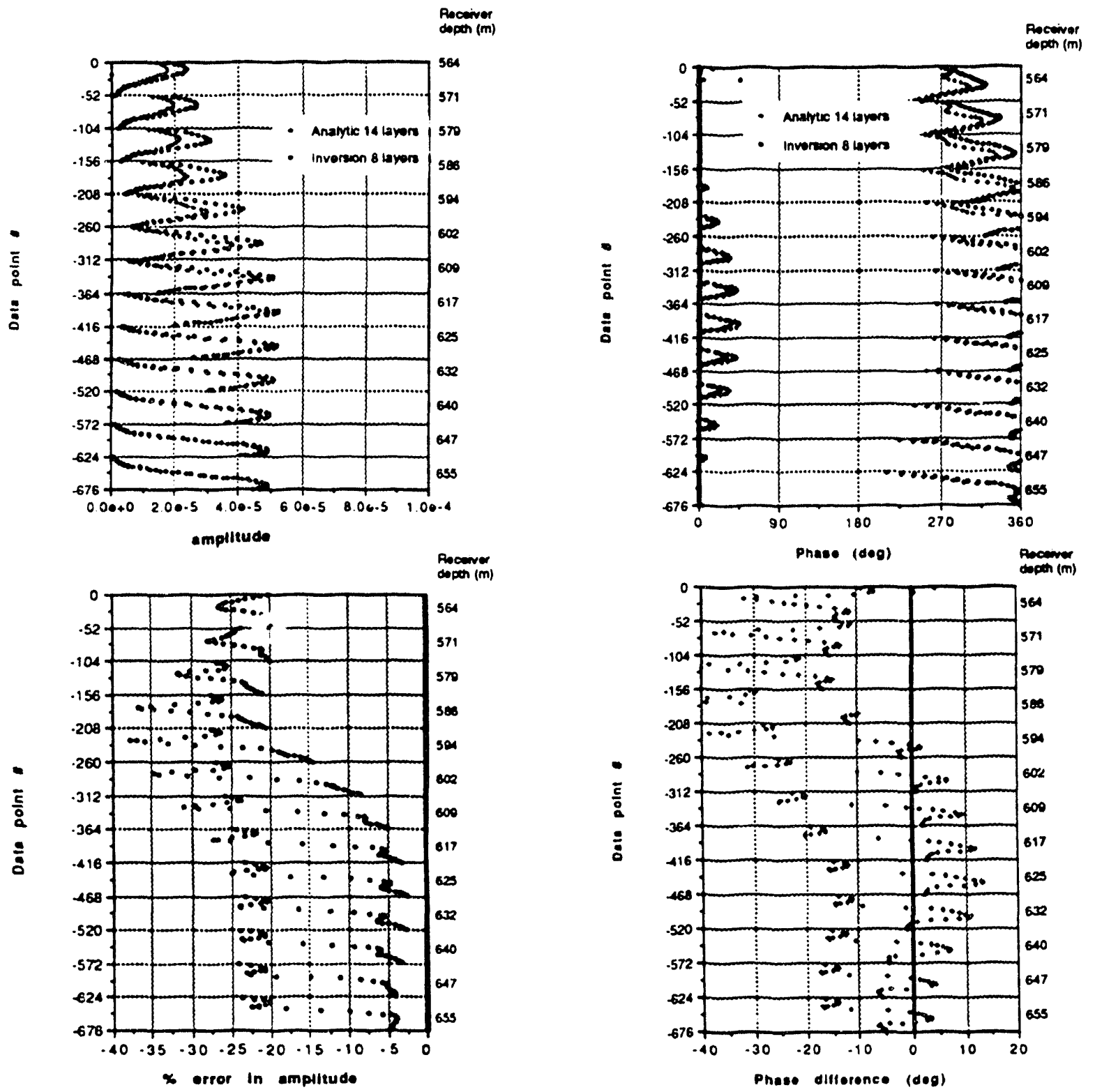

Fig. 5.3.2-13 The inversion of analytic data calculated using a 14 layer model and assuming 8 layer model in inversion at $2048 \mathrm{~Hz}$. The inversion was performed jointly on amplitude and phase data using all 13 receiver location and points spaced $4.5 \mathrm{~m}$ apart. 


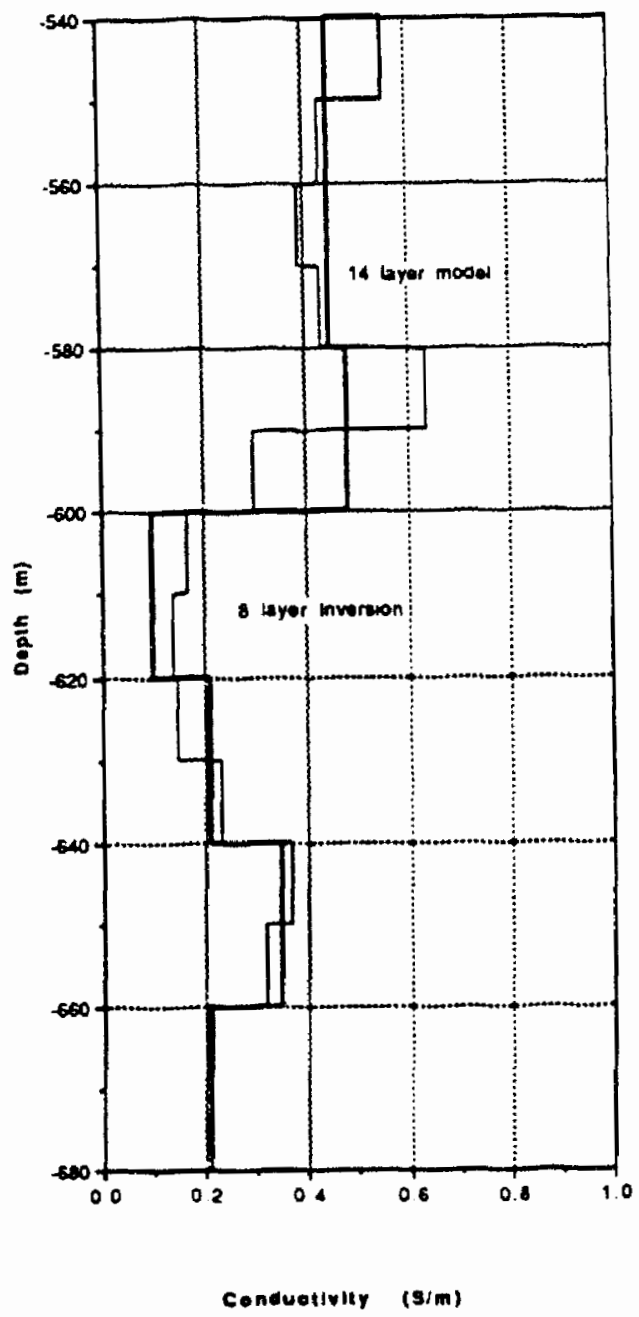

Fig. 5.3.2-14 The comparison of the 14 layer model that was used to calculate the analytic data for the inversion with the 8 layer model that resulted from the inversion. 

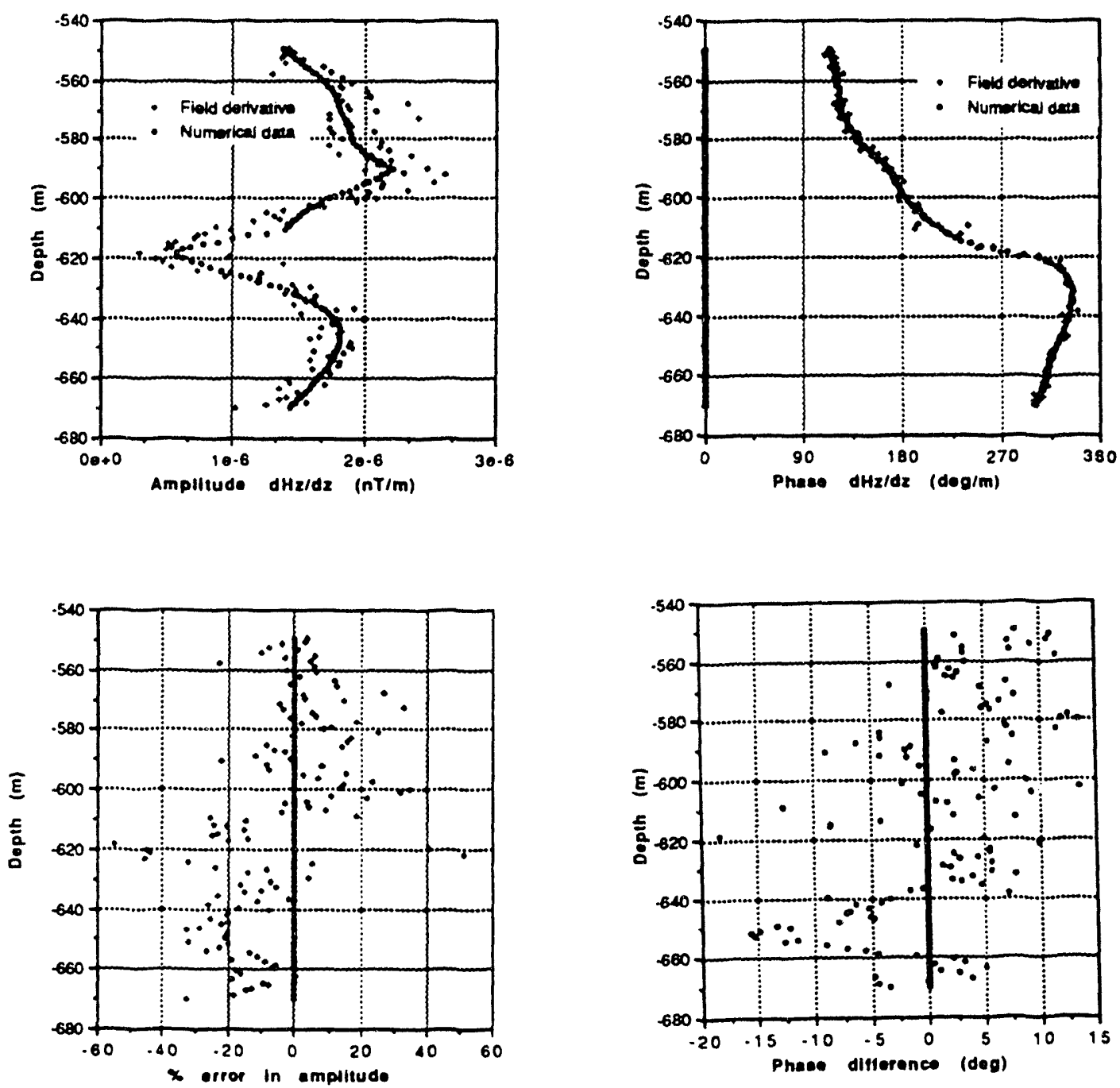

Fig. 5.3.3-1 The least squares inversion at $512 \mathrm{~Hz}$ using amplitude and phase of the first vertical derivative $\delta \mathrm{H}_{z} / \delta \mathrm{z}$ from receiver located at $609.6 \mathrm{~m}(2000 \mathrm{ft})$. 

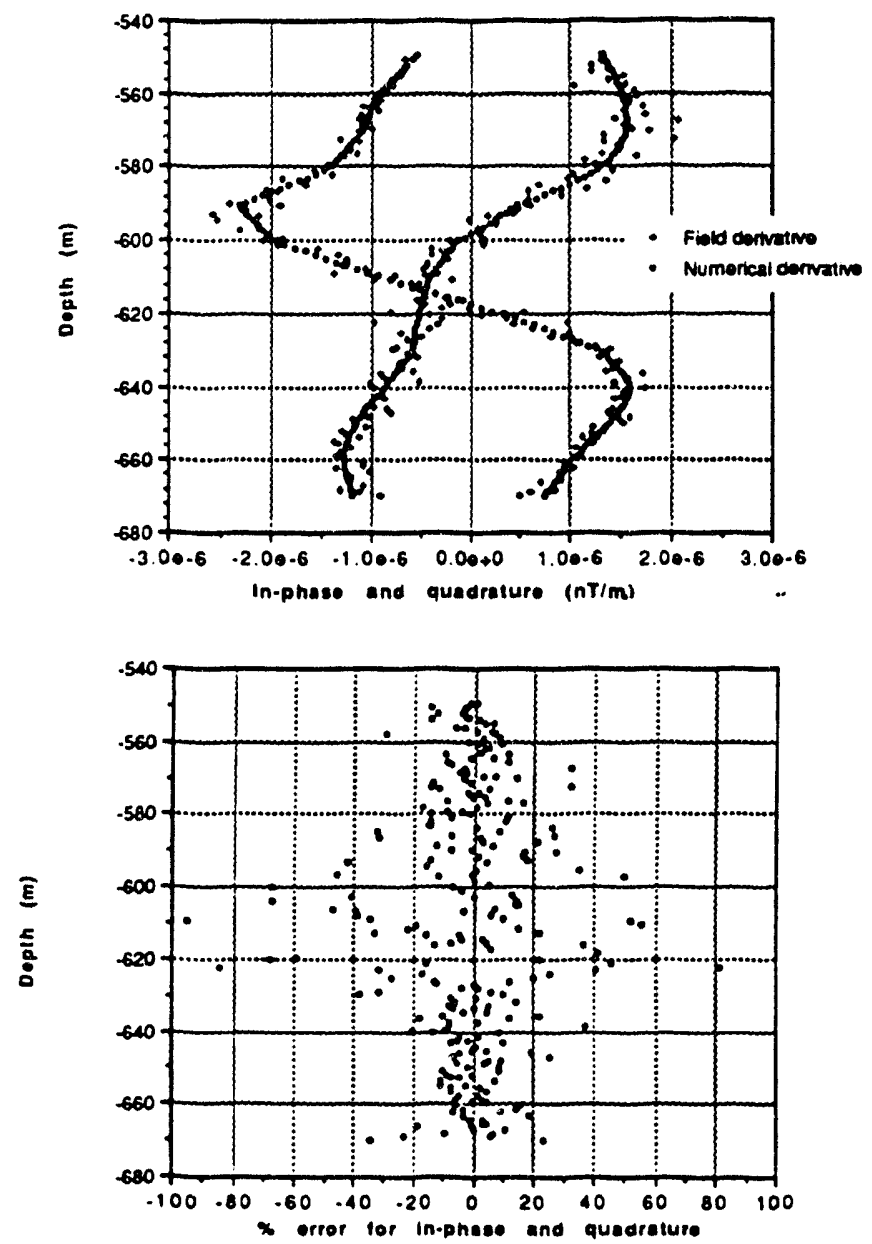

Fig. 5.3.3-2 The least squares inversion at $512 \mathrm{~Hz}$ using in-phase and quadrature of the first vertical derivative $\delta \mathrm{H}_{2} / \delta \mathrm{z}$ from receiver located at $609.6 \mathrm{~m}$ (2000 ft). 

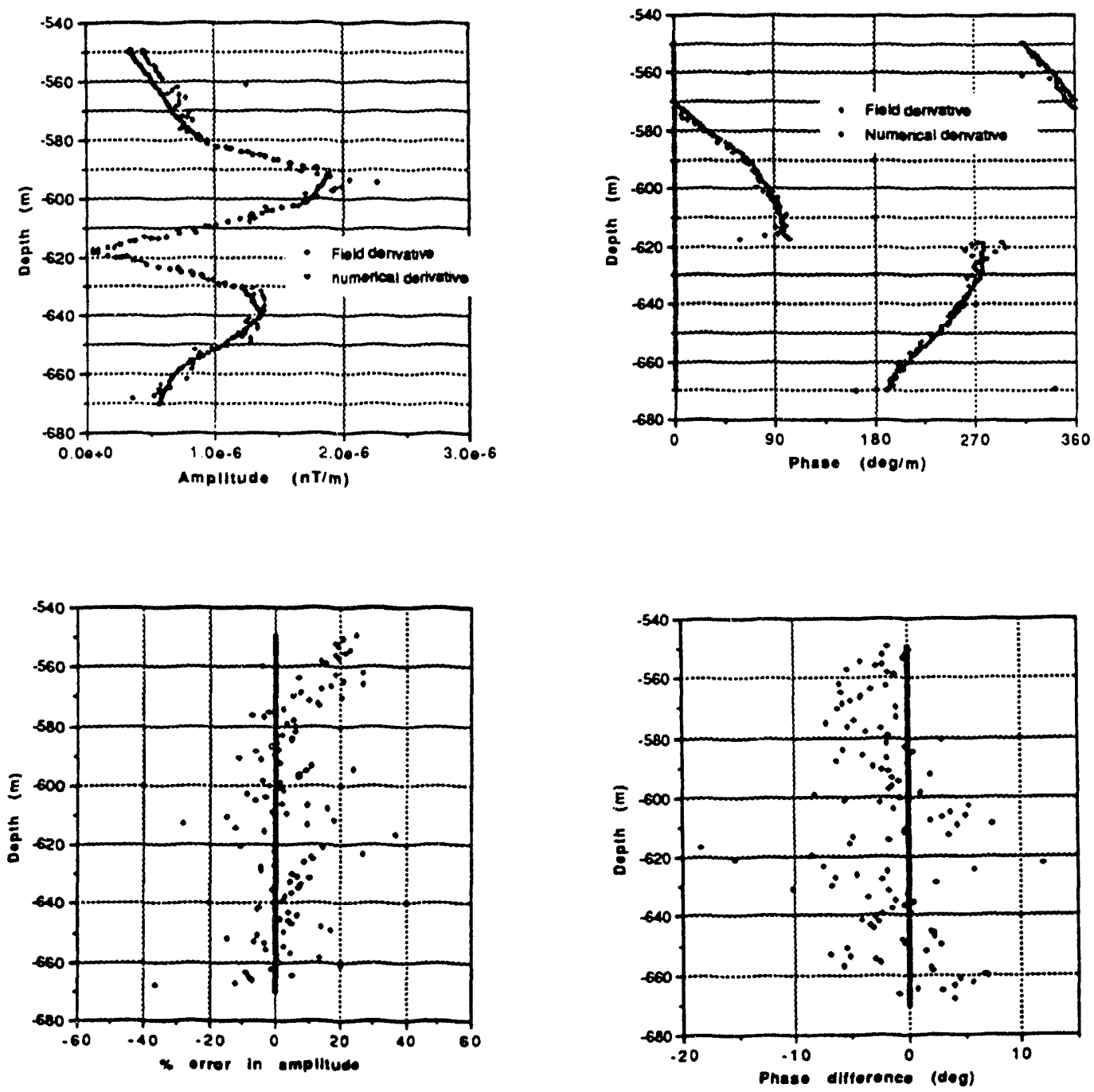

Fig. 5.3.3-3 The least squares inversion at $2048 \mathrm{~Hz}$ using amplitude and phase of the first vertical derivative $\delta \mathrm{H}_{\mathrm{z}} / \delta \mathrm{z}$ from receiver located at $609.6 \mathrm{~m}(2000 \mathrm{ft})$. 


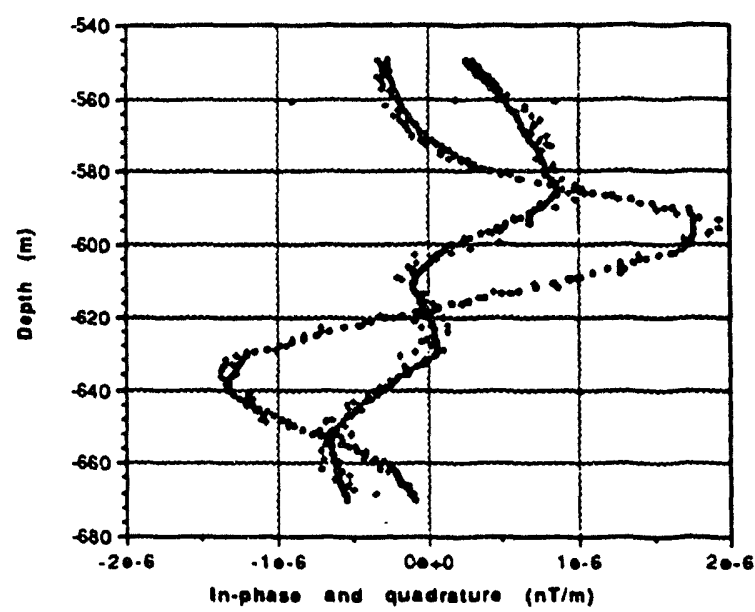

- Field comarive

- Numerteal convarime

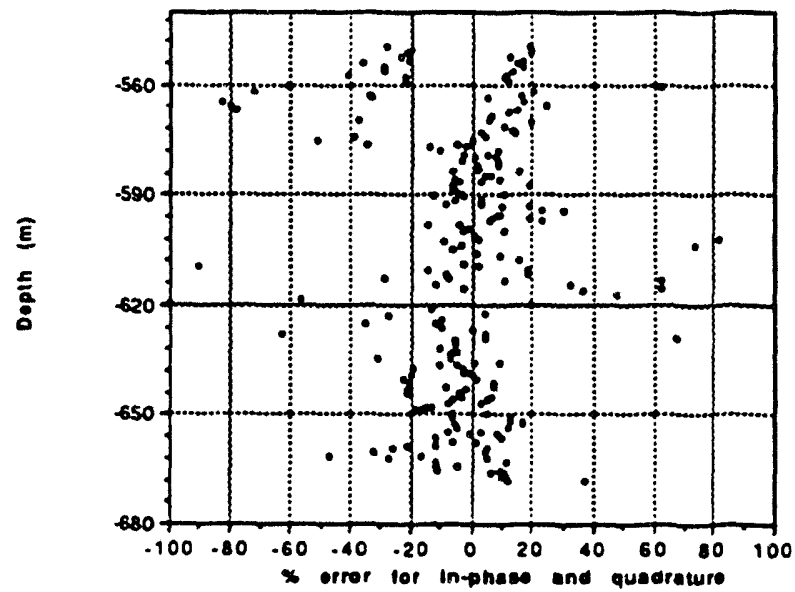

Fig. 5.3.3-4 The least squares inversion at $2048 \mathrm{~Hz}$ using in-phase and quadrature of the first vertical derivative $\delta \mathrm{H}_{z} / \delta \mathrm{z}$ from receiver located at $609.6 \mathrm{~m}(2000 \mathrm{ft})$. 


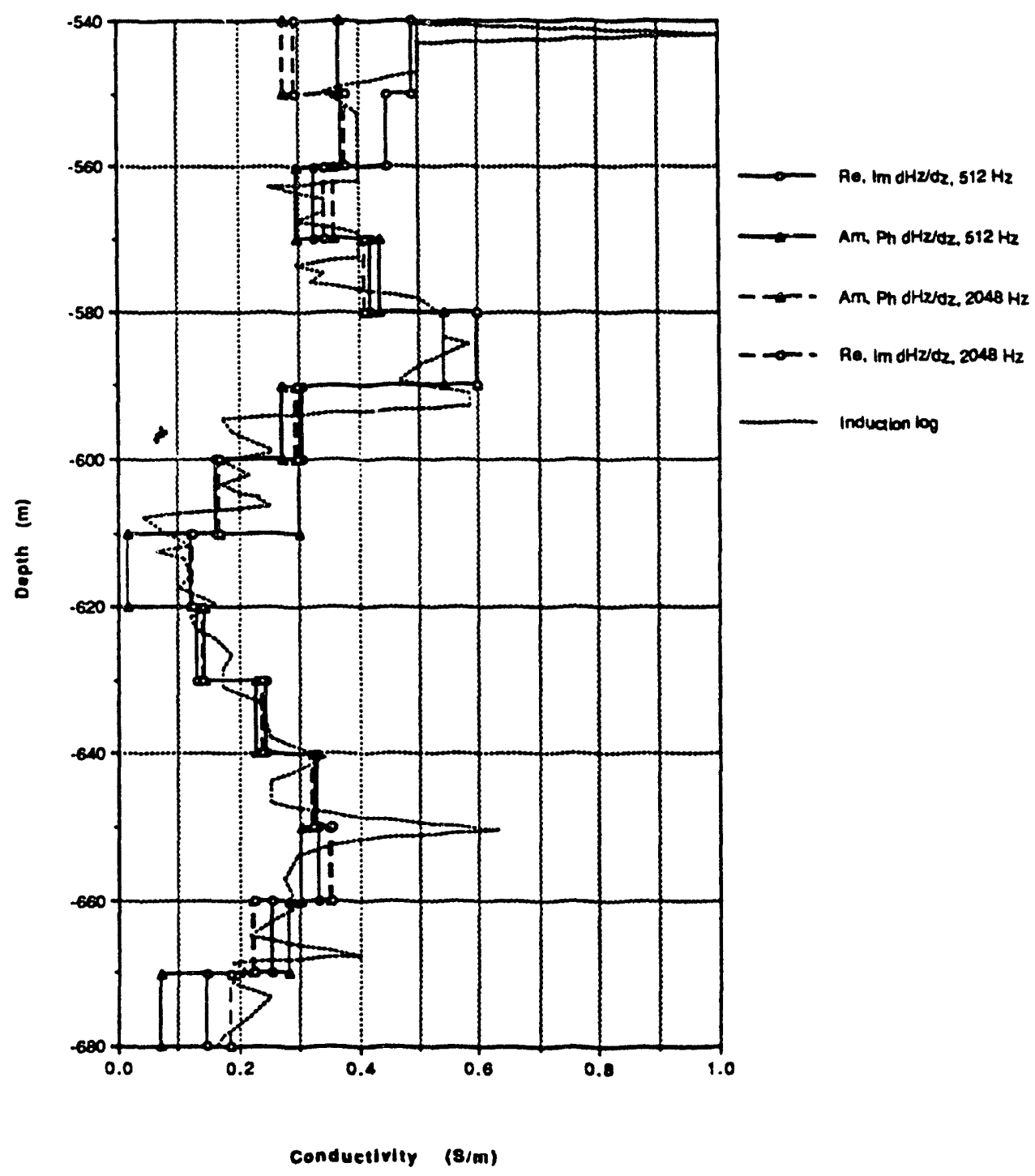

Fig. 5.3.3-5 The fourteen layer model of conductivity distribution in Devine obtained from the least-squares inversions using first vertical derivatives. 

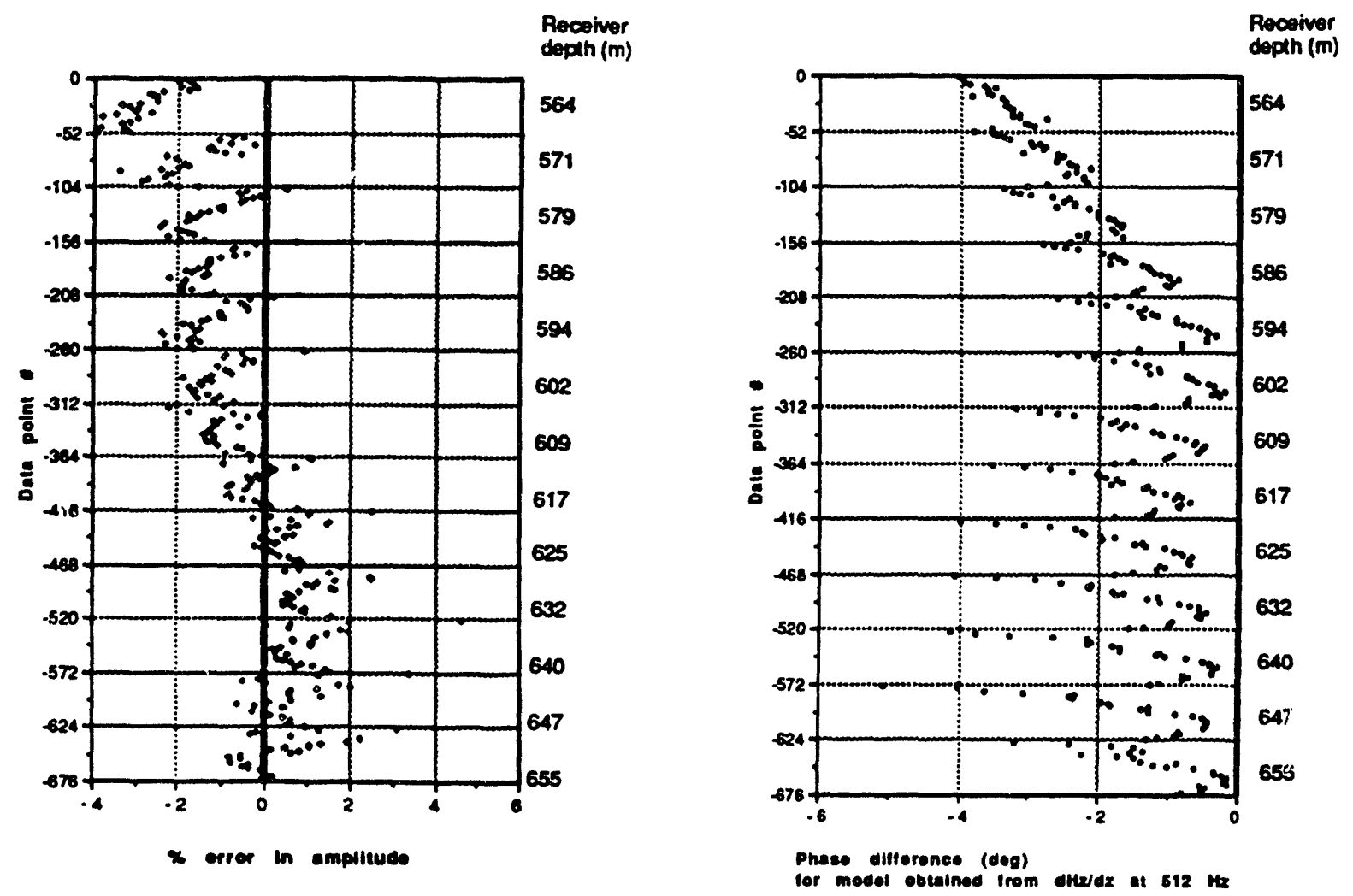

Fig. 5.3.3-6 The fit for amplitude and phase components at $512 \mathrm{~Hz}$ between measured values and the data calculated using the 14 layer model obtained from the vertical derivative inversions of data collected at $512 \mathrm{~Hz}$. 

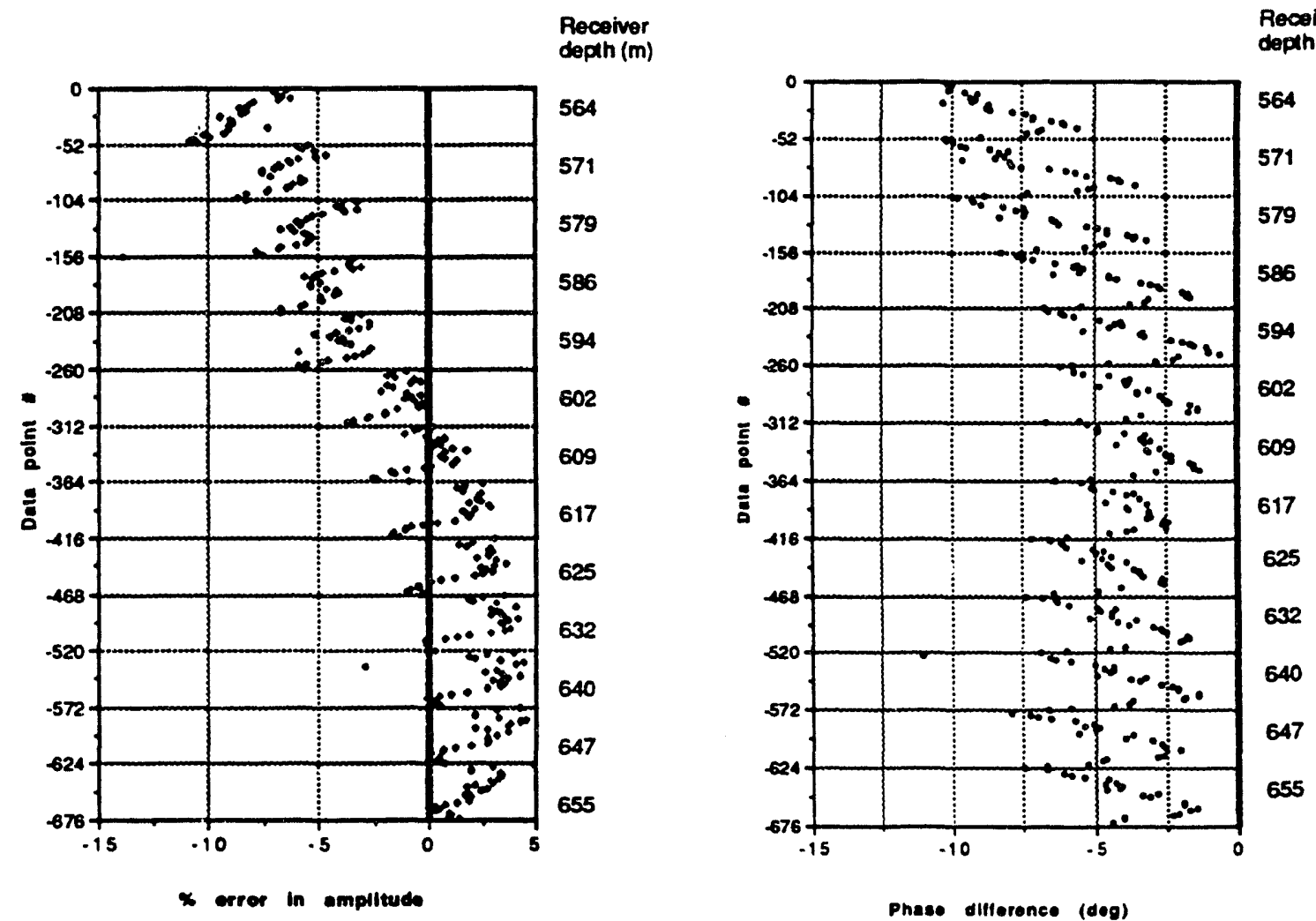

Fig. 5.3.3-7 The fit for amplitude and phase components at $2048 \mathrm{~Hz}$ between measured values and the data calculated using the 14 layer model obtained from the vertical derivative inversions of data collected at $512 \mathrm{~Hz}$. 

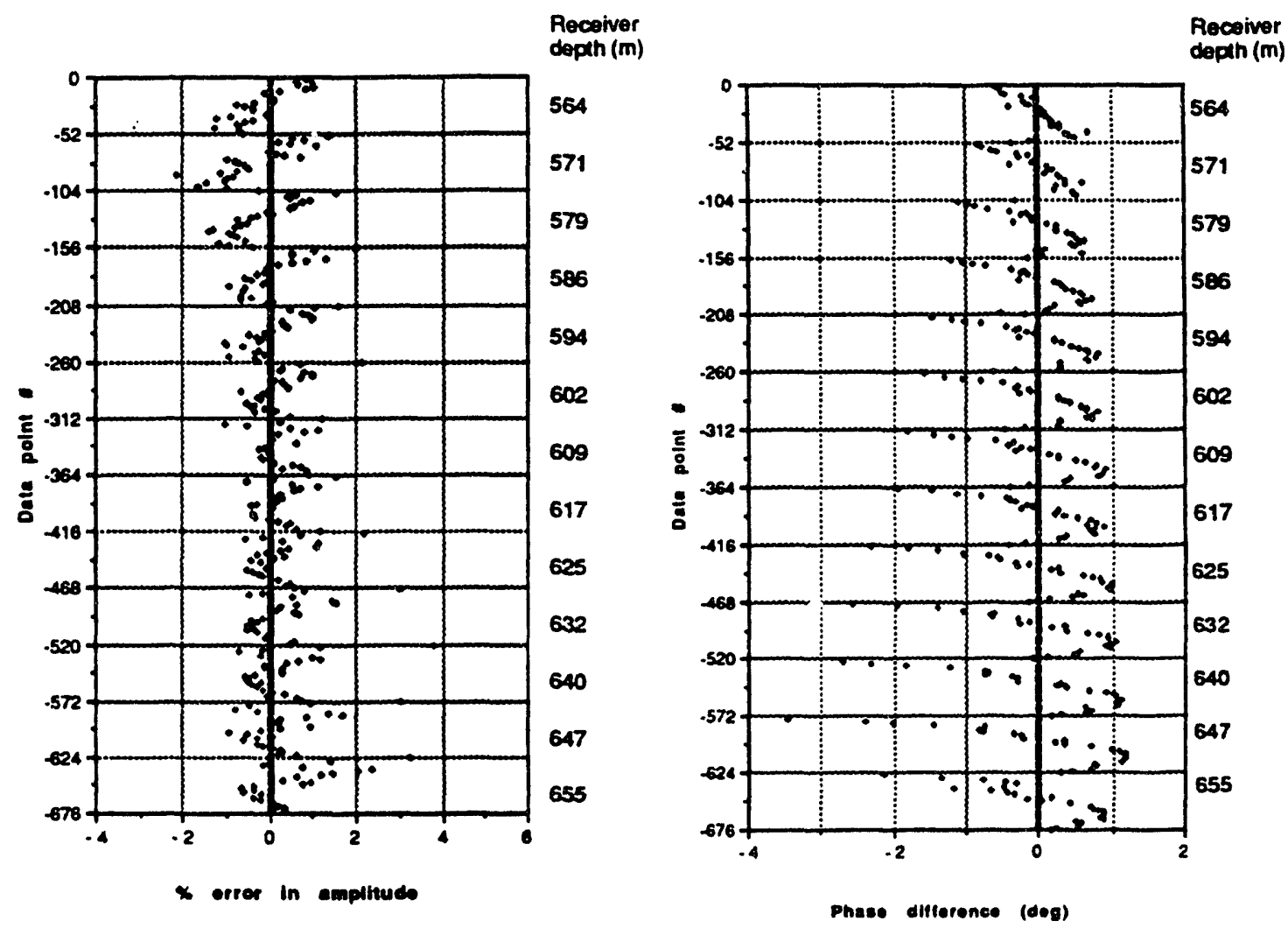

Fig. 5.3.3-8 The change in fit for amplitude and phase components at $512 \mathrm{~Hz}$ when the amplitude and phase corrections, different for each profile were incorporated into the inversion (compare with Fig. 5.3.3-6) 


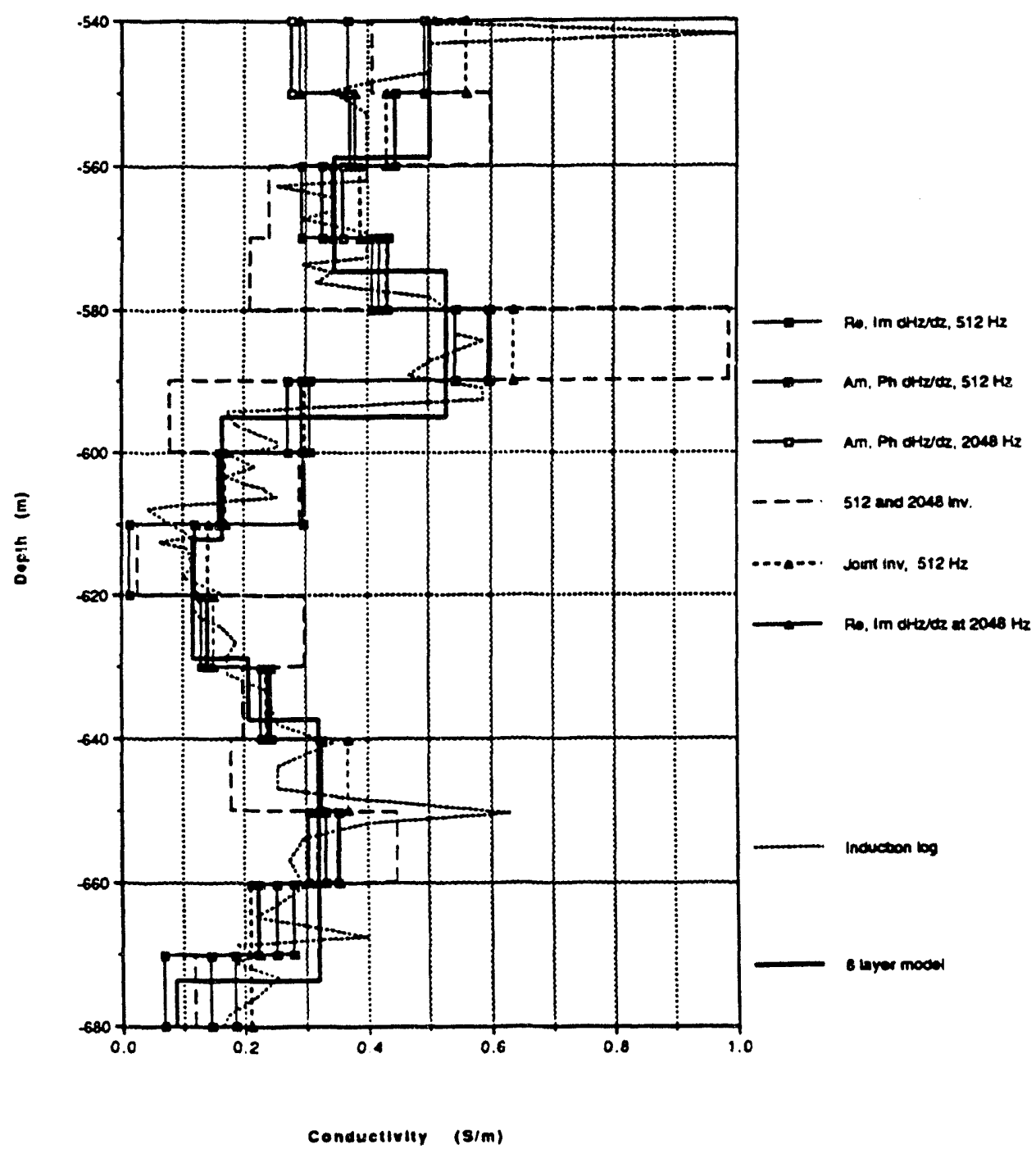

Fig. 5.3.3-9 The comparison of conductivity models for Devine obtained using different inversion schemes and frequencies. 

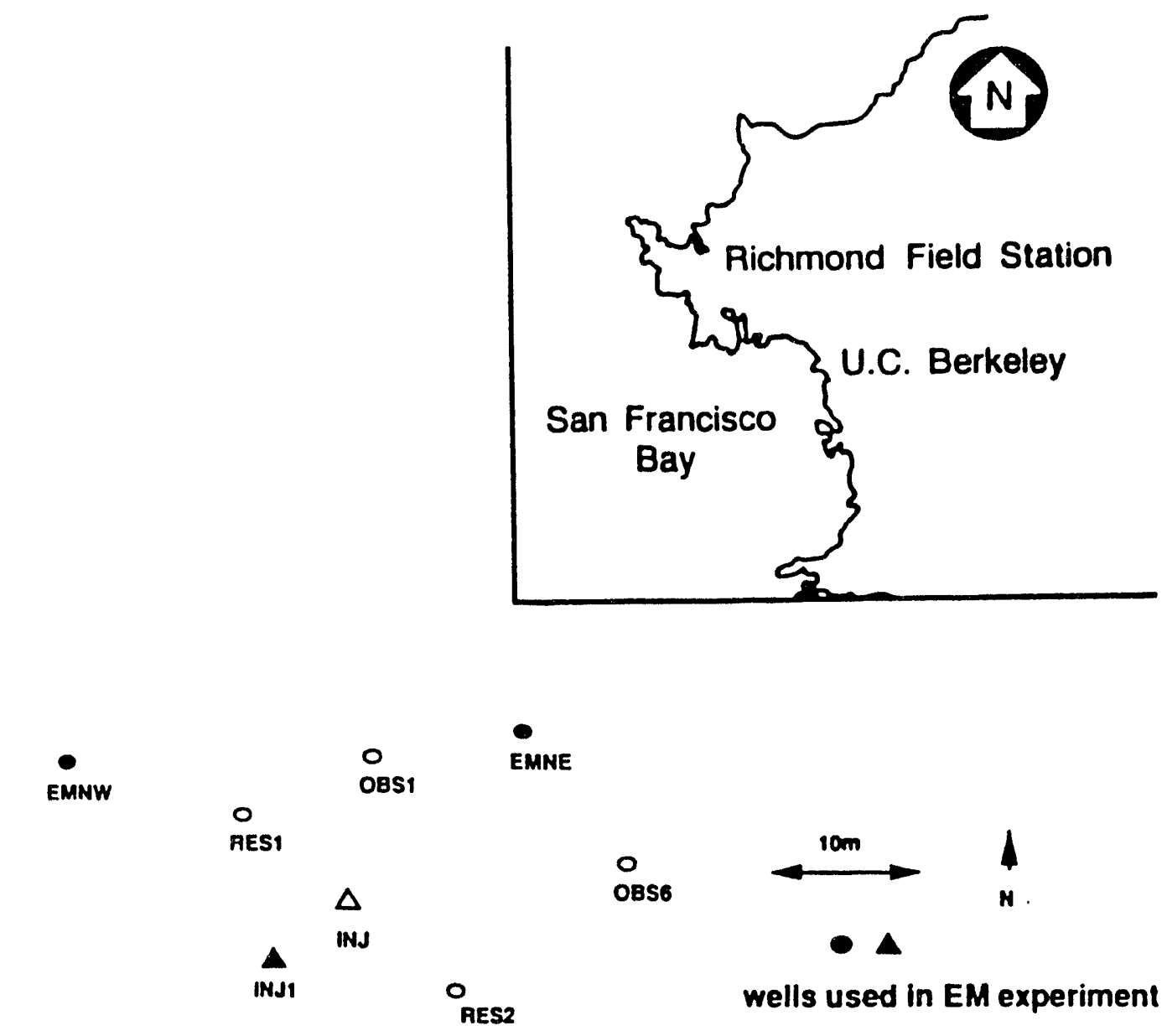

O OBSA

$\Delta$

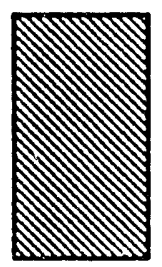

Bullding 300

Fig. 5.4-1 The location and the experimental set-up of the Richmond Field Station, California. 
Borehole Log EMNE

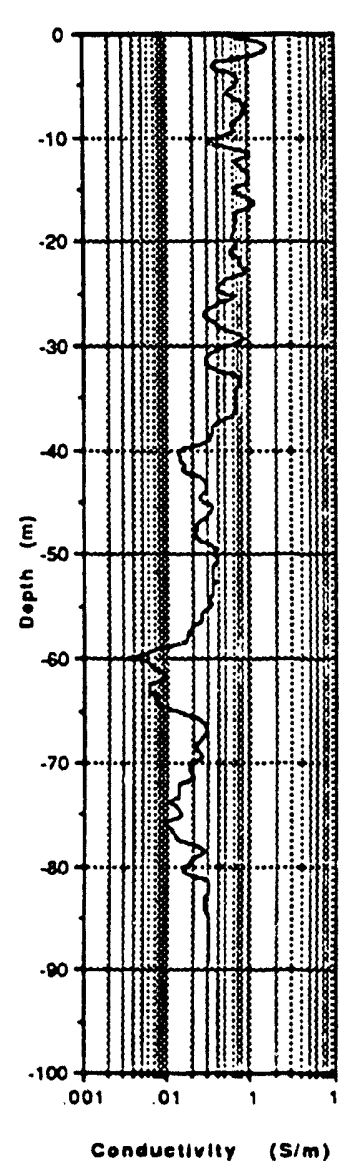

40 I
Borehole Log EMSW

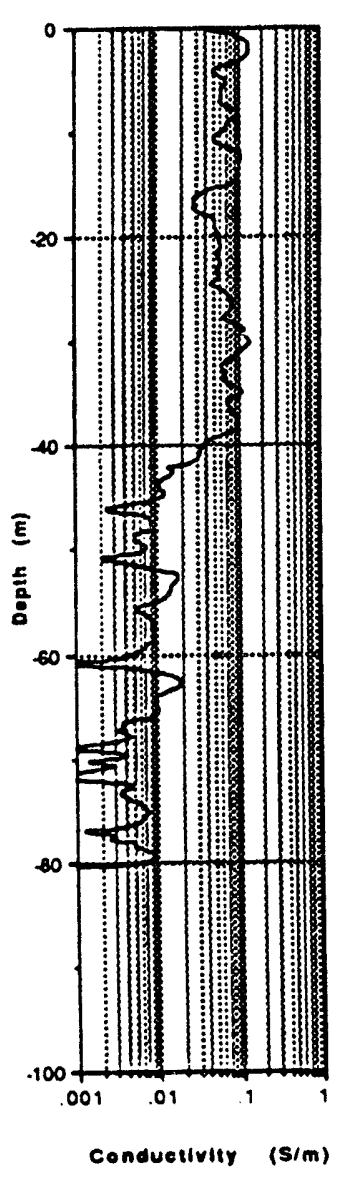

0

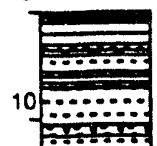

\%?

$\because \because \because 3$

$2 0 \longdiv { 1 0 }$

………

30

-

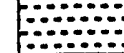

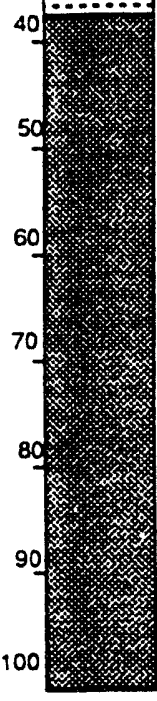

(sim)

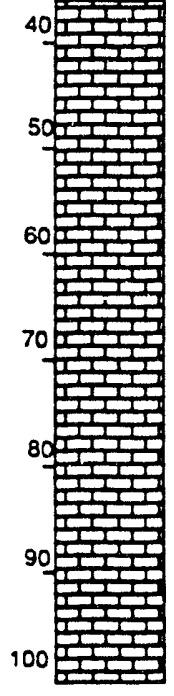

(1)

$\ldots$
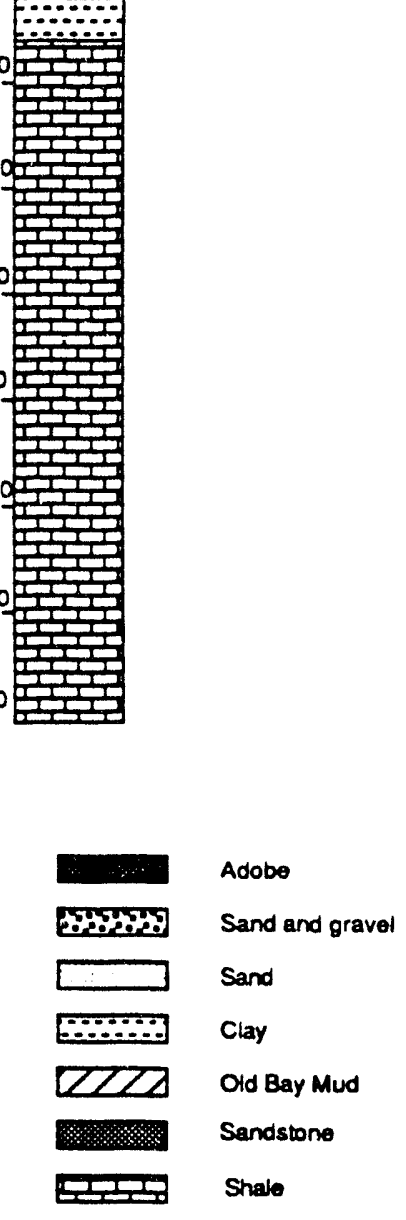

Fig. 5.4-2 The borehole induction logs and the geologic sections of the Richmond Field Station. 

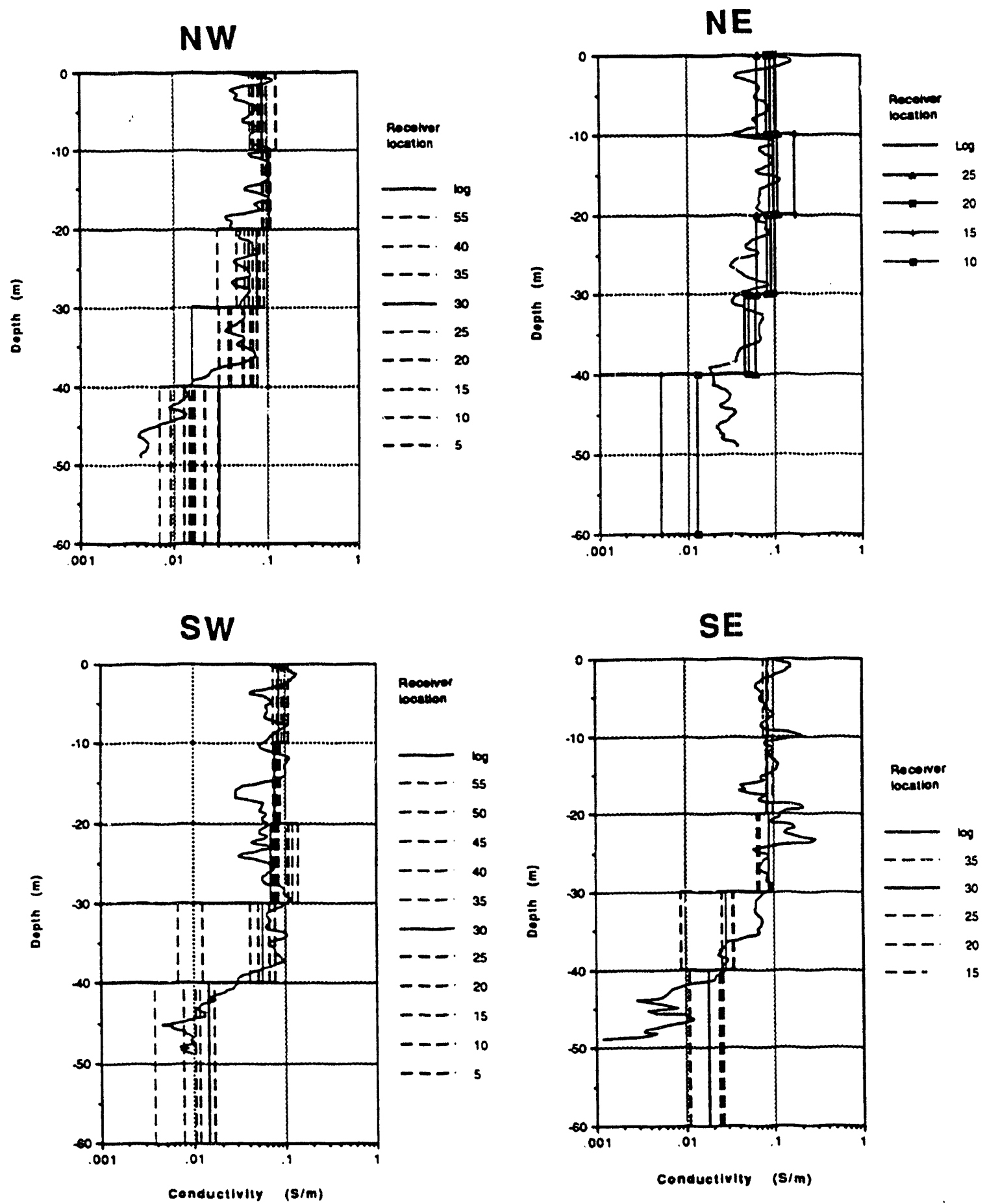

Fig. 5.4-3 The illustration of conductivity variations obtained from individual inversions of profiles on different receiver locations superimposed on the induction log. The numbers on the right side represent the location of receiver. The best fitting profiles were used for NE and SE wells. 

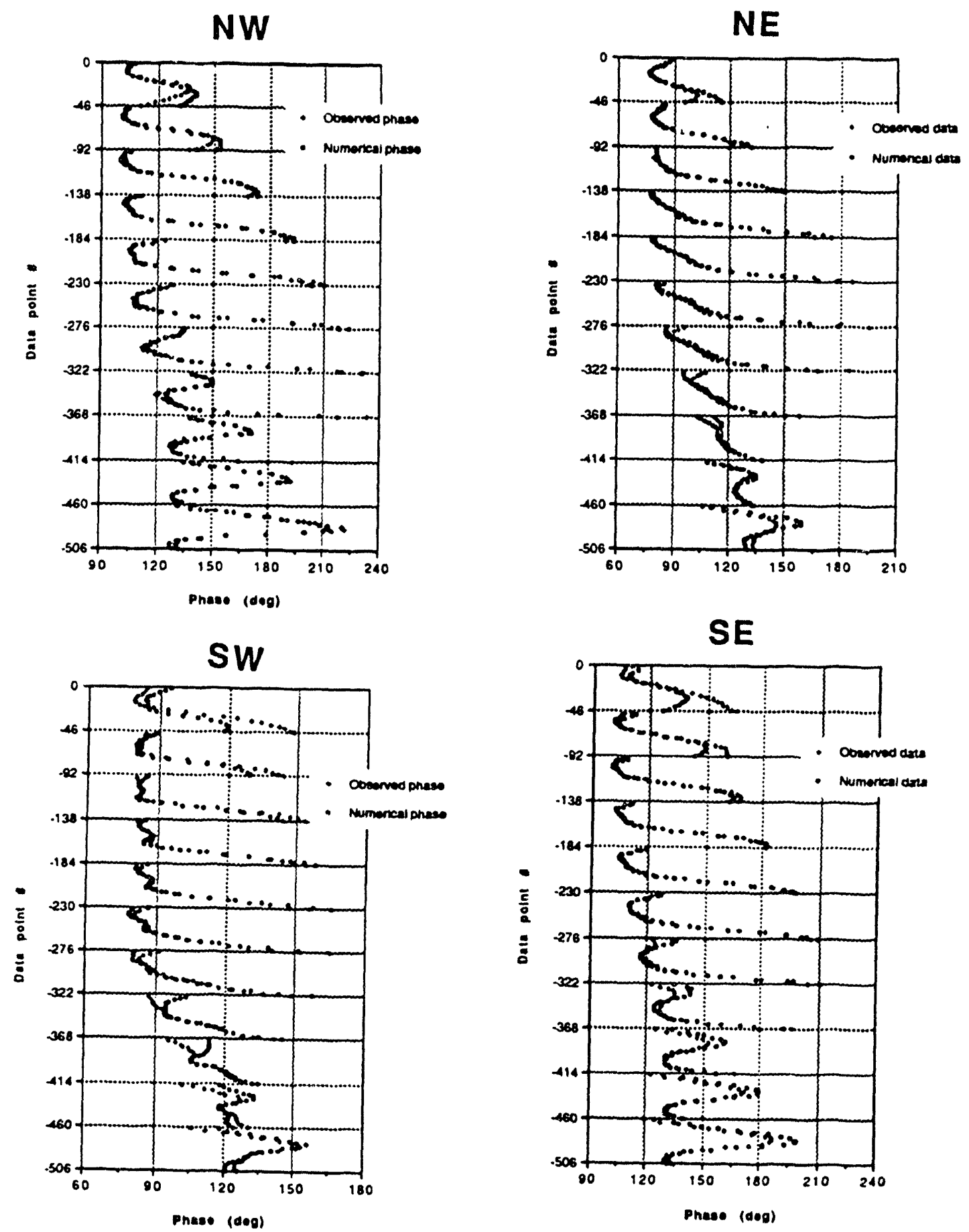

Fig. 5.4-4 The fit between observed and calculated phase based on the joint inversion of amplitude and phase data using simultaneous inversions of eleven profiles from all receiver locations for each of the observation wells: NW, NE, SE and SW. Each 46 point section represents one profile, starting from the profile collected with receiver located $5.0 \mathrm{~m}$ below surface and ending with the receiver located $55.0 \mathrm{~m}$ below surface. 
NW

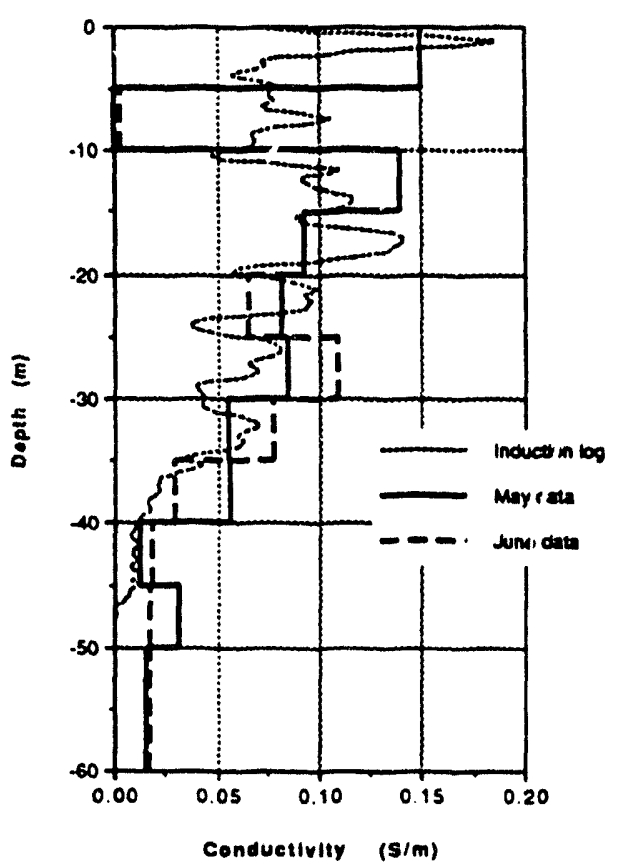

sw

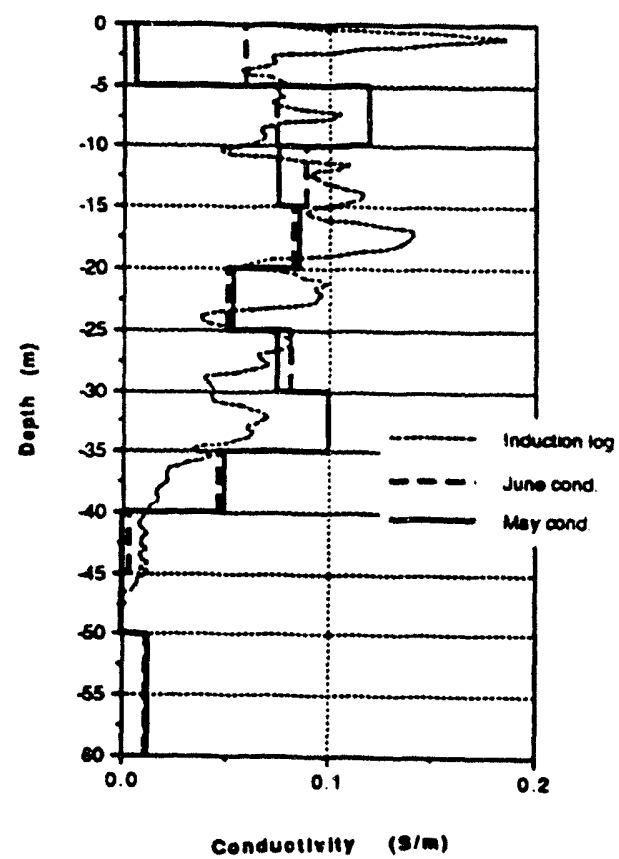

NE

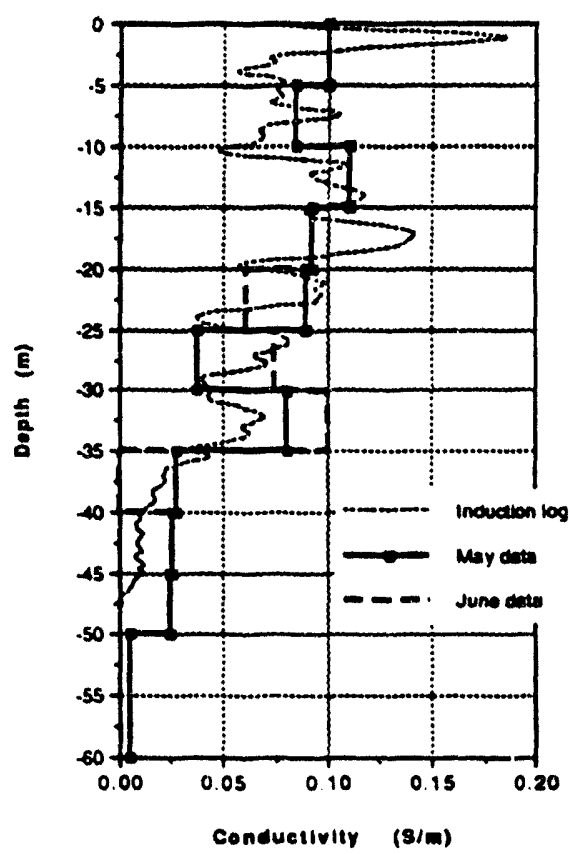

SE

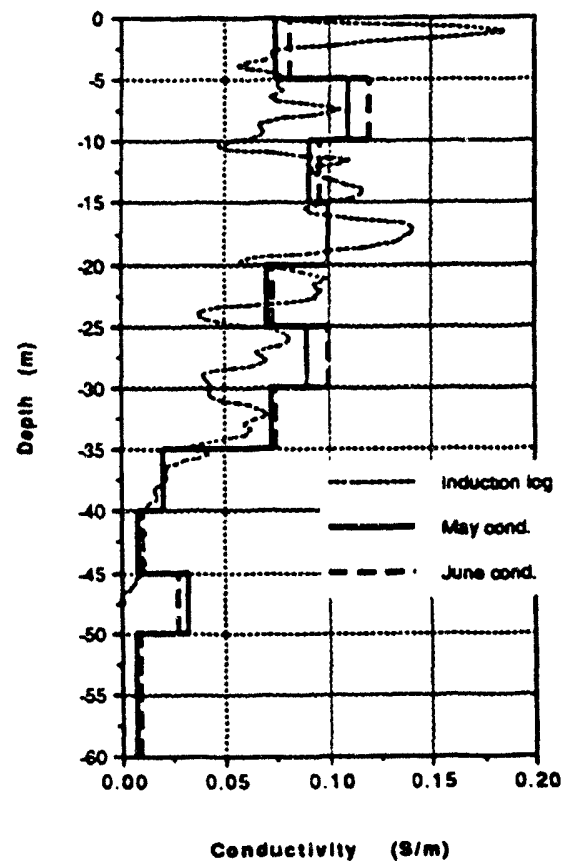

Fig. 5.4-5 Model of conductivity for NW, NE, SE and SW wells obtained from joint inversion of amplitude and phase data using 11 profiles simultaneously of two sets of data: one collected in May before injection of salt water, the second collected in June after the injection of salt water. 

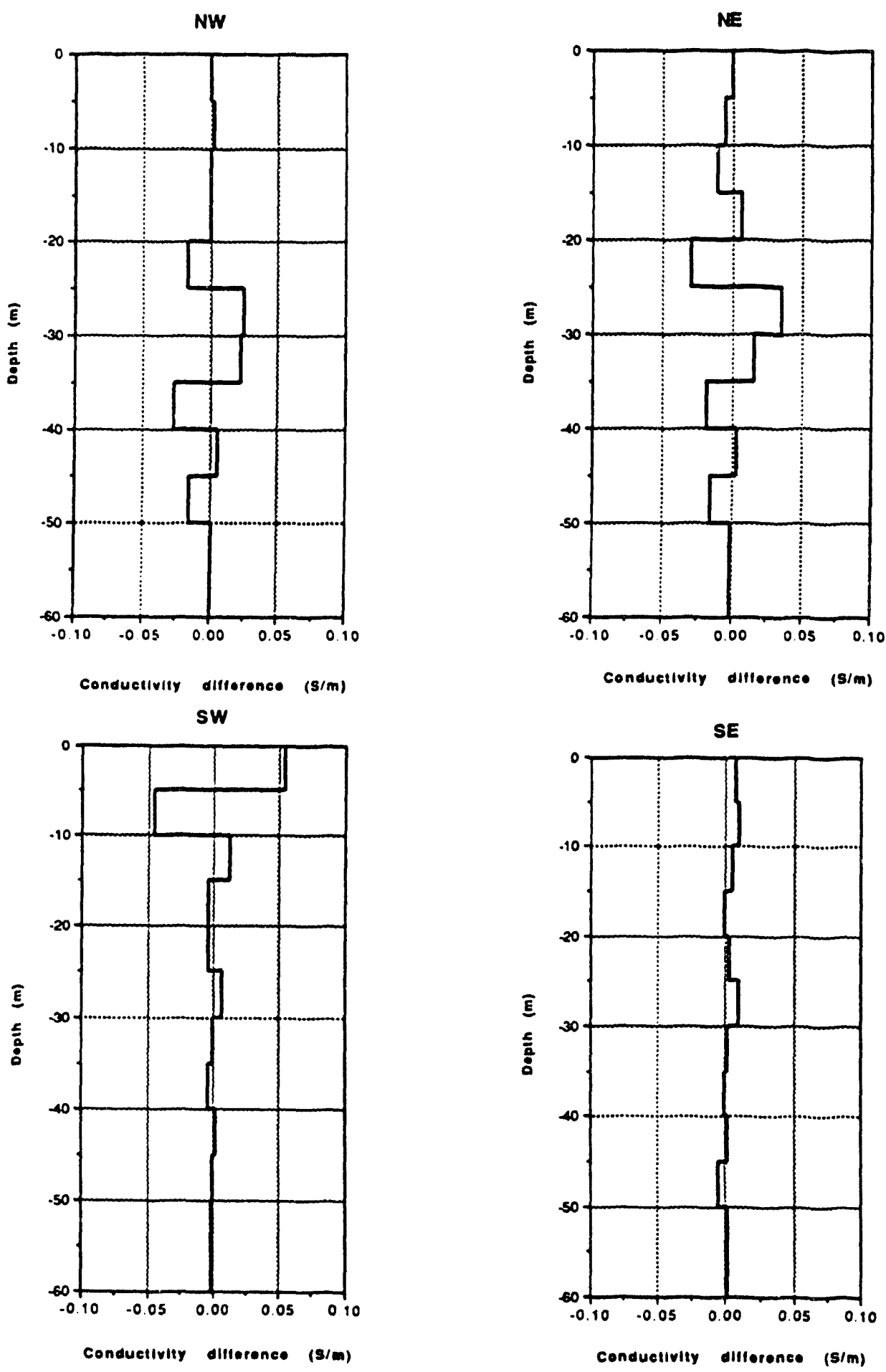

Fig. 5.4-6 The difference in conductivities before and after the injection of salt water for NW, NE, SE and SW wells obtained by subtracting the results presented in Figure 5.4-5. 

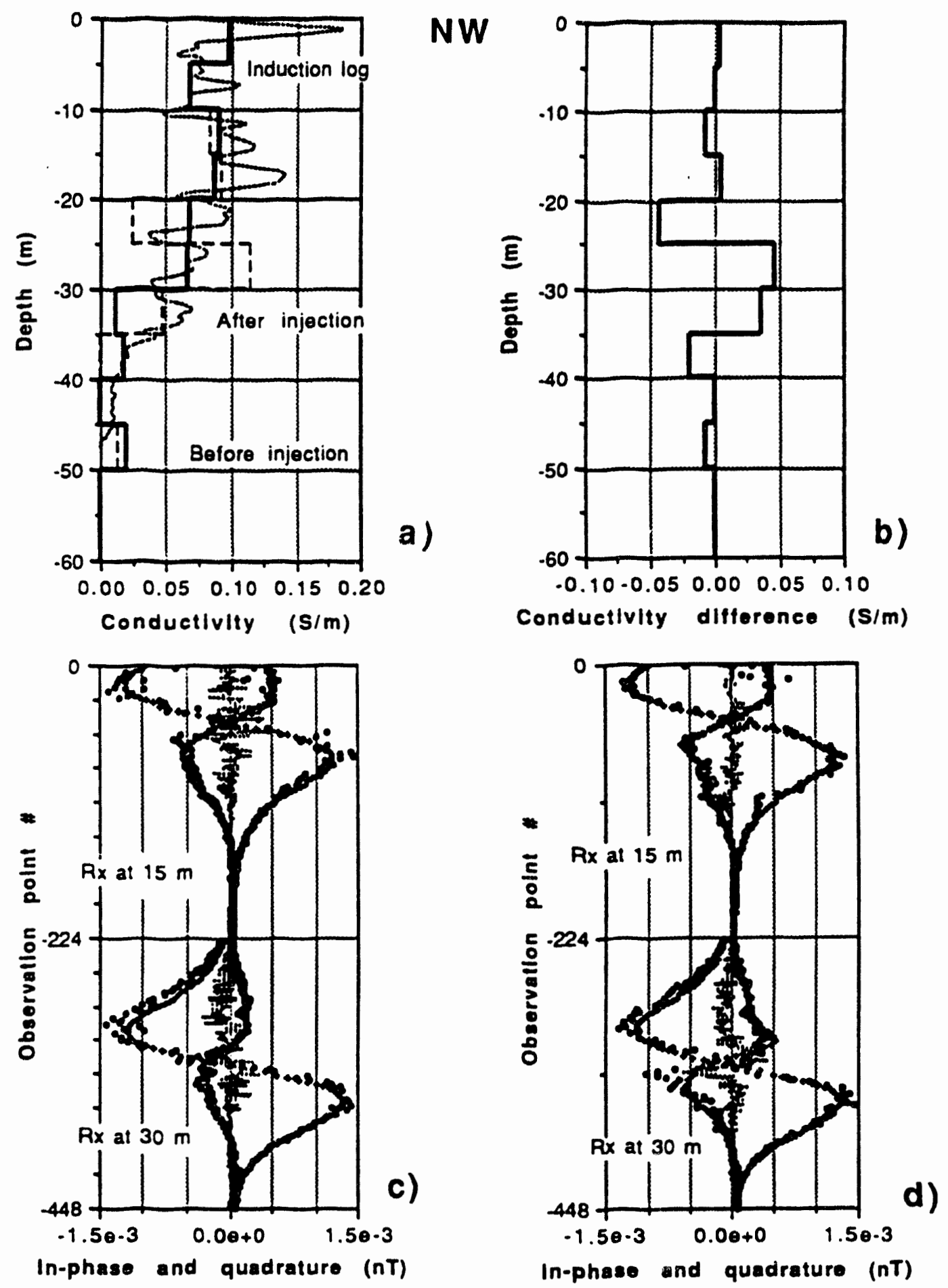

Fig. 5.4-7 The results of the least-squares inversion using the first vertical derivative $\Delta \mathrm{H}_{\mathrm{z}} / \Delta \mathrm{z}$ for the NW well; a) shows the model of conductivity of the data before and after the injection of salt water superimposed on the induction log from injection well; b) represents the conductivity difference before and after the injection; c) and d) show the observed and numerical in-phase and quadrature components with misfit error for data before and after the injection, respectively. 

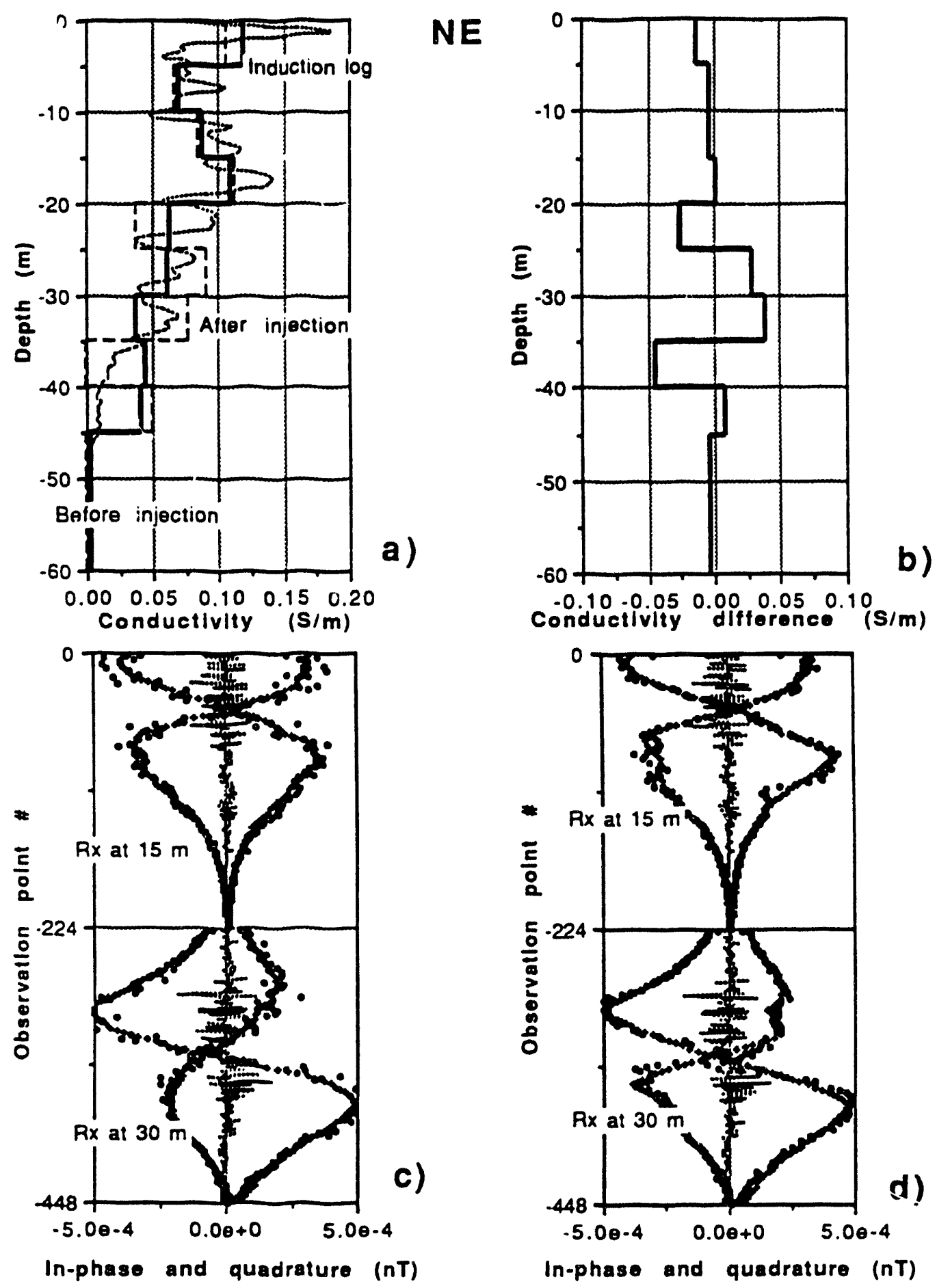

Fig. 5.4-8 The results of the least-squares inversion using the first vertical derivative $\Delta \mathrm{H}_{2} / \Delta \mathrm{z}$ for the NE well; a) shows the model of conductivity of the data before and after the injection of salt water superimposed on the induction log from injection well; b) represents the conductivity difference before and after the injection; c) and d) show the observed and numerical in-phase and quadrature components with misfit error for data before and after the injection, respectively. 

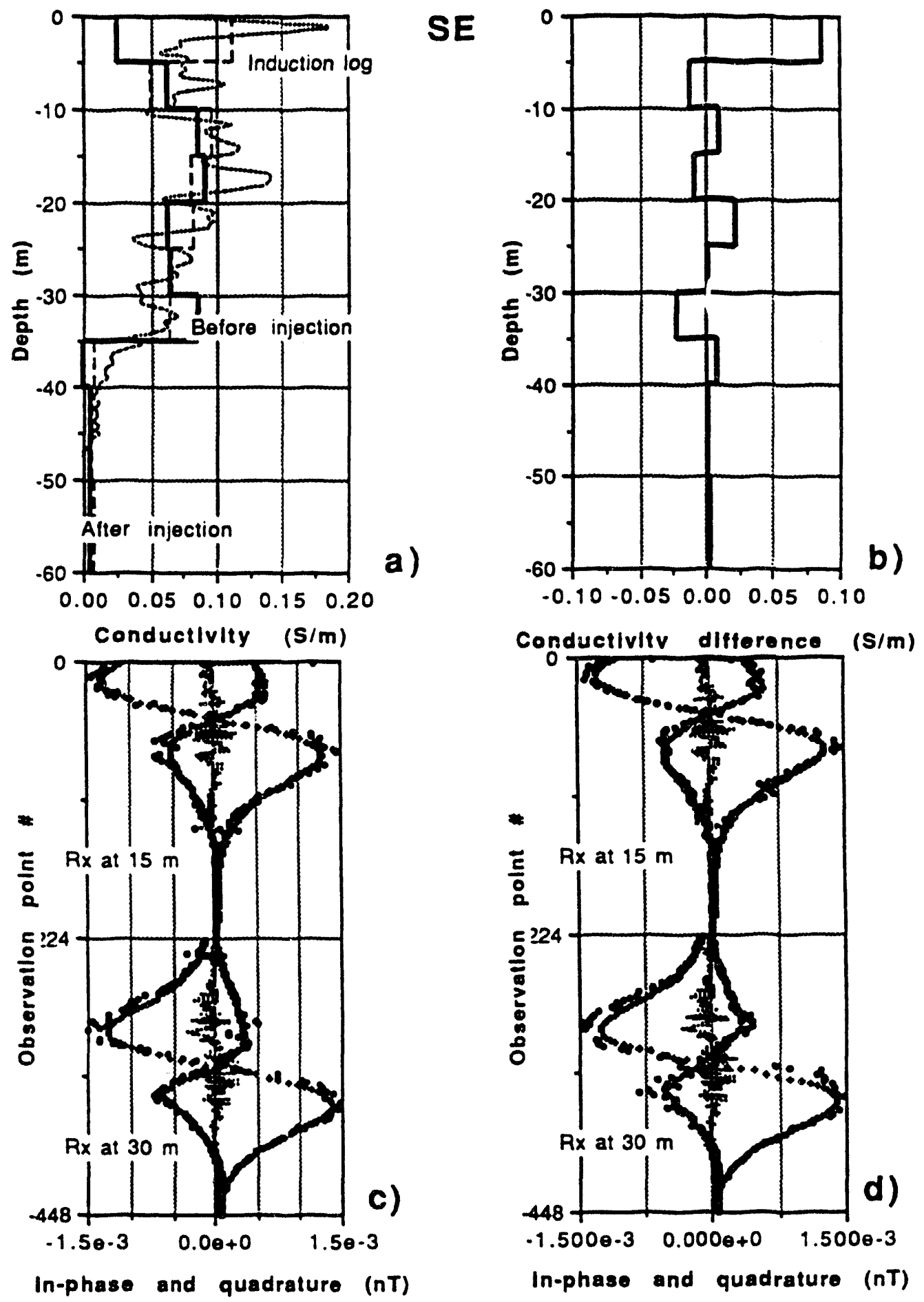

Fig. 5.4-9 The results of the least-squares inversion using the first vertical derivative $\Delta \mathrm{H}_{\mathrm{z}} / \Delta \mathrm{z}$ for the SE well; a) shows the model of conductivity of the data before and after the injection of salt water superimposed on the induction log from injection well; b) represents the conductivity difference before and after the injection; c) and d) show the observed and numerical in-phase and quadrature components with misfit error for data before and after the injection, respectively. 

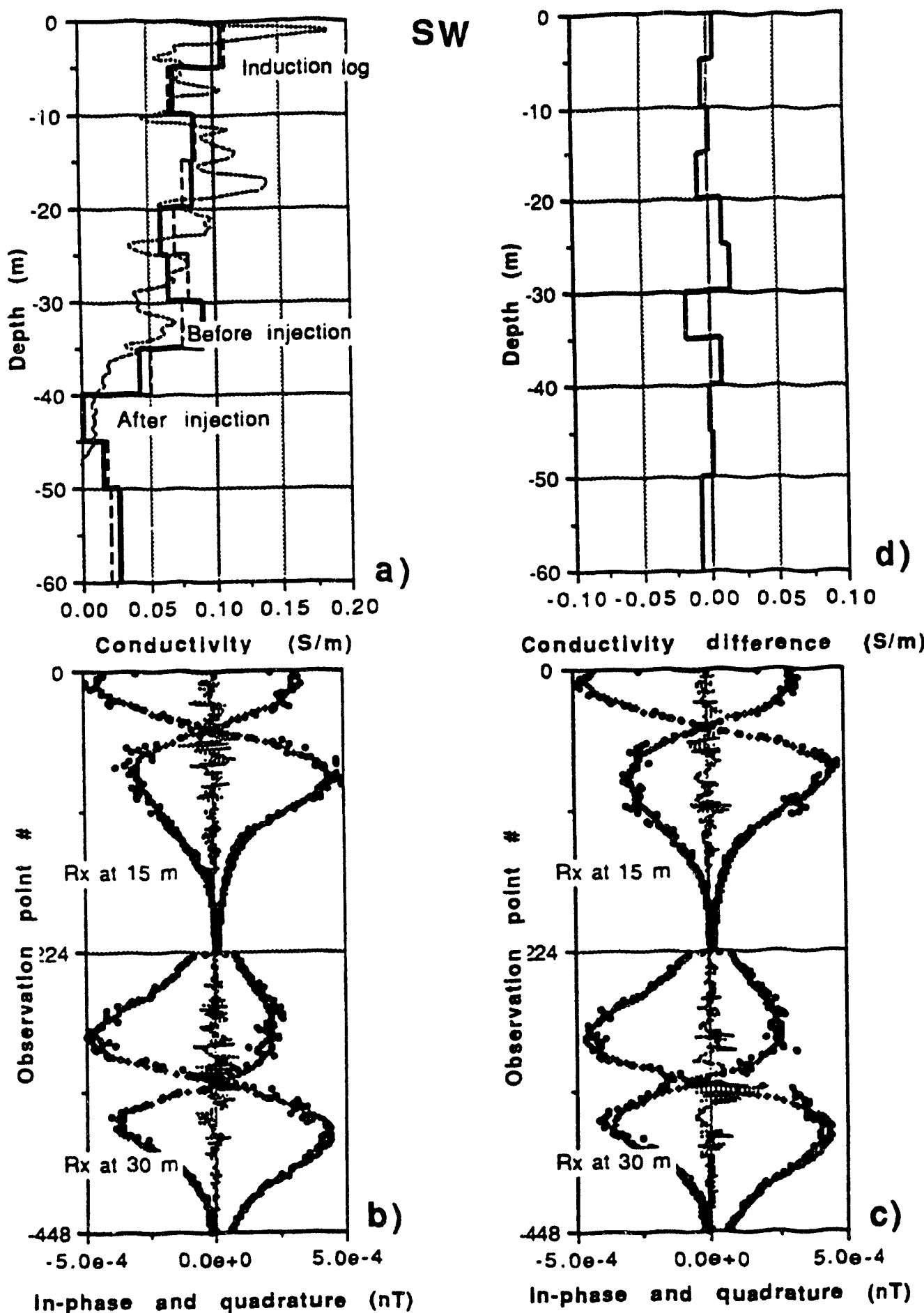

Fig. 5.4-10 The results of the least-squares inversion using the first vertical derivative $\Delta \mathrm{H}_{2} / \Delta \mathrm{z}$ for the SW well; a) shows the model of conductivity of the data before and after the injection of salt water superimposed on the induction log from injection well; b) represents the conductivity difference before and after the injection; c) and d) show the observed and numerical in-phase and quadrature components with misfit error for data before and after the injection, respectively. 


\section{Chapter 6 \\ Conclusions and recommendations for future research.}

\subsection{Conclusions}

This work has examined some basic characteristics of electromagnetic measurements inside and between boreholes. In Chapter 1 we summarized the evidence of laboratory research and field experience which shows the importance of using electrical conductivity maps for defining the distribution of subsurface fluids. Furthermore we showed that the conductivity measurements seem to be more suited for this purpose than the seismic methods, because the corductivity variations are larger than the corresponding velocity variations when temperature, saturation or salinity vary.

In Chapter 2 we examined the fields in a conductive whole-space to evaluate the range of penetration, sensitivity to conductivity and the influence of errors in the transmitter and the receiver location. We showed that different components of the magnetic fields respond differently to the properties of the medium and that the response of a particular component depends on the geometry between the transmitter and the receiver. For example close to the transmitter the fields vary rapidly with distance and for this reason it is more important to have an accurate and stable measurements of positions closer to the transmitter than far away from it.

In Chapter 3 we considered the boundary between two conductive whole-spaces, and a layer sandwiched between two conductive half-spaces as well. Using analytic expressions on the boundary between two conductive half-spaces we concluded that the first vertical derivative of the fields is more sensitive to the layer boundaries than the vertical $\mathrm{H}_{z}$ component itself, and is as sensitive as the horizontal field $\mathrm{H}_{\rho}$. Using fields on the boundary we showed that the more conductive half-space dominates the response and it is necessary to use higher induction numbers to detect conductivity variations inside the resistive half-space. Using the explicit expressions that describe the fields above the boundary we showed that the response above the boundary depends, among other parameters, on the total transmitter-boundary and receiver-boundary distances and not on the particular distance of the transmitter or receiver from the boundary. We also showed that when the transmitter and the receiver are placed on the opposite sides of the layer, in a medium that has the same conductivity on both sides, then the response from the layer is independent of its location.

In Chapter 4 we ased the second vertical derivative to recover the conductivity distribution on the axis of the transmitter and it a crosshole environment. We showed 
that the conductivity closely correlates with the induction log measurements and we used this fact to correct for the depth difference between the induction log and our measurements at the Devine. We showed that the conductivity distribution on the axis of a transmitter is best recovered if the vertical transmitter-receiver separations are small. However at close transmitter-receiver separations the fields vary rapidly with distance and even a small error in position can cause large error in the calculations of the second vertical derivative. This suggests that the measurements of the gradient rather than the field itself would be more practical. Furthermore we observed that the calculation of the second vertical derivative using finite differences is more accurate at higher frequencies because the fields vary faster with distance and for this reason the measurements do not require so many significant digits in order to calculate the difference accurately.

In Chapter 5 the inversion results show that the crosshole measurements are very sensitive to the parameters of the medium. However they are also very sensitive to the mutual geometry between the transmitter and the receiver. If the geometrical parameters of the measurements are not known exactly, or are different from these assumed, than the resulting model is biased. In such case it is difficult or impossible to obtain consistent models for every profile or frequency combination inside a 1-D medium.

The requirement of fitting the data within the measurement error is important when the conductivity model in the inversion adequately represents the conductivity distribution at the field site. The Devine test site is an example of excellent agreement where the fit between the numerical data and field data was close to the measurement error. If the model is highly complicated and not one-dimensional, as was the case in the Richmond Field Station experiment, then it is still possible to obtain useful information about the conductivity variations from the crosshole measurements using the onedimensional inversion. In this case the apparent conductivity distribution shows the largest variations in the direction of fluid propagation. The inversion tests also proved that the higher frequencies are more sensitive to the model parameters than the lower frequencies but at the same time it is more difficult to fit the model to the data. We also found that the least-squares inversion of the $\mathrm{H}_{2}$ fields works better if we use amplitude and phase data rather than in-phase and quadrature data. The reason for this is that the inphase and quadrature data are very noisy in the regions where they change sign because the fields are small in these regions.

The most important conclusion of our work using 1-D inversion is the discovery that the use of the vertical derivative in place of $\mathrm{Hz}$ field results in a faster convergence to the model that is consistent at high and low frequencies. The model is also almost 
independent on the initial guess if the inversion is performed on the in-phase and quadrature derivatives jointly.

\subsection{Recommendations for future research}

In Chapter 4 we showed the possibility of using the second vertical derivative to determine the conductivity of the medium in the immediate neighborhood of the borehole. We showed that the method can work at frequencies as low as $100 \mathrm{~Hz}$. Because these low frequencies still show a good sensitivity to the conductivity of the medium, they should be examined for application inside cased boreholes. The possibility of measurements inside cased boreholes is currently examined by LLNL and the first preliminary measurements inside cased boreholes (M. Wilt et al., 1990) showed that the fields are attenuated, but still measurable.

We also showed that it is possible to recover the induction log information in the crosshole configuration using the second derivative of the $\mathrm{H}_{2}$ component. These results were found to be sensitive to the amplitude and phase of the $\mathrm{H}_{\mathrm{z}}$ fields. For this reason we feel that there is a possibility of using the crosshole measurements together with induction logs to obtain an independent estimate of the amplitude and phase calibration of the field system. Working with the second vertical derivative (and first vertical derivative as well) we observed that profiles collected with different receiver locations are strongly correlated. This fact can be used in data processing to get rid of the some of the random noise that is not obvious in the $\mathrm{H}_{2}$ fields themselves.

In Chapter 5 we presented the results of least-squares inversion for the Devine test site. We feel that this site can be a perfect place to calibrate the crosshole-electromagnetic field systems. For this purpose a set of very accurate crosshole measurements (more accurate that our data set) should be completed, preferably at high and low frequency. This is already a realistic task since the system used for collecting the Devine data was very recently modified by O. Tseng and M. Wilt (October 1993) and is capable of repeating the data within $0.2 \%$ in amplitude and 0.1 degrees in phase. The absolute measurements of depths and transmitter - receiver separations (including the corrections for the possible change in geometry due to the cable stretching) within $0.1 \mathrm{~m}$ are as important as the EM measurements themselves. The data should be inverted to produce an equivalent conductivity distribution in the similar way as we attempted to do for our data set, but without relaxing the requirements on the misfit errors.

We recommend that further work on crosshole measurements include a calibration of the equipment at Devine and be extended to measurements through casing. 


\section{References.}

Abramovitz, M., and Stegun, I. A., 1965, Handbook of mathematical functions: Dover, New York.

Al-Chalabi, M., 1992, When least-squares squares least: Geophys. Prosp., 40, 359 378.

Alumbaugh, D. L., and Morrison, H. F., 1992, Tomographic imaging of cross-well EM data: 62nd Ann. Internat. Mtg., Soc. Expl. Geophys., Expanded Abstracts, SEG, Session EM4-4.

Alumbaugh, D. L., 1993, Iterative electromagnetic Born inversion applied to earth conductivity imaging: Ph.D. thesis, University of California at Berkeley.

Alumbaugh, D. L., and Becker, A., 1990, Cross-borehole scale-modeling experiment: Lawrence Berkeley Laboratory, Annual Report LBL-2970, 127-131.

Anderson, W. L., 1982, Adaptive nonlinear least- - squares solution for constrained or unconstrained minimization problems (Subprogram NLSOL): U.S. Department of the Interior Geological Survey, Open-File Report 82-68.

Archie, G.E., 1942, The electrical resistivity $\log$ as an aid in determining some reservoir characteristics: Trans. AIME, 146, p.54062.

Asch, T., and Morrison, H. F., 1989, Mapping and monitoring electrical resistivity with surface and subsurface electrode arrays: Geophysics, 54, 235-244.

Augustine, A. M., Kennedy, W. D., Morrison, H. F., and Lee, K. H., 1989, A theoretical study of surface-to-borehole electromagnetic logging in cased holes: Geophysics, 54, 90-99.

Bannister, P. R., 1968, Determination of the electrical conductivity of the sea bed in shallow waters: Geophysics, 33, 995-1003.

Bannister, P. R., after 1977, Quasi-static electromagnetic fields: Naval Underwater Systems Center.

Banos, A., 1966, Dipole radiation in the presence of a conducting half-space: Pergamon Pres, Inc.

Bamett, C. T., 1984, Simple inversion of time-domain electromagnetic data: Geophysics, 49, 925-933.

Bartel, L. C., and Ranganayaki, R. P., 1989, Acquisition of controlled-source audiofrequency magnetotelluric (CSAMT) data at an active steam drive site. 59'th Ann. Int. Mtg., Soc. Explor. Geophys., Dallas, Esp. Abstr., 213-218. 
Bevc, D., and Morrison, H. F., 1991, Borehole-to-surface electrical conductivitiy monitoring of a salt water injection experiment: Geophysics, 56, 769-777.

Bishop, J. R., and Lewis, R. J. G., 1987, Down-hole electromagnetic surveys at Renison Bell, Tasmania: Exploration Geophys., 18, 265-277.

Boyd, G. W., and Wiles, C. J., 1984, The Newmont drill-hole EM pulse system Examples from eastern Australia: Geophysics, 49, 949-956.

Brekhovskikh, L. M., 1960, Waves in layered media: Academic Press. Inc.

Bubenik, D. M., and Fraser-Smith, A. C., 1978, ULF/ELF electromagnetic fields generated in a sea of finite depth by a submerged vertically-directed harmonic magnetic dipole: Radio Sci., 13, 1011-1020.

Bussian, A. E., 1983, Electrical conductance in porous medium: Geophysics, 48, 12581268.

Chave, A. D., Constable, S. C., and Edwards, R. N., 1990, Electrical exploration methods for the seafloor: in M. N. Nabighian, ed., Electromagnetic Methods in Applied Geophysics-Theory, Vol. 1: Soc. Explor. Geophys.

Chave, A. D., and Cox, C. S., 1982, Controlled electromagnetic sources for measuring the electrical conductivity beneath the oceans: J. Geophys. Res., 87, 5327-5338.

Cheesman, S. J., Edwards, R. N., and Chave, A. D., 1987, On the theory of sea-floor conductivity mapping using transient electromagnetic systems: Geophysics, 52, 204 217.

Coggon, H. H., and Clarke, E. H., 1987, The fixed receiver electromagnetic (FREM) method for drill hole surveys: Exploration Geophys., 18, 305-311.

Coggon, J. H., and Morrison, H. F., 1970, Electromagnetic investigation of the sea floor: Geophysics, 35, 476-429.

Constable, S. C., Parker, R. L., and Constable, C. G., 1987, Occam's inversion; a practical algorithm for generating smooth models from electromagnetic sounding data: Geophysics, 52, 289-300.

Cull, J. P., and Cobcroft, R., 1986, Omni-directional downhole EM probe: Geophys. Prosp., 34, 569-579.

Daily, W., 1984, Underground oil-shale retort monitoring using geotomography: Geophysics, 49, 1701-1707.

Dennis, J. E., Gay, D. M., and Welsch, R. E., 1981, An adaptive nonlinear leastsquares algorithm: ACM Trans. on Math. Software, 7, No. 3, 348-368. 
Deszcz-Pan, M., Lee, Ki Ha, and Morrison, H. F., 1989, One-dimensional least-squares inversion of surface-to-borehole and crosshole electromagnetic (EM) data: Lawrence Berkeley Laboratory, Annual Report, 149-150.

Dyck, A. V., 1975, Drill-hole electromagnetic methods: in M. N. Nabighian, ed., Electromagnetic Methods in Applied Geophysics-Theory, Vol. 1: Soc. Explor. Geophys., 1-50.

Dyck, A. V., and West, G. F., 1984, The role of simple computer models in interpretation of wide-band, drill-hole electromagnetic surveys in mineral exploration: Geophysics, 49, 957-980.

Dyck, A. V., 1975, Electrical borehole methods applied to metallic mineral prospecting, in Borehole geophysics applied to metallic mineral prospecting: A review, Dyck, A.V., Ed., Geol. Surv. Can., paper 75-31, 113-29.

Eadie, T., and Staltari, G., 1987, Introduction to downhole electromagnetic methods: Exploration Geophys., 18, 247-351.

Eadie, T., 1987, The downhole EM response of the Hellyer Ore deposit: Exploration Geophys., 18, 255-264.

Eaton, P.A., and Hohmann, G. W., 1984, The influence of a conductive host on twodimensional borehole transient electromagnetic responses: Geophysics, 49, 861-869.

Edwards, R. N., 1988, Two-dimensional modeling of a towed in-line electric dipoledipole sea-floor electromagnetic system: The optimum time delay or frequency for target resolution: Geophysics, 53, 846-853.

Edwards, R. N., and Cheesman, S. J., 1987, Two-dimensional modeling of a towed transient dipole-dipole sea floor EM system: J. Geophys., 61, 110-121.

Edwards, R. N., and Chave, A. D., 1986, A transient electrid dipole-dipole method for mapping the conductivity of the sea floor: Geophysics, 51, 984-987.

Eaton, P. A., and Hohmann, G. W., 1984, The influence of a conductive host on twodimensional borehole transient electromagnetic responses: Geophysics, 49, 861-869.

Fullagar, P. K., 1987, Inversion of downhole TEM data using circular current filaments: Geophysical Prosp., 18, 341-344.

Greaves, R. J., Beydoun, W. B., and Spies, B. R., 1991, New dimensions in geophysics for reservoir monitoring: SPE Formation Evaluation, 141-150.

Hardman, R., and Shen, 1986, Induction sonde in dipping beds: Geophysics, 51, 799809.

Harrington, R. F., 1961, Time harmonic electromagnetic fields: McGrow-Hill Book Co. 
Hearst, J.R., and Nelson, P.H., 1985, Well logging for physical properties: R.R. Donnelley@ Sons Co.

Hohmann, G.W., Vanvoorhis, G.D., and Nelson, P.H., 1989, A vector EM system and its field applications: Geophysics, 43, 1418-1440.

Howard, A. Q. Jr., 1983, On resolution in geophysical probing using electromagnetic methods: IEEE trans. on Geosc. and Remote Sensing, GE-21, 102-108.

Kauffman, A. A., and Keller, G. R., 1983, Frequency and transient sounding: Elsevier Science Publ. Co.

Keller, G. V., and Frischknecht,***

Keller, G. V, 1988, Rock and mineral properties, in M. N. Nabighian, ed., Electromagnetic Methods in Applied Geophysics-Theory, Vol. 1: Soc. Explor. Genphys

King, R. W. P., Bettencourt J. T. de, and Sandler, B. H., 1979, Lateral-wave propagation of electromagnetic waves in the lithosphere: IEEE Trans. Geosci. Remote Sensing, 17, 86-92.

Kraichman, M. B., 1970, Handbook of electromagnetic propagation in conducting media: U. S. Government printing office, 0-401-469.

Labson, V. F., Becker, A., Morrison, H. F., and Conti, U., 1985, Geophysical exploration with audiofrequency natural magnetic fields: Geophysics, 50, 656-664.

Lee, K. H., and Deszcz-Pan, M., 1991,***Lawrence Berkeley Laboratory, Annual Report LBL-, .

Llera, F. J., Sato, M., Nakatsuka, K., and Yokoyamas, 1990, Temperature dependence of the electrical resistivity of water-saturated rocks: Geophysics, 55, 576-585.

Lytle, R. J., Lager, D. L., Laine, E. F., Salisbury, J. D., and Okada, J. T., 1981, Fluidflow monitoring using electromagnetic probing, Geophys. Prosp., 29, 627-638.

Lytle, R. J., Laine, E. F., Lager, D. L., and Davis, D. T., 1979, Cross-borehole electromagnetic probing to locate high-contrast anomalies, Geophysics, 44, 16671676.

Macnae, J., and Staltari, G., 1987, Classification of sign changes in borehole TEM decays: Exploration Geophys., 18, 331-339.

Mahmond, S. F., Batros, A. Z., and Wait, J. R., 1970, Transient electromagnetic fields of a vertical magnetic dipole on a two-layer earth: Proc. IEEE, 67, no. 7, 1022-1029.

Mansure, A. J., and Meldau, R. F., 1990, Steam-zone electrical characteristics for geodiagnostics evaluation of steamflood performance: SPE Formation Evaluation, 241-247. 
Nekut, A. G., and Spies, B. R., 1989, Petroleum exploration using controlled-source electromagnetic methods: Proc. IEEE, 77, No. 2, 338-363.

Oristaglio, M. L., and Worthington, M. H., 1978, Inversion of surface and borehole electromagnetic data for two-dimensional electrical conductivity models: Geophys. Prosp., 28, 633-657.

Parkhomenko, E. I, 1967, Electrical properties of rocks (trans. by G. V. Keller), Plenum.

Poddar, M., Caleb Dhanasekaran, P., and Prabhakar Rao, K., 1980, Comparison between moving and stationary transmitter systems in induction logging, PAGEOPH, 123, 707-731.

Raiche, A. P., and Bennett, L. A., 1987, Layered earth models using downhole electromagnetic receivers: Exploration Geophys., 18, 325-329.

Ramirez, A. L., Daily, W., LaBrecque, D. J., Owen, E. W., and Chesnut, D. A, 1992, Electrical resistance tomography used to monitor subsurface steam injection: 62nd Ann. Internat. Mig., Soc. Expl. Geophys., Expanded Abstracts, SEG, Session EM43.

Ranganayaki R. P., Arkturk, S. E., and Fryer, S. M., 1992, Formation resistivity variation due to steam flooding: A log study: Geophysics, 57, 488-494.

Roy, A., and Dhar, R. L., 1970, Relative contribution to signal by ground elements in two-coil induction logging system: Geophys. Prosp., 18, 389-404.

Schlubmerger log analy

Sen, P. N., and Goode, P. A., 1992, Influence of temperature on electrical conductivity on shaly sands: Geophysics, 57, 89-96.

Sen, P. N., and Goode, P. A., 1988, Shaly sands conductivity at low and high salinities: Paper f, TRANS. 29th Ann. Symp. Soc. Prof. Well Log Analysts.

Sen, P. N., 1991, Correspondence between membrane potential and conductivity: Geophysics, 56, 461-471.

Sen, P. N., 1989, Influence of microgeometry on membrane potential of shaly sands: Geophysics, 54, 1543-1553.

Shankland, T. J., and Waff, H. S., 1974, Conductivity in fluid-bearing rocks: J. G. R., 79, 4863-4868.

Sinha, A. K., and Bhattaharaya, P. K., 1966, Vertical magnetic dipole buried inside a homogeneous earth: Radio Science, 1, No. 3, 379-385.

Stinson, D.C., 1976, Intermediate mathematics of eluctromagnetic: Prentice-Hall, Inc. 
Spies, B. R., 1992, Survey design considerations for cross-well electromagnetics: 62nd Ann. Intemat. Mtg., Soc. Expl. Geophys., Expanded Abstracts, SEG, Session EM45.

Spies, B. R., and Habashy, T. M., 1992, Sensitivity analysis of cross-well electromagnetics: 62nd Ann. Internat. Mtg., Soc. Expl. Geophys., Expanded Abstracts, SEG, Session EM4-6.

Spies, B.R., and Greaves, R. J., 1991, Numerical modeling of surface-to-borehole electromagnetic surveys for monitofing thermal enhanced oil recovery: Geoexploration, 28, 293-311.

Stoyer, C. H., 1976, Electromagnetic fields of dipoles in stratified media: IEEE Trans. on Antennas and Propagation, 547552.

Vallee, M. A., and Ng, T. P., 1992, Electromagnetic response of a resistive target detected from a distant borehole: 62nd Ann. Internat. Mtg., Soc. Expl. Geophys., Expanded Abstracts, SEG, Session EM4-8.

Wait, J. R., 1981, (Lectures on) Wave propagation Theory: Pergamon Press Inc.

Wait, J. R., and Spies, K. P., 1972, Electromagnetic propagation in an idealized Earth crust waveguide, Part I: Pure Appl. Geophys., 101, 174-187.

Wait, J. R., and Spies, K. P., 1972, Electromagnetic propagation in an idealized Earth crust waveguide, Part II: Pure Appl. Geophys., 101, 188-193.

Wait, J. R., and Spies, K. P., 1972, Dipole excitation of ultralow-frequency waves in the Earth crust waveguide: J. Geophys. Res., 77, 7118-7121.

Wait, J. R., 1970, Electromagnetic waves in stratified media: Pergamon Press Inc.

Ward, S. H., and Fraser, D. C., 1967, Conduction of electricity in rocks: in Mining Geophysics, SEG.

Waxman, M. H., and Smits, L. J. M., 1968, Electrical conduction in oil bearing shaly sands: Soc. Petr. Eng. J., 8, 107-122.

West, G. F., and Edwards, R. N., 1985, A simple parametric model for the electromagnetic response of an anomalous body in a host medium: Geophysics, 50, 2542-2557.

Wilt, M., and Schenkel, C., 1992, Cross-borehole electromagnetic induction for steam flooding monitoring: 62nd Ann. Internat. Mtg., Soc. Expl. Geophys., Expanded Abstracts, SEG, Session EM4-1.

Wilt, M. J., Morrison, H. F., Becker, A., and Lee, K. H., 1991, Cross-borehole and surface-to-borehole electromagnetic induction for reservoir characterization: U.S. Dept. og Energy Report DOE/BC/91002253. 
Wilt, M. J., Alumbaugh, D. L., Mcrrison, H. F., Becker, A., Lee, K. H., and DeszczPan, Maria, 1993, Crosshole electromagnetic imaging for reservoir definition and monitoring: to be submitted to Geophysics.

Wilt, M. J., and Ranganayaki, R. P., 1990, Surface-to-borehole electromagnetic logging for enhanced oil recovery (EOR) applications: 60'th Ann. Int. Mtg., Soc. Explor. Geophys., San Francisco, Exp. Abstr., 532-534.

Worthington, M. H., Kuckes, A., and Oristaglio, M., 1981, A borehole induction procedure for investigating electrical conductivity within the broad vicinity of a hole: Geophysics, 46, 65-67.

Zhou, Q., 1989, Audio-frequency electromagnetic tomography for reservoir evaluation: Ph. D. thesis, University of California, Berkeley. 
Appendix A

Mathematical derivations.

\section{A.1 General solution.}

Assume that the impressed source (transmitter) of electromagnetic waves is a magnetic dipole located at $\left(0,0, z_{s}\right)$ and oriented alorg the $z$-axis. The observation point (receiver) is placed at an arbitrary depth $z$ (Figure A.1-1).

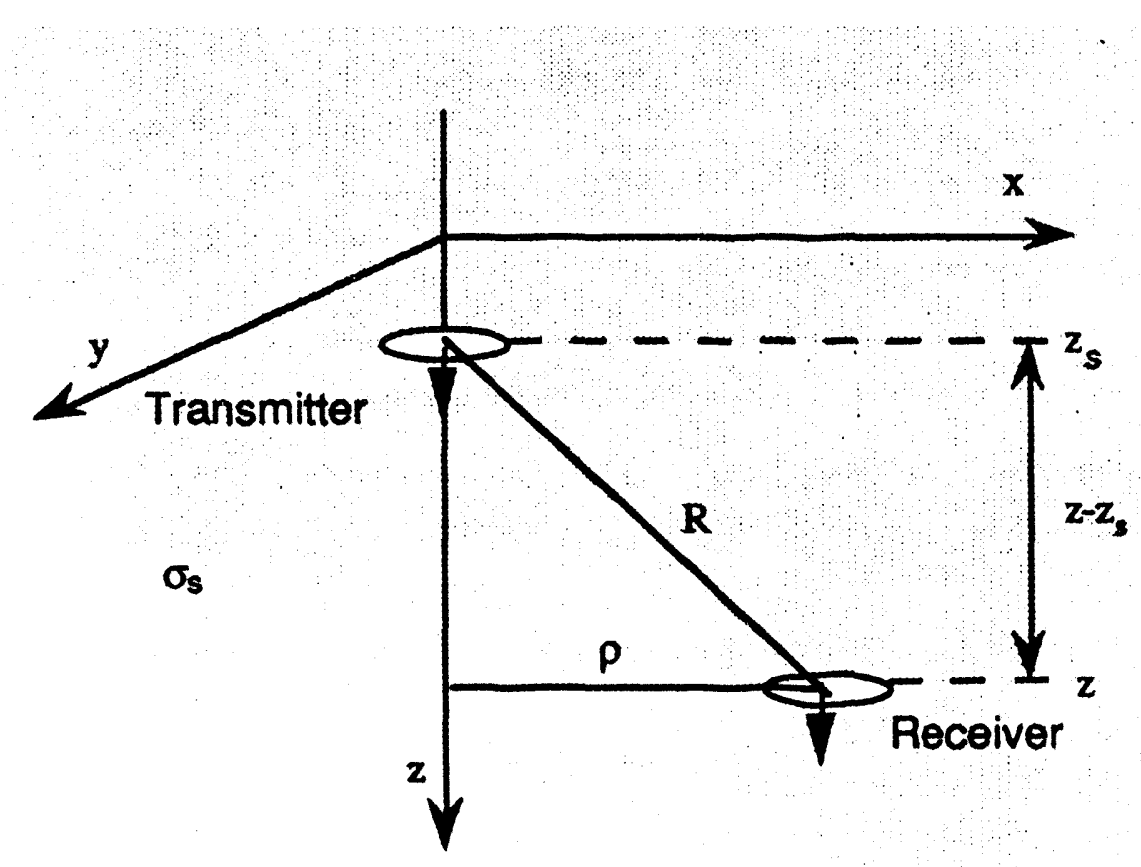

Fig. A.1-1. Configuration for the magnetic dipole inside a conductive medium

This impressed source can be written as:

$$
\mathbf{M}=\mathbf{M} \delta(\mathbf{R}) \vec{z}
$$

where $M$ is magnetic dipole moment density $(A / m), M$ is magnetic dipole moment ( $A$ $\mathrm{m}^{2}$ ), $\mathbf{R}$ is a vector from transmitter to receiver, and $\vec{z}$ is a unit vector in the $z$-direction. With $\rho$ denoting the horizontal transmitter receiver separation, the total distance between the transmitter and the receiver is given by: 


$$
R=\sqrt{p^{2}+\left(z-z_{s}\right)^{2}}
$$

The electromagnetic fields, source and the medium are related by Maxwell's equations, that in a linear and isotropic medium are given by:

$$
\begin{aligned}
& \nabla \times \mathbf{E}=-\frac{\partial}{\partial t} \mathbf{B} \\
& \nabla \times \mathbf{H}=\mathbf{J}+\frac{\partial \mathbf{D}}{\partial t} \\
& \nabla . \mathbf{B}=0 \\
& \nabla . \mathbf{D}=\rho
\end{aligned}
$$

and consitiutive relations: $B=\mu(\mathbf{H}+\mathbf{M}), \mathbf{D}=\boldsymbol{\varepsilon} \mathbf{E}, \mathbf{J}=\boldsymbol{\sigma E}$, where, $\mathbf{H}$ and $\mathbf{B}$ are magnetic field vectors in $(\mathrm{A} / \mathrm{m})$ and $\left(\mathrm{Wb} / \mathrm{m}^{2}\right)$ respectively, $\mathbf{E}$ and $\mathbf{D}$ are electric field vector in $(\mathrm{V} / \mathrm{m}), J$ is conductive current density $\left(\mathrm{A} / \mathrm{m}^{2}\right), \mathbf{M}$ is magnetic dipole moment density $(\mathrm{A} / \mathrm{m})$ defined earlier. The constants $\varepsilon, \mu, \sigma$, describe the medium where $\varepsilon$ is a dielectric constant $(F / m), \mu$ is the magnetic permeability $(H / m)$, and $\sigma$ is the conductivity $(\mathrm{S} / \mathrm{m})$.

If the medium does not have discontinuities in horizontal direction, then $\mathrm{H}_{2}, \mathrm{H}_{\mathrm{p}}$ and $\mathrm{E}_{\phi}$ are the only components of electromagnetic fields vectors produced by a vertical magnetic dipole (VMD). It is then convenient to use cylindrical system of coordinates in which the Maxwell's equations and source term have the following form:

$$
\begin{aligned}
& \frac{\partial E_{\phi}}{\partial z}=i \omega \mu H_{\rho} \\
& \frac{1}{\rho} \frac{\partial}{\partial \rho} \rho E_{\phi}=-i \omega \mu\left[H_{z}+\frac{M}{2 \pi} \frac{\delta(\rho)}{\rho} \delta\left(z-z_{s}\right)\right] \\
& \frac{\partial H_{p}}{\partial z}-\frac{\partial H_{z}}{\partial \rho}=(\sigma+i \omega \varepsilon) E_{\phi}
\end{aligned}
$$


where $\omega$ is angular frequency (rad/s). In the regions without the source the second term in square parenthesis in equation (A.1-8) vanishes. In the above equations we assumed $\mathrm{e}^{+ \text {iwol }}$ time dependence.

Maxwell's equations are conveniently solved with the help of the magnetic Hertz vector $\Pi^{*}$, that is derived using equation (A.1-6) which leads to the assumption that $\mathbf{E}=-\mathbf{i} \omega \mu \nabla \times \Pi^{*}$. This in turn provides: $\mathbf{H}=\nabla \nabla \cdot \Pi^{*}+\mathbf{k}^{2} \Pi^{*}$. In solutions involving the vertical magnetic dipole, Hertz vector has only $z$-component. The relations between Hertz vector and fields are given by:

$$
\begin{aligned}
& \mathrm{H}_{\mathbf{z}}=\frac{\partial^{2} \Pi_{z}^{*}}{\partial \mathrm{z}^{2}}+\mathrm{k}^{2} \Pi_{\mathrm{z}}^{*} \\
& \mathrm{H}_{\rho}=\frac{\partial^{2} \Pi_{z}^{*}}{\partial \rho \partial z} \\
& \mathrm{E}_{\phi}=i \omega \mu \frac{\partial \Pi_{z}^{*}}{\partial \rho}
\end{aligned}
$$

If we eliminate $\mathrm{H}_{2}$, and $\mathrm{H}_{\rho}$ from the cylindrical Maxwell's equations and express $E_{\phi}$ in terms of Hertz potential, then after integration with respect to $\rho$ one obtains the following relation:

$$
\frac{\partial^{2}}{\partial \rho^{2}} \Pi_{z}^{*}+\frac{1}{\rho} \frac{\partial}{\partial \rho} \Pi_{z}^{*}+\frac{\partial^{2}}{\partial z^{2}} \Pi_{z}^{*}+k^{2} \Pi_{z}^{*}=-\frac{M}{2 \pi} \frac{\delta(\rho)}{\rho} \delta\left(z-z_{s}\right)
$$

where

$$
k=\sqrt{\omega^{2} \mu \varepsilon-i \omega \mu \sigma}
$$

is a propagation constant of the medium. The general solution of this equation is given by:

$$
\Pi_{z}^{*}=\int_{0}^{-}\left[A(\lambda) e^{-i \sqrt{k^{2}-\lambda^{2}} z}+B(\lambda) e^{+i \sqrt{k^{2}-\lambda^{2}}}\right] \lambda J_{0}(\lambda \rho) d \lambda
$$


The coefficients $A(\lambda)$ and $B(\lambda)$ are found by matching the source and boundary conditions. The boundary conditions state that the tangential electric and magnetic fields are continuous across the interface if no surface charges or surface currents are present. The source condition tells that at the source the vertical derivative of electromagnetic fields is discontinuous by the source strength. Furthermore the fields must satisfy the radiation condition, i.e. they must vanish at infinity.

The boundary conditions on the Hertz potential are derived from the relations between the Hertz potential and the electromagnetic fields. Assuming thet there is no discontinuity in $\mu$, the boundary conditions at any given interface " $i$ " located at depth $z=$ $\mathrm{z}_{\mathrm{i}}$ are given by:

$$
\begin{aligned}
& \Pi_{z i-1}^{*}=\Pi_{z i}^{*} \\
& \frac{\partial}{\partial z} \Pi_{z i-1}^{*}=\frac{\partial}{\partial z} \Pi_{z i}^{*}
\end{aligned}
$$

where $\Pi_{z i-1}^{*}$ is a Hertz vector in the medium above the boundary and $\Pi_{z i}^{*}$ is a Hertz vector below the boundary. The source conditions are given by:

$$
\begin{aligned}
& \Pi_{z s-0}^{*}=\Pi_{z s+0}^{*} \\
& \frac{\partial}{\partial z} \Pi_{z s+0}^{*}-\frac{\partial}{\partial z} \Pi_{z s-0}^{*}=-\frac{M}{2 \pi} \frac{\delta(\rho)}{\rho} \delta\left(z-z_{s}\right)
\end{aligned}
$$

where subscript "s+0" and "s-0" indicate the regions below and above the source respectively.

\section{A.2 Whole space.}

In a whole space only the source conditions and radiation conditions are needed to obtain the coefficients of equation (A.1-15). Thus $A(\lambda)=0$ for $z<z_{s}$ and $B(\lambda)=0$ for $\mathrm{z}_{\mathrm{s}}<\mathrm{z}$ in order to satisfy the radiation condition. Matching the source condition the solution for $\Pi^{*}$ in a whole space is given by:

$$
\Pi_{z}^{*}\left(k_{s}, R\right)=\frac{M}{4 \pi} \int_{0}^{\infty} \frac{\lambda e^{-i \sqrt{k_{0}^{2}-\lambda^{2}|z-z|}}}{i \sqrt{k_{0}^{2}-\lambda^{2}}} J_{0}(\lambda \rho) d \lambda
$$


In the above equation subscript " $s$ " denotes the medium with the source.

In this thesis the whole-space fields are frequently involved in more complicated expressions. To describe the whole space medium and geometry, we always indicate in parenthesis the propagation constant and total transmitter-receiver separation.

The above solution is linked to the another form of solution through Sommerfield's identity:

$$
\Pi_{z}^{*}\left(k_{s}, R\right)=\frac{M}{4 \pi} \frac{e^{-i k_{s} R}}{R}=\frac{M}{4 \pi} \int_{0}^{-} \frac{\lambda e^{-i \sqrt{k_{s}^{2}-\lambda^{2}}|z-z|}}{i \sqrt{k_{0}^{2}-\lambda^{2}}} J_{0}(\lambda \rho) d \lambda
$$

The whole space fields $\mathrm{H}_{\mathrm{z}}, \mathrm{H}_{p}, \delta \mathrm{H}_{\mathrm{z}} / \delta \mathrm{z}$ and $\delta^{2} \mathrm{H}_{\mathrm{z}} / \delta \mathrm{z}^{2}$ are obtained by differentiating the Hertz vector according to equations (A.1-10), (A.1-11) and (A.1-12):

$$
\begin{aligned}
& H_{z}\left(k_{s}, R\right)=\frac{M}{4 \pi}\left(\frac{\partial^{2}}{\partial z^{2}}+k_{s}^{2}\right) \frac{e^{-i k_{s} R}}{R}=\frac{M}{4 \pi} \int_{0}^{-} \frac{\lambda^{3} e^{-i \sqrt{k_{s}^{2}-\lambda^{2}}\left|z-z^{2}\right|}}{i \sqrt{k_{s}^{2}-\lambda^{2}}} J_{0}(\lambda \rho) d \lambda \\
& H_{\rho}\left(k_{s}, R\right)=\frac{M}{4 \pi} \frac{\partial^{2}}{\partial z \partial \rho} \frac{e^{-i k_{s} R}}{R}=\frac{M}{4 \pi} \int_{0}^{-} \lambda e^{-i \sqrt{k_{s}^{2}-\lambda^{2}}|z-z|} \frac{\left(z-z_{s}\right)}{\left|z-z_{s}\right|} J_{1}(\lambda \rho) d \lambda . \\
& \frac{M}{4 \pi}\left(\frac{\partial^{3}}{\partial z^{3}}+k_{s}^{2} \frac{\partial}{\partial z}\right) \frac{e^{-i k_{s} R}}{R}=-\frac{M}{4 \pi} \int_{0}^{-} \lambda^{3} e^{-i \sqrt{k_{s}^{2}-\lambda^{2}} \mid z-z d} \frac{\left(z-z_{s}\right)}{\mid z-z_{s}} J_{0}(\lambda \rho) d \lambda .
\end{aligned}
$$




$$
\begin{gathered}
\frac{\partial^{2}}{\partial z^{2}} H_{z}\left(k_{s}, R\right)= \\
\frac{M}{4 \pi}\left(\frac{\partial^{4}}{\partial z^{4}}+k_{s}^{2} \frac{\partial^{2}}{\partial z^{2}}\right) \frac{e^{-i k_{s} R}}{R}=\frac{M}{4 \pi} \int_{0}^{\infty} \lambda^{3}\left(i \sqrt{k_{s}^{2}-\lambda^{2}} e^{-i} \sqrt{k_{s}^{2}-\lambda^{2}}|z-z| J_{0}(\lambda \rho) d \lambda .\right.
\end{gathered}
$$

Taking the prescribed derivatives of $\frac{M}{4 \pi} \frac{e^{-i k_{s} R}}{R}$, the whole space fields are given by:

$$
\begin{aligned}
& H_{z}\left(k_{s}, R\right)=\frac{M}{4 \pi} \frac{e^{-i k_{s} R}}{R^{5}}\left[\left(1+i k_{s} R\right)\left(-\rho^{2}+2\left(z-z_{s}\right)^{2}\right)+\left(i k_{s} R\right)^{2}\left(-\rho^{2}\right)\right], \\
& H_{\rho}\left(k_{s}, R\right)=\frac{M}{4 \pi} \frac{e^{-i k_{s} R}}{R^{5}} \rho\left(z-z_{s}\right)\left[3\left(1+i k_{s} R\right)+\left(i k_{s} R\right)^{2}\right], \\
& \frac{\partial}{\partial z} H_{z}\left(k_{s}, R\right)=\frac{M}{4 \pi} \frac{e^{-i k_{s} R}}{R^{7}}\left(z-z_{s}\right)\left\{\left(1+i k_{s} R\right) 3\left[3 p^{2}-2\left(z-z_{s}\right)^{2}\right]\right. \\
& \left.+\left(i k_{s} R\right)^{2} 2\left(2 p^{2}-z^{2}\right)+\left(i k_{s} R\right)^{3} \rho^{2}\right\} \text {. } \\
& \frac{\partial^{2} H_{z}\left(k_{s}, R\right)}{\partial z^{2}}=\frac{M}{4 \pi} \frac{e^{-i k_{s} R}}{R^{9}}\left\{\left(1+i k_{s} R\right) 3\left[3 \rho^{4}-24 \rho^{2}\left(z-z_{s}\right)^{2}+8\left(z-z_{s}\right)^{4}\right]\right. \\
& +\left(i k_{s} R\right)^{2}\left[\left(4 \rho^{4}-31 \rho^{2}\left(z-z_{s}\right)^{2}+10\left(z-z_{s}\right)^{4}\right)\right] \\
& +\left(i k_{s} R\right)^{3}\left[\begin{array}{lll}
( & \rho^{4} & \left.-7 \rho^{2}\left(z-z_{s}\right)^{2}+2\left(z-z_{s}\right)^{4}\right)
\end{array}\right] \\
& \left.+\left(\mathrm{ik}_{\mathrm{s}} \mathrm{R}\right)^{4}\left[\left(-\rho^{2}\left(\mathrm{z}-\mathrm{z}_{\mathrm{s}}\right)^{2}\right)\right]\right\}
\end{aligned}
$$

The above relations are useful in developing the asymptotic expansions. A more complete listing of these expansions is given in Appendix C.2. 


\section{A.3 Single boundary}

Let's consider a vertical magnetic dipole of moment $M$ placed above the interface located at depth $z=z_{s}$. The interface is located at depth $z=z_{s+1}$. This boundary separates two half-spaces: one with the source in it of conductivity $s_{8}$, and the other below with conductivity $\sigma_{s+1}$. The subscript $" s+1$ " is used to indicate that it is the first boundary below source. The first boundary above source will have subscript "s-1", the next one "s2 " in later chapters. The horizontal separation between the transmitter and observation point is $\rho$. The total distance between transmitter and receiver is $R=\left[\rho^{2}+\left(z-z_{s}\right)^{2}\right]^{1 / 2}$. The geometry of the problem is illustrated in Figure (A.3-1a).

The solutions can be obtained by matching the source and boundary conditions in three regions: above the source where $z<z_{s}$, below the source but above the boundary where $z_{s} \leq z<z_{s+1}$, and below the interface, where $z_{s}<z \leq z_{s+1}$. The solutions to wave equation in this three regions have the forms:

for $\mathrm{z}<\mathrm{z}_{\mathrm{s}}$,

$$
\left.\Pi_{z s-0}^{*}=\int_{0}^{-} B_{s-0} e^{+i \gamma_{j} z} \lambda J d \lambda \rho\right) d \lambda
$$

for $z_{s} \leq z<z_{s+1}$

$$
\Pi_{z s+0}^{*}=\int_{0}^{-}\left(A_{s+0} e^{-i \gamma_{s} z}+B_{s+0} e^{+i \gamma_{s} z}\right) \lambda J_{d}(\lambda \rho) d \lambda,
$$

for $z_{s+1} \leq z$,

$$
\Pi_{z s+1}^{*}=\int_{0}^{\infty} A_{s+1} e^{-i \gamma_{t+1} z} \lambda J_{0}(\lambda p) d \lambda
$$

where: $\gamma_{s}=\sqrt{k_{s}^{2}-\lambda^{2}}, \gamma_{s+1}=\sqrt{k_{s+1}^{2}-\lambda^{2}}$. The subscript "s-0" describes the medium with the source, but above the transmitter, subscript " $s+0$ " describes the same source medium but below the transmitter, subscript " $s+1$ " describes the medium below the first boundary.

After matching boundary and source conditions, the solutions for Hertz potential are as follows: 
for $\mathrm{z}<\mathrm{z}_{\mathrm{s}}$ (above the interface),

$$
\Pi_{z s-0}^{*}=\frac{M}{4 \pi i} \int_{0}^{\infty}\left(\frac{e^{-i} \gamma_{\alpha}\left(z_{0}-z\right)}{\gamma_{i}}+R_{s, s+1} \frac{e^{-i \gamma\left(2 z_{0+1}-z_{-}-z\right)}}{\gamma_{b}}\right) \lambda J_{0}(\lambda \rho) d \lambda,
$$

for $z_{s} \leq z<z_{s+1}$ (below the source and above the interface),

$$
\Pi_{z s+0}^{*}=\frac{M}{4 \pi i} \int_{0}^{-}\left(\frac{e^{-i} \gamma_{\alpha}\left(z-z_{s}\right)}{\gamma_{z}}+R_{s, s+1} \frac{e^{-i \gamma_{s}\left(2 z_{s+1}-z_{s}-z\right)}}{\gamma_{s}}\right) \lambda J_{0}(\lambda \rho) d \lambda
$$

for $z_{s+1} \leq z$ (below the source),

$$
\Pi_{z s+1}^{*}=\frac{M}{4 \pi i} \int_{0}^{-} 2 \frac{e^{-i \gamma_{o+1}\left(z-z_{o+1}\right) \mathrm{e}^{-i} \gamma_{\alpha}\left(z_{o+1}-z_{s}\right)}}{\gamma_{\mathrm{a}}+\gamma_{t+1}} \lambda \mathrm{J}_{0}(\lambda \rho) \mathrm{d} \lambda
$$

where $R_{s, s+1}$ is a reflection coefficient given by:

$$
R_{s, s+1}=\frac{\gamma_{s}-\gamma_{s+1}}{\gamma_{s}+\gamma_{s+1}}
$$

The equations for transmitter and receiver above the interface can be converted to another form that is more suitable for analysis and is useful in the development of the asymptotic solutions.

The first terms in the equations (A.3-4) and (A.3-5) can be written using the Sommerfield's identity:

$$
\frac{M}{4 \pi} \frac{e^{-i k_{0} R}}{R}=\frac{M}{4 \pi} \int_{0}^{-} \frac{e^{-i \gamma_{j} \mid z-z d}}{i \gamma_{0}} \lambda J d(\lambda \rho) d \lambda
$$

The coefficients in the second terms in equations (A.3-4) and (A.3-5) can be written as (Kauffman and Keller, 1983):

$$
\frac{1}{\gamma_{s}} \frac{\gamma_{s}-\gamma_{s+1}}{\gamma_{s}+\gamma_{s+1}}=-\frac{1}{\gamma_{s}}+\frac{2}{\gamma_{s}+\gamma_{s+1}}=-\frac{1}{\gamma_{s}}+\frac{2\left(\gamma_{s}-\gamma_{s+1}\right)}{k_{s}^{2}-k_{s+1}^{2}}
$$


As a result the expressions for the Hertz potentials above the interface can be written as:

$$
\begin{gathered}
\Pi_{z s+0}^{*}=\frac{M}{4 \pi i} \int_{0}^{-} \frac{e^{-i \gamma\left(z-z_{s}\right)}}{\gamma_{s}} \lambda J_{0}(\lambda \rho) d \lambda-\frac{M}{4 \pi i} \int_{0}^{-} \frac{e^{-i \gamma_{2} z_{1}}}{\gamma_{s}} \lambda J_{0}(\lambda \rho) d \lambda+ \\
\frac{M}{4 \pi i} \frac{2}{k_{s}^{2}-k_{s+1}^{2}} \int_{0}^{-}\left(\gamma_{s}-\gamma_{s+1}\right) e^{-i \gamma_{z_{1}}} \lambda J_{0}(\lambda \rho) \mathrm{d} \lambda,
\end{gathered}
$$

where $\left(2 z_{s+1}-z_{s}-z\right)=z_{1}$.

The above equation can be simplified further if one uses the relation obtained by differentiating twice the Sommerfield's identity:

$$
\frac{\partial^{2}}{\partial z_{1}^{2}} \frac{M}{4 \pi} \frac{e^{-i k_{1} R_{1}}}{R_{1}}=\frac{M}{4 \pi} \int_{0}^{-} i \gamma_{1} e^{-i \gamma_{s} z_{1}} \lambda J_{0}(\lambda \rho) d \lambda
$$

where $R_{1}=\rho^{2}+z_{1}^{2}$

Finally the solution for Hertz vector when transmitter and receiver are both above the interface is given by:

$$
\begin{aligned}
& \Pi_{s+0}^{*}=\frac{M}{4 \pi} \frac{e^{-k_{s} R}}{R}-\frac{M}{4 \pi} \frac{e^{-i c_{s} R_{1}}}{R_{1}} \\
& \left.-\frac{2}{k_{s}^{2}-k_{s+1}^{2}} \frac{M}{4 \pi}\left[\frac{\partial^{2}}{\partial z_{1}^{2}} \frac{e^{-i k_{1} R_{1}}}{R_{1}}-\int_{0}^{\infty} \lambda\left(i \gamma_{s+1}\right) e^{-i \gamma_{s} z_{1}} J d \lambda \rho\right) d \lambda\right]
\end{aligned}
$$

A.3-1 Exact expressions when transmitter and receiver are both above the interface.

The fields $\mathrm{H}_{z}, \mathrm{H}_{\rho}, \delta \mathrm{H}_{z} / \delta z$ and $\delta^{2} \mathrm{H}_{z} / \delta z^{2}$ on both sides of the interface are calculated from the Hertz potential using relations (A.1-10), (A.1-11) and (A.1-12)

In the medium where the source and receiver are located the exact expressions for $\mathrm{H}_{2}, \mathrm{H}_{\mathrm{r}}$ and $\mathrm{dHz} / \mathrm{dz}$ have the form:

$$
\begin{aligned}
H_{z_{+0}+0}= & H_{z}\left(k_{s}, R\right)-H_{z}\left(k_{s}, R_{1}\right) \\
& -\frac{2}{k_{s}^{2}-k_{s+1}^{2}}\left[\frac{\partial^{2}}{\partial z_{1}^{2}} H_{z}\left(k_{s}, R_{1}\right)-\frac{M}{4 \pi} \int_{0}^{\infty} \lambda^{3}\left(i \gamma_{s+1}\right) e^{-i \gamma_{s} z_{1}} J_{0}(\lambda \rho) d \lambda\right]
\end{aligned}
$$




$$
\begin{aligned}
& \mathrm{H}_{\rho_{\text {oto }}}=\mathrm{H}_{\mathrm{\rho}}\left(\mathrm{k}_{\mathrm{s}}, \mathrm{R}\right)-\mathrm{H}_{\mathrm{\rho}}\left(\mathbf{k}_{\mathbf{s}}, \mathrm{R}_{1}\right) \\
& -\frac{2}{k_{s}^{2}-k_{s+1}^{2}}\left[\frac{\partial^{2}}{\partial z_{1}^{2}} H_{\rho}\left(k_{s}, R_{1}\right)-\frac{M}{4 \pi} \frac{\partial}{\partial \rho} \int_{0}^{-} \lambda\left(i \gamma_{s+1}\right)\left(i \gamma_{s}\right) e^{-i \gamma_{2} z_{1}} J_{0}(\lambda \rho) d \lambda\right] \\
& \frac{\partial}{\partial z} H_{z_{0+0}}=\frac{\partial}{\partial z} H_{z}\left(\mathbf{k}_{s}, \mathbf{R}\right)-\frac{\partial}{\partial z} H_{z}\left(k_{s}, R_{1}\right) \\
& +\frac{2}{k_{0}^{2}-k_{s+1}^{2}}\left[\frac{\partial^{3}}{\partial z_{1}^{3}} H_{2}\left(k_{s}, R_{1}\right)+\frac{M}{4 \pi} \int_{0}^{-} \lambda^{3}\left(i \gamma_{s+1}\right)\left(i \gamma_{s}\right) e^{-i \gamma_{s} z_{1}} J_{0}(\lambda \rho) d \lambda\right] \text {. } \\
& \frac{\partial^{2}}{\partial z^{2}} H_{z_{s+0}}=\frac{\partial^{2}}{\partial z^{2}} H_{z}\left(k_{s}, R\right)-\frac{\partial^{2}}{\partial z^{2}} H_{z}\left(k_{s}, R_{1}\right) \\
& -\frac{2}{k_{s}^{2}-k_{s+1}^{2}}\left[\frac{\partial^{4}}{\partial z_{1}^{4}} H_{2}\left(k_{s}, R_{1}\right)-\frac{M}{4 \pi} \int_{0}^{-} \lambda^{3}\left(i \gamma_{s+1}\right) \gamma_{s}^{2} e^{-i \gamma_{s} z_{1}} J_{0}(\lambda \rho) d \lambda\right] \text {. }
\end{aligned}
$$

where $z_{1}=\left(2 z_{s+1}-z_{s}-z\right)$ and $R_{1}=\left(\rho^{2}+z_{1}^{2}\right)^{1 / 2}$

\section{A.3-2 Exact integral expressions when transmitter is above and receiver} is below the interface.

On the opposite side of the boundary, in the region without the source, the exact, analytical expression for $\mathrm{H}_{z}, \mathrm{H}_{\rho}, \delta \mathrm{H}_{2} / \delta \mathrm{z}$ and $\delta^{2} \mathrm{H}_{z} / \delta \mathrm{z}^{2}$ are given by the following:

$$
\begin{aligned}
& H_{z_{s+1}}=-\frac{2}{k_{s}^{2}-k_{s+1}^{2}} \frac{M}{4 \pi} \int_{0}^{-} \lambda^{3}\left(i \gamma_{s}-i \gamma_{s+1}\right) e^{-i \gamma_{s+1}\left(z-z_{s+1}\right)} e^{-i \gamma\left(z_{s+1}-z_{s}\right)} J_{0}(\lambda \rho) d \lambda \\
& H_{\rho_{s+1}}=\frac{2}{k_{s}^{2}-k_{s+1}^{2}} \frac{M}{4 \pi} \frac{\partial}{\partial \rho} \int_{0}^{-} \lambda\left(i \gamma_{s+1}\right)\left[\left(i \gamma_{s}\right)-\left(i \gamma_{s+1}\right)\right] e^{-i \gamma_{o+1}\left(z-z_{o+1}\right)} e^{-i \gamma_{\alpha}\left(z_{o+1}-z_{0}\right)} J_{0}(\lambda \rho) d \lambda \\
& \frac{\partial}{\partial z} H_{z_{s+1}}=\frac{2}{k_{s}^{2}-k_{p+1}^{2}} \frac{M}{4 \pi} \int_{0}^{-} \lambda^{3}\left(i \gamma_{s+1}\right)\left(\left(i \gamma_{s}\right)-\left(i \gamma_{s+1}\right)\right] e^{-i \gamma_{s+1}\left(z-z_{o+1}\right)} e^{-i \gamma_{\alpha}\left(z_{o+1}-z_{s}\right)} J_{0}(\lambda \rho) d \lambda \\
& \frac{\partial^{2}}{\partial z^{2}} H_{z_{s+1}}= \\
& \frac{2}{k_{s}^{2}-k_{s+1}^{2}} \frac{M}{4 \pi} \int_{0}^{-} \lambda^{3}\left(i \gamma_{s+1}\right)^{2}\left[\left(i \gamma_{s}\right)-\left(i \gamma_{s+1}\right)\right] e^{-i \gamma_{s+1}\left(z-z_{s+1}\right)} e^{-i \gamma_{\alpha}\left(z_{s+1}-z_{s}\right)} J_{0}(\lambda \rho) d \lambda
\end{aligned}
$$




\section{A.4 Transmitter below the layer}

Let's consider a vertical magnetic dipole of moment $M$ placed below the layer at depth $z=z_{s}$. The first interface above the transmitter is located at $z=z_{s-1}$. The second (upper) interface above the transmitter is located at depth $z=z_{s-2}$. The layer thickness is: $z_{s-1}-z_{s-2}$. Figure A.4-1 shows the geometry of the problem.

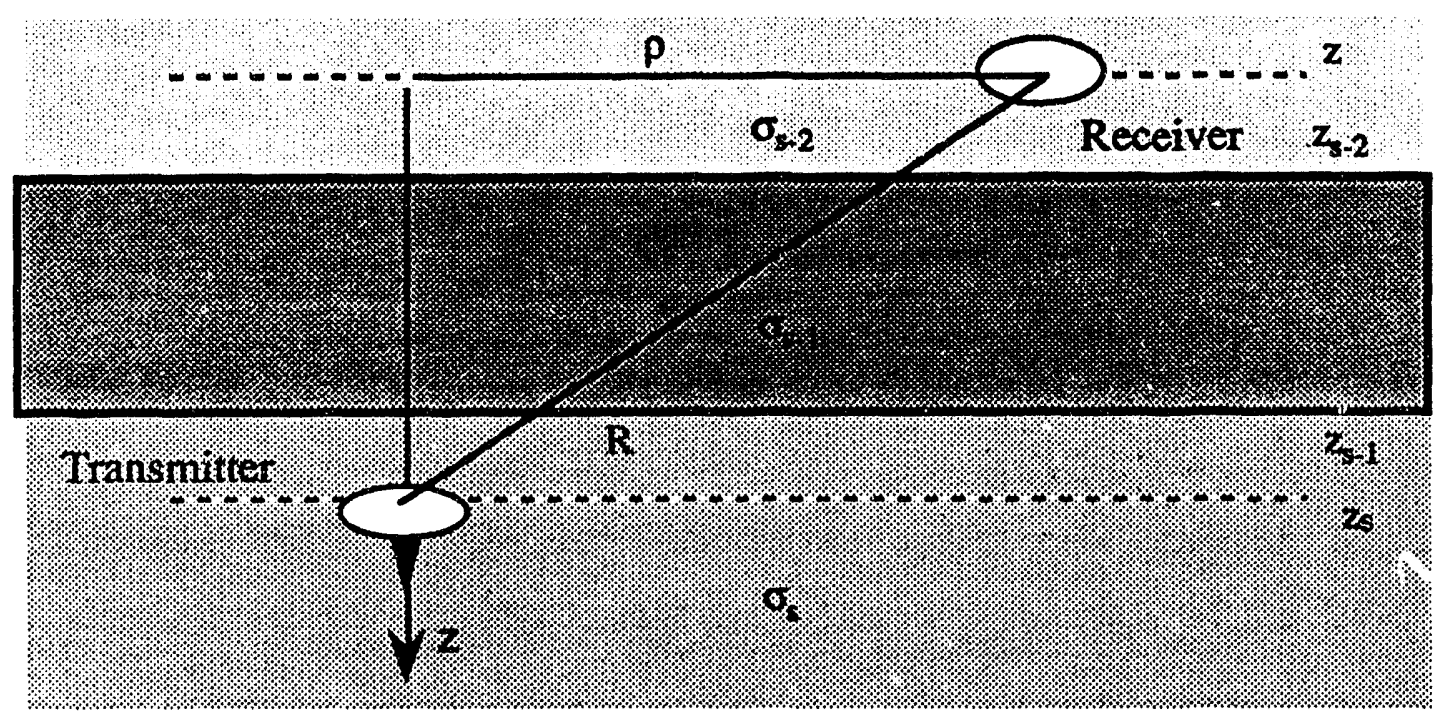

Fig. A.4-1. Configuration for transmitter below a layer.

The solutions are obtained by matching the source and boundary conditions in four regions: below the transmitter where $z \geq z_{s}$, above the source but below the lower interface where $z_{s}>z \geq z_{s-1}$, inside the layer where $z_{s-1}>z \geq z_{s-2}$, and above the source where $z_{s-2}>z$ The solutions to wave equation in this four regions have the forms: for $z_{s-2}>z$,

$$
\left.\Pi_{z s-2}^{*}=\int_{0}^{\infty} B_{s-2} e^{+i y-2 z} \lambda J d \lambda \rho\right) d \lambda
$$

for $z_{s-1}>z \geq z_{s-2}$

$$
\left.\Pi_{z s-1}^{*}=\int_{0}^{\infty}\left(A_{s-1} e^{-\dot{\gamma}_{p-1} z}+B_{3-1} e^{+\dot{\gamma}_{-1-1} z}\right) \lambda J d \lambda \rho\right) d \lambda,
$$


for $z_{s}>z \geq z_{s-1}$,

$$
\Pi_{z s-0}^{*}=\int_{0}^{-}\left(A_{s-0} e^{-i \gamma_{s} z}+B_{s-0} e^{+i \gamma_{s} z}\right) \lambda J_{0}(\lambda \rho) d \lambda,
$$

for $z \geq z_{s,}$

$$
\left.\Pi_{z s+0}^{*}=\int_{0}^{\infty} A_{s+0} e^{-\eta_{\alpha} z} \lambda J d \lambda \rho\right) d \lambda
$$

where: $\gamma_{s}=\sqrt{k_{s}^{2}-\lambda^{2}}, \quad \gamma_{s-1}=\sqrt{k_{s-1}^{2}-\lambda^{2}}, \quad \gamma_{s-2}=\sqrt{k_{s-2}^{2}-\lambda^{2}}$. The subscript "s-0" describes the medium with the source and above the transmitter, the subscript " $s+0$ " describes the same source medium and below the transmitter, the subscript "s-1" describes the medium above the first boundary, and finaly the subscript "s-2" describes the medium above the second boundary. After matching boundary and source conditions, the coefficients inside the integrals are as follows:

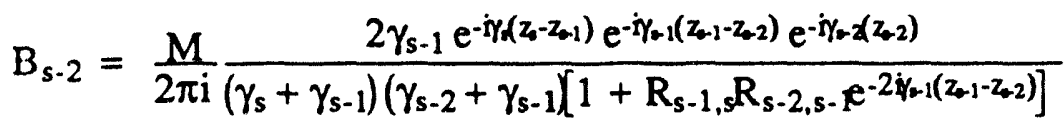

$A_{s-1}=-\frac{M}{2 \pi i} \frac{R_{s-2, s-1} e^{-i \gamma_{s}\left(z_{s-2}-z_{s-1}\right)} \mathrm{e}^{-\dot{\gamma} \gamma_{s-1}\left(z_{s+1}-2 z_{-2}\right)}}{\left(\gamma_{s}+\gamma_{s-1}\right)\left[1+R_{s-1, s} R_{s-2, s-} \mathrm{e}^{-2 \gamma_{s-1}\left(z_{s-1-1}-z_{s-2}\right)}\right]}$

$B_{s-1}=\frac{M}{2 \pi i} \frac{e^{-i \gamma_{\alpha}\left(z_{s}-z_{s-1}\right)} e^{-\gamma_{z_{-1}}\left(z_{-1}\right)}}{\left(\gamma_{s}+\gamma_{s-1}\right)\left[1+R_{s-1, s} R_{s-2, s-1} e^{\left.-2 \gamma_{-1}\left(z_{-1}-z_{s-2}\right)\right]}\right.}$

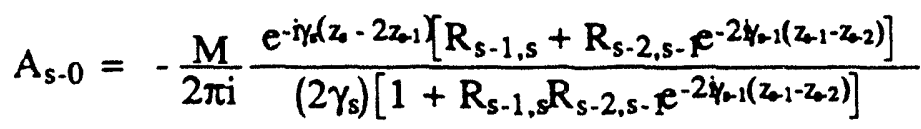

$B_{s-0}=\frac{M}{2 \pi i} \frac{e^{-i \gamma_{s}\left(z_{s}\right)}}{\left(2 \gamma_{s}\right)}$

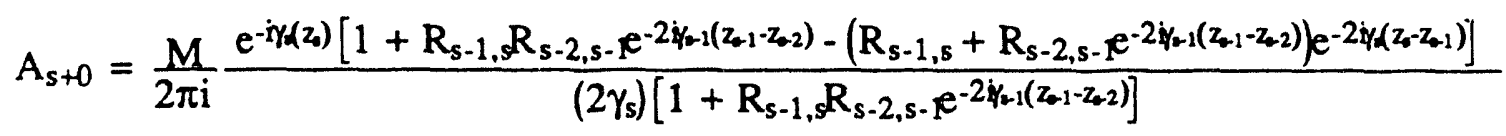


where

$$
R_{s-1, s}=\frac{\gamma_{s-1}-\gamma_{s}}{\gamma_{s-1}+\gamma_{s}}
$$

and

$$
R_{s-2, s-1}=\frac{\gamma_{s-2}-\gamma_{s-1}}{\gamma_{s-2}+\gamma_{s-1}}
$$

Let's consider the $\mathrm{H}_{2}$ fields in the region above the source, but below the layer. After substituting the $A_{s-0}$ and $B_{s-0}$ coefficients into the integral for this region and using the Sommerfield's identity (Equation A.3-8) we obtain:

$$
\begin{aligned}
& \mathrm{H}_{\mathrm{zS}-0}=\mathrm{H}_{\mathrm{d}}\left(\mathrm{k}_{\mathrm{s}} \mathrm{z}_{\mathrm{s}}-\mathrm{z}\right)
\end{aligned}
$$

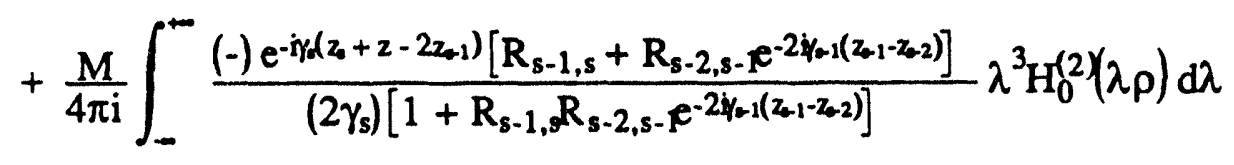

The expression under the integral sign can be further simplified by using the relation:

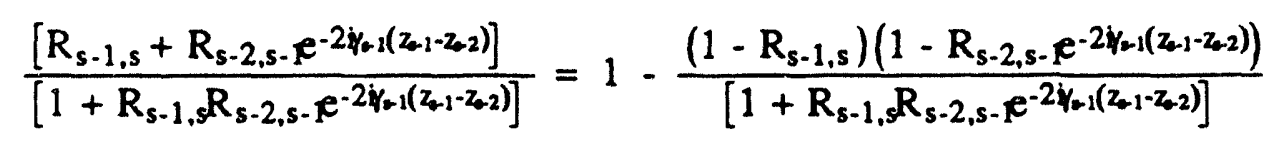

then,

$$
\begin{aligned}
& H_{z s+0}=H_{d}\left(k_{s s} z_{s}-z\right)-H_{d}\left(k_{s} z_{s}+z-2 z_{s-1}\right)
\end{aligned}
$$

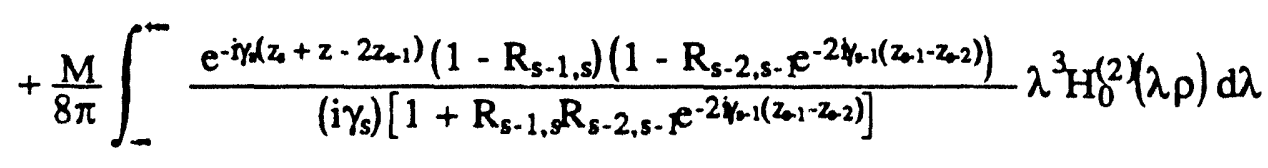




\section{A.5 Transmitter inside the layer}

A vertical magnetic dipole of moment $M$ placed inside a layer at depth $z=z_{3}$. The first interface above the transmitter is located at $z=z_{s-1}$. The first interface below the transmitter is located at depth $z=z_{s+1}$. The layer thickness is $z_{s+1}-z_{s-1}$. Figure A.5-1 gives the geometry of the problem.

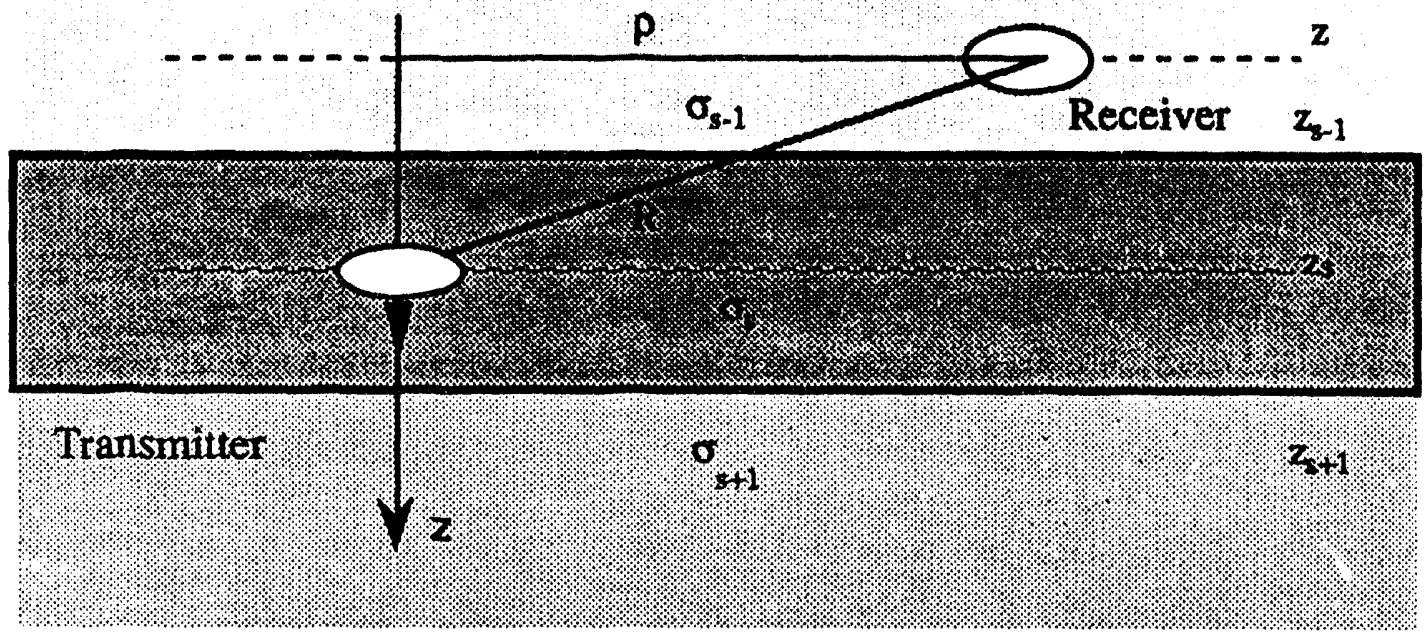

Fig. A.5-1. Configuration for transmitter inside a layer.

The solution is obtained by matching the source and boundary conditions in four regions: below the layer where $z \geq z_{s+1}$, inside the layer below the source where $z_{s} \leq z<$ $z_{s+1}$, inside the layer above the transmitter where $z_{s-1} \leq z<z_{s}$, and above the layer where $z_{s-1}>z$. The solutions to wave equation in this four regions have the forms:

for $z_{s-1}>z$,

$$
\left.\Pi_{z s-1}^{*}=\int_{0}^{-} B_{s-1} e^{+\dot{y_{-1}-2}} J d \lambda \rho\right) d \lambda,
$$

for $z_{s-1} \leq z<z_{s}$

$$
\left.\Pi_{z s-0}^{*}=\int_{0}^{\infty}\left(A_{s-0} e^{-i \gamma_{0} z}+B_{s-0} e^{+i_{2} z}\right) \lambda J d \lambda \rho\right) d \lambda,
$$

for $z_{s} \leq z<z_{s+1}$

$$
\left.\Pi_{z s+0}^{*}=\int_{0}^{\infty}\left(A_{s+0} \mathrm{e}^{-\dot{\gamma} z z}+B_{s+0} \mathrm{e}^{+\dot{\gamma} \gamma z z}\right) \lambda \mathrm{J} d \lambda \rho\right) \mathrm{d} \lambda,
$$


224

for $z \geq z_{8+1}$,

$$
\Pi_{z s+1}^{*}=\int_{0}^{\infty} A_{s+1} e^{-i b_{s+1} z \lambda J} J(\lambda \rho) d \lambda .
$$

where: $\gamma_{s}=\sqrt{k_{s}^{2}-\lambda^{2}}, \gamma_{s-1}=\sqrt{k_{s-1}^{2}-\lambda^{2}}, \gamma_{s+1}=\sqrt{k_{s+1}^{2}-\lambda^{2}}$. The subscript "s-0" describes the medium in which the source is locked, and above the transmitter, subscript. "st" describes the same medium below the transmitter, subscript "ss" describes the medium above the first boundary above the transmitter , " $8+1$ " describes the medium below the first boundary below the source.

Matching boundary and source conditions leads to the coefficients inside the integrals that are as follow:

$$
\begin{aligned}
& B_{s-1}=\frac{M}{2 \pi i} \frac{\left[1+R_{s, s+1} e^{\left.-2 i \gamma_{s}\left(z_{s+1}-z_{s}\right)\right]} e^{-i \gamma_{(}\left(z_{s}-z_{s-1}\right)\left(1-R_{s-1, s}\right) e^{-i \gamma_{s-1}\left(z_{s-1}\right)}}\right.}{2 \gamma_{s}\left[1+R_{s, s+1} R_{s-1, s} e^{\left.-2 i \gamma_{(}\left(z_{s+1}-z_{s+1}\right)\right]}\right.} \\
& A_{s-0}=-\frac{M}{2 \pi i} \frac{\left[1+R_{s, s+1} e^{-2 i \gamma\left(z_{s+1}-z_{s}\right)}\right] R_{s-1, s} e^{-i \gamma\left(z_{s}-2 z_{s+1}\right)}}{2 \gamma_{s}\left[1+R_{s, s+1} R_{s-1, s} e^{-2 i \gamma_{s}\left(z_{s+1}-z_{s+1}\right)}\right]} \\
& B_{s-0}=\frac{M}{2 \pi i} \frac{\left[1+R_{s, s+1} e^{\left.-2 i \gamma_{d}\left(z_{s+1}-z_{s}\right)\right]} e^{-i \gamma_{(}\left(z_{s}\right)}\right.}{2 \gamma_{s}\left[1+R_{s, s+1} R_{s-1, s} e^{\left.-2 i \gamma_{(}\left(z_{s+1}-z_{s+1}\right)\right]}\right.} \\
& A_{s+0}=\frac{M}{2 \pi i} \frac{\left[1-R_{s-1, s} e^{\left.-2 i \gamma_{(}\left(z_{s}-z_{-1}\right)\right]} e^{+i \gamma\left(z_{s}\right)}\right.}{2 \gamma_{s}\left[1+R_{s, s+1} R_{s-1, s} e^{\left.-2 i \gamma_{s}\left(z_{s+1}-z_{s-1}\right)\right]}\right.} \\
& B_{s+0}=\frac{M}{2 \pi i} \frac{\left[1-R_{s-1, s} e^{-2 i \gamma\left(z_{s}-z_{s+1}\right)}\right] R_{s, s+1} e^{-i \gamma\left(2 z_{s+1}-z_{s}\right)}}{2 \gamma_{s}\left[1+R_{s, s+1} R_{s-1, s} e^{-2 i \gamma_{(}\left(z_{s+1}-z_{+1}\right)}\right]}
\end{aligned}
$$

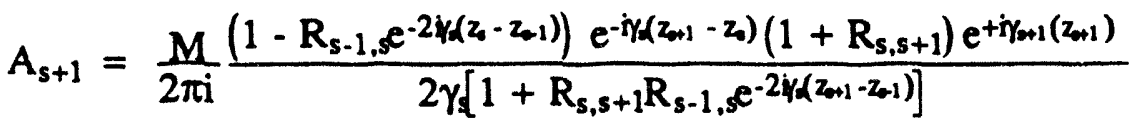

where

$$
R_{s-1, s}=\frac{\gamma_{s-1}-\gamma_{s}}{\gamma_{s-1}+\gamma_{s}}
$$

and

$$
R_{s, s+1}=\frac{\gamma_{s}-\gamma_{s+1}}{\gamma_{s}+\gamma_{s+1}}
$$




\section{Appendix B}

\section{Evaluation of the integralsby the method of steepest descent.}

This derivation follows the method given in Kauffman and Keller (1983). However the integrals evaluated here are simpler because the integral

$$
I=\int_{0}^{\infty} \frac{\lambda^{n} e^{-i \gamma_{z}}}{i \gamma} \frac{\left(\gamma_{s}-\gamma_{s+1}\right)}{\left(\gamma_{s}+\gamma_{s+1}\right)} J_{0}(\lambda \rho) d \lambda
$$

can be converted to:

$$
-\frac{M}{4 \pi} \frac{e-k_{G} R_{1}}{R_{1}}-\frac{2}{k^{2}-k_{k+1}^{2}} \frac{M}{4 \pi}\left[\frac{\partial^{2}}{\partial z_{1}^{2}} \frac{e-k_{1} R_{1}}{R_{1}}-\int_{0}^{-} \lambda\left(i \gamma_{s+1}\right) e^{-i \gamma_{j 4}} J_{d}(\lambda \rho) d \lambda\right]
$$

if $n=1$, or to:

$$
\left.-H_{d}\left(k_{s} R_{1}\right)-\frac{2}{k_{s}^{2}-k_{s+1}^{2}} \frac{M}{4 \pi}\left[\frac{\partial^{2}}{\partial z_{1}^{2}} H_{d}\left(k_{s} R_{1}\right)-\frac{M}{4 \pi} \int_{0}^{-} \lambda^{3}\left(i \gamma_{s+1}\right) e^{-i \gamma_{s i}} J d \lambda \rho\right) d \lambda\right]
$$

if $n=3$ following derivations in Appendix A using equations (A.3-8), (A.3-9) and (A.311). As a consequence of this simplification the expansion of the integrand into a power series as described in Kauffman and Keller (1983) was unnecessary. Let's consider the integral:

$$
I_{1}=\int_{0}^{-} \lambda^{n} i \gamma_{s+1} e^{-i \gamma_{2} z_{1}} J_{0}(\lambda \rho) d \lambda
$$

The value of the integral will not change if we deform the path of integration into the complex plane taking care of all branch points. The branch points of the integrand are located at $\lambda= \pm k_{s}$ and $\lambda= \pm k_{s+1}$, but the radiation condition implies that the $\operatorname{Im}\left(k_{s}\right)<0$ and $\operatorname{Im}\left(k_{s+1}\right)<0$, so only the points with negative imaginary parts have to be considered.

If we change the limits of integration from 0 to $\infty$ to $-\infty$ to to by applying the relations: 


$$
J_{d}(\lambda \rho)=\frac{1}{2}\left[H_{0}^{(2)}\left(\lambda_{\rho}\right)+H_{0}^{(1)}\left(\lambda_{\rho}\right)\right],
$$

and

$$
H_{0}^{(2)}(\lambda \rho)=-H_{0}^{(1)}(-\lambda \rho)
$$

then this integral takes a form:

$$
I_{1}=\frac{1}{2} \int_{-}^{+\infty} \lambda^{n} i \gamma_{s+1} e^{-i} \gamma_{z} H_{0}^{(2)}(\lambda \rho) d \lambda
$$

The $H_{0}^{(2)}\left(\lambda_{\rho}\right)$ was chosen over $H_{\delta}^{(1)}\left(\lambda_{\rho}\right)$ because $H_{0}^{(2)}\left(\lambda_{\rho}\right) \rightarrow 0$ if $\left|\lambda_{\rho}\right| \rightarrow \infty$ in the sector where $-\pi<\arg \lambda \rho<0$ and this is implied by radiation condition (Abramovitz, 1980).

In the complex plane the deformed integration path is given in Figure B-1.

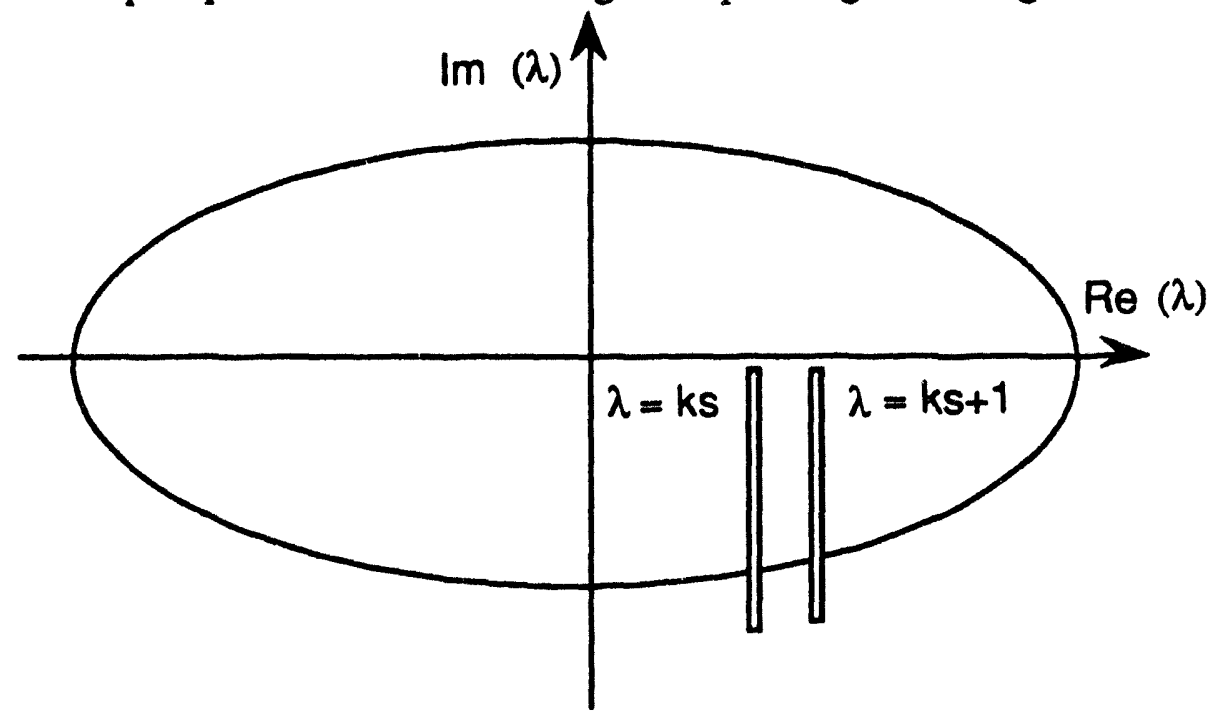

Fig. B-1. Deformation of the contour of integration in the complex plane

When the path of integration is as in Figure B.1, then the integral is given by: 


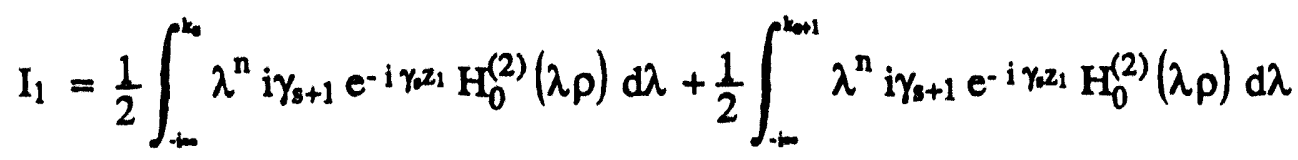

and the integration takes place on both sides of the branch points.

Let's assume as in Kauffman and Keller (1983) that the main contribution to the integral comes from the integrand near the branch points. This is true if all terns involved decay exponentially with increasing distance of $\lambda$ to the branch points.

The term $e^{-\gamma_{2} z_{1}}$ indeed decreases exponentially when $\lambda \neq \pm k_{8}$, but in addition we must ensure the exponential decay of $\mathrm{H}_{0}(2)(\lambda r)$, which happens at large arguments $\lambda \rho$. So our asymptotic expansions are valid in the far zone where the distance between the transmitter and receiver is greater than the skin depth in the medium. With the change of integration variable $\lambda, \mathrm{H}_{0}{ }^{(2)}(\lambda r)$ is the largest where it's argument $(\lambda \rho)$ is the smallest. So $\mathrm{H}_{0}{ }^{(2)}(\lambda \rho)$ should have a large contribution to the integral at small values of $\lambda$. But from Figure B.1 it can be seen, that the smallest $\lambda$ on the integration path around the branch point from -ios to $k_{s}$ or $k_{s+1}$ is at $\lambda=k_{s}$ and at $\lambda=k_{s+1}$. So the largest contribution to the integral occurs at branch points and decays exponentially away from this point.

In consequence, for integration around $k_{s+1}$ we have the following approximate values: $g_{s} \approx\left(k_{s}{ }^{2}-k_{s+1}{ }^{2}\right)^{1 / 2}$ and $\gamma_{s+1}$ is very small.

For integration around $k_{s+1}$ we have: $\gamma_{s+1}=\left(k_{s+1}{ }^{2}-k_{s}^{2}\right)^{1 / 2}$ and $\gamma_{s}$ is very small. The integral becomes:

$I_{1}=\frac{1}{2} \int_{-\infty}^{k_{0+1}} \lambda^{n} i \gamma_{s+1} e^{-i \sqrt{k_{s}^{2}-k_{s+1}^{2}} z_{1}} H_{0}^{(2)}(\lambda \rho) d \lambda+\frac{1}{2} \int_{-\infty}^{k_{\infty}} \lambda^{n} i \sqrt{L_{s+1}^{2}-k_{s}^{2}} e^{-i} \gamma_{s} z_{1} \quad H_{0}^{(2)}(\lambda \rho) d \lambda$

After taking $i \sqrt{k_{0+1}^{2}-k_{0}^{2}}$ and $e^{-i \sqrt{k_{1}^{2}-k_{0+1}^{2}}} z_{1}$ outside the integrals and using the relations C.2-4 and C.2-3 from the Appendix $C$ for the first and second integrals respectively, the final asymptotic value of the integral is given by:

$$
I_{1} \frac{M}{4 \pi} \approx e^{-i \sqrt{k_{s}^{2}-k_{s+1}^{2}} z_{1}} \frac{\partial^{2}}{\partial z^{2}} H_{z}\left(k_{s+1}, R\right) I_{z=0}-i \sqrt{k_{s+1}^{2}-k_{s}^{2}} \frac{\partial}{\partial z_{1}} H_{z}\left(k_{s}, R_{1}\right) .
$$


In the above final form we assumed that $n=3$. If $n=1$, then the same relations hold, but instead of $H_{z}\left(k_{8}, R\right)$ whole space fields we have $\frac{e^{-k_{0+1} R}}{R}$ terms [from equations (C.2-1) and (C.2-2) in Appendix C]:

$$
\left.I_{1} \approx e^{-i \sqrt{k_{s}^{2}-k_{+1}^{2}}} z_{1} \frac{\partial^{2}}{\partial z^{2}} \frac{e^{-i k_{s+1} R}}{R}\right|_{z=0}-i \sqrt{k_{s+1}^{2}-k_{s}^{2}} \frac{\partial}{\partial z_{1}} \frac{e^{-i k_{s} R_{1}}}{R_{1}}
$$

The integrals given below are estimated by the same procedure. And thus:

$$
\begin{aligned}
& I_{2}=\frac{1}{2} \int_{-}^{-} \lambda\left(i \gamma_{s+1}\right)\left(i \gamma_{s}\right) e^{-i \gamma_{s+1} z_{s}} e^{-i \gamma_{\alpha z}} H_{0}^{(2)}(\lambda \rho) d \lambda \approx \\
& \frac{1}{2} \int_{-\infty}^{k_{s}} \lambda i \sqrt{k_{s+1}^{2}-k_{s}^{2}}\left(i \gamma_{s}\right) e^{-i \sqrt{k_{s+1}^{2}-k_{k_{3}^{2}}^{2}}} e^{-i \gamma_{\sigma_{2}}} \mathrm{H}_{0}^{(2)}(\lambda \rho) \mathrm{d} \lambda+ \\
& \frac{1}{2} \int_{-\infty}^{k_{s+1}} \lambda\left(\mathrm{i} \gamma_{s+1}\right) i \sqrt{\mathrm{k}_{s}^{2}-\mathrm{k}_{s+1}^{2}} \mathrm{e}^{-\mathrm{i} \gamma_{s+1} z_{2}} \mathrm{e}^{-\mathrm{i} \sqrt{\mathrm{k}_{s}^{2}-k^{2}+1} \mathrm{z}_{3}} \mathrm{H}_{0}^{(2)}(\lambda \rho) \mathrm{d} \lambda \approx \\
& i \sqrt{k_{s+1}^{2}-k_{s}^{2}} e^{-i \sqrt{k_{s+1}^{2}-k_{2}^{2} z_{3}}} \frac{\partial^{2}}{\partial z_{2}^{2}} \frac{e^{-k_{s} R_{2}}}{R_{2}}+i \sqrt{k_{s}^{2}-k_{s+1}^{2}} e^{-i \sqrt{k_{s}^{2}-k_{+1}^{2} z_{2}}} \frac{\partial^{2}}{\partial z_{3}^{2}} \frac{e^{-i k_{s+1} R_{3}}}{R_{3}} \\
& I_{3}=\frac{1}{2} \int_{-}^{-} \lambda \mathrm{e}^{-\mathrm{i} \gamma_{\alpha+1} z_{3}} \mathrm{e}^{-\mathrm{i} \gamma_{\alpha 2}} \mathrm{H}_{0}^{(2)}(\lambda \rho) \mathrm{d} \lambda=
\end{aligned}
$$

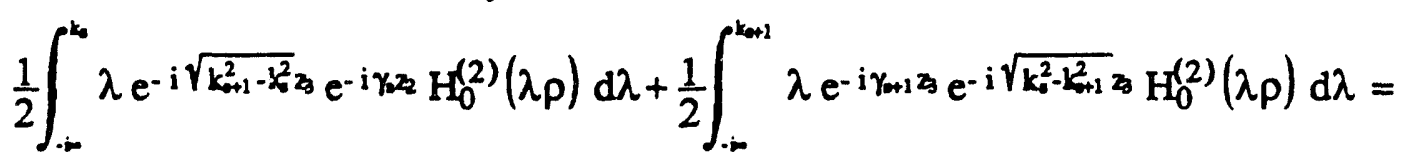

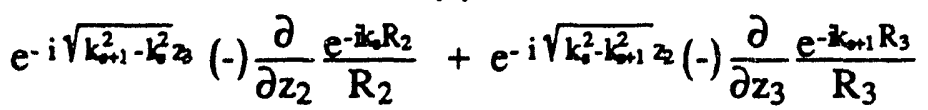

$$
\begin{aligned}
& \mathrm{I}_{4}=\frac{1}{2} \int_{-}^{-} \lambda \mathrm{e}^{-\mathrm{i} \gamma_{+1} z_{3}} \mathrm{e}^{-\mathrm{i} \gamma_{\alpha 2}} \mathrm{H}_{0}^{(2)}(\lambda \rho) \mathrm{d} \lambda \approx
\end{aligned}
$$

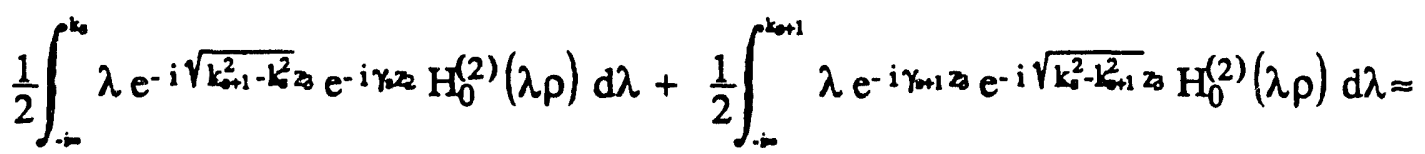

$$
\begin{aligned}
& e^{-i \sqrt{k_{0+1}^{2}-k_{2}^{2}}}(-) \frac{\partial}{\partial z_{2}} \frac{e^{-i k_{2} R_{2}}}{R_{2}}+e^{-i \sqrt{k_{2}^{2}-k_{2+1}^{2}}}(-) \frac{\partial}{\partial z_{3}} \frac{e^{-k_{0+1} R_{3}}}{R_{3}}
\end{aligned}
$$




$$
\begin{aligned}
& I_{4} \frac{M}{4 \pi}=\frac{M}{4 \pi} \frac{1}{2} \int_{-}^{\infty} \lambda^{3} e^{-i \gamma_{w+1} z_{3}} e^{-i \gamma_{2}} H_{0}^{(2)}(\lambda \rho) d \lambda \approx
\end{aligned}
$$

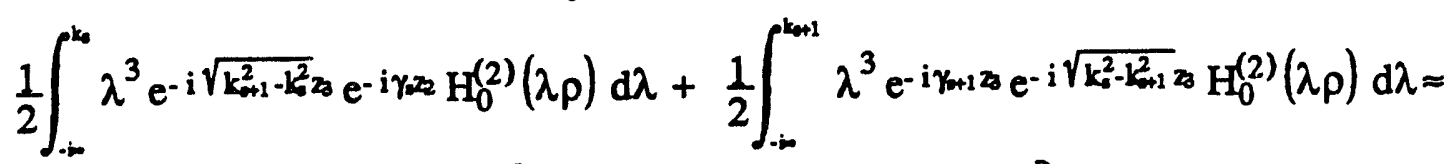

$$
\begin{aligned}
& e^{-i \sqrt{k_{s+1}^{2}-k_{s}^{2}} z_{3}}(-) \frac{\partial}{\partial z_{2}} H_{2}\left(k_{s} R_{2}\right)+e^{-i \sqrt{k_{s}^{2}-k_{s+1}^{2}} z_{2}}(-) \frac{\partial}{\partial z_{3}} H_{2}\left(k_{s+1}, R_{3}\right)
\end{aligned}
$$

$$
\begin{aligned}
& I_{5}=\frac{1}{2} \int_{-}^{-} \lambda\left(i \gamma_{s+1}\right)\left(i \gamma_{s}\right) e^{-i \gamma_{s} z} H_{0}^{(2)}(\lambda \rho) d \lambda \approx \\
& \frac{1}{2} \int_{-\infty}^{k_{s}} \lambda i \sqrt{k_{s+1}^{2}-k_{s}^{2}}\left(i \gamma_{s}\right) e^{-i \gamma_{s} H_{s}} \mathrm{H}_{0}^{(2)}(\lambda \rho) \mathrm{d} \lambda+ \\
& \frac{1}{2} \int_{-\infty}^{k_{s+1}} \lambda\left(i \gamma_{s+1}\right) i \sqrt{k_{s}^{2}-k_{s+1}^{2}} e^{-i \sqrt{k_{s}^{2}-k_{s+1}^{2}} z_{1}} H_{0}^{(2)}(\lambda \rho) d \lambda \approx \\
& i \sqrt{k_{s+1}^{2}-k_{s}^{2}} \frac{\partial^{2}}{\partial z_{1}} \frac{e^{-i k R_{1}}}{R_{1}}+\left.i \sqrt{k_{s}^{2}-k_{s+1}^{2}} e^{-i \sqrt{k_{s}^{2}-k_{s+1}^{2}} \frac{\partial^{2}}{\partial z}} \frac{e^{-i k_{s+1} R}}{R}\right|_{z=0}
\end{aligned}
$$

If in the above equations we have $\lambda^{3}$, instead of $\lambda$, then instead of the Hertz potentials in a whole space fields given by $e^{-i k_{s} R} / R$ terms we should use the whole space fields $\mathrm{H}_{\mathbf{z}}(\mathbf{k}, \mathbf{R})$. 


\section{Appendix C}

Summary of the expressions used in the derivations

\section{C.1 General expressions}

$$
\begin{aligned}
& \frac{\partial^{n+2}}{\partial z^{n+2}} \Pi_{z}^{*}=\frac{\partial^{n}}{\partial z^{n}} H_{z}-k^{2} \frac{\partial^{n}}{\partial z^{n}} \Pi_{z}^{*} \text { (Kaufman and Keller, 1983) } \\
& \frac{\partial^{2 n}}{\partial z^{2 n}} \frac{1}{R}=\frac{(-1)^{n}[(2 n-1) ! !]^{2}}{\rho^{2 n+1}} \quad \text { for } z=0
\end{aligned}
$$

(Kaufman and Keller,1983, p. 438)

$$
\begin{gathered}
\left.\int_{0}^{\infty} \frac{\lambda}{\gamma_{s} \gamma_{s+1}}\left(\frac{\gamma_{s}-\gamma_{s+1}}{\gamma_{s}+\gamma_{s+1}}\right)^{\nu} J d \lambda \rho\right) d \lambda=I V\left(\rho \frac{i k_{s}-i k_{s+1}}{2}\right) K_{V}\left(\rho \frac{i k_{s}+i k_{s+1}}{2}\right) \\
\text { with } \operatorname{Re}\left(i k_{s}\right)>\operatorname{Re}\left(i k_{s+1}\right) \geq 0 \\
\text { (Kaufman and } \operatorname{Keller,1983,~p.~415)~}
\end{gathered}
$$

$$
\int_{-\infty}^{k_{i}}\left(i \sqrt{k^{2}-\lambda^{2}}\right)^{n-1} e^{-i \sqrt{k^{2}-\lambda^{2}}} \mid \lambda \lambda H_{0}^{(2)}(\lambda \rho) d \lambda=2(-1)^{n} \frac{\partial^{n}}{\partial z^{n}} \frac{e^{-i k R}}{R}
$$

where $k_{i}$ is a branch point for which $\operatorname{Re}\left[i\left(k^{2}-\lambda^{2}\right)^{1 / 2}\right]>0$, (Kaufman and Keller,1983, p. 435) 
C.2 Integrals that can be reduced to whole space field values

$$
\begin{aligned}
& \Pi^{*}(k, R)=\frac{M}{4 \pi} \frac{e^{-i k R}}{R}=\frac{M}{4 \pi} \int_{0}^{\infty} \frac{\lambda e^{-i \sqrt{k^{2}-\lambda^{2}}|z|}}{i \sqrt{k^{2}-\lambda^{2}}} J_{0}(\lambda \rho) d \lambda \\
& =\frac{M}{8 \pi} \int_{-}^{-} \frac{\lambda e^{-i \sqrt{k^{2}-\lambda^{2}}|z|}}{i \sqrt{k^{2}-\lambda^{2}}} H_{0}^{(2)}(\lambda \rho) d \lambda \\
& H_{z}(k, R)=\frac{\partial^{2} \Pi^{*}(k, R)}{\partial z^{2}}+k^{2} \Pi^{*}(k, R)=-\frac{\partial^{2} \Pi^{*}(k, R)}{\partial \rho^{2}}-\frac{1}{\rho} \frac{\partial \Pi^{*}(k, R)}{\partial \rho} \\
& =\frac{M}{4 \pi} \int_{0}^{-} \frac{\lambda^{3} e^{-i \sqrt{k^{2}-\lambda^{2}}|\lambda|}}{i \sqrt{k^{2}-\lambda^{2}}} J_{0}(\lambda \rho) d \lambda \\
& =\frac{M}{4 \pi} \frac{e^{-i k R}}{R^{5}}\left[(1+i k R)\left(-\rho^{2}+2 z^{2}\right)+(i k R)^{2}\left(-\rho^{2}\right)\right] \\
& \frac{\partial}{\partial z} H_{z}(k, R)=-\frac{M}{4 \pi} \int_{0}^{-} \lambda^{3} e^{-i \sqrt{k^{2}-\lambda^{2}} \mid \lambda} J_{0}(\lambda \rho) d \lambda \\
& \frac{\partial^{2}}{\partial z^{2}} H_{z}(k, R)=\frac{M}{4 \pi} \int_{0}^{\infty} \lambda^{3}\left(i \sqrt{k^{2}-\lambda^{2}}\right) e^{-i \sqrt{k^{2}-\lambda^{2}}|d|} J_{0}(\lambda \rho) d \lambda \\
& \frac{\partial^{3}}{\partial z^{3}} H_{z}(k, R)=-\frac{M}{4 \pi} \int_{0}^{-} \lambda^{3}\left(i \sqrt{k^{2}-\lambda^{2}}\right)^{2} e^{-i \sqrt{k^{2}-\lambda^{2}}|z|} J_{0}(\lambda \rho) d \lambda \\
& -\left[k^{2} \frac{\partial H_{z}(k, R)}{\partial z}+\frac{\partial^{3} H_{z}(k, R)}{\partial z^{3}}\right]=\frac{M}{4 \pi} \int_{0}^{-} \lambda^{5} e^{-i \sqrt{k^{2}-\lambda^{2}}|z|} J_{0}(\lambda \rho) d \lambda
\end{aligned}
$$


C.3a Explicit expressions for whole space field: $H_{z}(k, R)$

$$
\begin{aligned}
& H_{d}(k, R)=\frac{M}{4 \pi} \frac{e^{-i k R}}{R^{5}}\left[(1+i k R)\left(-\rho^{2}+2 z^{2}\right)+(i k R)^{2}\left(-\rho^{2}\right)\right] \\
& \begin{aligned}
\frac{\partial H_{z}(k, R)}{\partial z}=\frac{M}{4 \pi} \frac{e^{-i k R}}{R^{7}}(z) & {\left[(1+i k R) 3\left(3 \rho^{2}-2 z^{2}\right)\right.} \\
& +(i k R)^{2} 2\left(2 \rho^{2}-z^{2}\right) \\
& \left.+(i k R)^{3}\left(\rho^{2}\right)\right]
\end{aligned}
\end{aligned}
$$

$$
\begin{aligned}
\frac{\partial^{2} \mathrm{H}_{2}(\mathrm{k}, \mathrm{R})}{\partial z^{2}}=\frac{M}{4 \pi} \frac{\mathrm{e}^{-i k R}}{\mathrm{R}^{9}} & {\left[(1+\mathrm{ikR}) 3\left(3 \rho^{4}-24 \rho^{2} \mathrm{z}^{2}+8 \mathrm{z}^{4}\right)\right.} \\
& +(\mathrm{ikR})^{2}\left(4 \rho^{4}-31 \rho^{2} z^{2}+10 z^{4}\right) \\
& +(\mathrm{ikR})^{3}\left(\rho^{4}-7 \rho^{2} z^{2}+2 z^{4}\right) \\
& \left.+(\mathrm{ikR})^{4}\left(-\rho^{2} z^{2}\right)\right]
\end{aligned}
$$

$$
\begin{aligned}
\frac{\partial^{3} H_{d}(k, R)}{\partial z^{3}}=\frac{M}{4 \pi} \frac{e^{-i k R}}{R^{11}}(z) & {\left[(1+i k R) 15\left(-15 \rho^{4}+40 p^{2} z^{2}-8 z^{4}\right)\right.} \\
& +(i k R)^{2} 3\left(-33 p^{4}+89 \rho^{2} z^{2}-18 z^{4}\right) \\
& +(i k R)^{3}\left(-24 \rho^{4}+67 \rho^{2} z^{2}-14 z^{4}\right) \\
& +(i k R)^{4}\left(-3 p^{4}+10 \rho^{2} z^{2}-2 z^{4}\right) \\
& \left.+(i k R)^{5}\left(+\rho^{2} z^{2}\right)\right]
\end{aligned}
$$

$$
\begin{aligned}
\frac{\partial^{4} H_{z}(k, R)}{\partial z^{4}}=\frac{M}{4 \pi} \frac{e^{-i k R}}{R^{13}} & {\left[(1+i k R) 45\left(-5 \rho^{6}+90 \rho^{4} z^{2}-120 \rho^{2} z^{4}+16 z^{6}\right)\right.} \\
& +(i k R)^{2} 3\left(-33 p^{6}+606 \rho^{4} z^{2}-824 \rho^{2} z^{4}+112 z^{6}\right) \\
& +(i k R)^{3} 12\left(-2 \rho^{6}+39 p^{4} z^{2}-56 \rho^{2} z^{4}+8 z^{6}\right) \\
& +(i k R)^{4} 3\left(-\rho^{6}+24 \rho^{4} z^{2}-39 \rho^{2} z^{4}+6 z^{6}\right) \\
& +(i k R)^{5}\left(6 p^{4} z^{2}-13 \rho^{2} z^{4}+2 z^{6}\right) \\
& \left.+(i k R)^{6}\left(-\rho^{2} z^{4}\right)\right]
\end{aligned}
$$

$$
\begin{aligned}
\frac{\partial H_{z}(k, R)}{\partial \rho}=\frac{M}{4 \pi} \frac{e^{-i k R}}{R^{7}}(\rho) & {\left[(1+i k R) 3\left(\rho^{2}-4 z^{2}\right)\right.} \\
& +(i k R)^{2} 2\left(\rho^{2}-2 z^{2}\right) \\
& \left.+(i k R)^{3}\left(\rho^{2}\right)\right]
\end{aligned}
$$


C.3b Explicit expressions for whole space field: $H_{\rho}(k, R)$

$$
\begin{aligned}
& \mathrm{H}_{\rho}(\mathrm{k}, \mathrm{R})=\frac{\mathrm{M}}{4 \pi} \frac{\mathrm{e}^{-k \mathrm{kR}}}{\mathrm{R}^{5}} \rho \mathrm{z}\left[3(1+\mathrm{ikR})+(\mathrm{ikR})^{2}\right] \\
& \begin{aligned}
\frac{\partial \mathrm{H}_{\rho}(\mathrm{k}, \mathrm{R})}{\partial \rho}=\frac{M}{4 \pi} \frac{e^{-i k R}}{\mathrm{R}^{7}}(\mathrm{z}) & {\left[(1+\mathrm{ikR}) 3\left(\mathrm{z}^{2}-4 \rho^{2}\right)\right.} \\
& +(\mathrm{ikR})^{2}\left(\mathrm{z}^{2}-5 \rho^{2}\right) \\
& \left.+(\mathrm{ikR})^{3}\left(-\rho^{2}\right)\right]
\end{aligned}
\end{aligned}
$$

$$
\begin{aligned}
\frac{\partial H_{\rho}(k, R)}{\partial z}=\frac{M}{4 \pi} \frac{e^{-i k R}}{R^{7}}(\rho) & {\left[(1+i k R) 3\left(\rho^{2}-4 z^{2}\right)\right.} \\
& +(i k R)^{2}\left(\rho^{2}-5 z^{2}\right) \\
& \left.+(i k R)^{3}\left(-z^{2}\right)\right]
\end{aligned}
$$

$$
\begin{aligned}
\frac{\partial^{2} H_{\rho}(k, R)}{\partial z^{2}}=\frac{M}{4 \pi} \frac{e^{-i k R}}{R^{9}} \rho z & {\left[(1+i k R) 15\left(-3 p^{2}+4 z^{2}\right)\right.} \\
& +(i k R)^{2} 9\left(-2 p^{2}+3 z^{2}\right) \\
& +(i k R)^{3}\left(-3 \rho^{2}+7 z^{2}\right) \\
& \left.+(i k R)^{4}\left(z^{2}\right)\right]
\end{aligned}
$$


C.4 Explicit expressions for whole space $\Pi^{*}{ }_{z}(k, R) /(M / 4 \pi)$

$\frac{\partial}{\partial z} \frac{e^{-i k R}}{R}=\frac{e^{-i k R}}{R^{3}}(-z)[1+i k R]$

$\frac{\partial^{2}}{\partial z^{2}} \frac{\mathrm{e}^{-i k R}}{R}=\frac{\mathrm{e}^{-i k R}}{\mathrm{R}^{5}}\left[(1+i k R)\left(-\rho^{2}+2 z^{2}\right)+(i k R)^{2}\left(z^{2}\right)\right]$

$\frac{\partial^{3}}{\partial z^{3}} \frac{e^{-i k R}}{R}=\frac{e^{-i k R}}{R^{7}}(z)\left[(1+i k R) 3\left(3 p^{2}-2 z^{2}\right)\right.$

$+(\mathrm{ikR})^{2} 3\left(\rho^{2}-\mathrm{z}^{2}\right)$

$\left.+(\mathrm{ikR})^{3}\left(-\mathrm{z}^{2}\right)\right]$

$\frac{\partial^{4}}{\partial z^{4}} \frac{e^{-i k R}}{R}=\frac{e^{-i k R}}{R^{9}}\left[(1+i k R) 3\left(3 p^{4}-24 p^{2} z^{2}+8 z^{4}\right)\right.$

$+(\mathrm{ikR})^{2} 3\left(\rho^{4}-10 \rho^{2} z^{2}+4 z^{4}\right)$

$+(\mathrm{ikR})^{3} 2\left(-3 p^{2} z^{2}+2 z^{4}\right)$

$\left.+(\mathrm{ikR})^{4}\left(z^{4}\right)\right]$

$$
\begin{aligned}
\frac{\partial^{5}}{\partial z^{5}} \frac{e^{-i k R}}{R}=\frac{e^{-i k R}}{R^{11}}(z)[ & (1 \\
+i k R) & 15\left(-15 p^{4}+40 p^{2} z^{2}-8 z^{4}\right) \\
& +(i k R)^{2} 30\left(-3 p^{4}+9 p^{2} z^{2}-2 z^{4}\right) \\
& +(i k R)^{3} 5\left(-3 p^{4}+14 p^{2} z^{2}-4 z^{4}\right) \\
& +(i k R)^{4} 5\left(\quad 2 p^{2} z^{2}-z^{4}\right) \\
& \left.+(i k R)^{5}\left(-z^{4}\right)\right]
\end{aligned}
$$

$$
\begin{aligned}
\frac{\partial^{6}}{\partial z^{6}} \frac{e^{-i k R}}{R}=\frac{e^{-i k R}}{R^{13}}[( & 1 \\
& +(i k R) 45\left(-5 \rho^{6}+90 \rho 4 z^{2}-120 \rho^{2} z^{4}+16 z^{6}\right) \\
& +(i k R)^{2} 45\left(-2 \rho^{6}+39 \rho^{4} z^{2}-56 \rho^{2} z^{4}+8 z^{6}\right) \\
& +(i k R)^{3} 15\left(-\rho^{6}+27 \rho{ }^{4} z^{2}-48 \rho^{2} z^{4}+8 z^{6}\right) \\
& +(i k R)^{4} 15\left(++3 \rho^{4} z^{2}-9 \rho^{2} z^{4}+2 z^{6}\right) \\
& +(i k R)^{5} 3\left(-5 \rho^{2} z^{4}+2 z^{6}\right) \\
& \left.+(i k R)^{6} \quad\left(z^{6}\right)\right]
\end{aligned}
$$

